\title{
Large Block Test Final Report
}

W. Lin, S.C. Blair, D. Wilder, S. Carlson, J. Wagoner, L. DeLoach, G. Danko, A.L. Ramirez, K. Lee

\section{December 1, 2001}




\section{DISCLAIMER}

This document was prepared as an account of work sponsored by an agency of the United States Government. Neither the United States Government nor the University of California nor any of their employees, makes any warranty, express or implied, or assumes any legal liability or responsibility for the accuracy, completeness, or usefulness of any information, apparatus, product, or process disclosed, or represents that its use would not infringe privately owned rights. Reference herein to any specific commercial product, process, or service by trade name, trademark, manufacturer, or otherwise, does not necessarily constitute or imply its endorsement, recommendation, or favoring by the United States Government or the University of California. The views and opinions of authors expressed herein do not necessarily state or reflect those of the United States Government or the University of California, and shall not be used for advertising or product endorsement purposes.

This work was performed under the auspices of the U. S. Department of Energy by the University of California, Lawrence Livermore National Laboratory under Contract No. W-7405-Eng-48.

This report has been reproduced directly from the best available copy.

Available electronically at http://www.doe.gov/bridge

Available for a processing fee to U.S. Department of Energy and its contractors in paper from

U.S. Department of Energy Office of Scientific and Technical Information

P.O. Box 62

Oak Ridge, TN 37831-0062

Telephone: (865) 576-8401

Facsimile: (865) 576-5728

E-mail: reports@adonis.osti.gov

Available for the sale to the public from

U.S. Department of Commerce

National Technical Information Service 5285 Port Royal Road Springfield, VA 22161

Telephone: (800) 553-6847

Facsimile: (703) 605-6900

E-mail: orders@ntis.fedworld.gov

Online ordering: http://www.ntis.gov/ordering.htm

OR

Lawrence Livermore National Laboratory

Technical Information Department's Digital Library http://www.llnl.gov/tid/Library.html 


\title{
Large Block Test Final Report
}

\author{
by \\ Wunan Lin \\ Stephen C. Blair \\ Dale Wilder, Steve Carlson, Jeff Wagoner, Laura DeLoach, George Danko \\ (University of Nevada, Reno), Abelardo L. Ramirez, and Ken Lee
}

December 2001

\section{Lawrence Livermore National Laboratory \\ Livermore, California}




\section{DISCLAIMER}

This document was prepared as an account of work sponsored by an agency of the United States Government. Neither the United States

Government nor the University of California nor any of their employees, makes any warranty, express or implied, or assumes any legal liability or responsibility for the accuracy, completeness, or usefulness of any information, apparatus, product, or process disclosed, or represents that its use would not infringe privately owned rights. Reference herein to any specific commercial product, process, or service by trade name,

trademark, manufacturer, or otherwise, does not necessarily constitute or imply its endorsement, recommendation, or favoring by the United States Government or the University of California. The views and opinions of authors expressed herein do not necessarily state or reflect those of the United States Government or the University of Califormia, and shall not be used for advertising or product endorsement purposes.

This report has been reproduced

directly from the best available copy.

Available to DOE and DOE contractors from the Office of Scientific and Technical Information

P.O. Box 62, Oak Ridge, TN 37831

Prices available from (615) 576-8401, FTS 626-8401

Available to the public from the National Technical Information Service

U.S. Department of Commerce 5285 Port Royal Rd., Springfield, VA 22161

This is an informal report intended primarily for internal distribution.

The opinions and conclusions stated are those of the author and may or may not be those of the Laboratory.

This work was preformed under the auspices of the U.S. Department of Energy by University of California, LLNL under Contract W-7405-Eng-48. 


\section{QUALITY ASSURANCE INFORMATION}

This document was prepared under the Technical Work Plan for Nearfield Environment Thermal Analyses and Testing, TWP-NBS-TH-000001 REV 00 (CRWMS M\&O 2000d). The Large Block Test was conducted under the control document of Activity Plan, AP-LBT-01 (Lin 1994). This technical report was prepared in accordance with procedure AP 3.11Q, REV 2. In accordance with the Activity Evaluation for Work Package \#4301213NML, the Large Block Test Final Report is subject to the requirements of the Quality Assurance Requirements and Description Document (QARD). The specific requirements that apply to the report are described in OCRWM Administrative Procedure AP-3.11Q, Technical Products, and procedures referenced therein. 
THIS PAGE INTENTIONALLY LEFT BLANK 


\section{ACKNOWLEDGMENTS}

The authors gratefully acknowledge the assistance of Geoffrey S. Siemering, Marion Capobianco, Gina V. Kaiper and the Technical Productions Group of NAI in editing and preparing the manuscript, and of Paul Harding and Treva Carey for creation and preparation of the graphics. The following persons played important roles at various phases of the Large Block Test: Jim Blink, Tom Buscheck, Patricia Berge, Eric Carlberg, Richard Carlson, Joanne Horn, Annemarie Meike, Mark Owens, Ron Pletcher, Jeff Roberts, Dave Ruddle, Don Watwood, and Troy Williams. Bob Glass of Sandia National Laboratories conducted the fracture flow visualization test during the excavation phase of the LBT. Joe Wang of Lawrence Berkeley National Laboratory conducted pre-heating cross-hole air permeability measurements and tracer tests in the block. Work performed under the auspices of the U.S. Department of Energy by University of California, Lawrence Livermore National Laboratory, under contract No. W-7405Eng-48. 
THIS PAGE INTENTIONALLY LEFT BLANK 


\section{EXECUTIVE SUMMARY}

The Large Block Test (LBT) was one of the thermal field tests conducted by the Yucca Mountain Site Characterization Project (YMP) to support confidence in the building and testing of the coupled process models used in analysis of the feasibility of Yucca Mountain as a potential repository site of high-level nuclear waste. The main objective of the LBT was to conduct a controlled, one-dimensionally-heated thermal test so that its results could be readily modeled. Through the comparison with model calculations, the results of the LBT help build confidence in the models of the coupled thermal-hydrological-mechanical-chemical (THMC) processes. The LBT was also used to test the use of optical multiple-point borehole extensometers and to evaluate the measurement of in-situ thermal conductivity and thermal diffusivity through the use of a Rapid Estimation of K and Alpha (REKA) probe. The LBT was also used to test the survivability and migration of local microbes in a heated partially saturated rock mass. The goal of the LBT was to heat the block from within so that a dryout zone was created and so that the thermal-hydrological (TH) processes associated with the heating, including moisture movement and condensate refluxing, could be monitored.

Pre-test scoping calculations indicated that a $3 \times 3 \times 4.5-\mathrm{m}$ block of Topopah Spring tuff would be required to serve the purpose of the test. The pre-test scoping calculations also determined that a minimum bulk permeability of $10^{-15} \mathrm{~m}^{2}$ and a minimum initial water saturation of about $50 \%$ would be required. Fracture mapping, air permeability measured by single-hole injection, and moisture content measured by neutron logging determined that the non-lithophysal Topopah Spring tuff outcrop at Fran Ridge, Nevada, would provide a test block suitable for the purpose.

After vertical boreholes were drilled and the boundary of a $3 \times 3-\mathrm{m}$ column was cut by a belt saw, neutron logging was used to confirm that the cutting did not change the moisture content in the block significantly. The block was retained horizontally by $10.2-\mathrm{cm}$-wide trucker straps and wooden blocking. The rock adjacent to the block was excavated using a hydraulic jackhammer. Some of the small blocks were selected, labeled, and stored for future tests in the laboratory. The fractures in the block were then mapped on the exposed five surfaces, and horizontal instrument boreholes were drilled. The rock core from each hole was preserved and managed by the Sample Management Facility. A video log of each hole was obtained, and the fractures in the holes were mapped from those video logs. Fracture characterization in the block was performed by combining results of fracture mapping on the block surfaces with analysis of fractures in the boreholes.

Instruments were installed in the boreholes and on the block surfaces. One electrical resistance heater was installed in each of the five heater holes. The vertical block surfaces were covered with 0.5-cm-thick Ultratemp insulation panels with embedded temperature monitors to determine heat flux out of the block. The trucker straps and wooden blocking were then reinstalled. A weatherproof cover was installed over the outside of the entire block assembly. During the test, additional insulation was blown in between the Ultratemp and outer cover, thereby filling voids between the trucker straps and wooden blocks. Thus, the block surface was insulated with three layers of materials with blown-in insulation between the wooden blocks. The first layer was the 5-cm-thick Ultratemp layer. Next, there was a $7.5-\mathrm{cm}$-thick layer of fiberglass. Finally the block was wrapped with a layer of Reflectix. Construction activities are detailed in Section 3. 
The five heater holes formed a heater plane about $2.74 \mathrm{~m}$ from the top of the block. The power output from each heater was about $450 \mathrm{~W}$.

Temperatures at the top of the block were maintained at about $60^{\circ} \mathrm{C}$ by circulating fluid through a heat exchanger. Heat loss from the sides was maintained by the insulation. Temperature was measured within the block in two vertical holes (TT1 and TT2), four horizontal holes from the north face (NT1-NT4), and three horizontal holes from the west face (WT1-WT3). In each of these holes, electrical resistance temperature devices (RTD) were used to measure temperatures every $20 \mathrm{~cm}$. Temperatures at three locations in each of the five heater holes were measured by RTDs. The temperature holes were sealed with cement grout. The five heater holes were left open with a plug at the collar, so that the heaters could be replaced if necessary. The temperature on top of the block was measured by five RTDs mounted on an aluminum plate, and these measurements were used to control the heat exchanger to maintain the temperature at about $60^{\circ} \mathrm{C}$ on the top. On the vertical faces, the temperature was measured at 32 locations on the block surface and on both sides of the 1.2-cm-thick Ultratemp sheet insulation. The rock surface and Ultratemp surface temperatures were used to calculate the heat flux from the block.

Moisture content was measured along holes using neutron logging and in planes using electrical resistance tomography (ERT). There were 15 neutron holes: five vertical holes (TN1-TN5), six horizontal holes from the north face (NN1-NN6), and four horizontal holes from the west face (WN1-WN4). Each of the neutron holes were kept open with a Teflon liner. The annular space between the liner and the borehole wall was sealed with cement grout. ERT electrodes were mounted in the block and on the block surface such that two vertical and two horizontal imaging planes were formed. The two vertical planes dissected the block faces and intersected at the center of the block. The two horizontal ERT planes were located at 1.74 and $4.35 \mathrm{~m}$ from the block top, thus defining planes $1 \mathrm{~m}$ above and $1.61 \mathrm{~m}$ below the plane of the heaters.

Hydrological parameters (relative humidity, gas pressure, and temperature) were measured in four hydrological holes (one vertical [TH1], and three horizontal [NH1, WH1, and WH2]). Packers were used to seal the hydrological holes, and the sensors were mounted between the packers. The block deformation was monitored in boreholes and on the surface. Multiple-point borehole extensometers (MPBX), including an experimental optical extensometer, were used to monitor the block deformation in eight holes (two vertical ones [TM1, TM2] and six horizontal ones [NM1-NM3 and WM1-WM3]). Fracture gauges were used to monitor the deformation of specifically selected fractures on the block surface. A REKA probe was used to measure in-situ thermal conductivity and thermal diffusivity in three holes (TR1, WR1, and WR2).

The heaters were energized to their full power on February 28, 1997. The heating phase lasted more than one year, until March 10, 1998. The block was allowed to cool naturally without removing the insulation materials, from March 10, 1998, until the end of monitoring on September 30, 1998, when data acquisition ceased. Post-test drilling was conducted in 1999 to core selected portions of the block and to over-core one of the heater holes.

The discussion of temperature, thermal conductivity, and thermal diffusivity measurements is presented in Section 5. The greatest temperature measured in the block reached about $142^{\circ} \mathrm{C}$ at TT1-14. This RTD was about $5 \mathrm{~cm}$ below the heater plane. In general, a one-dimensional temperature distribution was achieved within the block. However, heterogeneity was evident. For 
example, TT1-14 showed that the temperature rose through the boiling point of water without showing the evidence of boiling, but TT2-14 showed a significant effect of boiling/rapid evaporation on the temperature. This is probably due to the combination of edge effects (deviation from one-dimensional heating) and heterogeneous hydrologic properties. The measured temperatures in those two vertical temperature holes showed the effects of TH process. Two such events were recorded: one on June 13, 1997, and the other on September 2, 1997. In both events, many of the measured temperatures appear to have been affected by flowing water along the holes. The temperatures tended to converge to the boiling point of water and remained at that level for an extended period of time, followed by high-frequency fluctuations attributed to condensate refluxing. There was evidence that the September 2, 1997, event was triggered by a heavy rainstorm, but there was no clear evidence that the June 13,1997, event was associated with rain. The temperature variations in those two events were sufficiently different to attribute the June 13, 1997, event to a sudden release of overheated water in the rock rather than to rainfall infiltration. In-situ thermal conductivity and thermal diffusivity, as measured by REKA probes, were consistent with the expected values for this rock, but no correlation between the thermal properties measured by a REKA probe and the TH processes indicated by the two TH events could be established. The measured temperatures in the block agreed well with that calculated by dual-permeability models using NUFT codes.

Section 6 presents the variation of the moisture content in the block as measured by neutron logging and ERT. Neutron logging provided accurate measurement of the moisture content along a borehole within a region of about $12 \mathrm{~cm}$ in radius around the borehole. ERT provided less accurate imaging of the moisture content but in cross sections of the entire block. Both measurements showed that a dry-out region about $1 \mathrm{~m}$ in thickness developed at the heater plane. As was the case with temperatures, the drying of the block was approximately one-dimensional, but deviation from the uniform one-dimensional drying was evident. The deviation was probably caused by the fractures. The rock in the dry-out zone was not totally dried. The neutron results showed residue moisture content of about $1 \%$ fraction volume. There was no significant rewetting in the block during the cool-down phase. The moisture might have left the rock top as steam/vapor, drained through the bottom, and/or been held in pores in the condensate zone. The measured moisture content agreed well with that calculated by dual-permeability models using NUFT codes.

As presented in Section 7, borehole extensometers and fracture gauges were used to monitor the deformation of the block. The results showed that fractures had significant effect on the deformation. The upper two-thirds of the block deformed differently than the lower one-third, likely reflecting the lack of rigid lateral restraint. The discrete element model of the LBT more accurately predicts the deformation behavior than does the continuum model. Not all fractures were active; the deformation was controlled by a subset of 6-10 major fractures. A coefficient of thermal expansion value of $5.27 \times 10^{-6} /{ }^{\circ} \mathrm{C}$ is appropriate for the LBT. This is consistent with the value determined for the Single Heater Test and with preliminary analysis of the Drift-Scale Test data. The transient response of the deformation is correlated more closely with temperature of the heaters than with temperature at the measurement point. This indicates that a mechanism translating force at a distance is active. Fracture gauge monitoring showed that fractures moved in association with the major thermal-hydrological events that occurred in the LBT. As also indicated in Section 7, there was no change between the pre-heat and the post-test mechanical 
properties. The assessment of the chemical process in the LBT depended on the comparison of the mineralogical composition of the pre-test cores and the post-test cores.

Section 8 shows that the mineralogical composition of the matrix of the block was not altered by the heating and the associated TH processes. This finding is consistent with that of the mechanical property, as shown in Section 7. There were no significant alterations in the minerals on the fracture surface either.

Section 9 presents the results of other measurements. Those include the observations in boreholes near the bottom of the block designated as observation holes (EO3, NO1, NO2, and WO5); measurements of relative humidity, temperature, and gas pressures in the packer holes (TH1, NH1, WH1, and WH2); and microbial survivability and migration. Observations conducted in the observation holes were considered scoping in nature and therefore should be treated as non-Q. The observations indicated that moisture moved downward from the heaters. The water left some discrete markers on the observation assemblies, but the marks were not sufficiently definitive to distinguish between fracture flow and condensation dripping. Microbe migration indicates that at least a portion of the water was from fracture flow. The gas pressure measurements did not produce consistent results. The temperature measured by Humicaps agreed well with that measured by RTDs. The variations in relative humidity measured by Humicaps were in agreement with the variation of moisture measured in the rock. None of those measurements registered the TH events shown by the temperature measurements by the RTDs. Local microbes were labeled to be double-drug-resistant and emplaced in the heater holes before the start of the heating. Samples collected in the observation holes, which were about $1.5 \mathrm{~m}$ below the heater plane, showed that the microbes had survived the heating and had migrated, by being transported by water flow fractures, at least to that distance.

Section 10 presents conclusions and summary of some specific findings of the LBT. The LBT met its objectives and provided adequate testing of coupled process models. The drying of the block and the movement of water by the heat agreed well with the conceptual model of onedimensional heating in a partially saturated rock mass. The measured temperature and moisture content in the LBT agreed well with model calculations. The LBT showed that condensate can reflux under the idealized conditions imposed on the block, as predicted. The LBT also indicates that the condensate could penetrate the boiling zone. Under conditions represented by the block, it has not been determined whether this conclusion would apply to the potential repository, where high percolation flux events would be moderated by the non-welded tuff above and where a much larger zone of heated rock might be present. TH models identify this as a possibility. There was no significant rewetting during the cool-down phase. The LBT found that the effect of fractures on the THM process was significant. The test seemed have no effect on the matrix of the rock mass. 


\section{CONTENTS}

EXECUTIVE SUMMARY ................................................................................................................... vii

ACRONYMS AND ABBREVIATIONS ............................................................................... xxvii

1. INTRODUCTION ............................................................................................................. 1-1

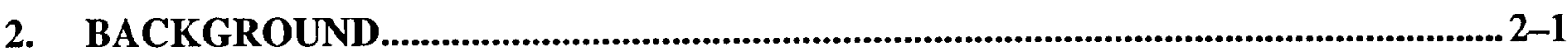

2.1 THE TESTING STRATEGY .............................................................................................................. 2-1

2.2. TEST SCALES ......................................................................................................................... 2-2

3. TEST DESCRIPTION......................................................................................................... 3-1

3.1 OBJECTIVES OF THE LARGE-BLOCK TEST .............................................................................. 3-1

3.1.1 Overall Objectives...................................................................................................................... $3-1$

3.1.2 Specific LBT Design and Implementation Objectives ................................................................... 3-2

3.2 CONFIGURATION OF THE LBT ......................................................................................................3-4

3.3 INSTRUMENTATION, MONITORING AND CHARACTERIZATION..................................3-6

3.3.1 Installation of Instrumentation and Data Collection Systems ............................................................ 3-8

3.4 MEASUREMENTS...........................................................................................................................................3-9

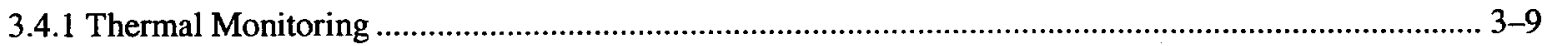

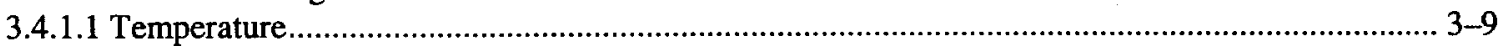

3.4.1.2 In-situ Thermal Conductivity and Thermal Diffusivity ………….......................................... 3-9

3.4.1.2.1 REKA Measurement and Evaluation Principles ………................................................ 3-9

3.4.1.2.2 The Measurement Arrangement...................................................................................... 3-10

3.4.2 Hydrological Monitoring................................................................................................................... 3-10

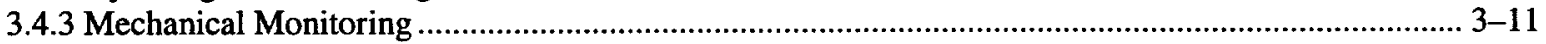

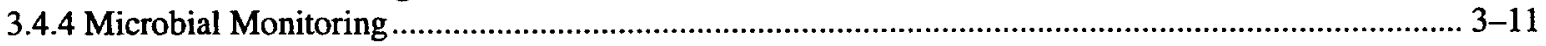

3.4.5 Assessment of Rock-Water Interaction .......................................................................................... 3-11

3.4.6 Other Monitoring.......................................................................................................................... 3-11

3.5 OVERVIEW OF CHRONOLOGICAL ACTIVITIES ...............................................................3-12

3.5.1 Block Preparation and Pre-test Characterization ........................................................................ 3-12

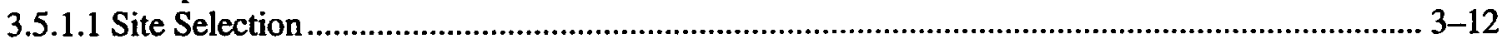

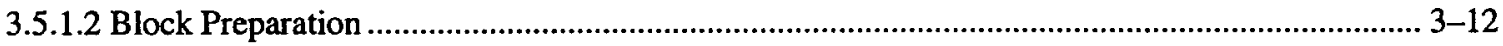

3.5.1.3 Block Characterization ………………....................................................................... 3-13

3.5.1.3.1 Laboratory-Determined Water Permeability of the Matrix.................................................. 3-14

3.5.1.3.2 Moisture Retention Curves ……………………………….......................................... 3-15

3.5.1.3.3 The Porosity and Micro-Pore Structure ……………………………………………... 3-17

3.5.1.3.4 Visualization of Fracture Flow Versus Matrix Imbibition Using X-ray Radiography.......... 3-17

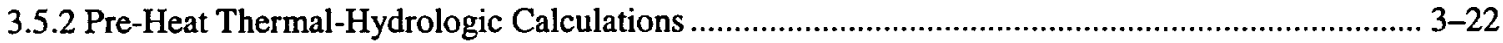

3.5.2.1 Homogeneous and Heterogeneous Cases....................................................................... 3-22

3.5.2.2 Thermal-Hydrologic Discrete Fracture Model...................................................................... 3-22

3.5.3 Pre-Heat Thermal-Mechanical Modeling ............................................................................... 3-23

3.5.3.1 Description of Problem......................................................................................................... 3-23

3.5.3.2 Description of Models ………………............................................................................... 3-23

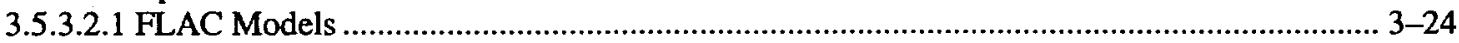

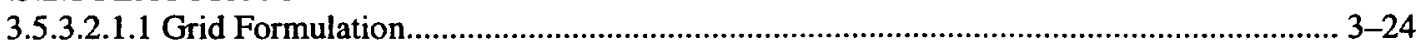

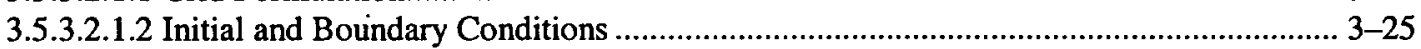

3.5.3.2.1.3 Heaters ............................................................................................................ 3-26 


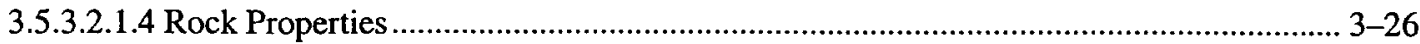

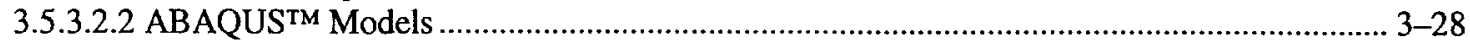

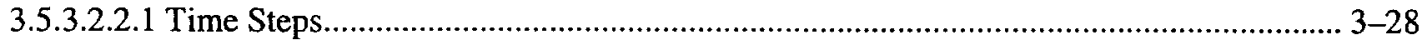

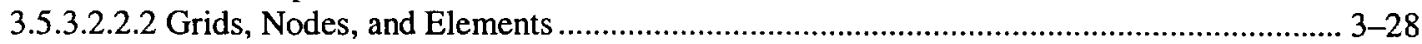

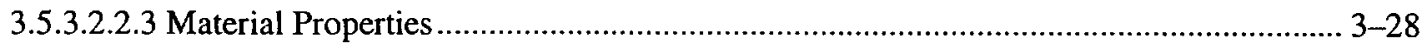

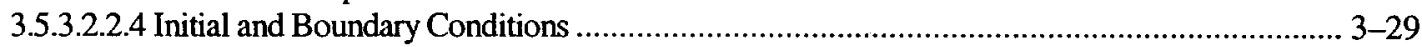

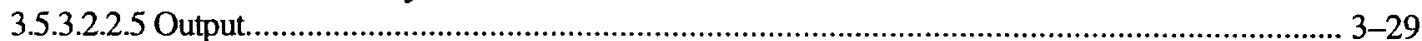

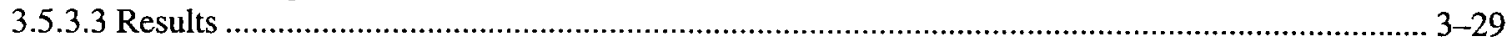

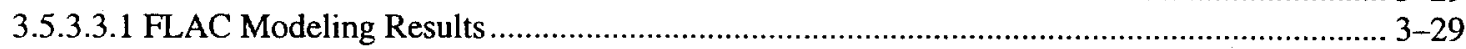

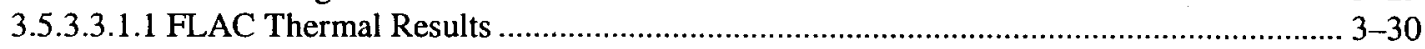

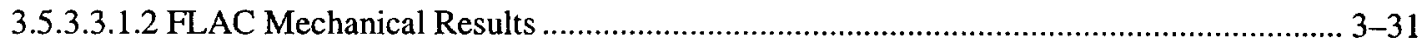

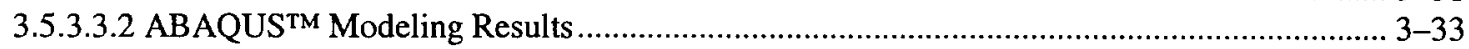

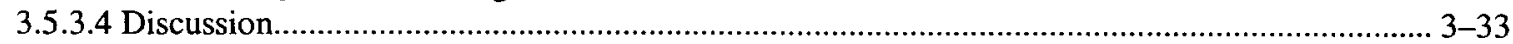

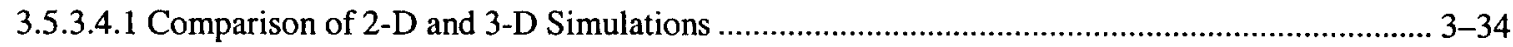

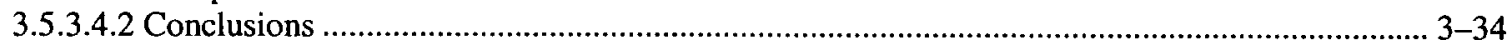

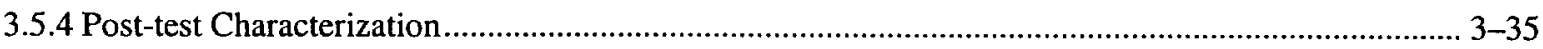

3.5.4.1 Boreholes for Post-test Characterization ................................................................................ 35

\section{DOCUMENTATION AND ANALYSIS OF FRACTURES IN THE LARGE}

BLOCK TEST .......................................................................................................................................4-1

5. THERMAL MEASUREMENTS..........................................................................................5-1

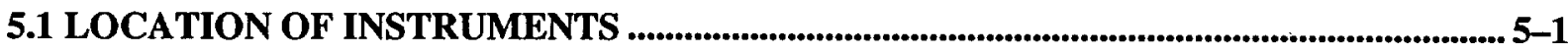

5.2 BASELINE DATA COLLECTION AND HEATER TURN-ON ............................................. 5-1

5.3 THE POWER OUTPUT FROM THE HEATERS ............................................................... 5-1

5.4 TEMPERATURE OBSERVATIONS ...................................................................................... 5-1

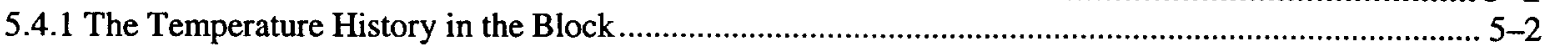

5.4.2 The Spatial Temperature Distribution in the Block ............................................................................

5.4.3 Temperature as an Indicator of Thermal-Hydrological Events ........................................................ 5-3

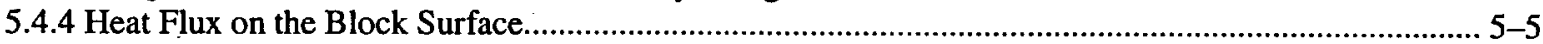

5.5 THERMAL CONDUCTIVITY AND THERMAL DIFFUSIVITY ........................................ 5-6

5.6 THERMAL-HYDROLOGICAL MODEL ANALYSES OF THE

TEMPERATURE DATA

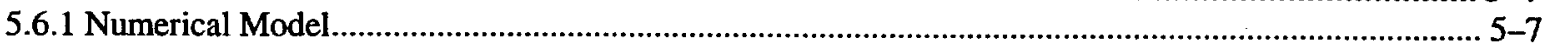

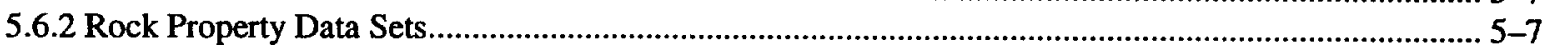

5.6.3 Drift-scale Simulation Results Versus Field Data ............................................................................ 5-8

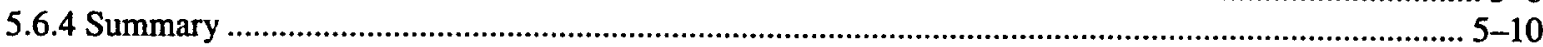

6. THERMAL HYDROLOGIC MEASUREMENTS AND ANALYSIS.............................6-1

6.1 THERMAL HYDROLOGIC MEASUREMENTS ................................................................................6-1

6.1.1 Bulk Air Permeability and Flow Path Measurements ....................................................................... 6-1

6.1.1.1 Pre-heating Permeability Measurements ................................................................................... 6-1

6.1.1.1.1 LLNL Single Hole Permeability Test .................................................................................. 6-1

6.1.1.1.2 Lawrence Berkeley National Laboratory Cross Hole .............................................................. 6-1

6.1.1.1.3 Lawrence Berkeley National Laboratory Trace Test ................................................................ 6-2

6.1.1.2 Post-Heating Air Permeability Measurement .............................................................................. 6-2

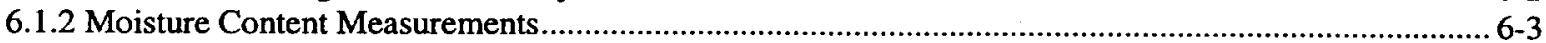

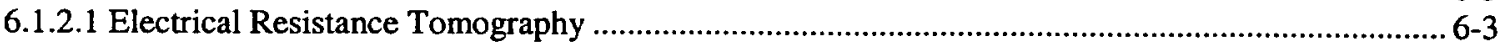

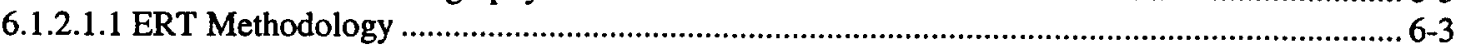

6.1.2.1.2 Changes in Moisture Content ......................................................................................... 6-4

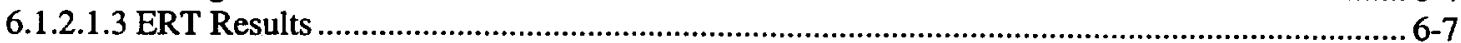

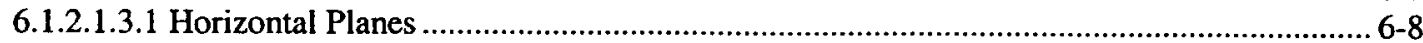


6.1.2.1.3.2 Vertical Planes ............................................................................................................... 6-9

6.1.2.1.4 Comparison of ERT with Other Data Sets ....................................................................... 6-10

6.1.2.1.4.1 Comparison of ERT and Fracture Distribution ......................................................... 6-10

6.1.2.1.4.2 Comparison of ERT and Neutron Logs.................................................................. 6-10

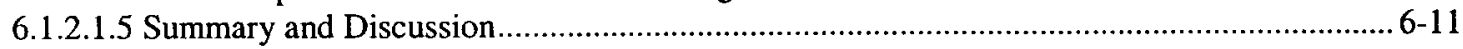

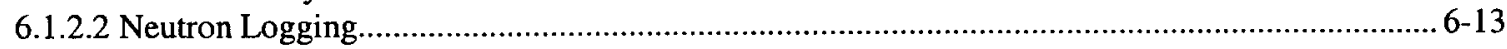

6.1 2.2.1 Pre-Cut and Post-Cut Moisture Contents ......................................................................... 6-13

6.1.2.2.2 Pre-Heat Baseline Moisture Content............................................................................ 6-13

6.1.2.2.3. Heating Phase and Cooling Phase Moisture Content..................................................... 6-14

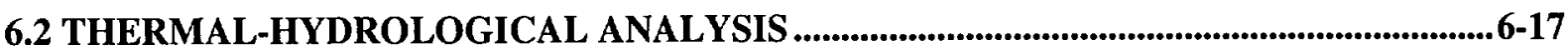

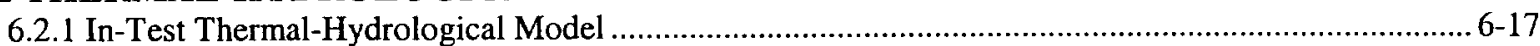

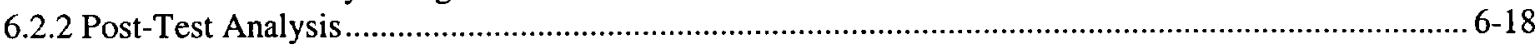

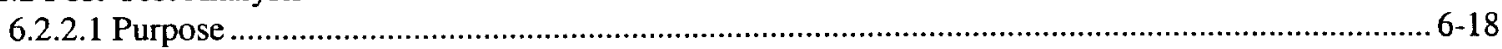

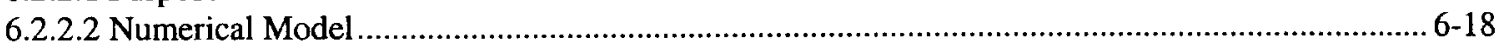

6.2.2.3 Rock Property Data Sets................................................................................................ 6-18

6.2.2.4 Drift-scale Simulation Results Versus Field Data .......................................................................... 6-19

6.2.2.6 Drift-scale Versus Mountain-scale Simulation Results ............................................................ 6-19

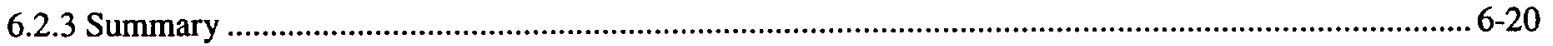

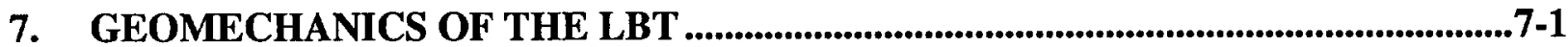

7.1 DEFORMATION MEASUREMENTS.............................................................................................7-1

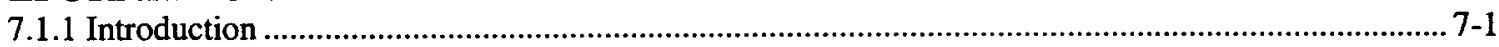

7.1.1.1 The First 100 Days ................................................................................................................. 7-1

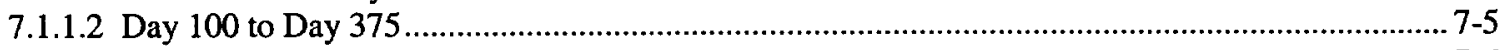

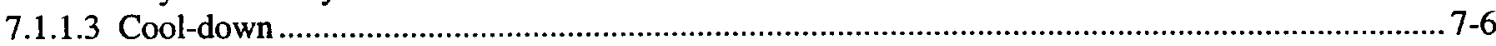

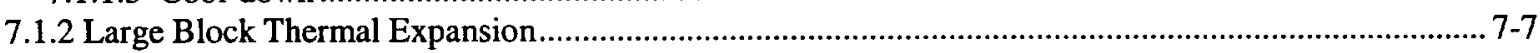

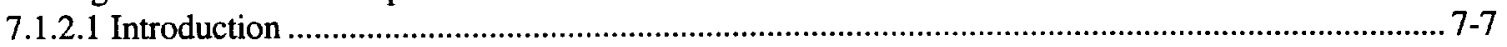

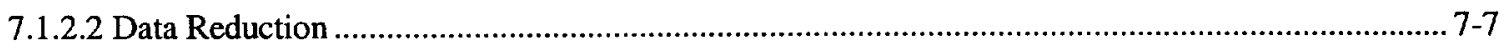

7.1.2.3 Results ...............................................................................................................................

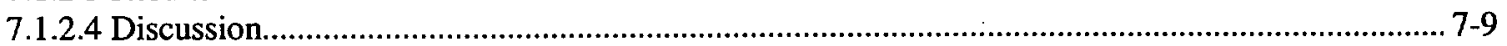

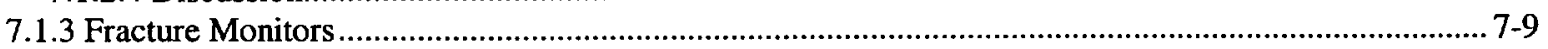

7.1.3.1 Deformation on Vertical Fractures ....................................................................................

7.1.3.2 Deformation of Horizontal Fractures ...................................................................................... 7-12

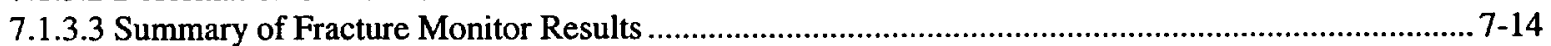

7.1.4 Discussion and Summary …......................................................................................................... 74

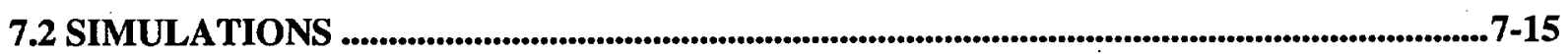

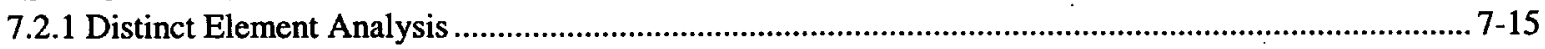

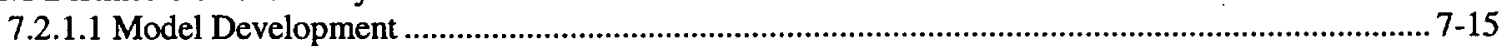

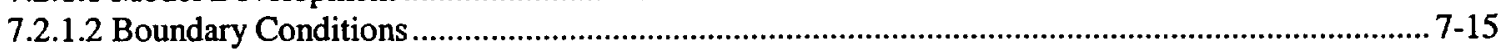

7.2.1.3 Temperature Field and Calculation Times............................................................................. 7-16

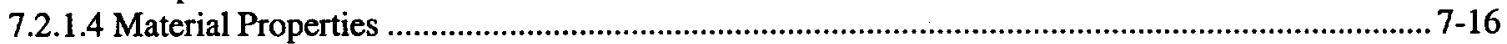

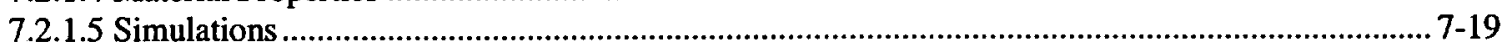

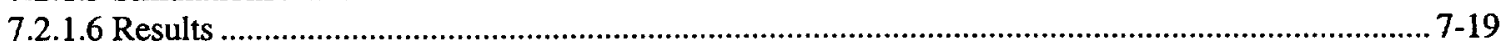

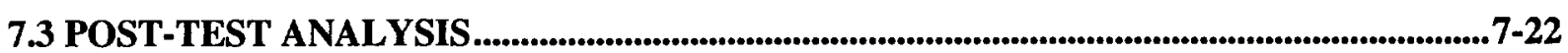

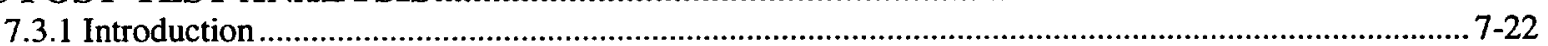

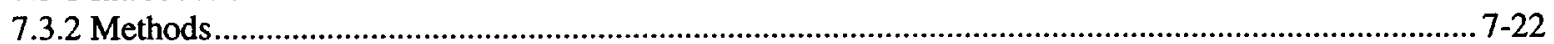

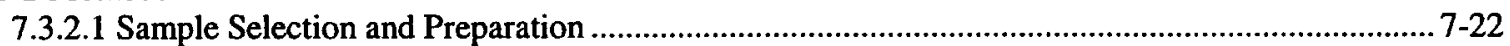

7.3.2.2. Ultrasonic Velocity Measurements............................................................................................. 7-23

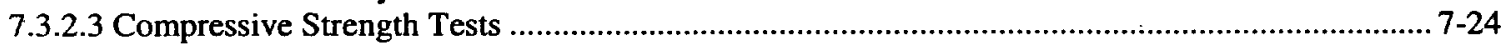

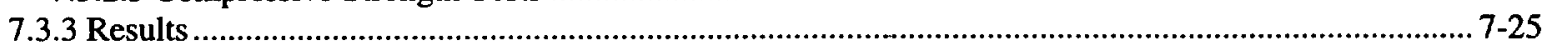

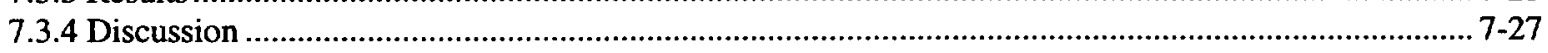

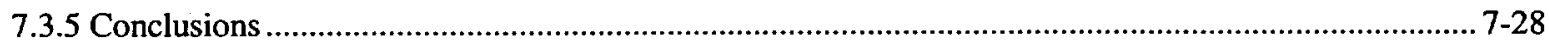


8. ROCK-WATER INTERACTION IN THE LBT...............................................................8-1

8.1 INTRODUCTION...................................................................................................................................... 8-1

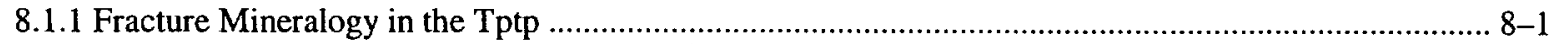

8.1.2 Test-Induced Geochemical Processes in the Large Block ................................................................. 8-2

8.2 EVALUATION OF ALTERATION IN THE THERMAL TEST BLOCK ........................... 8-2

8.2.1 Large Block Mineralogical Characterization ............................................................................... 8-2

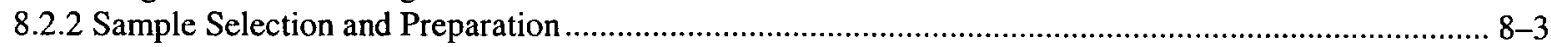

8.2.2.1 SEM and EDX Sample Preparation.........................................................................................

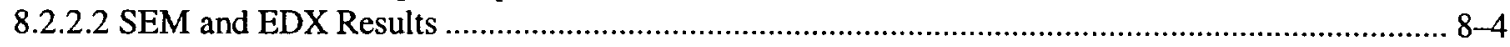

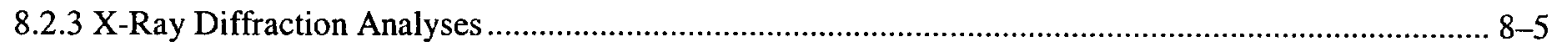

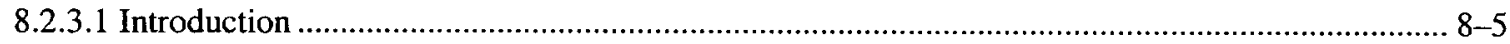

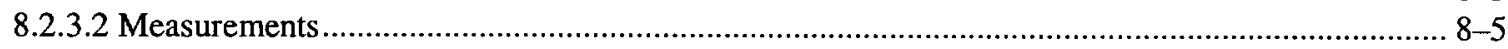

9. OTHER MEASUREMENTS AND OBSERVATIONS.....................................................9-1

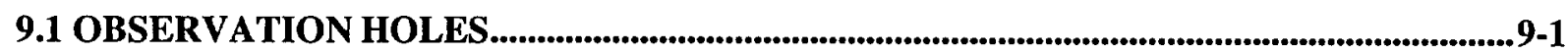

9.1.1 Construction of the Observation Assembly

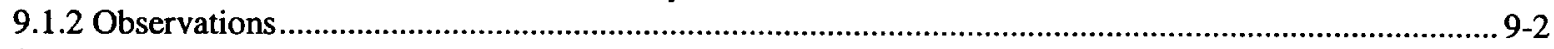

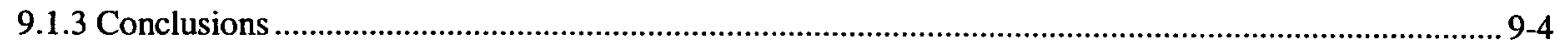

9.2 RELATIVE HUMIDITY AND TEMPERATURE RESULTS FROM HYDROLOGY BOREHOLES ..........................................................................................................................................................99-5

9.3 MICROBIAL SURVIVABILITY AND MIGRATION...............................................................................9-7

10. LARGE BLOCK TEST CONCLUSION AND FINDINGS...........................................10-1

10.1 GENERAL CONCLUSION................................................................................................................10-1

10.2 SPECIFIC FINDINGS............................................................................................................. 10-1

11. REFERENCES .......................................................................................................11-1

APPENDIX A: BOREHOLE LISTING AND INSTRUMENT TYPE BY BLOCK

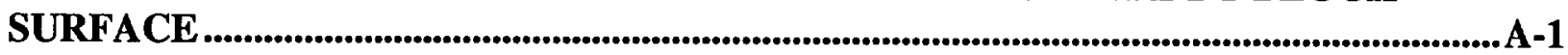

APPENDIX B: THE X-Y-Z COORDINATES (IN CM), WITH RESPECT TO THE SOUTHWESTERN CORNER ON TOP OF THE BLOCK, OF THE COLLAR (THE FIRST LINE) AND THE TOTAL DEPTH (THE SECOND LINE) OF ALL OF THE HOLES IN THE LBT. THE HOLE I.D. IN PARENTHESES IS THE CORRESPONDING INSTRUMENTATION HOLE AS LISTED IN APPENDIX A....... B-1 APPENDIX C: STRUCTURES DOCUMENTED IN THE BOREHOLE VIDEOS............C-1 


\section{FIGURES}

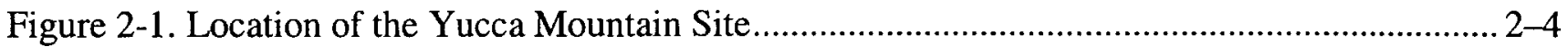

Figure 3-1. The Topopah Spring Tuff Block of the LBT........................................................ 3-37

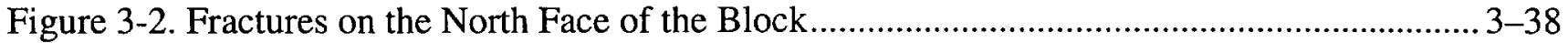

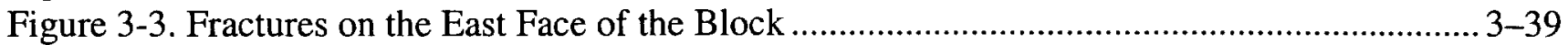

Figure 3-4. A Diagram Showing All Holes in the Block of the Large Block Test .......................3-40

Figure 3-5. Fractures on the South Face of the Block .......................................................... 3-41

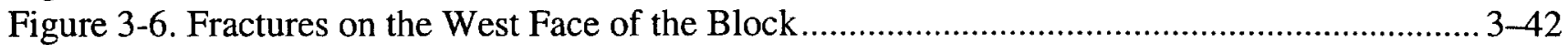

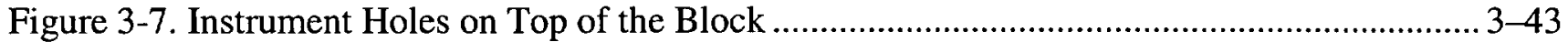

Figure 3-8. Instrument Holes on the North Face of the Block ................................................... 3-44

Figure 3-9. Instrument Holes on the East Face of the Block ..................................................... 3-45

Figure 3-10. Instrument Holes on the West Face of the Block ............................................... 3-46

Figure 3-11. Three REKA Probe Locations in the Large Heated Block.....................................3-47

Figure 3-12. Measurement Flow Chart for One Probe ............................................................ 3-48

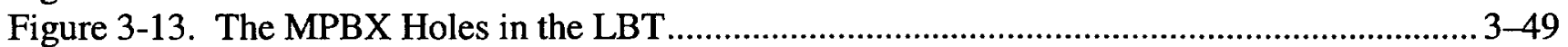

Figure 3-14. Initial Fracture Mapping at Fran Ridge ........................................................... 3-50

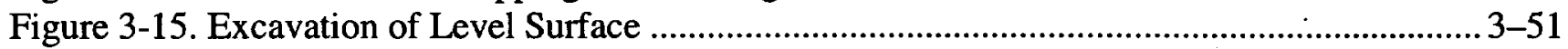

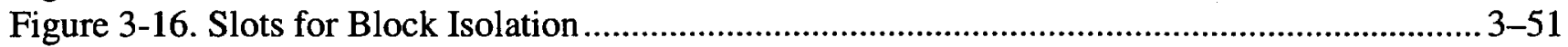

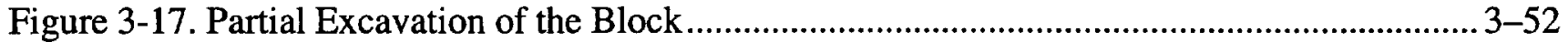

Figure 3-18. Drilling Rig Mounted on a Steel Platform to Drill Horizontal Boreholes .................. 3-53

Figure 3-19. Percent Saturation as a Function of Suction Potential at $20^{\circ} \mathrm{C}$ for Nine Topopah

Spring Tuff Samples During Wetting Phase ............................................................... 3-54

Figure 3-20. Percent Saturation as a Function of Suction Potential at $70^{\circ} \mathrm{C}$ for Six Topopah

Spring Tuff Samples During Drying Phase........................................................................ 3-55

Figure 3-21. Percent Saturation as a Function of Suction Potential at $70^{\circ} \mathrm{C}$ for Three Topopah

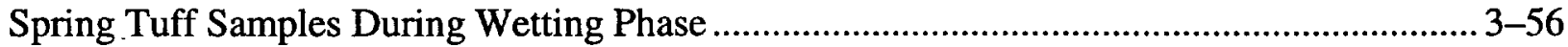

Figure 3-22. Percent Saturation as a Function of Suction Potential at $20^{\circ} \mathrm{C}$ for Three Topopah

Spring Tuff Samples During Drying Phase............................................................... 3-57

Figure 3-23. Schematic Diagram Showing the Set-up of Using X-ray Radiography to Investigate

Fracture Flow and Matrix Imbibition ...........................................................................5

Figure 3-24. Difference Images of Experiment 5 (a) and 6 (b) at 7.2 and 0.67 Hours Respectively

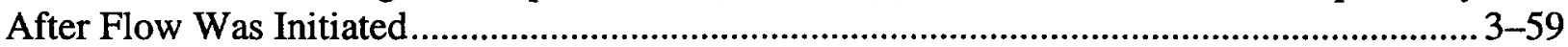

Figure 3-25. Schematic of Large Block Showing Locations of Fractures and Heater Boreholes .... 3-60

Figure 3-26. Predicted Temperature and Water Saturation Profiles Along a Vertical Line

Through the Matrix at One-year Heating .................................................................... 3-61

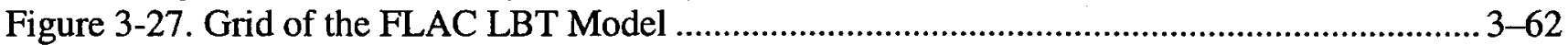

Figure 3-28. Temperature Contours in a Vertical Cross Section of the LBT After (a) 1 Day, (b)

10 Days, (c) 25 Days, (d) 50 Days, and (e) 75 Days of Heating, Calculated by FLAC Model 3-64

Figure 3-29. Temperature Profiles for (a) the Top and (b) Heater of the LBT, Calculated by FLAC

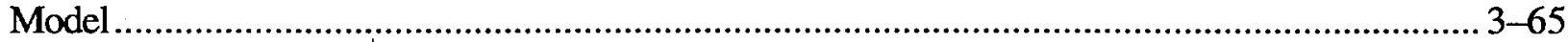

Figure 3-30. Temperature Near the Heater Horizon of the LBT Calculated by V-TOUGH

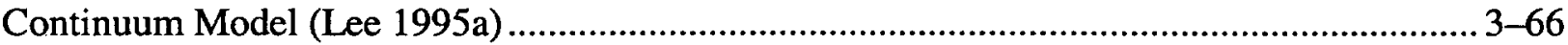


Figure 3-31. Displacements and Stresses Predicted by the First FLAC Model at 75 Days of Heating, for (a) Horizontal, x, Displacement; (b) Vertical, y, Displacement; (c) $\sigma_{\mathrm{XX}}$ Stress;

(d) $\sigma_{y y}$ Stress; and $(e) \sigma_{x y}$ Stress ........................................................................... 3-68

Figure 3-32. Model Configuration of the Third FLAC Model with One Horizontal Fracture and

One Vertical Fracture

Figure 3-33. Displacements and Stresses Predicted by the Third FLAC Model of the LBT at 75

Days of Heating, (a) Horizontal, x, Displacement; (b) Vertical, y, Displacement; (c) $\sigma_{\mathrm{Xx}}$

Stress; (d) $\sigma_{y y}$ Stress; and (e) $\sigma_{x y}$ Stress.

Figure 3-34. The (a) $\sigma_{x x}$ and (b) $\sigma_{y y}$ Contours in the LBT Predicted by the Fourth FLAC Model

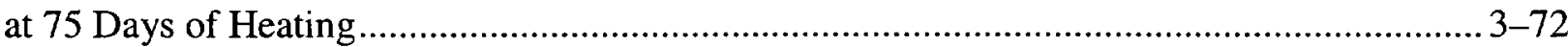

Figure 3-35. Temperature and Normal Stress Contours Predicted by ABAQUS Model at (a) 10,

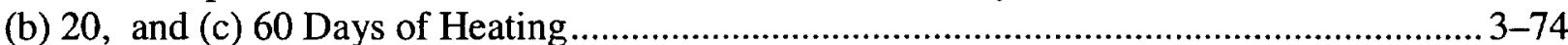

Figure 3-36. Displacements in the LBT Predicted by the ABAQUS Model at (a) 10, (b) 20, and

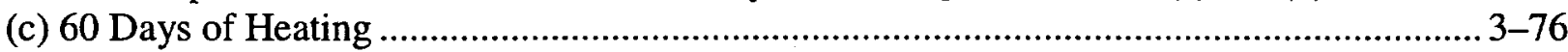

Figure 3-37. Post-test Coring Holes Drilled from the West Face of the Block ............................3-77

Figure 3-38. Post-test Coring Holes Drilled from the North Face of the Block ........................... 3-78

Figure 4-1. Mapped Surface Fractures on the Large Block ...................................................... 4-5

Figure 4-2. All Boreholes Drilled in the Large Block, Viewed at Various Angles ........................... 4-6

Figure 4-3. Equal-Area Diagram of Pole to the Major Fractures in the Large Block....................... 4-7

Figure 4-4. 3-D Depiction of the Major Mappable Fractures Cutting the Large Block ................... 4-8

Figure 4-5. Fracture System \#1 Contains Three Fractures That Strike N50E and Dip 40-45

Northwest

Figure 4-6. Fracture System \#2 Contains Two Fractures That Strike N30-40W and Dip 60-80

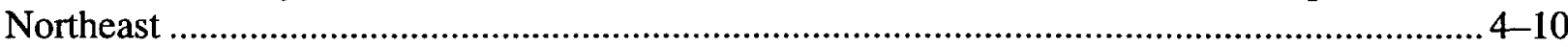

Figure 4-7. Fracture System \#3 Contains Six Subhorizontal Fractures ...................................... 4-11

Figure 4-8. Fracture System \#4 Contains 13 Mapped Fractures and Is the Dominant System in the Block $4-12$

Figure 4-9. Fracture System \#5 Contains Four Approximately Vertical Fractures That Strike East-West

Figure 4-10. Fracture System \#6 Contains Two Mappable Fractures That Strike Northeast and

Dip to the Southeast .................................................................................................... 4-14

Figure 4-11. Equal-area Net of Poles to the Major Fractures Mapped on Top of the Block........... 4-15

Figure 4-12. Equal-Area Contour Diagram of Poles to 90 Fractures Mapped in the Climax Stock

Granite

4-16

Figure 4-13. Fractures Mapped at the Fran Ridge Site Prior to Construction of the LBT (Square).4-17

Figure 4-14. Mapped Fractures on Top of the LBT ............................................................... 4-18

Figure 4-15. Mapped Fractures on the West Face of the LBT ................................................. 4-19

Figure 4-16. Mapped Fractures on the South Face of the LBT .................................................. 4-20

Figure 4-17. Mapped Fractures on the North Face of the LBT ............................................... 4-21

Figure 4-18. Mapped Fractures on the East Face of the LBT .................................................... 4-22

Figure 5-1. The Power Output of the Heater in EH1 as a Function of Time ...............................5-11

Figure 5-2. The Power Output of the Heater in EH2 as a Function of Time ................................. 5-11

Figure 5-3. The Power Output of the Heater in EH3 as a Function of Time ................................5-12

Figure 5-4. The Power Output of the Heater in EH4 as a Function of Time ................................ 5-12

Figure 5-5. The Power Output of the Heater in EH5 as a Function of Time ................................. 5-13 
Figure 5-6. Temperature at TT1-14 as a Function of Time .................................................... 5-13

Figure 5-7. Temperature at TT2-14 as a Function of Time .................................................... 5-14

Figure 5-8. Quarterly Temperature Snapshots in TT1 to Show the Spatial Temperature Variation 5-15

Figure 5-9. Quarterly Temperature Snapshots in TT2 to Show the Spatial Temperature Variation 5-16

Figure 5-10. Quarterly Temperature Snapshots in NT1 to Show the Spatial Temperature

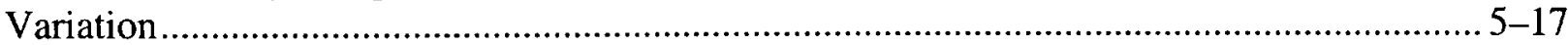

Figure 5-11. Quarterly Temperature Snapshots in NT2 ..................................................... 5-18

Figure 5-12. Quarterly Temperature Snapshots in NT3 to Show the Spatial Temperature

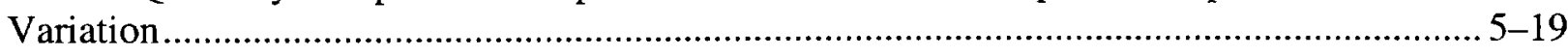

Figure 5-13. Quarterly Temperature Snapshots in NT4 to Show the Spatial Temperature

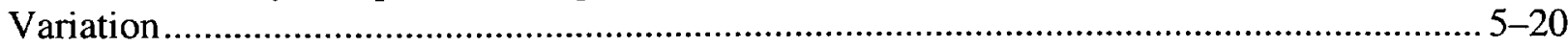

Figure 5-14. Quarterly Temperature Snapshots in WT1 to Show the Spatial Temperature

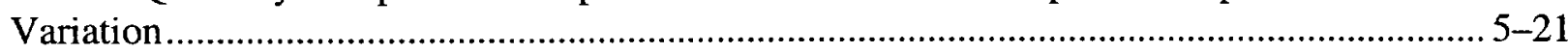

Figure 5-15. Quarterly Temperature Snapshots in WT2 to Show the Spatial Temperature

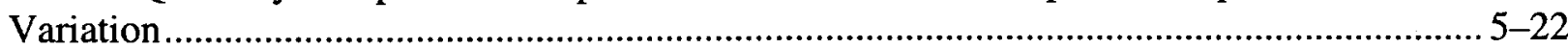

Figure 5-16. Quarterly Temperature Snapshots in WT3 to Show the Spatial Temperature

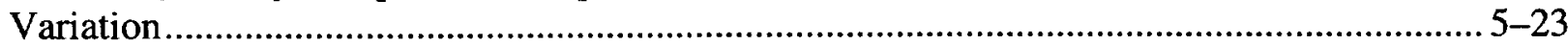

Figure 5-17. The Vertical Cross Section Along TT1 of the 3-D Temperature Field in the Block

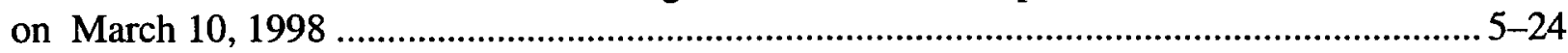

Figure 5-18. The Temperature at TT1-7 to TT1-11, Showing the Effect of the TH event on June

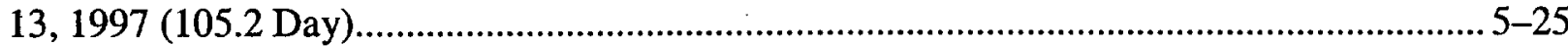

Figure 5-19. The Temperature at TT1-12 to TT1-17, Showing the Effect of the TH Event on

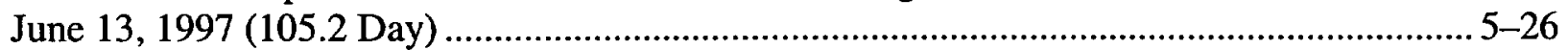

Figure 5-20. The Temperature at TT1-18 to TT1-23, Showing the Effect of the TH Event on

June 13, 1997 (105.2 Day) ............................................................................................27

Figure 5-21. Temperature at TT1-24 to TT1-28, Showing the Effect of the TH Event on June 13,

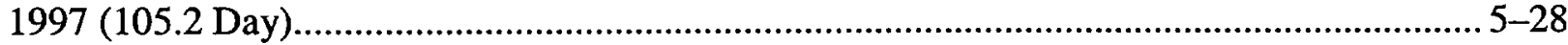

Figure 5-22. The Temperature at TT1-7 to TT1-11 as a Function of Time, Showing the Effect of

the TH Event on September 2, 1997 (186.5 Day) ............................................................ 5-29

Figure 5-23. The Temperature at TT1-12 to TT1-17 as a Function of Time, Showing the TH

Event on September 2, 1997 (186.5 Day) .................................................................. 5-30

Figure 5-24. The Temperature at TT1-18 to TT1-23, Showing the Effect of the TH Event on

September 2, 1997 (186.5 Day) ......................................................................... 5-31

Figure 5-25. The Temperature at TT1-24 to TT1-28, Showing the Effect of the TH event on

September 2, 1997 (186.5 Day) ............................................................................ 5-32

Figure 5-26. The Heat Flux per Unit Area in Zone $1(\mathrm{z}=-3.2$ to $-4.42 \mathrm{~m})$ of the Block as a

Function of Time from February 18, 1998, to February 25, 1998 .................................. 5-33

Figure 5-27. The Heat Flux per Unit Area in Zone $2(\mathrm{z}=-1.981$ to $-3.2 \mathrm{~m})$ of the Block as a

Function of Time from February 18, 1998, to February 25, 1998.

Figure 5-28. The Heat Flux per Unit Area in Zone $3(z=-0.762$ to $-1.981 \mathrm{~m})$ of the Block as a

Function of Time from February 18, 1998, to February 25, 1998 ................................... 5-35

Figure 5-29. The Heat Flux per Unit Area in Zone $4(\mathrm{z}=0$ to $-0.762 \mathrm{~m})$ of the Block as a

Function of Time from February 18, 1998, to February 25, 1998.

Figure 5-30. Rock Thermal Conductivity, K [W/(mK)], Diffusivity, Alpha $\left[\mathrm{m}^{2} / \mathrm{s}\right]$, and

Temperature $\left[{ }^{\circ} \mathrm{C}\right]$ with Time [Days] for the Horizontal REKA Probe 1 Installed $0.584 \mathrm{~m}$

Below the Large Block's Horizontal Heater Layer. 
Figure 5-31. Rock Thermal Conductivity, K [W/(mK)], Diffusivity, Alpha $\left[\mathrm{m}^{2} / \mathrm{s}\right]$, and

Temperature [ ${ }^{\circ} \mathrm{C}$ ] with Time [Days] for the Horizontal REKA Probe 2 Installed $0.889 \mathrm{~m}$

Below the Large Block's Horizontal Heater Layer.

Figure 5-32. Rock Thermal Conductivity, K [W/(mK)], Diffusivity, Alpha $\left[\mathrm{m}^{2} / \mathrm{s}\right]$, and

Temperature $\left[{ }^{\circ} \mathrm{C}\right]$ with Time [Days] for the Horizontal REKA Probe 3 Installed $1.38 \mathrm{~m}$

Below the Large Block's Horizontal Heater Layer..

Figure 5-33. The Measured Temperatures in the LBT in TT1 Compared with Model Calculation

Using NUFT...

Figure 5-34. Comparison of the Measured Temperature at TT1-14 and TT1-19 with the Model

Calculation Using the Drift-Scale Property Set

Figure 5-35. Predicted Temperature Profiles Along Hole \#TT1 of the LBT Using the DS and MS

Property Sets, Compared with the Measured Temperature ................................................. 5-42

Figure 6-1. Air Permeability Measured in a Single Hole Before Cutting (D) and After Cutting

(B) the Block as a Function of Depth.

Figure 6-2. Air Permeability Variations Along Five Heater Boreholes at the LBT Site

Figure 6-3. Air Permeability Variations Along Three Horizontal, Hydrologic, Monitoring

Boreholes at the LBT Site.

Figure 6-4. Tracer Breakthrough Curves from Heater Borehole Intervals to a Hydrologic,

Monitoring Borehole WH1 at the LBT Site

Figure 6-5. Air Permeability Measured by Single-hole Injection Along Hole TH1 in the Block

Before Heating (Cold) and During Heating (Hot)

Figure 6-6. Layout of ERT Electrodes for the Large Block Test.

.6-24

Figure 6-7. Changes in the Distribution of Moisture Content in Two Horizontal Image Planes

Figure 6-8. Changes in the Distribution of Moisture Content in Two Vertical Image Planes.... .6-26

Figure 6-9. Interpolated Temperature Isotherms $\left({ }^{\circ} \mathrm{C}\right)$ Superimposed on ERT-Derived Images of

Volumetric Water Change in the West-to-East Plane

Figure 6-10. Comparison of Neutron Log and ERT Measurements of Changes in Moisture

Content

Figure 6-11. Pre-Cut and Post-Cut Water Saturation as a Function of Depth

Figure 6-12. The Baseline Moisture Content Measured in Hole TN1

Figure 6-13. The Baseline Moisture Measured in Hole TN2.

Figure 6-14. The Baseline Moisture Content Measured in Hole TN3 .6-31

Figure 6-15. The Baseline Moisture Content Measured in Hole TN4 .6-32

Figure 6-16. The Baseline Moisture Content Measured in Hole TN1 .6-33

Figure 6-17. The Baseline Moisture Content Measured in Hole NN1. $6-34$

Figure 6-18. The Baseline Moisture Content Measured in Hole NN2.

Figure 6-19. The Baseline Moisture Content Measured in Hole NN3. Figure 6-20. The Baseline Moisture Content Measured in Hole NN4. Figure 6-21. The Baseline Moisture Content Measured in Hole NN5. Figure 6-22. The Baseline Moisture Content Measured in Hole NN6. Figure 6-23. The Baseline Moisture Content Measured in Hole WN1 $6-35$ .6-36 .6-37

Figure 6-24. The Baseline Moisture Content Measured in Hole WN2

Figure 6-25. The Baseline Moisture Content Measured in Hole WN3

Figure 6-26. The Baseline Moisture Content Measured in Hole WN4 as a Function of Depth from the West Face of the Block 
Figure 6-27. Difference Fraction Volume Water in Hole TN1 from March 11, 1997, to June 11, 1997

Figure 6-28. Difference Fraction Volume Water, Between the In-heat and the Baseline, in Hole

TN1 as a Function of Depth from Top of the Block, from June 8, 1997, to September 15, 1998.

Figure 6-29. Difference Fraction Volume Water, Between the In-heat and the Baseline, in Hole

TN2 as a Function of Depth from Top of the Block, from March 11, 1997, to June 11, 1997 .6-46

Figure 6-30. Difference Fraction Volume Water, Between the In-heat and the Baseline, in Hole

TN2 as a Function of Depth from Top of the Block, from July 8, 1997, to September 15, 1998.

Figure 6-31. Difference Fraction Volume Water, Between the In-heat and the Baseline, in Hole

TN3 as a Function of Depth from Top of the Block, from March 11, 1997, to June 11, 1997 .6-48

Figure 6-32. Difference Fraction Volume Water, Between the In-heat and the Baseline, in Hole

TN3 as a Function of Depth from Top of the Block, from July 8, 1997, to September 15, 1998.

Figure 6-33. Difference Fraction Volume Water, Between the In-heat and the Baseline, in Hole

TN4 as a Function of Depth from Top of the Block, from March 11, 1997, to June 11, 1997 .6-50

Figure 6-34. Difference Fraction Volume Water, Between the In-heat and the Baseline, in Hole

TN4 as a Function of Depth from Top of the Block, from July 8, 1997, to September 15, 1998.

Figure 6-35. Difference Fraction Volume Water, Between the In-heat and the Baseline, in Hole

TN5 as a Function of Depth from Top of the Block, from March 11, 1997 to June 11, 1997 ..6-52

Figure 6-36. Difference Fraction Volume Water, Between the In-heat and the Baseline, in Hole

TN5 as a Function of Depth from Top of the Block, from July 8, 1997, to September 15,

1998.

Figure 6-37. Difference Fraction Volume Water, Between the In-heat and the Baseline, in Hole

NN1 as a Function of Depth from the North Face of the Block, from March 11, 1997, to

July 8,1997

Figure 6-38. Difference Fraction Volume Water, Between the In-heat and the Baseline, in Hole

NN1 as a Function of Depth from the North Face of the Block, from July 22, 1997,

September 15, 1998

Figure 6-39. Difference Fraction Volume Water, Between the In-heat and the Baseline, in Hole

NN2 as a Function of Depth from the North Face of the Block, from March 11, 1997, to

July 8, 1997

Figure 6-40. Difference Fraction Volume Water, Between the In-heat and the Baseline, in Hole

NN2 as a Function of Depth from the North Face of the Block, from July 22, 1997, to

September 15, 1998.

Figure 6-41. Difference Fraction Volume Water, Between the In-heat and the Baseline, in Hole

NN3 as a Function of Depth from the North Face of the Block, from March 11, 1997, to

July 8, 1997

Figure 6-42. Difference Fraction Volume Water, Between the In-heat and the Baseline, in Hole

NN3 as a Function of Depth from the North Face of the Block, from November 6, 1997, to

September 15, 1998.

Figure 6-43. Difference Fraction Volume Water, Between the In-heat and the Baseline, in Hole

NN4 as a Function of Depth from the North Face of the Block, from March 11, 1997, to

July 8, 1997 
Figure 6-44. Difference Fraction Volume Water, Between the In-heat and the Baseline, in Hole NN4 as a Function of Depth from the North Face of the Block, from July 22, 1997, to September 15, 1998.

Figure 6-45. Difference Fraction Volume Water, Between the In-heat and the Baseline, in Hole

NN5 as a Function of Depth from the North Face of the Block, from March 11, 1997, to

July 8, 1997

Figure 6-46. Difference Fraction Volume Water, Between the In-heat and the Baseline, in Hole

NN5 as a Function of Depth from the North Face of the Block, from January 29, 1998, to

September 15,1998 .

Figure 6-47. Difference Fraction Volume Water, Between the In-test and the Baseline as a

Function of Depth from the North face of the Block, in Hole NN6 from March 11, 1997, to

November 6, 1997

Figure 6-48. Difference Fraction Volume Water, Between the In-heat and the Baseline, in Hole

NN6 as a Function of Depth from the North Face of the Block, from January 29, 1998, to

September 15, 1998 .

Figure 6-49. Difference Fraction Volume Water, Between the In-heat and the Baseline, in Hole

WN1 as a Function of Depth from the West Face of the Block, from March 11, 1998, to

July 8, 1997

Figure 6-50. Difference Fraction Volume Water, Between the In-heat and the Baseline, in Hole

WN1 as a Function of Depth from the West Face of the Block, from January 22, 1998, to

September 15, 1998

Figure 6-51. Difference Fraction Volume Water, Between the In-heat and the Baseline, in Hole

WN2 as a Function of Depth from the West Face of the Block, from March 11, 1997, to

July 8, 1997

Figure 6-52. Difference Fracture Volume Water, Between the In-heat and the Baseline, in Hole

WN2 as a Function of Depth from the West Face of the Block, from January 22, 1998, to

September 15, 1998.

Figure 6-53. Difference Fraction Volume Water, Between the In-heat and the Baseline, in Hole

WN3 as a Function of Depth from the West Face of the Block, from March 11, 1997, to

July 8,1997

Figure 6-54. Difference Fraction Volume Water, Between the In-heat and the Baseline, in Hole

WN3 as a Function of Depth from the West Face of the Block, from November 5, 1997, to

September 15, 1998

Figure 6-55. Difference Fraction Volume Water, Between the In-heat and the Baseline, in Hole

WN4 as a Function of Depth from the West Face of the Block, from March 11, 1997, to

July 8,1997

Figure 6-56. Difference Fraction Volume Water, Between the In-heat and the Baseline, in Hole

WN4 as a Function of Depth from the West Face of the Block, from January 29, 1998, to

September 15, 1998.

Figure 6-57. Pre-test Temperature and Liquid Saturation Calculated in Model Case A

Figure 6-58. The Moisture Content Measured by Neutron in Hole TN3 Compared with the

Model Calculations Using DS Property Set

Figure 6-59. Comparison of Calculated Liquid Saturation of the LBT Along Hole TN3 Using

MS Property Set and DS Property Set

Figure 7-1. Multiple-Point Borehole Extensometer (MPBX).

Figure 7-2. MPBX Borehole Locations, Viewed from the South Face 
Figure 7-3. Extensometer NM-1 Collar and Invar Rod Installation ............................................. 7-30

Figure 7-4. Invar Rod Corrosion ............................................................................................ 7-31

Figure 7-5. Horizontal Displacements During the First Two Weeks of the Large Block Test..........7-32 Figure 7-6. Displacement Rates for the Horizontal Extensometers During the First 40 Days of the

LBT

Figure 7-7. East-West Anchor Displacements During the First 100 Days for WM-1 .......................7-34

Figure 7-8. North-South Anchor Displacements During the First 100 Days for NM-1 .................... 7-35

Figure 7-9. North-South Anchor Displacements During the First 100 Days for NM-2 .....................7-36

Figure 7-10. East-West Anchor Displacements During the First 100 Days for WM-2 ..................... 7-37

Figure 7-11. North-South Anchor Displacements During the First 100 Days for NM-3 .................7-38

Figure 7-12. Vertical Anchor Displacements During the First 100 Days for TM-1 .......................... 7-39

Figure 7-13. Anchor 4 Strains for Each Extensometer During the First 100 Days.............................7-40

Figure 7-14. East-West Displacement for WM-1 Anchor 4 and Temperature at 4-m Depth .............7-41

Figure 7-15. East-West Displacement for WM-2 Anchor 4 and Temperature at 1.2-m Depth ..........7-41

Figure 7-16. North-South Displacement for NM-3-m Anchor 4 and Temperature at 1.2-m Depth..7-42

Figure 7-17. WM-2 Anchor Displacements and Borehole TT1 Temperature Near the Heater

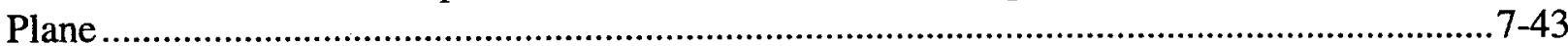

Figure 7-18. WM-2 Anchor 4 Displacements and Temperatures at 1.2 m Depth Around Day 105 .7-44 Figure 7-19. WM-2 and NM-3 Anchor Displacements and Borehole TT1 Temperature Near the

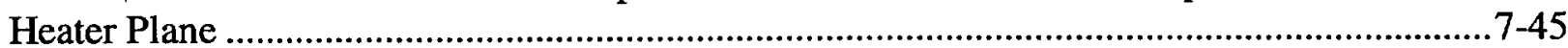

Figure 7-20. WM-1East-West Anchor Di ……………............................................................ 7-46

Figure 7-21. NM-1North-South Anchor Displacements During Cool Down .....................................7-47

Figure 7-22. WM-2 East-West Anchor Displacements During Cool-Down ......................................7-48

Figure 7-23. NM-3 North-South Anchor Displacements During Cool-Down ...................................7-49

Figure 7-24. TM-1 Vertical Anchor Displacements During Cool-Down ......................................... 7-50

Figure 7-25. Anchor 4 Strains During Cool-Down...................................................................... 7-51

Figure 7-26. Strain Histories for Horizontal Extensometers............................................................ 7-52

Figure 7-27. Strains, Calculated from MPBX Displacements, Regressed Against Temperature

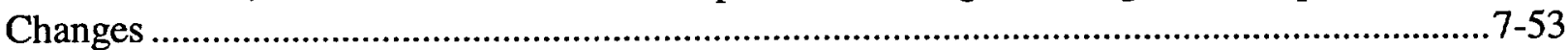

Figure 7-28. Linear Coefficients of Thermal Expansion with Depth into the Large Block ...............7-54

Figure 7-29. Cutaway View of LBT Surface Fracture Monitors ........................................................ 7-55

Figure 7-30. Fracture Monitor Locations on the East Side of the Large Block ...................................7-56

Figure 7-31. Fracture Monitor Locations on the North Side of the Large Block ...............................7-57

Figure 7-32. Fracture Monitor Locations on the West Side of the Large Block................................ 7-58

Figure 7-33. Fracture Monitor Locations on the South Side of the Large Block ..............................7-59

Figure 7-34. Photo of LBT with Fracture Monitor Grooves................................................................ 7-60

Figure 7-35. Photo of Installed Fracture Monitor ......................................................................... 7-61

Figure 7-36. Fracture Aperture Data for All Vertical Fracture Monitors and Temperature Near

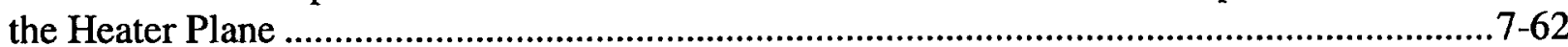

Figure 7-37. Fracture Aperture Data Between Days 100 and 140 for All Vertical Fracture

Monitors and Temperature Near the Heater Plane ..................................................................... 7-63

Figure 7-38. Fracture Slip for All Vertical Fractures and Temperature Near the Heater Plane ........7-64

Figure 7-39. Aperture Change for Vertical Fractures .....................................................................7-65

Figure 7-40. Slip Along Vertical Fractures ........................................................................................ 7-66

Figure 7-41. Fracture Aperture Data for All Horizontal Fracture Monitors and Temperature Near

the Heater Plane ................................................................................................................ 
Figure 7-42. Temperature and Fracture Aperture Data for All Horizontal Fracture Monitors

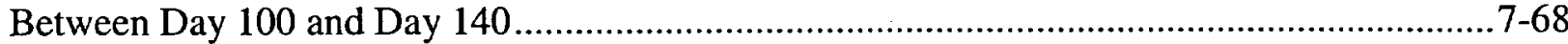

Figure 7-43. Fracture Slip for All Horizontal Fractures and Temperature Near the Heater Plane .... 7-69 Figure 7-44. Fracture Slip for All Horizontal Fractures and Temperature Near the Heater Plane,

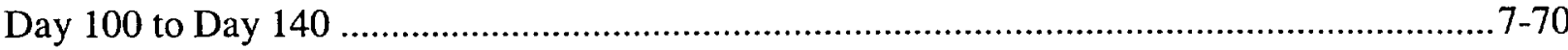

Figure 7-45. Deformation Across Horizontal Fractures....................................................... $7-71$

Figure 7-46. Slip Along Horizontal Fractures............................................................. $7-72$

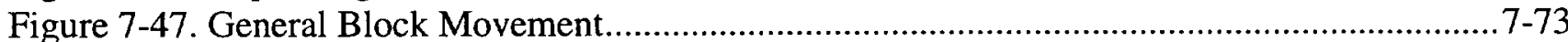

Figure 7-48. Anchor 4 Strains for Each Extensometer During the First 100 Days........................ 7-74

Figure 7-49. Fracture Aperture Data for All Vertical Fracture Monitors ...................................... 7-75

Figure 7-50. Large Block Test TM Model Domains .......................................................... 7-76

Figure 7-51. TM1 Anchor 4 Displacements and Model Results for the First 100 Days .................7-77

Figure 7-52. TM1 Anchor 4 Displacements and Model Results for the Cool-Down .......................7-78

Figure 7-53. NM1 Anchor 4 Displacements and Model Results for the First 100 Days .................7-79

Figure 7-54. NM1 Anchor 4 Displacements and Model Results for the Cool-Down....................... 7-80

Figure 7-55. WM1 Anchor 4 Displacements and Model Results for the First 100 Days ..................7-81

Figure 7-56. WM1 Anchor 4 Displacements and Model Results for the Cool-Down......................7-82

Figure 7-57. NM2 Anchor 4 Displacements and Model Results for the First 100 Days .................. 7-83

Figure 7-58. WM2 Anchor 4 Displacements and Model Results for the Entire Test .......................7-84

Figure 7-59. NM3 Anchor 4 Displacements and Model Results for the Entire Test....................... 7-85

Figure 7-60. WM2 Anchor 4 Displacements, Heater Plane (TT1-14) and WM2-Level (TT1-22)

Temperatures and Results of Models 3 and 4 for the First 100 Days .......................................7-86

Figure 7-61. Post-Test Core Drilling Activities, Overview .....................................................7-87

Figure 7-62. Post-Test Core Drilling Activities, Detail ....................................................... 7-88

Figure 7-63. Representative Stress-Strain Plots for (a) Pre-test Core HC_076_61 and (b) Post-

test Core OC_874B_01 .................................................................................................... 7-89

Figure 7-64. Compressional Velocities for (a) Pre-test and (b) Post-test Specimens ........................7-90

Figure 7-65. Shear Velocities for (a) Pre-test and (b) Post-test Specimens .....................................7-91

Figure 7-66. Compressive Strength for (a) Pre-test and (b) Post-test Specimens............................7-92

Figure 7-67. Static Young's Modulus for (a) Pre-test and (b) Post-test Specimens ..........................7-93

Figure 7-68. Uniaxial Compressive Strength for Topopah Spring Tuff .........................................7-94

Figure 7-69. Static Young's Modulus for Topopah Spring Tuff .................................................. 7-95

Figure 8-1. Sample SPC0039799: Pre-Test Fracture of a Crusty-Coating of Al, Mn-rich

Composition. The Grain Appearance and Analysis (Figure 8-2) Are Consistent with the

Layer-Structure Mn-oxides Lithiophorite.

Figure 8-2. EDX Spectrum Corresponding to Area Shown in Figure 8-1 (Presumably the

Reported Mineral Is Lithiophorite, Where Li Is Too Light for EDX Detection)

Figure 8-3. Sample SPC0039806: Pre-test Fracture Sample Exhibits Well-developed Crystalline

Morphology. The Blocky Crystals Are Consistent with Calcite. Small Rounded Mounds,

Lighter in Color and Scattered Around the Bases of the Calcite Are Determined to Be Silica

Polymorph (See Figures 8-5 and 8-6)

Figure 8-4. EDX Spectrum, Generally Corresponding to Area Photographed in Figure 8-3. The

Spectrum Is Likely a Composite from Two Phases: a Calcium Carbonate and a Silica

Polymorph 
Figure 8-5. Sample SPC0039806: Pre-Test Fracture, Magnified View of Rounded Masses

Similar to Those Shown in Figure 8-3 (Bases of Blocky Crystals). EDX Is Consistent with

a Silica Polymorph

Figure 8-6. EDX of Rounded Nodular Crystal Mass. Spectrum Is That of an $\mathrm{Sio}_{2}$ Phase ..............8-12

Figure 8-7. Sample SPC0039806: Strand Lying on the Surface of Other Minerals. The

Morphology and Relative Chemistry Suggest a Clay Mineral

Figure 8-8. EDX Spectrum for Strand Shown in Figure 8-7, Possibly a Clay, Such as Smectite .....8-14

Figure 8-9. Sample SPC0039799 Pre-Test Sample with Calcite Grain (EDX Not Shown)

Showing Pronounced Dissolution

Figure 8-10. Sample SPC02015843 Post-Test Sample with Significant Dissolution of a K-Rich

Feldspar (EDX Not Shown)

Figure 8-11. Sample SPC02015843 Post-Test Sample (Different Area Than Imaged in Figure 8-

10): Chevron Features are Sub-Micron Sized, Appear to Be Further Evidence of Dissolution

(Grain Not Identified by EDX)

Figure 8-12. Sample SPC02015679 Post-Test Sample: an Abundance of the Matted Fibrous

Material Covers Surfaces of Different Grains, Appears to Have a Morphology and

Chemistry Consistent with a Clay

Figure 8-13. Sample SPC039806 Pre-Test Sample: the Same Fibrous Mineral Is Observed

Coating Minerals of Fracture Surfaces Before Thermal Testing

Figure 8-14. Sample SPC02015679 Post-Test Sample: Overview of Post-Test Mineral Surface

Exhibits Several Minerals - the Fibrous Matted Mineral, the Spherical Silica Polymorphs,

Possibly Ribbons of Pore-Bridging Illite

Figure 9-1. Temperature Measured by the Humicap in Hole NH-1 as a Function of Time

Figure 9-2. Relative Humidity Measured by the Humicap in Hole NH-1 as a Function of Time.....9-10

Figure 9-3. Temperature Measured by the Humicap in Hole WH-2 as a Function of Time 9-11

Figure 9-4. Relative Humidity Measured by the Humicap in Hole WH-2 as a Function of Time ....9-12

Figure 9-5. Temperature Measured by the Humicap in Hole WH-1 as a Function of Time .9-13

Figure 9-6. Relative Humidity Measured by the Humicap in Hole WH-1 as a Function of Time ....9-14

Figure 9-7. Temperature Measured by the Humicap in the Third Zone of Hole TH-1 as a

Function of Time $9-15$

Figure 9-8. Relative Humidity Measured by the Humicap in the Third Zone of Hole TH-1 as a

Function of Time $9-16$ 
THIS PAGE INTENTIONALLY LEFT BLANK 


\section{TABLES}

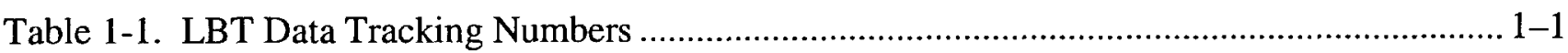

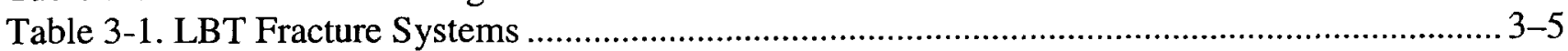

Table 3-2. Borehole and Instrument Listing by Instrument Type ............................................ 3-7

Table 3-3. Permeability Measurements on Intact Core Sample SPC00504573.4 ........................ 3-15

Table 3-4. The Porosity of LBT Samples Determined by Gravimetric Method............................ 3-18

Table 3-5. Pore Size Distribution of the LBT Samples Determined by Mercury Injection

Porosimetry Method .................................................................................................. 3-19

Table 3-6. Sample Descriptions-Fracture Flow Versus Matrix Imbibition Test...........................3-19

Table 3-7. Rock Property Values Used for Modeling of the LBT ...........................................3-27

Table 3-8. The xyz Coordinates (with Respect to the Southwestern Corner on Top of the Block) of the Collar, and Other Characteristics of the Post-test Holes............................................... 3-36

Table 4-1. Number of Fractures Per Each Length Interval and the Percentage of Fractures Occurring in Each Interval ............................................................................................... 4-2

Table 4-2. All Major Fracture Planes That Have Been Modeled for the LBT ............................. 4-3

Table 5-1. The DS and MS Rock Properties and the Thermal Properties of the Insulation

Material Used in the TH Modeling of the LBT. (Source: Table 7 of CRWMS M\&O 2000c). 5-8

Table 5-2. RMSD and MD for Temperature Profile Along TT1 Using Drift-scale Data Set............ 5-9

Table 5-3. RMSD and MD for Temperature Profile Along TT1 Using Drift-scale and Mountainscale Property Sets ................................................................................................... 5-10

Table 6-1. X-Y-Z Coordinates of the Collar of the Neutron Holes ..............................................6-15

Table 6-2. Hydraulic and Thermal Properties of Rock Units ...................................................6-18

Table 7-1. MPBX Extensometer Collar and Anchor Coordinates. The Origin of the Coordinates Is the Southwestern Corner on Top of the Block

Table 7-2. MPBX Orientations, Baseline Lengths, and Number of Anchors .................................. 7-2

Table 7-3. Interpolated Average Temperatures for Horizontal MPBX Extensometers ......................7-8

Table 7-4. Thermal Expansion Coefficients (CTE) and Fitting Statistics ..................................... 7-9

Table 7-5. Maximum and Residual Fracture Slip and Aperture .................................................. 7-12

Table 7-6. Input Parameters and Data Tracking Numbers.................................................... $7-17$

Table 7-7. Calculated or Assumed Model Parameters ..................................................................17

Table 7-8. Summary of LBT TM Simulations ................................................................... 7-19

Table 7-9 Pre-test Specimen Densities, Velocities, and Dynamic Moduli ..................................... 7-25

Table 7-10 Post-test Specimen Densities, Velocities, and Dynamic Moduli...................................7-25

Table 7-11 Pre-test Specimen Strain Rates and Static Mechanical Properties ................................ 7-26

Table 7-12. Post-test Specimen Strain Rates and Static Mechanical Properties..............................7-26

Table 8-1. Samples Selected for SEM Imaging and Analysis .................................................. 8-4

Table 8-2. Qualitative Results for the 19 Solid Samples from the Large Block Test Resulting from Analyses using Jade Software Package.................................................................. 8-6

Table 9-1, X-Y-Z Coordinates of the Collar, C, and Bottom, TD, of the Observation Holes of the

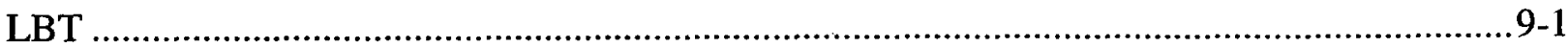

Table 9-2. The Location of Humicap in the Hydrology Holes ................................................9-5 
THIS PAGE INTENTIONALLY LEFT BLANK 


\section{ACRONYMS AND ABBREVIATIONS}

2-D Two-dimensional

3-D Three-dimensional

AMR Analysis Model Report

ASTM American Society for Testing and Materials

AZ

Altered Zone

$\mathrm{CD}$

Compact Disk

CD-ROM Compact Disk - Read Only Memory

CTE

Coefficient of Thermal Expansion

DAS Data acquisition system

DOE Department of Energy

DS

Drift-scale

DST

Drift Scale Test

DTN

Data tracking number

EBS Engineered Barrier System

ECM Equivalent continuum model

EDX Energy Dispersive X-ray

ERT Electrical resistance tomography

ESF Exploratory Studies Facility

FLAC Fast Lagrangian Analysis of Continua

FM Fracture monitor

HHL Horizontal heater layer

HP Hewlett Packard

KI Potassium iodide

LANL Los Alamos National Laboratory

LBNL Lawrence Berkeley National Laboratory

LBT Large Block Test

LLNL Lawrence Livermore National Laboratory

LVDT Linear Variable Differential Transformer

MD Mean difference

MPBX Multiple point borehole extensometer

MS Mountain-scale

NF Near Field

NFE Near Field Environment

NI National Instruments

NIST National Institute of Standards and Testing

OCRWMS Office of Civilian Radioactive Waste Management System 


$\begin{array}{ll}\text { PVC } & \text { Polyvinyl chloride } \\ \text { QA } & \text { Quality Assurance } \\ \text { REKA } & \text { Rapid Evaluation of K and Alpha } \\ \text { RH } & \text { Relative humidity } \\ \text { RIB } & \text { Reference Information Base } \\ \text { RMS } & \text { Root-mean-square } \\ \text { RMSD } & \text { Root-mean-squared difference } \\ \text { RTD } & \text { Resistance temperature device } \\ \text { RTV } & \text { Room temperature vulcanized rubber } \\ \text { SEM } & \text { Scanning Electron Microscope } \\ \text { SHT } & \text { Single Heater Test } \\ \text { SMF } & \text { Sample Management Facility } \\ \text { TC } & \text { Thermal-chemical } \\ \text { TCO } & \text { Test Coordination Office } \\ \text { TDMS } & \text { Technical Data Management System } \\ \text { TH } & \text { Thermal-hydrological } \\ \text { THC } & \text { Thermal-hydrological-chemical } \\ \text { THM } & \text { Thermal-hydrological-mechanical } \\ \text { THMC } & \text { Thermal-hydrological-mechanical-chemical } \\ \text { TM } & \text { Thermal-mechanical } \\ \text { TSPA } & \text { Total System Performance Assessment } \\ \text { Tptp } & \text { Topapah Spring tuff } \\ \text { Tptpmn } & \text { Topopah Spring tuff, middle nonlithophysical } \\ \text { TSw2 } & \text { Topopah Spring tuff thermal-mechanical unit } \\ \text { USGS } & \text { United States Geological Survey } \\ \text { UZ } & \text { Unsaturated Zone } \\ \text { W } & \text { Watt } \\ \text { WF } & \text { Waste Form } \\ \text { WP } & \text { Waste package } \\ \text { XRD } & \text { X-ray diffraction } \\ \text { YM } & \text { Yucca Mountain } \\ \text { YMP } & \text { Tuountain Site Characterization Program } \\ & \end{array}$




\section{INTRODUCTION}

This report documents the Large-Block Test (LBT) conducted at Fran Ridge near Yucca Mountain, Nevada. The LBT was a thermal test conducted on an exposed block of middle nonlithophysal Topopah Spring tuff (Tptpmn) and was designed to assist in understanding the thermal-hydrological-mechanical-chemical (THMC) processes associated with heating and then cooling a partially saturated fractured rock mass. The LBT was unique in that it was a large $(3 \times$ $3 \times 4.5 \mathrm{~m}$ ) block with top and sides exposed. Because the block was exposed at the surface, boundary conditions on five of the six sides of the block were relatively well known and controlled, making this test both easier to model and easier to monitor. This report presents a detailed description of the test as well as analyses of the data and conclusions drawn from the test. The rock block that was tested during the LBT was exposed by excavation and removal of the surrounding rock. The block was characterized and instrumented, and the sides were sealed and insulated to inhibit moisture and heat loss. Temperature on the top of the block was also controlled. The block was heated for 13 months, during which time temperature, moisture distribution, and deformation were monitored. After the test was completed and the block cooled down, a series of boreholes were drilled, and one of the heater holes was over-cored to collect samples for post-test characterization of mineralogy and mechanical properties.

Section 2 provides background on the test. Section 3 lists the test objectives and describes the block site, the site configuration, and measurements made during the test. Section 3 also presents a chronology of events associated with the LBT, characterization of the block, and the pre-heat analyses of the test. Section 4 describes the fracture network contained in the block. Section 5 describes the heating/cooling system used to control the temperature in the block and presents the thermal history of the block during the test. Sections 5 through 9 report the measurements made on the block during the preheating, heating, and cooling phases. These measurements include temperature, thermal conductivity and diffusivity, hydrological measurements (electrical resistivity, neutron logging, gas pressure, and relative humidity), geomechanics, selected chemical analyses, and microbial activity. These sections also include analyses and simulations of the block behavior.

Finally, conclusions are presented in Section 10. Complete data sets were submitted during the time the test was conducted. The data tracking numbers (DTNs) of all of the data are presented in Table 1-1.

Table 1-1. LBT Data Tracking Numbers

\begin{tabular}{|l|l|l|}
\hline \multicolumn{1}{|c|}{ Description } & Status & \multicolumn{1}{|c|}{ Data Tracking Number } \\
\hline Effect of Rock-Water Interaction on Permeability & QA & LL950916504242.018 \\
\hline Relative Humidity, Saturation, Lab Tests & QA & LL960100604244.007 \\
\hline Fracture Map of East Face & QA & LL960400404244.012 \\
\hline Fracture Map of South Face & QA & LL960400504244.013 \\
\hline Fracture Map of West Face & QA & LL960400604244.014 \\
\hline Fracture Map of North Face & QA & LL960400704244.015 \\
\hline
\end{tabular}




\begin{tabular}{|c|c|c|}
\hline Permeability, Lab Tests on Core & QA & LL 960905204244.022 \\
\hline Geomechanical Properties, Small Blocks & QA & LL970407204243.011 \\
\hline Geomechanical Properties, Small Blocks & QA & LL970407304243.012 \\
\hline Temperature & QA & LL970803004244.036 \\
\hline Rock Displacement, MPBX & QA & LL970803104244.037 \\
\hline Relative Humidity & QA & LL970803204244.038 \\
\hline Moisture Content & QA & LL970803404244.040 \\
\hline Thermal Conductivity, Thermal Diffusivity & QA & LL.970803504244.041 \\
\hline Neutron Log Data, Water Content & QA & LL971204304244.047 \\
\hline Geomechanical Properties, Small Blocks & QA & LL980204504243.016 \\
\hline Geomechanical Properties, Small Blocks & QA & LL980207304243.018 \\
\hline Geomechanical Properties, Small Blocks & QA & LL980208104243.019 \\
\hline Air Permeability & QA & LL980706604244.063 \\
\hline Electrical Resistivity, Saturation, ERT & QA & LL980913304244.072 \\
\hline Thermal Gradient, X-ray Diffraction & QA & Líg80916004242.055 \\
\hline Heater Power, Temperature Profiles & QA & LL.980918904244.074 \\
\hline Neutron Logs, Water Content & QA & LL980919304244.075 \\
\hline Rock and Fracture Displacements & QA & LL980919404244.076 \\
\hline Electrical Resistivity, Saturation, ERT & QA & LL981001604244.079 \\
\hline Rock and Fracture Displacements & QA & LL981004604243.024 \\
\hline Air Permeability & QA & LL981106204244.083 \\
\hline Thermal Expansion & QA & LL9881202104243.028 \\
\hline Permeability & QA & LL981202904242.079 \\
\hline Geomechanical Properties, Small Blocks & QA & LL981204004243.030 \\
\hline Compressive Strength, Modulus, Effect of Radiation & QA & LL981208504243.031 \\
\hline Fracture Frequency, Location & QA & LL981211004244.093 \\
\hline Compressive Strength, Modulus, Effect of Radiation & QA & LL990205304243.032 \\
\hline Fracture Attitude, Frequency, Gradient & QA & LL990707004244.101 \\
\hline Compressive Strength, Modulus, Lab Tests & QA & LLO00209404243.036 \\
\hline Permeability, NUFT & QA & LLO00314304242.094 \\
\hline Barometric Pressure & QA & LLO00317504244.108 \\
\hline Permeability, NUFT & QA & LLO000321204242.092 \\
\hline Invar Rod Thermal Expansion Coefficients & QA & SNL22100196001.003 \\
\hline Drift-Scale Hydraulic and Thermal Properties & NQ & LB990861233129.001 \\
\hline Mountain-Scale Hydraulic and Thermal Properties & NQ & LB997141233129.001 \\
\hline Insulation Properties & $\mathrm{NQ}$ & LL940800104244.000 \\
\hline Hydrologic Prop., Effect of Thermal Gradient, Lab Tests & NQ & LL940800504242.000 \\
\hline Hydrologic Prop., Effect of Thermal Gradient, Lab Tests & NQ & LL940800704242.002 \\
\hline Preliminary Characterization & Na & LL940800804244.001 \\
\hline Gas Permeability, Temperature, Lab Tests & NQ & LL940909304242.007 \\
\hline
\end{tabular}




\begin{tabular}{|l|l|l|}
\hline Moisture Content, Permeability, Porosity & NQ & LL950102904244.003 \\
\hline Lab Geomechanical Tests, Load, Strain, Temperature & NQ & LL950803104243.001 \\
\hline Electrical Resistivity & NQ & LL950812304244.006 \\
\hline Rock Strain, Small Blocks & NQ & LL950812404243.002 \\
\hline Porosity, Density, Drying and Re-Wetting Tests & NQ & LL950812704242.017 \\
\hline Relative Humidity, Lab Tests & NQ & LL960201304244.010 \\
\hline Electrical Resistivity. Lab Tests & NQ & LL960201404244.011 \\
\hline Water Imbibition & NQ & LL971204204242.025 \\
\hline Electrical Resistivity, Saturation, ERT & NQ & LL980916704244.073 \\
\hline Water Content, Air Perm., Displacement, Resistivity & NQ & LL981110604244.084 \\
\hline Fracture Frequency, Saturation, Temperature & NQ & LL981110804244.086 \\
\hline Bacterial Types, Abundance, Growth Rates & NQ & LL981202305912.004 \\
\hline Hydro. Prop., Effect of Thermal Gradient, Lab Tests & NQ & LL981203004242.078 \\
\hline Rock Strength, Small Blocks & NQ & LL981203504243.029 \\
\hline Rock Physical Properties, FLAC Model & NQ & LL990201804243.031 \\
\hline
\end{tabular}


THIS PAGE INTENTIONALLY LEFT BLANK 


\section{BACKGROUND}

The U.S. Department of Energy (DOE) is investigating the suitability of Yucca Mountain (YM) as a potential site for the nation's first high-level nuclear waste repository. As shown in Figure $2-1$, the site is located about $120 \mathrm{~km}$ northwest of Las Vegas, Nevada.

Favorable aspects of YM as a potential repository site include its arid environment and the sorptive properties of the rock materials. The arid environment results in unsaturated conditions at the potential emplacement horizon, which is the Topopah Spring tuff (Tptp) of the Paintbrush Group. The major advantages of unsaturated conditions are that waste package material corrosion, waste-form leaching, and radionuclide-transport mechanisms are minimized because of the lack of water in the waste package environment.

Regulations require that a repository must isolate radioactive wastes for long periods of time. Specifically, evaluation must be made of the repository system's isolation potential, composed of both natural and engineered components, for a minimum of 10,000 years and up to one million years.

Direct testing of the processes and performance of a repository can only be conducted in limited regions for a very short time. Therefore, analyses based on conceptual models using computer codes to evaluate or predict the performance will be the basis for determining the potential for the repository to properly function (that is, to provide isolation) over the long times required. Such an analysis entails more than merely achieving a scientifically acceptable view of the repository. It must provide sufficient rigor in evaluation of the models and assumptions to be useful in a regulatory process wherein the analyses will be subject to challenge by the regulatory agency, the supervisory agencies, and the interveners. Thus, the models need to be tested and verified to the greatest extent possible.

Among the processes considered in the LBT were thermally driven hydrologic processes in unsaturated fractured porous rock, plus coupling to mechanical and chemical responses.

\subsection{THE TESTING STRATEGY}

The testing strategy developed to address coupled processes is designed to evaluate these processes by accelerating portions of the testing to address different segments of the time frames of interest and to look at the functional relationships of different geometric scales. The LBT was designed to be one of a series of tests performed at different scales and conditions that assist in defining the physical processes that need to be considered in models of a potential repository in Yucca Mountain and to provide real-world testing of the conceptualizations and model approaches used to evaluate the behavior or development of the environment that interacts with the waste components and other elements of the engineered system. A more detailed discussion of the processes that are considered to be important in terms of testing is contained in Volume 1 of the Near-Field and Altered-Zone Environment Report (Wilder 1997, pp. 1-109) and in the Near Field Environment Process Model Report (CRWMS 2000b). Planning for field testing that includes the basis for the LBT is documented in Buscheck and Nitao (1995, p. 11). 
Because no single test can address all of the issues that must be studied for a potential repository, several different test approaches are being used to assess the models. The types of tests, identified in order from the smallest geometric scale to the largest, and generally from the shortest duration to the longest, fall into the following categories:

- Laboratory tests of core-size samples

- Laboratory tests of $\sim 1$-m scale block samples (small-block tests)

- Large block test

- In-situ tests, such as the Single Heater Test (CRWMS M\&O 1999a), the Drift Scale Test (CRWMS M\&O 1997), and the possible Cross Drift Thermal Test

- Performance confirmation tests

\subsection{TEST SCALES}

Laboratory testing on core-sized samples has been used to measure properties and processes of intact samples and intact core samples containing one single fracture. The duration of such tests tends to be short, usually a few days or months.

The next scale of tests includes those performed in the laboratory on block samples that are as large as $1 \mathrm{~m}$ on a side. These samples are large enough to allow testing of fractures or discontinuities and even some multiple-fracture responses and interactions. To distinguish the larger scale represented by the LBT from the 1-m-scale block tests, these latter tests are sometimes designated as the small-block tests (although their dimensions are not small). The small-block tests measure block properties, including fractures. They provide an understanding of the processes and properties of a fractured rock mass and help develop a functional relationship in terms of the influence of scale of testing on properties and processes.

The next scale of testing is represented by the LBT, which is unique in both size and test conditions. The LBT is a critical test because it is of sufficient size to incorporate a fracture system that is representative of the distribution of fracture dimensions and characteristics that would likely be present in a repository-with the possible exception of major geologic structures, such as faults. The LBT location was chosen to include large, through-going fractures as well as small, healed fractures that are of limited extent. The LBT location also includes a variety of fracture sizes, connectivities, and characteristics that fall between the bounds of the large and the very small test dimensions. The LBT allows for boundary controls and monitoring that are somewhat similar to those typical of laboratory studies, and it allows for threedimensional characterization and monitoring. The unique combination of size with boundary controls of the LBT allows processes to be evaluated and models to be tested more completely than in tests of any other scale. The focus of the LBT is, thus, to evaluate and test process understandings, conceptualizations, and models. This test is not intended to characterize YM or to measure responses of potential repository horizon rock mass. Rather, its purpose is to provide 
testing and data related to the conceptual understanding of processes and to build confidence in models by testing against appropriately sized rock-mass responses.

The next in scale of the planned tests are in-situ tests such as the Single Heater Test (SHT) and the Drift Scale Test (DST). These are relatively large tests that involve hundreds of cubic meters and extend for many months or years. They incorporate sufficient volumes of rock mass to be representative of total rock-mass responses (with the caveat that fracture domains can vary, and faulting is a localized phenomena that might not be well represented in field studies). These tests have boundary conditions that are less controlled than those of the LBT. Thus, they are focused more on hypotheses-testing for processes that are scale-dependent (thus cannot be tested at the LBT) and on characterization of repository rock-mass behavior. The in-situ tests focus on testing of the actual repository rock mass under conditions of stress, and so forth, that are more representative of the repository.

Although the in-situ tests last several years, they are nonetheless highly accelerated compared with the rates and other processes that will be typified by an actual repository. In a repository, processes such as heating, moisture redistribution, and rock-water interaction will occur at time scales of hundreds to thousands of years. The final type or scale of tests are performance confirmation tests, wherein the rock mass is monitored for the processes and parameters that are associated with the actual emplacement waste.

Confirmation testing does not involve issues of scale because the actual repository and its associated process rates will be monitored. Thus, one of the primary purposes of such testing is to confirm that the testing performed at partial repository scales and abbreviated time frames accurately reflects or predicts the behavior of the system. However, even this type of testing or monitoring will not address all of the issues related to the coupled thermal-hydrologicalmechanical-chemical (THMC) response (Buscheck and Nitao 1995, p. 3) of the rock mass to waste emplacement because only very early heating phase responses can be monitored (100 to 200 years as opposed to 10,000 to $1,000,000$ years). 


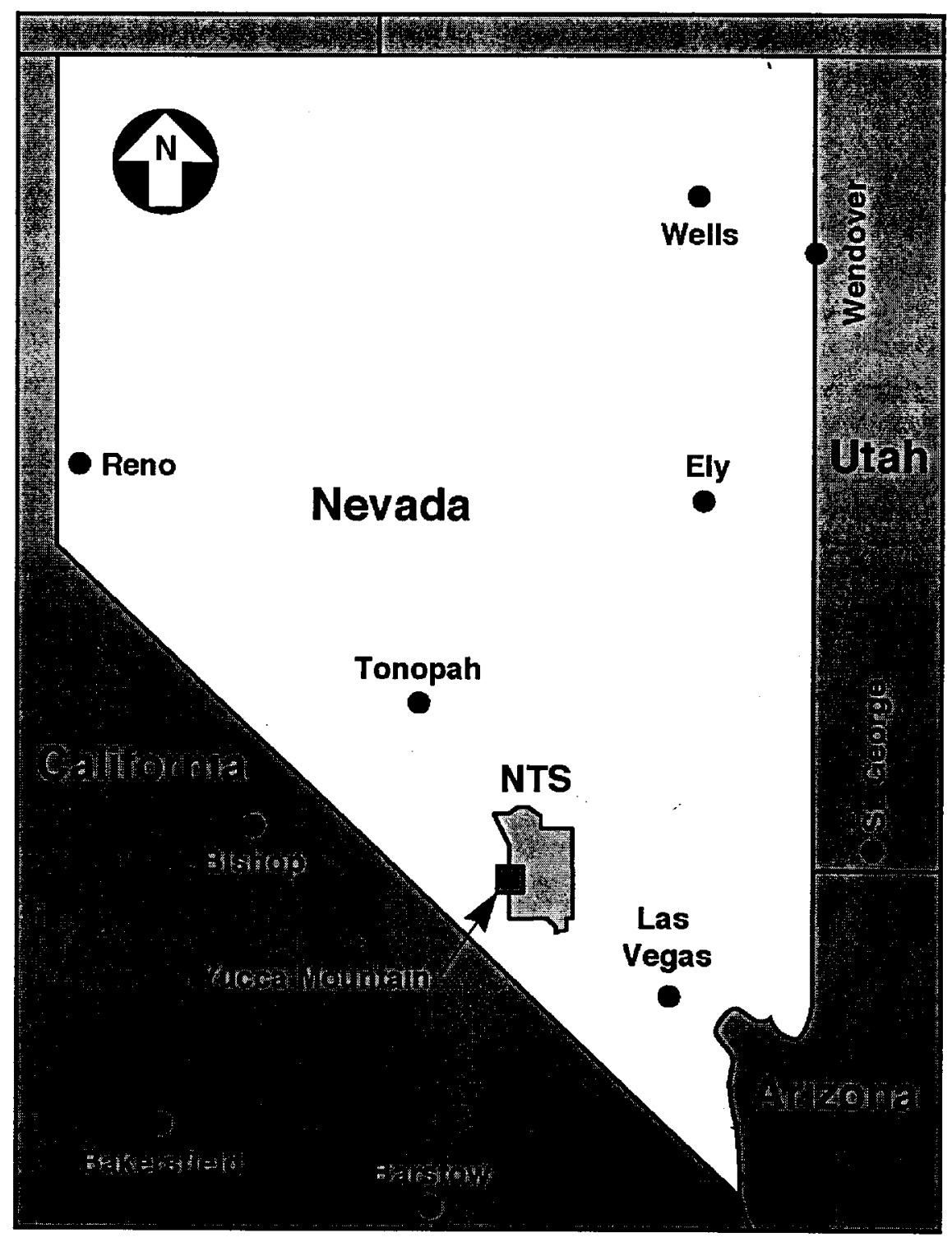

Figure 2-1. Location of the Yucca Mountain Site 


\section{TEST DESCRIPTION}

\subsection{OBJECTIVES OF THE LARGE-BLOCK TEST}

\subsubsection{Overall Objectives}

The main determinant of how the Near Field Environment (NFE) evolves is the coupling of heat from the radioactive decay of the waste to the geologic materials and water present within those materials. As noted in Volume I of the Near-Field and Altered-Zone Environment Report (Wilder et al. 1997, pp. 1-109), many coupled processes must be understood to determine the environmental conditions that interact with the waste and other components of the potential repository system.

The LBT was one of a series of tests intended to assist in defining the physical processes that must be considered in models of the evolution of the environmental conditions of a potential repository at Yucca Mountain. As such, it provided real-world testing of the conceptualizations and model approaches used to evaluate the behavior or development of the environment that interacts with the waste and engineered components, called the Engineered Barrier System (EBS). The LBT focused on the in-rock processes of what has been defined as the NFE. The primary objective of the LBT was to provide observations of the coupled THMC processes that took place within a fractured rock mass, representative of the potential repository rock mass, in response to imposition of one-dimensional heating. One-dimensional heating was chosen so that the test data could be readily usable for testing processes predicted by one-dimensional models, which will eventually be incorporated into three-dimensional models for predicting the processes in a potential repository. Thus, its purpose was to assist in identifying of the processes that must be incorporated in the models and to provide real-world testing of the conceptualizations and model approaches used to evaluate the behavior or development of the environment that interacts with the EBS.

The LBT was not intended to characterize properties or even processes that are unique to the waste emplacement horizon in the YM potential repository. Rather, its purpose was to test conceptualizations in a representative rock mass so that the tools used for the characterization and analyses of YM are appropriate, and so that models are sufficiently valid (Section 1.4 of Wilder et al. 1997). This is in contrast to the important role of the in-situ thermal tests identified by Hardin (1998, Section 3.4.1) in the calibration of thermohydrologic properties that are required by models supporting the Total System Performance Analysis for Viability Assessment (TSPA-VA) (CRWMS M\&O 1998) and the Total System Performance Analysis (TSPA) for Site Recommendation and License Application (CRWMS M\&O 1999b).

The question of how best to build confidence in models has been the focus of considerable discussion over the years. Validation of models was discussed frequently in the Yucca Mountain Site Characterization Plan (DOE 1988, Section 8.3.5.20.), and considerable debate developed over how to validate models (see, for example, Buscheck and Nitao [1995, p. 9]). The concept of testing to build confidence in models replaced the concept of validation, and this was the objective of the LBT. As noted by Konikow and Bredehoeft (1992), models "...provide a means to organize our thinking, test ideas for their reasonableness, and indicate which are the sensitive 
parameters. They point the way for further investigation. They help formulate critical experiments with which to test hypotheses."

Buscheck and Nitao (1995, p. 9) note that no individual model is itself a "valid" representation. However, the combined use of suites of model calculations provides a means to identify critical dependencies, evaluate worst-case scenarios, and develop fundamental hypotheses that can be addressed by subsequent analysis and testing. Buscheck and Nitao (1995, p. 9) identified a list of major hypotheses, based on model analyses, that should be tested in field tests. Buscheck noted in Volume II of Wilder (1996, Section 1.9.2, p. 1.9-4) that field tests-such as the LBTperformed at Fran Ridge and the in-situ thermal tests-such as SHT and DST-at the Exploratory Studies Facility (ESF) will provide the most conclusive means of evaluating issues associated with thermal loading, including resolution of the major hypotheses.

The objectives of the LBT, as stated in the activity plan, AP-LBT-01, (Lin 1994), are to:

- Assist in understanding the processes that are expected to occur within the NF and the Altered Zone (AZ).

- Provide a test of the models and conceptualizations.

- Allow evaluation of techniques and equipment that will be used in subsequent in-situ tests. (This objective is less applicable because the LBT did not start early enough to provide these data prior to fielding of the in-situ tests.)

\subsubsection{Specific LBT Design and Implementation Objectives}

As noted, the objectives of the LBT were to build confidence in the models used in assessing the evolution of the NFE. Understanding the coupled THMC processes was one of the primary objectives of the LBT. Developing a proper understanding of that coupling required that the test be conducted within a rock mass with characteristics as similar as possible to those of the potential repository horizon. Therefore, specific objectives were developed to determine the selection of the test location and the specific rock units that would be tested, the size and configuration of the test, and the basic operation and layout of the test.

Location and rock unit selection were based on the following criteria:

- Rock unit would be representative of that of the potential repository horizon. On this basis, the middle non-lithophysal zone of the Topopah Spring welded unit was selected as the target. This was consistent with the unit that would be used for underground testing.

- The bulk air permeability in the block be greater than $0.1 \mathrm{md}\left(10-16 \mathrm{~m}^{2}\right)$.

- The initial water saturation in the block should be at least $50 \%$. 
- Fracture sets should be similar to those of the potential repository. It was judged that the majority of fractures would be high-angle fractures with fracture density of $\sim 40$ fractures $/ \mathrm{m}^{3}$.

- Block size should be sufficient to include major and minor fractures. It had been noted that the majority of flow in fracture-dominated flow systems occurs only within a small percentage of the fractures $(5-10 \%$ at most). Therefore, to increase the potential to measure the effect of fracture on flow, an objective was to include sufficient number of fractures within the rock volume so that at least a few major fractures would be present. Thus, the block should be of sufficient size to contain about 100 fractures. Based on 40 fractures $/ \mathrm{m}^{3}$ (mostly high-angle fractures so that there would be approximately 30 fractures $/ \mathrm{m}^{2}$ along a horizontal plane), a block with $3.5 \mathrm{~m}^{2}$ in the horizontal plane would be the minimum size.

- Three-dimensional characterization and monitoring would be provided. This objective was achieved by exposing the block on all sides and on the top. The fractures in the block could be characterized from the five exposed surfaces and the boreholes. This fracture information facilitated the analysis of the test results and a better understanding of the completed processes.

- A zone of dryout sufficient to mobilize water and to support a reflux zone would be developed. A dryout zone of approximately $0.75-1 \mathrm{~m}$ was desired.

Detailed site-selection processes are presented in Section 3.5.1.1.

To build confidence in the models, the LBT needed to be designed to properly monitor the processes that would be included in the models. Therefore, one of the specific objectives for the LBT was to focus the design of the test on those coupled processes of concern identified by model analyses. Thus, the LBT design was based on numerical modeling of the important aspects of a potential repository, on previous laboratory and field studies, and on recommendations derived from the results of those studies. The LBT objectives were to address those issues identified by modeling as significant to the evolution of the NFE. The specific objectives were to design a test that could help determine: (1) the dominant heat-transfer mechanism, (2) if there is coincidence of the dryout zone and the boiling-point isotherm, (3) refluxing of condensate water above the heated zone, (4) the change of water chemistry in the condensate zone, (5) the mechanical responses of the block, and (6) the relation between rewetting and cooling of the dryout zone.

One major issue, related to objectives 2 and 3, was to determine the likelihood of refluxing and the conditions under which it can occur-more specifically, to determine whether condensate can build up above the heated zone (and thus the repository drifts) or if it would be transported by shedding to regions below the heated zone. Another issue was to determine if condensate will penetrate the boiling zone, whether dryout can occur, and whether temperatures will be limited to the boiling point. 
Although the potential for condensate buildup and shedding is design-specific, another objective was to design the LBT to maximize the potential for observations while reflecting the likely overall repository design. The basic repository feature that was considered in the design objective was a series of parallel emplacement drifts that might develop a horizontal planar region of boiling. This would be the condition that would result in the greatest likelihood of condensate buildup, rather than shedding between drifts. Therefore, a specific objective was to design the LBT to create and monitor one-dimensional heating.

The one-dimensional heating objectives were:

- Fracture permeability in the range of those expected at the potential repository horizon.

- Sufficient vertical extent to allow heat pipe zones to form and to have a large enough zone for geochemical/mineral alteration to be sampled. It was judged that a reflux model analysis of a heat zone of approximately $1 \mathrm{~m}$ would be required.

One specific objective of the LBT, in contrast with other field tests, was to provide controlled boundary conditions. This allowed observation and testing of some of the specific coupled THMC processes that could not be controlled or facilitated in a more open system. As an example, the heat loss could be either controlled or monitored. Also, the temperatures at the top could be maintained so that reflux cycles can be provided and monitored.

One of the major advantages of the LBT was that geochemical processes could be evaluated by carefully observing the mineralogy that develops within the block in response to the refluxing, because the refluxing zone can be well identified. The LBT is the only field-scale test that allows for such analysis. Although this was not the only, or even the primary, objective of the test, it was nonetheless a critically important one.

The LBT had as a secondary objective to evaluate the responses of introduced materialsspecifically cement and steels typical of waste packages (WPs)-to the potential environmental conditions of the repository. Coupons of certain candidate WP materials placed in the block were examined before and after the test for their response to the environment. Furthermore, the LBT provided an opportunity to evaluate microbial survival and mobility.

\subsection{CONFIGURATION OF THE LBT}

The test location was selected at Fran Ridge to reflect the specific objectives regarding rock unit, fracture characteristics, and permeability. Detailed discussions of the site selection and characterization can be found in Sections 2.1 and 2.2 of the Large Block Test Status Report (Wilder et al. 1997) and are summarized in Section 3.5 of this report.

To observe coupled THMC behavior, the size of the test was chosen so that the block of rock to be heated was large enough to contain several fractures but still small enough so that boundary conditions and rock heterogeneity could be adequately controlled and/or characterized without undue cost. 
A rectangular prism of rock $3 \mathrm{~m} \times 3 \mathrm{~m}$ in cross-section and $4.5 \mathrm{~m}$ high was exposed from an outcrop of fractured rock at Fran Ridge by excavating the surrounding rock, as shown in Figure 3-1.

In general, two subvertical sets (one dominant) of major fractures and one set of subhorizontal fractures intersect the block (Figures 3-2 and 3-3). The subvertical fracture sets are approximately orthogonal, with spacing of 0.25 to $1 \mathrm{~m}$, and are oriented generally in the NW-SE (dominant set) and NE-SW. Moreover, a major subhorizontal fracture is located approximately $0.5 \mathrm{~m}$ below the top surface. This fracture is visible in Figure 3-3. More detailed analyses (Wagoner 1999) identified a total of six fracture systems within the LBT rock mass, which are summarized in Table 3-1. The fracture analysis is presented in Section 4.

Table 3-1. LBT Fracture Systems

\begin{tabular}{|c|c|}
\hline System \# & Strike and Dip Direction \\
\hline 1 & Strike: N50E \\
& Dip: $40-45^{\circ} \mathrm{NW}$ \\
\hline 2 & Strike: $\mathrm{N30-40 \textrm {W }}$ \\
& Dip: $60-80^{\circ} \mathrm{NE}$ \\
\hline 3 (contains the most significant fracture in the LBT) & Strike: generally NW \\
& Dip: mainly $20 \mathrm{SW}$ \\
\hline 4 (contains the greatest number of fractures) & Strike: range from N03 to $34 \mathrm{~W}$ \\
& Dip: $77-89^{\circ} \mathrm{SW}$ \\
\hline 5 & Strike: general east-west \\
& Dip: near vertical \\
\hline 6 & Strike: northeast \\
& Dip: near vertical \\
\hline
\end{tabular}

The heating duration of the test was designed to provide a sufficient length of time for thermalhydrological-chemical (THC) processes to develop. The heating phase of the LBT began on February 28, 1997, and ended on March 10, 1998, when the heaters were turned off to begin a natural cooling phase. The cooling of the block was monitored until September 30, 1998, when it was determined that the block had returned to ambient temperature and the Data Acquisition System was turned off. During the heating phase the block was heated from within to reach a temperature of about $140^{\circ} \mathrm{C}$ at the heater horizon, and a heat exchanger was used to keep the top temperature at about $60^{\circ} \mathrm{C}$. During the last four months of the heating phase, the heater power was reduced to maintain an almost constant temperature.

The desired thermal regime was determined by scoping TH calculations, as described in Section 3.5.2.1. For ease of thermal modeling, a one-dimensional thermal field within the block was created by line heaters used to simulate a planar heat source located at a height of one-third of the total height of the block ( $1.75 \mathrm{~m}$ from the base of the block). A heat exchanger system was used to maintain a constant temperature on the top surface of the block. This system consisted of an aluminum plate fitted with heating/cooling coils mounted on the top of the block. This plate was connected to a heat exchanger to allow thermal control of the top surface. The sides of the block were insulated. A planar zone of boiling was created by imposing and maintaining this 
one-dimensional thermal gradient within the rock mass. Details of the heating/cooling system are given in Section 5.1.

In order to achieve a one-dimensional thermal-hydrological process, a layer of room temperature vulcanized rubber (RTV) and Viton were installed on the block sides to minimize moisture flux. Three layers of thermal insulation materials were installed on the outside of the moisture barrier. All of the instrument holes were sealed by cement grout, packers, or an RTV/Teflon membrane.

\subsection{INSTRUMENTATION, MONITORING AND CHARACTERIZATION}

Because the primary purpose of the LBT was to provide data to test model conceptualizations and applicability to the near field (NF) analyses for the YMP, the LBT was very thoroughly monitored by instrumentation emplaced in boreholes and on the block surface. These boreholes, as shown in Figure 3-4, also provided core and borehole video images to augment the characterization of the block from the five exposed surfaces. This provided excellent threedimensional characterization of the fractures within the block, as shown in Figures 3-5 and 3-6, as well as in Figure 3-3.

The instrumentation installed was intended to give data for three principal process models: thermal, hydrological, and mechanical. For purposes of monitoring and discussion of the instrumentation, these process models are considered as single coupled models. In reality, the coupling is much more complex and fully coupled, but for ease of discussion as well as instrumentation design the monitoring considered the single coupling. The models for which monitoring through instrumentation was performed were those that addressed the thermal responses of the rock mass (including fluids) to imposition of heat, simulating that from emplaced waste; secondly, the models used to analyze hydrological coupling to the resulting thermal regime; and third, the mechanical response to the thermal regime. In addition to these primary areas of monitoring, geochemical coupling was considered. However, there were no boreholes or instrumentation provided for active monitoring for geochemistry because there were no chemical sensors available for monitoring unsaturated medium, and sampling procedures would impact the test. Microbial monitoring was also provided for holes drilled for other monitoring purposes.

The boreholes and instrumentation were categorized in accordance to the objectives of that monitoring (e.g., hydrology, temperature, mechanical). The boreholes, with instrumentation types installed and the size of the boreholes, are listed in Table 3-2. The holes listed according to the block faces are shown in Appendix A. The $x-y-z$ coordinates of all of the sensors in the LBT are shown in Appendix B.

Figures 3-7 to 3-10 show the location of instrumentation holes within the block (on top, north, east, and west faces respectively). There is no instrumentation hole in the south face. 
Table 3-2. Borehole and Instrument Listing by Instrument Type

\begin{tabular}{|c|c|c|c|}
\hline $\begin{array}{c}\text { Hole or } \\
\text { instrument } \\
\text { number }\end{array}$ & Description & Installation method & $\begin{array}{l}\text { Borehole } \\
\text { size }(\mathrm{cm})\end{array}$ \\
\hline \multicolumn{4}{|c|}{ Heater holes } \\
\hline $\mathrm{EH} 1$ & Power and temperature & Open hole with a plug at the collar & 3.81 \\
\hline $\mathrm{EH} 2$ & (same) & (same) & 3.81 \\
\hline $\mathrm{EH} 3$ & (same) & (same) & 3.81 \\
\hline $\mathrm{EH} 4$ & (same) & (same) & 3.81 \\
\hline EH5 & (same) & (same) & 3.81 \\
\hline Total $=5$ & & & \\
\hline \multicolumn{4}{|c|}{ Neutron holes } \\
\hline TN1 & Neutron liner grouted in place & $1 / 16 / 97$, grouted & 3.81 \\
\hline TN2 & Neutron liner grouted in place & $1 / 16 / 97$, grouted & 3.81 \\
\hline TN3 & Neutron liner grouted in place & $1 / 16 / 97$, grouted & 3.81 \\
\hline TN4 & Neutron liner grouted in place & $1 / 16 / 97$, grouted & 3.81 \\
\hline TN5 & Neutron liner grouted in place & $1 / 16 / 97$, grouted & 3.81 \\
\hline NN1 & Neutron liner grouted in place & $1 / 16 / 97$, grouted & 3.81 \\
\hline NN2 & Neutron liner grouted in place & $1 / 16 / 97$, grouted & 3.81 \\
\hline NN3 & Neutron liner grouted in place & $1 / 16 / 97$, grouted & 3.81 \\
\hline NN4 & Neutron liner grouted in place & $1 / 16 / 97$, grouted & 3.81 \\
\hline NN5 & Neutron liner grouted in place & $1 / 16 / 97$, grouted & 3.81 \\
\hline NN6 & Neutron liner grouted in place & 1/16/97, grouted & 3.81 \\
\hline WN1 & Neutron liner grouted in place & 1/16/97, grouted & 3.81 \\
\hline WN2 & Neutron liner grouted in place & $1 / 16 / 97$, grouted & 3.81 \\
\hline WN3 & Neutron liner grouted in place & $1 / 16 / 97$, grouted & 3.81 \\
\hline WN4 & Neutron liner grouted in place & 1/16/97, grouted & 3.81 \\
\hline Total $=15$ & & & \\
\hline \multicolumn{4}{|c|}{ RTD holes } \\
\hline TT1 & RTDs grouted & 30-RTD bundle & 3.81 \\
\hline TT2 & RTDs grouted & 30-RTD bundle & 3.81 \\
\hline NT1 & RTDs grouted & 14-RTD bundle & 3.81 \\
\hline NT2 & RTDs grouted & 14 RTDs-larger borehole to grout & 7.62 \\
\hline NT3 & RTDs grouted & 14 RTDs-larger borehole to grout & 7.62 \\
\hline NT4 & RTDs grouted & 14-RTD bundle & 3.81 \\
\hline WT1 & RTDs grouted & 14-RTD bundle & 3.81 \\
\hline WT2 & RTDs grouted & 14-RTD bundle & 3.81 \\
\hline WT3 & RTDs grouted & 14-RTD bundle & 3.81 \\
\hline Total $=9$ & & & \\
\hline \multicolumn{4}{|c|}{ MPBX holes } \\
\hline TM1 & Mech MPBX grouted in & & 7.62 \\
\hline NM1 & Mech MPBX & Grouted after install & 7.62 \\
\hline NM2 & Mech MPBX & Grouted after install & 7.62 \\
\hline NM3 & Mech MPBX & Grouted after install & 7.62 \\
\hline WM1 & Mech MPBX & Grouted after emplacement & 7.62 \\
\hline WM2 & Mech MPBX & Grouted after emplacement & 7.62 \\
\hline WM3 & Mech MPBX & Grouted after emplacement & 7.62 \\
\hline Total $=7$ & & & \\
\hline
\end{tabular}




\begin{tabular}{|c|c|c|c|}
\hline $\begin{array}{c}\text { Hole or } \\
\text { instrument } \\
\text { number }\end{array}$ & Description & Installation method & $\begin{array}{l}\text { Borehole } \\
\text { size }(\mathrm{cm})\end{array}$ \\
\hline \multicolumn{4}{|c|}{ Optical extensometer } \\
\hline TM2 & $\begin{array}{c}\text { Optical extensometer with } \\
\text { liner }\end{array}$ & & 7.62 \\
\hline Total $=1$ & & & \\
\hline \multicolumn{4}{|c|}{ ERT hole } \\
\hline ERT & ERT electrodes & Grouted after installation & 3.81 \\
\hline \multicolumn{4}{|l|}{ Total $=1$} \\
\hline \multicolumn{4}{|c|}{ Hydro packers } \\
\hline TH1 & Hydrol w/ packer & $\begin{array}{c}\text { High-temperature packers with gas } \\
\text { pressure transducers, humidity } \\
\text { sensors, and introduced material } \\
\text { coupons }\end{array}$ & 7.62 \\
\hline $\mathrm{NH} 1$ & Hydrol w/ packer & (Same as above) & 7.62 \\
\hline WH1 & Hydrol w/ packer & (Same as above) & 7.62 \\
\hline WH2 & Hydrol w/ packer & (Same as above) & 7.62 \\
\hline Total $=4$ & & & \\
\hline \multicolumn{4}{|c|}{ Observation } \\
\hline NO1 & Observation hole & Pyrex liner w/crape paper-pvc support & 3.81 \\
\hline NO2 & Observation hole & Pyrex liner w/crape paper-pvc support & 3.81 \\
\hline WO5 & Observation hole & Pyrex liner w/crape paper-pvc support & 3.81 \\
\hline EO3 & Observation hole & Pyrex liner w/crape paper-pvc support & 3.81 \\
\hline \multicolumn{4}{|l|}{ Total $=4$} \\
\hline $\begin{array}{c}\text { TR1 } \\
\text { WR1 } \\
\text { WR2 }\end{array}$ & $\begin{array}{l}\text { REKA (Rapid Evaluation of K } \\
\text { and Alpha) }\end{array}$ & Grouted after install & 1.27 \\
\hline
\end{tabular}

\subsubsection{Installation of Instrumentation and Data Collection Systems}

Monitoring instruments were installed within the block during the period from December 10, 1996, to February 28, 1997. This included RTDs, ERT electrodes, MPBX systems, neutron hole liner, fracture monitors, REKA probes, packers, heaters, and heat-exchange unit.

The block was sealed with thermal and moisture barriers on its four sides. The moisture barrier included a layer of high-temperature RTV silicone sealant overlain with a layer of hightemperature Neoprene rubber sheeting (Viton). The RTV was troweled into the block, with special attention to sealing fractures along the block surface. RTV-sealed surfaces acted as a bind for the Neoprene, which was applied while RTV was still curing. The LBT featured two data acquisition modes: automated data acquisition by a data acquisition system (DAS) and manual data acquisition. The data acquired by the DAS included temperature, gas pressure, displacement, wattage, humidity, and REKA probes. Data being collected manually included neutron logging, ERT, and air permeability. Data from the DAS were transferred to the Test Coordination Office (TCO) for incorporation into their data base and transferred to Lawrence Livermore National Laboratory (LLNL) via compact disks (CDs) to be downloaded to the LLNL database. 


\subsection{MEASUREMENTS}

\subsubsection{Thermal Monitoring}

\subsubsection{Temperature}

The temperature measurements included the spatial and temporal variation of the temperature in the block and the thermal gradient on the block surfaces. Resistance temperature devices (RTDs) were used to measure temperatures in the block, and temperature was measured in nine RTD holes and five heater holes (see Table 3-2). The RTD holes were instrumented with RTDs at 20 -cm spacing. This was accomplished by grouting a bundle of RTDs with cement in each of the temperature holes. In addition, five RTDs were placed in a thin-walled stainless-steel tube so that they could be calibrated or replaced during the test. The stainless-steel tube was grouted along with the RTD bundle in-hole TT1. Three RTDs were placed in each of the five heater holes at about $0.6,1.5$, and $2.4 \mathrm{~m}$ from the collar. The thermal gradient to determine heat flux out of the block across the block surface was measured by a pair of RTDs on both sides of a 1.2-cmthick Ultratemp insulation panel. Ultratemp panels were mounted on the four vertical faces of the block, on the outside of the Viton sheet.

\subsubsection{In-situ Thermal Conductivity and Thermal Diffusivity}

In-situ measurements of the thermal conductivity and thermal diffusivity of the large block were done by using the Rapid Evaluation of K and Alpha (REKA) thermal probe method (Danko and Mousset-Jones 1991). The REKA probe method incorporated the application of three small probes $(0.013-\mathrm{m}$ diameter and $0.125-\mathrm{m}$ length) that were cemented in the REKA holes Table 3-2). The probes were heated by built-in heaters of $2 \mathrm{~W}$ during the measurement period of 24 hours. When not activated, the probes were not heated.

The temperature fields along the probes were recorded by a Hewlett Packard data acquisition system that was remotely controlled from Reno. The readings were recorded on disks, as well as entered into the YMP TCO database. After the original data disks were brought to Reno, the temperature fields were evaluated against the unknown thermophysical properties, such as heat conductivity (k) and thermal diffusivity (alpha).

The $\mathrm{k}$ and alpha values, as well as the undisturbed rock temperatures during the REKA measurements, were plotted versus the time of measurement, measured in days from the start (i.e., day zero) of the main heating of the rock.

\subsection{REKA Measurement and Evaluation Principles}

The REKA method involves a single borehole probe with a heater and a temperature measurement section. An elliptical temperature field is generated by the heater, and the temperature distribution along the length of the probe is recorded at several locations and at given time intervals for a period of 24 hours. An optimization procedure is used to determine the unknown thermophysical properties by minimizing the root-mean-square (RMS) error between the measured and the calculated temperature fields. The rock mass and its moisture content affect the thermophysical properties, and the values represent effective properties. 
The unknown $\mathrm{k}$ and alpha are determined by finding the values that satisfy the best least-squarefit between the measured (TM) and predicted (ts) temperature fields using all the temperature stations and measurement readings with respect to time:

$$
\sum_{1}^{8} \sum_{1}^{100}(T M-t s)^{2}=\min
$$

where

$$
\text { ts }=f(k, \text { alpha, geometry, time })
$$

The $\mathrm{k}$ and alpha values in Equation 3-2 are systematically varied until the left-hand-side of Equation 3-1 is minimized. These values for the minimum error are the results of the measurement evaluation.

\subsection{The Measurement Arrangement}

Three permanent REKA probes were grouted into the large rock block at Fran Ridge. The locations of the three probes are given in Figure 3-11. The horizontal REKA Probe 1 (WR2 in Figure 3-10) was installed $0.584 \mathrm{~m}$ below the rock block's horizontal heater layer (HHL); the horizontal REKA Probe 2 (WR1 in Figure 3-10) was installed $0.889 \mathrm{~m}$ above the HHL; and the vertical REKA Probe 3 (TR1 in Figure 3-7) was installed with its representative sensor location $1.38 \mathrm{~m}$ above the HHL. Each probe reading approximately represents a $0.1-\mathrm{m}$-diameter spherical rock volume. The measurement system, shown in Figure 3-12, consisted of an HP75000 data acquisition unit and a personal computer controller with a phone modem for remote communication. The REKA probe consisted of a linear array of nine thermocouples, centered $0.0125 \mathrm{~m}$ apart and systematically arranged around the heater, and a thin-foil heater powered by $2 \mathrm{~W}$ using an HP3611A DC power supply. The thermocouples were mounted on a flexible, perforated, Kapton foil for sufficient grout bonding.

\subsubsection{Hydrological Monitoring}

Spatial distribution and temporal variation of moisture content in the rock were monitored using Electrical Resistance Tomography (ERT) and neutron logging. Relative humidity and gas pressure were measured in the packed hydrological holes. ERT is a geophysical imaging technique that can be used to map subsurface resistivity (Daily and Owen 1991). The ERT measurements consisted of a series of voltage and current measurements from buried electrodes using an automated data collection system. The data were then processed to produce electrical resistivity tomographs. The images of resistivity change can be used, along with the measured temperature field and what is known of initial conditions in the rock mass, to estimate moisture change during heating. ERT electrodes were placed on the surface of the block and in one borehole, as shown in Table 3-2.

Neutron logging was conducted periodically in 15 holes (see Table 3-2). These holes were lined with a Teflon tube liner that was grouted in place. The annular space between the liner and borehole was sealed with cement grout. Neutron logs of these holes were taken approximately once per month during the test. 
Relative humidity $(\mathrm{RH})$ and gas pressure were measured in four packed hydrologic holes (TH1, NH1 and WH1, and WH2), as shown in Figures 3-7, 3-8, and 3-10. The results of those measurements are discussed in Section 9.2.

In addition, four horizontally oriented observation boreholes were drilled near the bottom of the block for observing water drainage away from the block (see Table 3-2).

\subsubsection{Mechanical Monitoring}

The overall three-dimensional geomechanical response of the rock to the heating was monitored using six multiple-point borehole extensometers (MPBX) (Figure 3-13). Three were oriented in the north-south direction, two were oriented in the east-west direction, and one was oriented vertically (see Table 3-3). In addition, deformation of several major fractures that intersect the block sides was monitored using 3-component fracture gauges. These were installed at 17 locations on the surface of the block. The fracture gauges measured movement in directions across the fracture and along the trace of the fracture, both parallel and perpendicular to the face.

A prototype optical extensometer was also deployed in one borehole, TM2, as shown in Table 3-2.

\subsubsection{Microbial Monitoring}

Microbes were introduced in the block so that their survivability and migration could be studied. The transport of bacteria in the LBT was investigated by first isolating two bacterial species, Bacillus subtilis and Arthrobacter oxydans, from the local tuff at the block site. Natural mutants that can grow under the simultaneous presence of the two antibiotics, streptomycin and rifampicin, were selected from these species by laboratory procedures. The characteristics of double-drug resistance distinguish the natural mutants from the indigenous species. The mutants were cultured and then injected into the five heater boreholes of the large block hours before heating was initiated.

Bacterial samples were then collected from the observation boreholes that were approximately $1.5 \mathrm{~m}(5 \mathrm{ft}$ ) below the injection (heater) holes. Cells that were possibly present in the heater boreholes were collected when the heaters were removed after the entire LBT experiment was completed. The result of the microbial monitoring is presented in Section 9.3.

\subsubsection{Assessment of Rock-Water Interaction}

There was no in-situ chemical monitoring in the LBT. There was no plan to sample the water in the block during the test, because no significant amount of water was expected to be collected. Geochemical processes were investigated by comparing the mineralogical composition of the pre-test and post-test rock samples.

\subsubsection{Other Monitoring}

Coupons of waste package material and manmade material were placed in the packed hydrological boreholes in the block so that they could be examined. The analysis of those coupons has not been performed. 


\subsection{OVERVIEW OF CHRONOLOGICAL ACTIVITIES}

\subsubsection{Block Preparation and Pre-test Characterization}

\subsubsection{Site Selection}

The work site at Fran Ridge, Nevada Test Site, was selected for the LBT because of its desirable rock type, fracture characteristics, and accessibility. The LBT was first located, in general terms, on the basis of a location that was cleared from an environmental standpoint for development but that was also in the general vicinity of the two U.S. Geological Survey (Sweetkind et al. 1995, p. 36) test pits that provided fracture characterization data and observation access. The location chosen is on a slope on the side of Fran Ridge.

The general location was stripped of vegetation and soil covering to expose the rock. The area was then mapped, noting observed fractures. The mapping was corrected for the slope of the outcrop by use of levels, plumb bob, and tape measures. The results of the mapping are shown in Figure 3-14. Based on this mapping, it was judged that the general site was adequate for the LBT, specifically that the rock fracturing and matrix block sizes were consistent with what was anticipated to exist at YM (Wilder et al. 1997, Section 2.1). Block preparation proceeded by excavating a level surface from which to work (Figure 3-15) and providing a sump to collect and recirculate water that would be used during sawing of the sides of the block. After the surface was leveled, more detailed mapping was performed.

As noted in Section 3.1.2, it was judged that approximately 100 fractures needed to be present to have a few fractures that would be open to flow of the magnitudes of interest. Possible locations for the LBT were then selected where a sufficient number of fractures would be included in the block to account for observed flow in fractured rock wherein less than $10 \%$ of the fractures were responsible for $90 \%$ of the flow. Pre-test thermal-hydrological (TH) and thermal-mechanical (TM) calculations, documented in Section 3.5.2 of this report, were performed to verify that the block size was suitable. It was deemed important to measure permeabilities to ensure that there were adequate zones for the tests and that the fractures were neither too open, because of being near the surface, nor too plugged by calcite. (The top portion of rock was removed to obtain a level surface and to place the test beneath a possible zone where calcite filled the fractures, as was observed in the two USGS test pits or holes.) A pattern of vertical instrumentation and measurement boreholes was laid out. These holes also served as exploratory boreholes. The pattern was arranged so that the LBT could be centered around one of two different boreholes that would become either the center or the edge of the array of instrument boreholes. Air permeability tests were then conducted to assist in selecting a final location that had sufficient permeability for conducting the LBT.

\subsubsection{Block Preparation}

Once the site for the LBT was selected, numerous vertical instrumentation boreholes were drilled and cored within the block for vertical rock bolts at one midpoint on each side and one in the center of the block as well as numerous instrumentation boreholes. Neutron logging was conducted in a few of those vertical holes to determine the initial moisture content in the rock. (See Section 6.1.2.2.1 for greater details.) The initial water saturation was determined to be about 
60-80\% (Figure 2-22 of Wilder et al. 1997), which was greater than the required $50 \%$, as stated in Section 3.1.2. Neutron logging was repeated after the cutting of the block was completed to determine the effect of the wet-cutting of the block on the moisture content, and to determine the actual initial moisture content before heating was started. The block was then isolated by cutting slots, as shown in Figure 3-16, along its boundary. A belt saw was used to saw four vertical slots that formed the boundary of the large block. At the completion of cutting of each of these slots, the slots were filled with expanding foam in plastic bags to support the block and to isolate it from the effects of excavation-induced damage (e.g., from vibration). A large hydraulic jack hammer was used to excavate the surrounding rocks in about 2-to-3-ft vertical sections (see Figure 3-17, which shows the partial excavation). After excavation of the upper level (about $1 \mathrm{~m}$ ) was completed, the top of the block was trimmed to its final surface using a commercial wire saw, and a top cross member (fabricated from welded steel I-beams) was placed on the top and connected to rock bolts on the four sides and center. (The rock bolt holes were N4 to N7 and E4 in Figure 2-7 of Wilder et al. [1997]). Excavation then proceeded in 2-to-3-ft stages with the emplacement of trucker-type straps. Some smaller blocks of rock, about $30 \mathrm{~cm}$ in size, were collected within a 1-m region around the large block for laboratory tests. By this excavation process, a block of Topopah Spring tuff measuring $3 \times 3 \times 4.5 \mathrm{~m}$ was isolated at Fran Ridge.

After block excavation was completed, a concrete pad was poured around the base of the block. As illustrated in Figure 3-1, all block surfaces were mapped, and horizontal boreholes were located for the emplacement of instrumentation and heaters. The horizontal instrumentation boreholes were drilled using a drill rig mounted on a built-up steel platform (Figure 3-18). The location of boreholes and types of instruments were discussed in Section 3.3. The heater plane consisted of a series of five heater boreholes drilled from the east face approximately $1.8 \mathrm{~m}(6 \mathrm{ft})$ above the pad at the base of the block and equally spaced to form a line of horizontal heater boreholes.

\subsubsection{Block Characterization}

Characterization of the test block is very important for helping the understanding of the test observations and results. The characterization of the block was treated as an integral part of the LBT. The characterization began with mapping the fractures on the Topopah Spring tuff outcrop at Fran Ridge, as mentioned in Section 3.5.1.1, and reported in Section 2.1 of the LBT Status Report (Wilder et al. 1997). The characterization of the test block included mapping and analysis of the distribution of fractures, determination of the mechanical and hydrologic properties in the laboratory, the initial moisture content, the bulk air permeability, and the mineralogical composition of the matrix and the fracture coating. The fracture characterization of the block is presented in Section 4.0 of this report. The measurement of the bulk air permeability of the block is presented in Section 6.1.1.1.1 of this report. The determination of the initial moisture content of the block is presented in Section 6.1.2.2.1 as the baseline moisture content of the neutron logging. The determination of the mineralogical composition is presented in Section 8. The pre-test and post-test characterization of the mechanical properties and the ultrasonic wave velocity are presented in Section 7.2. This section presents the laboratorydetermined hydrological properties and processes of the LBT samples. Those hydrological properties include porosity, water permeability, and moisture retention curves. The hydrological process investigated was fracture flow and matrix imbibition. 


\subsection{Laboratory-Determined Water Permeability of the Matrix}

Permeability is one of the basic hydrologic properties required for any hydrological modeling. The water permeability of the Topopah Spring tuff samples obtained from Fran Ridge was measured in the laboratory as a function of temperature. The technique of measuring the permeability was the steady-state flow-through method. A detailed description of the methodology and permeability measuring techniques was reported in Lin and Daily (1984). Core samples of about $2.54 \mathrm{~cm}$ in diameter and $5.1 \mathrm{~cm}$ in length were prepared from small block SPC00504573 collected at Fran Ridge during the excavation of the large block, with core identification numbers SPC00504573.4 and SPC00504573.5. The test sample was first saturated with water. Then the sample was encapsulated in membrane, which separated the sample from the confining pressure fluid. The sample assembly was placed within a pressure vessel with independently controlled confining pressure, pore water pressure, and temperature. The sample was brought to an equilibrium of certain temperature, confining pressure, and pore pressure. A differential pressure across the length of the sample was created to cause a flow. The steadystate flow rate was measured. The permeability was calculated using Darcy's equation, assuming the pore pressure gradient is linear. For water flow along the sample axis,

$$
k=\mu \frac{Q l}{A \Delta P}
$$

where $\mathrm{k}$ is the permeability, $\mu$ is the viscosity of water (cp), $\mathrm{Q}$ is the flow rate $\left(\mathrm{cm}^{3} / \mathrm{s}\right), \mathrm{A}$ is the area of the sample $\left(\mathrm{cm}^{2}\right)$, and 1 is the sample length $(\mathrm{cm})$. The viscosity of water at each temperature was obtained from Eisenberg and Kauzmann (1969). The permeability has units of $\mathrm{m}^{2}$ and was converted to the standard unit of permeability, the Darcy, where one Darcy equals $9.87 \times 10^{-13} \mathrm{~m}^{2}$. The error in the permeability measurement depends on a number of factors and, through the propagation of errors in the above equation (e.g., Bevington and Robinson 1992), is estimated to be less than $3 \%$.

The measurement equipment used in the permeability measurement included a confining pressure transducer, pore pressure transducer, differential pressure transducer, and thermocouple to measure temperature. The flow rate was determined by letting water flow into a container on a balance. The weight of the balance corresponds to the volume of water that has flowed through the sample and is recorded by a computer, along with all the other data such as time, temperature, differential pressure, pore pressures, and confining pressure. Because the flow rate is low, it was necessary to consider the rate of evaporation from the collection bottle. This was found to be linear with time over a period of about one week. The water lost due to evaporation was $4.13 \mathrm{mg} /$ hour. This lost water was added to the balance reading for a specific period of time when calculating permeability.

The water preliminary data are summarized in Table 3-3. The permeability of the intact Topopah Spring tuff sample was less than $10^{-18} \mathrm{~m}^{2}$. This permeability value is consistent with that measured in cores from Yucca Mountain (Lin and Daily 1984). It is also shown in Table 3-3 that intact sample permeability was not a strong function of temperature. This is also consistent with that reported by Lin and Daily (1984). 
Table 3-3. Permeability Measurements on Intact Core Sample SPC00504573.4

\begin{tabular}{|c|c|c|c|}
\hline Temperature, ${ }^{\circ} \mathbf{C}$ & Confining Pressure, $\mathbf{M P a}$ & $\begin{array}{c}\text { Differential Pressure, } \\
\mathbf{M P a}\end{array}$ & Permeability, $\mu \mathrm{D}$ \\
\hline 23 & 5.06 & 1.92 & 0.12 \\
\hline 25 & 5.07 & 2.47 & 0.14 \\
\hline 53 & 5.06 & 2.42 & 0.11 \\
\hline 53 & 5.06 & 1.91 & 0.15 \\
\hline 91 & 5.06 & 2.17 & 0.14 \\
\hline 92 & 5.06 & 1.60 & 0.14 \\
\hline 154 & 5.06 & 1.61 & 0.09 \\
\hline 130 & 5.05 & 1.46 & 0.13 \\
\hline 130 & 5.05 & 2.04 & 0.11 \\
\hline 83 & 5.06 & 2.02 & 0.17 \\
\hline 26 & 5.06 & 2.59 & 0.67 \\
\hline 26 & 5.06 & 2.61 & 0.20 \\
\hline
\end{tabular}

\subsection{Moisture Retention Curves}

The moisture retention curve of rocks is needed for modeling the hydrological processes in a partially saturated medium. The determination of the moisture retention curves was described in Roberts and Lin (1996a). The moisture retention curve is the relationship between matric potential and water saturation. In tuff we assume that the suction potential is equal to the matric potential. Matric potential is defined by Kelvin's Law as

$$
\psi=\frac{\beta R T \ln \left(e / e_{0}\right)}{M}
$$

where

$$
\begin{aligned}
& \psi=\text { matric potential in } \mathrm{MPa} \\
& \beta=\text { density of water at the temperature of interest in } \mathrm{g} / \mathrm{cm}^{3} \\
& \mathrm{R}=\text { universal gas constant }(8.314 \mathrm{~J} / \mathrm{Kmol}) \\
& \mathrm{T}=\text { temperature in } \mathrm{K} \\
& \mathrm{e} / \mathrm{e}_{0}=\text { relative humidity, and } \\
& \mathrm{M}=\text { molecular weight of water }(18 \mathrm{~g} / \mathrm{mole})
\end{aligned}
$$

Samples from the LBT were sub-cored to a diameter of $19.1 \mathrm{~mm}$ and cut into disks approximately $2.5 \mathrm{~mm}$ thick. Samples with obvious large cavities and inhomogeneous inclusions were avoided. The average porosity of the 12 samples is $10.4 \pm 1.3 \%$ and was determined by subtracting the dry density from the saturated density and dividing by the water density. The sample identification and the porosity were listed in Table 1 of Roberts and Lin (1996a), which is also represented as Table 3-4 in this report. To determine the moisture retention curve, samples were placed in the humidity chamber at a specific temperature and $\sim 20 \% \mathrm{RH}$. The measurement and test equipment for the measurement of moisture retention curves are RH sensors and balances. Measurements began on dry samples. When the weights 
reached a constant value for several days (samples were weighed daily), it was assumed that equilibrium was established. When a stable weight was achieved, the $\mathrm{RH}$ was increased and the process repeated at the same temperature. The maximum saturation achieved at the highest $\mathrm{RH}$ $(\sim 98 \%)$ was $\sim 25 \%$. A balance with a sensitivity of $0.01 \mathrm{mg}$ calibrated to a traceable standard was used to weigh the samples. Saturation was calculated by comparing weights with dry weights and taking into account porosity. The process was then repeated for the drying portion of the measurement. This cycle of measurement was then repeated at a different temperature.

One difficulty was the establishment of steady weight values at the highest humidities, particularly at high temperatures. The reasons for this difficulty are that the RH is difficult to control at the highest settings and the weight of the samples is more sensitive to changes in RH at the highest settings. Refinement of the control parameters on the humidity chambers aided in the solution of this problem.

The measurement uncertainty involved in the determination of the moisture retention curves include the measurements of weights, relative humidity, and sample size. The sample dimensions are used to determine sample wet and dry densities. It is estimated that the thickness of the sample can be determined to $\pm 0.005 \mathrm{~mm}$ and diameter to $\pm 0.05 \mathrm{~mm}$. For the samples used here, this results in an error in sample volume of $\sim \pm 0.3 \%$. The uncertainty in dry weight is estimated to be $\sim 0.00002 \mathrm{~g}$ and for wet weight $\sim 0.0001 \mathrm{~g}$. The error in the wet weight is higher than that of the dry condition because of the difficulty in achieving and maintaining saturation levels of $100 \%$. These uncertainties result in errors in dry and wet densities of $\sim 0.3 \%$. When propagated through to porosity, the error is $\sim 1.0 \%$ porosity, or $\sim 7-11 \%$ of the measurement.

When repetitive measurements are made on samples over a period of several days, such as the determination of weights at a specified $\mathrm{RH}$, for example, the uncertainty in the measurement is often less than the statistical uncertainty in the mean of the measured parameter. In such cases, the error is taken as one standard deviation of the mean. The errors in saturation determined at specific temperature and RH vary from $\sim 0.07$ to $0.5 \%$ water saturation. Thus, the relative uncertainty is between $\sim 1$ and $10 \%$, with a $1-2 \%$ error most common.

The uncertainty in the relative humidity is approximately $\pm 2 \% \mathrm{RH}$. When propagated through equation 1 to matric potential, the absolute uncertainties are fairly low, but the relative uncertainties are high at the matric potentials closest to zero (as much as $200 \%$ at $\psi=-1.36 \pm$ $2.73 \mathrm{MPa}$ ).

The results of the moisture retention curves of the LBT samples are shown in Figures 3-19 to 3-21. For comparison, the moisture retention curves for the USW H-1 samples at $20^{\circ} \mathrm{C}$ during the drying phase are presented in Figure 3-22. The USW H-1 was one of the surface-based deep holes drilled very early in the project; therefore, its cores were not Q-cores. The moisture retention curves of the LBT samples were within the range of the USW H-1 cores. The moisture retention curve of those samples seemed to be temperature dependent. The saturation at room temperature was greater than that at $70^{\circ} \mathrm{C}$. Hysteresis was observed between the wetting and drying curves, with the drying curve slightly more saturated at any suction than the wetting curve, for the USW H-1 samples at $20^{\circ} \mathrm{C}$. However, at $70^{\circ} \mathrm{C}$ the hysteresis seemed to be insignificant for both the USW H-1 samples and the LBT samples. 


\subsection{The Porosity and Micro-Pore Structure}

The porosity and the pore size distribution of rock are important parameters in the modeling of the hydrological process to determine the variation of water saturation. Carlberg and Roberts (2000) reported the determination of the effective (connected) porosity and the micro-pore structure of the Topopah Spring tuff samples obtained from the LBT site. The porosity was determined by the drying-and-saturation (gravimetric) method (Roberts and Lin 1996a). The porosity was determined by calculating the difference between the dry density and the watersaturated density (assuming that the water density is $1.0 \mathrm{~g} / \mathrm{cm}^{3}$ ). The micro-pore size distribution was determined by mercury injection porosimetry, another conventional method in the study of the pore structure of rocks. Table 3-4 shows the porosity of the LBT samples. The porosity ranged from 0.08 to 0.14 , with a mean of 0.104 for the 36 samples. The results of the mercury injection porosimetry are summarized in Table 3-5. The mean porosity of the 33 samples used in the mercury injection porosimetry ranged from 0.08 to 0.20 , with a mean of 0.115 , which agreed well with that determined by dry-and-saturation method.

\subsection{Visualization of Fracture Flow Versus Matrix Imbibition Using X-ray Radiography}

Roberts and Lin (1996b and 1997a) reported using x-ray radiography to investigate and image fracture flow and matrix imbibition in blocks of Topopah Spring tuff with a tensile fracture. Later, Roberts and Kneafsy (in Wildenschild et al. 1998) reported adding video imaging and infrared temperature imaging to $\mathrm{x}$-ray radiography to visualize the fracture flow and matrix imbibition processes. The samples used in those investigations were machined from small blocks obtained at the LBT. The block identification number, sample identification number, and dimensions, of these samples are listed in Table 3-6.

In the earlier work (Roberts and Lin 1996b and 1997a), a vertical tensile fracture was induced in the middle of the sample, oriented so that the plane of the fracture was parallel to the direction of $\mathrm{x}$-ray transmission. X-rays were transmitted through the smallest dimension. Thus, in a typical $\mathrm{x}$-ray radiograph, the fracture was perpendicular to the plane of the image. The sample was dried in a vacuum oven at $35^{\circ} \mathrm{C}$ until the weight did not change appreciably for several days. The sample was then coated with a latex moisture barrier on all sides, leaving the top and bottom of the sample exposed. A lucite frame was constructed that surrounded the sample on all sides and held the sample together. Approximately $25 \mathrm{in}-\mathrm{lb}$ of torque was applied to nylon screws holding the fracture together. At the top and bottom of the sample were chambers for ponding and collection of water.

Figure 3-23 shows a diagram of the sample assembly in the $\mathrm{x}$-ray scanning machine. X-ray radiographs were taken periodically to image water movement into the fracture and rock matrix by translating the sample vertically through the $160 \mathrm{kVp}$ linear $\mathrm{x}$-ray source. Water flowing down the fracture could be imbibed into the matrix on both sides of the fracture. X-rays passing through the sample were converted to a digital signal by a photo-diode linear array detector and stored by a computer. X-ray attenuation contrast was enhanced by adding potassium iodide (KI) to the J-13 water. X-ray linear scans were taken periodically while water flowed along the fracture and imbibed into the matrix. 
Table 3-4. The Porosity of LBT Samples Determined by Gravimetric Method

\begin{tabular}{|c|c|c|c|c|c|c|c|}
\hline Sample ${ }^{\dagger}$ & Sample ID & Depth, m & Dry wt, $\mathbf{g}$ & Wet wt, $\mathbf{g}$ & $\begin{array}{c}\text { Dry density, } \\
\mathrm{g} / \mathrm{cm}^{3}\end{array}$ & $\begin{array}{c}\text { Wet density, } \\
\mathrm{g} / \mathrm{cm}^{3}\end{array}$ & Porosity \\
\hline $\mathrm{N} 1-6.3$ & 0032079.3 & 1.92 & 1.5352 & 1.6111 & 2.23 & 2.35 & 0.110 \\
\hline N1-6.3A & $0032079.3 A$ & 1.92 & 1.7890 & 1.8680 & 2.26 & 2.36 & 0.0997 \\
\hline N1-6.3B & $0032079.3 B$ & 1.92 & 1.6358 & 1.7098 & 2.28 & 2.39 & 0.103 \\
\hline N1-11.0 & 0032081.3 & 3.35 & 1.6352 & 1.7181 & 2.25 & 2.37 & 0.114 \\
\hline $\mathrm{N} 1-11.0 \mathrm{~A}$ & $0032081.3 \mathrm{~A}$ & 3.35 & 1.5920 & 1.6734 & 2.26 & 2.38 & 0.116 \\
\hline $\mathrm{N} 1-11.0 \mathrm{~B}$ & $0032081.3 B$ & 3.35 & 1.6762 & 1.7525 & 2.30 & 2.41 & 0.105 \\
\hline $\mathrm{N} 1-13.45$ & 0032082.3 & 4.10 & 1.4982 & 1.5752 & 2.27 & 2.39 & 0.117 \\
\hline N1-13.45A & $0032082.3 \mathrm{~A}$ & 4.10 & 1.7118 & 1.7951 & 2.27 & 2.38 & 0.110 \\
\hline N1-13.45B & $0032082.3 \mathrm{~B}$ & 4.10 & 1.6954 & 1.7781 & 2.26 & 2.37 & 0.110 \\
\hline $\mathrm{N} 1-16.9$ & 0032083.3 & 5.15 & 1.6499 & 1.7522 & 2.20 & 2.33 & 0.136 \\
\hline $\mathrm{N} 1-16.9 \mathrm{~A}$ & $0032083.3 \mathrm{~A}$ & 5.15 & 1.6094 & 1.6987 & 2.23 & 2.35 & 0.124 \\
\hline $\mathrm{N} 1-16.9 \mathrm{~B}$ & $0032083.3 B$ & 5.15 & 1.6885 & 1.7670 & 2.27 & 2.38 & 0.106 \\
\hline N1-20.3 & 0032084.3 & 6.19 & 1.5438 & 1.6133 & 2.22 & 2.32 & 0.0998 \\
\hline N1-20.3A & $0032084.3 \mathrm{~A}$ & 6.19 & 1.5567 & 1.6244 & 2.26 & 2.36 & 0.0982 \\
\hline N1-20.3B & $0032084.3 \mathrm{~B}$ & 6.19 & 1.5109 & 1.5849 & 2.21 & 2.32 & 0.108 \\
\hline N4-11.6 & 0032104.3 & 3.54 & 1.5429 & 1.6036 & 2.26 & 2.34 & 0.0887 \\
\hline $\mathrm{N} 4-11.6 \mathrm{~A}$ & $0032104.3 \mathrm{~A}$ & 3.54 & 1.6222 & 1.6864 & 2.24 & 2.33 & 0.0886 \\
\hline N4-11.6B & $0032104.3 \mathrm{~B}$ & 3.54 & 1.6375 & 1.6969 & 2.27 & 2.35 & 0.0823 \\
\hline N5-4.9 & 0032107.3 & 1.49 & 1.6998 & 1.7687 & 2.25 & 2.34 & 0.0911 \\
\hline N5-4.9A & $0032107.3 \mathrm{~A}$ & 1.49 & 1.6501 & 1.7104 & 2.28 & 2.37 & 0.0834 \\
\hline N5-4.9B & $0032107.3 \mathrm{~B}$ & 1.49 & 1.8818 & 1.9569 & 2.31 & 2.40 & 0.0922 \\
\hline N5-20.4 & 0032111.3 & 6.22 & 1.5230 & 1.5909 & 2.22 & 2.32 & 0.0992 \\
\hline N5-20.4A & $0032111.3 \mathrm{~A}$ & 6.22 & 1.4883 & 1.5593 & 2.21 & 2.32 & 0.106 \\
\hline N5-20.4B & $0032111.3 \mathrm{~B}$ & 6.22 & 1.4765 & 1.5463 & 2.22 & 2.32 & 0.105 \\
\hline N6-4.75 & 0032112.3 & 1.43 & 1.7549 & 1.8228 & 2.25 & 2.33 & 0.0869 \\
\hline N6-4.75A & $0032112.3 \mathrm{~A}$ & 1.43 & 1.6761 & 1.7374 & 2.29 & 2.37 & 0.0837 \\
\hline N6-4.75B & $0032112.3 \mathrm{~B}$ & 1.43 & 1.7136 & 1.7755 & 2.27 & 2.35 & 0.0819 \\
\hline N6-14.2 & 0032116.3 & 4.33 & 1.6590 & 1.7398 & 2.26 & 2.37 & 0.110 \\
\hline N6-14.2A & $0032116.3 A$ & 4.33 & 1.6869 & 1.7706 & 2.24 & 2.35 & 0.111 \\
\hline N6-14.2B & $0032116.3 B$ & 4.33 & 1.6285 & 1.7137 & 2.23 & 2.35 & 0.117 \\
\hline N7-5.7 & 0032120.3 & 1.74 & 1.6161 & 1.7003 & 2.24 & 2.36 & 0.117 \\
\hline N7-5.7A & $0032120.3 \mathrm{~A}$ & 1.74 & 1.6320 & 1.7051 & 2.29 & 2.39 & 0.102 \\
\hline N7-5.7B & $0032120.3 \mathrm{~B}$ & 1.74 & 1.7091 & 1.7834 & 2.28 & 2.38 & 0.0991 \\
\hline N7-11.0 & 0032123.3 & 3.35 & 1.5850 & 1.6705 & 2.25 & 2.37 & 0.121 \\
\hline N7-11.0A & $0032123.3 A$ & 3.35 & 1.6353 & 1.7171 & 2.26 & 2.38 & 0.113 \\
\hline N7-11.0B & $0032123.3 \mathrm{~B}$ & 3.35 & 1.6318 & 1.7112 & 2.27 & 2.38 & 0.110 \\
\hline mean $^{*}$ & 36 samples & & & & $2.25 \pm 0.03$ & $2.36 \pm 0.02$ & $0.104 \pm 0.013$ \\
\hline
\end{tabular}

Source: Roberts and Lin 1996a

NOTE: †Sample name consists of borehole designation followed by depth in feet below the template used to locate vertical boreholes.

*Statistical mean for 36 samples. Errors represent one standard deviation for all samples collectively. 
Table 3-5. Pore Size Distribution of the LBT Samples Determined by Mercury Injection Porosimetry Method

\begin{tabular}{|c|c|c|c|c|c|c|c|}
\hline Samplet & $\begin{array}{l}\text { Total pore } \\
\text { area }\left(\mathrm{m}^{2} / \mathrm{g}\right)\end{array}$ & $\begin{array}{l}\text { Median pore } \\
\text { diameter by } \\
\text { volume ( } \mathrm{\mu m} \text { ) }\end{array}$ & $\begin{array}{l}\text { Median pore } \\
\text { diameter by } \\
\text { area ( } \mu \mathrm{m})\end{array}$ & $\begin{array}{l}\text { Average pore } \\
\text { diameter by } \\
\text { 4V/A }(\mu \mathrm{m})\end{array}$ & $\begin{array}{c}\text { Bulk } \\
\text { density } \\
\left(\mathrm{g} / \mathrm{cm}^{3}\right)\end{array}$ & $\begin{array}{l}\text { Skeletal } \\
\text { density } \\
\left(\mathrm{g} / \mathrm{cm}^{3}\right)\end{array}$ & Porosity \\
\hline N1-5.7 to 6.3 & 4.6429 & 0.0932 & 0.0080 & 0.0350 & 2.3372 & 2.5825 & 0.0950 \\
\hline $\mathrm{N} 1-10.9$ to 11.5 & 6.9868 & 0.0611 & 0.0171 & 0.0345 & 2.24 .27 & 2.5930 & 0.1351 \\
\hline N1-11.0 & 8.8620 & 0.0303 & 0.0148 & 0.0224 & 2.3089 & 2.6081 & 0.1148 \\
\hline $\mathrm{N} 1-13.4 \mathrm{a}$ & 8.3196 & 37.8102 & 0.0180 & 0.0931 & 1.7272 & 2.5948 & 0.3344 \\
\hline N1-13.4b & 9.6257 & 0.0272 & 0.152 & 0.0207 & 2.3102 & 2.6113 & 0.1153 \\
\hline $\mathrm{N} 2-16.9$ & 4.0976 & 0.2434 & 0.0116 & 0.0589 & 2.2421 & 2.5927 & 0.1352 \\
\hline $\mathrm{N} 1-20.3$ & 11.1302 & 0.0513 & 0.0063 & 0.0212 & 2.2239 & 2.5595 & 0.1311 \\
\hline$N 2-4.0$ & 5.5900 & 0.1616 & 0.0071 & 0.0367 & 2.2850 & 2.5883 & 0.1172 \\
\hline$N 2-4.3$ & 8.7110 & 0.0383 & 0.0070 & 0.0169 & 2.2967 & 2.5088 & 0.0845 \\
\hline N2-11.1 & 8.2266 & 0.0570 & 0.0075 & 0.0231 & 2.2461 & 2.5150 & 0.1069 \\
\hline N2-11.25 & 5.8431 & 0.1435 & 0.0073 & 0.0362 & 2.2900 & 2.6057 & 0.1211 \\
\hline N2-13.9 & 6.3615 & 0.2336 & 0.0071 & 0.0469 & 2.1707 & 2.5894 & 0.1617 \\
\hline N2-19.2 & 8.4895 & 0.0480 & 0.0085 & 0.0224 & 2.2567 & 2.5277 & 0.1072 \\
\hline$N 3-4.5$ & 5.4111 & 0.2110 & 0.0068 & 0.0389 & 2.2894 & 2.6029 & 0.1204 \\
\hline N3-4.75 & 6.9874 & 0.0665 & 0.0081 & 0.0263 & 2.2555 & 2.5167 & 0.1038 \\
\hline NB-11.0 & 9.0228 & 0.0728 & 0.0073 & 0.0227 & 2.3177 & 2.6305 & 0.1189 \\
\hline$N 3-15.2$ & 7.2965 & 0.1026 & 0.0074 & 0.0325 & 2.2679 & 2.6196 & 0.1343 \\
\hline N3-20.4 & 9.4214 & 0.0339 & 0.0087 & 0.0187 & 2.2528 & 2.5019 & 0.0996 \\
\hline N4-5.3 & 8.9013 & 0.0544 & 0.0059 & 0.0200 & 2.2611 & 2.5135 & 0.1004 \\
\hline N4-11.6 & 6.9084 & 0.0505 & 0.0084 & 0.0235 & 2.2616 & 2.4903 & 0.0918 \\
\hline N4-14.5 & 6.0849 & 0.1298 & 0.0075 & 0.0316 & 2.3096 & 2.5983 & 0.1111 \\
\hline N4-19.0 & 7.4861 & 0.0456 & 0.0125 & 0.0266 & 2.2459 & 2.5281 & 0.1116 \\
\hline$\sqrt{5}-4.9$ & 6.5621 & 0.0476 & 0.0085 & 0.0232 & 2.3276 & 2.5534 & 0.0884 \\
\hline N5-11.15 & 5.7744 & 0.0889 & 0.0082 & 0.0360 & 2.2889 & 2.5981 & 0.1190 \\
\hline N5-14.8 & 5.9586 & 0.0911 & 0.0087 & 0.0378 & 2.2465 & 2.5717 & 0.1265 \\
\hline N5-20.1 & 9.2912 & 0.0457 & 0.0076 & 0.0196 & 2.2422 & 24963 & 0.1018 \\
\hline N6-4.8 & 7.6122 & 0.0371 & 0.0078 & 0.0188 & 2.3001 & 2.5065 & 0.0823 \\
\hline N6-11.0 & 6.6135 & 0.0515 & 0.0106 & 0.0275 & 2.2825 & 2.5464 & 0.1036 \\
\hline NG-14.5 & 6.4598 & 0.1186 & 0.0065 & 0.0338 & 2.2666 & 2.5861 & 0.1235 \\
\hline N6-20.2 & 8.7303 & 1.0908 & 0.0062 & 0.0464 & 1.9941 & 2.4982 & 0.2018 \\
\hline N7-5.0 & 8.1133 & 0.0536 & 0.0071 & 0.0212 & 2.2718 & 2.5177 & 0.0977 \\
\hline N7-10.9 & 6.4128 & 0.0682 & 0.0105 & 0.0310 & 23060 & 2.6045 & 0.1146 \\
\hline N7-14.3 & 7.2915 & 0.0543 & 0.0146 & 0.0301 & 2.2768 & 2.6013 & 0.1248 \\
\hline E2-19.5 & 8.3521 & 0.0552 & 0.0086 & 0.0235 & 2.2147 & 24850 & 0.1087 \\
\hline mean* & $\begin{array}{l}715 \\
\pm 1.5795\end{array}$ & & & & $\begin{array}{l}2.2633 \\
\pm 0.0596\end{array}$ & $\begin{array}{l}2.5591 \\
\pm 0.0456\end{array}$ & $\begin{array}{c}0.1154 \\
\pm 0.0228\end{array}$ \\
\hline
\end{tabular}

T Sample name consists of borehole designation followed by depth in feet below the template used to locate vertical boreholes.

- Statistical mean for 33 samples. Errors represent one standard deviation for all samples collectively. Sample N1-13.4a has been excluded from statistical analyses.

Source: Table 4 of Carlberg and Roberts 2000

Table 3-6. Sample Descriptions-Fracture Flow Versus Matrix Imbibition Test

\begin{tabular}{ccc}
\hline Block Number & Sample Number & Dimensions $(\mathrm{cm})$ \\
\hline SPC00501631 & SPC00501631.1 & $2.45 \times 10.13 \times 14.1$ block \\
SPC00504573 & SPC00504573.2 & $2.65 \times 15.14 \times 29.21$ block \\
SPC00504573 & SPC00504573.3 & $2.62 \times 14.94 \times 22.96$ block \\
\hline
\end{tabular}


A total of six experiments were reported by Roberts and Lin (1996b and 1997a):

1. Fracture flow at room temperature without shim.

2. Fracture flow at room temperature with $25-\mu \mathrm{m}$ shims in the fracture.

3. Dehydration of the sample after Experiment $\# 2$ with a thermal gradient of $\sim 95^{\circ} \mathrm{C}$ at the bottom and $\sim 33^{\circ} \mathrm{C}$ at the top.

4. Fracture flow in shimmed fracture with 3 heaters at the bottom to create a thermal gradient of $\sim 111^{\circ} \mathrm{C}$ at the bottom and $\sim 28^{\circ} \mathrm{C}$ at the top, and a water head of $\sim 0.02 \mathrm{~m}$.

5. Fracture flow in shimmed fracture with 3 heaters at the bottom and 4 heaters on sides to create a thermal gradient of $\sim 148^{\circ} \mathrm{C}$ at the bottom and $\sim 80^{\circ} \mathrm{C}$ at the top, and a water head of $\sim 0.26 \mathrm{~m}$.

6. Fracture flow in shimmed fracture with 3 heaters at the bottom and 4 heaters on sides to create a thermal gradient of $\sim 148^{\circ} \mathrm{C}$ at the bottom and $\sim 80^{\circ} \mathrm{C}$ at the top, and a water head of $\sim 0.46 \mathrm{~m}$.

The result of experiments 1 and 2 showed that, at room temperature, imbibition occurred chiefly through the matrix for the unshimmed fracture, and primarily horizontally from the fracture to the matrix during the shimmed fracture. A roughly v-shaped wetting front was observed for the unshimmed case, and after more than 2017 hours of ponding, water still had not flowed the length of the fracture. During the shimmed fracture experiment, water flowed the fracture length after only $\sim 0.15$ hours. Different (lateral) imbibition rates were observed in different regions of the sample, demonstrating the heterogeneous properties of the rock. Enhanced imbibition appeared to be controlled by the presence of lithic fragments that contain small microfractures. A significant difference in the two series of experiments is that water was imbibed laterally into the matrix (from the fracture) at a much higher rate when the fracture was shimmed open. For the shimmed experiment, after 1000 hours a large portion of the sample was highly saturated.

The dehydration experiment (3) began after no more imbibition into the matrix was observed (shimmed experiment, $>1200$ hours of imbibition). Dryout along the fracture was observed and was most pronounced between 4.5 and 23 hours after heating was initiated. At longer times dryout along the fracture was not apparent, and at times greater than $\sim 215$ hours the fracture was highly attenuating. Possible explanations include:

- The fracture increased in saturation at times greater than 215 hours.

- Evaporation of water continuously along the fracture resulted in the crystallization of potassium iodide $(\mathrm{KI})$ salt that attenuated the $\mathrm{x}$-rays.

Another feature was the development of a horizontal band of high attenuation approximately $1.5-2 \mathrm{~cm}$ from the bottom of the sample. This band appeared initially at about 0.5 hours after 
heating and became more pronounced with time. At about 25 hours after heating, two such bands were observed, again near the bottom of the sample.

Experiment 4 was the first fracture flow under a thermal gradient to be conducted in the series. The temperature in the bottom $2-3 \mathrm{~cm}$ of the sample was greater than the boiling point of water. The water head in this experiment was small, only about $1-2 \mathrm{~cm}$, but not zero. The sample was dry to start with. The result of this test showed that water flowed down the fracture quickly but stopped about $13 \mathrm{~cm}$ from the top. It seemed that the flow was stopped by a relatively highporosity clast that was intersected by the fracture. As time progressed, this more porous region became more saturated (attenuating) until flow proceeded around or through the region. During this time, there was significant lateral imbibition into the matrix from the fracture. After flow continued past the more porous region, the shape of the wetting front was less sharp and more rounded. This wetting front stopped about $2.6 \mathrm{~cm}$ from the bottom of the sample. A very narrow neck of high attenuation extended from the wetting front to another highly attenuating region at the bottom of the sample. This lower highly attenuating region was probably due to the deposition of the KI salt due to the boiling. In this test, the water never penetrated the entire fracture length. After nine days the heaters were turned off, and water still did not flow through the fracture.

Figure 3-24 shows two images to illustrate the effect of water head on the fracture flow and matrix imbibition. Those are the result of experiments 5 and 6 . In those two cases the lower 6-7 cm from the bottom of the sample was the boiling zone. The convention used for the difference images is that darker colors or shades indicate relatively high $\mathrm{x}$-ray attenuation and the presence of water, while the lighter areas correspond to lower attenuation and relatively dry areas. For experiment 5 the water head was about $0.26 \mathrm{~m}$. The water wetted almost the entire fracture first, followed by imbibition into the matrix, as shown in Figure 3-24a. Within 7.2 hours of ponding, the water penetrated about $3 \mathrm{~cm}$ into the boiling zone. The difference between this experiment and Experiment 4 above was that flow continued down the length of the fracture after the heaters were turned off. This was probably due either to the increased water head or the lack of sealing of the fracture by the salt deposit. Figure 3-24b shows the result of experiment 6 , in which the water head was increased to $0.46 \mathrm{~m}$. The water flowed through the entire length of the fracture within minutes and continued to flow through the boiling region. Not much imbibition into the matrix was observed in this case.

Roberts and Kneafsey (in Wildenschild et al. 1998) added visualization capability to the X-ray radiography. In the later experiments, the fracture was simulated by the interface between a sawcut tuff surface and a piece of glass. Water was flowed along the interface from the top to bottom of the block. X-ray radiography and video images were taken on the front face of the glass looking into the rock face. Temperatures were measured both within the block, using thermocouples, and on the glass surface, using infrared imaging. The water flow was also under a thermal gradient, similar to the earlier experiments. They concluded that observations in the visualization experiments supported many of the observations and conclusions made for the previous x-ray radiography experiments. For instance, in one of the early experiments, a highly attenuating band was observed above the heated region. The tentative conclusion was that this could be a region of either enhanced saturation or salt crystal formation. The visualization experiments confirmed that both the salt deposit and the condensation halo formed in the region above the boiling zone. The early experiments showed the development of a narrow, highly 
attenuating neck in or near the boiling region. The visualization experiments observed similar features. They also concluded that the processes and physical phenomena observed in the tuff tensile fractures also occurred in the tuff saw-cut/glass plate interface.

\subsubsection{Pre-Heat Thermal-Hydrologic Calculations}

Pre-heat scoping TH calculations were conducted to assist the design of the test. These calculations were reported in Section 3 of the LBT Status Report (Wilder et al. 1997). Those scoping calculations include a series of two-dimensional (2-D) and three-dimensional (3-D) TH calculations using V-TOUGH and NUFT codes and 3-D layer model and fracture model using TOUGH2. The TOUGH2 simulations were mainly for assisting the design of the tracer tests in the block. Only the NUFT simulations are summarized here, because they used input parameters that were more relevant to the LBT. Preliminary 2-D analyses and one 3-D analysis that modeled only thermal conduction were followed by TH 3-D analyses (Lee 1995a, 1995b). Modeling results assumed either a homogeneous block having the dominant bulk permeability as measured by single-borehole air injection, or a heterogeneous block reflecting the permeability profile. The heterogeneous permeability field had a "layer-cake" distribution because the permeability measured at any depth was assumed to be constant for that depth. This does not reflect the dominant vertical attitude of many of the fractures but does represent the horizontal sets.

The pre-test calculations are presented in detail in Section 3 of the LBT Status Report (Wilder et al. 1997).

\subsubsection{Homogeneous and Heterogeneous Cases}

For the homogeneous case, an equivalent continuum model was used. A distinct dryout zone was predicted in and around the heater plane, with well-developed condensation zones above and below the heaters. In contact, the heterogeneous case did not show a well-developed condensation zone above the heaters. A distinct dryout zone was predicted, and a well-developed condensation zone was formed only below the heater plane. For the heterogeneous case, there was clearly a net loss of liquid above the heater plane. The results suggest that the saturation changes might be sensitive to permeability distribution.

Higher gas pressures were generated for the heterogeneous case. Peak gas phase pressure for the homogeneous case was only $97 \mathrm{kPa}$ (14 psia), compared to $157 \mathrm{kPa}$ (23 psia) for the heterogeneous case. The higher pressure is caused by additional confinement of water vapor and air by layers of bulk permeability substantially lower than those for the homogeneous permeability case.

\subsubsection{Thermal-Hydrologic Discrete Fracture Model}

An analysis using the discrete fracture model was performed in which the fracture system was modeled as a series of $200-\mu \mathrm{m}$-aperture fractures at a uniform spacing of $30 \mathrm{~cm}$. The fractures were vertical and parallel to the heater borehole axes and to occur midway between two boreholes, as shown in Figure 3-25. It should be noted that the east-west orientation of these assumed fractures is not consistent with predominant fracture orientations, which is NW-SE. 
However, the purpose of these calculations was to determine sensitivity to equivalent continuum model (ECM) versus discrete approaches.

In these analyses, distinct dryout and recondensation zones were predicted both above and below the heater plane. The dryout zone was thickest at the fractures and thinnest in the matrix, midway between the fractures (at heaters). Figure 3-26 shows the predicted temperature and liquid saturation profiles along a vertical line through the matrix at one-year heating. Block temperatures were not significantly affected by fracture location. The peak gas pressure in the system was about $177 \mathrm{kPa}(26 \mathrm{psig})$, located in the matrix at the heater level.

\subsubsection{Pre-Heat Thermal-Mechanical Modeling}

This section presents results of thermal-mechanical (TM) simulations of the LBT. Pre-test continuum simulations were done using two different numerical codes that are commercially available, a 2-D finite difference model (FLAC) and a 3-D finite element model (ABAQUS ${ }^{\mathrm{TM}}$ ).

The goal of the initial numerical modeling was to calculate temperatures, stresses, and displacements in two and three dimensions for a simplified representation of the large block. In reality, numerous joints and fractures complicate the behavior of the large block significantly. Nonetheless, these simulations provide a general understanding of the TM behavior to be expected in the LBT.

\subsubsection{Description of Problem}

The FLAC and ABAQUS ${ }^{\mathrm{TM}}$ codes were used to perform TM simulation of the heat-up phase of the LBT. The numerical models are one-way weakly coupled in the sense that the temperature field produces thermal stresses, but mechanical stresses do not in turn influence temperatures. The temperature field is calculated as a function of time from a thermal conduction model. In simulations conducted using each code the sides and top of the block are assumed to be insulated. Also, the ground surface is assumed to be isothermal. The two codes are similar in that the mechanical model in each uses the temperature field at given times to apply thermal loading. For all simulations all of the surface stresses are assumed to be zero. For the 2-D (FLAC) analysis only one half of the block in cross-section is modeled, and for the 3-D case only one-quarter of the block is modeled; therefore, the two symmetry planes are given boundary conditions of zero displacement normal to the plane. The bottom of the model lies several meters below the ground surface, and it is also a zero displacement boundary. Up to 75 days, the temperature effects of the heating extended only a short $(<2 \mathrm{~m})$ distance below the block; therefore, the model bottom boundaries have little effect on the TM behavior of the large block itself.

\subsubsection{Description of Models}

This section describes the models used in the simulation of the LBT, including inherent assumptions, configuration, and input parameters. The 2-D FLAC models are described first, followed by the 3-D ABAQUS ${ }^{\mathrm{TM}}$ model. 


\subsection{FLAC Models}

FLAC is a time-dependent finite difference code capable of treating both mechanical and thermally induced stresses and deformations. It is a 2-D code in which materials are represented by arbitrarily-shaped, quadrilateral zones and is capable of using several built-in constitutive relations to describe material behavior.

Version 3.22 of the FLAC code was used in this study, and a detailed description of the code can be found in the FLAC User's Manual (Itasca Consulting Group, Inc. 1993). All the FLAC modeling described in this report assumes a 2-D, plane strain geometry. This is equivalent to having infinite thickness in the third dimension, or to being in a symmetry plane (no net strain) in the third dimension. The plane of the 2-D FLAC models is the vertical plane perpendicular to the long axis of the heaters for the LBT. The FLAC models all use linear mechanical and thermal properties where mechanical properties (e.g., elastic moduli) and thermal properties (e.g., thermal expansion coefficient) are independent of stress and temperature. Constitutive relations are either isotropic elastic or else make use of the ubiquitous joint model capability of the FLAC code. The thermal response in all models assumes isotropic heat conduction.

The procedure for running a FLAC model to simulate the LBT is to first create a file that provides information about the grid used in the model as well as the mechanical and thermal properties used to represent the rock, and the mechanical boundary conditions and initial conditions. Next, FLAC is run in the mechanical mode with this file as input, to bring the model to initial mechanical equilibrium. This usually requires several thousand steps. Mechanical steady-state is reached when additional steps do not produce significant additional displacement-e.g., an additional 1000 steps results in less than $1 \mathrm{~mm}$ displacement for a model that showed displacements on the order of $1 \mathrm{~cm}$ for initial steps. The equilibrium state is saved in a FLAC output file. Then, another input file is created to provide information about the thermal boundary conditions, initial conditions, and heat sources. FLAC is run in the thermal mode with the new input file and the saved file from the previous run for a number of time steps required to follow the desired heating schedule. The resulting temperature field in the model is saved in a new FLAC output file. Note that the size of a time step is set to be slightly smaller than the area of the smallest grid element divided by the thermal diffusivity of the rock, so that heat transfer can be resolved at the scale of the grid and yet the model will not require an excessive amount of computer time for computing the temperature field. Finally, FLAC is run again in the mechanical mode, to compute the displacements and stresses that result from the thermal loading. The mechanical and thermal modeling in FLAC can be described as a one-way weak coupling, with the mechanical state affected by the temperature field but with the thermal state independent of the displacements and the stress field.

\subsection{Grid Formulation}

Several important limitations of FLAC must be considered when developing a grid to represent the configuration of the LBT. According to the FLAC User's Manual, errors in stress and displacement due to edge effects will be small (below 6\%) if fixed model boundaries are placed at distances of at least five times the length of the region of interest in the model. For a large 
block that is $5 \mathrm{~m}$ high, the model must therefore have distant edges at least $23 \mathrm{~m}$ below and at least $23 \mathrm{~m}$ beyond the block. The model must represent the 2-D $3 \mathrm{~m} \times 4.5 \mathrm{~m}$ block while retaining enough small-scale detail to properly represent the $5 \mathrm{~cm}(2 \mathrm{in}$.) diameter heater holes. This suggests a grid with more gridlines concentrated in the block region, particularly around the heater holes, and fewer gridlines toward the far edges of the model. According to the FLAC User's Manual, grid elements must have aspect ratios that do not exceed 5:1, and the areas of two adjacent elements should not exceed a 4:1 ratio. The number of elements in the model, $\mathrm{N}$, determines how much computer time is required for a steady-state solution, with run time proportional to $\mathrm{N}^{3 / 2}$. For example, a 1000 -element model may require a few minutes to run on a Sun Sparc20 workstation, and a 4000-element model would take eight times as long to run on that same computer.

The gridding used in all of the FLAC models described in this report is almost the same as that used by Lee (1995a) for hydrothermal modeling. The only differences are that one gridline $1 \mathrm{~cm}$ below the top of the large block in Lee's model was eliminated to avoid aspect ratio problems, and gridlines near the far edges of the model were moved slightly to prevent areas of adjacent elements from exceeding the 4:1 ratio. There are 26 vertical and 37 horizontal gridlines, for a total of 900 elements. In the FLAC models, the large block is represented as a set of zones $4.5 \mathrm{~m}$ high and having a $1.5 \mathrm{~m}$ width for half of the block. (Only half of the large block is modeled, since the block is symmetrical for a vertical cross-section.) Grid elements within the block have sizes varying from $5 \mathrm{~cm} \times 5 \mathrm{~cm}$ to $10 \mathrm{~cm} \times 20 \mathrm{~cm}$. Below the block, the element sizes increase to a maximum of $16 \mathrm{~m} \times 16 \mathrm{~m}$ at the far edges of the model but are smaller than $1 \mathrm{~m} \times 1.5 \mathrm{~m}$ within a few meters of the large block. The heaters in the block are represented as $10 \mathrm{~cm} \times 10 \mathrm{~cm}$ elements, placed $3 \mathrm{~m}$ below the top of the block. These heater elements are larger than the actual heater holes, but the heater power was scaled accordingly, as will be described later in this section. Figure 3-27 shows a detail of the grid for the large block models.

\subsection{Initial and Boundary Conditions}

Initial conditions used in all the FLAC modeling set all initial stresses to zero within all grid elements, and set temperatures initially to $20^{\circ} \mathrm{C}$ in all elements. The gravitational acceleration is $9.8 \mathrm{~m} / \mathrm{s}^{2}$. A set of boundary conditions was used for all of the FLAC modeling, as follows: The axis of symmetry at the left edge of the model, for the center of the large block, was modeled as an adiabatic boundary fixed in the horizontal direction. The top of the block was also adiabatic and had an applied compressive stress of $0.1 \mathrm{MPa}$ (nominally zero) to simulate atmospheric pressure. The right edge of the block was adiabatic and also had an applied compressive stress of $0.1 \mathrm{MPa}$. The horizontal boundary representing the ground surface was an isothermal boundary set to a temperature of $20^{\circ} \mathrm{C}$ with an applied compressive stress of $0.1 \mathrm{MPa}$. The far right edge and bottom edge of the model were fixed in both the horizontal and vertical directions and were isothermal boundaries fixed at $20^{\circ} \mathrm{C}$. These conditions simulate the unconfined LBT with guard heaters to prevent heat flow from the sides of the block. Modeling of the top boundary of the block could be improved by allowing heat flux out until the top reaches a temperature of $60^{\circ} \mathrm{C}$, and then converting to an isothermal condition. This is a nontrivial problem in FLAC that will be addressed in future modeling. The present simple adiabatic condition allows the top of the block to reach $60^{\circ} \mathrm{C}$ too soon but avoids the problem of heat flux downward from the top that would occur if the top boundary were initially set at an isothermal condition at $60^{\circ} \mathrm{C}$ (e.g., Lee $1995 \mathrm{a}$ ). 


\subsection{Heaters}

All of the FLAC modeling described in this report represented the heat sources as $300-\mathrm{W}$ heaters placed at $60-\mathrm{cm}$ intervals at a level $3 \mathrm{~m}$ below the top of the large block. This configuration follows that used by Lee (1995a). Lee used a heating schedule that began with about 75 days of heating at constant power of $1500 \mathrm{~W}$ using five heaters, followed by reducing the power to $1200 \mathrm{~W}$ and finally achieving a steady-state at about $1050 \mathrm{~W}$ at an elapsed time of about 180 days. The FLAC modeling follows the first 75 days of Lee's heating schedule, using a constant power of $1500 \mathrm{~W}$.

Each heater is represented as an internal heat source in the model, specified in units of W per square $\mathrm{m}$ of grid element per $\mathrm{m}$ in the third (infinite) direction. The actual heater holes in the LBT will each have a radius of $2.5 \mathrm{~cm}$ and a length of $2.7 \mathrm{~m}-$ i.e., a cross-sectional area of $0.0020 \mathrm{~m}^{2}$ and a volume of $0.0053 \mathrm{~m}^{3}$. The ratio of power to volume for the heaters is then about $0.00057 \mathrm{~W} / \mathrm{m}^{3}$ for a $300-\mathrm{W}$ heater. For a $10 \mathrm{~cm} \times 10 \mathrm{~cm}$ grid element having an area of $0.010 \mathrm{~m}^{2}$, this $300-\mathrm{W}$ heater is represented by scaling the value of $0.00057 \mathrm{~W} / \mathrm{m}^{3}$ by the ratio of the heater hole cross-sectional area to the grid element area, to obtain a source of $0.00011 \mathrm{~W} / \mathrm{m}^{3}$ throughout the grid element. It is clear this is an appropriate procedure for representing the LBT heaters in the FLAC modeling, since results described in later sections of this report show temperature fields that are in agreement with the temperature field produced by Lee (1995a) for modeling the LBT.

\subsection{Rock Properties}

Input parameters for physical properties used in the FLAC modeling are given in Table 3-7. All of the FLAC modeling described in this report used the simplest thermal conduction model available in the FLAC code. This isotropic heat conduction modeling required three input parameters: thermal conductivity, specific heat, and the thermal expansion coefficient. FLAC requires that a bulk modulus, a shear modulus, and a density be specified for any mechanical model. This is the complete set of mechanical properties in the elastic case. The ubiquitous joint model is an anisotropic plasticity model that assumes a series of weak planes embedded in a Mohr-Coulomb solid. It requires eight additional parameters to specify where and how yield may occur. A detailed discussion of these parameters and the ubiquitous joint model can be found in the FLAC User's Manual. Four properties of the Mohr-Coulomb solid are the cohesion, dilation angle, tension limit, and angle of internal friction. The joints have four properties, including the angle of the parallel joints embedded in the solid (with respect to the horizontal), the joint cohesion, the joint tension limit, and the joint friction angle. Note that since the joints are ubiquitous plastic slip planes, the ubiquitous joint model does not have a crack density parameter or joint spacing associated with it. The values for the thermal properties listed in Table 3-7 are the same as those used by Lee (1995a). The thermal conductivity and specific heat values for wet tuff are given in the YMP Reference Information Base (RIB) (DOE 1990). Future modeling may use different values as laboratory data become available from tests on 1-m blocks of Topopah Spring tuff.

The bulk modulus and shear modulus values listed in Table 3-7 were determined from elastic wave velocity measurements, both preliminary measurements on small blocks of Topopah 
Spring tuff and also velocities reported from the G-tunnel heated block experiment (Zimmerman et al. 1986).

The density for tuff listed in Table 3-7 is from laboratory measurements on Topopah Spring tuff samples from the LBT site (Roberts and Lin 1996a). The tuff had a skeletal density of $2.56 \mathrm{~g} / \mathrm{cm}^{3}$ and a bulk density of $2.27 \mathrm{~g} / \mathrm{cm}^{3}$. The bulk density is appropriate for representing the density of the entire large block.

Values for the ubiquitous joint model parameters were mainly set using information from Arulmoli and St. John (1987). Their finite element TM modeling included ubiquitous joint modeling, and they report the model values they used to represent $80 \%$ saturated, devitrified Topopah Spring tuff. In Table 3-7, values for the cohesion of the solid and the internal angle of friction for the solid are those used by Arulmoli and St. John (1987). They used a value of 9.0 MPa for the tension limit of the tuff, but this is probably too high for the highly fractured large block. A value of 6.0 MPa was chosen for the FLAC modeling described in this report. Although Arulmoli and St. John (1987) do not report values for the dilation angle for tuff, the FLAC User's Manual says that values of about $10-15^{\circ}$ would be typical for concrete or sand. A value of $10^{\circ}$ was chosen for the FLAC modeling described in this report. Preliminary results of fracture mapping for the LBT show that most fractures in the block are subvertical, and there is a

Table 3-7. Rock Property Values Used for Modeling of the LBT

\begin{tabular}{lll}
\multicolumn{1}{c}{ Physical property } & \multicolumn{1}{c}{ Value } & \multicolumn{1}{c}{ Source } \\
\hline $\begin{array}{l}\text { Thermal properties } \\
\text { Thermal conductivity }\end{array}$ & $2.10 \mathrm{~W} /\left(\mathrm{m}^{\circ} \mathrm{K}\right)$ & YMP RIB (1990) \\
$\begin{array}{l}\text { Specific heat } \\
\text { Thermal expansion coeff }\end{array}$ & $840 \mathrm{~J} /\left(\mathrm{kg}-^{-} \mathrm{K}\right)$ & \\
\hline Mechanical properties & $9.1 \times 10^{-6} / \mathrm{K}^{\circ}$ & Estimate \\
Bulk modulus & & \\
Shear modulus & $2.7 \times 10^{9} \mathrm{~Pa}$ & Zimmerman et al. (1986) \\
Density & $14 \times 10^{9} \mathrm{~Pa}$ & \\
Cohesion & $2300 \mathrm{~kg} / \mathrm{m}^{3}$ & Roberts and Lin (1996a) \\
Dilation angle & $22 \times 10^{6} \mathrm{PA}$ & Arulmoli and St. John (1987) \\
Tension limit & $10^{\circ}$ & FLAC Users's Manual \\
Internal angle of friction & $6 \times 10^{6} \mathrm{~Pa}$ & Estimate \\
Joint angle (cc from horiz) & $29^{\circ}$ & Arulmoli and St. John (1987) \\
Joint cohesion & $10^{\circ} \& 85^{\circ}$ & Estimate \\
Joint tension & $6 \times 10^{5} \mathrm{~Pa}$ & Estimate \\
Joint friction angle & $5 \times 10^{5} \mathrm{~Pa}$ & Estimate \\
\hline $31^{\circ}$ & Price et al. (1993) \\
\hline
\end{tabular}

major subhorizontal fracture near the top of the block. A value of about $85^{\circ}$ is appropriate for representing the joint angle for subvertical fractures, and a value of $10^{\circ}$ is representative of subhorizontal fractures. For the joint cohesion, Arulmoli and St. John (1987) used values of 1.0 $\mathrm{MPa}$ and $0.05 \mathrm{MPa}$. An intermediate value of $0.6 \mathrm{MPa}$ was chosen for the FLAC modeling. Arulmoli and St. John (1987) used a joint friction angle of about $39^{\circ}$. Reported values of the average coefficient of friction for tuff (Price et al. 1993) suggest that a value of $31^{\circ}$ is more 
appropriate for the joint friction angle, and thus this lower value was used in the FLAC modeling. In FLAC, the tensile strength is limited by cohesion and the friction angle. If the tension limit exceeds the value found by dividing the cohesion by the tangent of the friction angle, the tension limit is reset to that value. Thus the maximum value of the joint tension limit found using a joint cohesion of $0.6 \mathrm{MPa}$ and a joint friction angle of $31^{\circ}$ would be about $1 \mathrm{MPa}$. The default joint tension limit in FLAC is zero, but that would be too small a value. The FLAC modeling used an intermediate value of $0.5 \mathrm{MPa}$ for the joint tension limit.

\subsection{ABAQUS ${ }^{\mathrm{TM}}$ Models}

The second method considered in the pre-test simulations is the 3-D finite element method, and the code ABAQUS ${ }^{\mathrm{TM}}$ was used to evaluate this method. ABAQUS ${ }^{\mathrm{TM}}$ is a 3-D code with capability for time-dependent analysis of thermal and mechanical behavior.

ABAQUS ${ }^{\mathrm{TM}}$ processes input in a batch fashion. The thermal model uses "therm.inp" as an independent input file, which writes a results file of nodal temperatures at specified time steps. The mechanical model uses "mech.inp" to read the results of the thermal model and calculates an equilibrium displacement field. The two models use the same nodes and linear, brick elements. The procedure for running ABAQUS ${ }^{\mathrm{TM}}$ is to first create a file that provides information about the grid used, the mechanical and thermal properties of the rock to be simulated, etc. This file is very similar to the input file used for the FLAC code. The following paragraphs discuss the input used for the simulations conducted for this study.

\subsection{Time Steps}

The thermal model in ABAQUS ${ }^{\mathrm{TM}}$ is run with one step and 75 increments, where each increment is one day. The mechanical model is run in seven steps. Each step represents 10 days. The mechanical problem is static and hence each step is one increment long. Altogether, the model contained 3912 nodes and 2864 elements. The thermal model took about 50 minutes to execute 75 time steps of one day each on a Sparc 10 workstation. The mechanical model was computed every 10 days, and the execution time was about 30 minutes on a Sparc10 workstation. Output files were stored after every time step.

\subsection{Grids, Nodes, and Elements}

The nodes are divided into a "block" set and a "ground" set. The grid for the faces of the block perpendicular to the heaters is the same as that used in thermal-hydrologic simulations of the LBT (Lee 1995a) and in the thermal-mechanical FLAC model simulations mentioned above. The grid for the ground does not extend as far as the previous models, because those results showed that effects of the heating are of limited extent into the ground. The thermal elements (DC3D8) and mechanical elements (C3D8R) are paired for thermomechanical problems, in which the temperature field is first computed at different times before solving for mechanical equilibrium.

\subsection{Material Properties}

ABAQUS ${ }^{\mathrm{TM}}$ requires many of the same material properties as FLAC, and for the simulations conducted in this study the mechanical and thermal properties input to ABAQUSTM were identical to 
those used in the FLAC elastic model and are given in Table 3-7. In the ABAQUSTM modeling, an elastic model was used to simulate the large block, and no viscoelastic properties or fracture zones were used.

\subsection{Initial and Boundary Conditions}

The initial temperature is $20^{\circ} \mathrm{C}$ for both the thermal and mechanical models. Thermal stresses arise as a result of thermal expansion relative to this reference state. The default condition for the temperature problem is that boundaries are adiabatic. In the model, all boundaries are insulated except the ground surface, which is maintained at $20^{\circ} \mathrm{C}$. The default condition for the mechanical problem is that boundaries are at zero stress. In the model, all boundaries are stress-free except the symmetry planes and the bottom of the model. The load for the thermal problem is the constant temperature heaters. (Modeling the heaters as constant power heat sources requires a FORTRAN subroutine HETVAL that was not available for this study.) The load for the mechanical problem is the thermal stresses arising from the nonuniform temperature field at different times.

\subsection{Output}

The nodal temperatures were placed in a special results file for use by the mechanical model. Also, temperatures along the side of the block parallel to the heaters were specified to be included in the *.dat ASCII file with other results normally produced by ABAQUS ${ }^{\mathrm{TM}}$. In the mechanical model, displacements on the outer face parallel to the heaters were specified to be included in the .dat file.

\subsubsection{Results}

\subsection{FLAC Modeling Results}

Four different 2-D FLAC models were run to model the LBT. The first model assumed the large block was a homogeneous elastic solid having the elastic moduli values listed in Table 3-7. The second model assumed a homogeneous elastic large block, but the values of the bulk and shear moduli were reduced by an order of magnitude to simulate the effect of many fractures in an elastic medium. In the third model, two fractures were simulated in the block by modeling the block as an elastic medium containing one set of vertical grid elements and one set of horizontal grid elements having elastic moduli reduced by one order of magnitude. The fourth model simulated these two fractures by using the ubiquitous joint model within these sets of horizontal and vertical grid elements and representing the rest of the block as a homogeneous elastic medium.

Each of these four models was first brought to initial mechanical equilibrium. The initial maximum unbalanced force had a magnitude of about $10^{6} \mathrm{~N} / \mathrm{m}$ as a result of the maximum stress in the initial mechanical model, $10^{5} \mathrm{~Pa}$, acting on the largest grid element, having 16-m sides. This initial unbalanced force was reduced by about four orders of magnitude after 9000 steps, requiring about four minutes of computer time on a Sun Sparc10 workstation. 


\subsection{FLAC Thermal Results}

For each of the four models, after mechanical equilibrium was attained, FLAC was run in the thermal mode to simulate heating for 75 days. Since the temperature field is not affected by mechanical properties, all of the four models had identical temperatures for any given number of days. Figure 3-28(a-e) shows the temperatures contours in a vertical cross section after 1 day, 10 days, 25 days, 50 days, and 75 days of heating, respectively. Figure 3-29a shows the temperature profile for the top of the block, and Figure 3-29b shows the profile for the grid element representing the central heater. Lee (1995a) shows a similar temperature profile for the heaters (see Figure 3-30), except that the temperatures in Lee's model are slightly lower at a given time, because he does not use an adiabatic boundary condition at the top of the block.

The figures show that the base of the block and the top $1 \mathrm{~m}$ of the block remain at the ambient temperature of about $20^{\circ} \mathrm{C}$ for the first 10 days of heating, at which time the heaters are at about $70^{\circ} \mathrm{C}$. The isotherms are horizontal and are symmetrical above and below the heater plane for the first 10 days of heating.

By the 25th day of heating, the top of the block has reached a temperature of about $35^{\circ} \mathrm{C}$ and the heaters are at about $95^{\circ} \mathrm{C}$. The isotherms are horizontal except for within about $30 \mathrm{~cm}$ of the heaters and the region near the bottom corner of the block where the adiabatic boundary condition for the side of the block meets the isothermal boundary condition for the ground surface. If the ground surface within about a meter of the block was adiabatic, these contours at the base of the block would be made more horizontal. After 25 days of heating, the temperature gradient is somewhat steeper below the heaters than above the heaters, because there is no heat flow out the top of the block. The base of the block is about $30-50^{\circ} \mathrm{C}$ at this time. No dryout zone forms in the first 25 days of heating, except for right in the plane of the heaters.

Note that the temperature change in the heater zone is very high for the first 10 days of heating, about $50^{\circ} \mathrm{C}$ as the heaters go from $20^{\circ} \mathrm{C}$ to $70^{\circ} \mathrm{C}$, and is only half as much for the next 15 days of heating as the heaters go up to about $95^{\circ} \mathrm{C}$ on the 25 th day of heating. This suggests that stresses due to thermal expansion of the rock will be developed fairly early in the 75 days of heating.

According to FLAC, the top of the block reaches $60^{\circ} \mathrm{C}$ at about the 45th day of heating, assuming the top is adiabatic. This is probably about $20-30$ days earlier than observed in the LBT, and some future modeling with a different boundary condition will be done to better simulate the top of the block. Lee (1995a) used an isothermal top boundary set at $60^{\circ} \mathrm{C}$, but this results in heat flow downward from the top of the block for the first 30 days in his hydrothermal modeling.

After 50 days of heating, the isotherms are horizontal above the heater zone, and slightly distorted and closer together below the heater zone. The base of the block is about $40-60^{\circ} \mathrm{C}$, the top of the block is slightly above $60^{\circ} \mathrm{C}$, and the heaters are at about $125^{\circ} \mathrm{C}$. Temperatures above the boiling point of water are found extending to about $50 \mathrm{~cm}$ above and $30 \mathrm{~cm}$ below the heaters, suggesting that a dryout zone nearly $1 \mathrm{~m}$ wide could form in the large block at this time. Between the 25th and the 50th day of heating, temperatures at the base of the block have only changed about $10-20^{\circ} \mathrm{C}$, while temperatures of the heaters and the top of the block change by about $30^{\circ} \mathrm{C}$. The stresses and displacements due to the thermal loading are expected to be higher 
above the heaters compared to the region below the heaters. Note that the temperatures beyond about $1 \mathrm{~m}$ below the base of the block remain low even after 50 days of heating in the block.

The heaters reach a temperature of about $140^{\circ} \mathrm{C}$ after 75 days of heating. The temperature $1 \mathrm{~m}$ below the base of the block is about $40^{\circ} \mathrm{C}$, and temperatures of regions more than about $0.5 \mathrm{~m}$ away from the block remain at the original temperature of $20^{\circ} \mathrm{C}$. Temperatures remain at about $20^{\circ} \mathrm{C}$ for regions more than about $2 \mathrm{~m}$ below the block. Lee (1995a) shows a similar temperature field in his hydrothermal modeling. The similarity of the temperature fields for the TM and TH modeling assures that appropriate parameters for simulating the LBT were used in the FLAC modeling, and suggests that results of Lee's modeling could be used as input for future FLAC modeling in order to model coupled thermal-hydrological-mechanical processes.

\subsection{FLAC Mechanical Results}

Once the temperature field was determined for different heating times, FLAC was run in the mechanical mode to determine what stresses and displacements developed in each of the four different mechanical models. Each of these runs required about 7000 steps and less than 10 minutes of computer time on the Sun Sparc10. The stresses and displacements differ for different heating times; this section concentrates on results from 75 days of heating.

Figure 3-31(a-e) shows the horizontal (x) displacements, vertical (y) displacements, $\sigma_{x x}, \sigma_{y y}$, and $\sigma_{x y}$ stresses respectively, after 75 days of heating for the first FLAC model in which the large block was represented as a homogeneous elastic medium having a bulk modulus of $27 \mathrm{GPa}$ and a shear modulus of $14 \mathrm{GPa}$. This model shows that the maximum horizontal displacement occurs in the heater plane, at the outside edge of the model, and has a magnitude of about $2 \mathrm{~mm}$. Vertical displacement is highest near the top of the block with a magnitude of about $5 \mathrm{~mm}$, decreasing to about $0.5 \mathrm{~mm}$ at the base of the block. Vertical displacement contours are approximately horizontal. Both the $\sigma_{x x}$ and the $\sigma_{y y}$ plots show that the region around the central heater develops compressive stresses with magnitudes up to about $8 \mathrm{MPa}$. The $\sigma_{\mathrm{xx}}$ plot also shows compressive stresses of this magnitude developing at the bottom corner of the block, while the $\sigma_{\mathrm{yy}}$ plot shows tensile stresses having magnitudes up to $10 \mathrm{MPa}$ developing at the outer edge of the model in the plane of the heaters. This is significant because this stress level is of the order of the tensile strength of the welded tuff, which is about $6 \mathrm{MPa}$ (see Table 3-7), and much greater than the tensile strength of any fractures in the block, which is estimated to be less than 1 MPa (see Table 3-7). The $\square x y$ plot for this elastic model shows that shear stresses having magnitudes up to about $4 \mathrm{MPa}$ may develop in the plane of the heaters. Plots of stresses for 25 days of heating the elastic model (not shown) are essentially the same as the plots for 75 days. The displacements are slightly lower, with a maximum of $1 \mathrm{~mm}$ horizontal displacement after 25 days of heating and a maximum of about $2.2 \mathrm{~mm}$ vertical displacement. The displacement contours are similar in shape for 25 days of heating compared to 75 days of heating, except that the very top of the block is slightly less disturbed. It is important to note that the displacements and stresses develop comparatively early in the heating, because the rates of temperature changes are higher early in the 75 days of heating.

The second FLAC mechanical model represented the large block as a homogeneous elastic medium having the same coefficient of thermal expansion but lower elastic moduli than the first model. A value of $2.7 \mathrm{GPa}$ was used for the bulk modulus, and a value of $1.4 \mathrm{GPa}$ was used for 
the shear modulus. Initial results of mechanical measurements made in the laboratory for $11-\mathrm{m}$ blocks of tuff from Fran Ridge suggest that these values may be appropriate for representing the highly fractured mass of rock that issuch as the large block. Horizontal and vertical displacements (not shown in the figure) found after 75 days of heating for this second model were identical to those found for the first model; this indicates that the thermal expansion coefficient controls displacements. For linear elasticity, it is expected that stresses for this second model should simply be reduced by an order of magnitude from those found for the first elastic model. Results of this modeling (not shown in the figure) found no significant compressive stresses or shear stresses developing anywhere in the model, and very small tensile stresses ( $<2 \mathrm{MPa})$ developing in the heater plane at the outer edge of the model. In this second model, the block is so compliant that it cannot build up significant stresses. An important implication of these results is that the presence of many fractures in the large block prevents stress from building to the high levels predicted for a homogeneous, elastic block.

To simulate the effect of discrete fractures on the thermomechanical behavior, the third and fourth mechanical models represented the large block as an elastic medium containing one horizontal set of grid elements and one vertical set of grid elements in which the properties were changed to simulate a horizontal and a vertical fracture. In the third model, these sets of grid elements were represented as elastic media with bulk and shear moduli lowered by one order of magnitude with respect to the rest of the block. The fourth model used the ubiquitous joint model for these elements. Figure 3-32 shows these elements that represent fractures, located in a horizontal line about $0.5 \mathrm{~m}$ below the top of the block and a vertical line about $1 \mathrm{~m}$ from the center of the block.

Stress and displacement results for the third model, for 75 days of heating, are shown in Figure 3-33(a-e). The $\square \mathrm{xx}$ plot is nearly the same as that for the original elastic model. Most of the compressive stress near the central heater (4-6 MPa) and most of the tensile stress at the outer edge of the model in the heater plane ( $>8 \mathrm{MPa}$ ) are already present after one day of heating (not shown). The $\square$ yy plot is almost the same as that for the first elastic model, except that the stress field is slightly distorted by the vertical fracture zone. The $\square x y$ plot shows that shear stresses are very low, below $2 \mathrm{MPa}$, for this model. The horizontal displacements are nearly the same as those found in the first elastic model, but the vertical displacements show contours that are offset slightly along the vertical fracture. Magnitudes of maximum displacements are the same for this model and the first elastic model.

The fourth model, which used the ubiquitous joint model to represent the horizontal and vertical fractures, had displacement results (not shown in the figure) that were nearly the same as those found usingin the third mechanical model-i.e., horizontal displacements the same as in the original elastic model and vertical displacements offset a small amount along the vertical fracture. The $\square$ xy results (not shown) were about the same for the third and fourth models. Figure 3-34(a,b) shows However, the $\square \mathrm{xx}$ plot and the $\square$ yy plot for the fourth model. These differ from results for the third model, as shown in Figure 3-34(a,b). Stresses are lower but are more affected by the presence of the vertical fracture. These results imply that the presence of fractures in the block has a strong affect effect on the distribution as well as the magnitudes of stresses. 


\subsection{ABAQUS ${ }^{\mathrm{TM}}$ Modeling Results}

This section presents temperatures, normal stresses, and normal displacements for 3-D ABAQUS $^{\mathrm{TM}}$ simulations at 10,20 , and 60 days. The results are presented graphically as perspective diagrams of the large block. The diagrams show the perspective of looking at the outside of the block from the point $(1,2,2)$. Only one-quarter of the block is displayed, and the two back surfaces are symmetry planes. Note that the back surface perpendicular to the long axis of the heaters corresponds to the 2-D cross-section modeled using FLAC. The figures showing the 3-D views are grouped into two sets. Figure 3-35 (a-c) shows predicted contours of temperature, and normal stresses at 10,20, and 60 days, respectively. In these figures, normal stresses on the faces perpendicular to the 1,2 , and 3 axes are denoted by $\sigma_{11}, \sigma_{22}$, and $\sigma_{33}$, respectively.

These results show that the predicted temperature field is approximately one-dimensional due to the insulated boundaries along the sides. The top of the block, which is also an insulated boundary in the model, reaches a temperature of about $65^{\circ} \mathrm{C}$ after 60 days. An alternative boundary condition for the top of the block would be a boundary in which the heat flux out of the top of the block would be proportional to the difference between the block and ambient air temperature (nominally, $20^{\circ} \mathrm{C}$ ).

The largest of the normal stresses is the vertical stress, $\sigma_{33}$, at the outside corner of the block near the heater plane. The largest value shown is about $14 \mathrm{Mpa}$, and it occurs at 10 days. This is consistent with the 2-D analysis that shows that the higher stresses occur early in the test. The maximum stress decreases to about $10 \mathrm{MPa}$ at 60 days because the thermal gradients decrease as the block becomes more uniformly heated. The horizontal stresses parallel and perpendicular to the heaters are almost an order of magnitude smaller than the vertical stress.

The second set of figures shows displacements that are predicted for the block at 10,20, and 60 days (Figures 3-36, a-c). In each of these figures the wire net diagrams in the upper left corner presents total displacements that have been exaggerated for purposes of clarity. Displacements in the 1,2, and 3 directions are given by U1, U2 and U3, respectively. The wirenet diagrams show how the block bulges in all directions, with the largest horizontal displacements normal to the vertical side in the heater plane. The horizontal displacements show at early times an approximate cylindrical shape about a horizontal axis orthogonal to the displacement component, but they become more planar at longer times. Similar patterns are seen in the 2-D FLAC analysis for the purely elastic case (Figure 3-31, a,b)

\subsubsection{Discussion}

The elastic model in 2-D and 3-D produced the highest stress levels, and these occurred during the first 10 days of heating. Tensile stress levels that approach the tensile strength of Topopah Spring tuff were predicted in the plane of the heaters and in the vertical direction in both the 2-D and 3-D cases for a homogeneous elastic block.

These simulations assumed nominally stress-free boundaries on the exposed faces of the block, and as a result the displacements (and strains) are dominated by the thermal expansion of the rock mass. The predicted temperature fields agree well with those predicted by Lee (1995a) 
using a code that incorporates a more sophisticated $\mathrm{TH}$ model. It is important to note that the LBT design incorporated a retention frame that surrounded the sides of the block and applied a nominal confining stress between 0.2 and $0.3 \mathrm{MPa}$ on the block. This retention frame also applied a shear stress on the sides of the block that opposed the tension generated in the vertical direction and reduced the level of tensile stress in the block in this direction, but the amount of reduction is difficult to estimate.

Two simulations were conducted in which discrete fractures present in the large block were simulated, and lower levels of stress were predicted in these simulations, indicating that the presence of compliant fractures may reduce the stress levels.

- 3.5.3.4.1 Comparison of 2-D and 3-D Simulations

The 2-D, plane strain, FLAC model was constructed in a plane orthogonal to the heater holes. The thermal predictions made with this code show a 2-D temperature field near the heaters at early times. At later times, the code predicts a temperature field that is nearly one-dimensional because all the walls are adiabatic. The temperature fields in the 3-D model were generated using $100^{\circ} \mathrm{C}$ constant-temperature heaters. This approach produced very similar temperature fields to those predicted by the $300-\mathrm{W}$ constant-power heaters used in the 2-D FLAC model. For example, the temperature at the top of the block is approximately $65^{\circ} \mathrm{C}$ after 60 days in the 3-D model and approximately $70^{\circ} \mathrm{C}$ after 55 days (not shown in the figure) in the 2-D model.

The differences are greater between the 2-D and 3-D mechanical models. The plane strain approximation used in the 2-D model assumes that the length of the heater direction is long compared with the height and width of the block. Further, the plane strain model does not incorporate the stress-free boundary faces parallel to the model plane. The plane strain model generates a large, compressive normal stress in the heater direction to suppress the thermal expansion in that direction.

Despite these caveats and the differences in the thermal models, the plane strain results and those from the mid-plane perpendicular to the heaters of the 3-D model are very similar because this plane is a symmetry plane on which the normal displacement is zero in both the 3-D and 2-D models. Additional analysis not included here shows that in this plane the 3-D model predicts that both $\square 11$ and $\square 33$ are compressive near the center of the block, just as they are in the 2-D model. In fact, the detailed contour patterns and approximate magnitudes for this plane in the 3-D model match those of the 2-D plane strain model rather closely. However, the patterns do not project to the outer surfaces of the block.

\subsection{Conclusions}

The main results of the modeling can be summarized as follows:

The thermal-mechanical FLAC and ABAQUS ${ }^{\mathrm{TM}}$ modeling produced temperature fields similar to that the TH model of Lee (1995a). who used a code which contains a more sophisticated thermal-hydrologic model. This suggests that the FLAC and ABAQUS ${ }^{\text {TM }}$ codes could be used with a TH model to better investigate coupled processes.

- Most of the thermal stress develops in the first 10 days of heating, when the rate of temperature change is highest. 
- Stress levels are highest in the heater plane and at the bottom outside edge of the block. Tensile stresses are highest at the surface of the block in the plane of the heaters, are oriented in the vertical direction, and may be capable of causing tensile fracture. However, results show that the presence of fractures in the large block may reduce the levels of stress in the block below those predicted for a purely elastic continuum. High tensile stresses may still occur locally in the plane of the heaters.

- Displacements are highest at the top of the block and at the outside edge of the block in the heater plane, with magnitudes on the order of a few millimeters for both horizontal and vertical directions.

The 2-D and 3-D modeling is are complementary. Results agree for the elastic constitutive model in the symmetry plane at the center of the block. The 2-D modeling was faster and was useful for exploring effects of different constitutive models, . but was unable to simulate the outside face of the large block where stresses are highest. This is because the plane strain assumption is equivalent to assuming that the 2-D model lies at the center of the block. Note that most of the instrumentation in the LBT will be located on the outside surface of the block. The 3-D modeling required an order of magnitude more in computer time but was able to estimate maximum stresses and displacements everywhere in the block's volume.

\subsubsection{Post-test Characterization}

Post-test characterization activities included drilling and coring and analyzing the post-test cores. The main purpose of the post-test analyses was to assess the effect of the test on the changes in mineralogy of the block. The results of the post-test mechanical analysis are presented in Section 7. The results of the post-test mineralogical analyses are presented in Section 8.

\subsubsection{Boreholes for Post-test Characterization}

Nineteen HQ-sized, core-drilled boreholes and one 10-in overcore of an existing heater borehole were dry-drilled to provide samples for post-test characterization of the LBT. The characteristics of each borehole are listed in Table 3-8. Most of these boreholes were drilled in two vertical fans. One fan of holes was drilled from the west side of the block and the other from the north side. The layouts of these boreholes are shown in Figure 3-37 and Figure 3-38.

Prior to drilling the boreholes, all the collar locations (except the overcore) were located and marked; the overcore is located concentrically with the heater hole EH-4, which served as a pilot hole for the drilling.

Of the 10 holes drilled into the west face of the block, nine were drilled in a fan-like pattern in a vertical plane that intersected borehole LBL-1. This borehole was intersected to provide core samples that might contain microbes placed in LBL-1 prior to the start of heating.

The tenth borehole drilled from the west side of the block (PTC-10) was oriented in the horiztontal plane and approximately $16^{\circ}$ clockwise from the vertical fan. This borehole was designed to intersect borehole LBL-2 and to obtain core in the horizontal plane at the level above the heaters in the block. 
Nine boreholes were also drilled into the north face of the block. Eight of these were oriented north-south and in a fan-shape pattern in a vertical plane aligned with vertical borehole TN-3. Aligning these holes with borehole TN-3 was done to obtain samples of grout used in the borehole that had been involved with rock/water interaction so that the effect of the THC environment on the grout could be determined. The ninth borehole on the north side was drilled at $16^{\circ}$ to the east of north and in the horizontal plane to penetrate borehole TM-2.

All core boreholes extended through the block so that edge effects, if any, could be observed. As-built surveys of the boreholes and video logging of each borehole were completed.

Table 3-8. The xyz Coordinates (with Respect to the Southwestern Corner on Top of the Block) of the Collar, and Other Characteristics of the Post-test Holes

\begin{tabular}{lcccccrc}
\hline Borehole ID \# & $\mathbf{X}(\mathbf{m})$ & $\mathbf{Y}(\mathbf{m})$ & $\mathbf{Z}(\mathbf{m})$ & Face & Orientation & Angle & $\begin{array}{c}\text { Length } \\
(\mathbf{m})\end{array}$ \\
\hline UE25-FR-PTC-1 & 1.22 & 3.05 & -2.29 & $\mathrm{~N}$ & $\mathrm{~N}$ & $0^{\circ}$ & 3.05 \\
UE25-FR-PTC-2 & 1.22 & 3.05 & -2.02 & $\mathrm{~N}$ & $\mathrm{~N}$ & $+10^{\circ}$ & 3.1 \\
UE25-FR-PTC-3 & 1.22 & 3.05 & -2.55 & $\mathrm{~N}$ & $\mathrm{~N}$ & $-10^{\circ}$ & 3.1 \\
UE25-FR-PTC-4 & 1.22 & 3.05 & -1.73 & $\mathrm{~N}$ & $\mathrm{~N}$ & $+20^{\circ}$ & 3.2 \\
UE25-FR-PTC-5 & 1.22 & 3.05 & -2.84 & $\mathrm{~N}$ & $\mathrm{~N}$ & $-20^{\circ}$ & 3.2 \\
UE25-FR-PTC-6 & 1.22 & 3.05 & -1.41 & $\mathrm{~N}$ & $\mathrm{~N}$ & $+30^{\circ}$ & 2.82 \\
UE25-FR-PTC-7 & 1.22 & 3.05 & -3.76 & $\mathrm{~N}$ & $\mathrm{~N}$ & $-44^{\circ}$ & 4.27 \\
UE25-FR-PTC-8 & 1.22 & 3.05 & -1.01 & $\mathrm{~N}$ & $\mathrm{~N}$ & $+40^{\circ}$ & 1.57 \\
UE25-FR-PTC-9 & 1.22 & 3.05 & -2.29 & $\mathrm{~N}$ & $\mathrm{~N} 16 \mathrm{~W}$ & $0^{\circ}$ & 3.32 \\
UE25-FR-PTC-10 & 0 & 1.83 & -1.10 & $\mathrm{~W}$ & $\mathrm{~W}$ & $+38^{\circ}$ & 1.78 \\
UE25-FR-PTC-11 & 0 & 1.83 & -1.30 & $\mathrm{~W}$ & $\mathrm{~W}$ & $+33^{\circ}$ & 1.39 \\
UE25-FR-PTC-12 & 0 & 1.83 & -1.51 & $\mathrm{~W}$ & $\mathrm{~W}$ & $+27^{\circ}$ & 3.33 \\
UE25-FR-PTC-13 & 0 & 1.83 & -1.73 & $\mathrm{~W}$ & $\mathrm{~W}$ & $+20^{\circ}$ & 3.25 \\
UE25-FR-PTC-14 & 0 & 1.83 & -1.91 & $\mathrm{~W}$ & $\mathrm{~W}$ & $+14^{\circ}$ & 3.14 \\
UE25-FR-PTC-15 & 0 & 1.83 & -2.26 & $\mathrm{~W}$ & $\mathrm{~W}$ & $+1^{\circ}$ & 3.05 \\
UE25-FR-PTC-16 & 0 & 1.83 & -2.55 & $\mathrm{~W}$ & $\mathrm{~W}$ & $-10^{\circ}$ & 3.1 \\
UE25-FR-PTC-17 & 0 & 1.83 & -2.84 & $\mathrm{~W}$ & $\mathrm{~W}$ & $-20^{\circ}$ & 3.25 \\
UE25-FR-PTC-18 & 0 & 1.83 & -3.35 & $\mathrm{~W}$ & $\mathrm{~W}$ & $-35^{\circ}$ & 3.73 \\
UE25-FR-PTC-19 & 0 & 1.83 & -2.18 & $\mathrm{~W}$ & $\mathrm{~W}$ & $+4^{\circ}$ & 3.06 \\
UE25-FR-PTC-19a & 0 & 1.83 & -2.29 & $\mathrm{~W}$ & $\mathrm{E} 16 \mathrm{~S}$ & $0^{\circ}$ & 3.32 \\
UE25-FR-PTC-OC-1 & 3.05 & 2.13 & -2.74 & $\mathrm{E}$ & $\mathrm{W}$ & $0^{\circ}$ & 3.05 \\
\hline
\end{tabular}




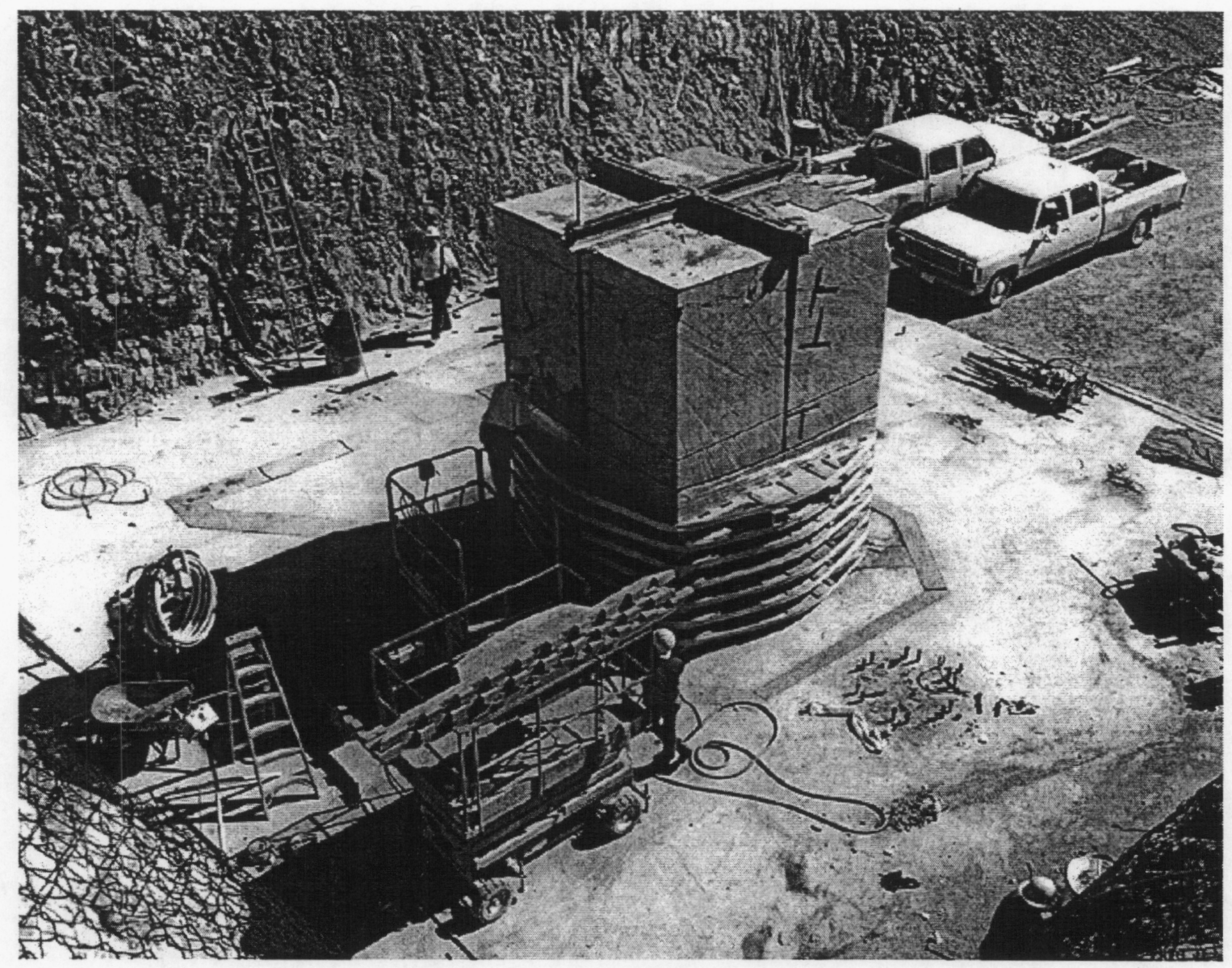

Figure 3-1. The Topopah Spring Tuff Block of the LBT 


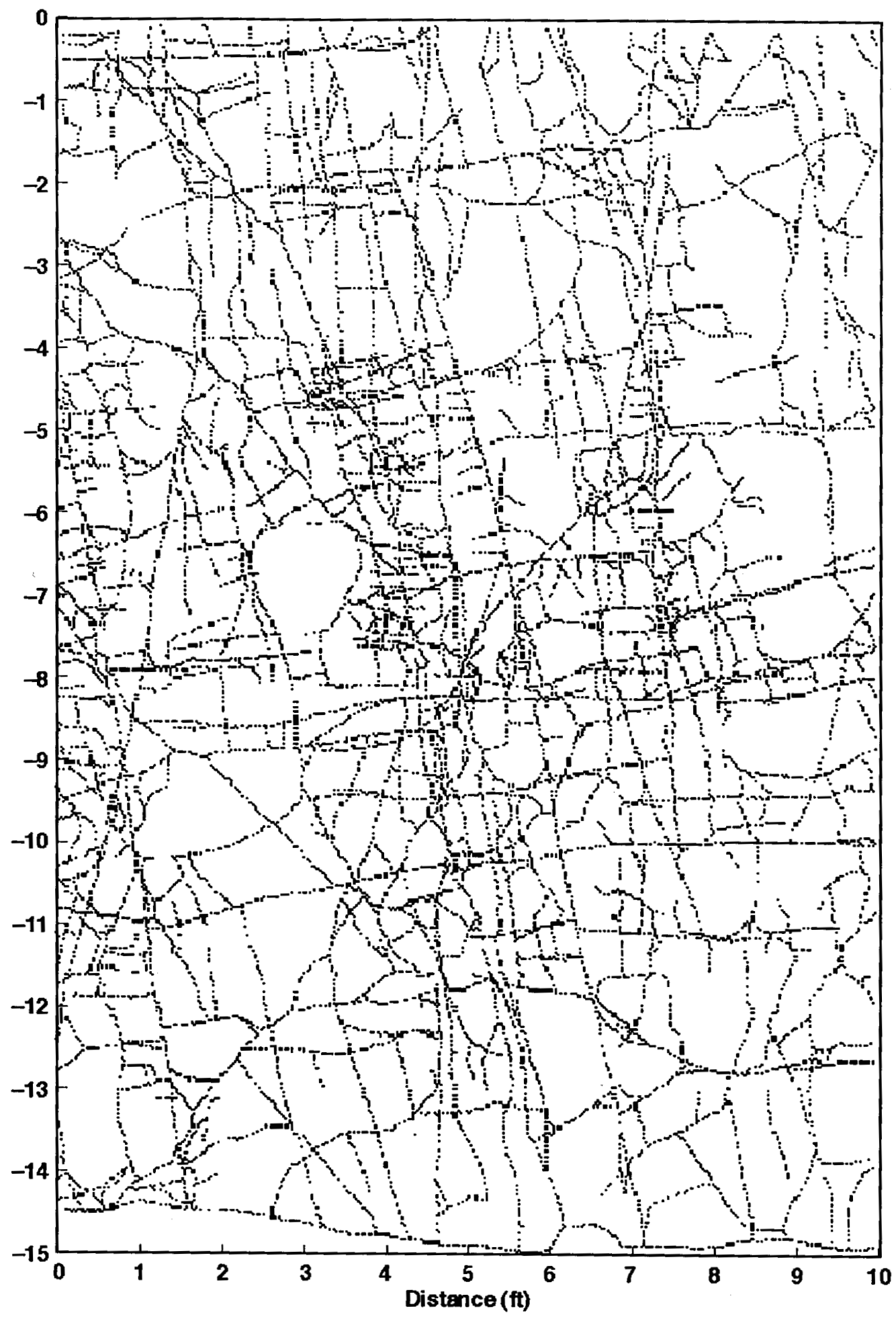

Figure 3-2. Fractures on the North Face of the Block 


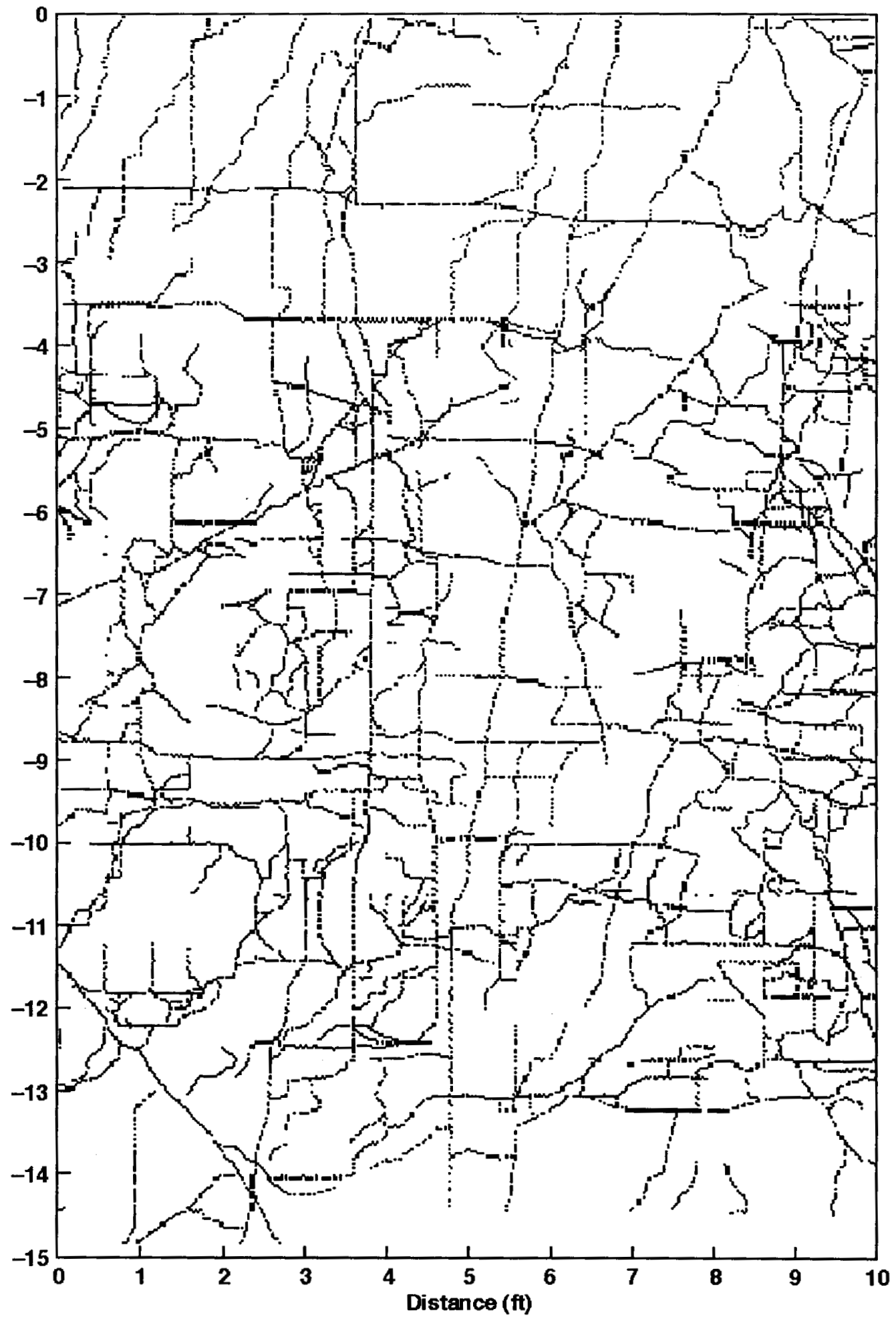

Figure 3-3. Fractures on the East Face of the Block 


\section{Large Block Test Instrument Holes}

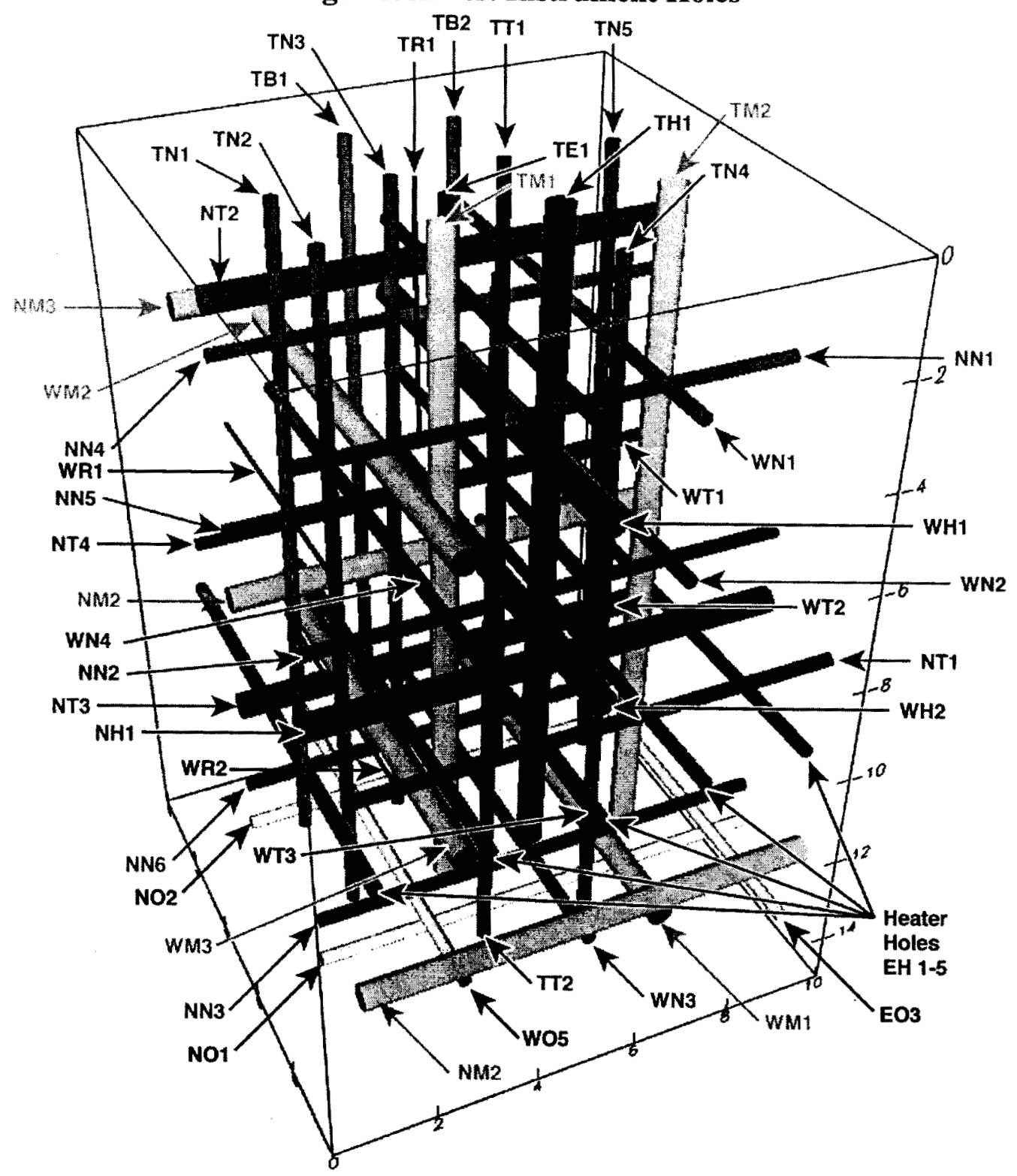

Figure 3-4. A Diagram Showing All Holes in the Block of the Large Block Test 


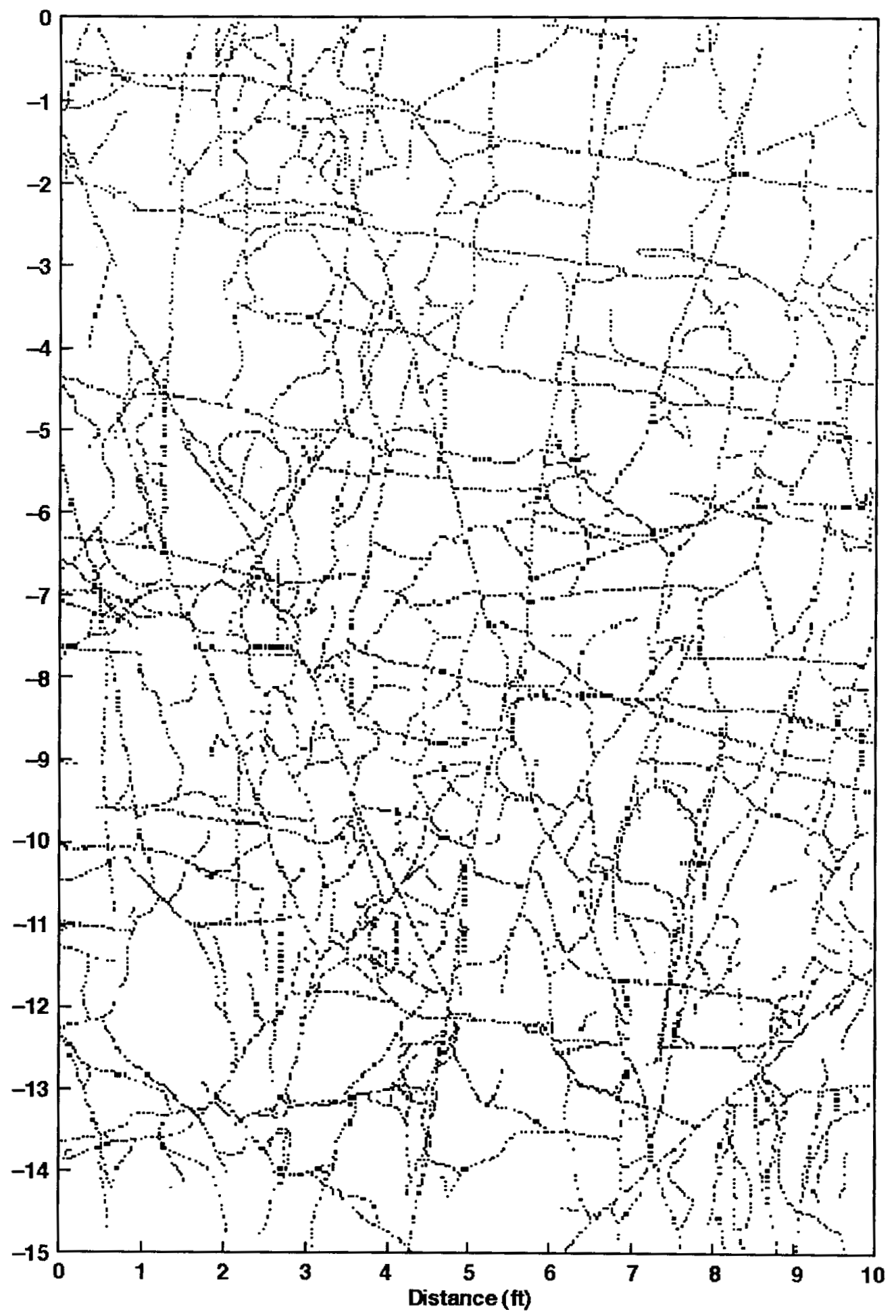

Figure 3-5. Fractures on the South Face of the Block 


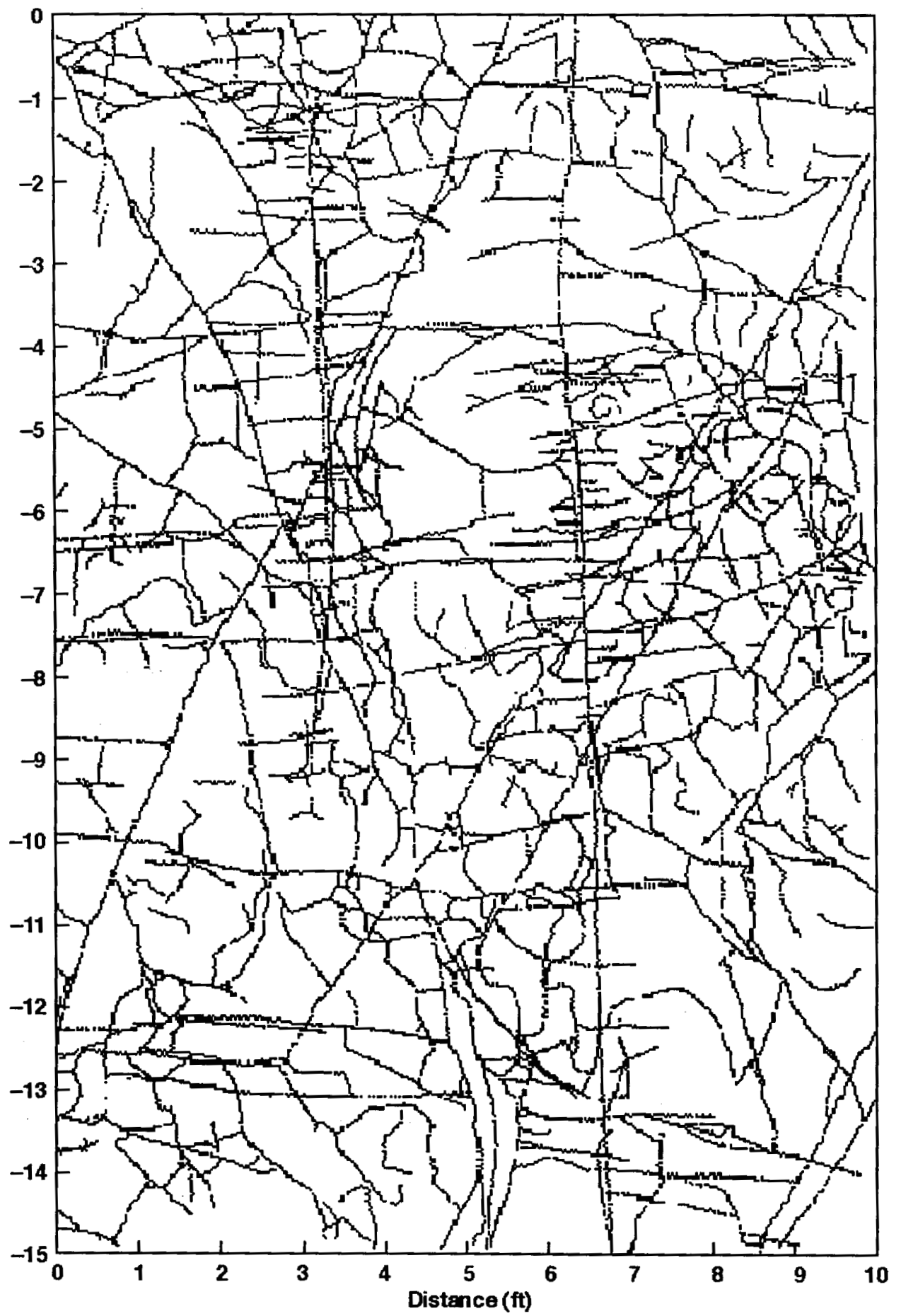

Figure 3-6. Fractures on the West Face of the Block 


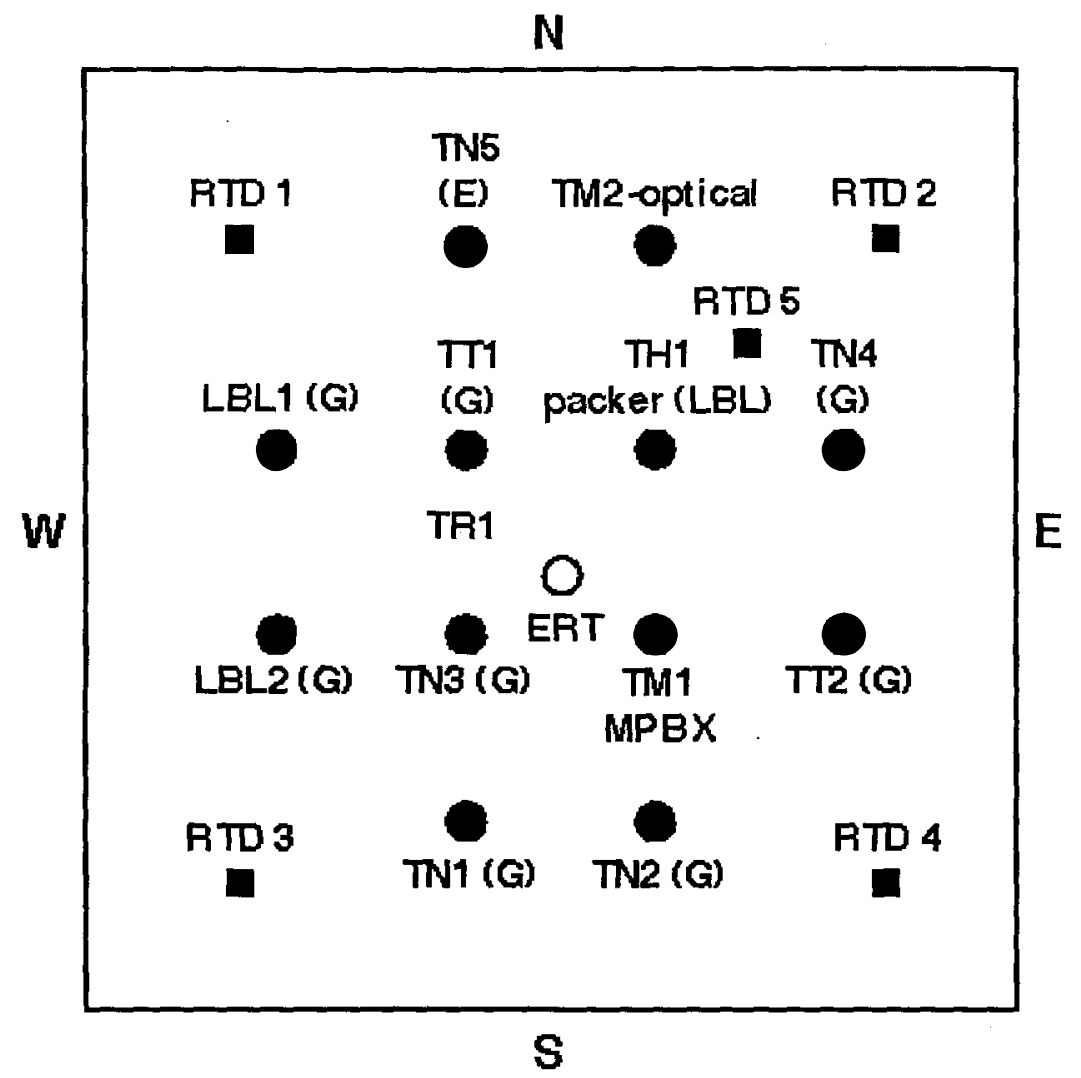

TTH - Temperature measurements - borehole

RTD\#- Temperature - Surface mounted RTD

LBL\# - Open holes to be used by LBL for air and then grouted

TNF - Neutron holes to be lined and grouted

TR1 - REKA probe - to be grouted

TM\# - Mechanical - MPBX borehole

Figure 3-7. Instrument Holes on Top of the Block 


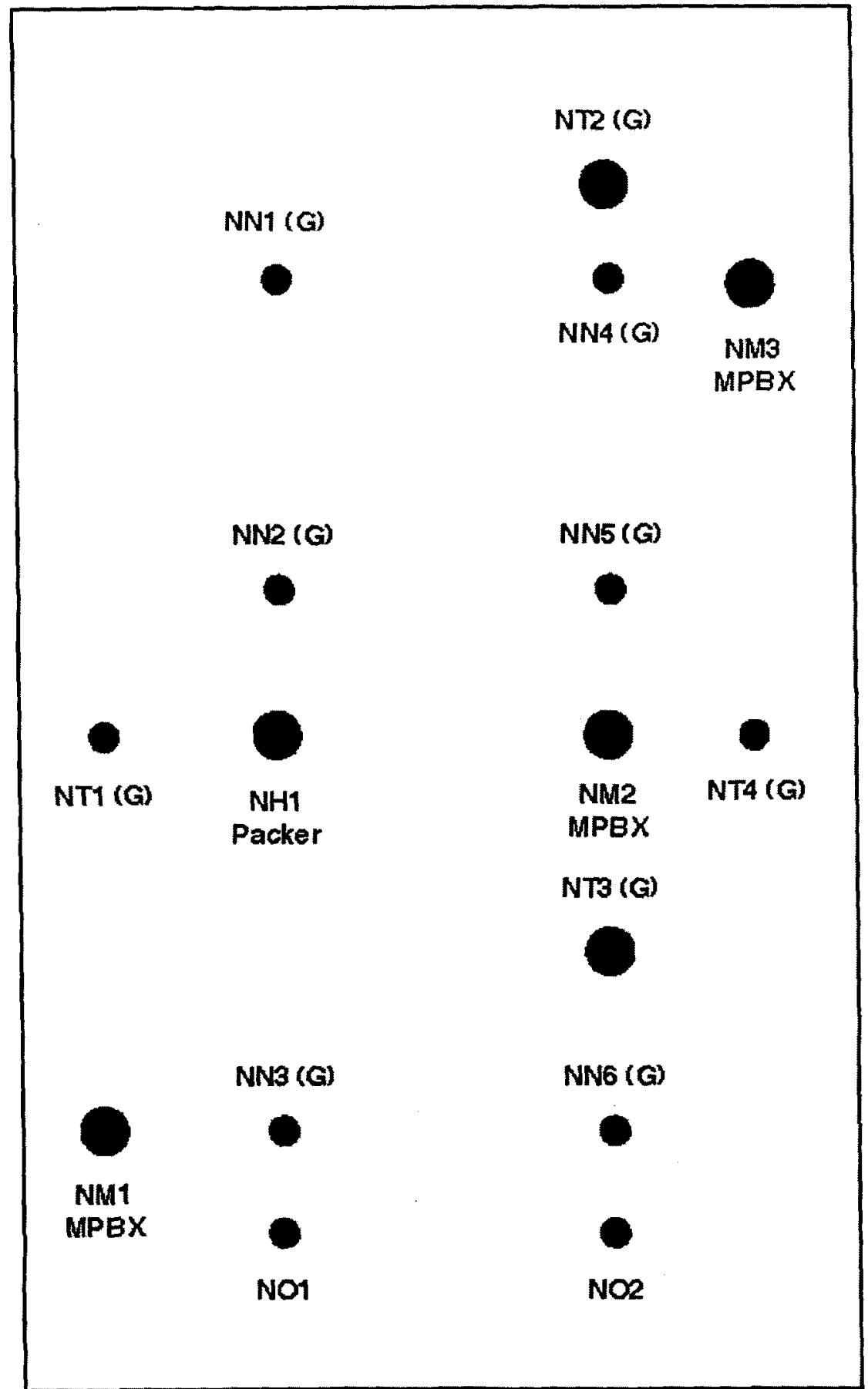

NNI - North side neutron hole - to be lined and grouted

NTH - North side temperature (RTD bundle) all to be grouted NM\# - North side mechanical

NO\# - North side observation hole

NHI - North side hydrology - packer assembly to be installed

Figure 3-8. Instrument Holes on the North Face of the Block 


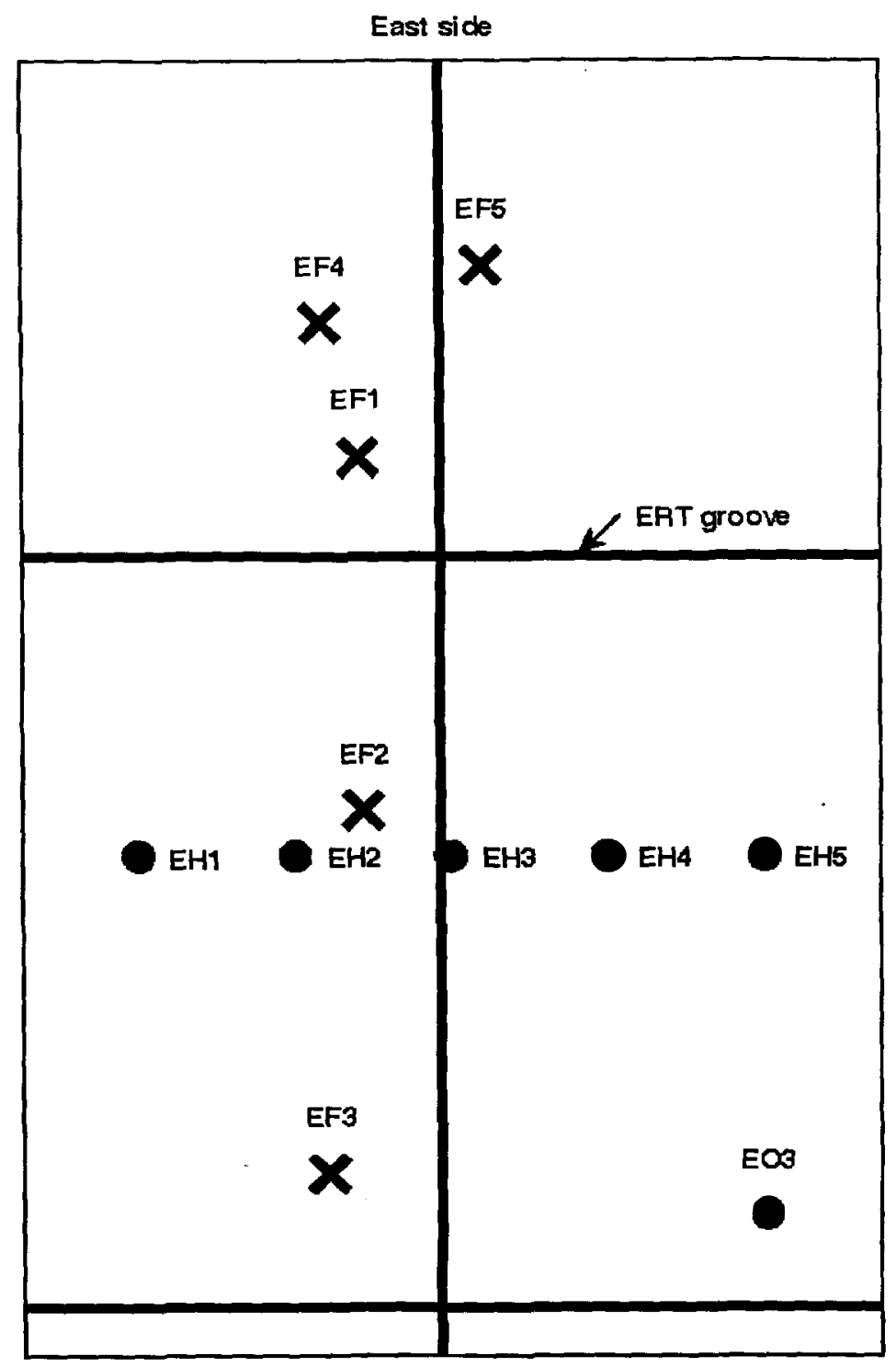

EH\# - East heater hole (heater assembly wERT) EO - East observation

EFF - Fracture monltors

Figure 3-9. Instrument Holes on the East Face of the Block 
West side

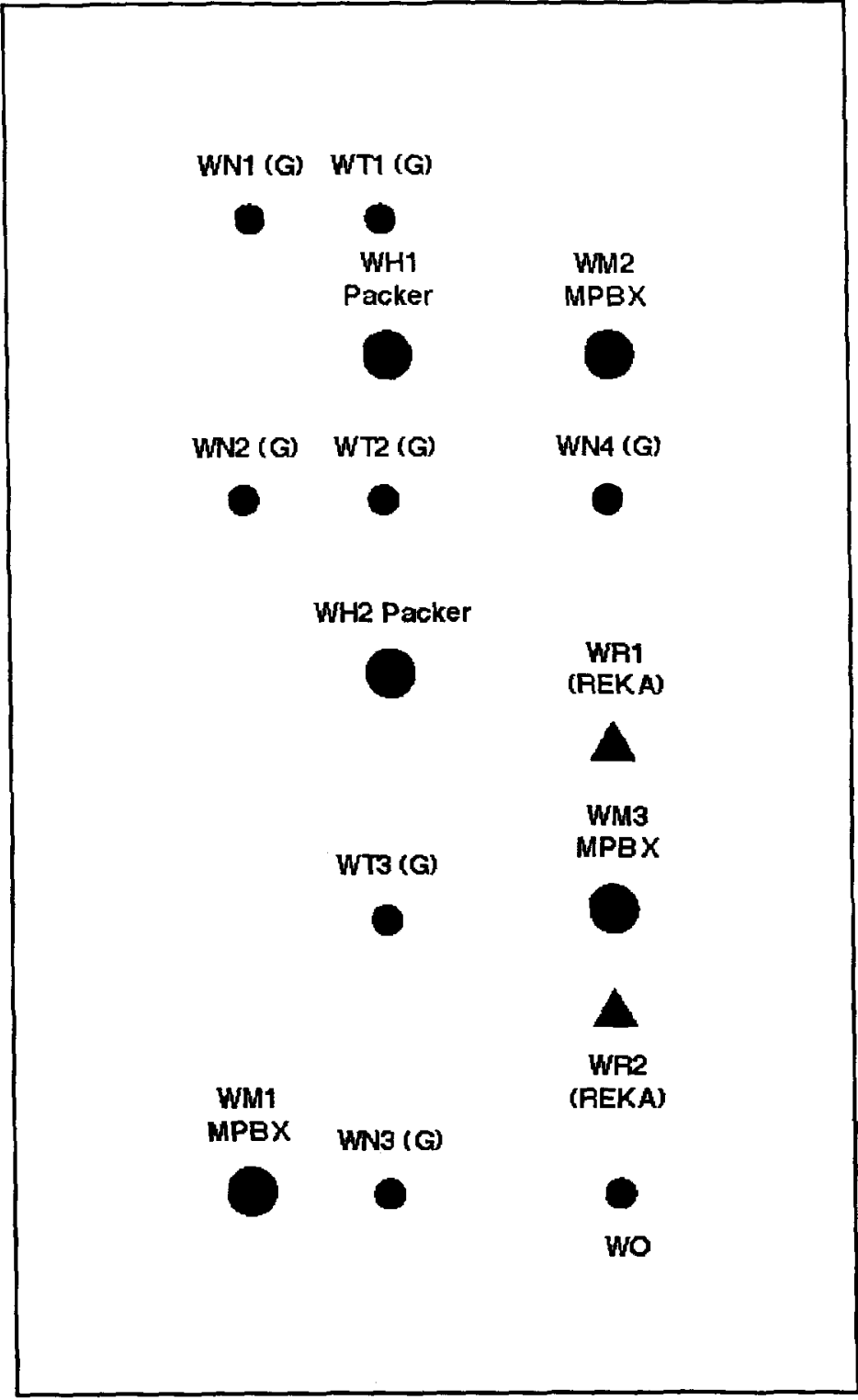

WNF - West side neutron hole - to be lined and grouted WTH - West sicle thermal (RTD bundle) to be grouted WH\# - West side horlzontal - packer assembly to be Installed WMI - West side mechanical (MPBXO hole - to be grouted WO\# - West side observation hole

Figure 3-10. Instrument Holes on the West Face of the Block 


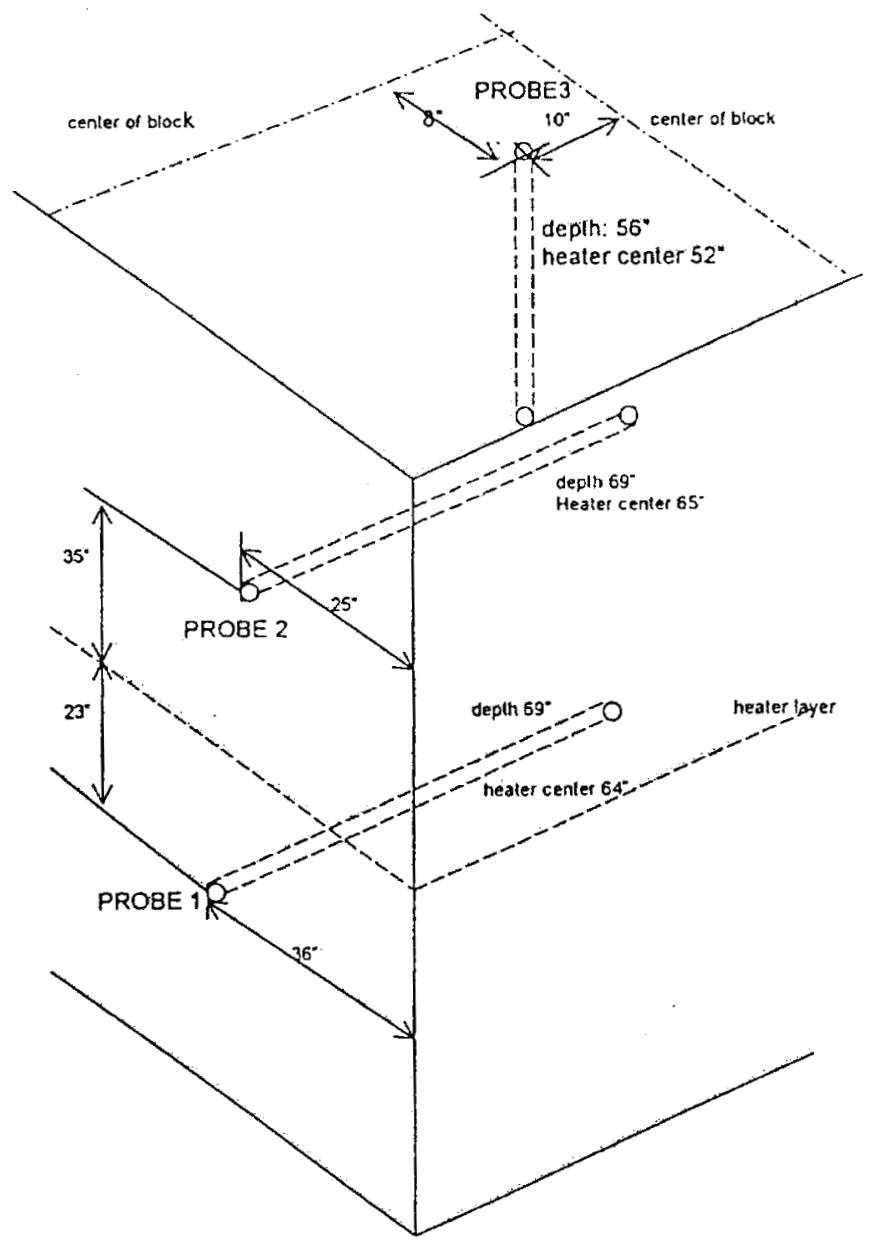

Figure 3-11. Three REKA Probe Locations in the Large Heated Block 


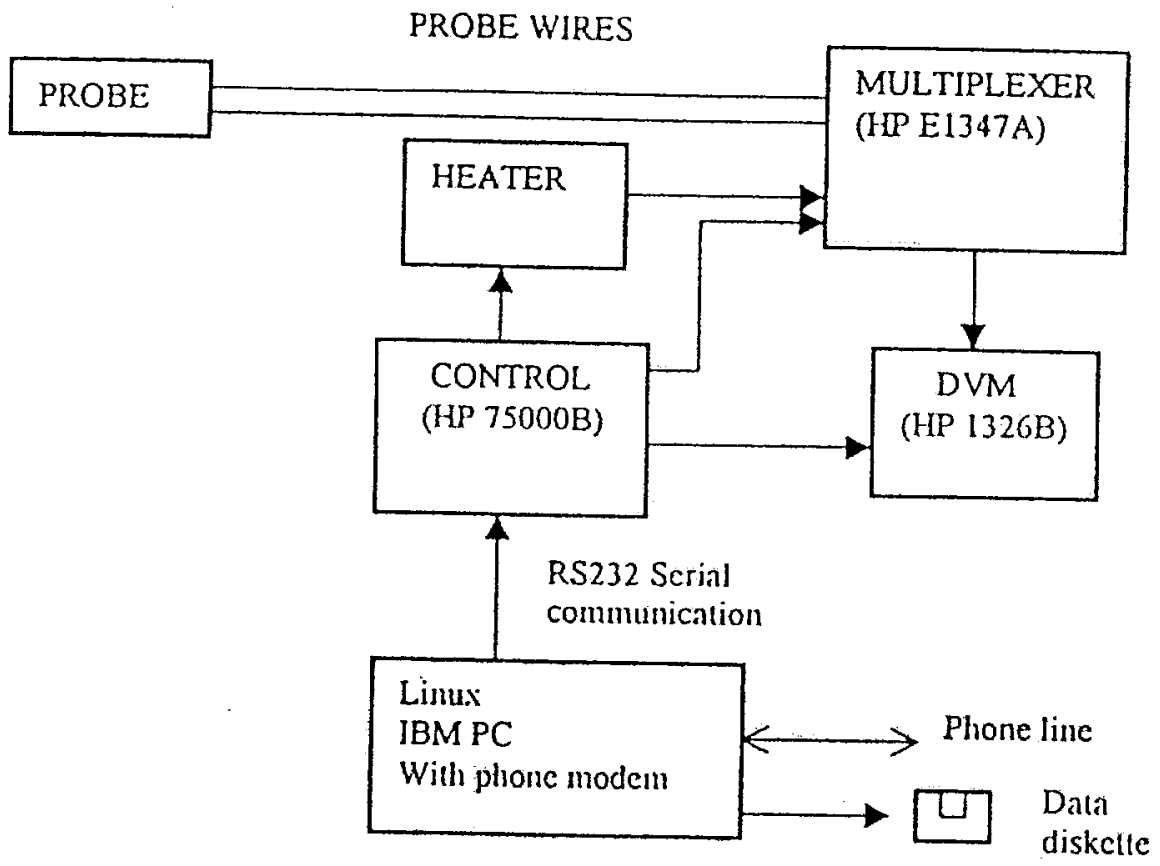

Figure 3-12. Measurement Flow Chart for One Probe 


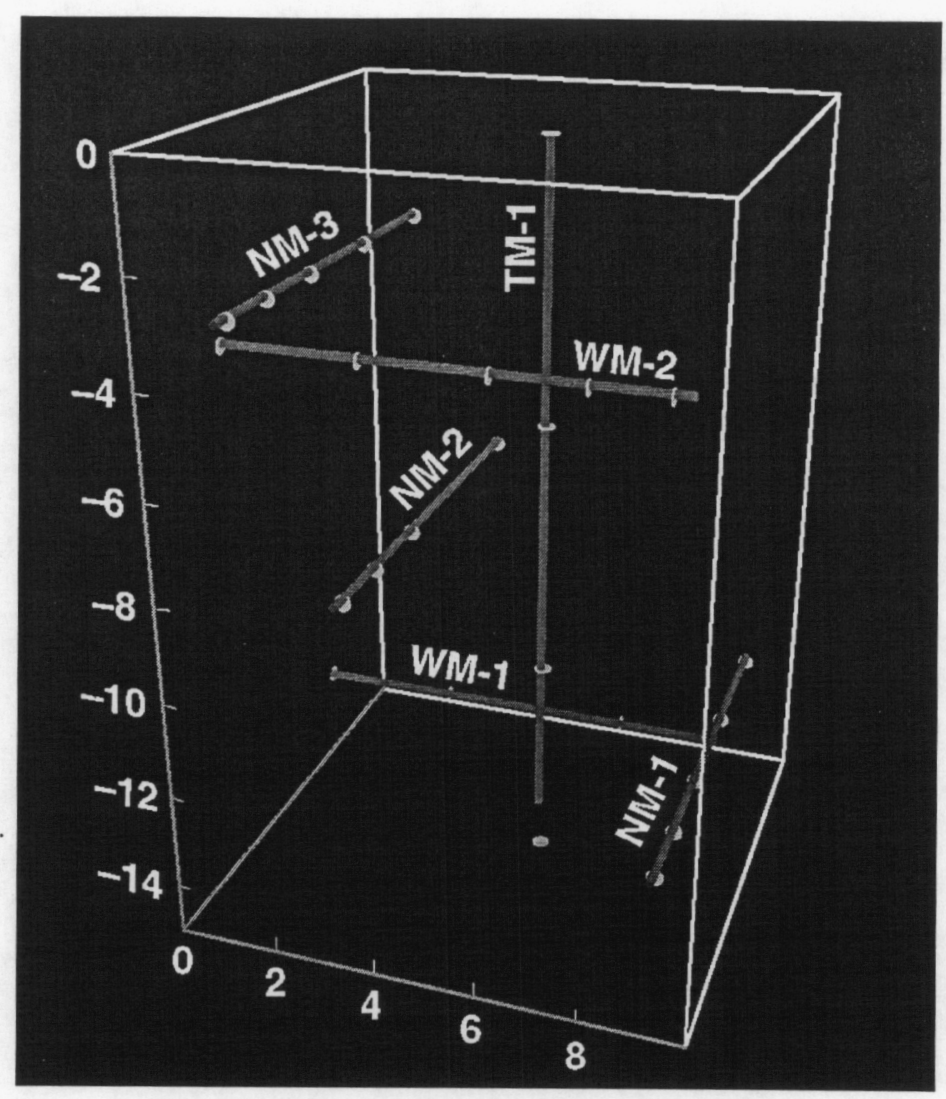

NOTE: Anchors are shown as discs in each hole.

Figure 3-13. The MPBX Holes in the LBT 


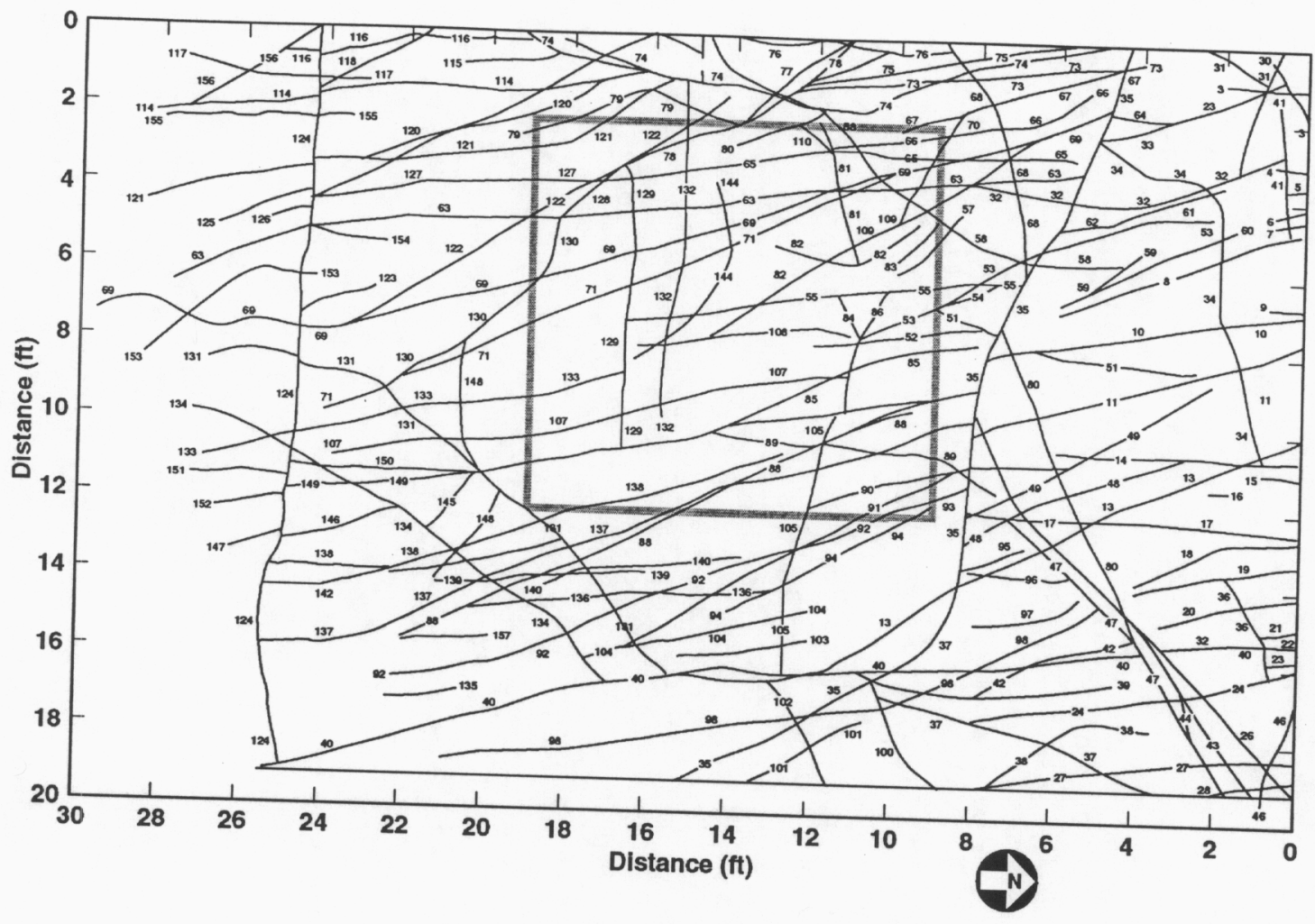

Figure 3-14. Initial Fracture Mapping at Fran Ridge 


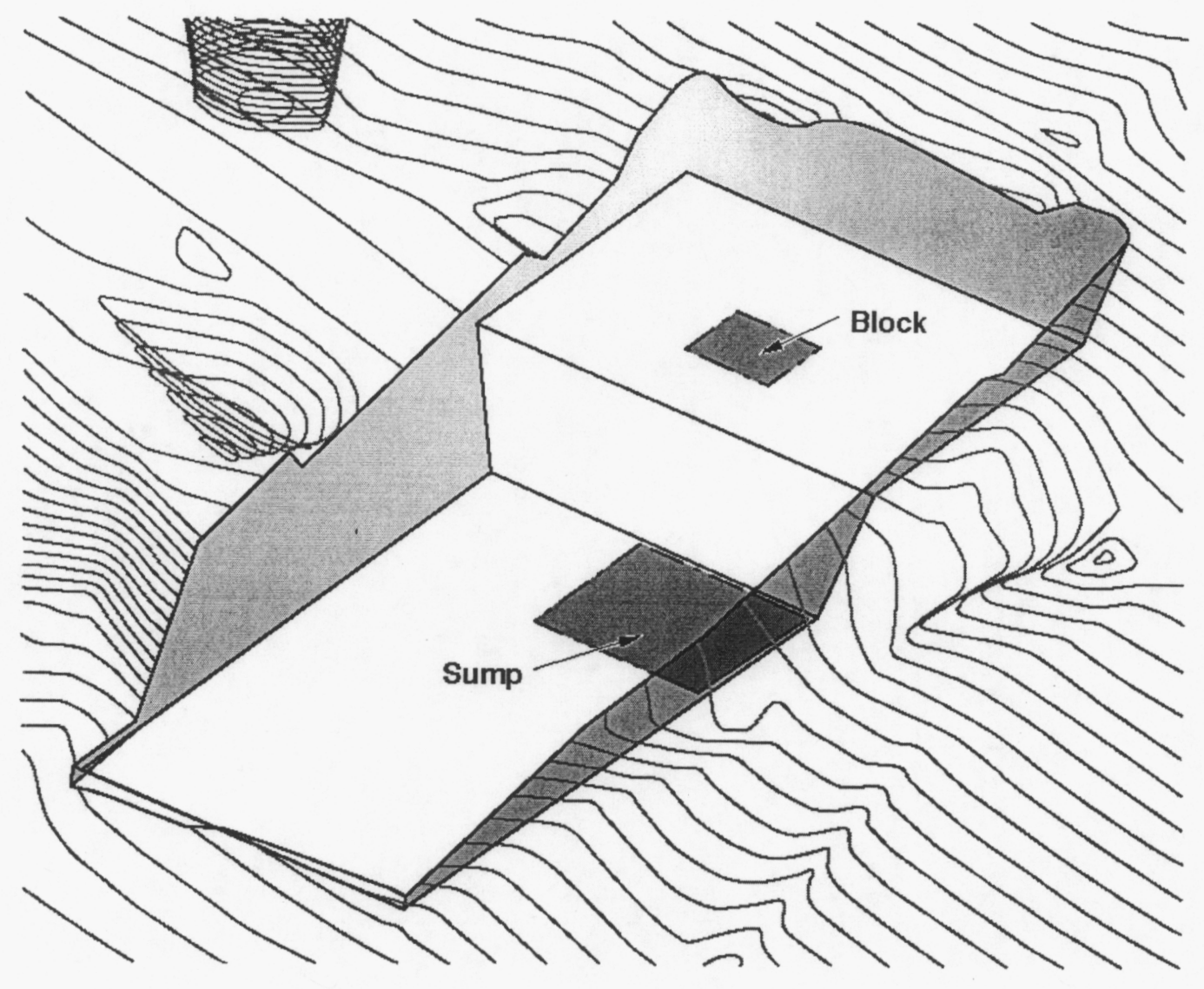

Figure 3-15. Excavation of Level Surface

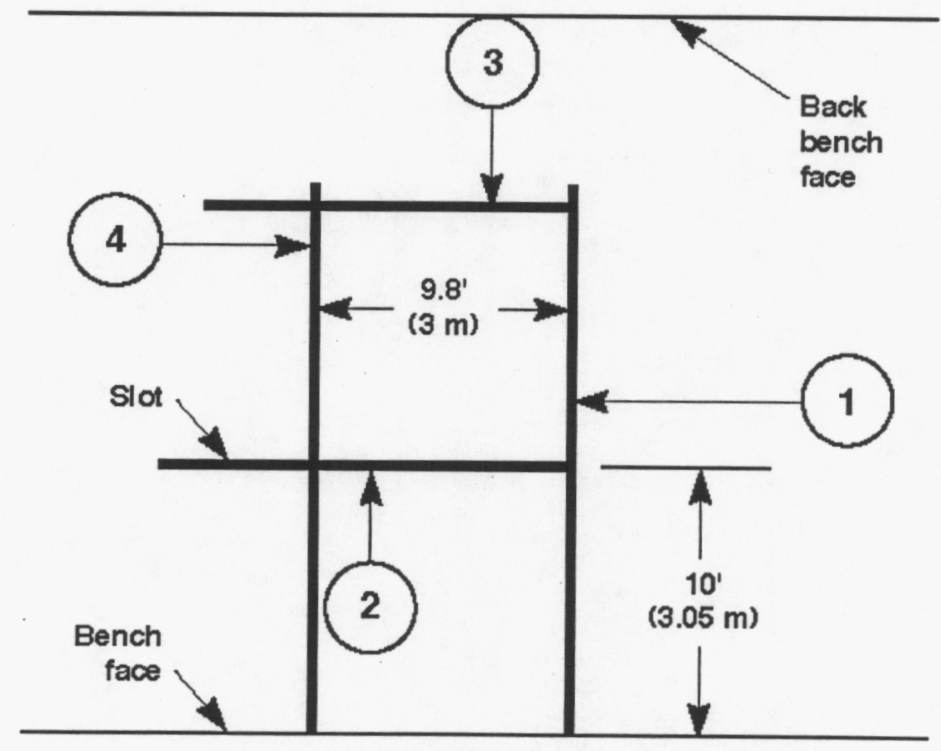

Figure 3-16. Slots for Block Isolation 


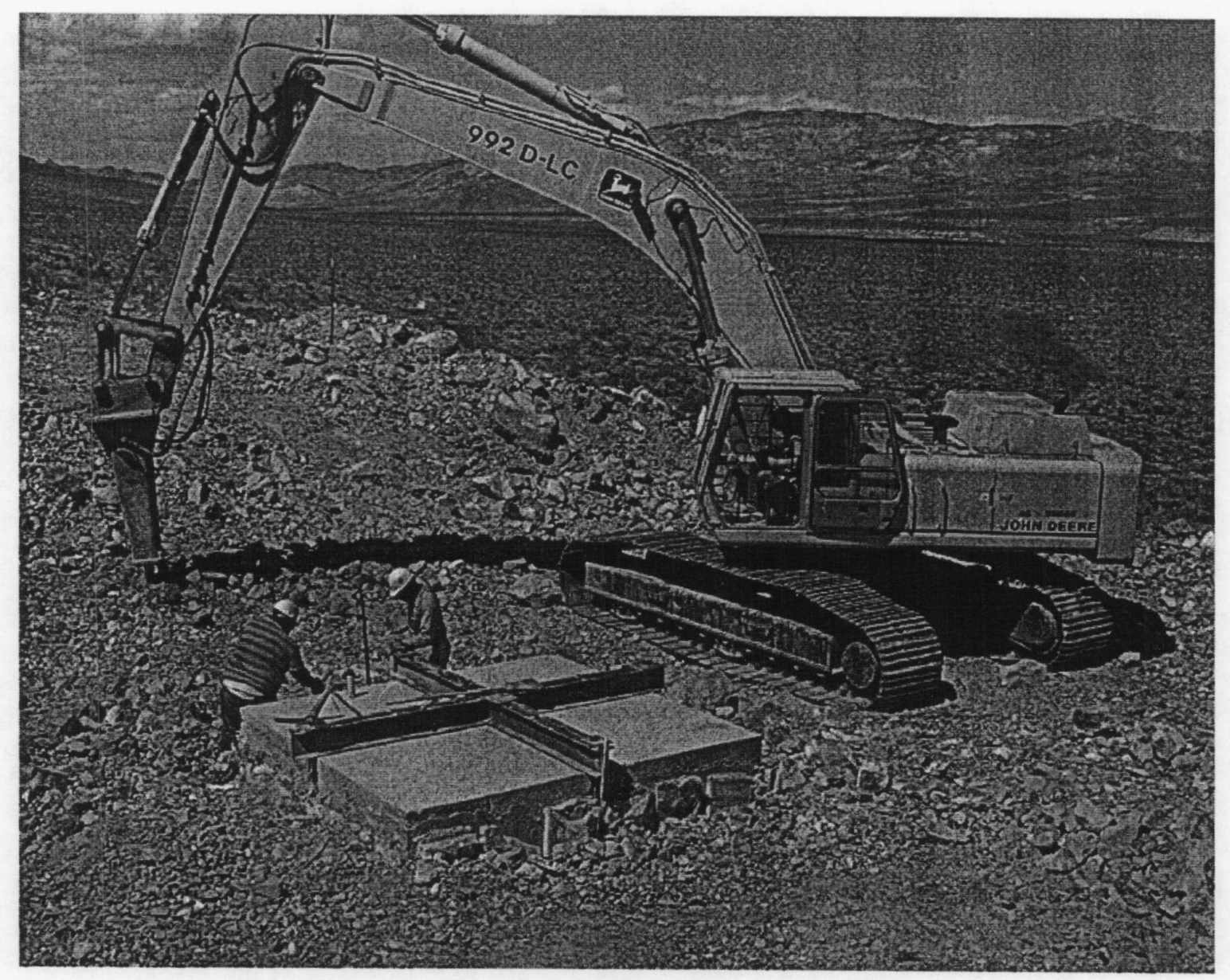

Figure 3-17. Partial Excavation of the Block 


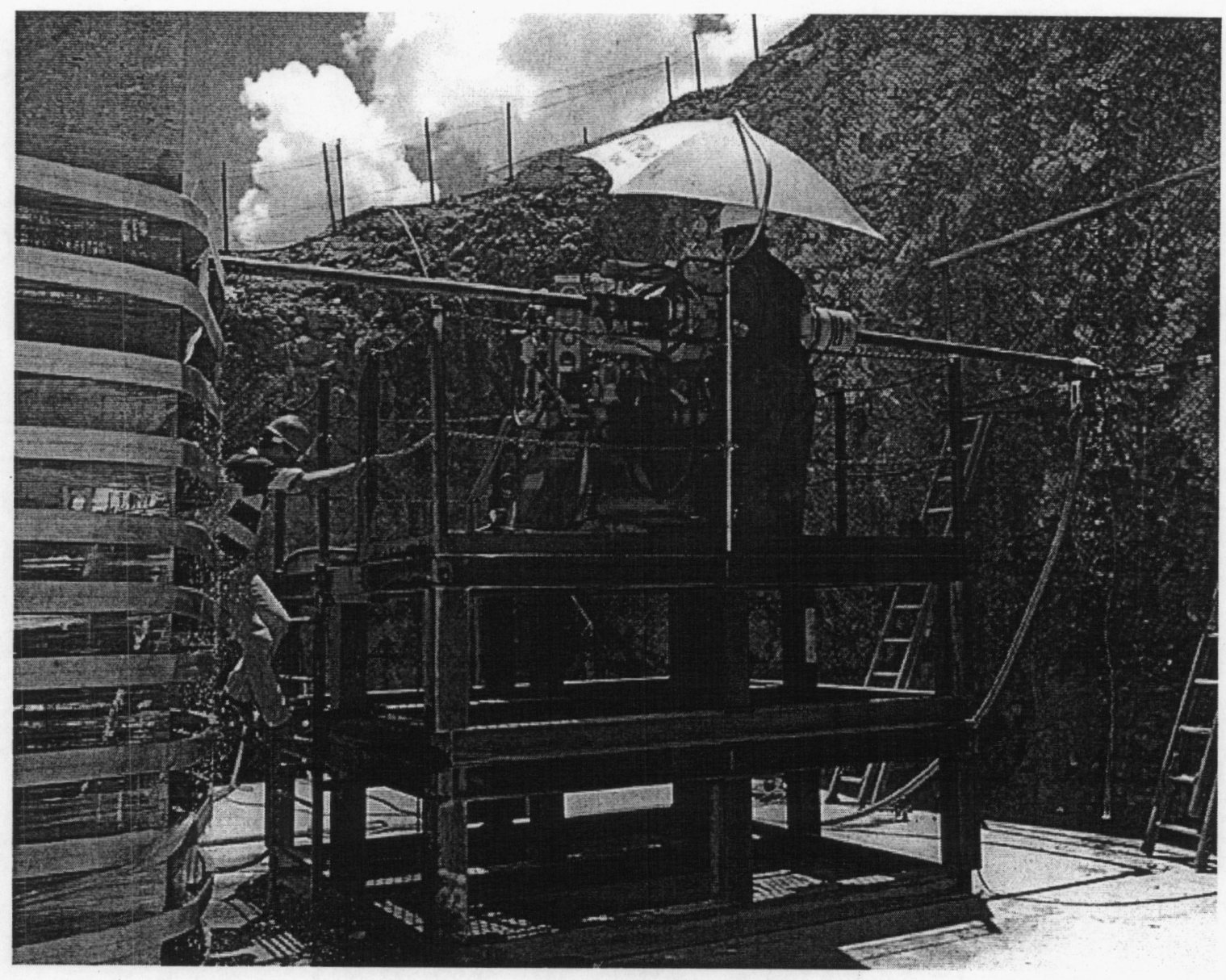

Figure 3-18. Drilling Rig Mounted on a Steel Plattorm to Drill Horizontal Boreholes 


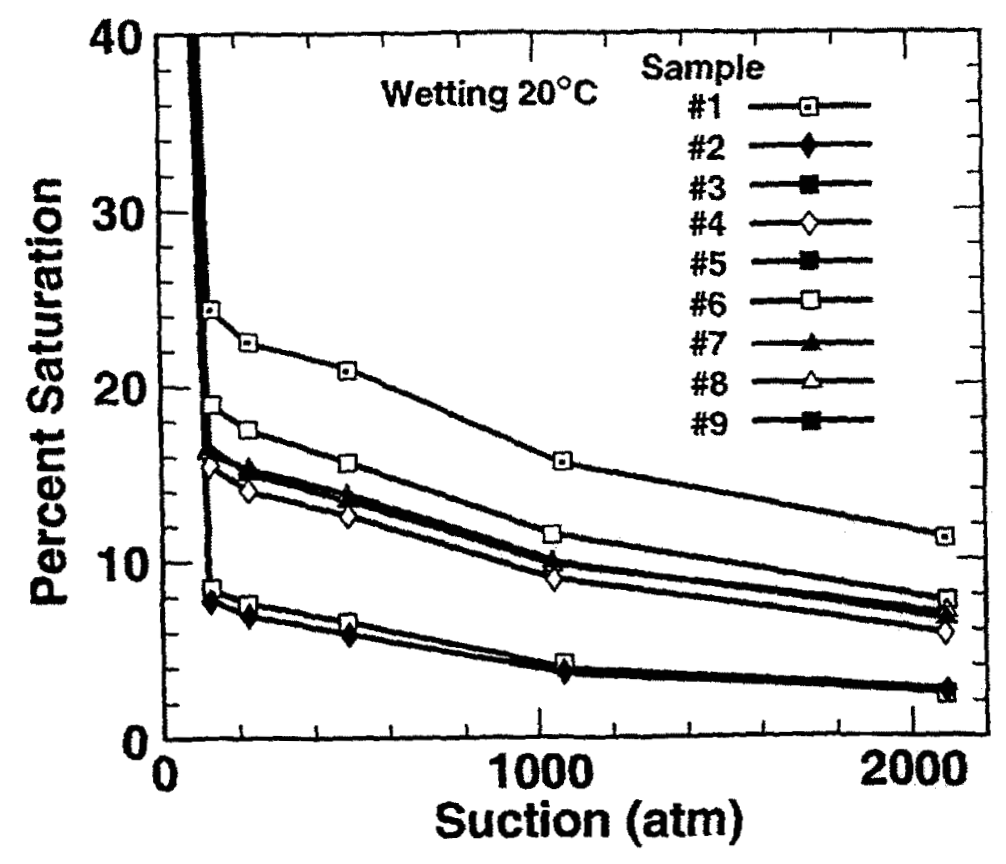

NOTE: Samples 1-3 were taken from USW H-1 core; samples 4-9 were taken from Fran Ridge outcrop.

Figure 3-19. Percent Saturation as a Function of Suction Potential at $20^{\circ} \mathrm{C}$ for Nine Topopah Spring Tuff Samples During Wetting Phase 


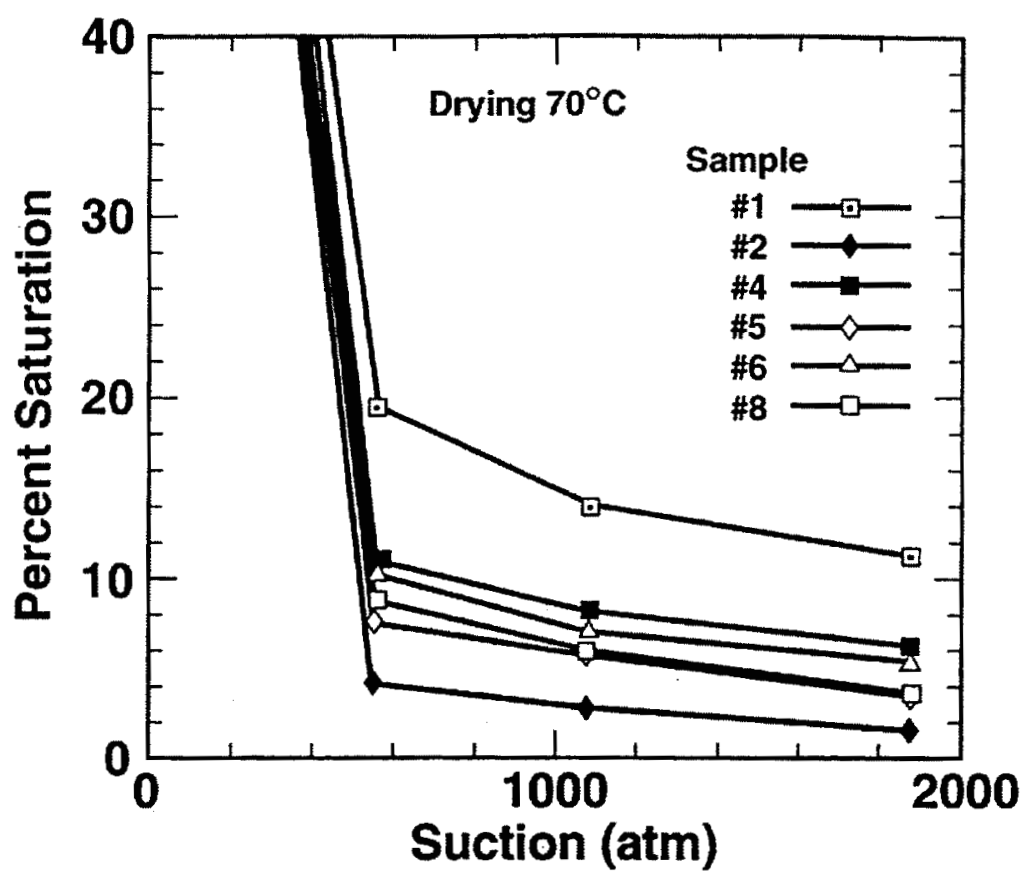

NOTE: Samples 1-2 were taken from USW H-1 core; samples 4-8 were taken from Fran Ridge outcrop.

Figure 3-20. Percent Saturation as a Function of Suction Potential at $70^{\circ} \mathrm{C}$ for Six Topopah Spring Tuff Samples During Drying Phase 


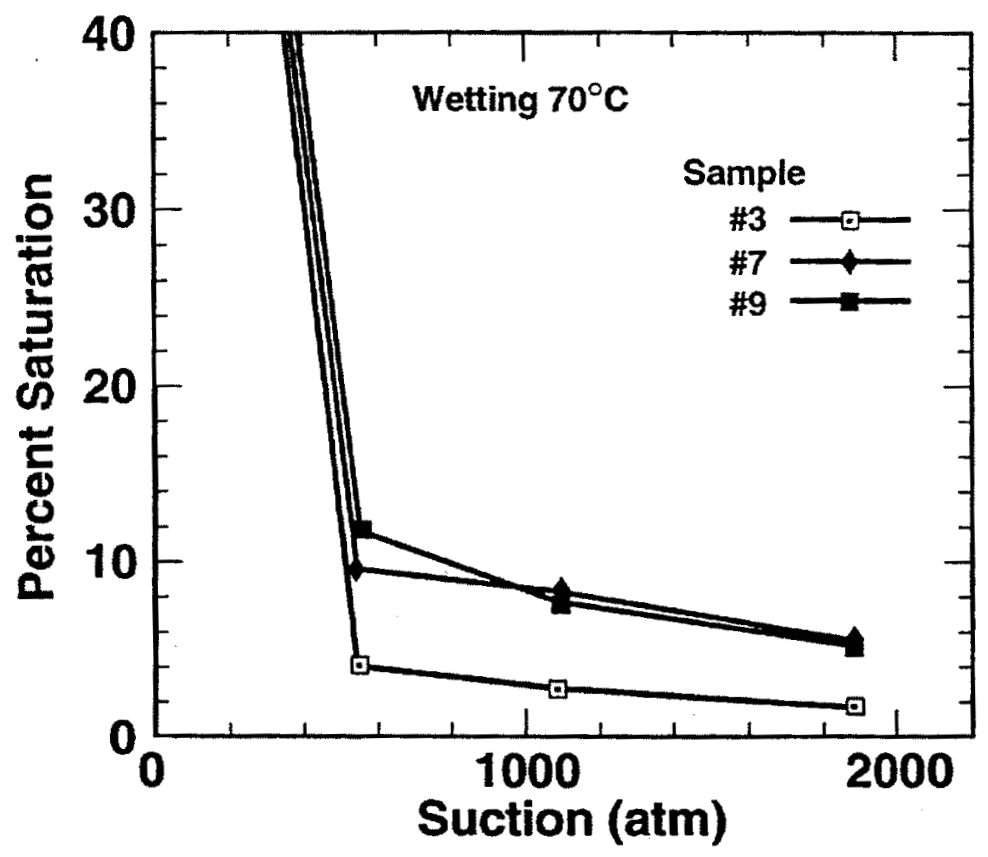

NOTE: Sample 3 was taken from USW H-1 core; samples 7 and 9 were taken from Fran Ridge outcrop.

Figure 3-21. Percent Saturation as a Function of Suction Potential at $70^{\circ} \mathrm{C}$ for Three Topopah Spring Tuff Samples During Wetting Phase 


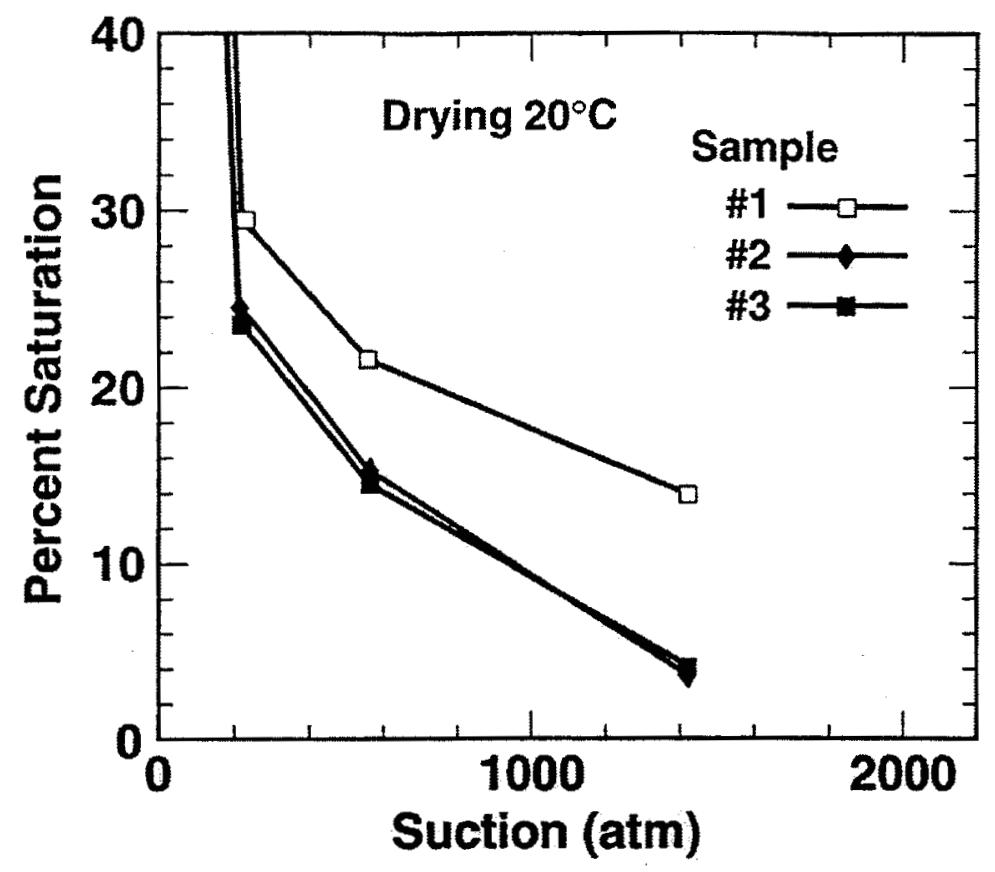

Figure 3-22. Percent Saturation as a Function of Suction Potential at $20^{\circ} \mathrm{C}$ for Three Topopah Spring Tuff Samples During Drying Phase 


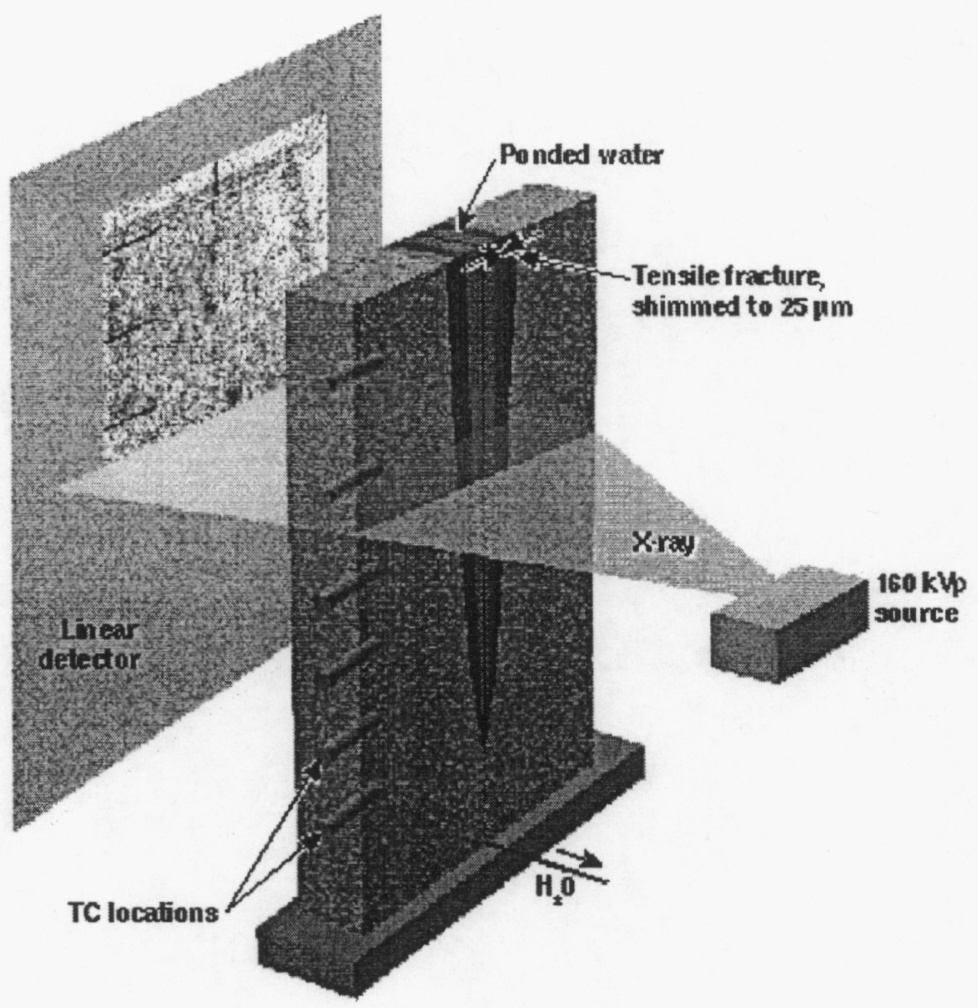

Figure 3-23. Schematic Diagram Showing the Set-up of Using X-ray Radiography to Investigate Fracture Flow and Matrix Imbibition 
(a)
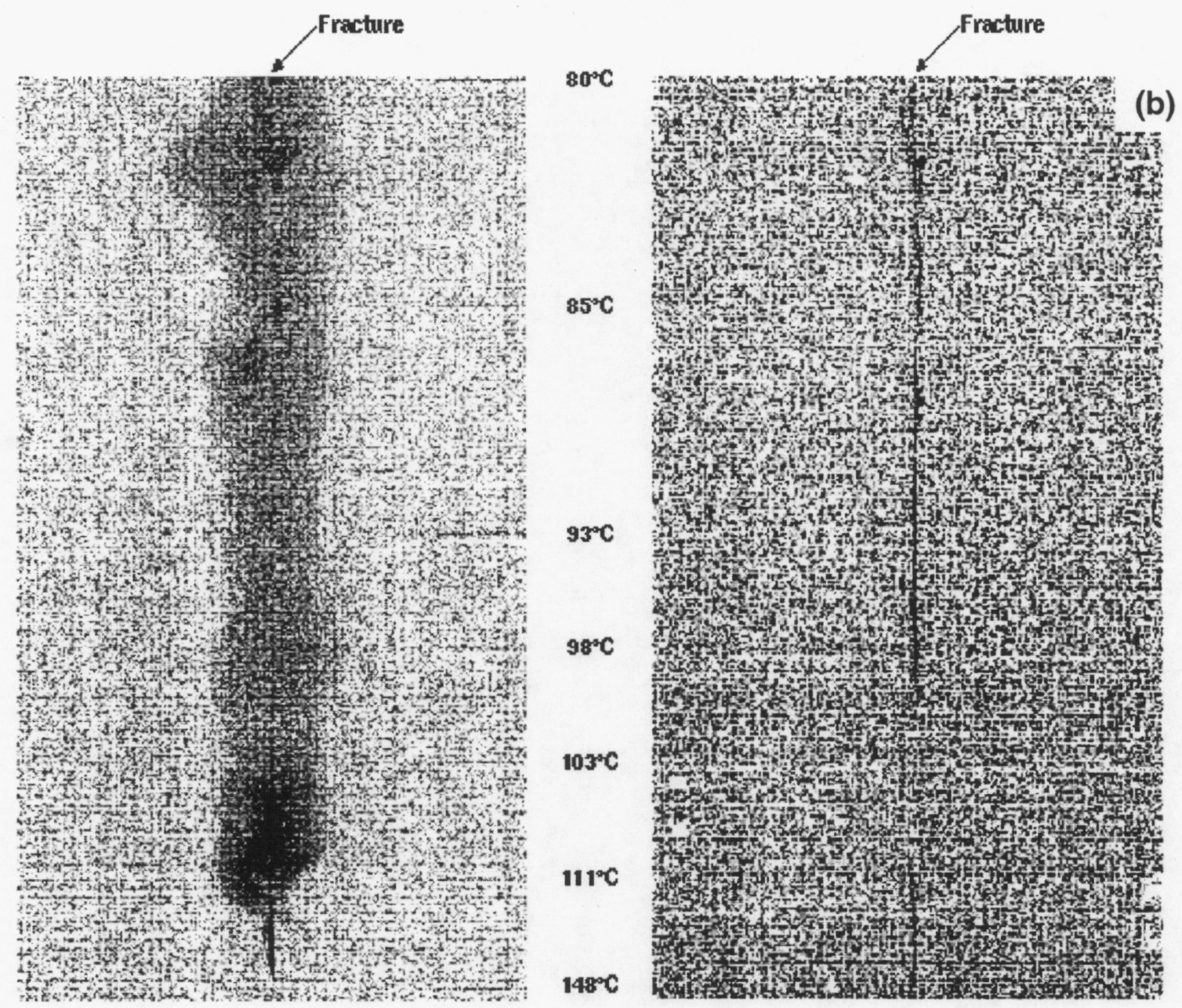

NOTE: Thermal gradient is indicated between the images. The difference between these two experiments was the height of the water column, 0.26 and $0.46 \mathrm{~m}$ for (a) and (b) respectively. The difference in the water head was enough to force flow through the boiling zone without causing much imbibition in (b).

Figure 3-24. Difference Images of Experiment 5 (a) and 6 (b) at 7.2 and 0.67 Hours Respectively After Flow Was Initiated 


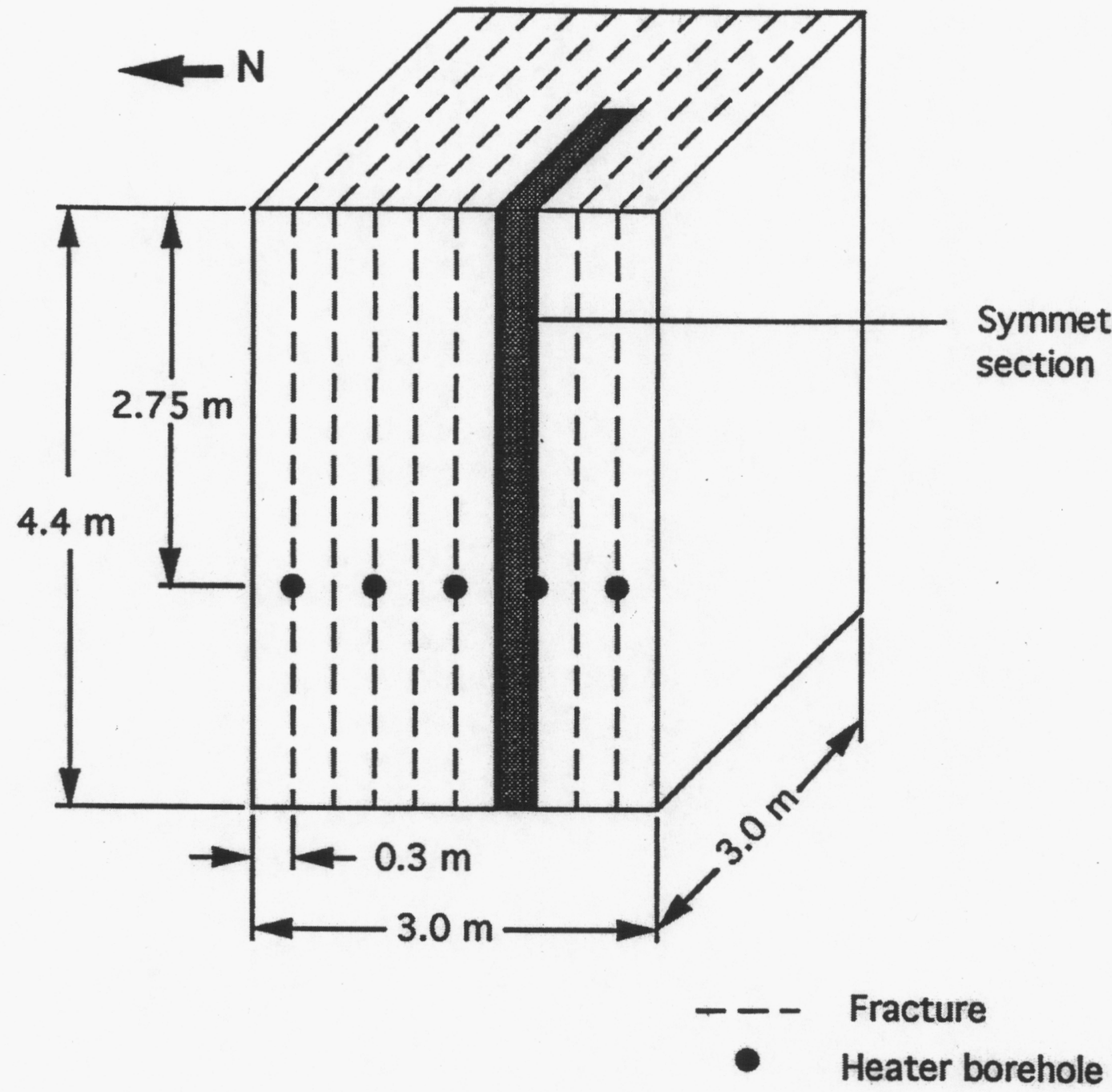

NOTE: The shaded area is the $1 / 20$ symmetry section modeled.

Figure 3-25. Schematic of Large Block Showing Locations of Fractures and Heater Boreholes 


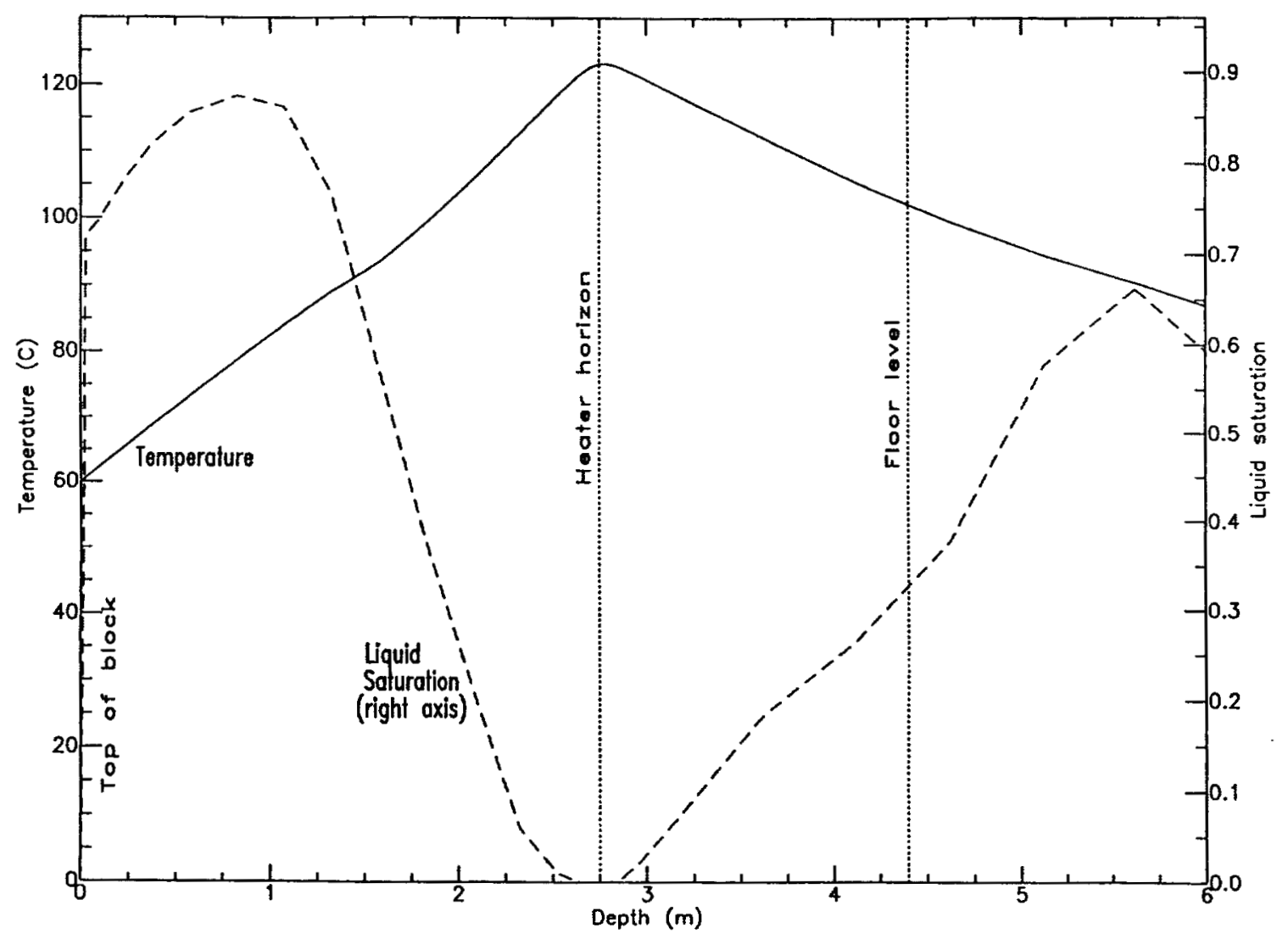

Figure 3-26. Predicted Temperature and Water Saturation Profiles Along a Vertical Line Through the Matrix at One-year Heating 


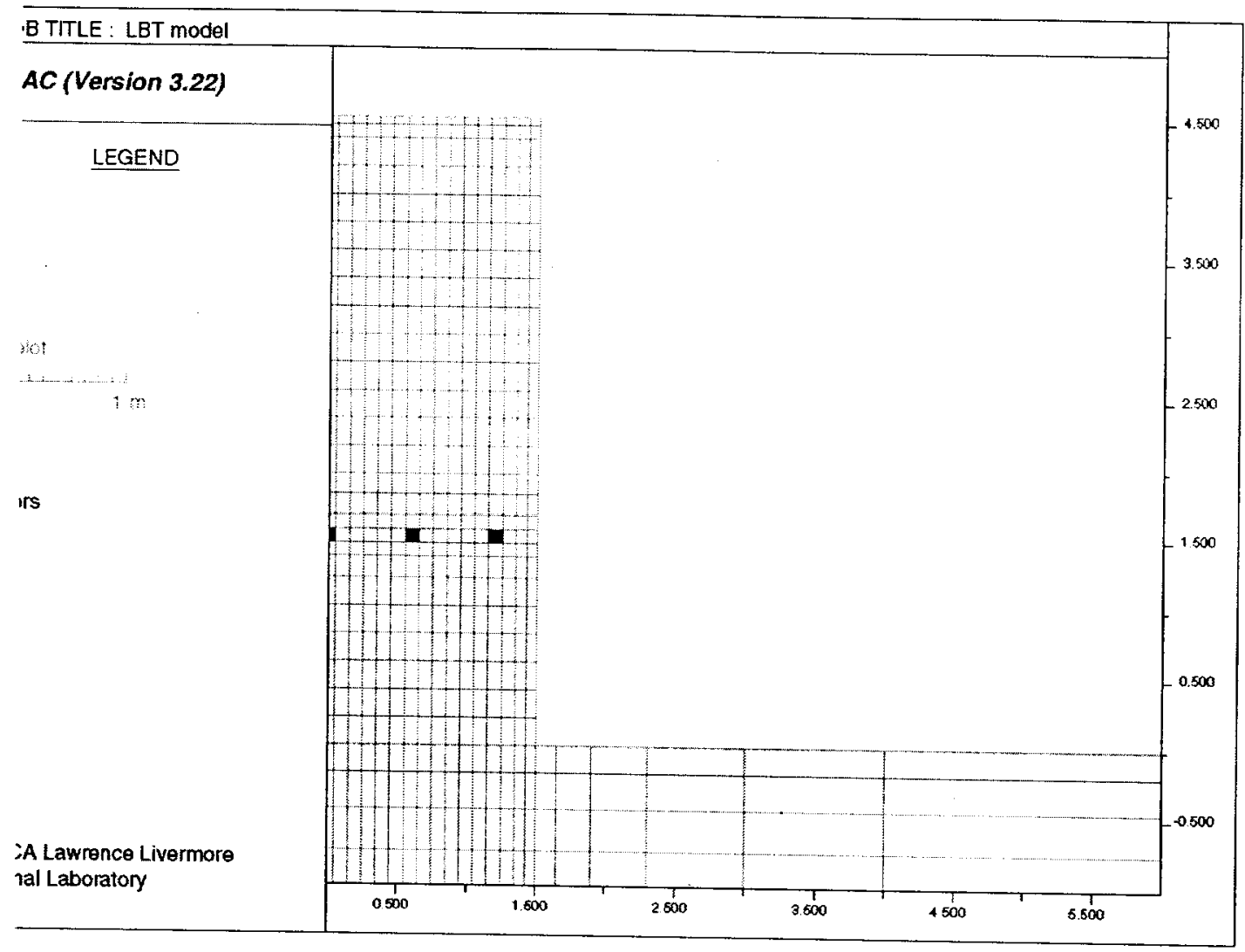

Figure 3-27. Grid of the FLAC LBT Model 


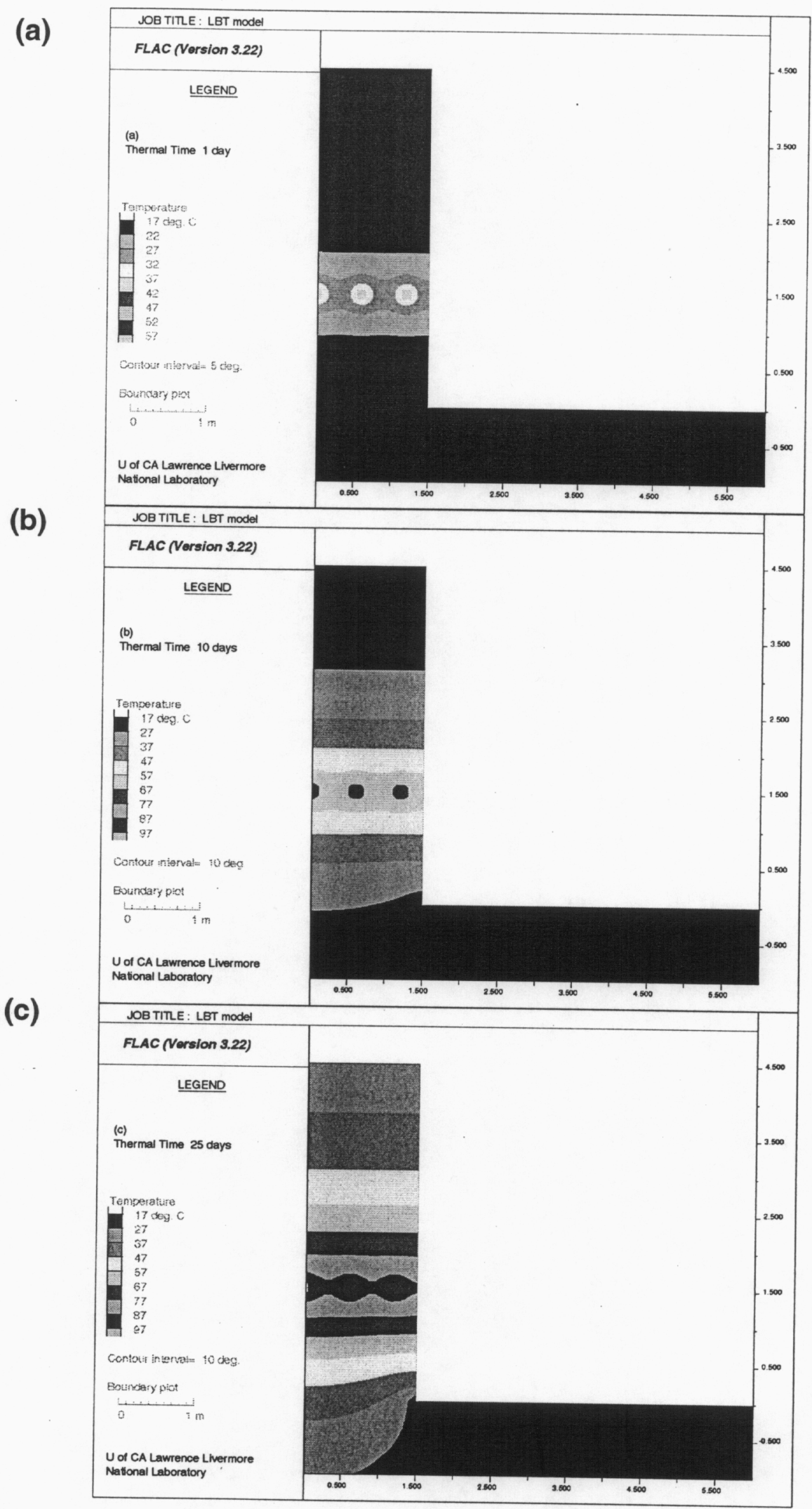


(d)

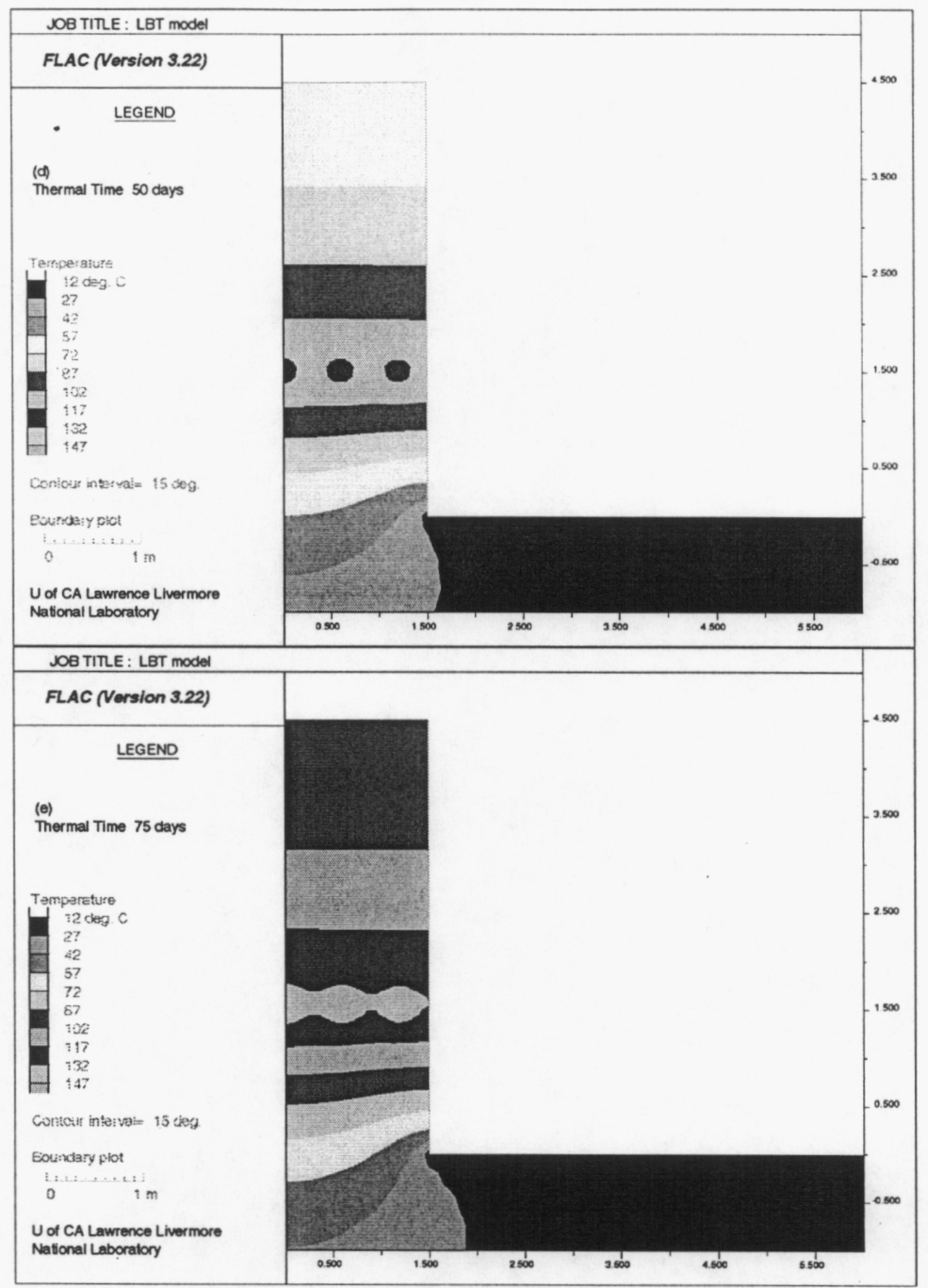

Figure 3-28. Temperature Contours in a Vertical Cross Section of the LBT After (a) 1 Day, (b) 10 Days, (c) 25 Days, (d) 50 Days, and (e) 75 Days of Heating, Calculated by FLAC Model 

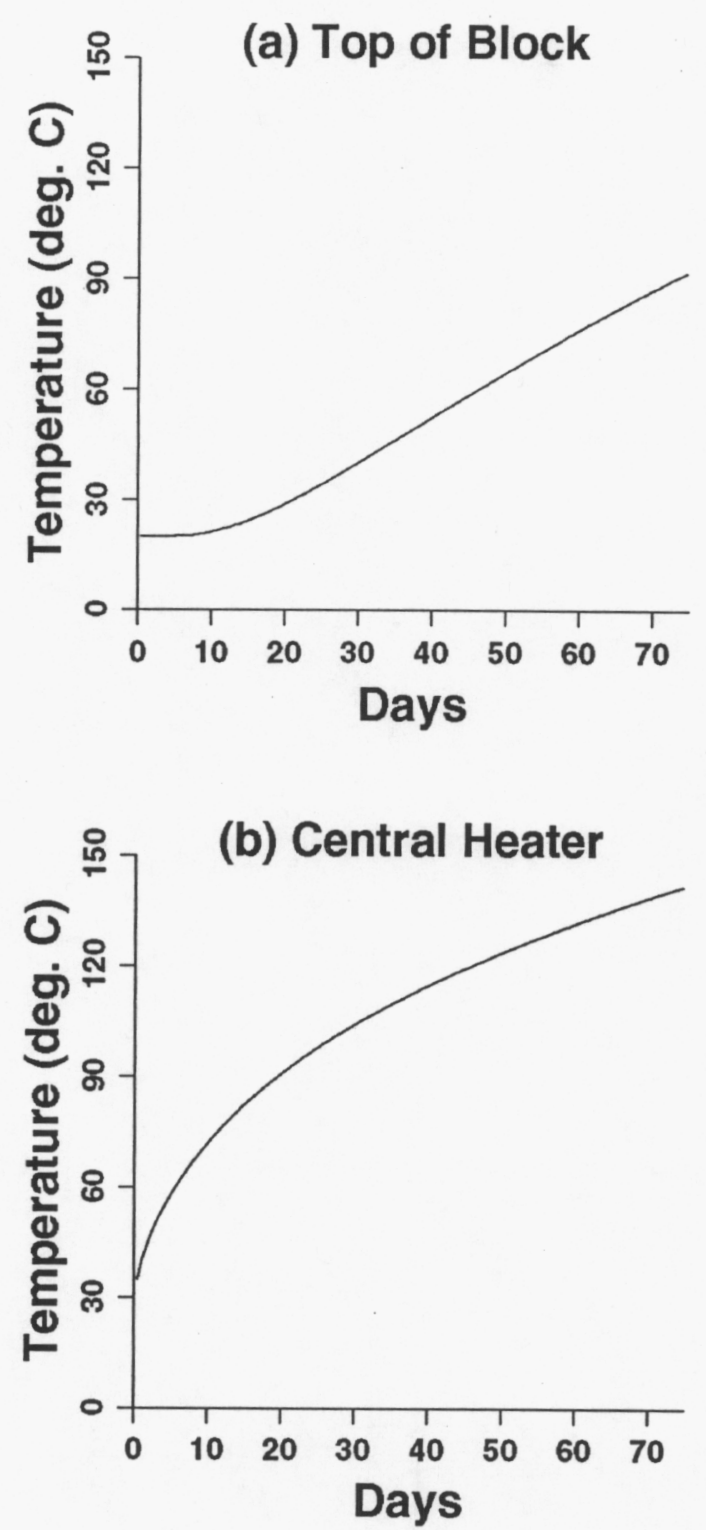

Figure 3-29. Temperature Profiles for (a) the Top and (b) Heater of the LBT, Calculated by FLAC Model 


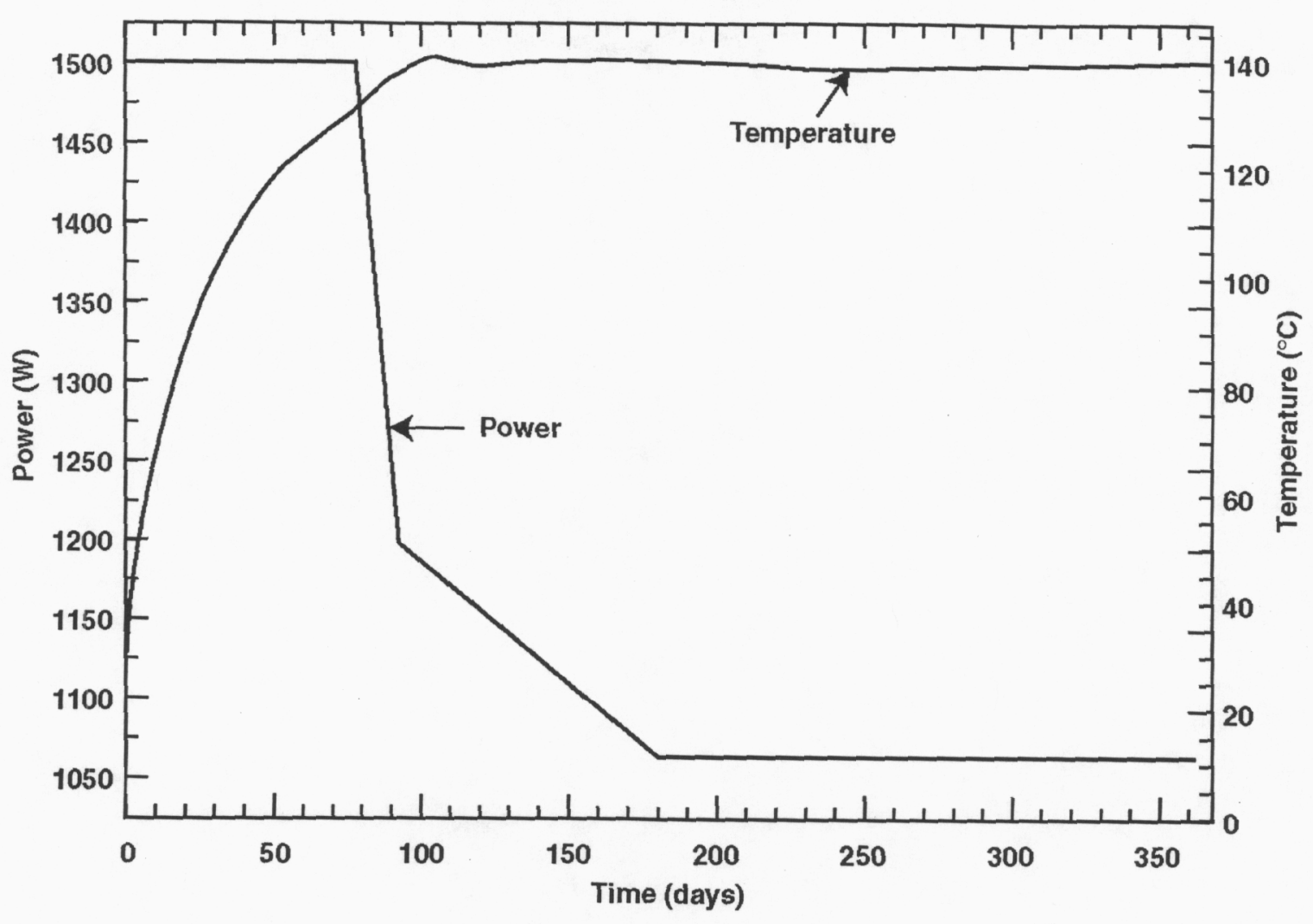

Figure 3-30. Temperature Near the Heater Horizon of the LBT Calculated by V-TOUGH Continuum Model (Lee 1995a) 
(a)

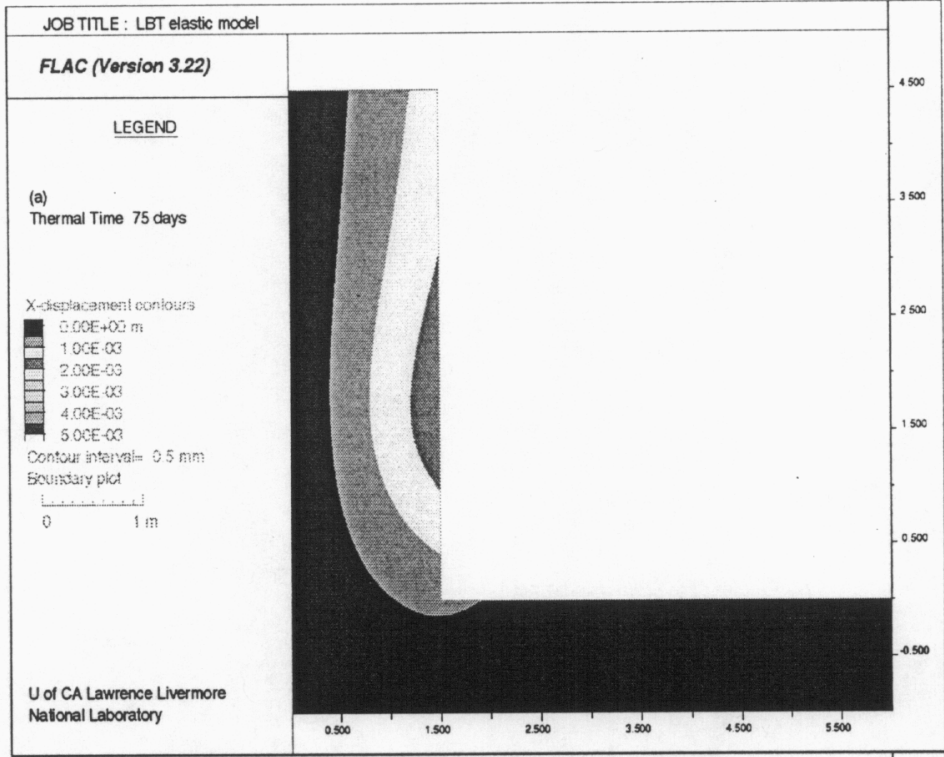

(b)

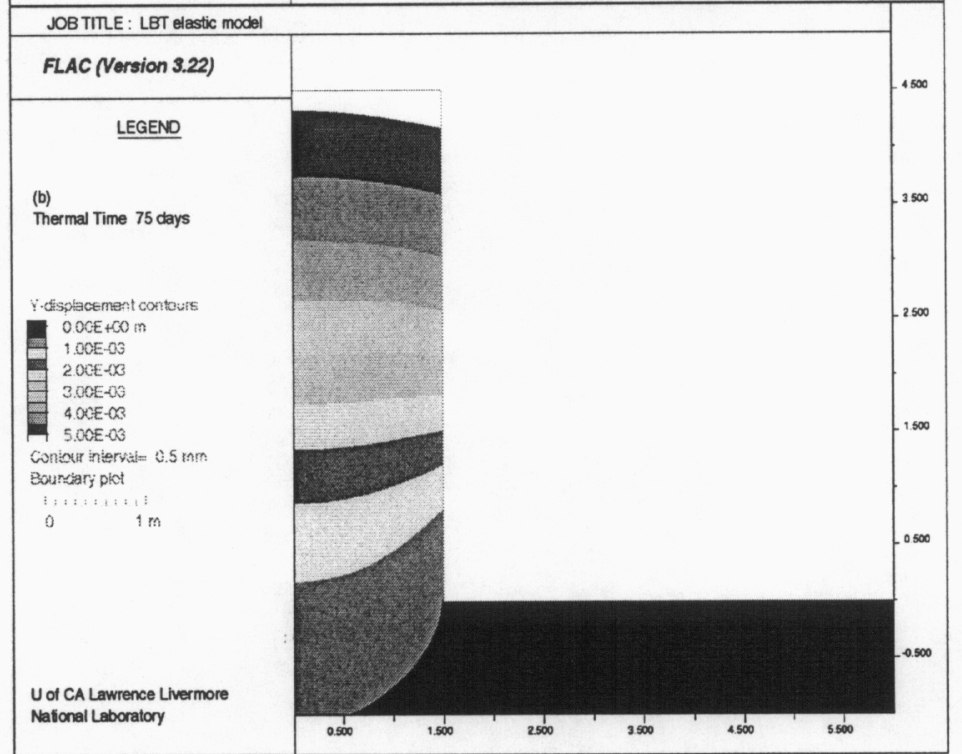

(c)

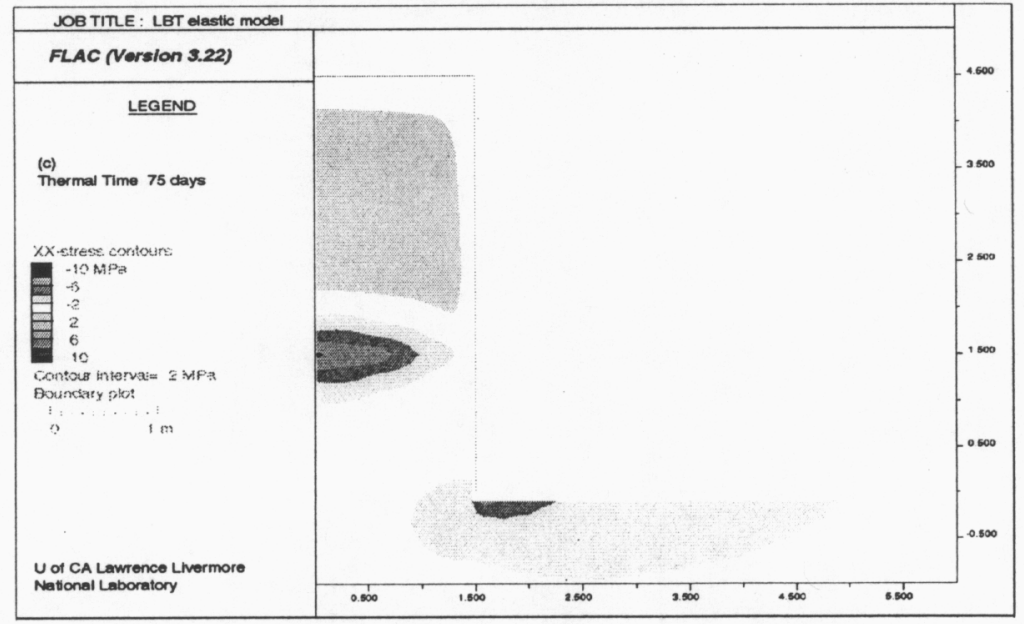


(d)

(e)

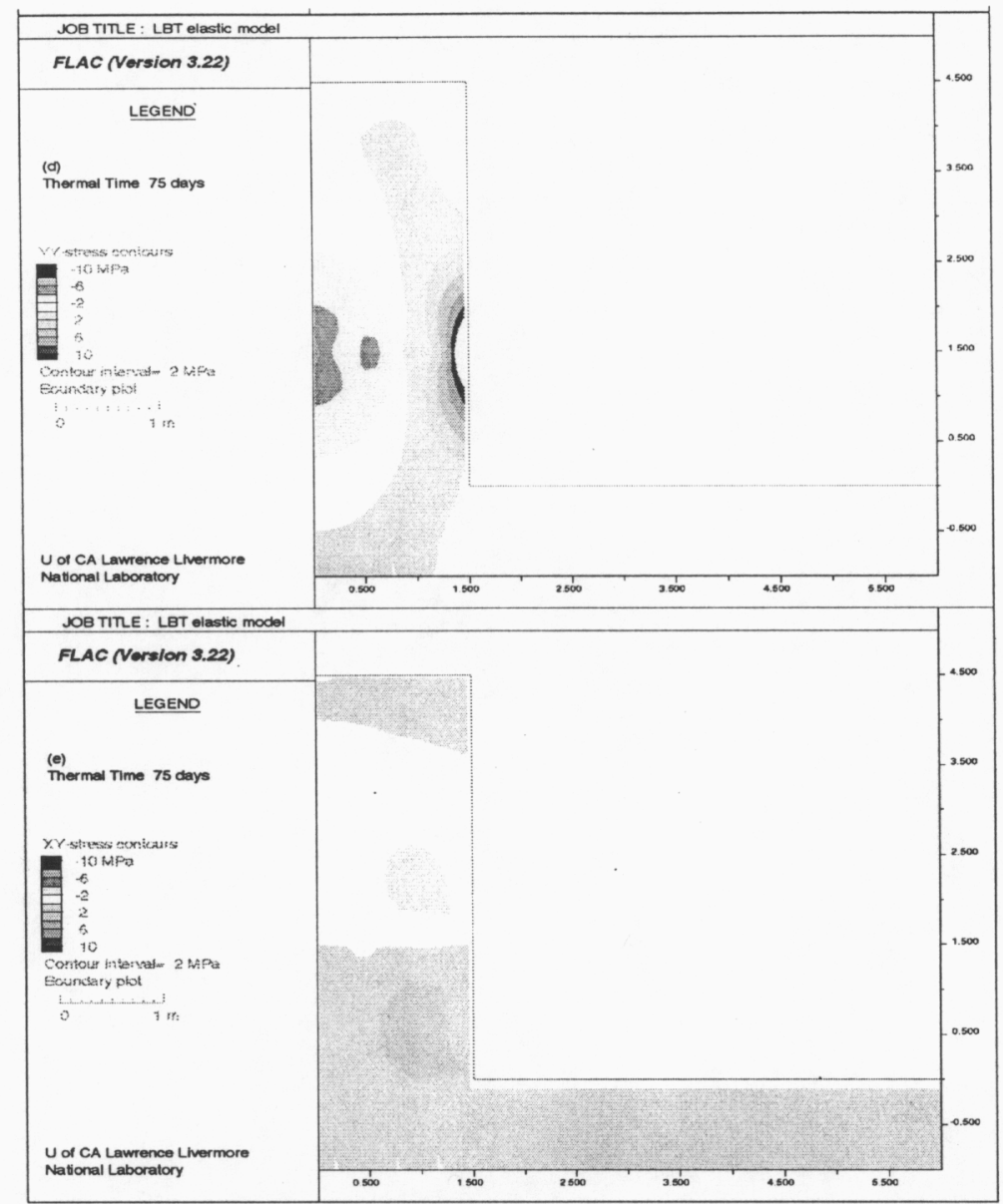

Figure 3-31. Displacements and Stresses Predicted by the First FLAC Model at 75 Days of Heating, for (a) Horizontal, x, Displacement; (b) Vertical, y, Displacement; (c) $\sigma_{x x}$ Stress; (d) $\sigma_{y y}$ Stress; and $(e) \sigma_{x y}$ Stress 


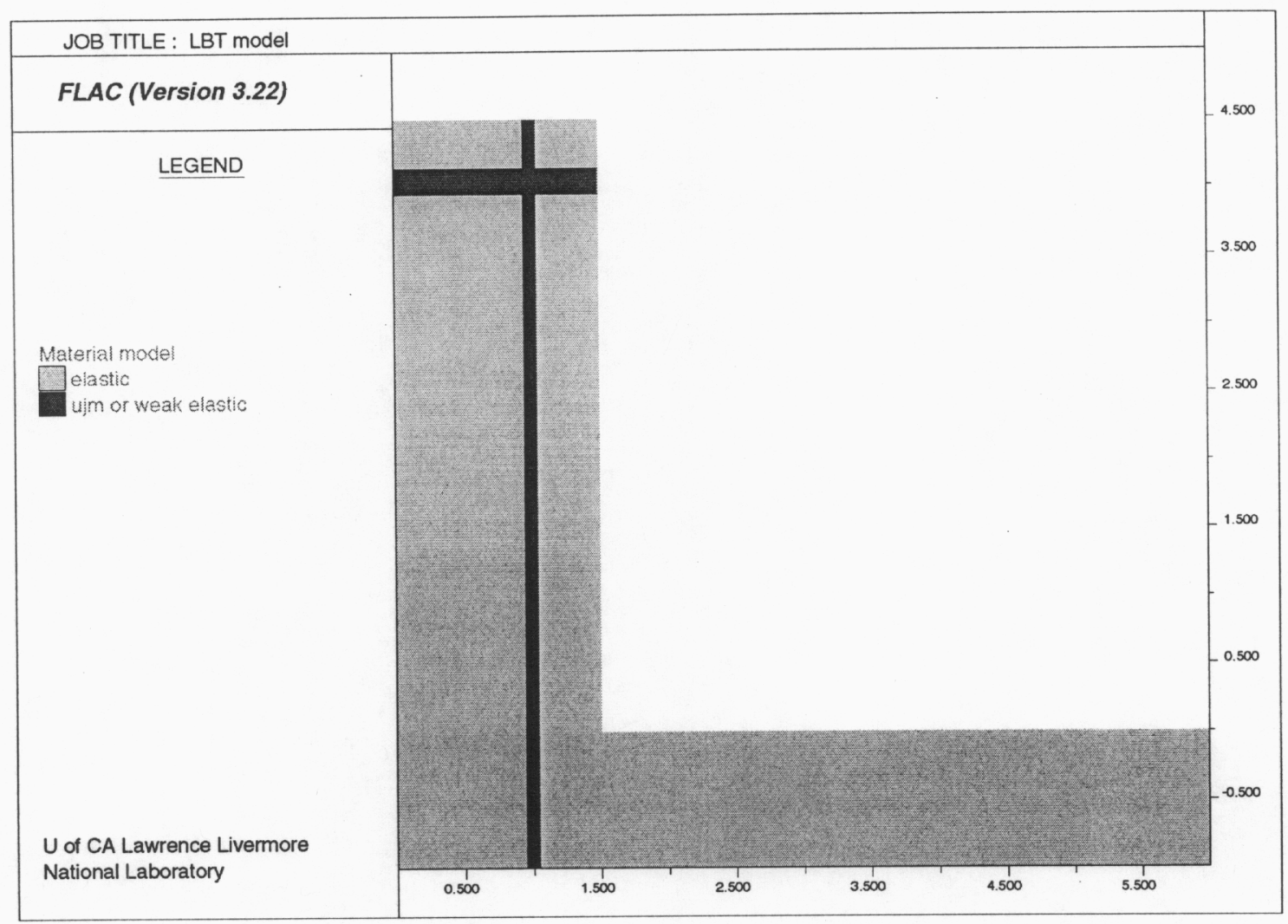

Figure 3-32. Model Configuration of the Third FLAC Model with One Horizontal Fracture and One Vertical Fracture 
(a)

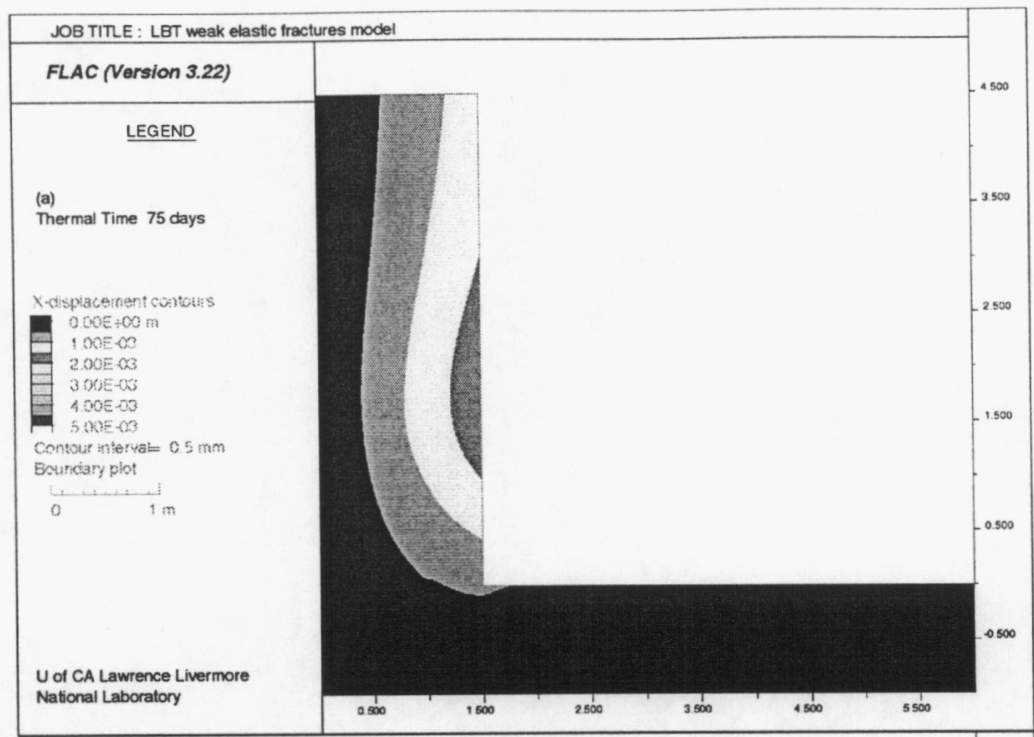

(b)

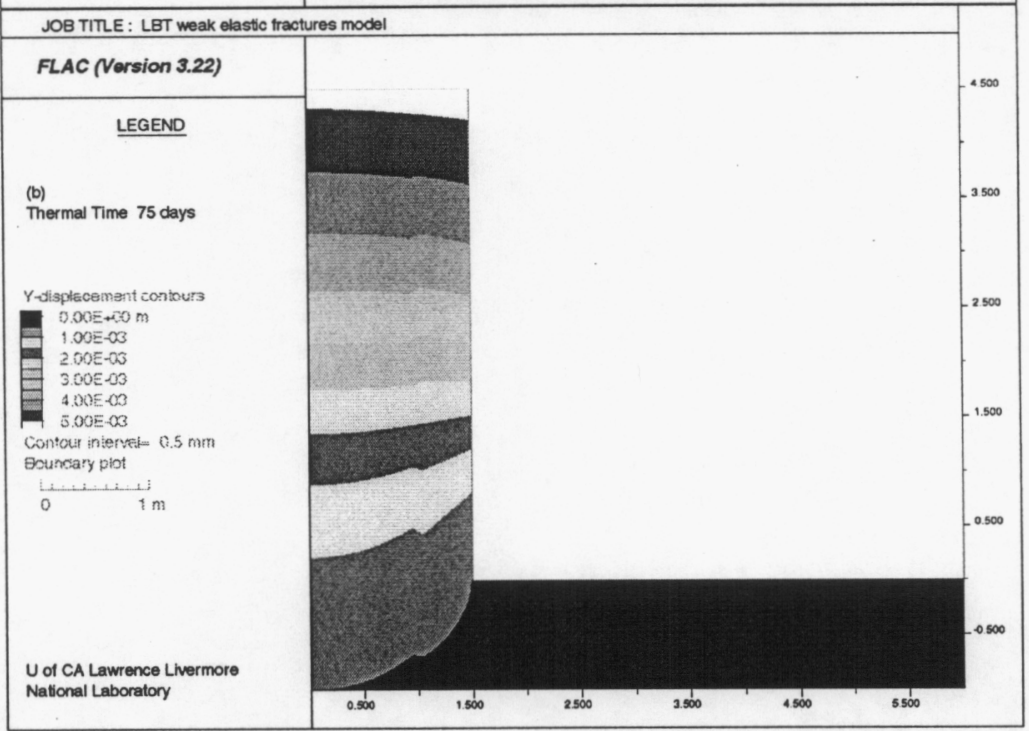

(c)

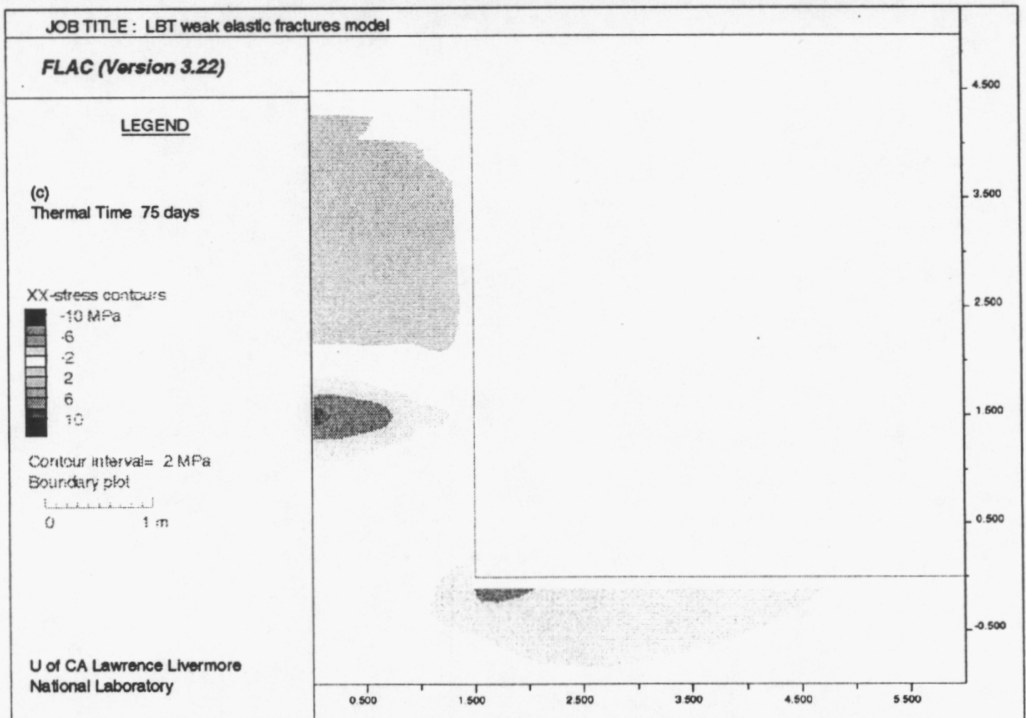




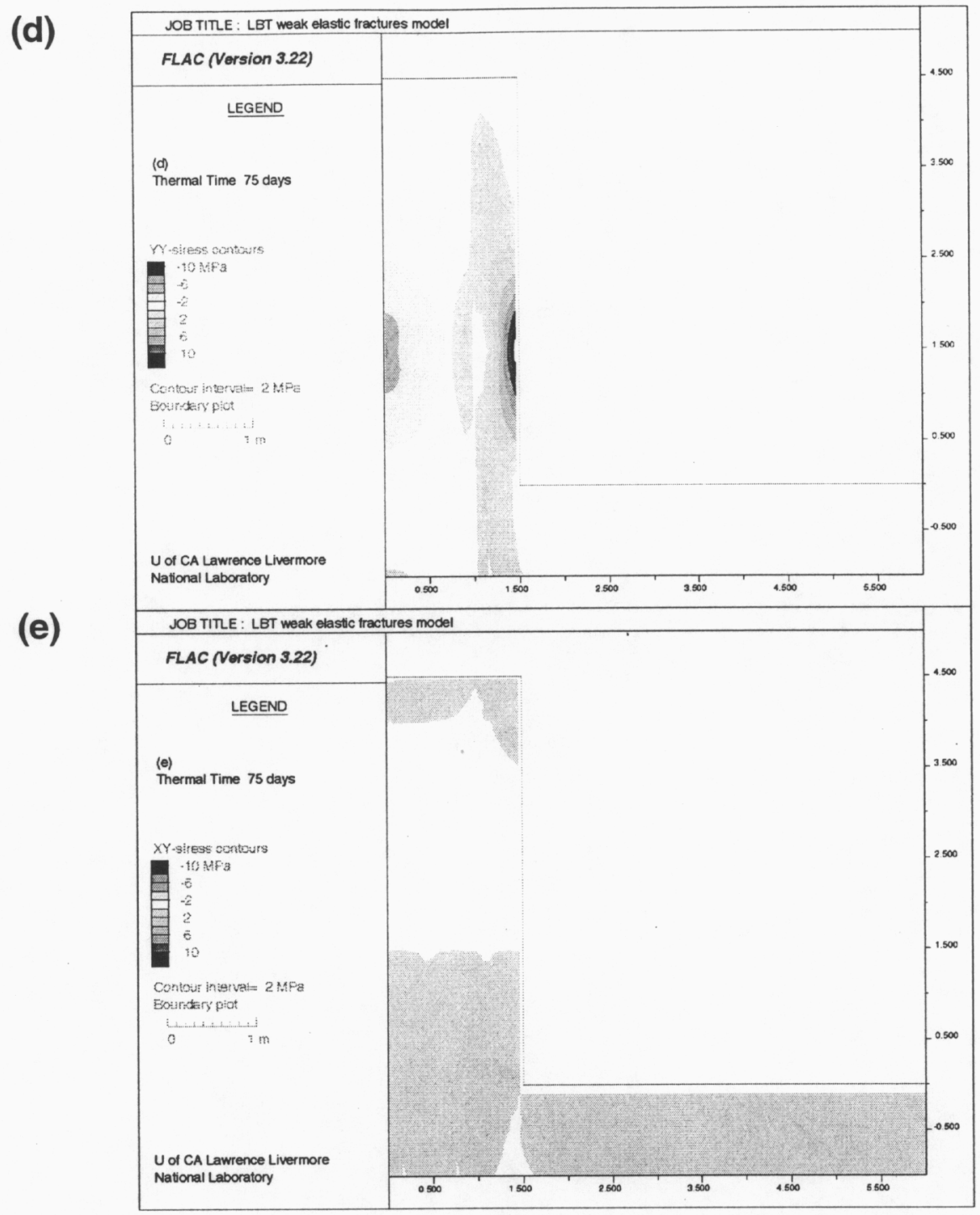

Figure 3-33. Displacements and Stresses Predicted by the Third FLAC Model of the LBT at 75 Days of Heating, (a) Horizontal, x, Displacement; (b) Vertical, y, Displacement; (c) $\sigma_{x x}$ Stress; (d) $\sigma_{y y}$ Stress; and (e) $\sigma_{x y}$ Stress. 
(a)

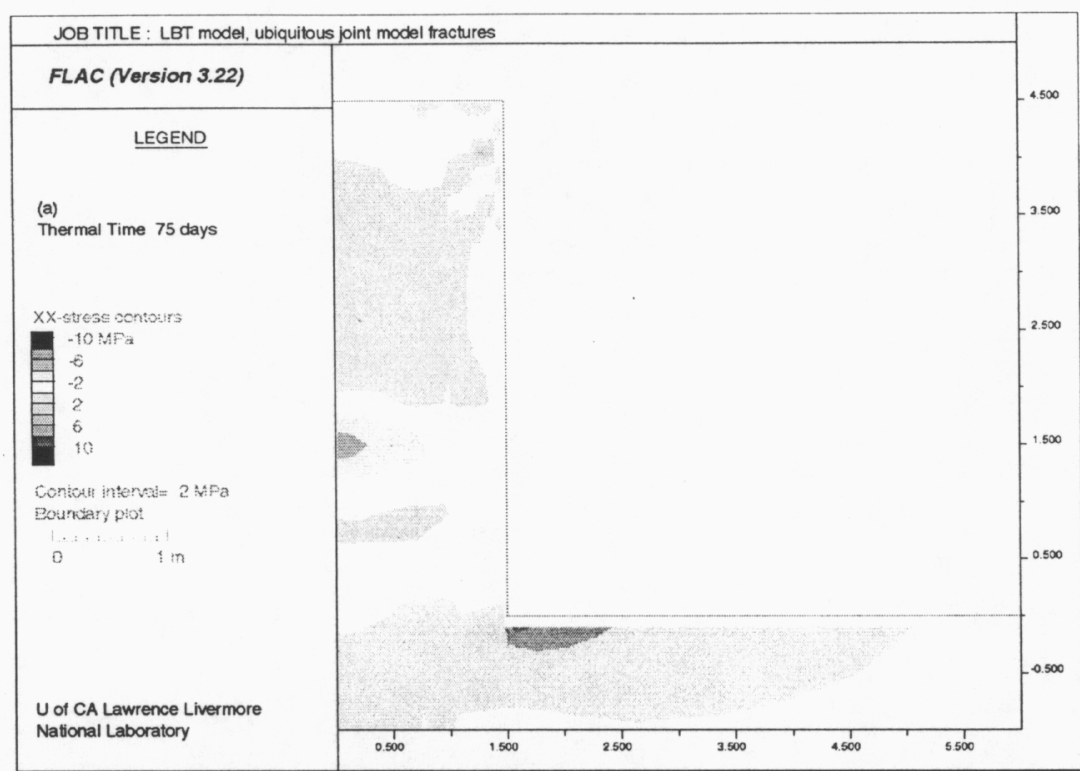

(b)

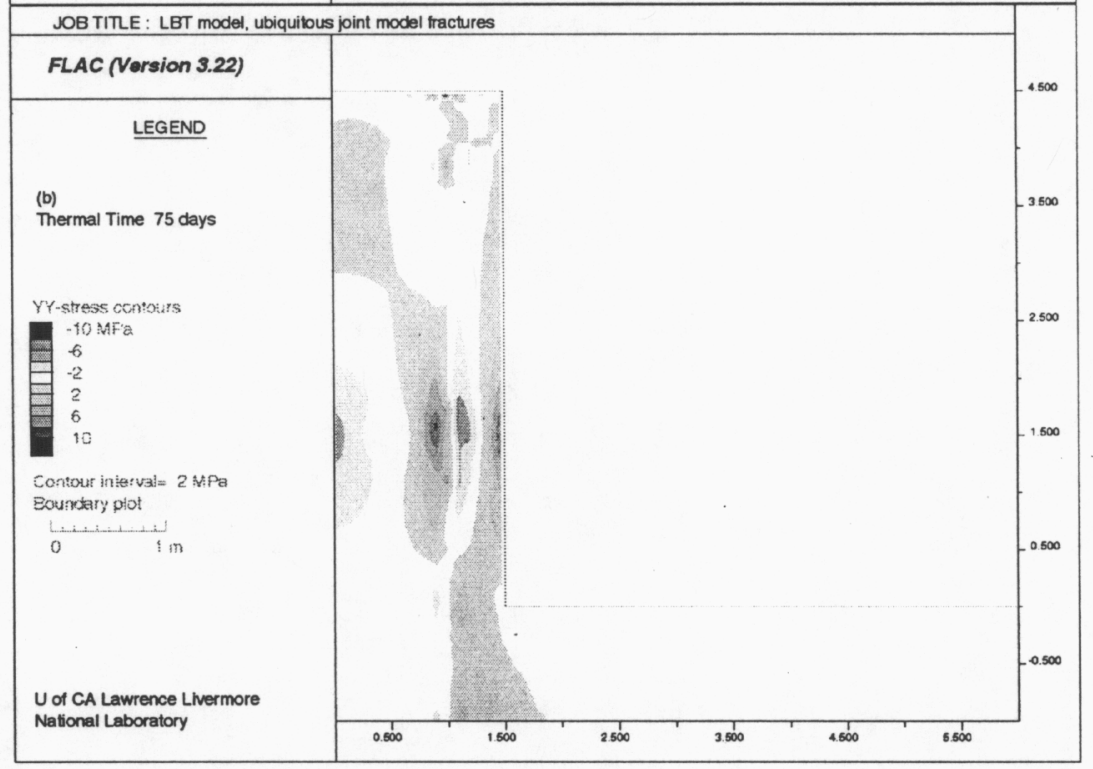

Figure 3-34. The (a) $\sigma_{x x}$ and (b) $\sigma_{y y}$ Contours in the LBT Predicted by the Fourth FLAC Model at 75 Days of Heating 
(a)

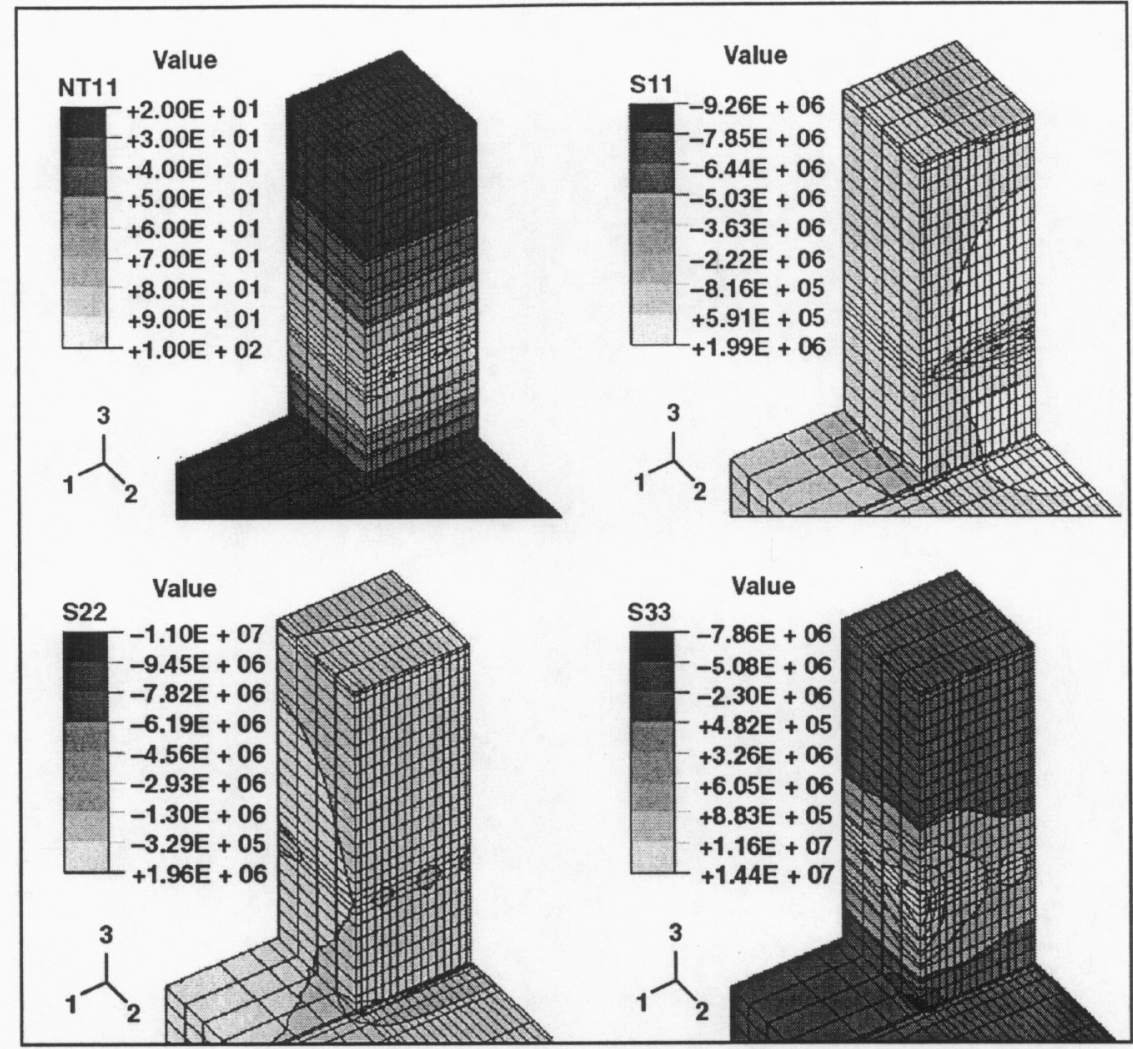

(b)

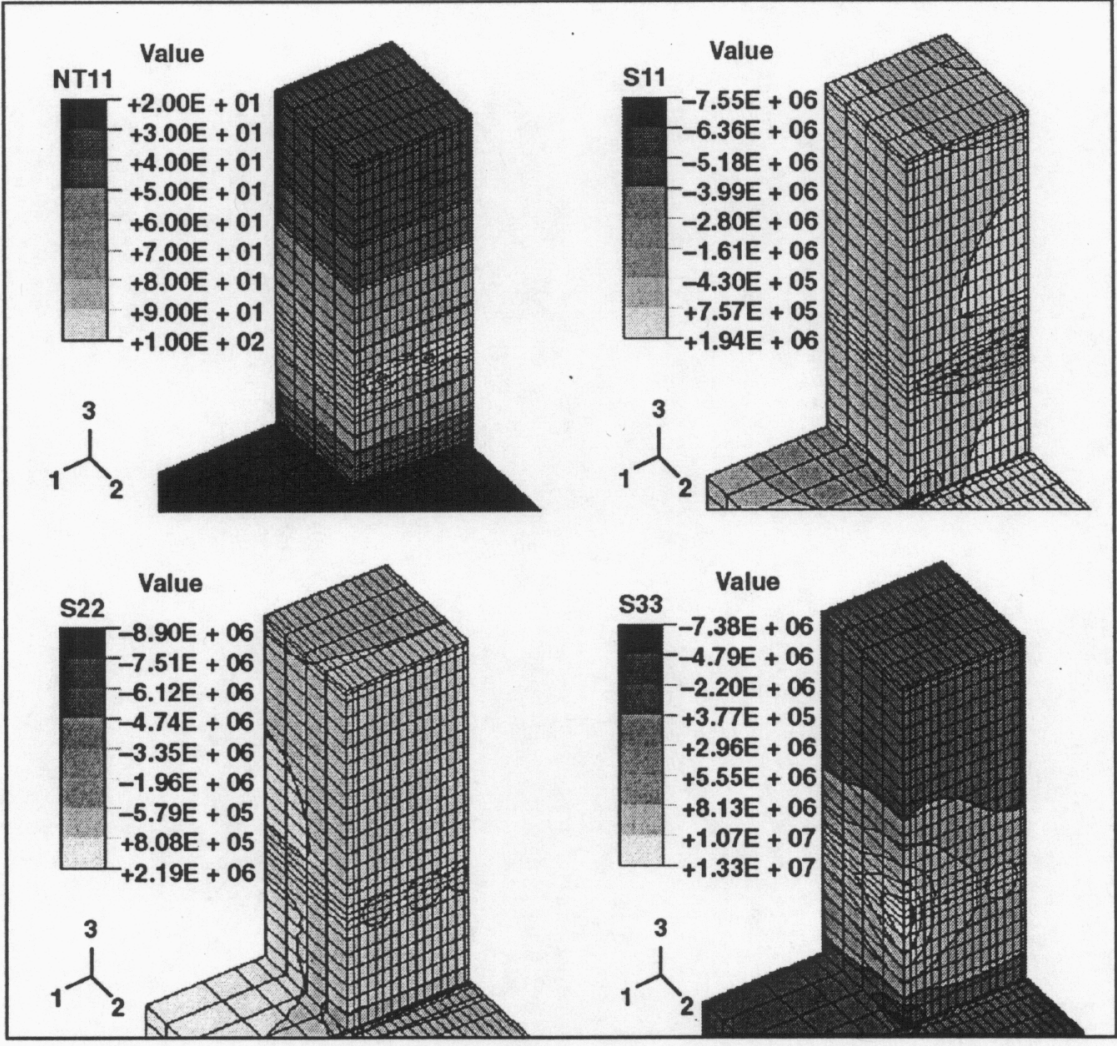


(c)

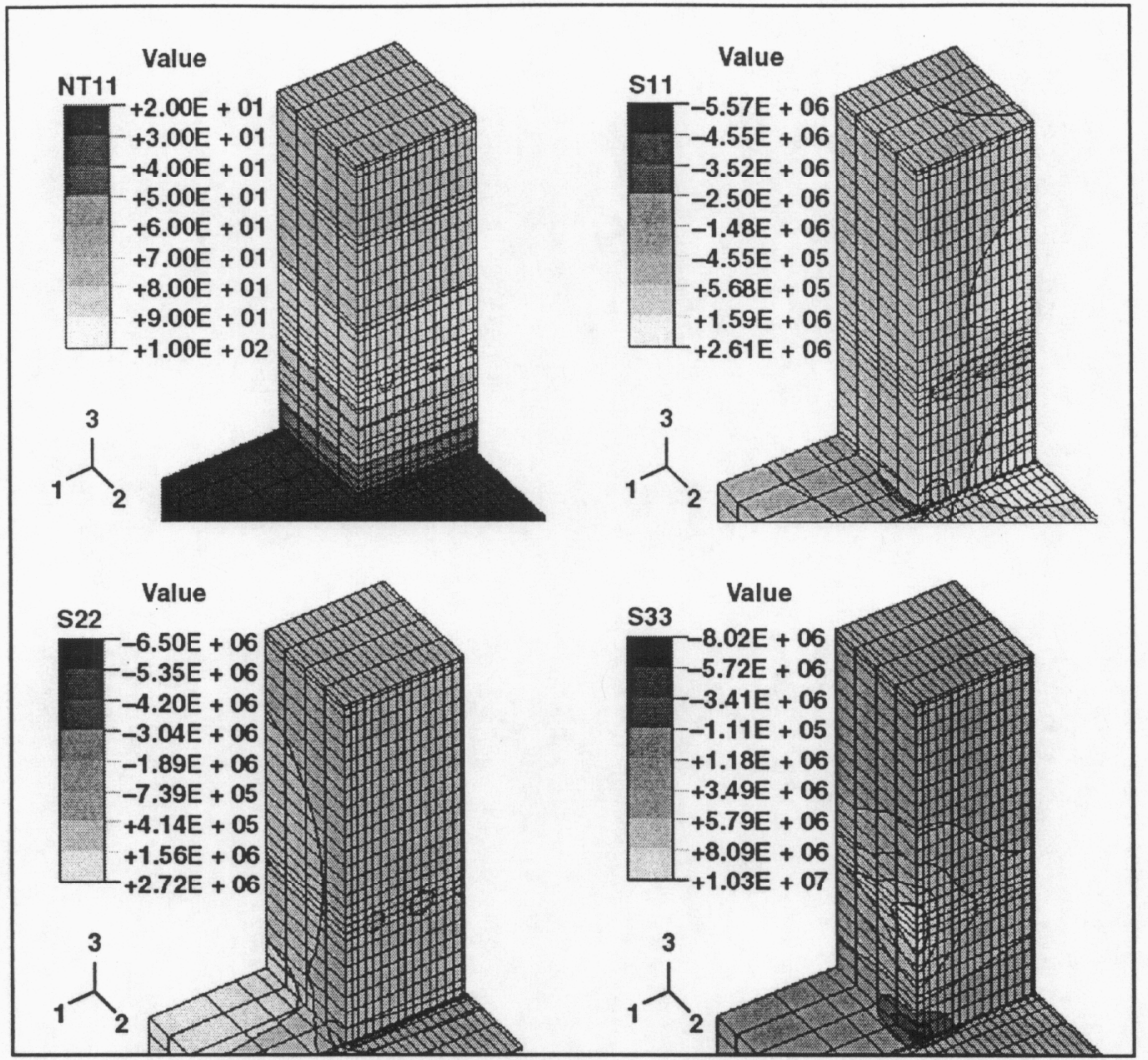

Figure 3-35. Temperature and Normal Stress Contours Predicted by ABAQUS Model at (a) 10, (b) 20, and (c) 60 Days of Heating 
(a)

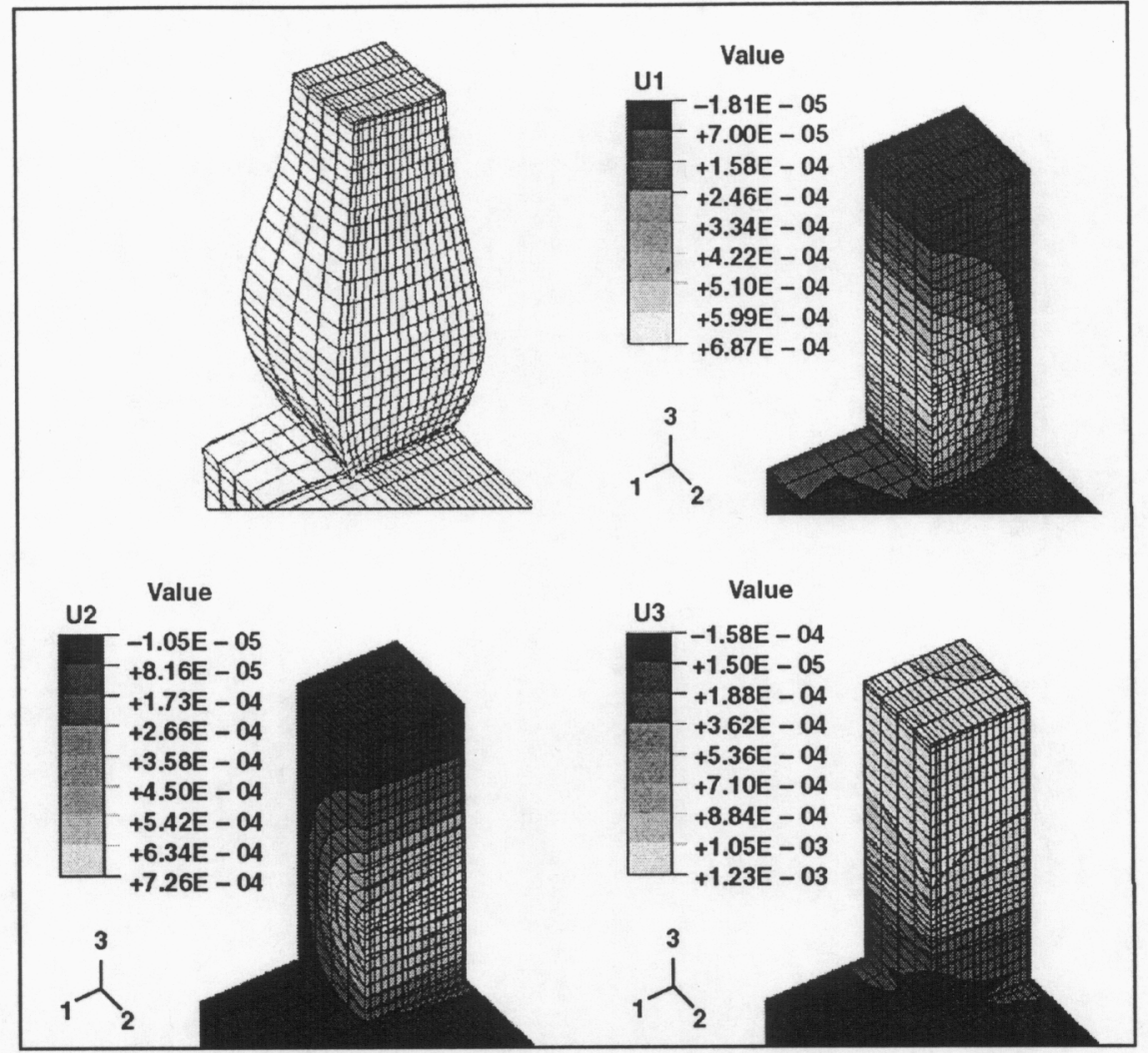

(b)

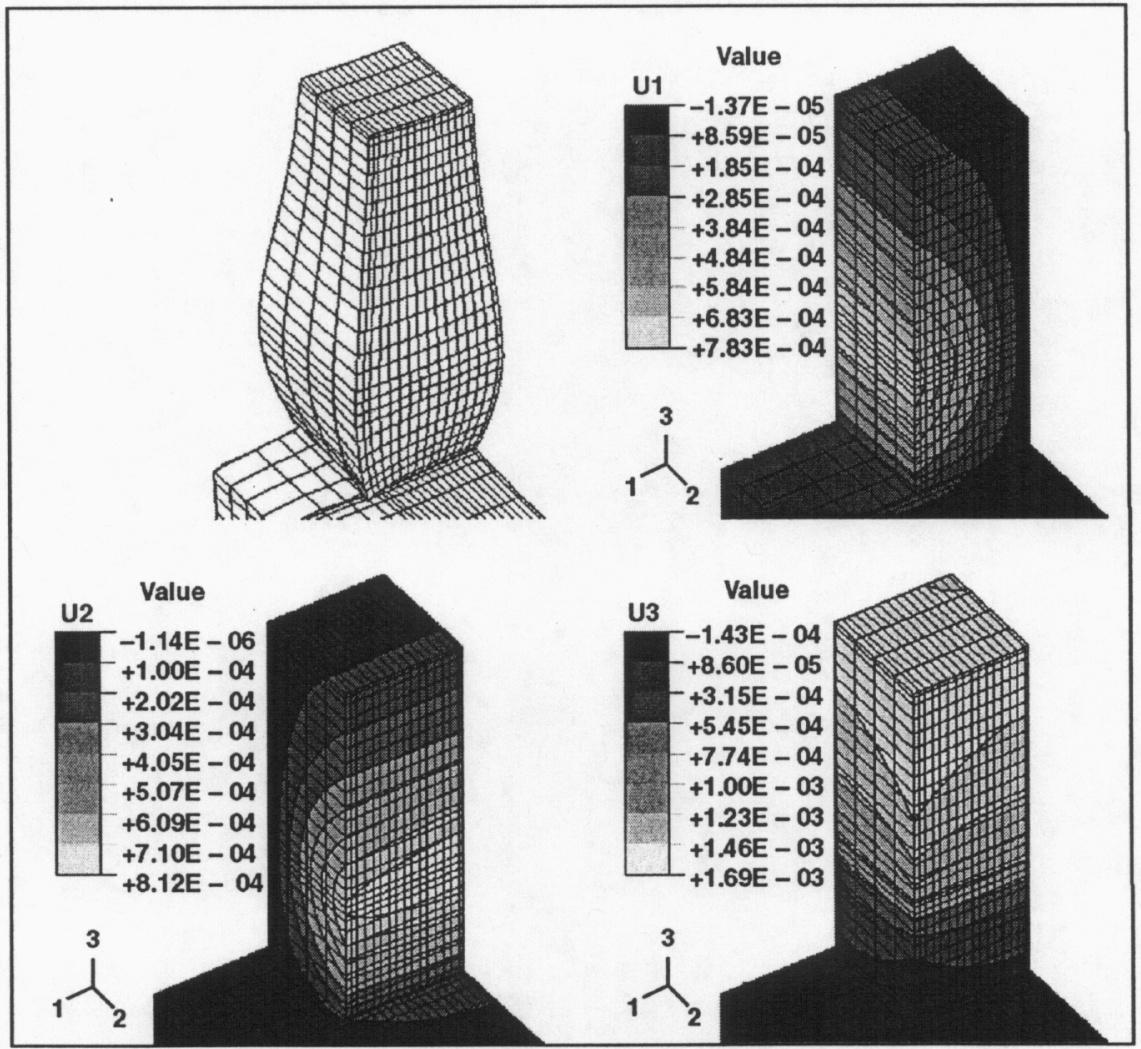


(c)

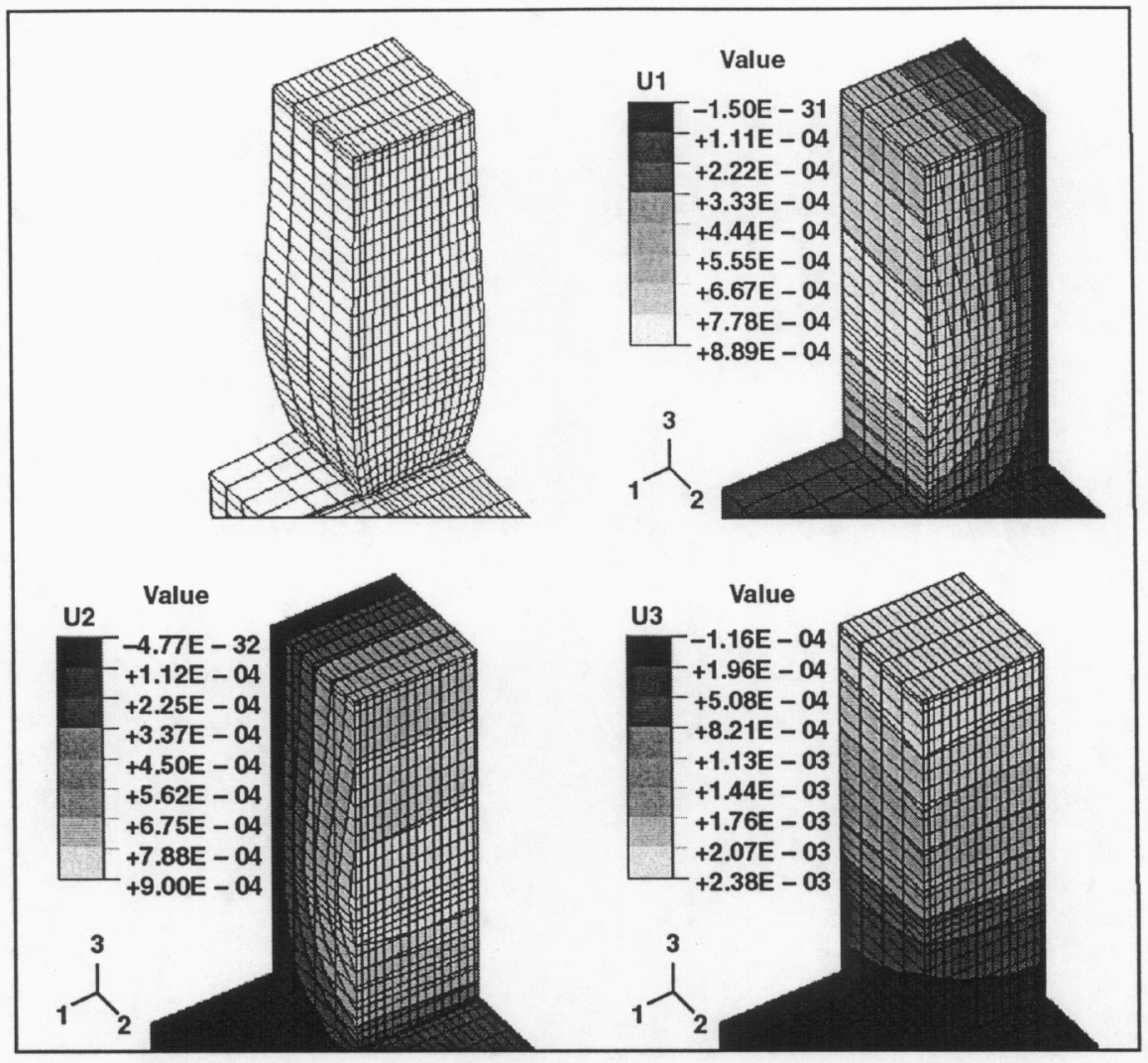

Figure 3-36. Displacements in the LBT Predicted by the ABAQUS Model at (a) 10, (b) 20, and (c) 60 Days of Heating 


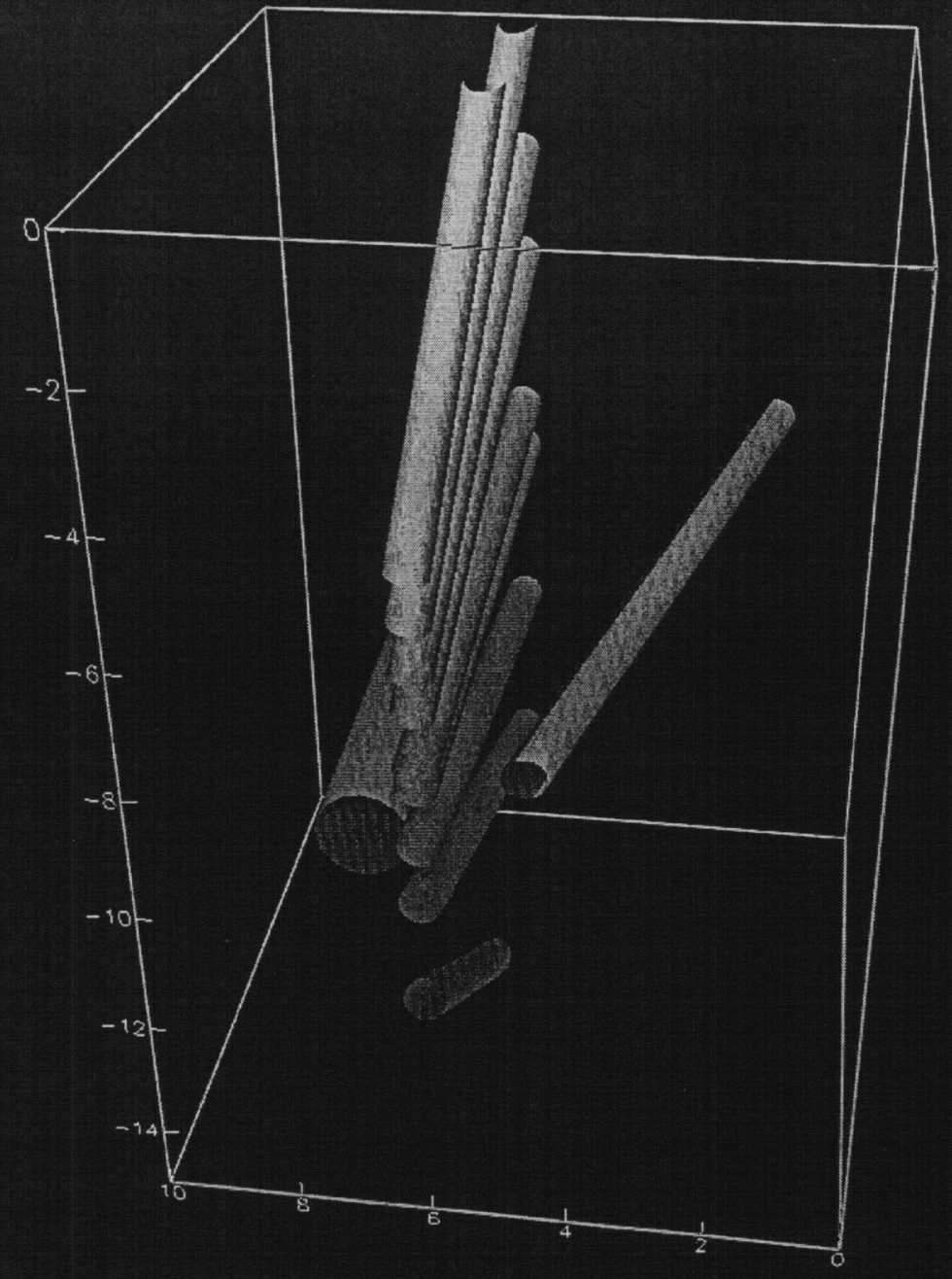

Figure 3-37. Post-test Coring Holes Drilled from the West Face of the Block 


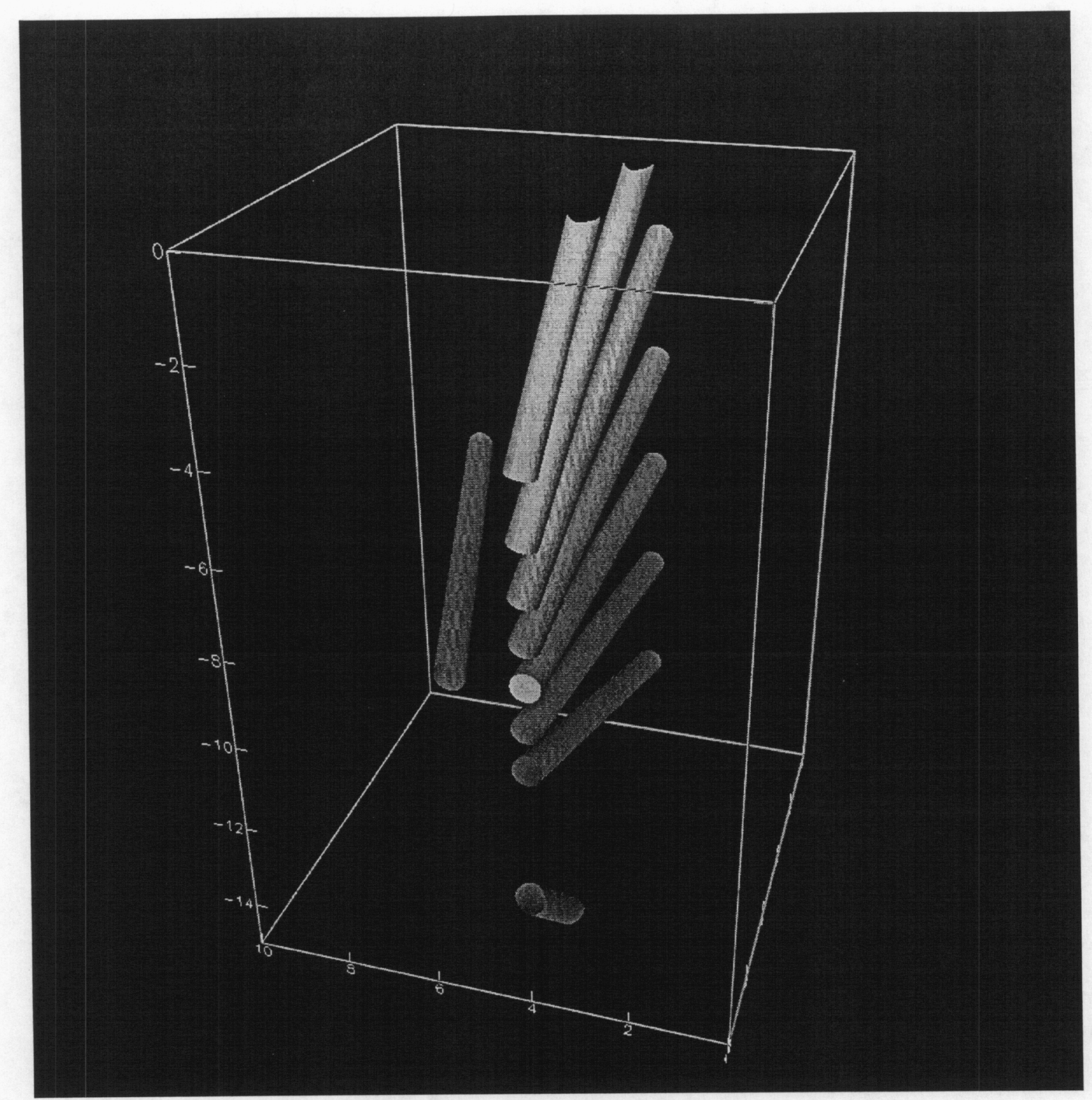

Figure 3-38. Post-test Coring Holes Drilled from the North Face of the Block 


\section{DOCUMENTATION AND ANALYSIS OF FRACTURES IN THE LARGE BLOCK TEST}

Fractures were carefully mapped on the block surface, and information on fracturing was also collected from cores and video logs of boreholes (Wagoner 1999; Wagoner 2000). The fractures were evaluated for their significance on the basis of size, extent, and other considerations, and the major fractures were selected to be included in a 3-D model. Although the LBT is not being used to characterize YM, its usefulness as a test of processes, models, and so forth is enhanced if the rock mass is similar to the repository horizon rock mass. For this reason, Fran Ridge was selected. Although the stress conditions are not the same and fracture apertures thus will not necessarily be the same, it is helpful if the fractures are similar in distribution to those in the repository. Therefore, the second analysis was of the fracture distribution compared with that of the main Exploratory Studies Facility (ESF) drift that is in the repository horizon. The fractures in the LBT were dominated by high-angle fractures. This is similar to the fractures in the ESF of Yucca Mountain. However, the fracture orientation in the block is somewhat different from that in the ESF (Section 2.2.1, Wilder et al. 1997).

Characterization of the block began with mapping and analysis of the distribution of fractures. Fractures were carefully mapped using a $1 \mathrm{ft} \times 1 \mathrm{ft}$ grid system on all four vertical sides and the top of the block (see Figure 4-1). Fracture mapping started September 19, 1994, and was completed October 6, 1994. Each fracture was assigned a unique number on each side of the block, and fracture attitudes were measured where possible. Fracture surface roughness was not recorded. More than 2400 individual fractures were mapped. The range and distribution of fracture lengths are recorded in Table 4-1. The fracture locations were digitized, and fracture segment nodes were assigned $x-y-z$ values. These scattered data points were then input into a 3-D modeling code (EarthVision ${ }^{\mathrm{TM}}$ version 5.0). The resulting surface fracture distribution is shown in Figure 4-1; Table 4-1 lists fractures ordered by length and shows that most of the fractures are less than $1 \mathrm{~m}$ long.

Numerous boreholes were drilled in the large block for installation of monitoring instrumentation and observation. Figure 4-2 shows the location of those boreholes. The angled boreholes (colorcoded yellow) are the post-test boreholes.

Fracture data were also collected from the borehole video logs of these holes. Information on the core is not included in this document. Detailed fracture information from the video logs is available in Appendix C. Seventy-one boreholes were videotaped. In that process, a measuring tape was placed in the borehole for location purposes. In the case of the vertical boreholes, the tape was hung along the north side of the hole, which provided an orientation in the borehole.

The depths at which the fracture enters and exits the borehole were recorded as were the strike, dip, dip direction, aperture, and magnitude of the features.

The goal of the fracture analysis is to identify and model the major through-going structures that penetrate the LBT. This involved correlating the surface fracture traces with the location of fractures intersecting the boreholes. Correlation of the borehole fractures with the surface 
fractures is confirmed by the location and the strike and dip of the fracture as measured in the video $\log$.

Table 4-1. Number of Fractures Per Each Length Interval and the Percentage of Fractures Occurring in Each Interval

\begin{tabular}{|c|c|c|c|}
\hline $\begin{array}{l}\text { Number of } \\
\text { fractures }\end{array}$ & Length ( $\mathrm{m}$ ) & Percentage & $\begin{array}{l}\text { Cumulative } \\
\text { percentage }\end{array}$ \\
\hline 1044 & $0.0-0.15$ & 43.41 & 43.41 \\
\hline 631 & $0.15-0.30$ & 26.24 & 69.65 \\
\hline 251 & $0.30-0.46$ & 10.44 & 80.08 \\
\hline 130 & $0.46-0.61$ & 5.41 & 85.49 \\
\hline 118 & $0.61-0.76$ & 4.91 & 90.40 \\
\hline 59 & $0.76-0.91$ & 2.45 & 92.85 \\
\hline 35 & $0.91-1.07$ & 1.46 & 94.30 \\
\hline 32 & $1.07-1.22$ & 1.33 & 95.63 \\
\hline 24 & $1.22-1.37$ & 1.00 & 96.63 \\
\hline 13 & $1.37-1.52$ & 0.54 & 97.17 \\
\hline 8 & $1.52-1.68$ & 0.33 & 97.51 \\
\hline 5 & $1.68-1.83$ & 0.21 & 97.71 \\
\hline 8 & $1.83-1.98$ & 0.33 & 98.05 \\
\hline 6 & $1.98-2.13$ & 0.25 & 98.30 \\
\hline 6 & $2.13-2.29$ & 0.25 & 98.54 \\
\hline 3 & $2.29-2.44$ & 0.12 & 98.67 \\
\hline 3 & $2.44-2.59$ & 0.12 & 98.79 \\
\hline 2 & $2.59-2.74$ & 0.08 & 98.88 \\
\hline 5 & $2.74-2.90$ & 0.21 & 99.09 \\
\hline 2 & $2.90-3.05$ & 0.08 & 99.17 \\
\hline 8 & $3.05-3.20$ & 0.33 & 99.50 \\
\hline 0 & $3.20-3.35$ & 0.00 & 99.50 \\
\hline 0 & $3.35-3.51$ & 0.00 & 99.50 \\
\hline 1 & $3.51-3.66$ & 0.04 & 99.54 \\
\hline 0 & $3.66-3.81$ & 0.00 & 99.54 \\
\hline 0 & $3.81-3.96$ & 0.00 & 99.54 \\
\hline 2 & $3.96-4.11$ & 0.08 & 99.63 \\
\hline 0 & $4.11-4.27$ & 0.00 & 99.63 \\
\hline 3 & $4.27-4.42$ & 0.12 & 99.75 \\
\hline 0 & $4.42-4.57$ & 0.00 & 99.75 \\
\hline 2 & $4.57-4.72$ & 0.08 & 99.83 \\
\hline 3 & $4.72-4.88$ & 0.12 & 99.96 \\
\hline 0 & $4.88-5.03$ & 0.00 & 99.96 \\
\hline 1 & $5.03-5.18$ & 0.04 & 100.00 \\
\hline
\end{tabular}

NOTE: Fractures mapped on all five sides.

Figure 4-3 is an equal-area diagram of pole to the major fractures that have been defined for the LBT. These fractures are defined by the surface mapping of the LBT and by the video mapping of the boreholes. Table 4-2 lists the major mappable fractures that have been modeled in the LBT. The individual modeled fractures are grouped into six fracture systems based on similarity in strike and dip. Figure 4-4 is a 3-D perspective of these six systems cutting the block. 
Table 4-2. All Major Fracture Planes That Have Been Modeled for the LBT

\begin{tabular}{|c|c|c|c|}
\hline Number & Strike & Dip & System number \\
\hline LBT21 & N32E & $82 N W$ & 1 \\
\hline LBT11 & N49E & $43 N W$ & 1 \\
\hline LBT22 & N52E & $58 \mathrm{NW}$ & 1 \\
\hline LBT2 & N30W & 78NE & 2 \\
\hline LBT15 & N37W & 62NE & 2 \\
\hline LBT14 & N26E & 01SE & 3 \\
\hline LBT1 & N36W & $11 \mathrm{NE}$ & 3 \\
\hline LBT30 & N53W & ogSW & 3 \\
\hline LBT32 & N68W & 13NE & 3 \\
\hline LBT31 & N70W & O9NE & 3 \\
\hline LBT33 & N78W & 23NE & 3 \\
\hline LBT38 & N03W & 89SW & 4 \\
\hline LBT12 & N04W & $895 W$ & 4 \\
\hline LBT16 & N05W & 89SW & 4 \\
\hline LBT13 & N10W & 87SW & 4 \\
\hline LBT37 & N11W & 88SW & 4 \\
\hline LBT42 & N16W & $80 S W$ & 4 \\
\hline LBT36 & N17W & 87SW & 4 \\
\hline LBT41 & N18W & $81 S W$ & 4 \\
\hline LBT20 & N1BW & 87SW & 4 \\
\hline LBT35 & N18W & $87 S W$ & 4 \\
\hline LBT6 & N22W & $78 S W$ & 4 \\
\hline LBT40 & N25W & $80 S W$ & 4 \\
\hline LBT5 & N34W & $77 \mathrm{SW}$ & 4 \\
\hline LBT34 & N70W & $80 S W$ & 5 \\
\hline LBT8 & N78W & $79 S W$ & 5 \\
\hline LBT39 & N78W & 87NE & 5 \\
\hline LBT3 & N89E & 90 & 5 \\
\hline LBT4 & N46E & 87SE & 6 \\
\hline
\end{tabular}

NOTE: These fractures are defined by the surface mapping of the LBT and by the video mapping of the boreholes.

Fracture system \#1 is defined by three major fractures called LBT11, LBT21, and LBT22. This system occurs on the south and north sides of the large block and strikes N50E, then dips $40-45^{\circ}$ to the northwest (Figure 4-5). System \#2 consists of two major mapped fractures called LBT2 and LBT15. These fractures strike N30-40 W and dip $60-80^{\circ}$ to the northeast. The two fractures are mapped on the south and west sides of the large block (Figure 4-6).

Fracture system \#3 contains six major mappable fractures. The fractures making up this system are LBT14, LBT30, LBT31, LBT32, LBT33, and LBT1, which is by far the most significant fracture in the LBT, because it completely cuts through the block and is identified in all vertical boreholes drilled from the top of the large block. As seen in the borehole videos, this feature generally has a wide aperture, common secondary mineralization, and, locally, alteration halos. These fractures are subhorizontal and have strikes that are generally northwest and dip about $20^{\circ}$ 
toward the southwest (Figure 4-7). These fractures occur on all four sides of the large block. The dip direction of these fractures is similar to the topographic slope of this part of Fran Ridge.

Fracture system \#4 contains the greatest number of fractures. This system is defined by 13 major mappable fractures that penetrate the large block. The fracture attitudes range from N03 to $34 \mathrm{~W}$, dipping $77-89^{\circ}$ to the southwest. This system is present on all sides of the LBT and contains a large number of subordinate, associated fractures with similar attitudes (Figure 4-8). Fracture system \#5 contains three major mappable fractures that have a general east-west strike and near vertical dips (generally $80-90^{\circ}$ ) (Figure 4-9). Fracture system \#6 is defined by two mappable fractures, LBT4 and LBT23. The fractures strike northeast with near-vertical dips (Figure 4-10).

Figure 4-11 is an equal-area net of poles to the major fractures that were mapped on top of the large block prior to construction of the LBT. The steeply dipping fractures correlate well with Figure 4-3, but the subhorizontal fractures are missing. These subhorizontal fractures were not observed in the initial mapping because of the shallow dip of the structures and probable subtle expression at the surface of the ground. This fracture pattern, including the subhorizontal fracture system, compares reasonably well with the fractures and faults that were mapped in the Climax Stock (Figure 4-12), located approximately $50 \mathrm{~km}$ northeast of Fran Ridge (Thorpe and Springer 1981, p. 14, Figure 6).

The fracture map of the ground surface above the area of the LBT prior to construction of the block is shown in Figure 4-13. The highlighted fractures have been identified in the large block and then projected to the land surface. Five of the six fracture systems have been identified; the subhorizontal fracture system \#3 is not identified on this map. Identification and correlation of the fractures was done by projecting the measured attitudes of the LBT fractures up to the land surface.

Figures 4-14 through Figure 4-18 are 2-D presentations of all fractures mapped on the five surfaces of the LBT. The fracture systems are color-coded for increased visual discrimination. 


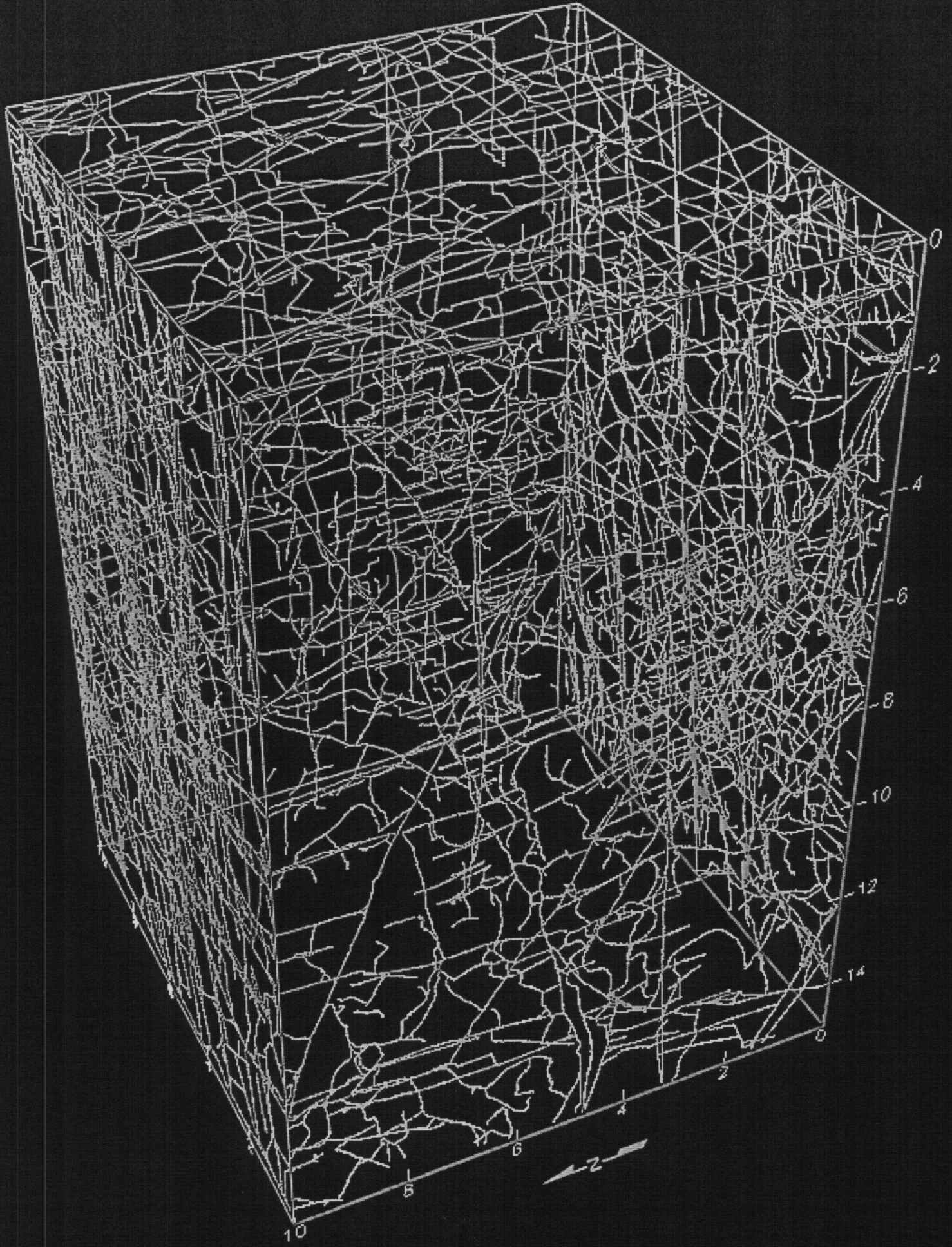

Figure 4-1. Mapped Surface Fractures on the Large Block 

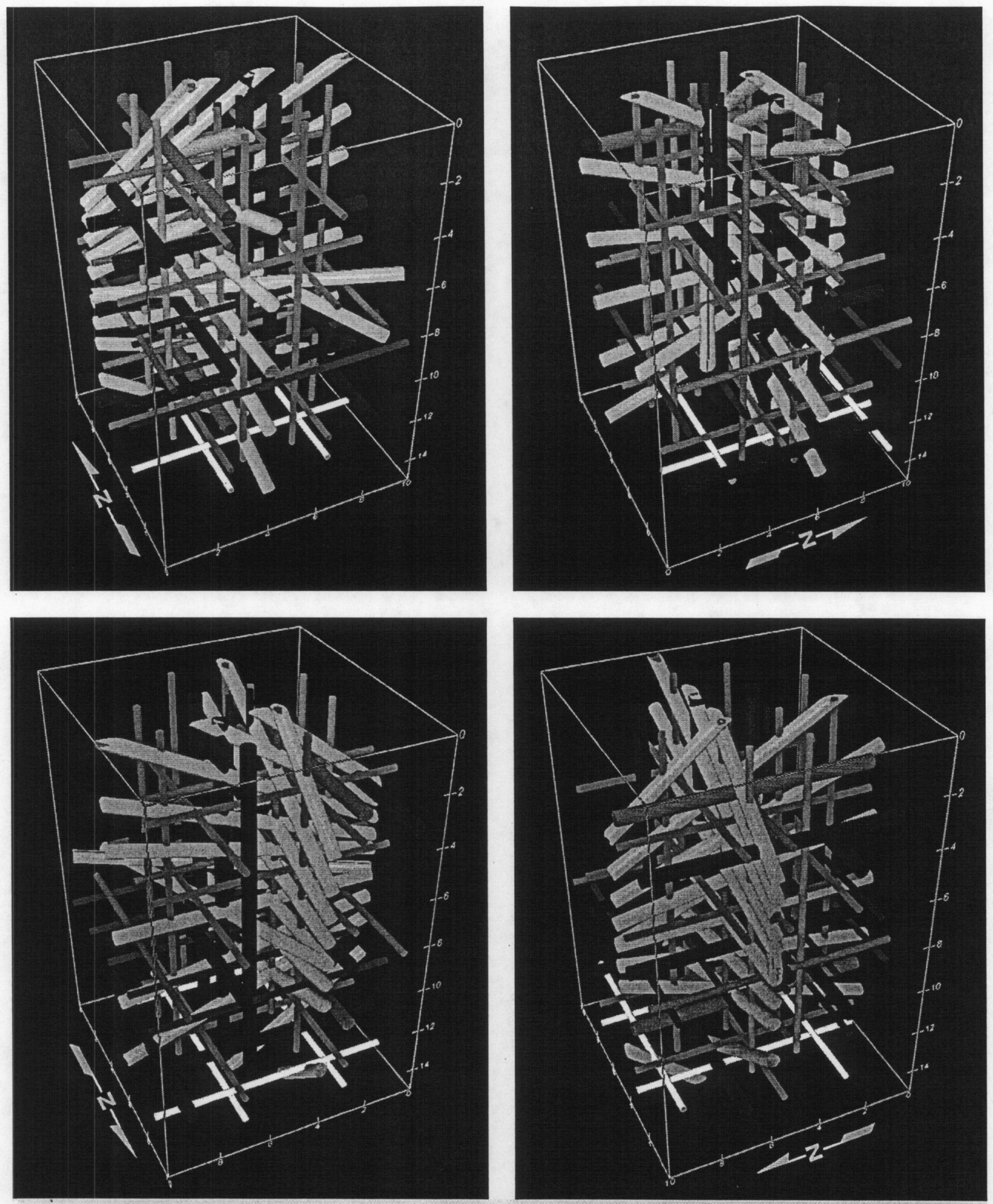

E9 E10 EH1 EH2 EH3 EH4 EH5 N1 N2 N3 NH1 NM1 NM2 NM3 WH1 WH2 WM1 WM2 WM3 E03 NO1 NO2 WO5 TE1 TR1 WR1 WR2 E1 E7 NT1 NT2 NT3 NT4 WT1 WT2 WT3 E2 E3 E5 E6 E8 NN1 NN2 NN3 NN4 NN5 NN6 WN1 WN2 WN3 WN4 Post-test holes

Figure 4-2. All Boreholes Drilled in the Large Block, Viewed at Various Angles 


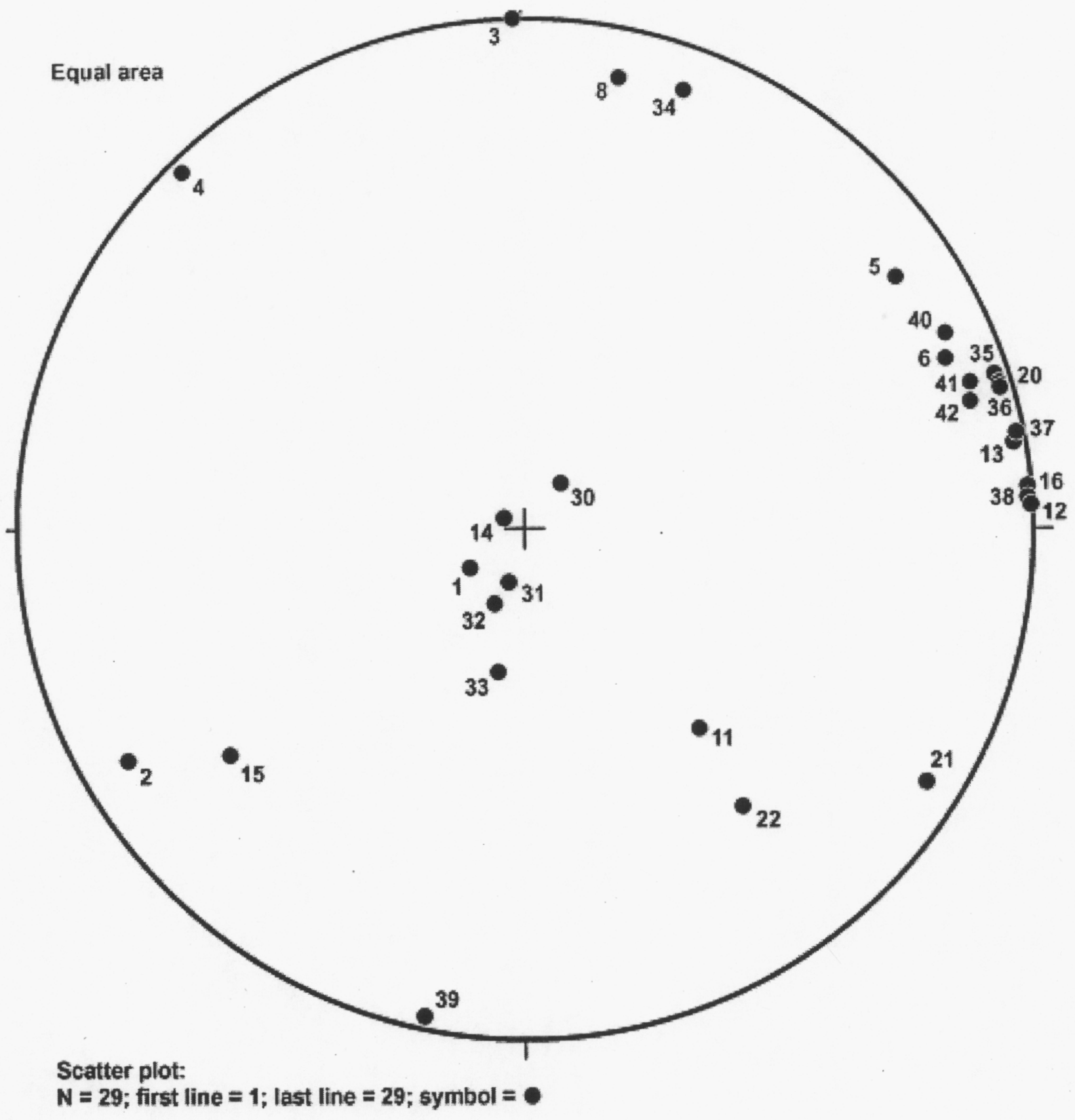

Figure 4-3. Equal-Area Diagram of Pole to the Major Fractures in the Large Block 

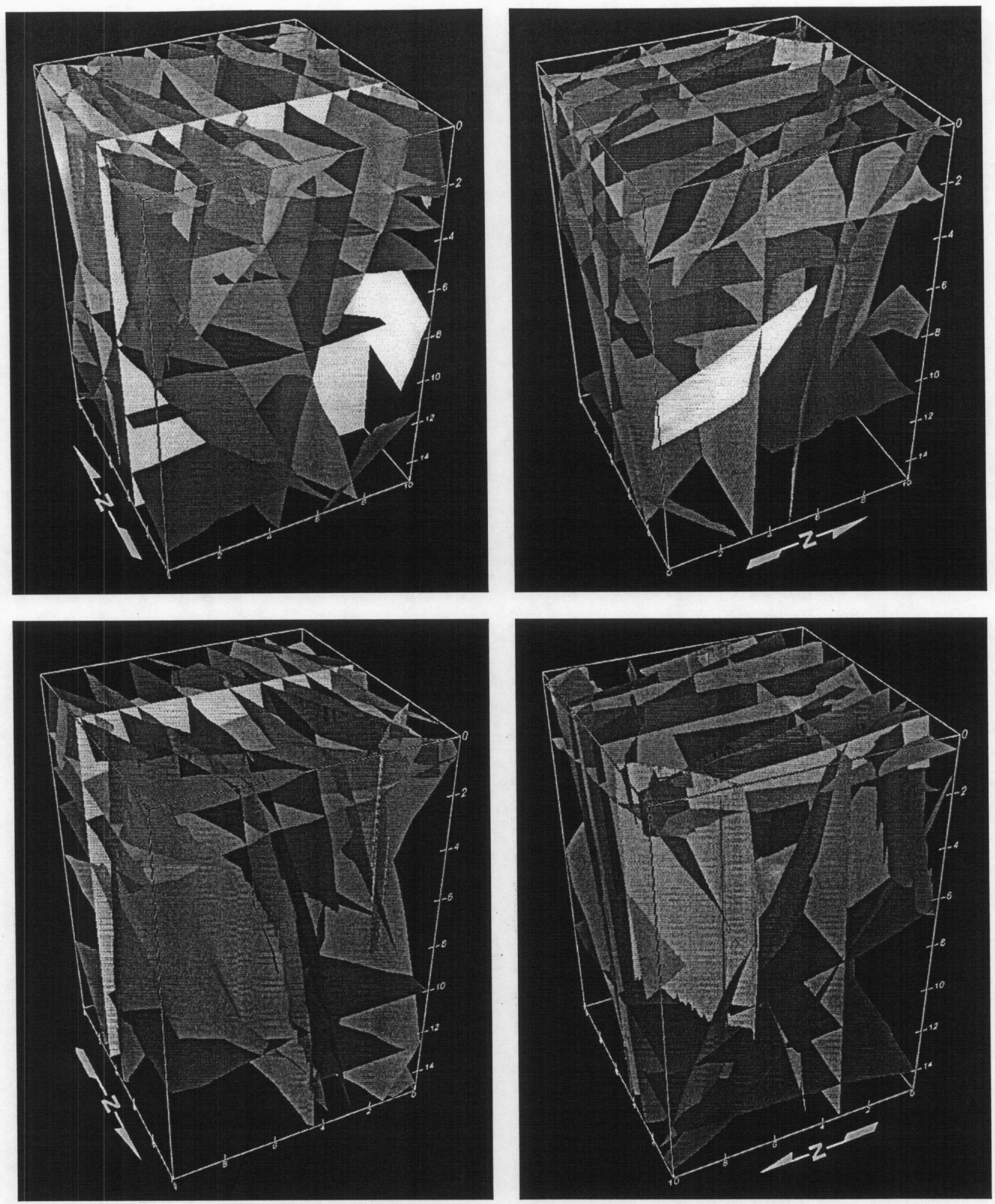

Figure 4-4. 3-D Depiction of the Major Mappable Fractures Cutting the Large Block 

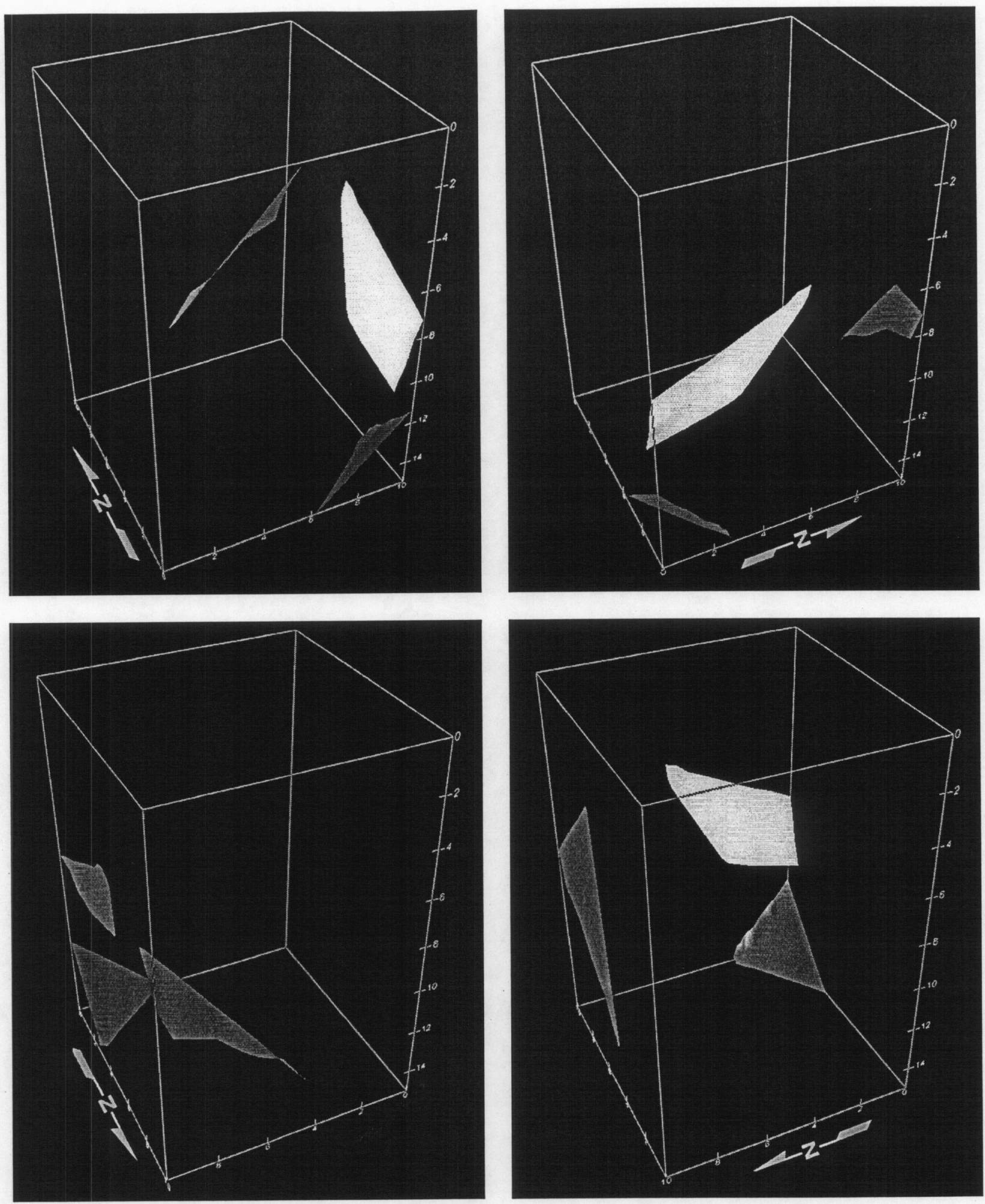

Figure 4-5. Fracture System \#1 Contains Three Fractures That Strike N50E and Dip $40-45^{\circ}$ Northwest 

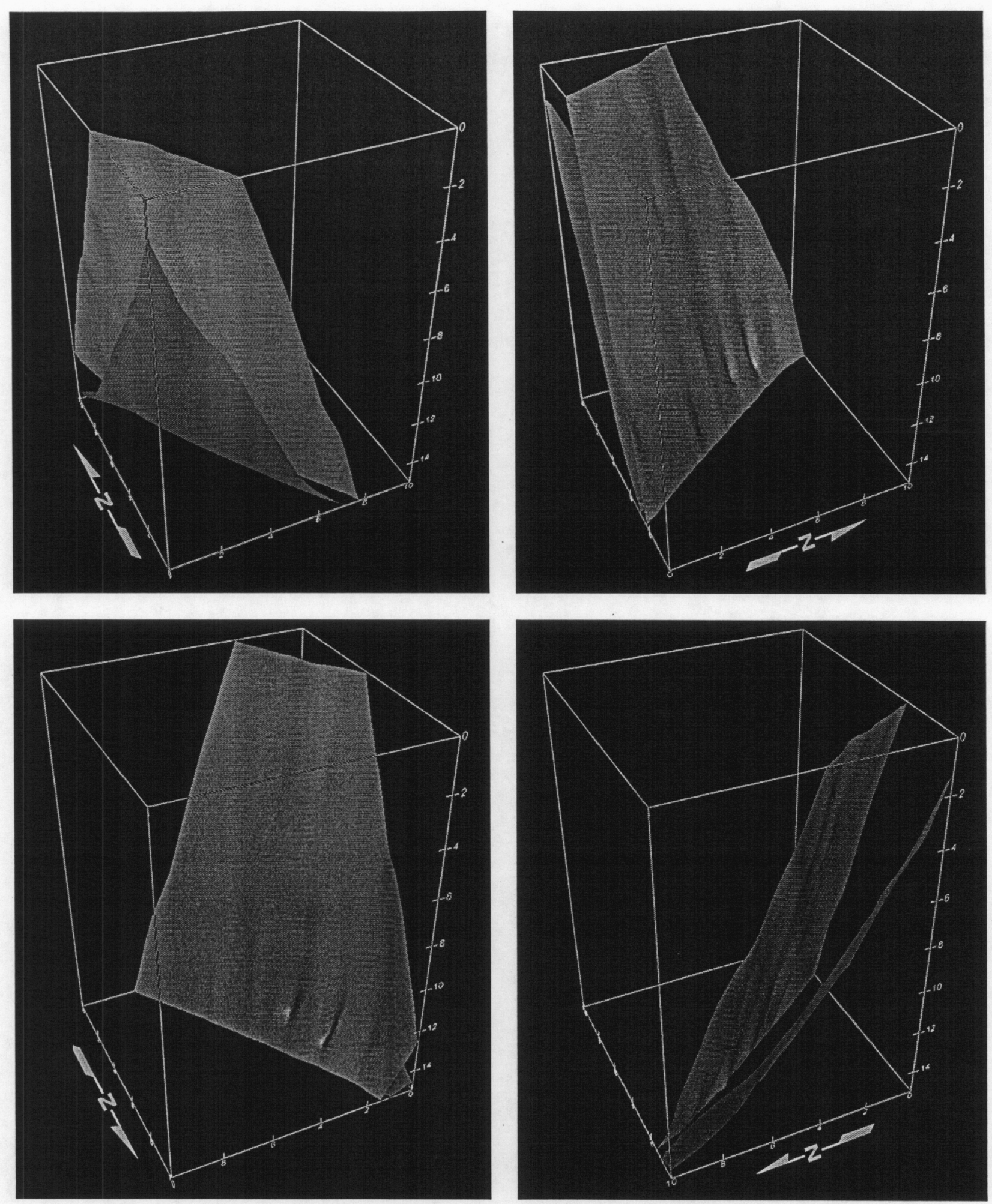

Figure 4-6. Fracture System \#2 Contains Two Fractures That Strike N30-40W and Dip 60-80 Northeast 

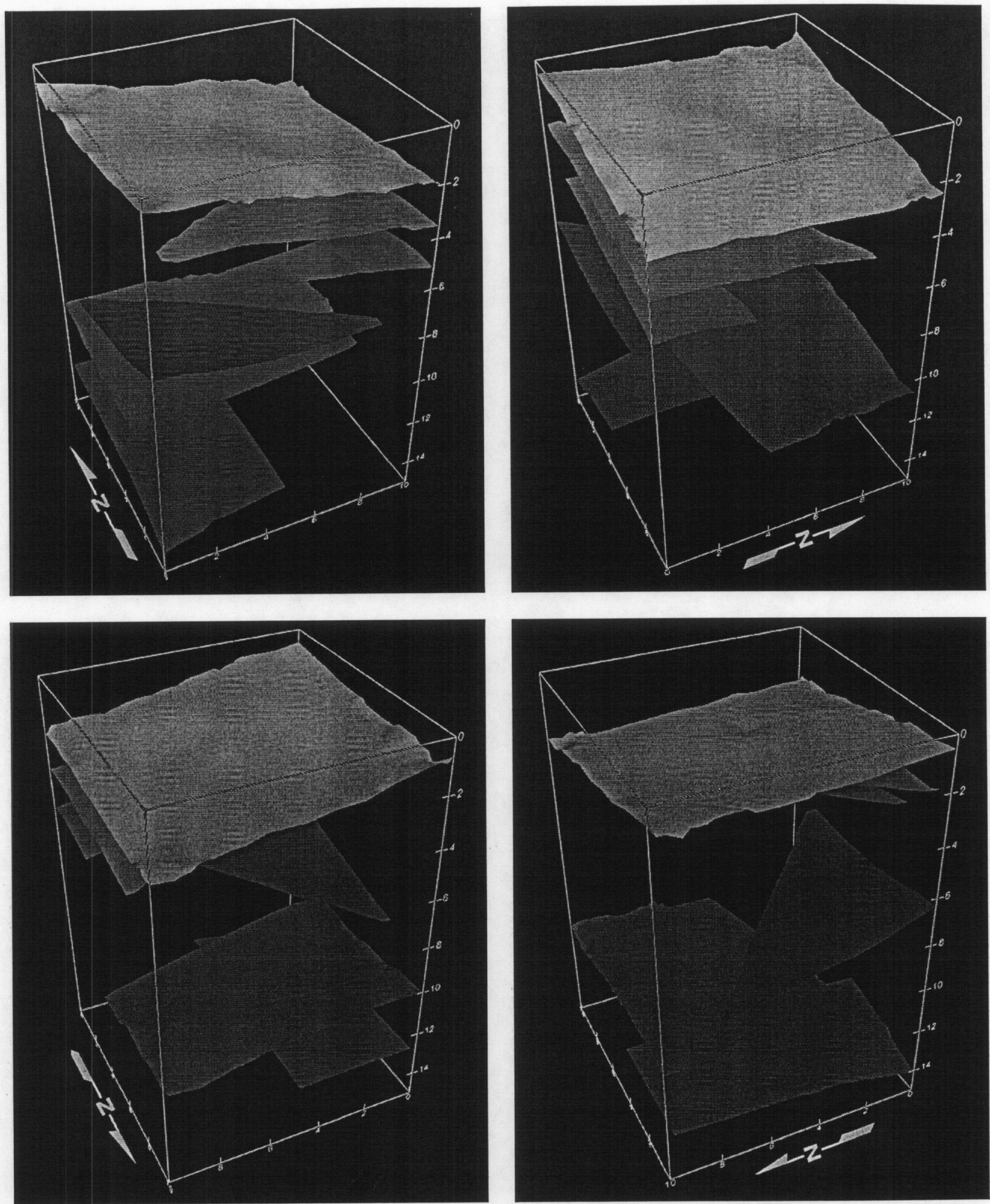

NOTE: Fracture LBT1 (green) is the largest, most significant fracture mapped in the block.

Figure 4-7. Fracture System \#3 Contains Six Subhorizontal Fractures 

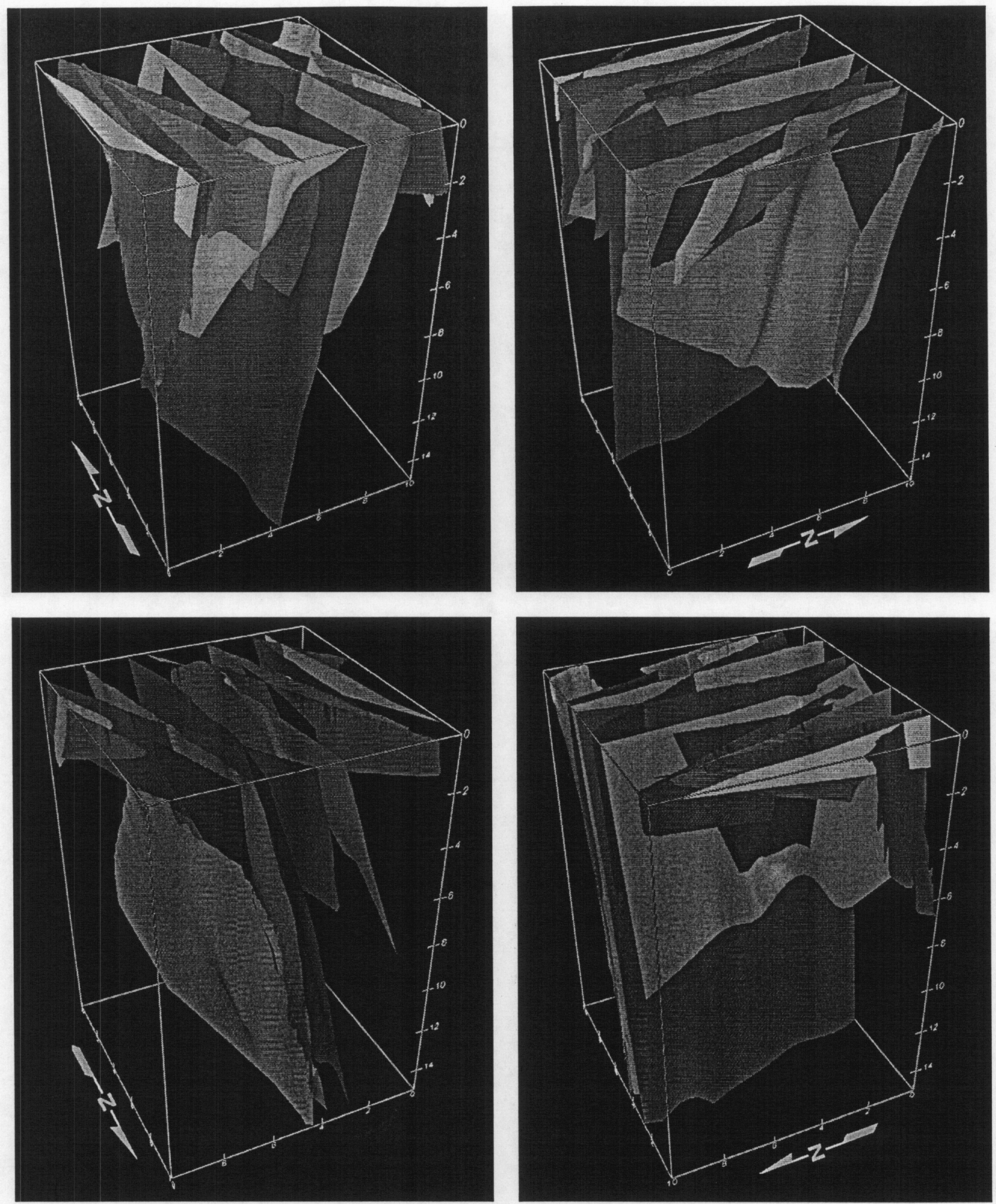

NOTE: This system strikes northwest and dips toward the southwest.

Figure 4-8. Fracture System \#4 Contains 13 Mapped Fractures and Is the Dominant System in the Block 

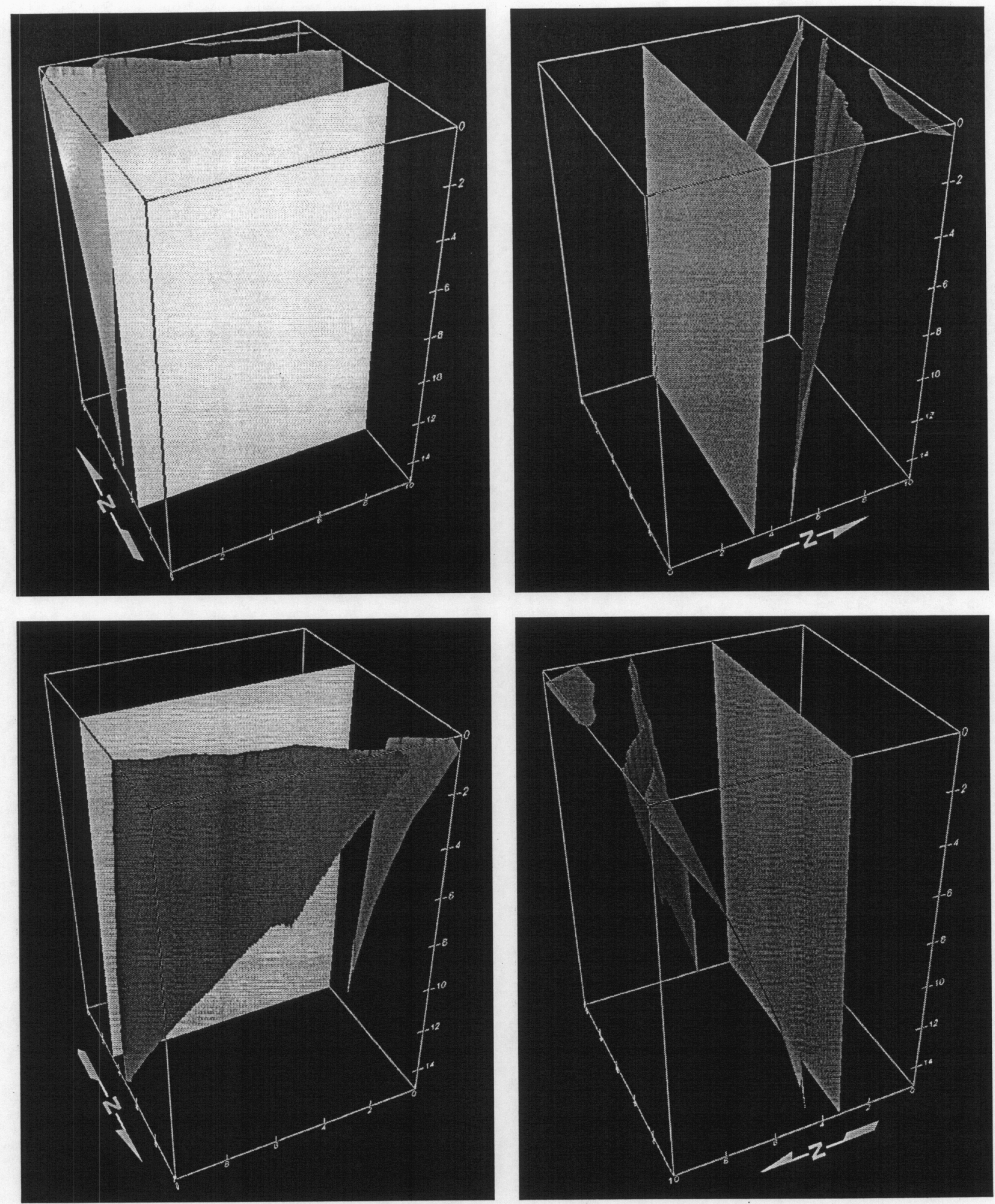

Figure 4-9. Fracture System \#5 Contains Four Approximately Vertical Fractures That Strike East-West 

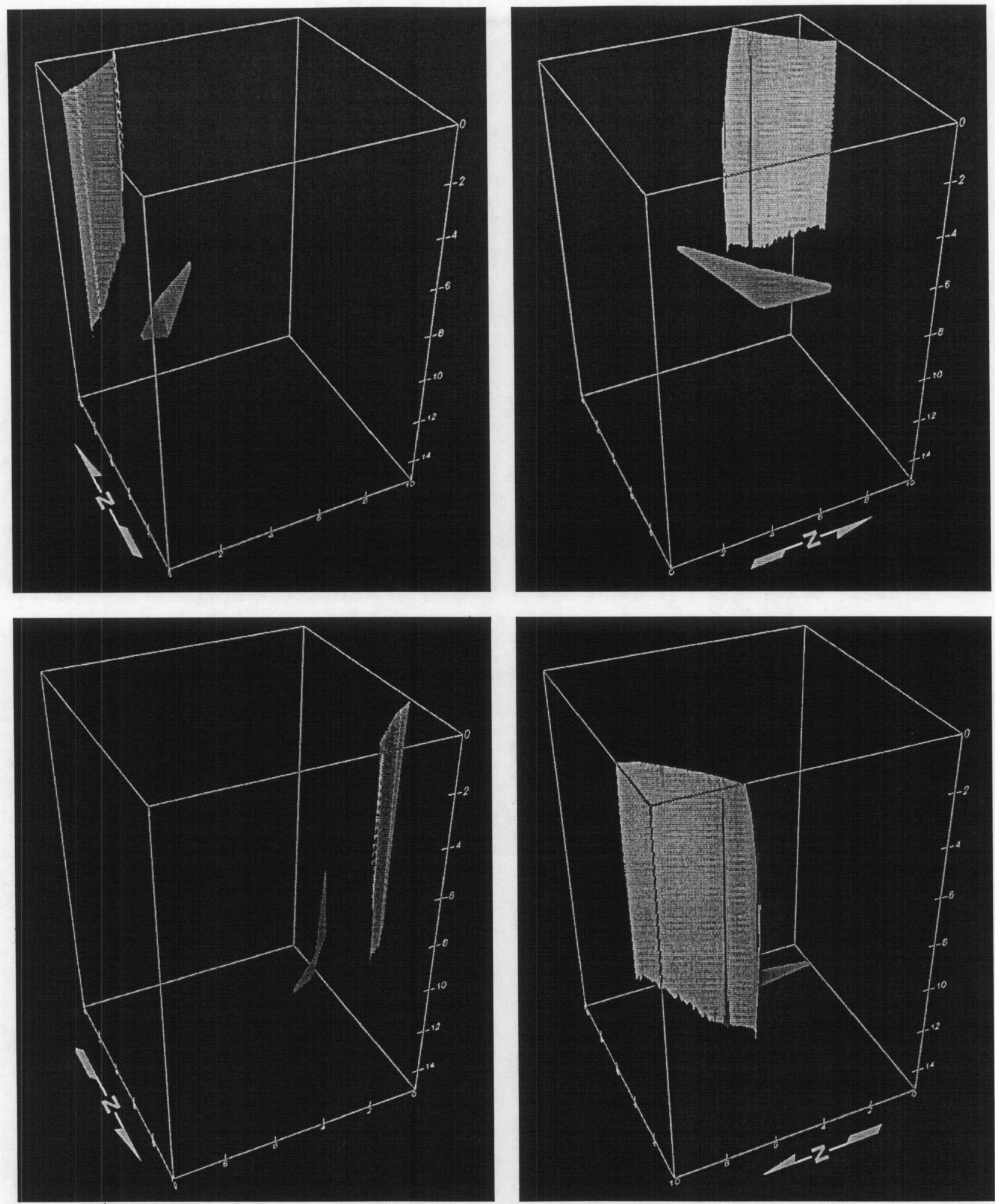

Figure 4-10. Fracture System \#6 Contains Two Mappable Fractures That Strike Northeast and Dip to the Southeast 


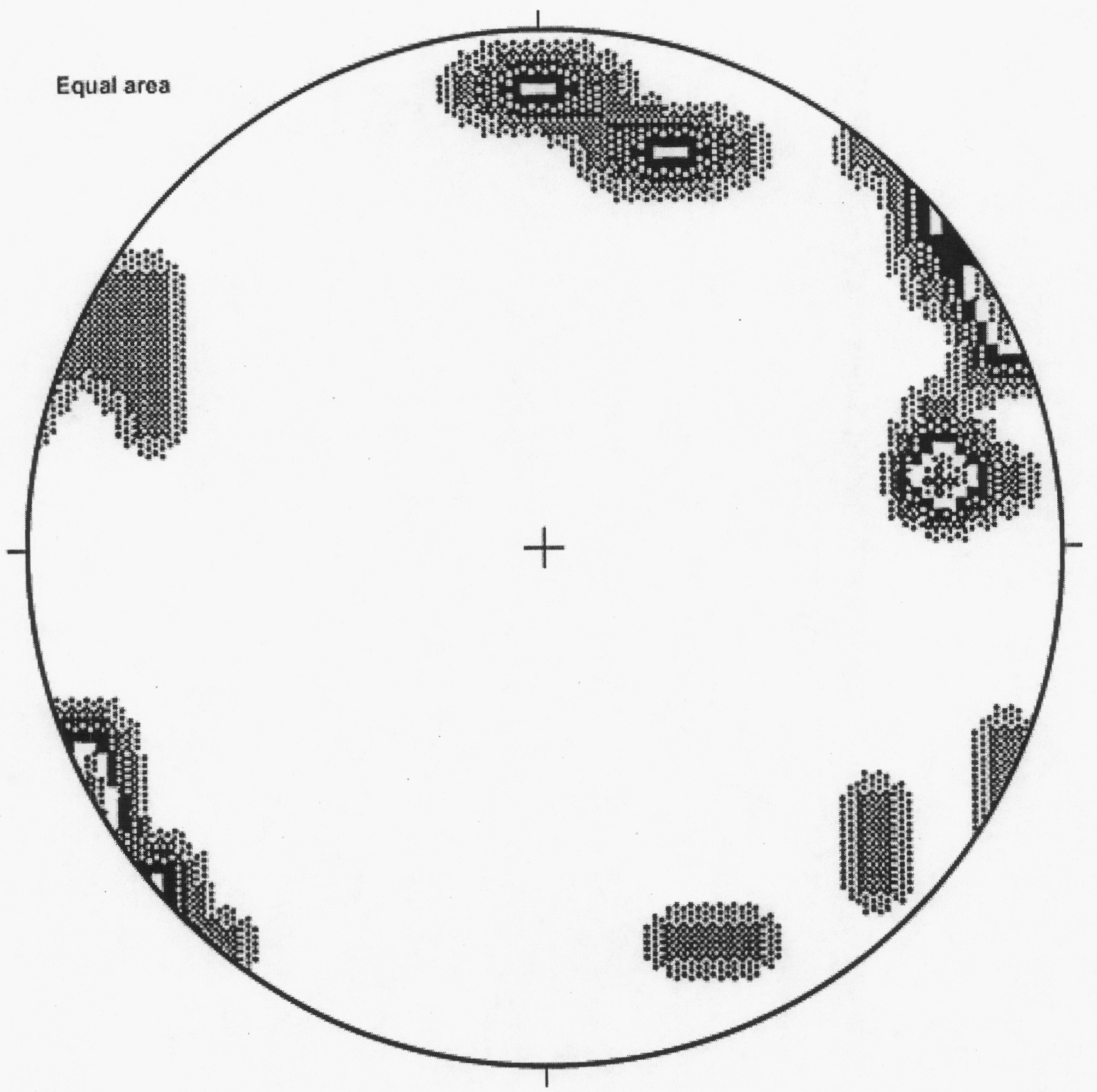

$1 \%$ area contour:

$N=17$; contour interval $=2 \% / 1 \%$ area

Figure 4-11. Equal-area Net of Poles to the Major Fractures Mapped on Top of the Block 


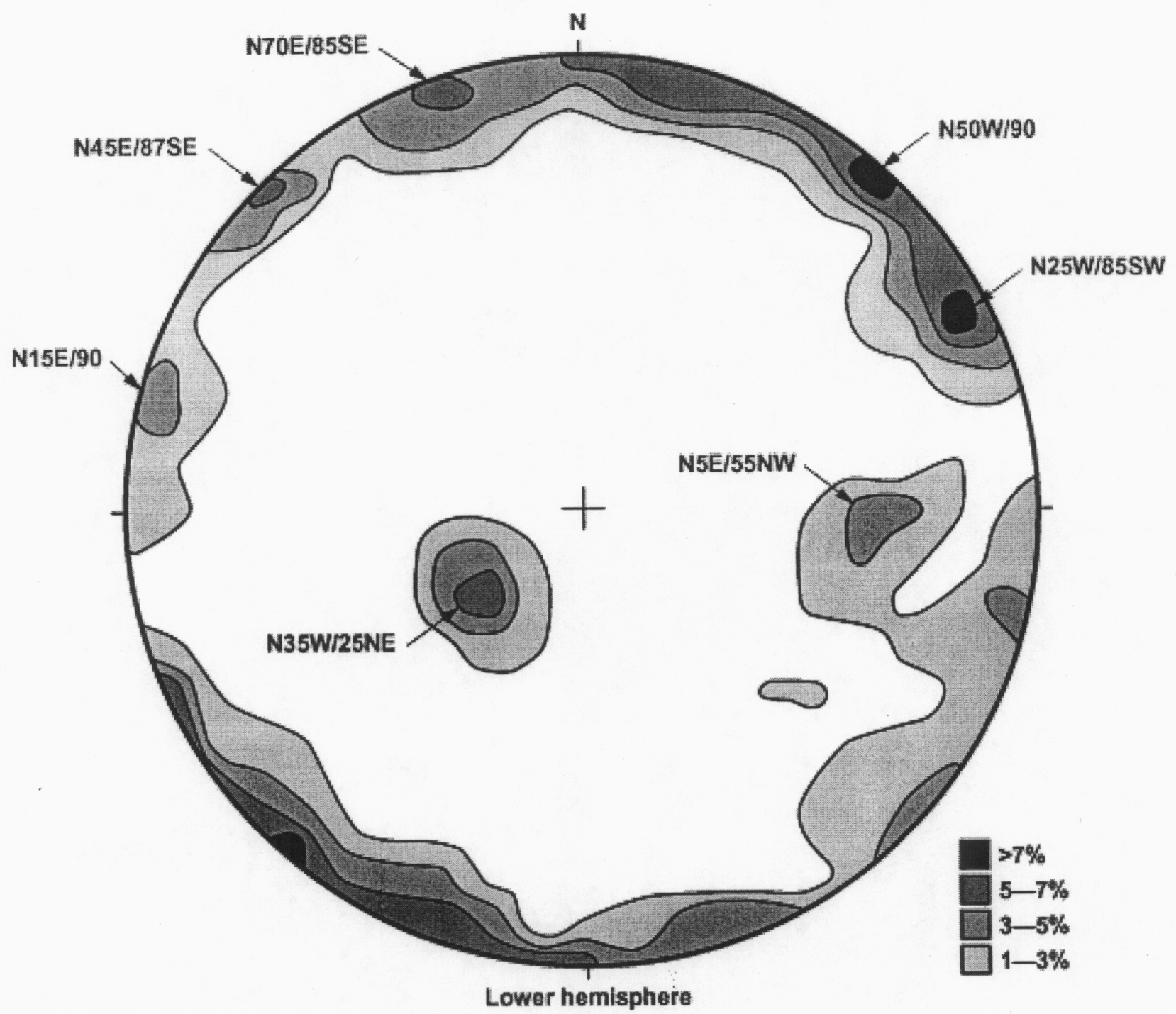

NOTE: Concentrations in percentage points within one percent area counting circle.

Figure 4-12. Equal-Area Contour Diagram of Poles to 90 Fractures Mapped in the Climax Stock Granite 


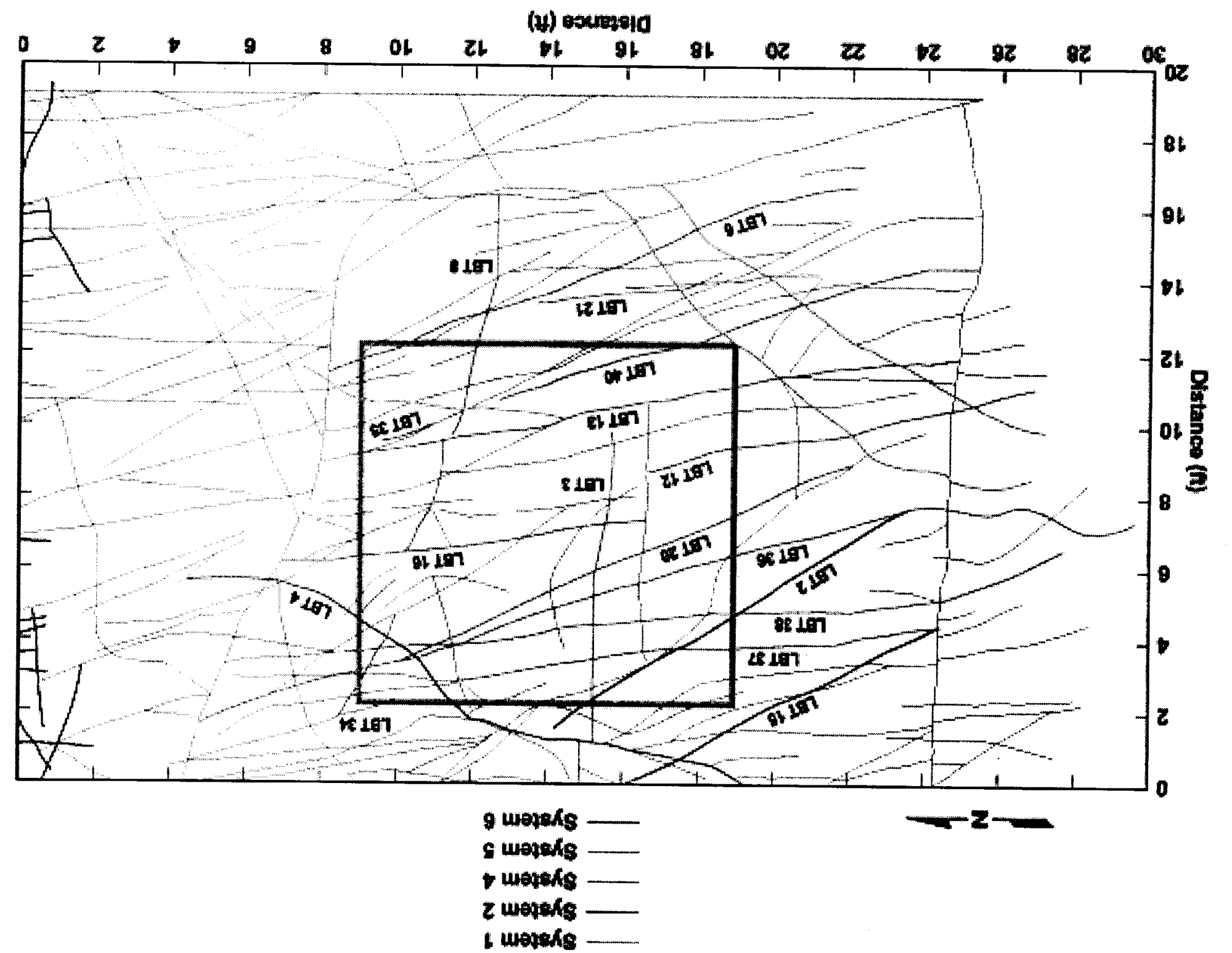

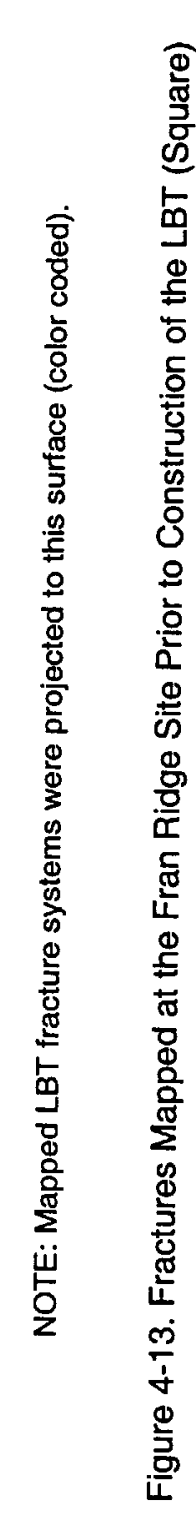

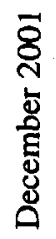




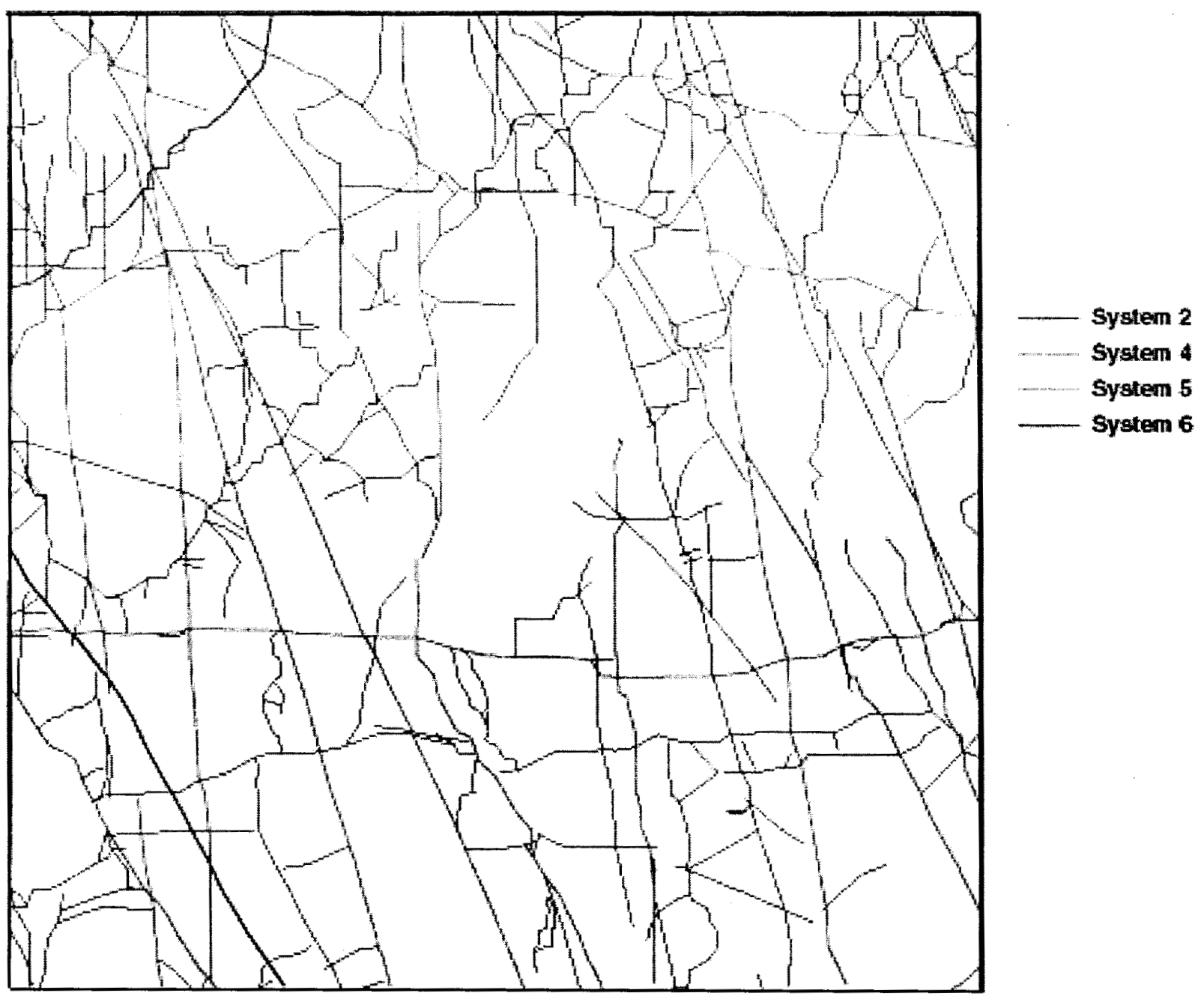

Figure 4-14. Mapped Fractures on Top of the LBT 


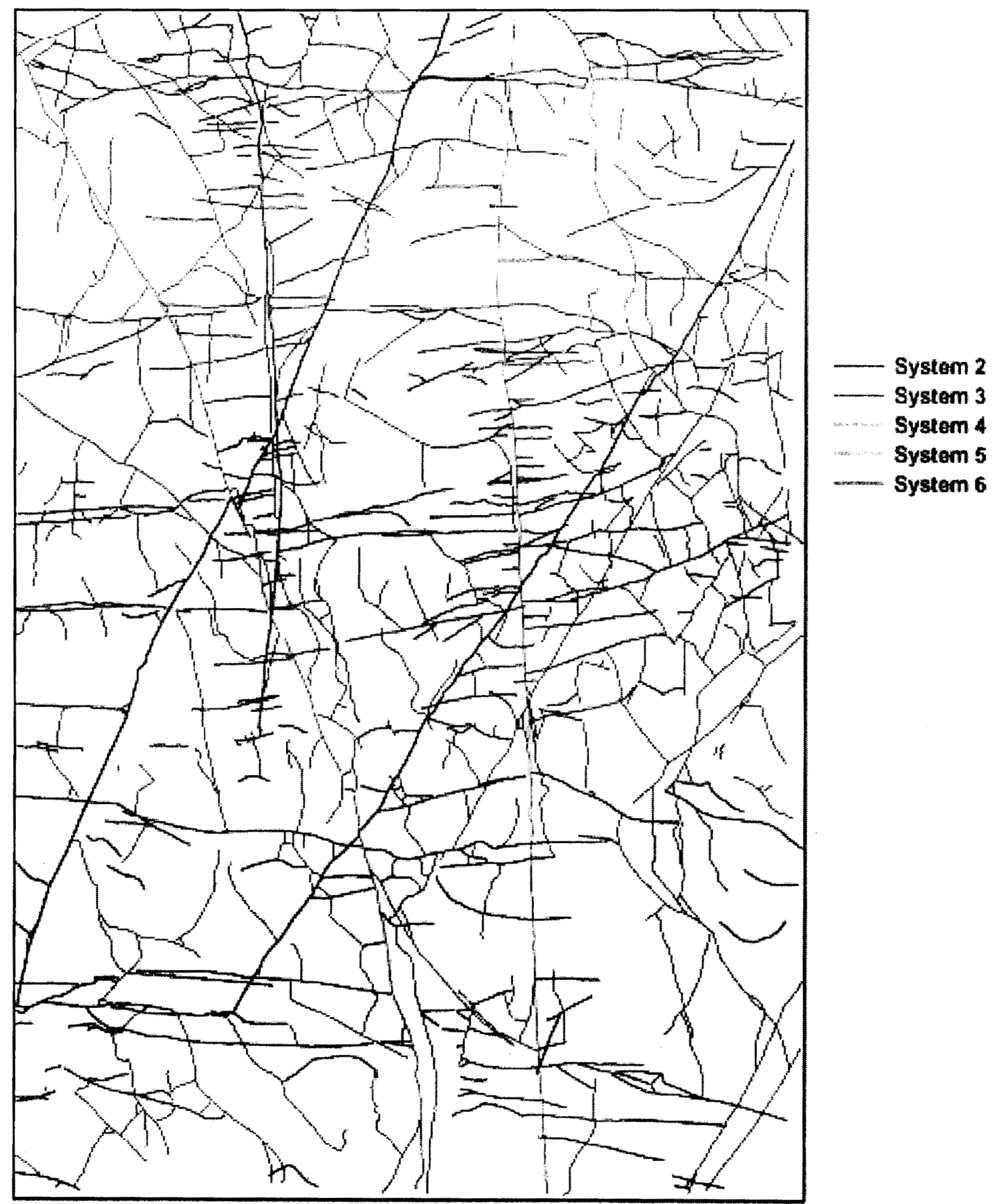

Figure 4-15. Mapped Fractures on the West Face of the LBT 


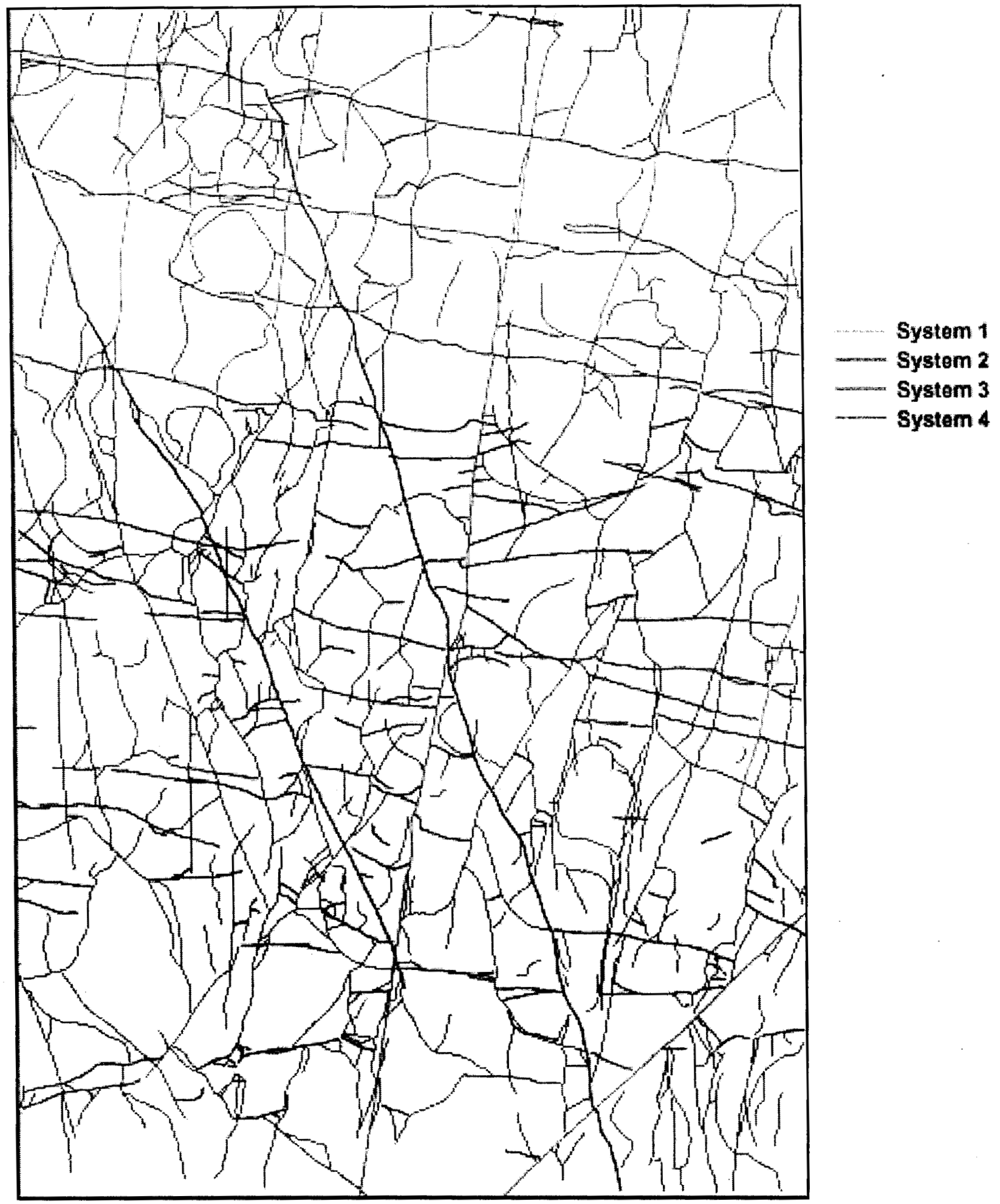

Figure 4-16. Mapped Fractures on the South Face of the LBT 
IIII

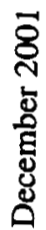

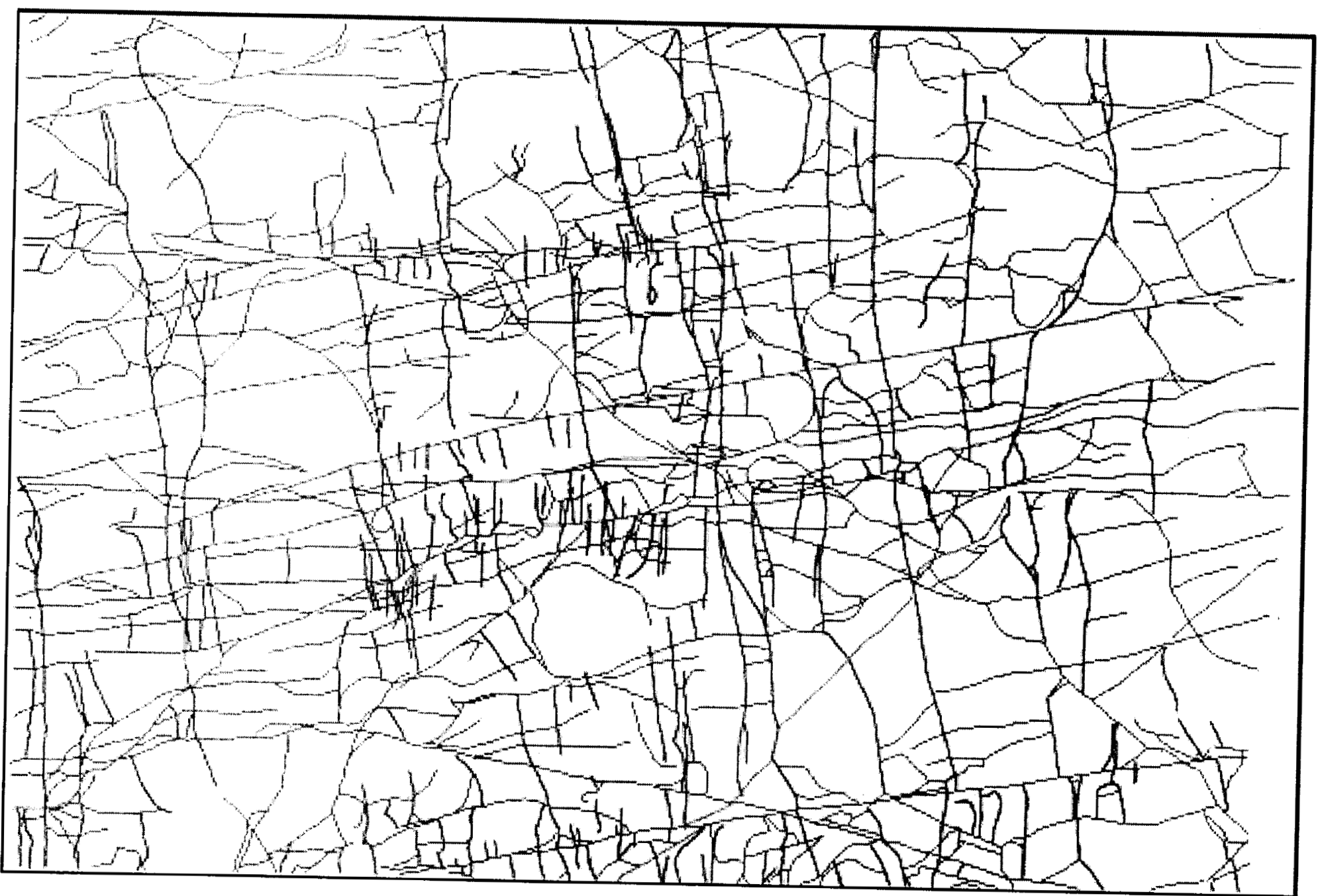

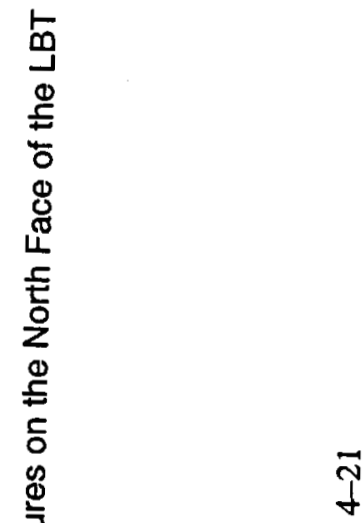




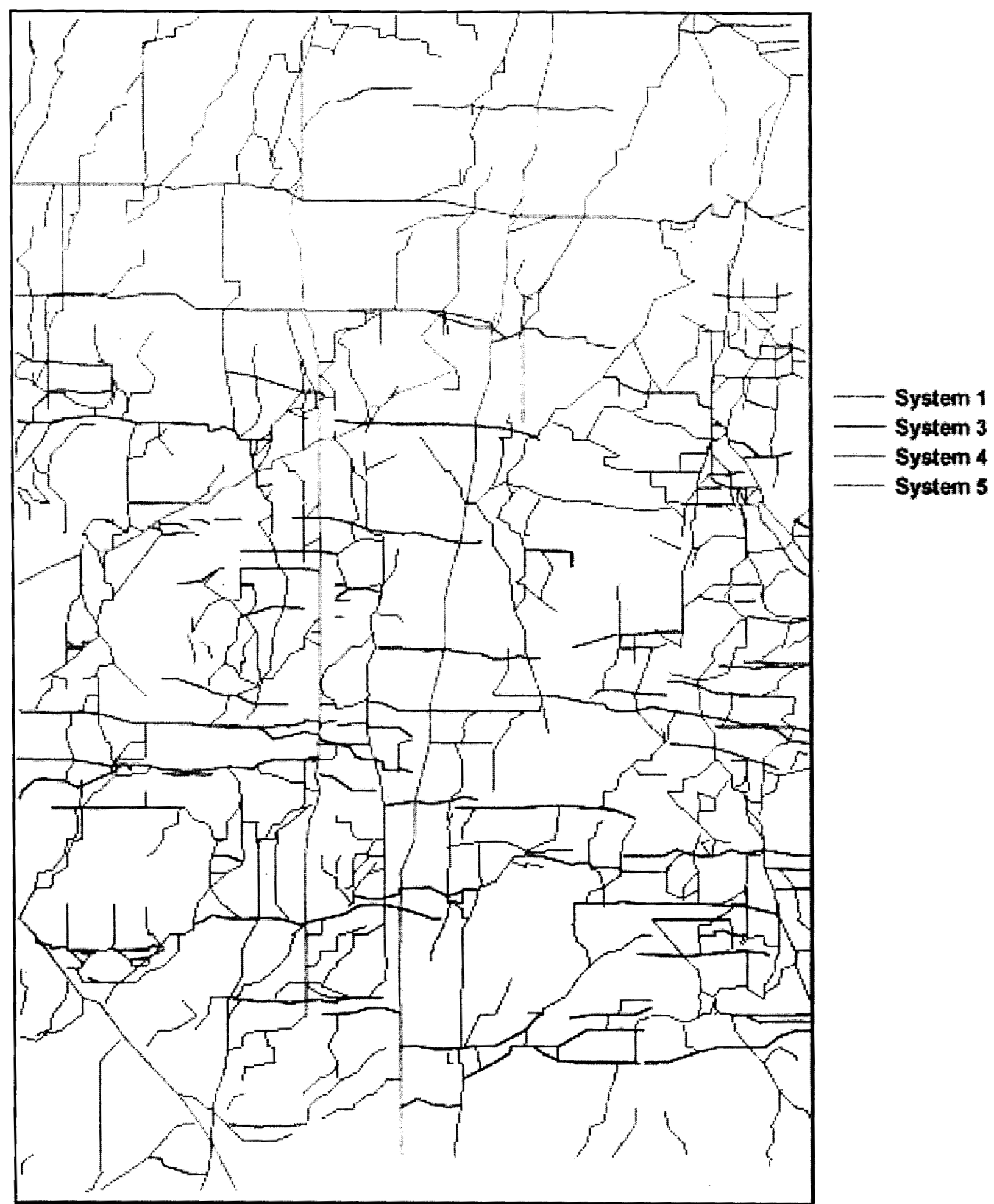

Figure 4-18. Mapped Fractures on the East Face of the LBT 


\section{THERMAL MEASUREMENTS}

To impose and maintain a one-dimensional thermal gradient within the block, the block was outfitted with heaters in five boreholes in a horizontal plane and with a heat exchange unit on top. The heaters were $450 \mathrm{~W}$ each and were installed in each of the five horizontal heater holes. Heater temperature was monitored using three RTDs mounted to each heater.

\subsection{LOCATION OF INSTRUMENTS}

The instrumentation of the LBT is discussed in Section 3.3. The boreholes for the temperature measurement in the block are shown in Figures 3-7, 3-8, and 3-10.

\subsection{BASELINE DATA COLLECTION AND HEATER TURN-ON}

After all instrumentation was installed in the block and the moisture barrier and insulation were installed outside of the block, final characterization was completed with the cross-hole permeability and tracer tests described earlier. When the final characterization was completed, heaters and packers were installed (heater holes and hydrology holes had been used in the permeability and tracer tests), and baseline monitoring was initiated. Two weeks of baseline data were collected. Some instrumentation was able to take baseline data earlier, and nearly a month of baseline temperature data were collected. The heaters were then turned on February 28, 1997.

\subsection{THE POWER OUTPUT FROM THE HEATERS}

The heater in each of the five heater holes (EH1 to EH5 in Figure 3-9) was energized to $450 \mathrm{~W}$ on February 28, 1997. The power output of those heaters as a function of time is shown in Figures 5-1 to 5-5. The spikes in those figures were due to short-duration power outages. Those power outages did not affect the test significantly. The data gaps at 133 to 136 days and at 280 to 283 days were due to malfunction of the data acquisition unit. The power outputs of those heaters were about the same. The power was maintained fairly constantly with respect to time, at about $450 \mathrm{~W}$ until about Day 222, when the power was reduced in order to reach a steady-state temperature. The temperature at TT1-14 was maintained fairly constantly at about $135^{\circ} \mathrm{C}$ (see Figure 5-6) for the remainder of the test. During the later part of the test the power had to be increased back to almost the $450-\mathrm{W}$ level in order to maintain a steady-state temperature. This was probably due to a cooler ambient temperature at that time.

\subsection{TEMPERATURE OBSERVATIONS}

Temperature in the block was measured using RTDs both in boreholes and on the surfaces of the block. As presented in Section 3.3, the borehole temperature measurements were conducted in 14 holes: two vertical holes (TT1 and TT2), seven horizontal holes (NT1, NT2, NT3, NT4, WT1, WT2, and WT3), and the five heater holes (EH1 to EH5). The temperature measurements on the top of the block were to verify that the heat exchanger controlled the top temperature at about $60^{\circ} \mathrm{C}$ during the test. The temperature measurements on the four vertical faces were for determining the thermal gradient on the surfaces, so that the heat flux away from the block could be determined. For the discussion of the temperatures within the block during the test, only the temperature measured in the boreholes will be used. The temperature measured on the block surface is discussed in Section 5.4.4. The two vertical RTD holes and the seven horizontal RTD 
holes were sealed with grout during the test. The five heater holes were not grouted but were plugged at the collar using insulation material. In this report, only the temperature measured in the nine sealed RTD holes are presented in figures to illustrate the thermal responses of the block due to heating. As mentioned in Section 3.3, the RTDs in those holes were separated by a spacing of $20 \mathrm{~cm}$. The RTD numbering was always starting from the bottom of a hole. For example, TT1-1 is the RTD at the bottom of the vertical RTD hole TT1, and NT1-14 is the RTD near the collar of the horizontal RTD hole NT1, which was drilled from the north face of the block and ended at a distance of about $30 \mathrm{~cm}$ from the south face of the block. All of the temperature data are available in Microsoft Excel spreadsheet form in the Technical Data Management System (TDMS) of the Yucca Mountain Site Characterization Project (YMP) and in the LLNL Large Block Test database. The DTNs for the data in the TDMS are listed in Table 1-1. The five heaters of the LBT were energized at about 10 a.m. on February 28, 1997, to a power level of about $450 \mathrm{~W}$ each. The preheat ambient temperature in the block was collected about 18 hours before the heaters were turned on. The data collection frequency was once per hour. The data acquisition frequency was increased to once per 10 minutes on June 30, 1997, in order to have a more accurate monitoring of the temperature fluctuations, which started on June 12,1997 , because of a TH event. The TH event is discussed in greater detail later in this section.

\subsubsection{The Temperature History in the Block}

The temperature in the two vertical RTD holes will be used to illustrate the temperature history in the block during the test. Figures 5-6 and 5-7 show the temperature history in holes TT1-14 and TT2-14 respectively. TT1-14 and TT2-14 are at 5 and $10 \mathrm{~cm}$ below the heater plane respectively. The location of holes TT1 and TT2 can be found in Figure 5-1. The temperatures at TT1-14 are about $10^{\circ} \mathrm{C}$ greater than those at TT2-14. This is mainly because TT1-14 is about $5 \mathrm{~cm}$ closer to the heater plane than TT2-14. All of the sharp drops in temperature that occurred before 100 days since heating are related to power outages. The short straight-line segment at about Day 135 was due to a temporary malfunction of the data acquisition system. TT1-14 represents the highest measured temperature in the rock of the LBT.

As shown in Figure 5-6, the temperature at TT1-14 increased with time rapidly at the early stage of the heating. The temperature increased with time mainly due to the conduction of heat from the heaters. The increasing rate of the temperature decreased with time, mainly due to the decrease of the thermal gradient at the RTD location as the thermal front expanded with time. A minor factor that also contributed to the decrease of the temperature rate is the consumption of energy in the evaporation of the in-situ pore water in the rock. When the temperature reached the boiling point of water, which is about $96.6^{\circ} \mathrm{C}$ at the elevation of Fran Ridge, the temperature rate of increase was significantly decreased. This was caused by rapid evaporation of the pore water in the rock. During the 20-day period between Day 30 and Day 50, the temperature at TT1-14 increased from about $96-98^{\circ} \mathrm{C}$, indicating a very rapid evaporation but not boiling. After Day 50, the temperature at TT1-14 increased faster with time, indicating that most of the pore water had been evaporated. Then at Day 104 (June 12,1997) the temperature dropped to near the boiling point of water. This is the on-set of the first of the two TH events, which are discussed below. The second TH event occurred at Day 186 (September 2, 1997). On Day 220 (October 6, 1997) the heater power started to ramp down, in order to keep the TT1-14 temperature at about $135-140^{\circ} \mathrm{C}$. The heaters were turned off on March 10,1998 , to start a natural cooling phase. The data acquisition was terminated on September 30, 1998. 
Figure 5-7 shows a very similar temperature history at TT2-14 to that of TT1-14, except for the apparent boiling of the pore water. The temperature at TT2-14 remained at $97.4^{\circ} \mathrm{C}$ for about 37 days (Day 75 to Day 112). Then the temperature increased to, and remained at, about $98.8^{\circ} \mathrm{C}$ for 16 days. This indicates that a process very close to boiling of the pore water was maintained at TT2-14. If boiling had actually occurred, the boiling point of the pore water is greater than the expected boiling point of pure water at the elevation of Fran Ridge $\left(96.6^{\circ} \mathrm{C}\right)$. The greater boiling point of the pore water may be caused by the dissolved solutes in the water, and the increased pore pressure caused by the increased vapor pressure before the boiling. The increase of the boiling point of water during the boiling process (from 97.4 to $98.8^{\circ} \mathrm{C}$ ) may be caused by the increased concentration of the solutes. In addition to boiling, another factor that may contribute to the constant temperature is a cooling effect by continuous flow of fluid to balance the energy. The mechanisms of maintaining such a continuous flow remain to be determined. Because the temperature at TT2-14 was at the boiling point of water when the first TH event occurred, the temperature at TT2-14 was not affected by that event. The rest of the temperature history at TT2-14 was very similar to that at TT1-14.

\subsubsection{The Spatial Temperature Distribution in the Block}

As mentioned in Section 3.1.1, the LBT was designed to perform one-dimensional heating of the block. Figures 5-8 and 5-9 show the temperature distribution along the two vertical RTD holes TT1 and TT2 respectively. To avoid cluttering the figure, only quarterly (since the heating) temperature snapshots are presented. As shown, the temperature was the highest at the heater plane and decreased with respect to distance away from the heater in both directions. The spatial temperature distribution in the vertical direction agrees well with the pre-test predictions, such as those shown in Figure 3-26.

Figures 5-10 through 5-16 show the spatial temperature variation in the seven horizontal RTD holes: NT1, NT2, NT3, NT4, WT1, WT2, and WT3 respectively. They show the horizontal spatial temperature variation in the block. The data gaps in Figures 5-11 and 5-16 are due to malfunction of RTDs NT2-9, NT2-13, and WT6. Generally speaking, the horizontal temperatures are fairly uniform in most parts of the block. The heating of the block was very close to one-dimensional.

All of the temperature data were input to EarthVision v5.0 to construct a 3-D contour of the temperature field within the block at certain instances. Figure 5-17 shows one example of such a temperature field in the block on March 10, 1998, before the end of the heating phase. This figure shows the temperature contours in a vertical cross section along TT1. With the 3-D temperature field, temperatures along any cross section in the block can be illustrated.

\subsubsection{Temperature as an Indicator of Thermal-Hydrological Events}

As shown in Figures 5-6 and 5-7, the temperature in the LBT illustrated two TH events on June 13, 1997, (Day 105.2) and September 2, 1997 (Day 186.5). There was no evidence that the June 13, 1997, event was associated with rain. It is known for certain that the September 2, 1997, event was associated with heavy rain, which had started the night before the event. The heat exchanger, which controlled the temperature on top of the block at about $60^{\circ} \mathrm{C}$, was out of function for a few days starting on June 12, 1997 (Day 104.2). During the week from June 12, 1997 (Day 104.2) to 
June 19, 1997 (Day 111.4), the temperature of the block top varied from $59^{\circ} \mathrm{C}$ to about $42^{\circ} \mathrm{C}$, then back to about $59^{\circ} \mathrm{C}$. During the September 2, 1997, event the heat exchanger functioned normally. The common features of these two events included: the temperature at some RTDs decreased sharply; the temperature at some RTDs increased sharply; the temperature at those RTDs near the heater plane tended to converge to the boiling point of water; the temperatures stayed at the boiling point of water for a while then increased with time sequentially, apparently when the rock began to dry out; and during the drying process the temperatures fluctuated with time with high frequency. However, there are differences between these two events, as discussed in the following paragraphs.

Figures 5-18 through 5-21 show the temperature along TT1 as a function of time during the June 13, 1997, event, (Day 105.2). At the beginning of this event, the temperature in TT1 at RTDs TT1-8 to TT1-12 increased (as shown in Figure 5-18); the temperature at TT1-13 to TT1-16 decreased (as shown in Figure 5-19); the temperature at TT1-17 to TT1-20 increased (as shown in Figure 5-20); and the temperature at TT1-24 and TT1-25 increased and the temperature at TT1-26 to TT1-28 decreased (as shown in Figure 5-21). The temperature at TT1-26 to TT1-28 was probably influenced by the malfunction of the heat exchanger. The temperatures at the RTDs below TT1-7 were not affected by this event. The different responses of the RTDs in TT1 show that this event was not strongly influenced by an exterior cause, such as rain water. Figures 5-19 and 5-20 show the main feature of this event: that is, the temperatures converged to near the boiling point of water, stayed at the boiling point of water for at least 14 days, then increased one by one, starting with TT1-14, which was closest to the heater plane. The increase of temperature with time, after staying at the boiling point of water, was probably due to the drying of the rock. When the temperatures were increasing with time, they fluctuated with high frequency. The temperature fluctuations extended beyond the time frame shown in the figures. The temperature fluctuation seemed to follow a similar sequence as the drying, because the temperature fluctuation at RTDs TT1-18 and TT1-23 extended into early September 1997. It was verified that the temperature fluctuation was not due to noise from the data acquisition system. Switching TT1-19 to a standard resistor on site did the verification; after the switch, TT1-19 read the resistance correctly. The temperature fluctuation was probably due to the reflux of the steam/water along the RTD column.

The influence of the June 13, 1997, event on the temperature in TT2 was not great. At the onset of the event, there was almost no significant variation in the temperature in TT2. The temperature at several RTDs in TT2 (TT2-13 to TT2-20) associated with the fluctuation as in TT1, but with much less amplitude, until September 1997. One of the possible causes of this event is that some mechanical effect caused the release of the overheated pressurized water to flow quickly along the RTD column in both TT1 and TT2. The location of this release of the pressurized water is not known but was maybe somewhere above the heater plane. When the water was released, its temperature decreased quickly but was still near the boiling point of water. The water could flow both upward (due to pressure gradient) and downward (due to both the pressure gradient and the gravity). The quick flow of this boiling water caused the temperatures along the RTD column to change. The boiling/rapid evaporation of this water caused the temperature to stay at near the boiling point of water for an extended period. Then the steam/condensate refluxing caused the temperature fluctuations. 
Figures 5-22 through 5-25 show the temperatures in TT1 as a function of time during the September 2, 1997, event (Day 186.5). Figure 5-22 shows that at the onset of the event the temperature at TT1-11 decreased; there was a slight increase at TT1-9 and TT1-8; there were no changes in the temperature at the RTDs below TT1-7. Figures 5-23 through 5-25 show that at the onset of the event (Day 186.5), the temperature at all RTDs decreased then increased, except at TT1-19, where the temperature only showed the later increase. This feature was different from that in the June 13,1997, event. This is an indication that the September 2, 1997, event was strongly influenced by an exterior source, such as rain. The effect of this event on the temperature in TT2 is similar to that on TT1. Figure 5-22 shows the main feature of this event. The temperatures converged to near the boiling point of water, similar to the June 13, 1997, event, and stayed at that temperature for about two days only. This "boiling" period was much shorter than that of the June 13,1997, event. The temperatures then began to increase in the order of distance from the heater plane, again similar to the June 13, 1997, event. The temperatures fluctuated with time after the boiling period, also similar to the June 13, 1997, event. Despite the apparent difference in the cause of the event, the TH feature of this event is very similar to that of the June 13,1997, event. The shorter "boiling" period in this case was because the rock mass was dry when this event occurred, and the rain provided most of the water, which drove the event. Another reason for the quicker drying out was because more insulation was put on the block surface before the event; therefore, more heat was available for the drying. Figure 5-25 shows that steam coming from below was enough to maintain the temperature at TT1-24 to TT1-28 at the boiling point of water for a few days. When the steam was gone, the temperatures decreased one-by-one, starting from the top. Most of the temperature fluctuations stopped by September 17, 1997 (Day 201.4), but the temperature at TT1-23 fluctuated until October 20, 1997 (Day 234.4). The effect of this event on the temperature in TT2 is very similar, with smaller amplitudes of the temperature fluctuations. The process of this event was similar to that of the June 13, 1997 event, but the cause was the rainstorm in the night before.

\subsubsection{Heat Flux on the Block Surface}

As mentioned earlier, the surface temperature of the block was measured in zones. The four vertical faces of the block were divided into four zones ( 1 to 4 ) from bottom to top. The first three zones were $1.219 \mathrm{~m}$ in height. The fourth zone (near the top) was about $0.762 \mathrm{~m}$ in height. Each face within each zone was further divided into three regions: one central region and two corner regions. The central regions were designated as N1 to N4, W1 to W4, E1 to E4, and S1 to S4. For example, N1 to N4 were for the central region in the north face, for zones 1 to 4 respectively. The corner regions were designated as NW1 to NW4, NE1 to NE4, SW1 to SW4, and SE1 to SE4. For example, NW1 to NW4 represented the north-west corner for zones 1 to 4 respectively. Each corner region included a portion of both faces that formed the corner. For example, NW1 included a portion of the north face at the corner and a portion of the west face at the corner, in zone 1 . The central region of zones 1 to 3 in each face was about $2.438 \times 1.219 \mathrm{~m}$. The corner regions of zones 1 to 3 in each face were $0.305 \times 1.219 \mathrm{~m}$ each. In the zone 4 , the central region in each face was $2.438 \times 0.762 \mathrm{~m}$, and each of the corner regions was $0.305 \times$ $0.762 \mathrm{~m}$. Within each region, two RTDs were mounted on the both sides of Ultratemp insulation sheet, which was then mounted against the vertical face of the block. In the corner regions, only one pair of RTDs were mounted. The RTD pair might be mounted on either side of the corner. 
For example, the RTD pair in NW1 might be mounted either on the Ultratemp insulation sheet of the north face or the west face.

Those surface temperatures were used to obtain the temperature field within the block, along with the temperatures measured in boreholes discussed earlier. The surface temperatures were also used to calculate the heat flux from the block during the test. The thermal gradient normal to the block face in each region was calculated by dividing the temperature difference, measured by the RTD pair, by the thickness of the Ultratemp sheet. The heat flux was calculated by multiplying the thermal gradient with the thermal conductivity of the Ultratemp. The thermal conductivity of the Ultratemp was provided by the manufacturer as $0.093 \mathrm{~W} / \mathrm{m}-\mathrm{K}$. The heat flux data can be used in TH modeling to account for the energy balance.

Figures 5-26 through 5-29 show some examples of the heat flux from the block as a function of time, in zones 1 to 4 respectively, during the steady-state temperature period (February 8-25, 1998) of the test. It is expected that the heat flux was dependent on the temperature in the block.

Zone 2 included the heater plane and therefore had the greatest heat flux in all regions. The north-west corner had the greatest heat flux in both zones 1 and 2 . This was probably related to a greater fracture intensity near the north-west corner than in other places in the block. Besides NW2, S2 had greater heat flux than other regions in zone 2. (There was no good data for W2.) This was probably due to more fractures intersecting the south face than the north and east faces in this zone. In zones 3 and 4 the heat flux was about the same in all regions. The nonuniform heat flux may have to be considered in order to fine-tune a TH model.

\subsection{THERMAL CONDUCTIVITY AND THERMAL DIFFUSIVITY}

As presented in Section 3.4.1.2, the thermal conductivity and thermal diffusivity of the block were measured in situ by using the REKA thermal probe method. The REKA method incorporated the application of three small probes $(0.013 \mathrm{~m}$ in diameter and $0.125 \mathrm{~m}$ in length) that were cemented into the rock block. The probes were heated by built-in heaters of $4 \mathrm{~W}$ during the measurement period of 24 hours. When not activated, the probes were not heated.

Three permanent REKA probes were grouted into the large rock block at Fran Ridge. As shown in Figure 3-11, horizontal REKA Probe 1 was installed $0.584 \mathrm{~m}$ below the rock block's heater plane; horizontal REKA Probe 2 was installed $0.889 \mathrm{~m}$ above heater plane; and the vertical REKA Probe 3 was installed with its representative sensor location $1.38 \mathrm{~m}$ above the heater plane. Each probe reading approximately represents a $0.1-\mathrm{m}$-diameter spherical rock volume.

The measured temperature fields were evaluated using the REKA 01 software that was qualified in 1998 in accordance with the LLNL quality assurance (QA) program. The effective conductivity and diffusivity results (Danko et al. 1998) are shown in Figures 5-30 to 5-32 for the three REKA probes. Day 0 in Figures 5-30 to 5-32 denotes the time of the start of the heating of the block. As shown in Figures 5-30 to 5-32, both $\mathrm{k}$ and alpha values are varying only slightly with time. There is an apparent correlation between the variations of the in-situ, independently obtained conductivity and diffusivity values. This correlation indicates that the specific heat and the density $(\rho)$ are approximately constant with time in the rock, since the equation 


$$
\rho \times c=k / a l p h a
$$

has to be satisfied. Therefore, the variations can be attributed to the change in the convective effects with time, caused by moisture and/or vapor migration. The assumption that the conductivity and diffusivity values are affected by the heat-driven convective effects seems to be supported by the apparent correlation between the $\mathrm{k}$, alpha, and rock temperature values, shown in Figures 5-30 through 5-32.

In summary, the results of the in-situ REKA probe measurements agree very well with the expected values at Fran Ridge (Nimick 1990). The REKA method is sensitive enough to detect convective effects. More advanced evaluations (Danko and Buscheck 1993) of the REKA measurements, using the NUFT hydrothermal model, are needed to quantify the TH effects upon the effective rockmass heat conductivity $(\mathrm{k})$ and thermal diffusivity (alpha).

\subsection{THERMAL-HYDROLOGICAL MODEL ANALYSES OF THE TEMPERATURE DATA}

The TH processes of the LBT were modeled using NUFT 3.0.1s (STN 10130-3.0.1s-00) using the implicit dual-permeability model and the active fracture concept, as reported in Section 6.2.4 of the Thermal Test TH AMR (CRWMS M\&O 2000c). The model analyses used two property sets: the drift-scale (DS) property set and mountain-scale (MS) property set, as shown in Table 5-1 (CRWMS M\&O 2000c, Table 7). Although the LBT was not designed to test various property sets of YM, it is interesting to see how well the property sets work. Statistical analyses of the goodness of fit between the measured and calculated temperatures in the block were reported in the AMR cited above. This section presents the comparison between the measured temperatures in the block and the calculated ones. The comparison of the measured moisture content and the model calculation are presented in Section 6.2.2.

\subsubsection{Numerical Model}

The model geometry, boundary and initial conditions, and heater power history are identical to those presented in Section 6.2.4 of the Thermal Test TH AMR (CRWMS M\&O 2000c) for the DS property set. The DTN for heater power histories used in the analysis is LL980918904244.074.

\subsubsection{Rock Property Data Sets}

Hydraulic and thermal properties of the tsw34 unit of the DS basecase property set (DTN LB990861233129.001) and the MS basecase property set (DTN LB997141233129.001) were used in model calculations. The tsw34 unit was used because the hydrogeologic unit of the LBT area is equivalent to the tsw34 model unit (Tptpmn) of the site-scale unsaturated zone (UZ) flow model. The only difference between the DS and MS properties for the tsw34 unit is a higher fracture permeability of $1.70 \times 10^{-11} \mathrm{~m}^{2}$ for the MS versus $2.76 \times 10^{-13} \mathrm{~m}^{2}$ for the DS. Properties of the LBT insulation for the simulations are also included in Table 5-1 (Table 7, Section 6.2.4 of the Thermal Test TH AMR [CRWMS M\&O 2000c]). 
Table 5-1. The DS and MS Rock Properties and the Thermal Properties of the Insulation Material Used in the TH Modeling of the LBT. (Source: Table 7 of CRWMS M\&O 2000c)

\begin{tabular}{|c|c|c|}
\hline Property & $\begin{array}{c}\text { DS }^{a} \text { Host rock (base case) and } \\
\text { insulation }\end{array}$ & $M^{b}$ host rock and insulation \\
\hline $\begin{array}{l}\text { Ultratemp thermal conductivity } \\
\left(\mathrm{W} / \mathrm{m}^{\circ} \mathrm{C}\right)\end{array}$ & $0.095^{\mathrm{c}}$ & $0.095^{\mathrm{d}}$ \\
\hline Ultratemp specific heat $\left(\mathrm{J} / \mathrm{kg}^{\circ} \mathrm{C}\right)$ & $1130^{c}$ & $1130^{d}$ \\
\hline $\begin{array}{l}\text { Insulator thermal conductivity } \\
\left(\mathrm{W} / \mathrm{m}^{\circ} \mathrm{C}\right) \text { (after } 125 \text { days) }\end{array}$ & $0.05^{\mathrm{c}}$ & $0.05^{\mathrm{d}}$ \\
\hline Matrix permeability $\left(\mathrm{m}^{2}\right)$ & $4.07 \times 10^{-18}$ & $4.07 \times 10^{-18}$ \\
\hline Fracture permeability $\left(\mathrm{m}^{2}\right)$ & $2.76 \times 10^{-13}$ & $1.70 \times 10^{-11}$ \\
\hline Matrix porosity & 0.11 & 0.11 \\
\hline Fracture porosity & $2.43 \times 10^{-4}$ & $2.43 \times 10^{-4}$ \\
\hline Matrix van Genuchten $\alpha \mathrm{m}(1 / \mathrm{Pa})$ & $3.86 \times 10^{-6}$ & $3.86 \times 10^{-6}$ \\
\hline Matrix van Genuchten $\beta \mathrm{m}$ & 0.291 & 0.291 \\
\hline Matrix residual saturation & 0.19 & 0.19 \\
\hline Fracture van Genuchten of (1/Pa) & $5.16 \times 10^{-4}$ & $5.16 \times 10^{-4}$ \\
\hline Fracture van Genuchten $\beta f$ & 0.608 & 0.608 \\
\hline Fracture residual saturation & $1.00 \times 10^{-2}$ & $1.00 \times 10^{-2}$ \\
\hline Initial liquid saturation & $75 \%^{\mathrm{c}}$ & $75 \%^{d}$ \\
\hline Dry thermal conductivity (W/m-K) & 1.56 & 1.56 \\
\hline Wet thermal conductivity $(\mathrm{W} / \mathrm{m}-\mathrm{K})$ & 2.33 & 2.33 \\
\hline Specific heat $(\mathrm{J} / \mathrm{kg}-\mathrm{K})$ & 948 & 948 \\
\hline Tortuosity & 0.7 & 0.7 \\
\hline $\begin{array}{l}\text { Gamma parameter for the active } \\
\text { fracture model }(\gamma)\end{array}$ & 0.41 & 0.41 \\
\hline Fracture frequency $\left(\mathrm{m}^{-1}\right)$ & 4.32 & 4.32 \\
\hline $\begin{array}{l}\text { Fracture to matrix interface area } \\
\left(\mathrm{m}^{2} / \mathrm{m}^{3}\right)\end{array}$ & $13.54^{\mathrm{C}}$ & 13.54 \\
\hline Grain density $\left(\mathrm{kg} / \mathrm{m}^{3}\right)$ & 2530 & 2530 \\
\hline
\end{tabular}

NOTE: ${ }^{a}$ DS (Drift-scale property set), DTN:LB990861233129.001

bMS (Mountain-scale property set), DTN:LB997141233129.001

'DTN:LL000321204242.092

¿DTN:LLO00314304242.094

\subsubsection{Drift-scale Simulation Results Versus Field Data}

Figure 5-33 shows the simulated versus measured temperature profile along Borehole TT1 at six times from 30 to 400 days. The DTN for all field temperatures data is LL970803004244.036. The model, with the DS property set, shows some overprediction of temperature at earlier times, but the difference between simulated and measured temperatures decreases at later times. At 300 and 400 days ( 25 days after power shutdown), the agreement is excellent.

Two statistical measures of goodness-of-fit, the root-mean-squared difference (RMSD) and mean difference (MD) between measured and simulated temperatures along Borehole TT1, were computed at various times from 30 days to 500 days. The results are tabulated in Table 5-2. As shown in the temperature profiles, the RMSD plot indicates a good fit between simulated and field temperatures. The match is good at 30 days, worsens at 100 days, and then consistently improves thereafter. The MD is always positive, which indicates that the DS data set, in general, 
overpredicts the temperatures. Again, the accuracy of the prediction consistently increases with time after 30 days, reducing to a mean error of less than $1^{\circ} \mathrm{C}$ at 500 days, 125 days into the cooldown phase.

Figure 5-34 shows comparisons of simulated and measured temperature histories at two sensor locations, TT1-14 and TT1-19, in TT1. The temperature history at TT1-14 (2.76 m below the top of the block) matches the field data fairly well in the time ranges 0 to 25 days and 225 to 375 days. The model, using the DS property set, overpredicts temperature in the time range 225 to 375 days. The simulated cool-down lags the measured cool-down by a few degrees. Model overprediction, especially between 25 and 225 days, may reflect that the rock properties do not adequately match the field conditions at the LBT. The overprediction might also be partially caused by a few unexplained events, where rainfall apparently percolated into the block causing cooling, or possibly by overestimating the effectiveness of the block wall insulation in the model.

Table 5-2. RMSD and MD for Temperature Profile Along TT1 Using Drift-scale Data Set

\begin{tabular}{|c|c|c|}
\hline Time (days) & RMSD $\left({ }^{\circ} \mathbf{C}\right)$ & MD $\left({ }^{\circ} \mathbf{C}\right)$ \\
\hline 30 & 7.25 & 6.90 \\
\hline 100 & 14.05 & 10.55 \\
\hline 200 & 10.56 & 4.34 \\
\hline 300 & 9.16 & 1.53 \\
\hline 400 & 5.82 & 3.53 \\
\hline 500 & 0.63 & 0.08 \\
\hline
\end{tabular}

The history for TT1-19 (1.76 m below the top of the block) is similar to that observed for TT1-14, except that the agreement with field data is somewhat improved. While the model predicts some superheating between 150 and 235 days, the field temperatures approach but never exceed boiling.

Figure 5-35 shows the comparison of the calculated temperature profiles along TT1, using the DS and the MS property sets, with the measured data. The MS property set overpredicts the temperature at the heater zone more than the DS property set. The degree of agreement with field temperatures between simulation results using the two property sets is examined by comparing the RMSD and MD for profiles along TT1 at various times from 30 days through 500 days. Histories of the RMSD and MD for the two property sets are tabulated in Table 5-3. As shown earlier in the temperature profiles, the DS data set consistently gives a lower RMSD than the MS data set. For both data sets, the match with field data is good at 30 days, worsens at 100 days, and then steadily improves thereafter. The MD is also generally smaller for the DS data set, suggesting better agreement with field data. Again, the accuracy of both predictions consistently increases with time, with a $\mathrm{MD}$ of less than $1^{\circ} \mathrm{C}$ at 500 days, 125 days into the cool-down phase. The TH modeling, as presented in Section 6.2.2.6, will shed some light on the temperature characteristics as shown in Figure 5-35. 
Table 5-3. RMSD and MD for Temperature Profile Along TT1 Using Drift-scale and Mountain-scale Property Sets

\begin{tabular}{|c|c|c|c|c|}
\hline \multirow{2}{*}{ Time (days) } & \multicolumn{2}{|c|}{ Drift-scale data } & \multicolumn{2}{c|}{ Mountain-scale data } \\
\cline { 2 - 5 } & RMSD $\left({ }^{\circ} \mathrm{C}\right)$ & MD $\left({ }^{\circ} \mathrm{C}\right)$ & RMSD $\left({ }^{\circ} \mathrm{C}\right)$ & MD $\left({ }^{\circ} \mathrm{C}\right)$ \\
\hline 30 & 7.25 & 6.90 & 7.37 & 7.02 \\
\hline 100 & 14.05 & 10.55 & 15.23 & 11.11 \\
\hline 200 & 10.56 & 4.34 & 12.60 & 6.05 \\
\hline 300 & 9.16 & 1.53 & 10.42 & 3.38 \\
\hline 400 & 5.82 & 3.53 & 6.42 & 4.27 \\
\hline 500 & 0.63 & 0.08 & 0.55 & -0.06 \\
\hline
\end{tabular}

\subsubsection{Summary}

Results of this comparative analysis to assess the performance of the model using the DS and MS data sets are summarized as follows:

- In general, the temperatures predicted by both property sets agree well with the measured data.

- Simulation results obtained using the DS data set show better agreement with the field temperatures than simulation results obtained using the MS data set.

- Both data sets generally overpredict temperature in and adjacent to the heater horizon, but the degree of overprediction is less for the DS data.

- The RMSD and MD for both data sets indicate that the agreement with field data generally increases as the test progresses.

- As mentioned earlier, the LBT was not designed to test rock properties, due to its size and the near-surface setting. Therefore, the conclusion inferred from these comparisons with property sets should be considered as for reference only. 


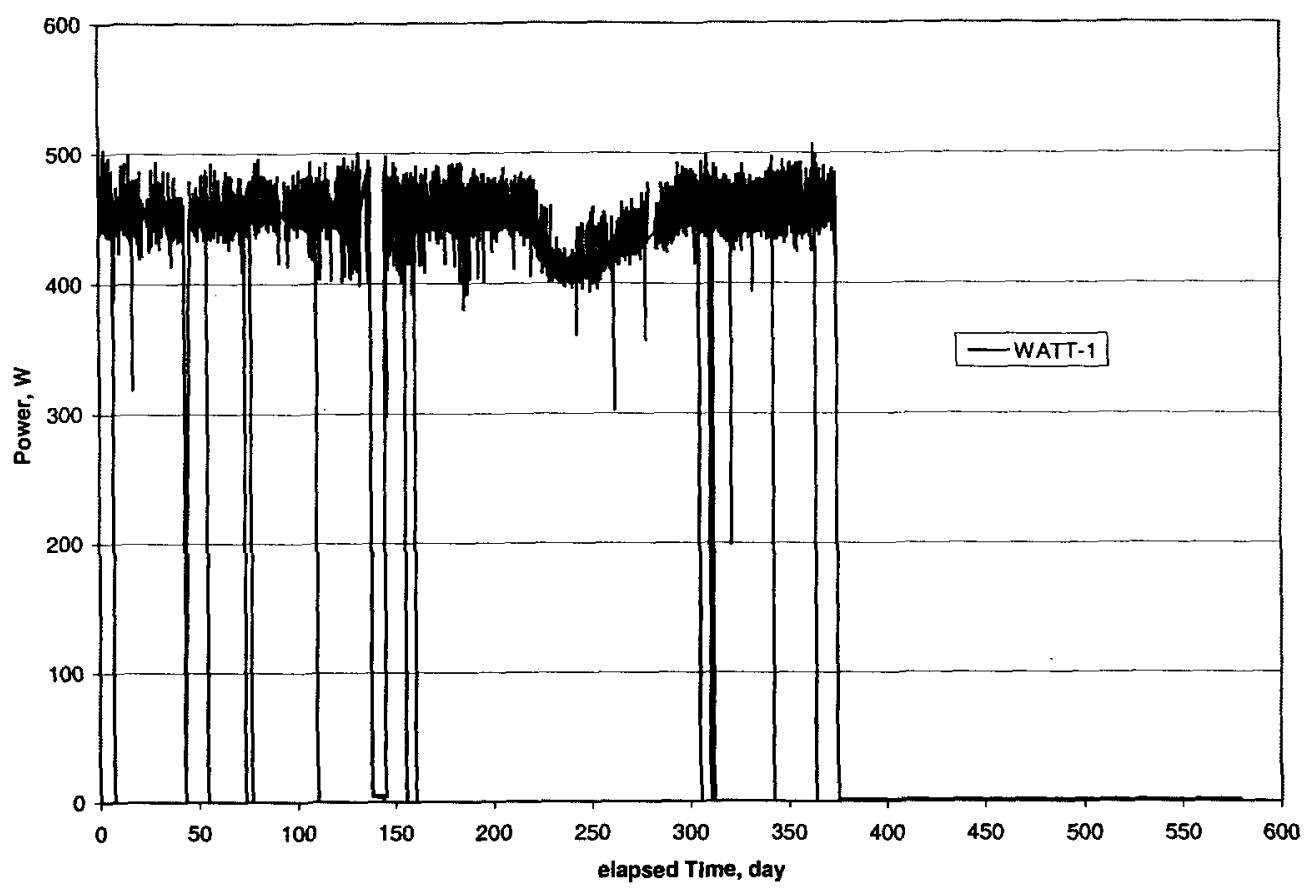

Figure 5-1. The Power Output of the Heater in EH1 as a Function of Time

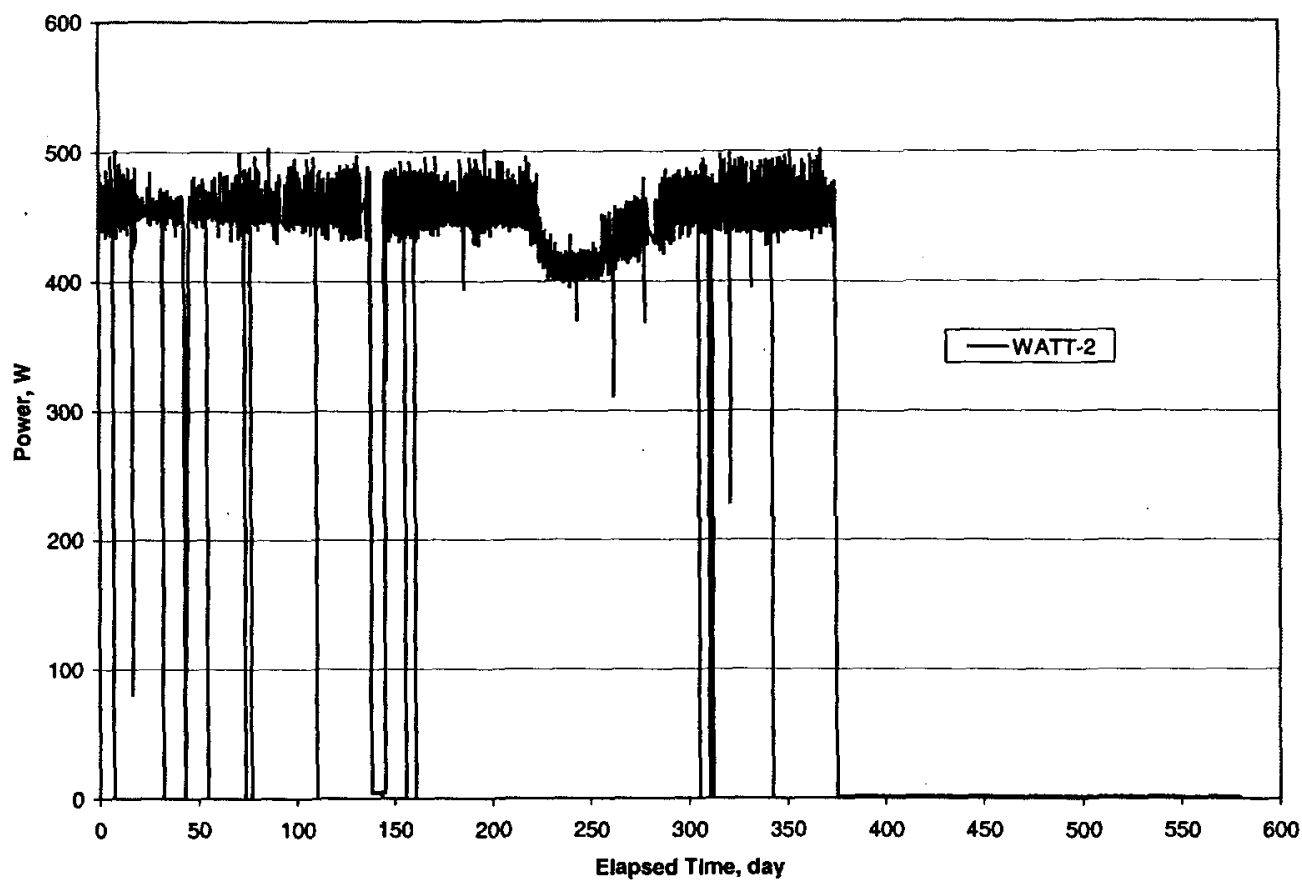

Figure 5-2. The Power Output of the Heater in EH2 as a Function of Time 


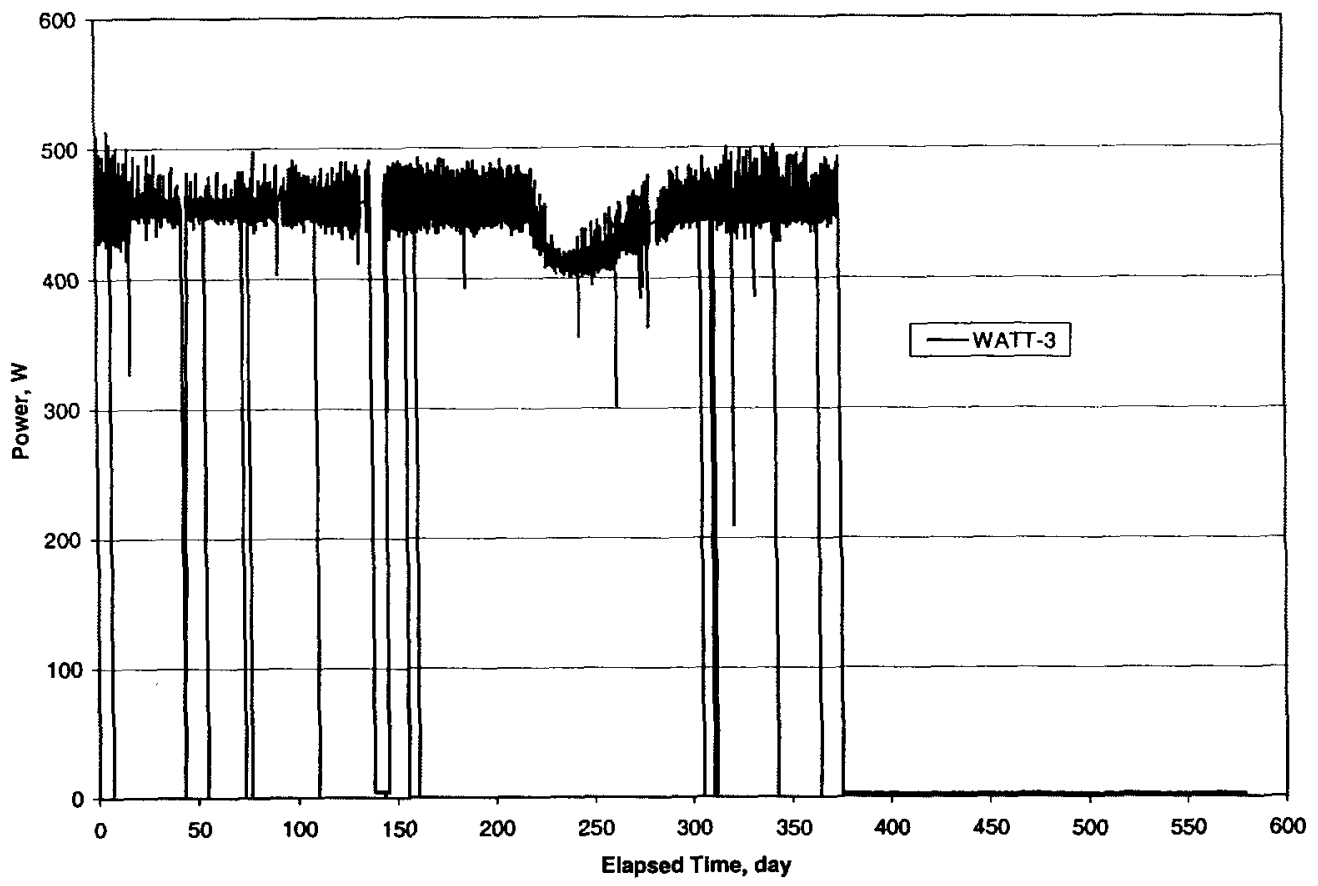

Figure 5-3. The Power Output of the Heater in EH3 as a Function of Time

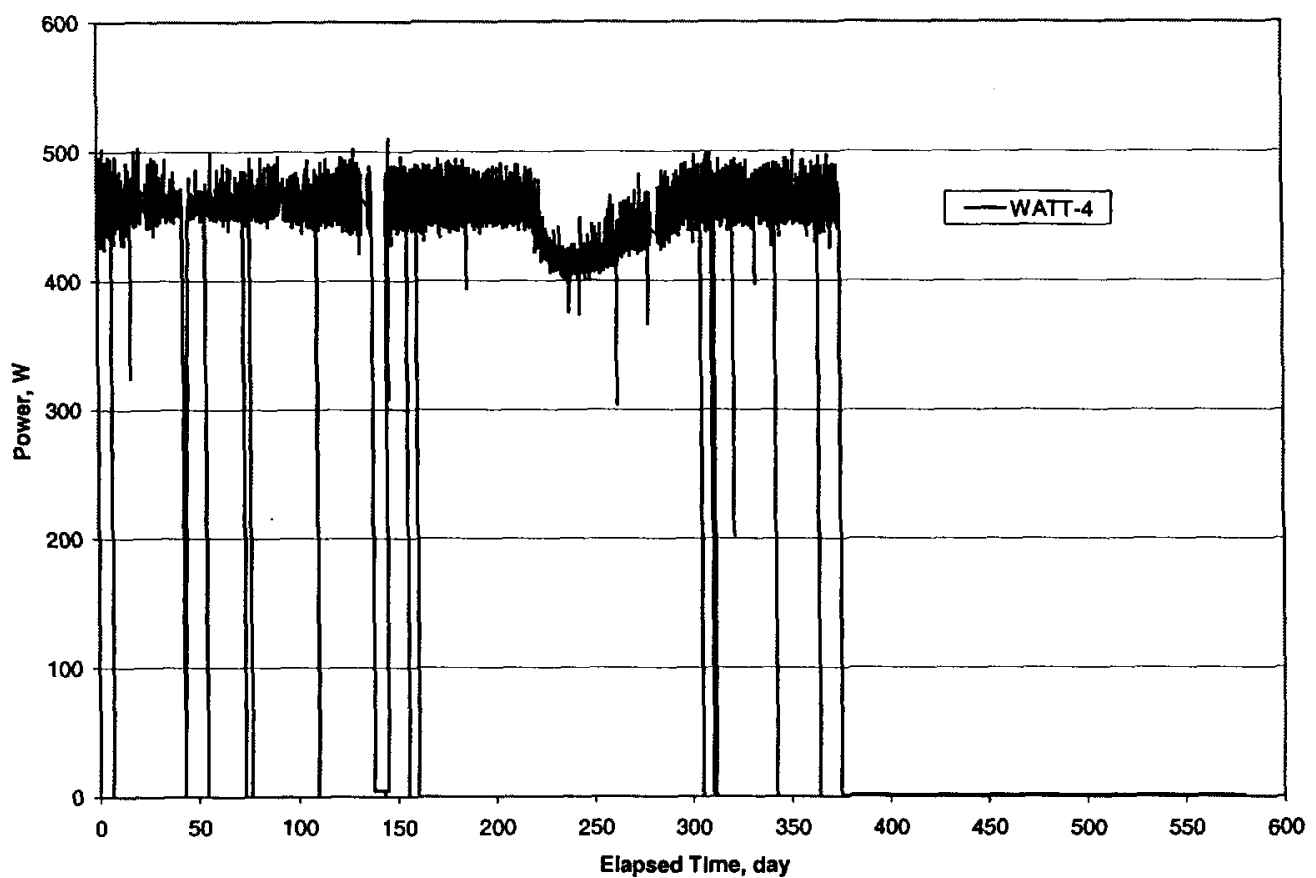

Figure 5-4. The Power Output of the Heater in EH4 as a Function of Time 


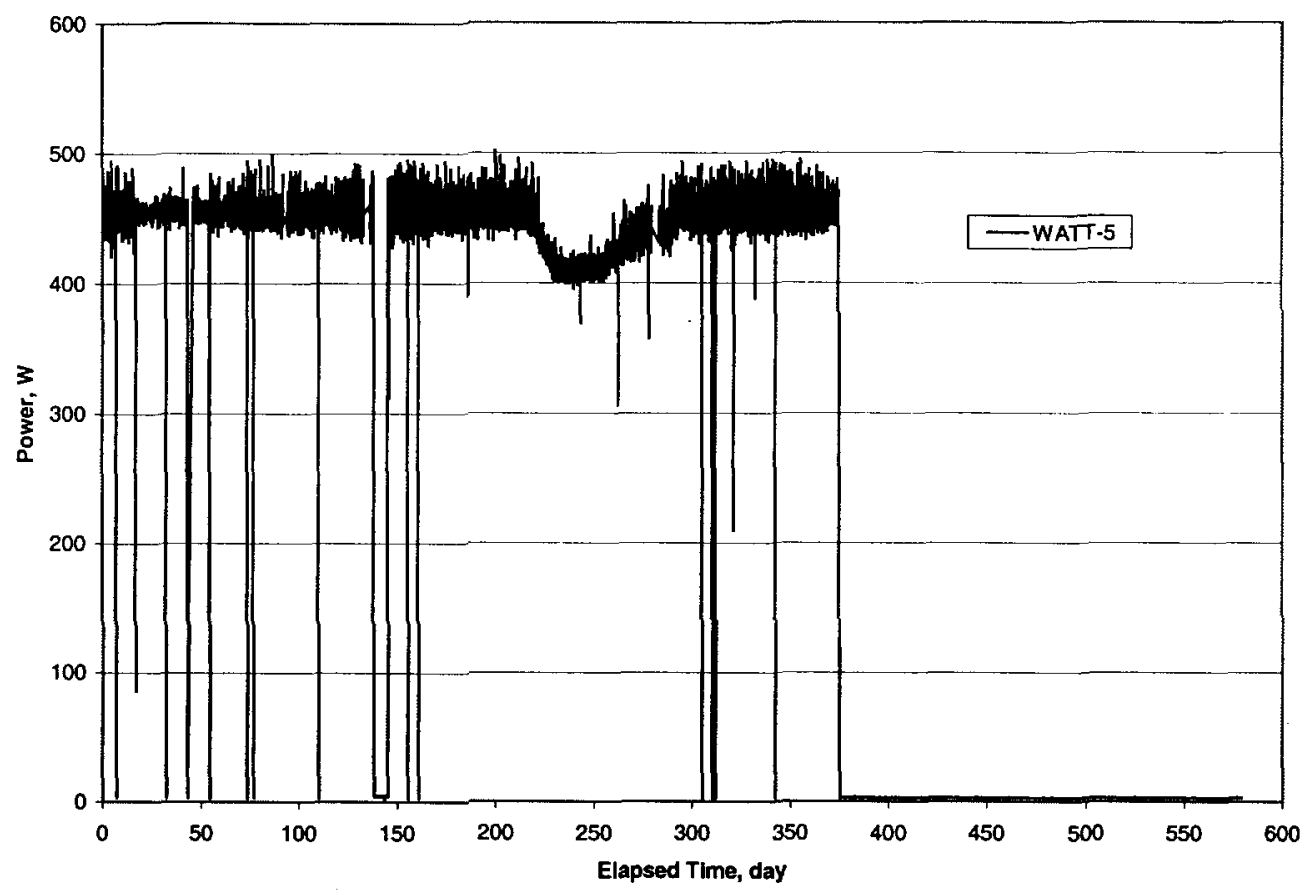

Figure 5-5. The Power Output of the Heater in EH5 as a Function of Time

TT1-14 of the Large Block Test.

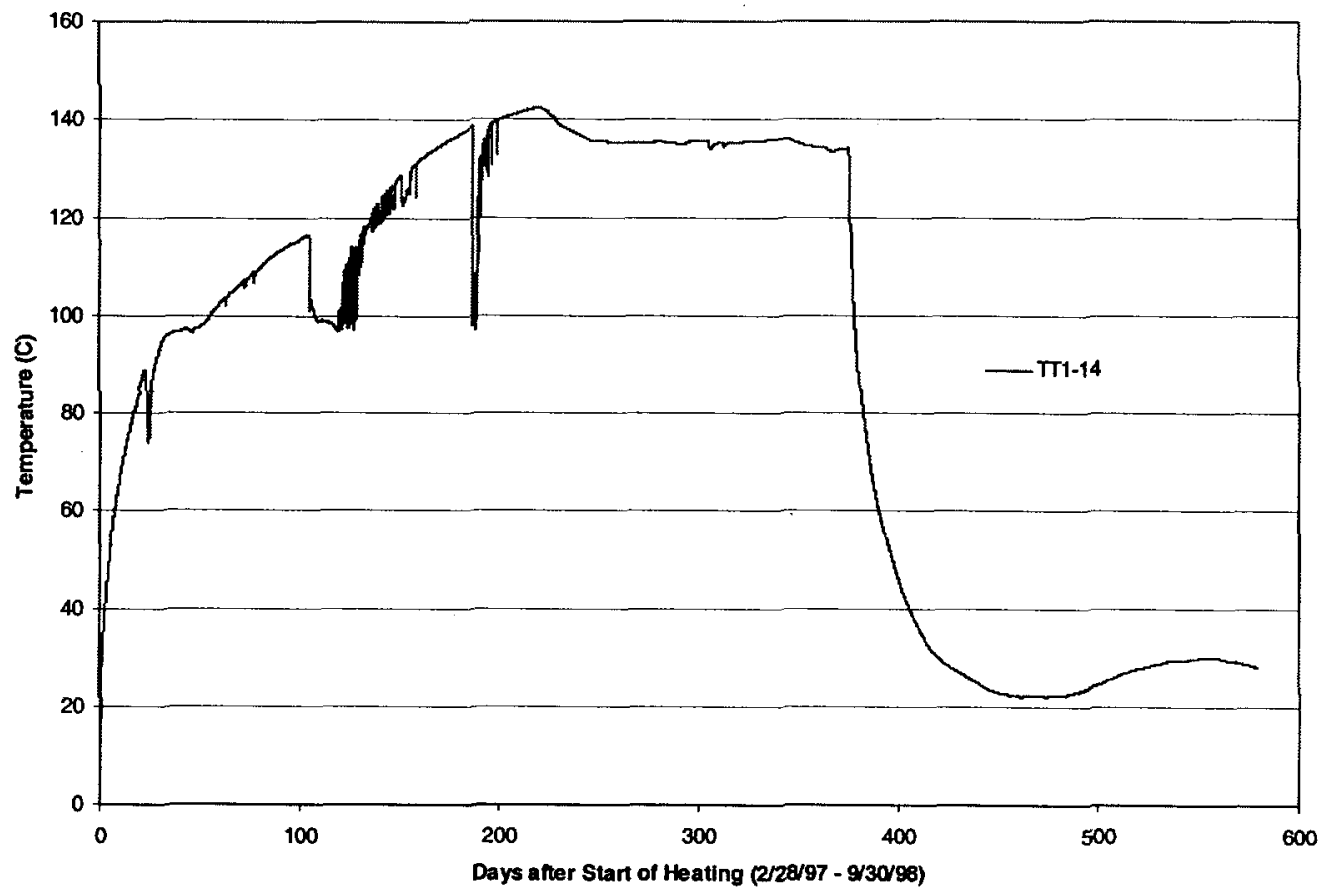

Figure 5-6. Temperature at $\mathrm{TT} 1-14$ as a Function of Time 
TT2-14 of the Large Block Test.

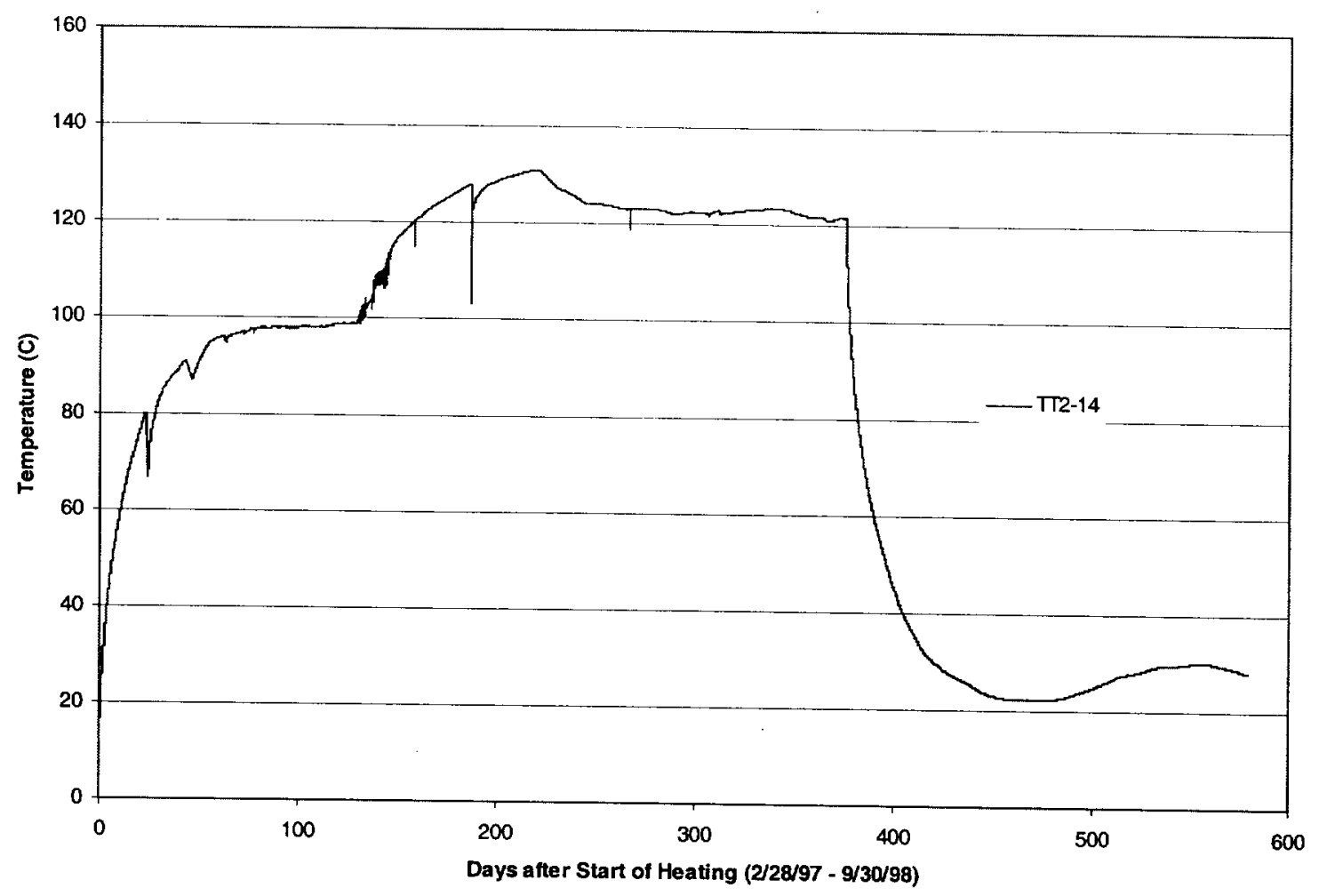

Figure 5-7. Temperature at $T \mathrm{~T} 2-14$ as a Function of Time 
Quarterly temperature snapshots in TT1.

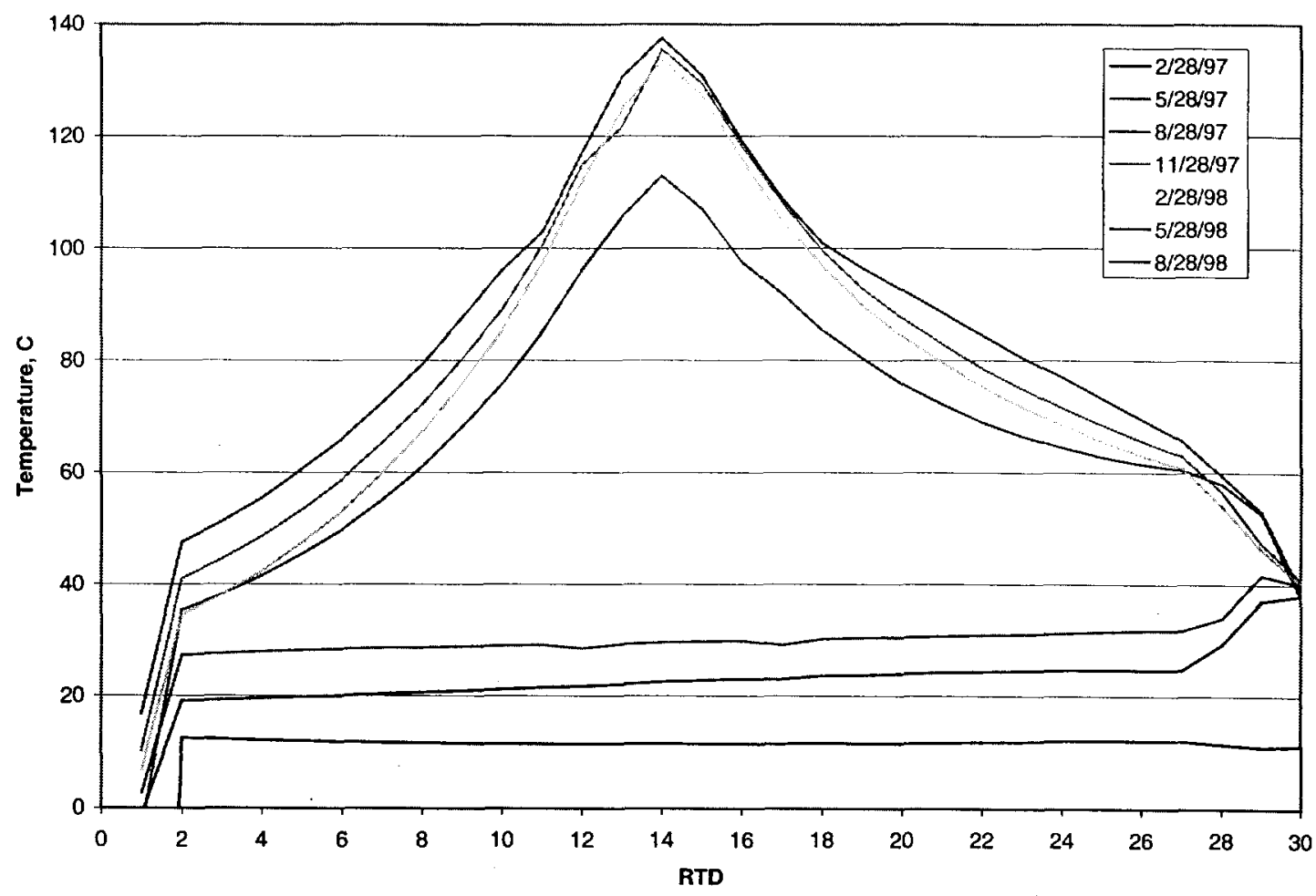

NOTE: RTD 1 is at the bottom of the hole.

Figure 5-8. Quarterly Temperature Snapshots in TT1 to Show the Spatial Temperature Variation 
Quarterly temperature snapshots in $\mathrm{TT2}$.

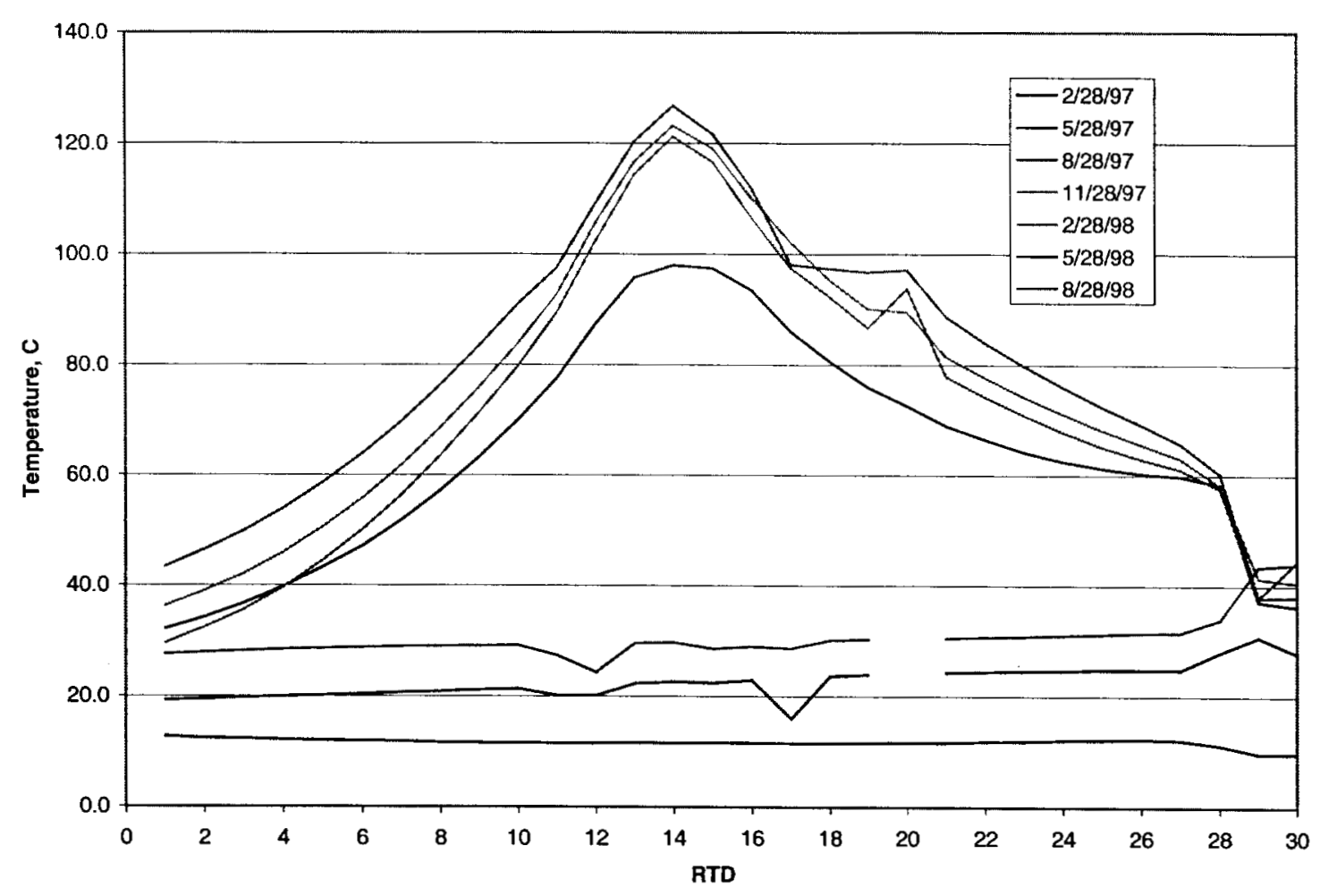

NOTE: RTD 1 is at the bottom of the hole.

Figure 5-9. Quarterly Temperature Snapshots in TT2 to Show the Spatial Temperature Variation 
Quarterly Temperature Snapshots in NT1.

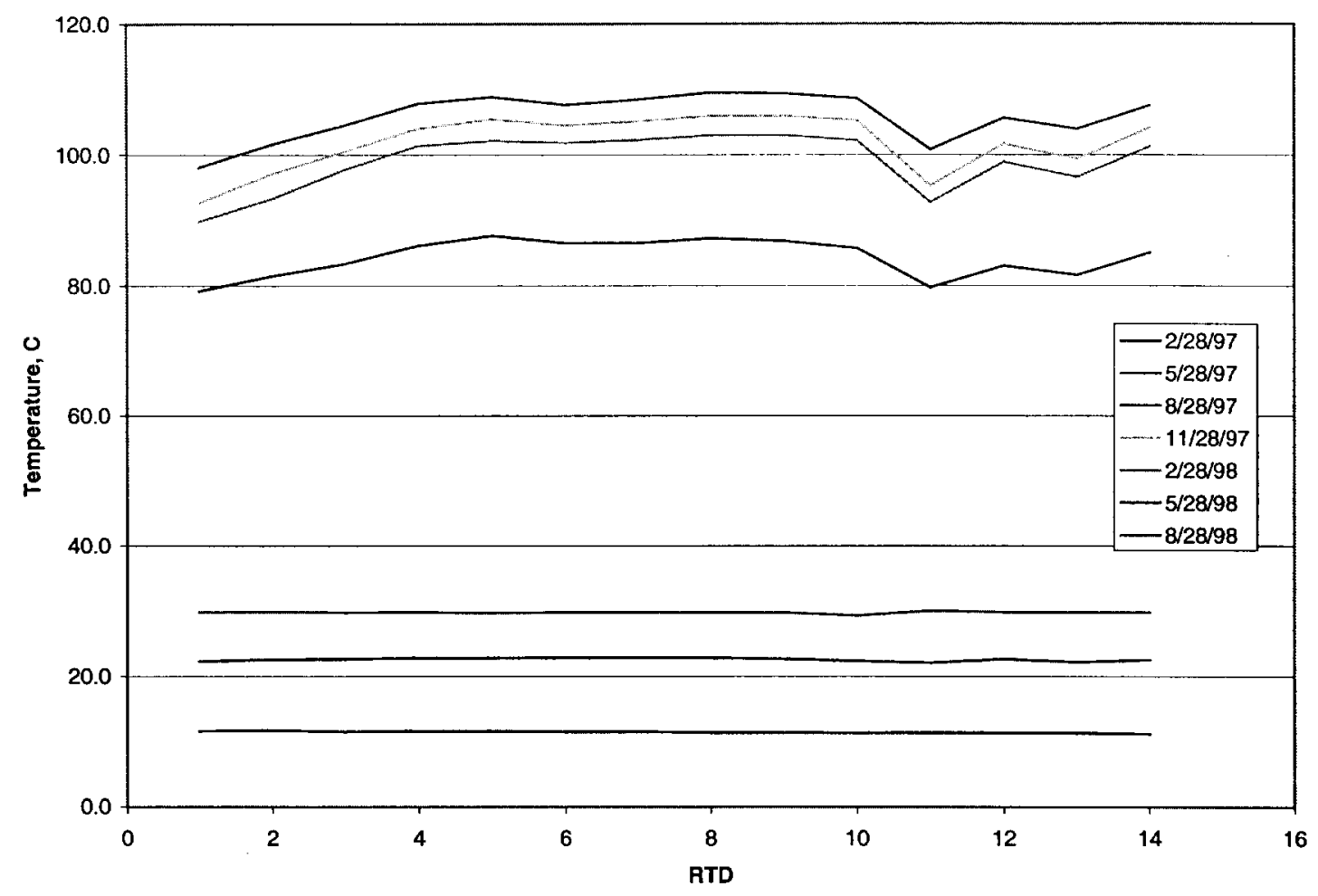

NOTE: RTD 1 is near the north face; RTD 14 is near the south face.

Figure 5-10. Quarterly Temperature Snapshots in NT1 to Show the Spatial Temperature Variation 
Quarterly Temperature Snapshots in NT2.

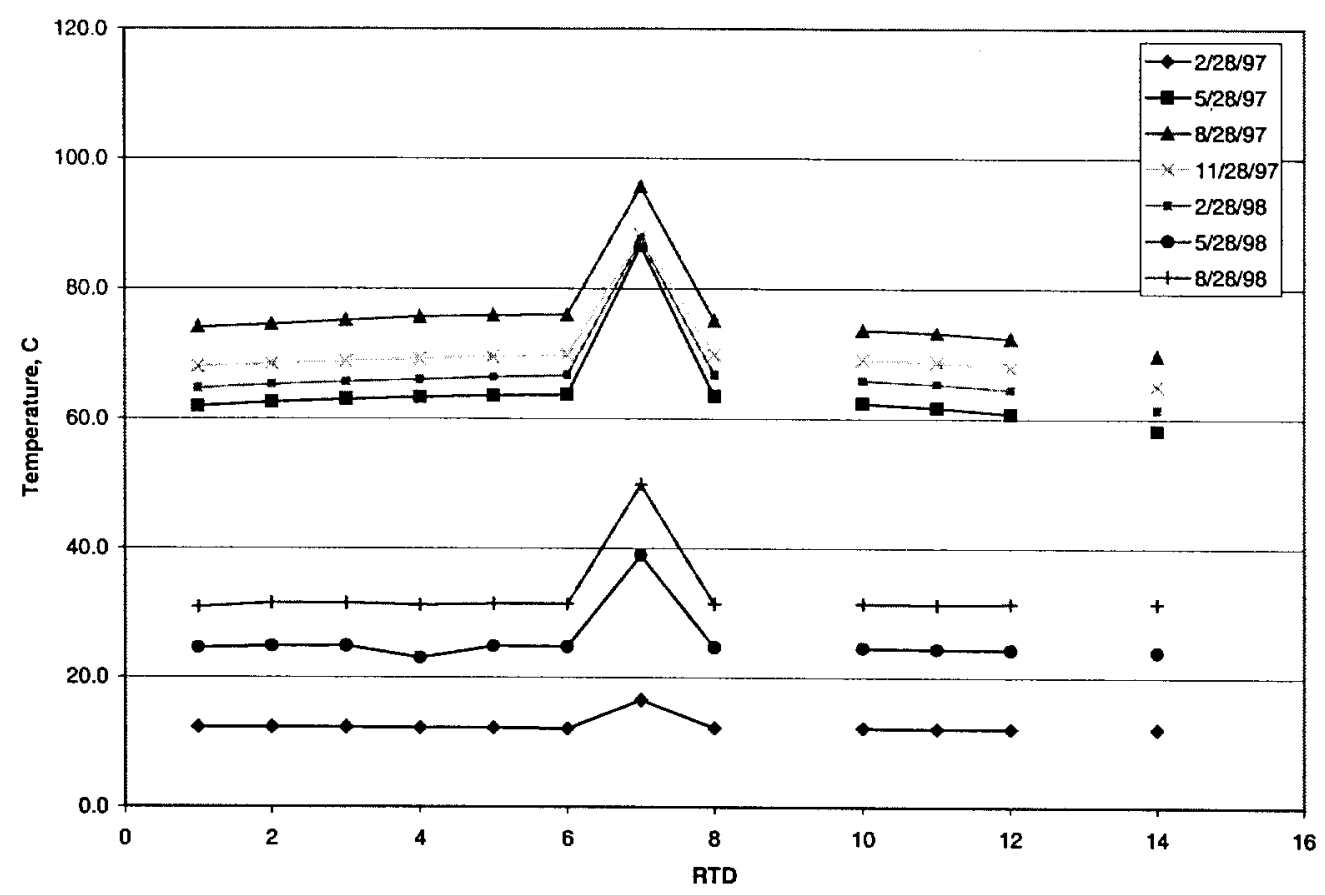

NOTE: RTD 1 is near the north face; RTD 14 is near the south face.

Figure 5-11. Quarterly Temperature Snapshots in NT2 
Quarterit Temperature Snapshots in NT3.

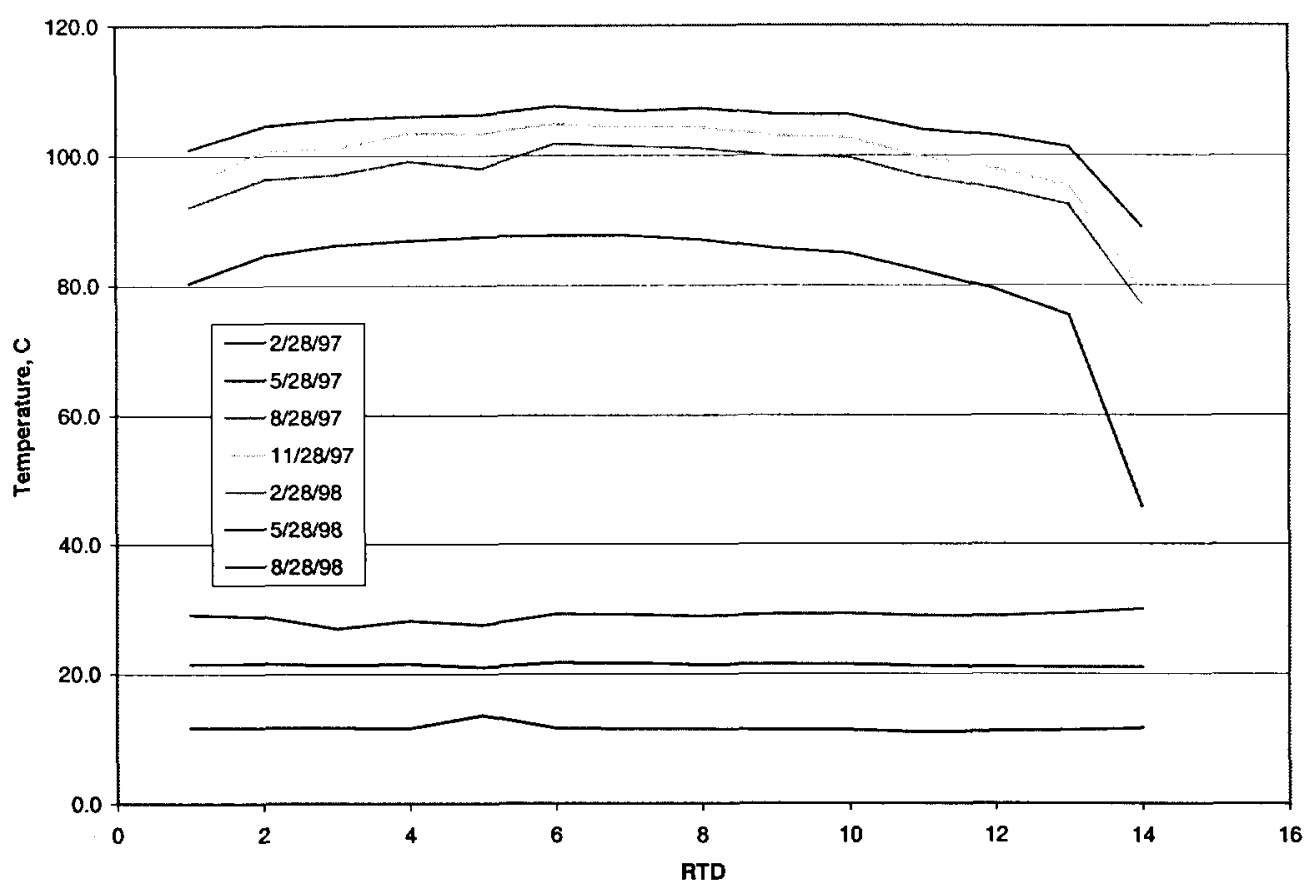

NOTE: RTD1 is near the north face; RTD 14 is near the south face.

Figure 5-12. Quarterly Temperature Snapshots in NT3 to Show the Spatial Temperature Variation 


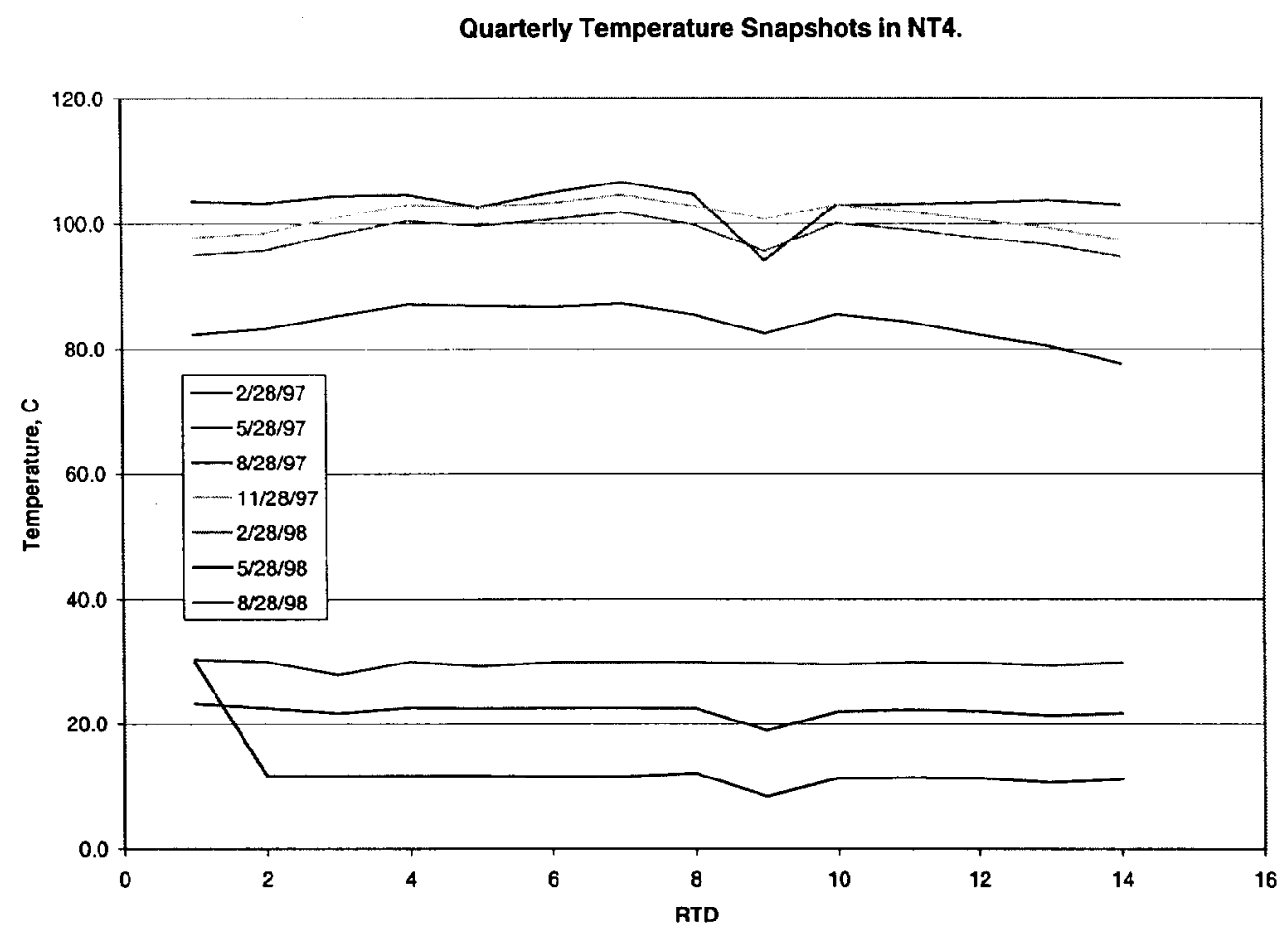

NOTE: RTD 1 is near the north face; RTD 14 is near the south face.

Figure 5-13. Quarterly Temperature Snapshots in NT4 to Show the Spatial Temperature Variation 
Quarterly Temperature Snapshots In WT1.

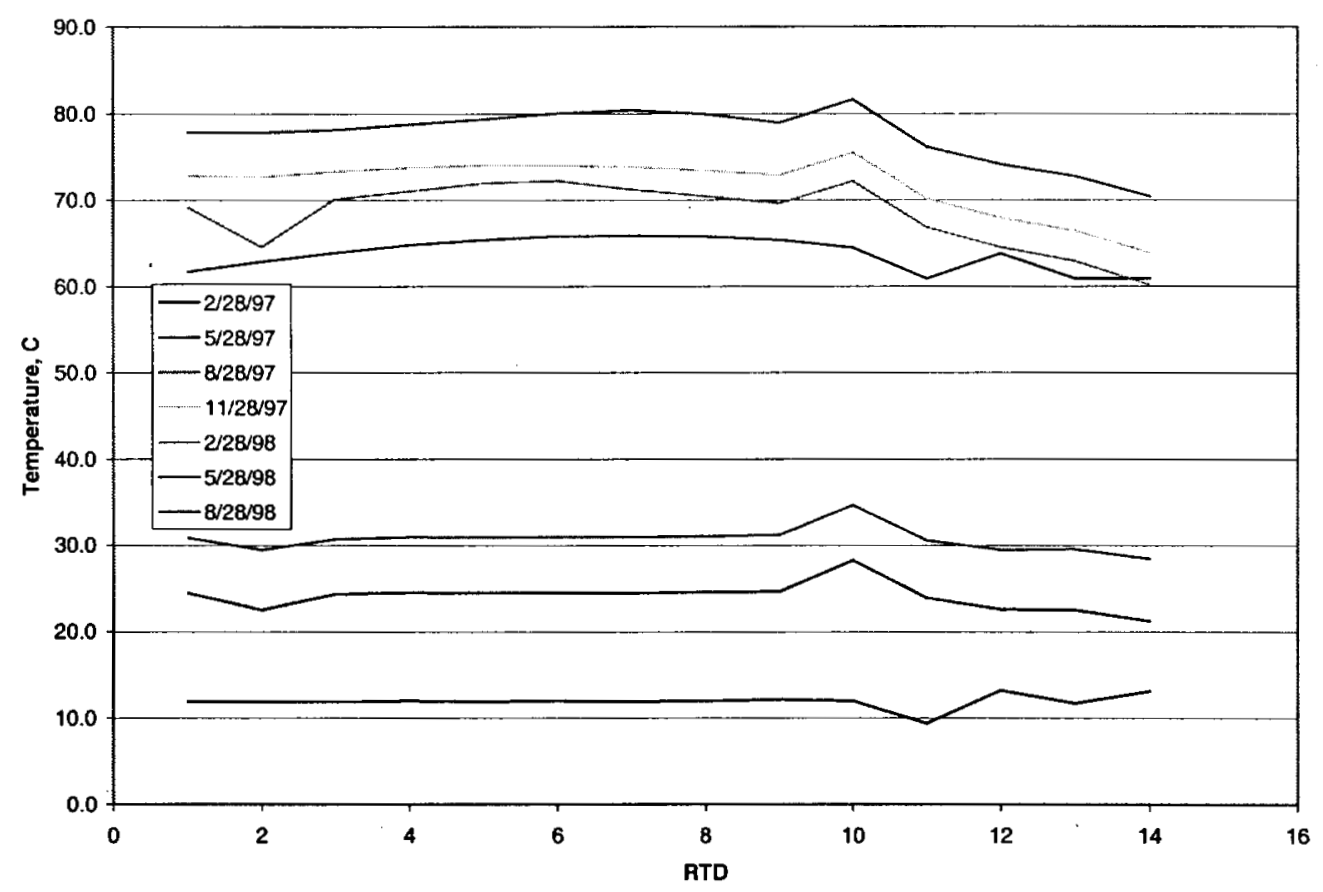

NOTE: RTD1 is near the west face; RTD 14 is near the east face.

Figure 5-14. Quarterly Temperature Snapshots in WT1 to Show the Spatial Temperature Variation 
Quarterly Temperature Snapshots in WT2.

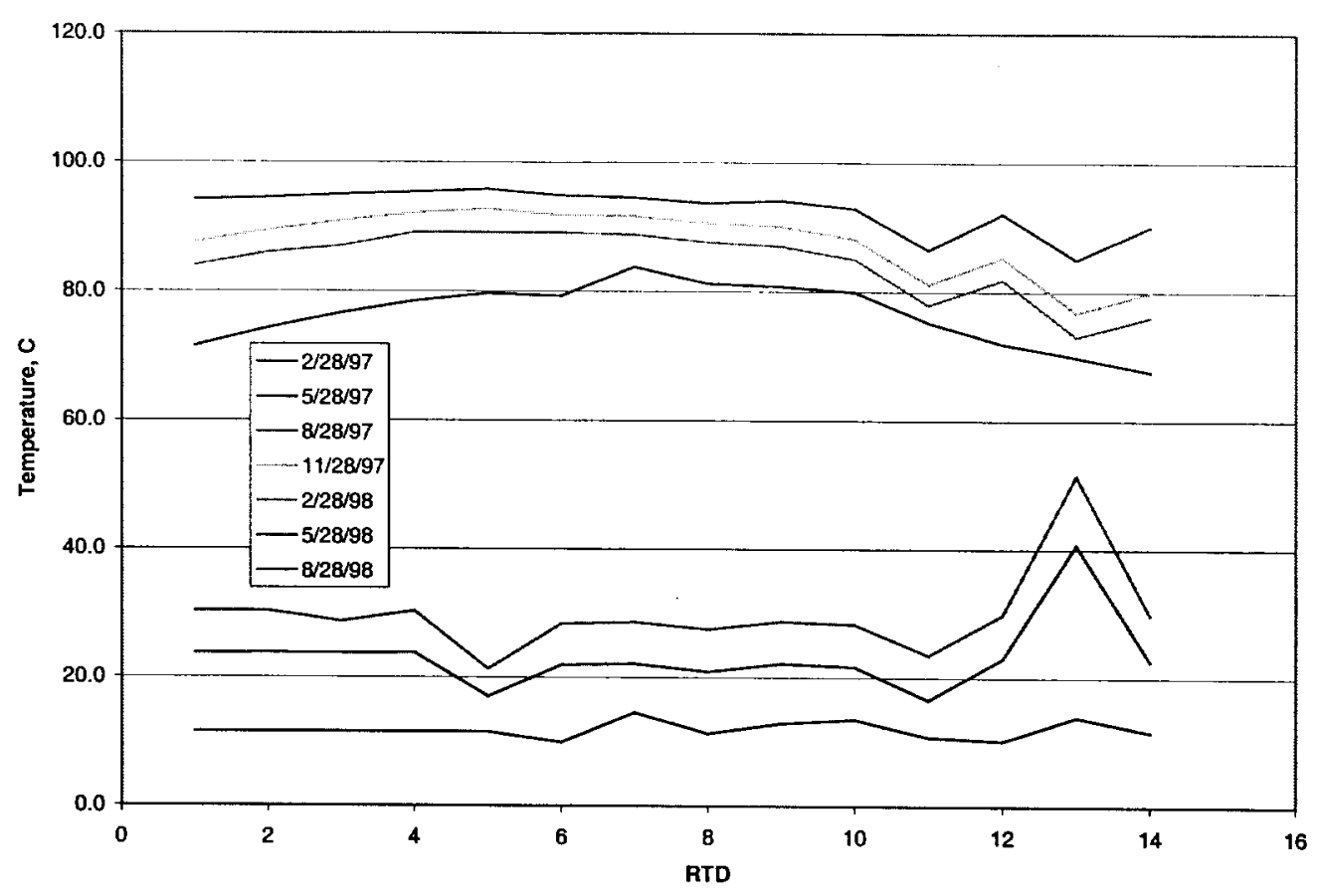

NOTE: RTD1 is near the west face; RTD 14 is near the east face.

Figure 5-15. Quarterly Temperature Snapshots in WT2 to Show the Spatial Temperature Variation 
Quarterly Temperature Snapshots in WT3.

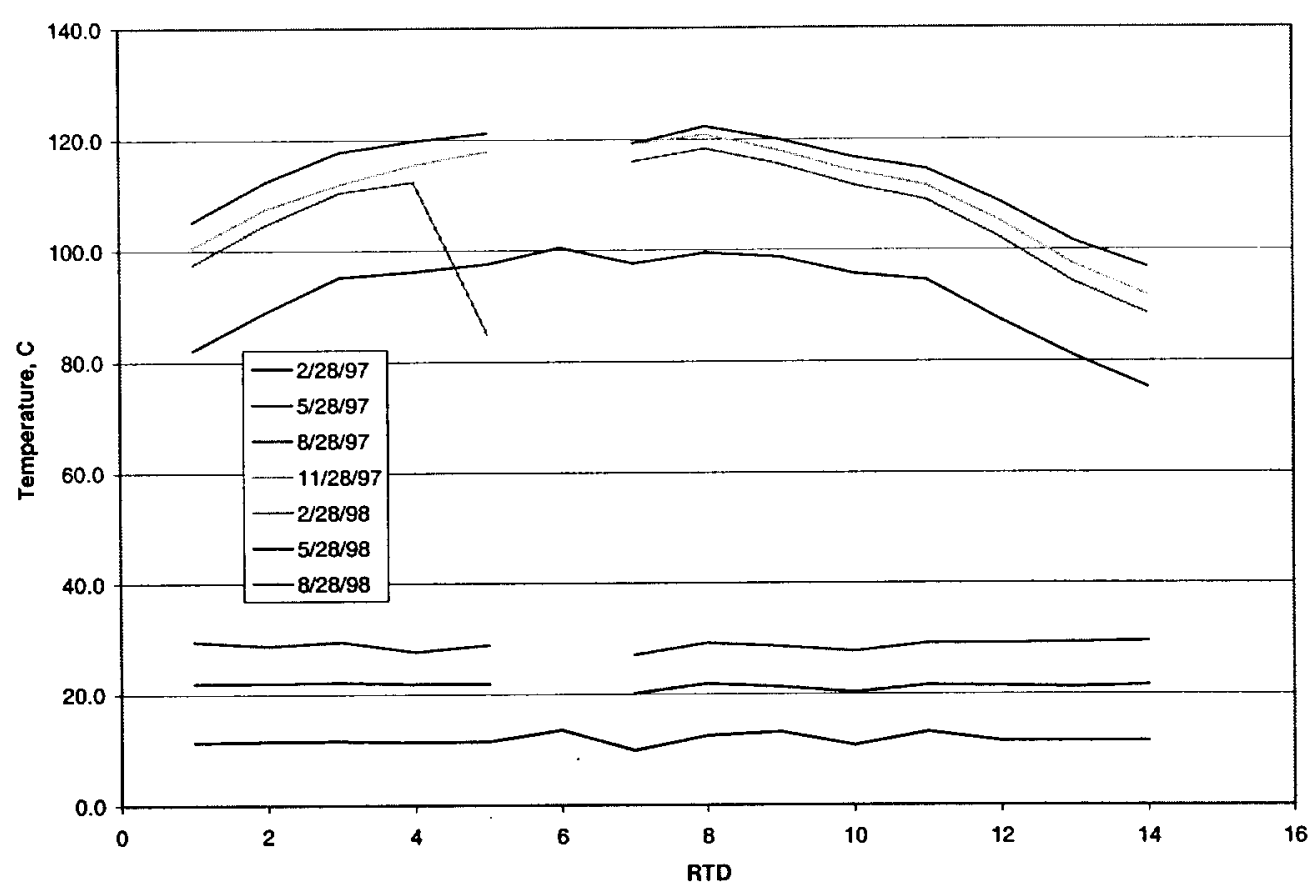

NOTE: RTD1 is near the west face; RTD 14 is near the east face.

Figure 5-16. Quarterly Temperature Snapshots in WT3 to Show the Spatial Temperature Variation 


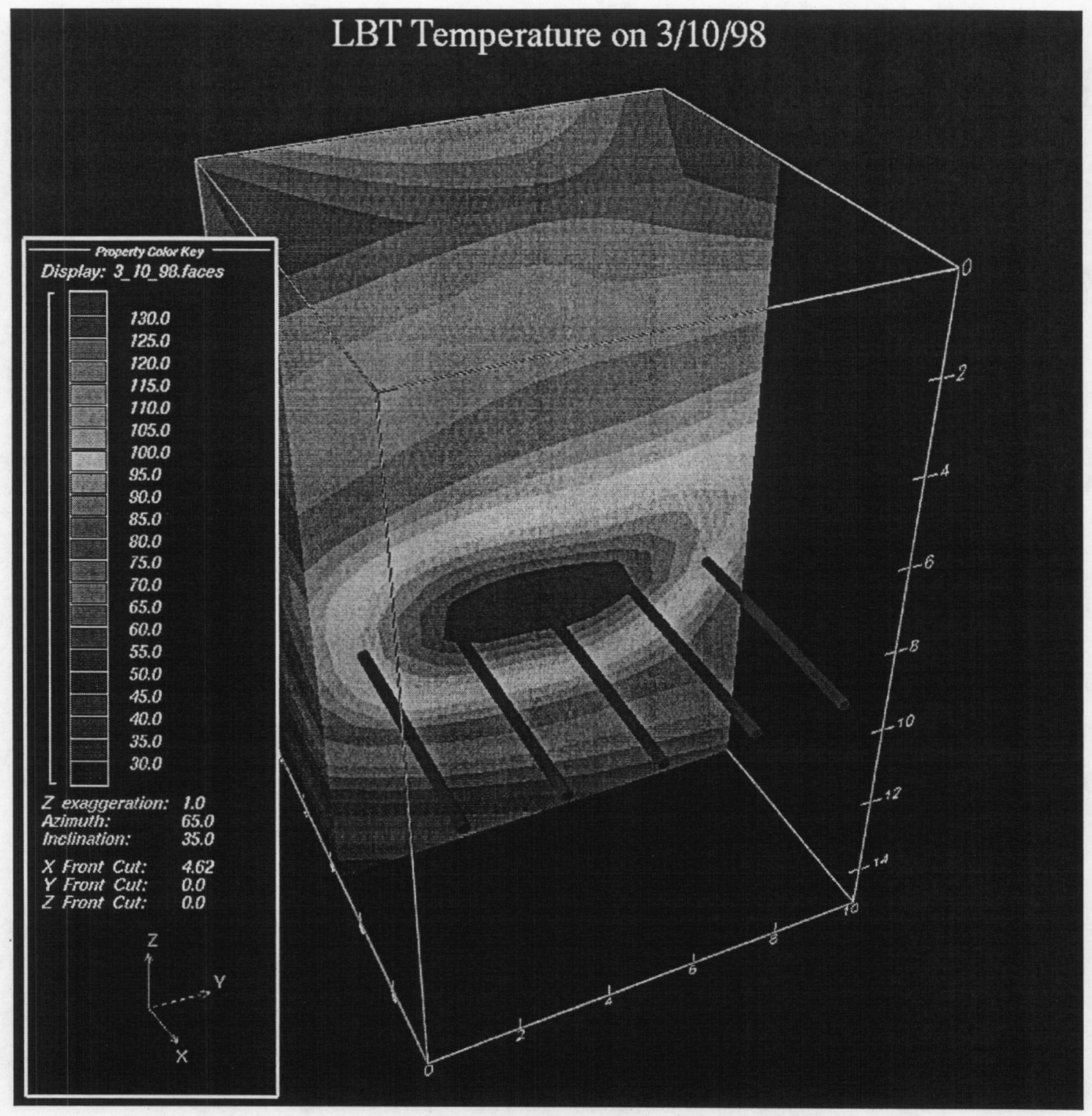

Figure 5-17. The Vertical Cross Section Along TT1 of the 3-D Temperature Field in the Block on March 10, 1998 
The temperature in TT1 as a function of time.

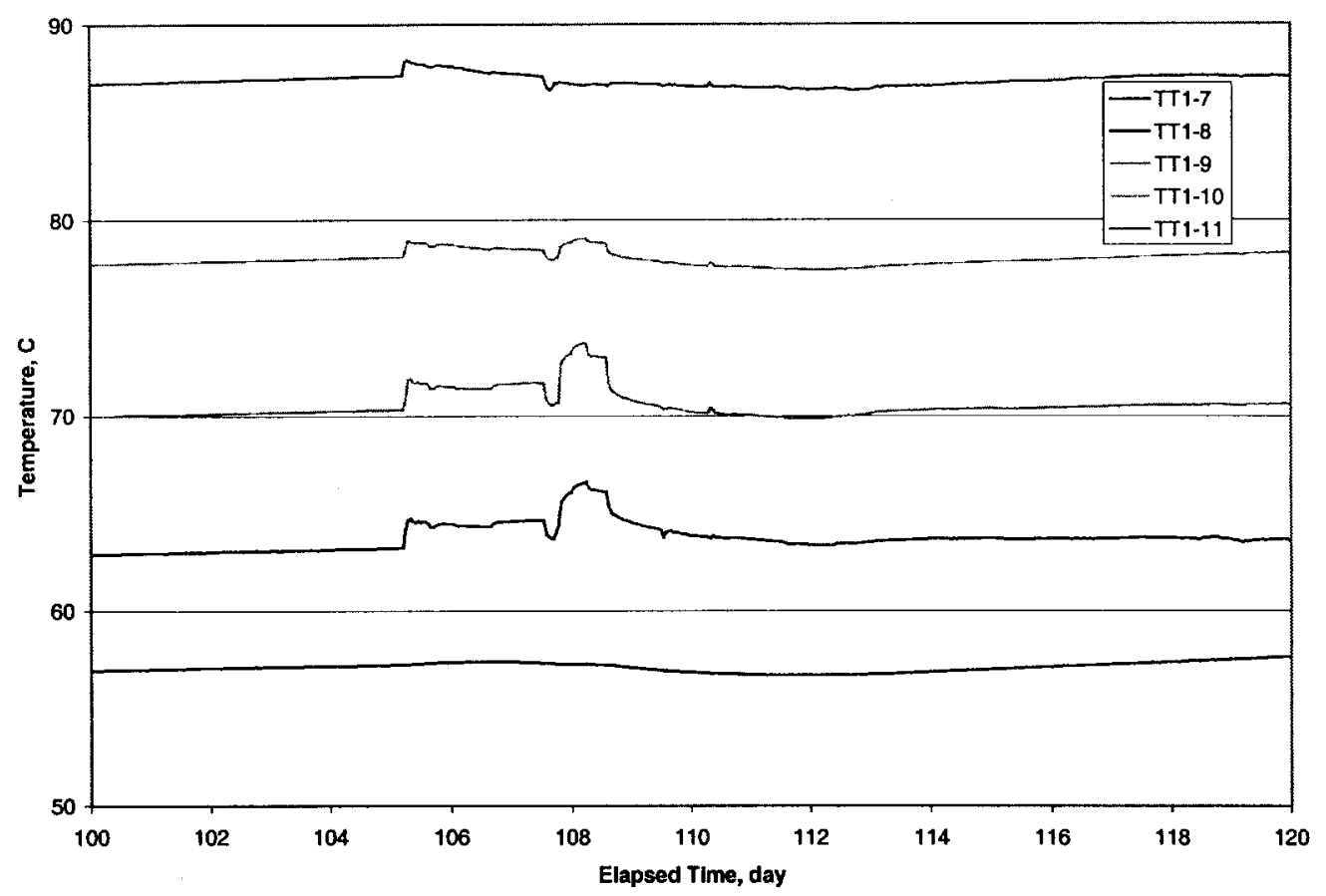

Figure 5-18. The Temperature at TT1-7 to $\pi 1-11$, Showing the Effect of the TH event on June 13, 1997 (105.2 Day) 
Temperatures in $\mathrm{T1}$ as a function of time.

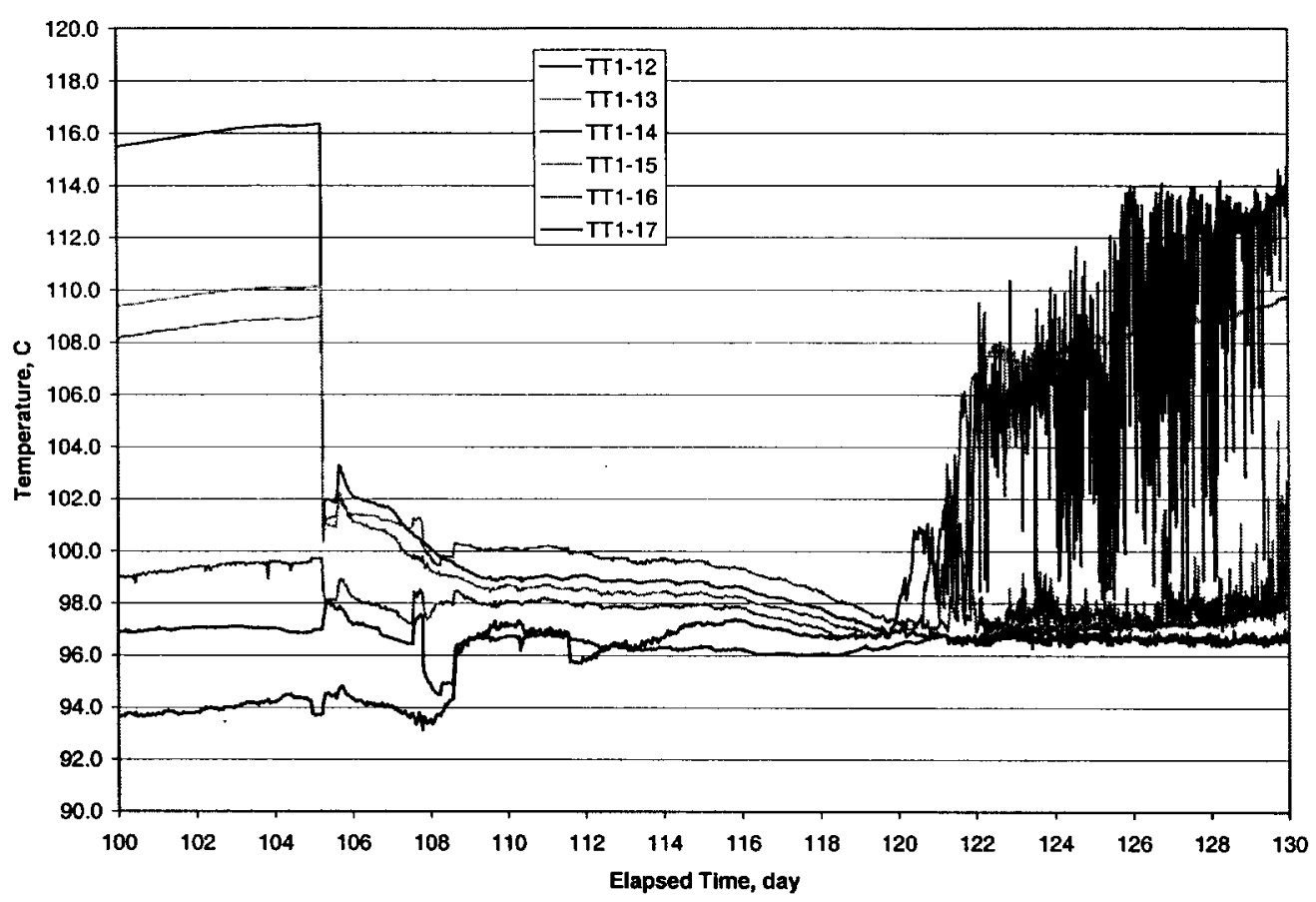

Figure 5-19. The Temperature at TT1-12 to $\pi T 1-17$, Showing the Effect of the TH Event on June 13, 1997 (105.2 Day) 
Temperature in TT1 as a function of time.

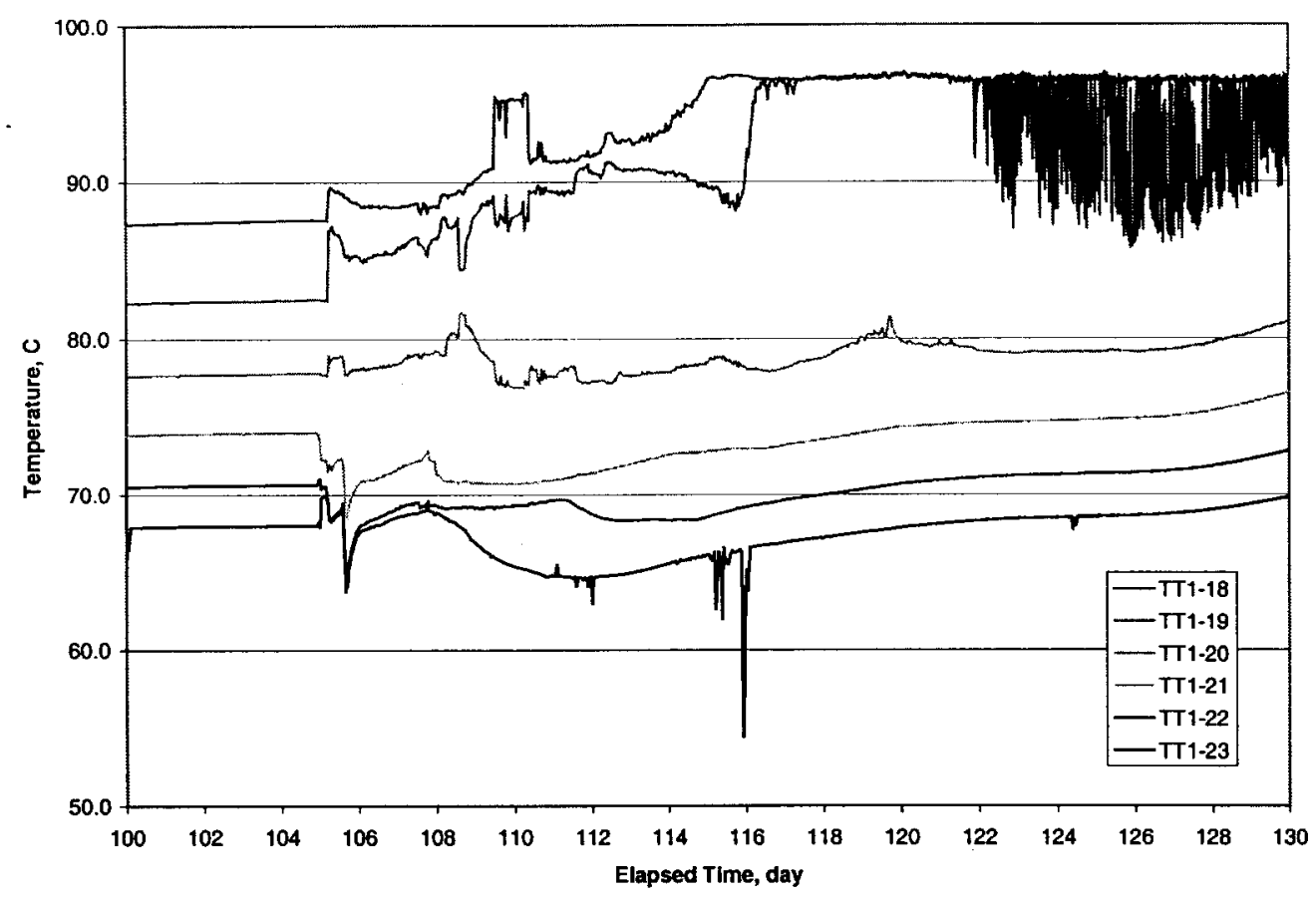

Figure 5-20. The Temperature at $\pi 1-18$ to $T T 1-23$, Showing the Effect of the TH Event on June 13, 1997 (105.2 Day) 
Temperature at $\mathrm{TT} 1$ as a function of time.

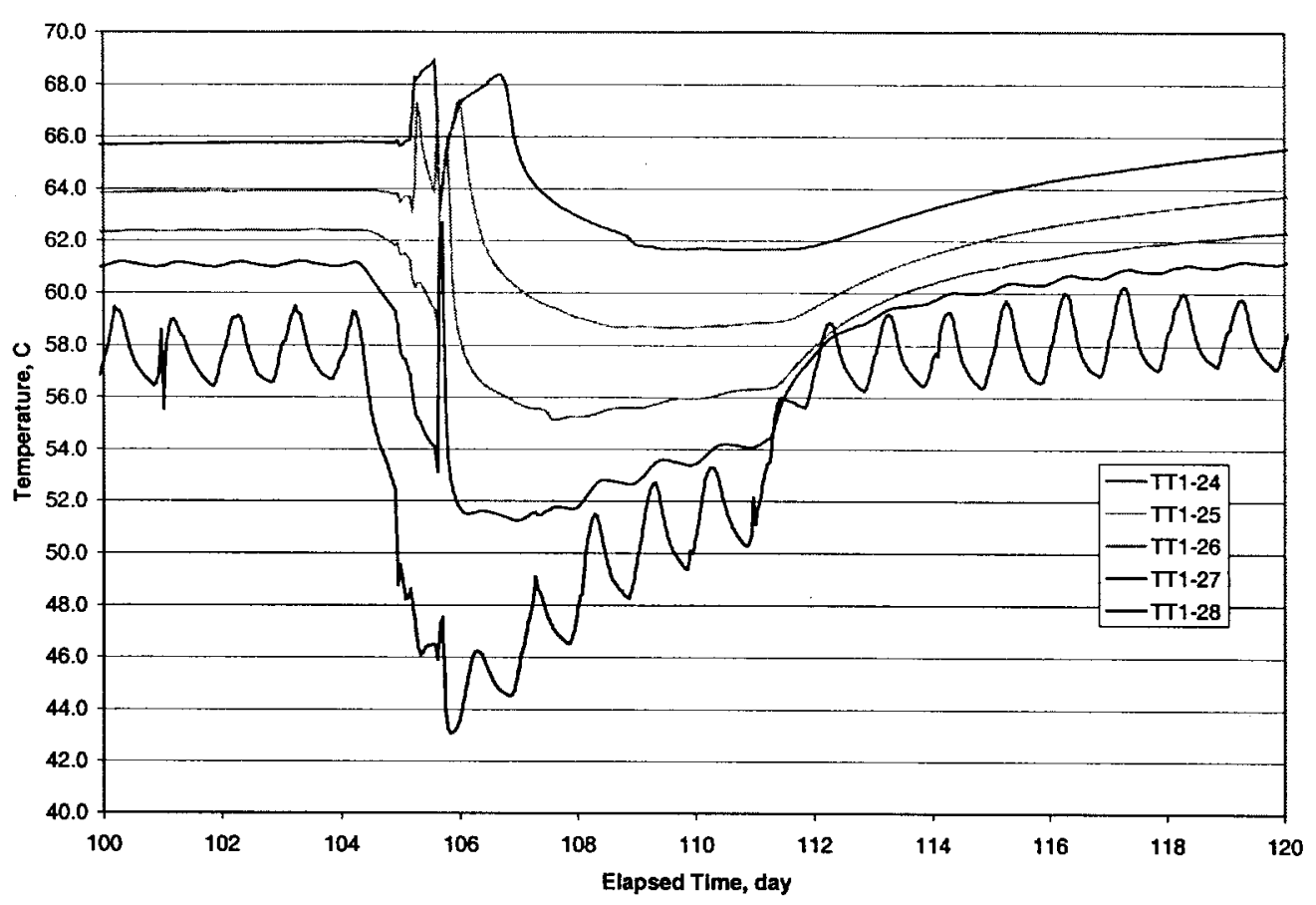

Figure 5-21. Temperature at TT1-24 to TT1-28, Showing the Effect of the TH Event on June 13, 1997 (105.2 Day) 
The temperature in $\mathrm{T1}$ as a function of time.

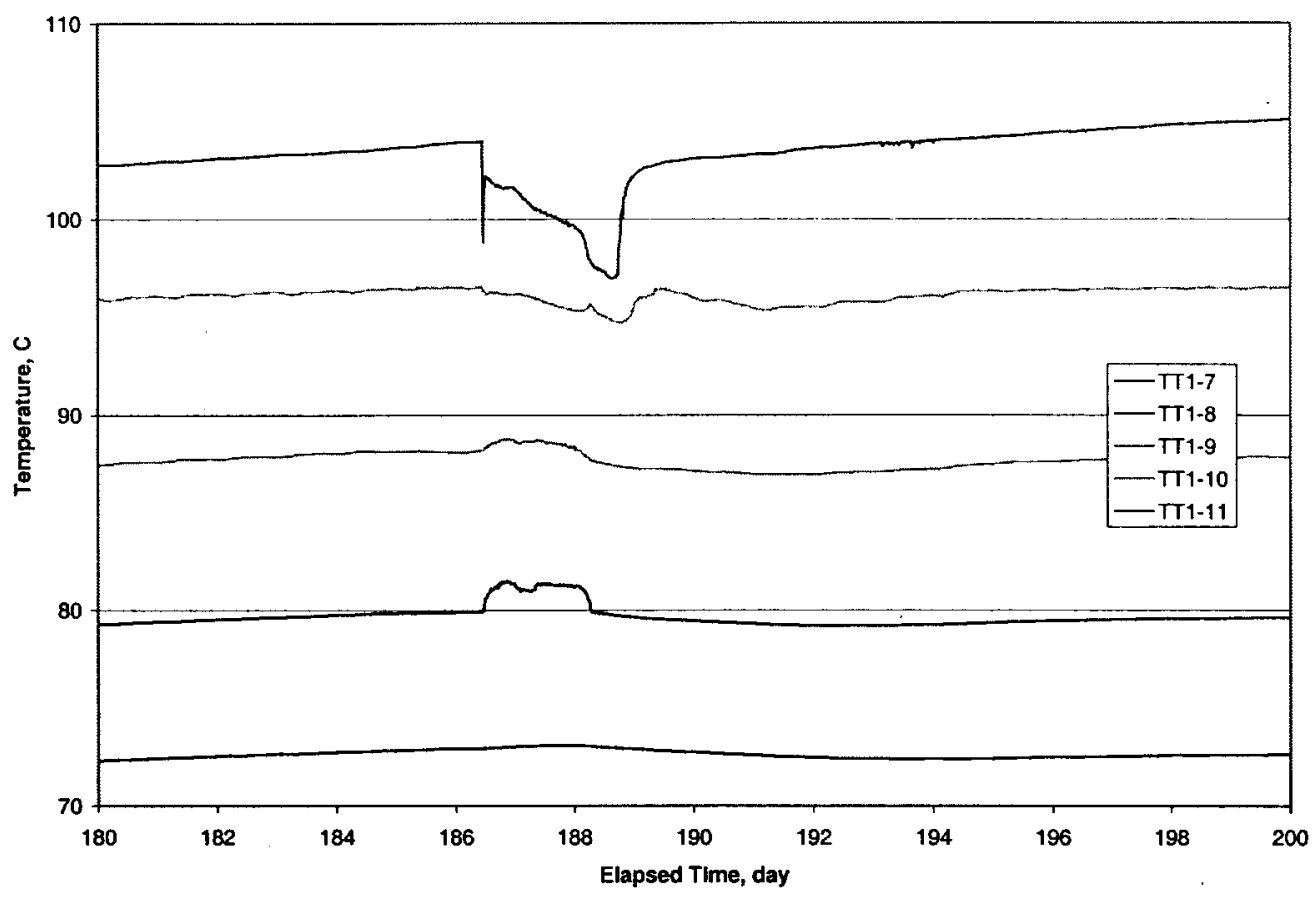

Figure 5-22. The Temperature at TT1-7 to TT1-11 as a Function of Time, Showing the Effect of the TH Event on September 2, 1997 (186.5 Day) 
Temperatures in TT1 as a function of time.

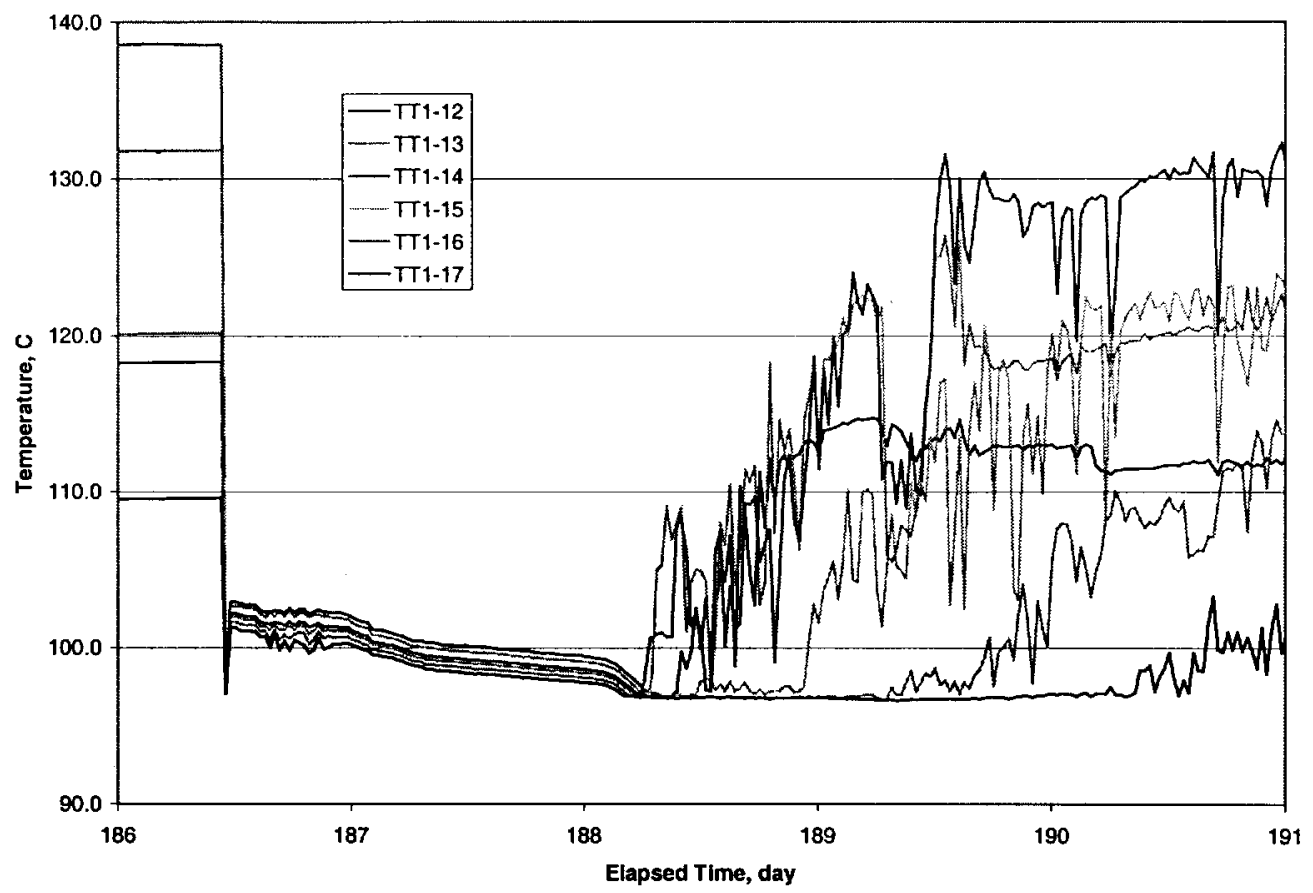

Figure 5-23. The Temperature at $\Pi 11-12$ to $T T 1-17$ as a Function of Time, Showing the TH Event on September 2, 1997 (186.5 Day) 
Temperature in $\mathrm{TT1}$ as a function of time.

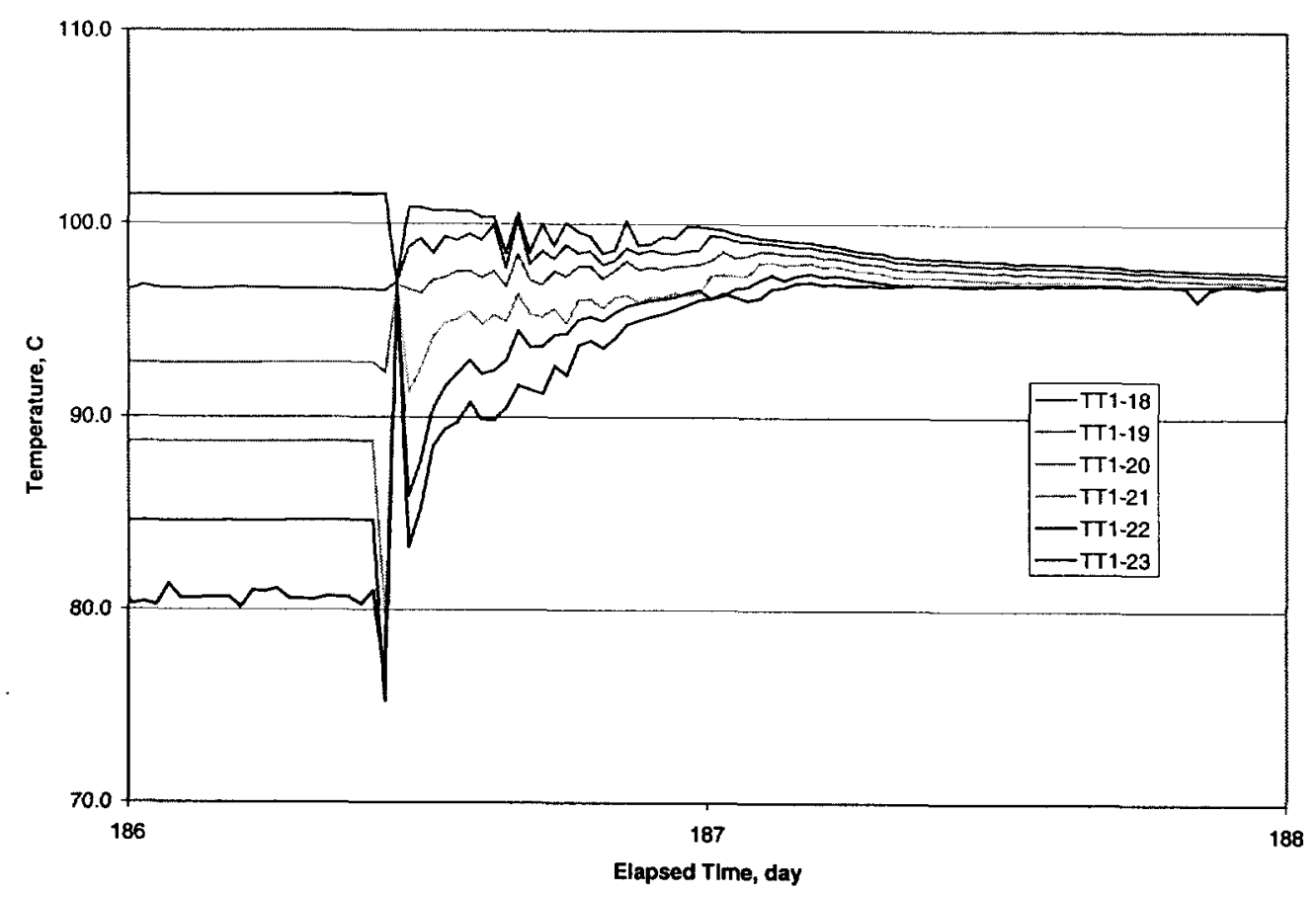

Figure 5-24. The Temperature at TT1-18 to TT1-23, Showing the Effect of the TH Event on September 2, 1997 (186.5 Day) 
Temperature at $\mathrm{TT} 1$ as a function of time.

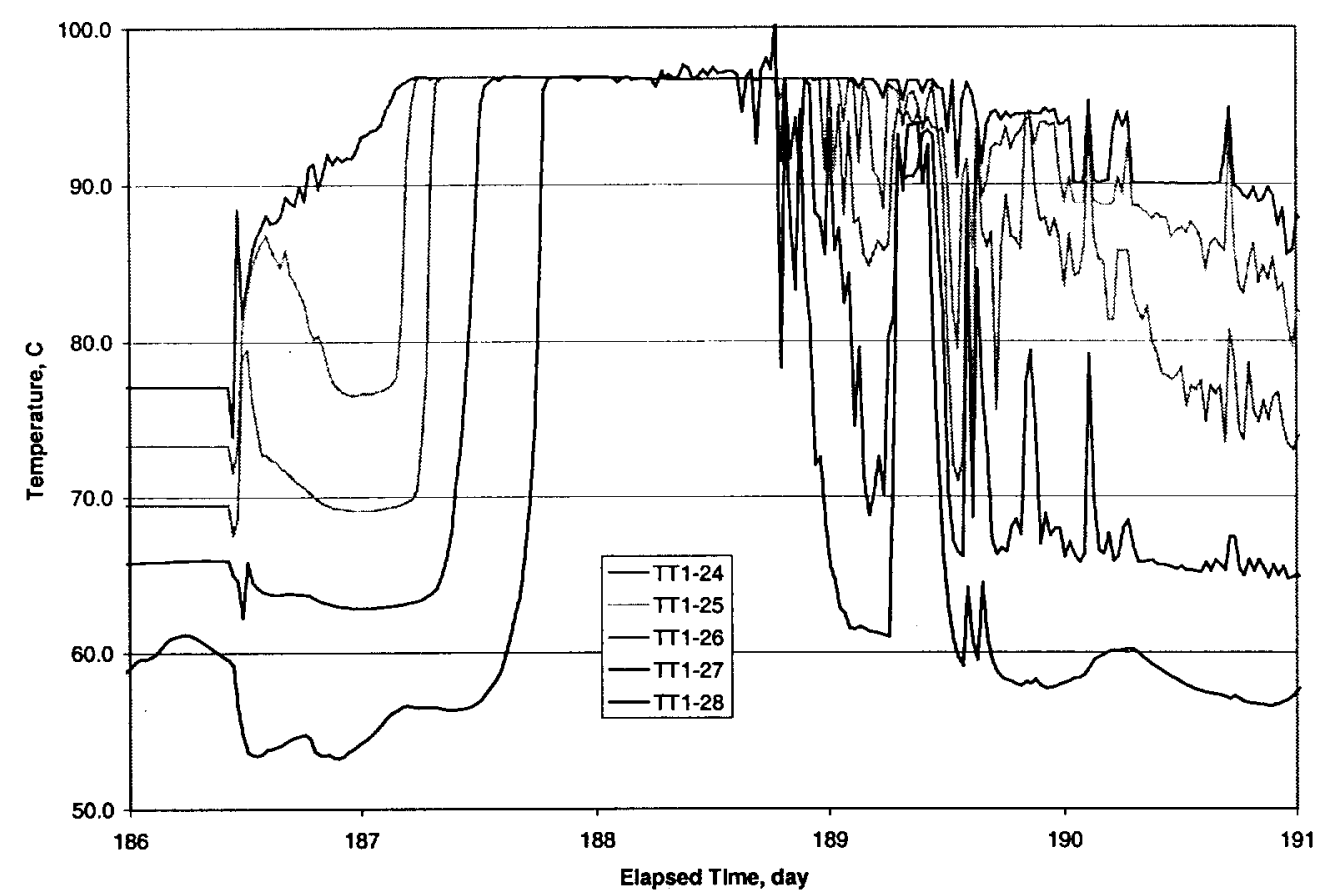

Figure 5-25. The Temperature at TT1-24 to TT1-28, Showing the Effect of the TH event on September 2, 1997 (186.5 Day) 
Heat loss per $\mathrm{m} 2$ in the first zone of the LBT

from $2 / 18 / 98$ to $2 / 25 / 98$.

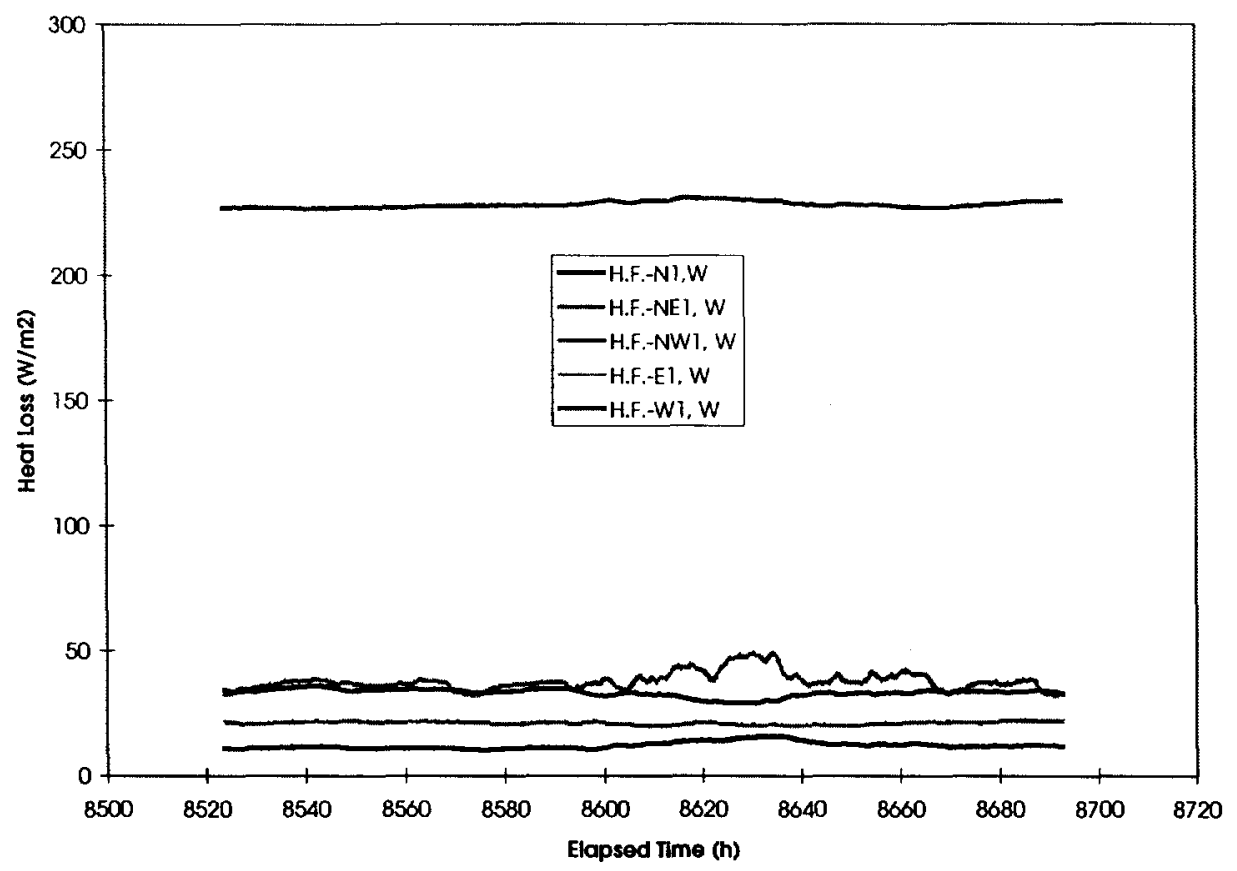

Figure 5-26. The Heat Flux per Unit Area in Zone $1(z=-3.2$ to $-4.42 \mathrm{~m})$ of the Block as a Function of Time from February 18, 1998, to February 25, 1998 


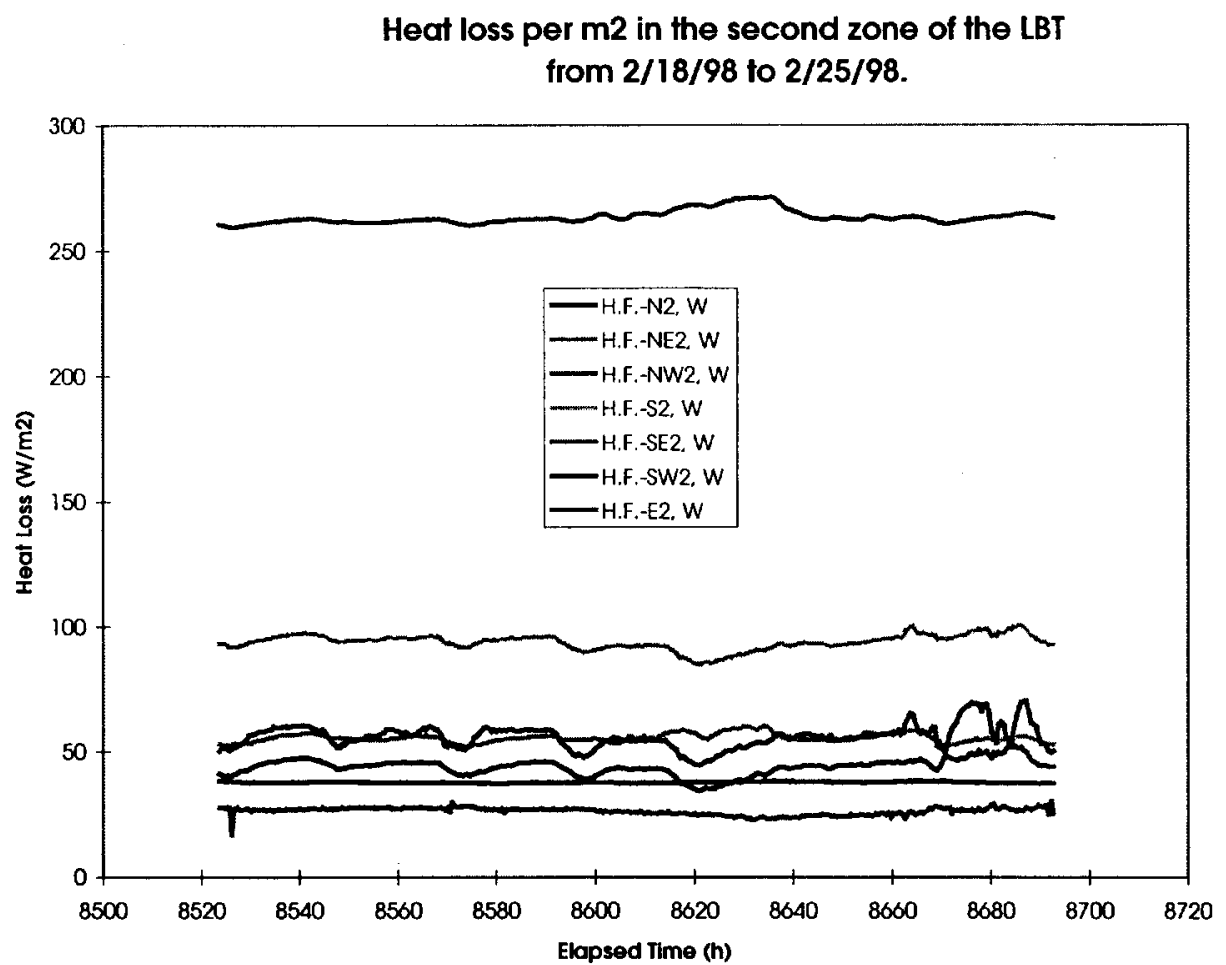

Figure 5-27. The Heat Flux per Unit Area in Zone $2(z=-1.981$ to $-3.2 \mathrm{~m})$ of the Block as a Function of Time from February 18, 1998, to February 25, 1998 
Heat loss per $\mathrm{m} 2$ in the third zone of the LBT from $2 / 18 / 98$ to $2 / 25 / 98$.

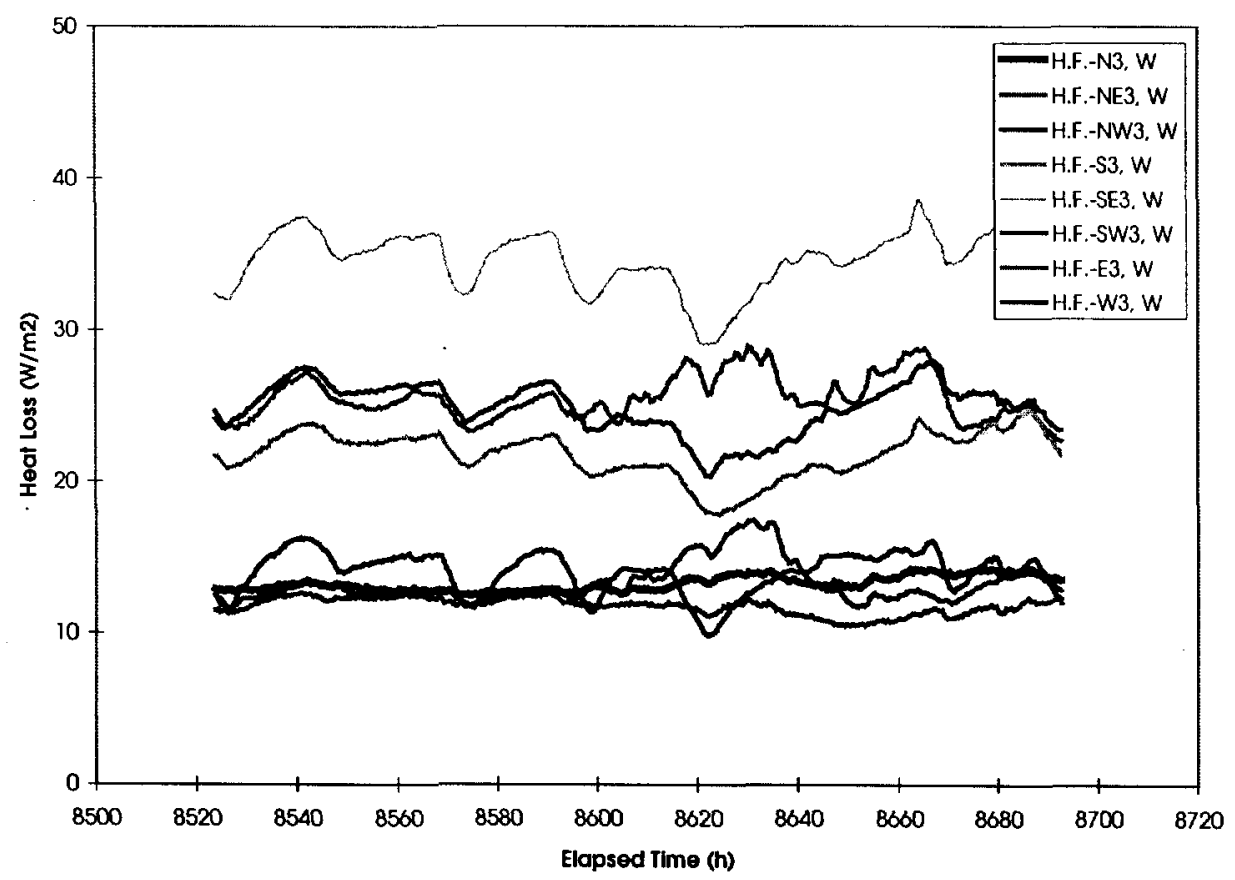

Figure 5-28. The Heat Flux per Unit Area in Zone $3(z=-0.762$ to $-1.981 \mathrm{~m})$ of the Block as a Function of Time from February 18, 1998, to February 25, 1998 
Heat loss per $\mathrm{m} 2$ in the fourth zone of the LBT from $2 / 18 / 98$ to $2 / 25 / 98$.

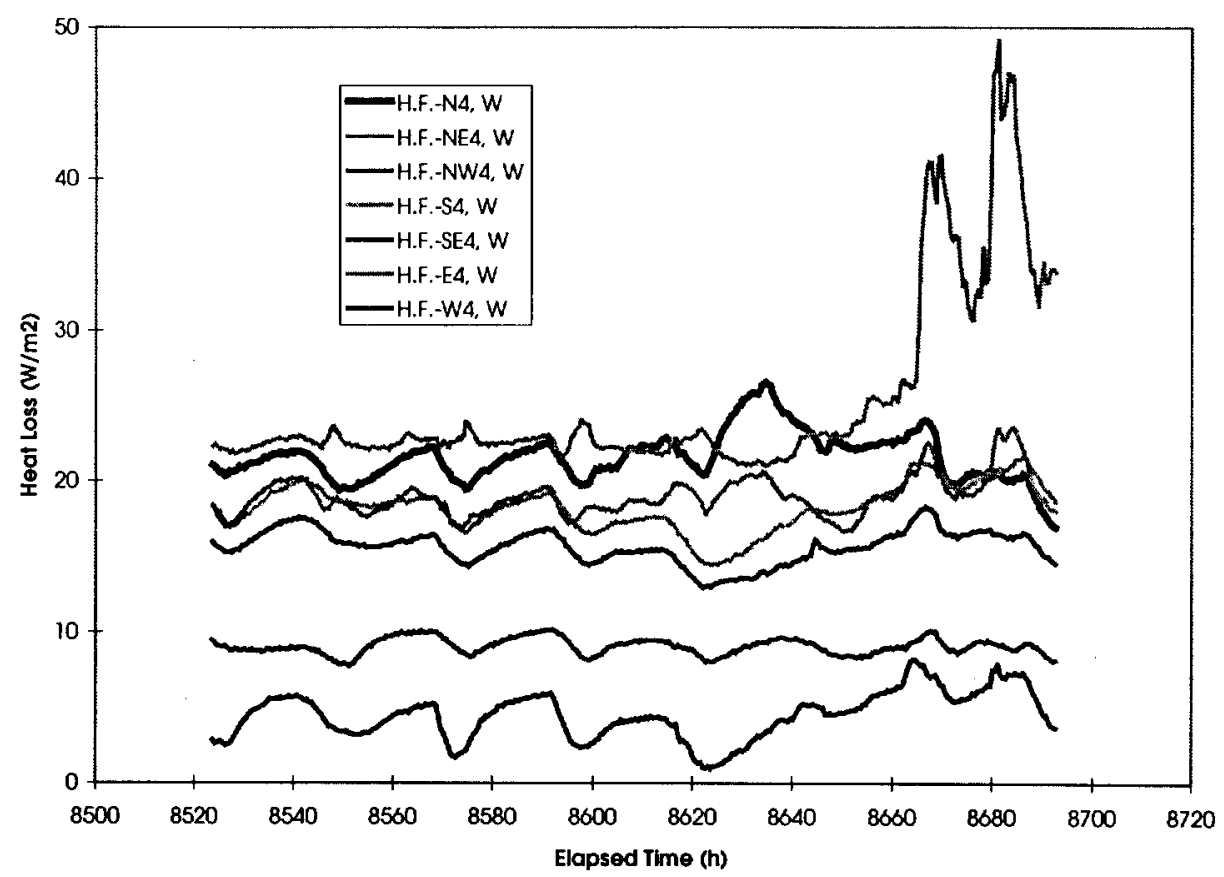

Figure 5-29. The Heat Flux per Unit Area in Zone $4(z=0$ to $-0.762 \mathrm{~m})$ of the Block as a Function of Time from February 18, 1998, to February 25, 1998 

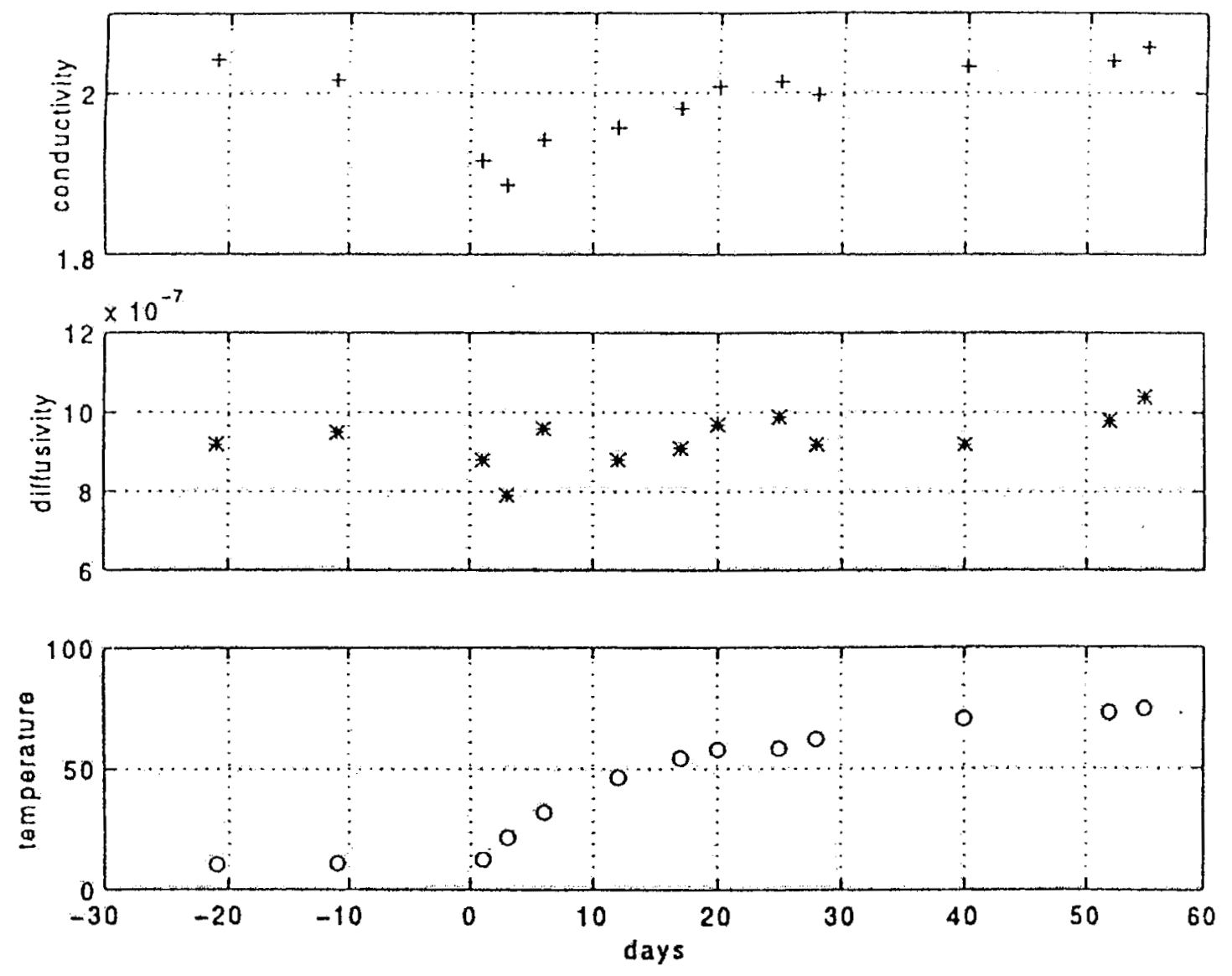

Figure 5-30. Rock Thermal Conductivity, $\mathrm{K}[\mathrm{W} /(\mathrm{mK})]$, Diffusivity, Alpha $\left[\mathrm{m}^{2} / \mathrm{s}\right]$, and Temperature $\left[{ }^{\circ} \mathrm{C}\right]$ with Time [Days] for the Horizontal REKA Probe 1 Installed $0.584 \mathrm{~m}$ Below the Large Block's Horizontal Heater Layer 

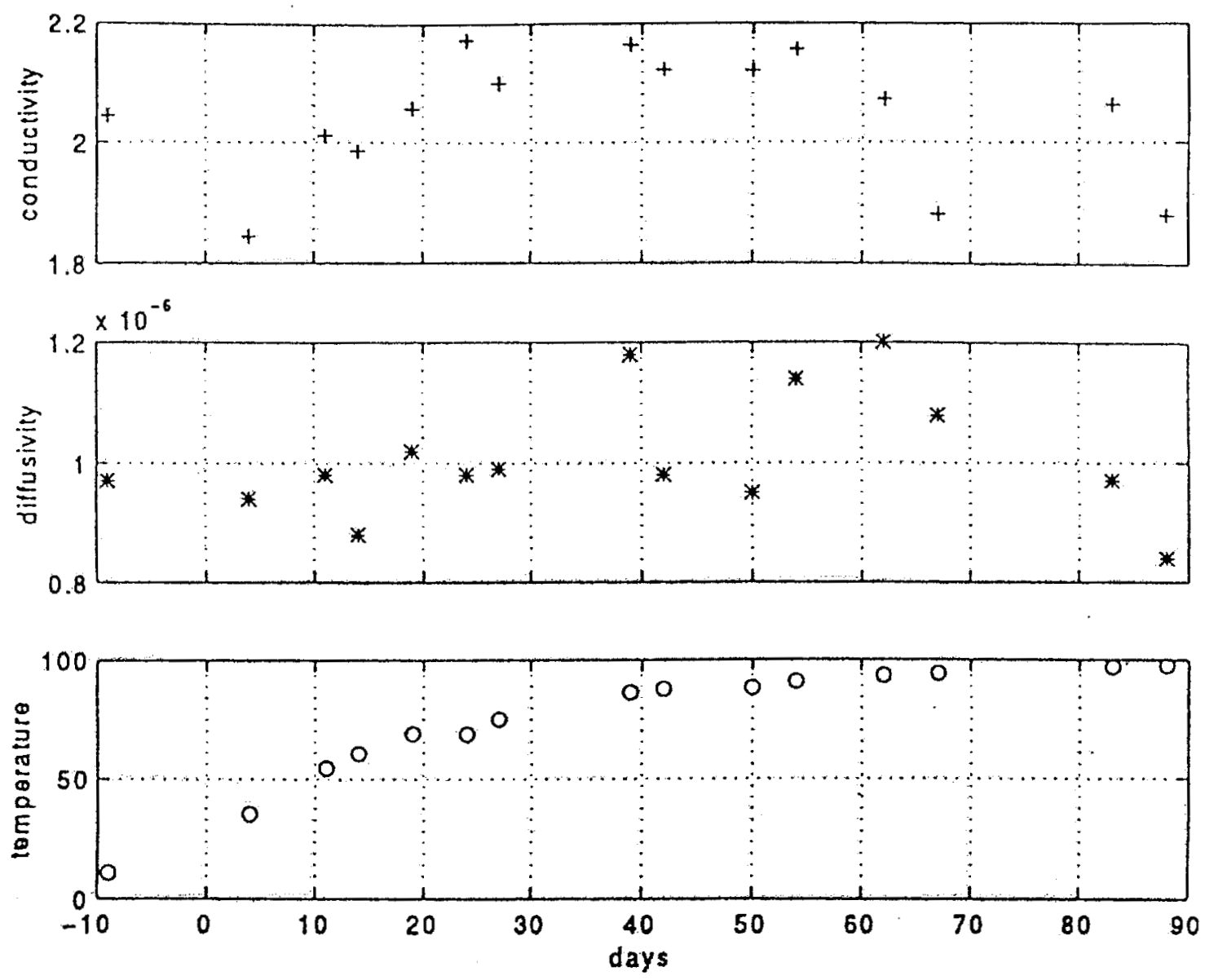

Figure 5-31. Rock Thermal Conductivity, K $[\mathrm{W} /(\mathrm{mK})]$, Diffusivity, Alpha $\left[\mathrm{m}^{2} / \mathrm{s}\right]$, and Temperature $\left[{ }^{\circ} \mathrm{C}\right]$ with Time [Days] for the Horizontal REKA Probe 2 Installed $0.889 \mathrm{~m}$ Below the Large Block's Horizontal Heater Layer 

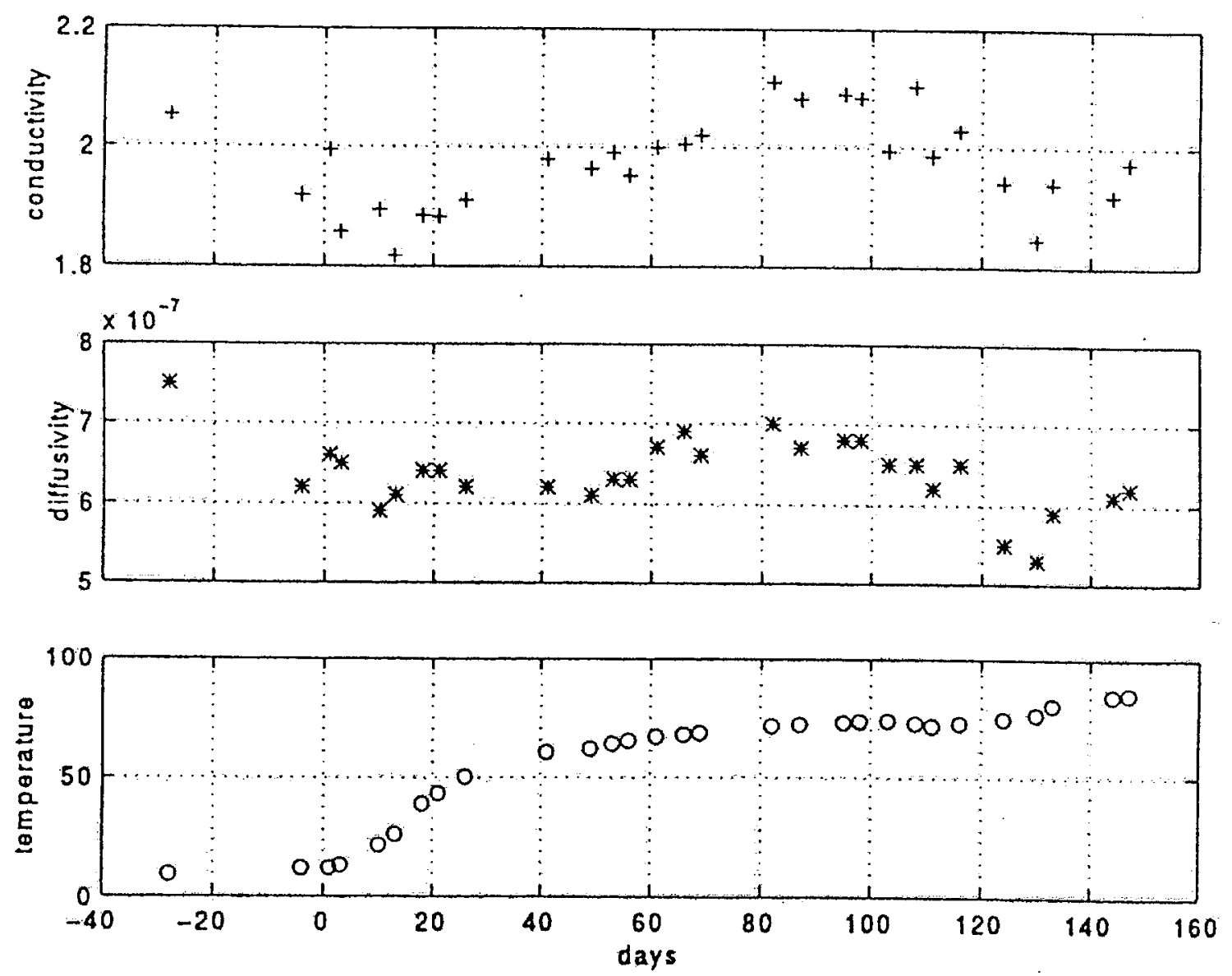

Figure 5-32. Rock Thermal Conductivity, $\mathrm{K}[\mathrm{W} /(\mathrm{mK})]$, Diffusivity, Alpha $\left[\mathrm{m}^{2} / \mathrm{s}\right]$, and Temperature $\left[{ }^{\circ} \mathrm{C}\right]$ with Time [Days] for the Horizontal REKA Probe 3 Installed $1.38 \mathrm{~m}$ Below the Large Block's Horizontal Heater Layer 


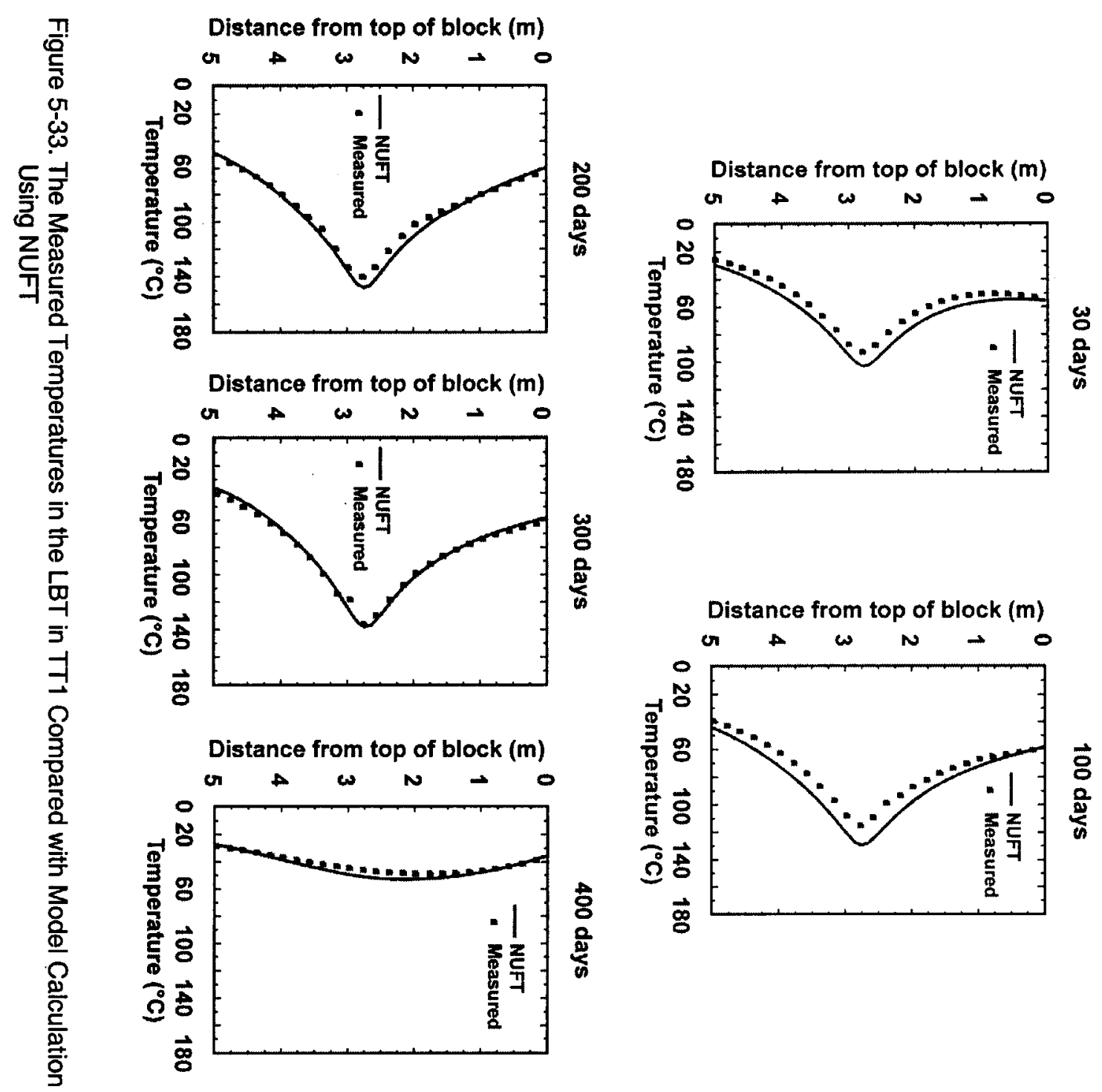


TT1-14

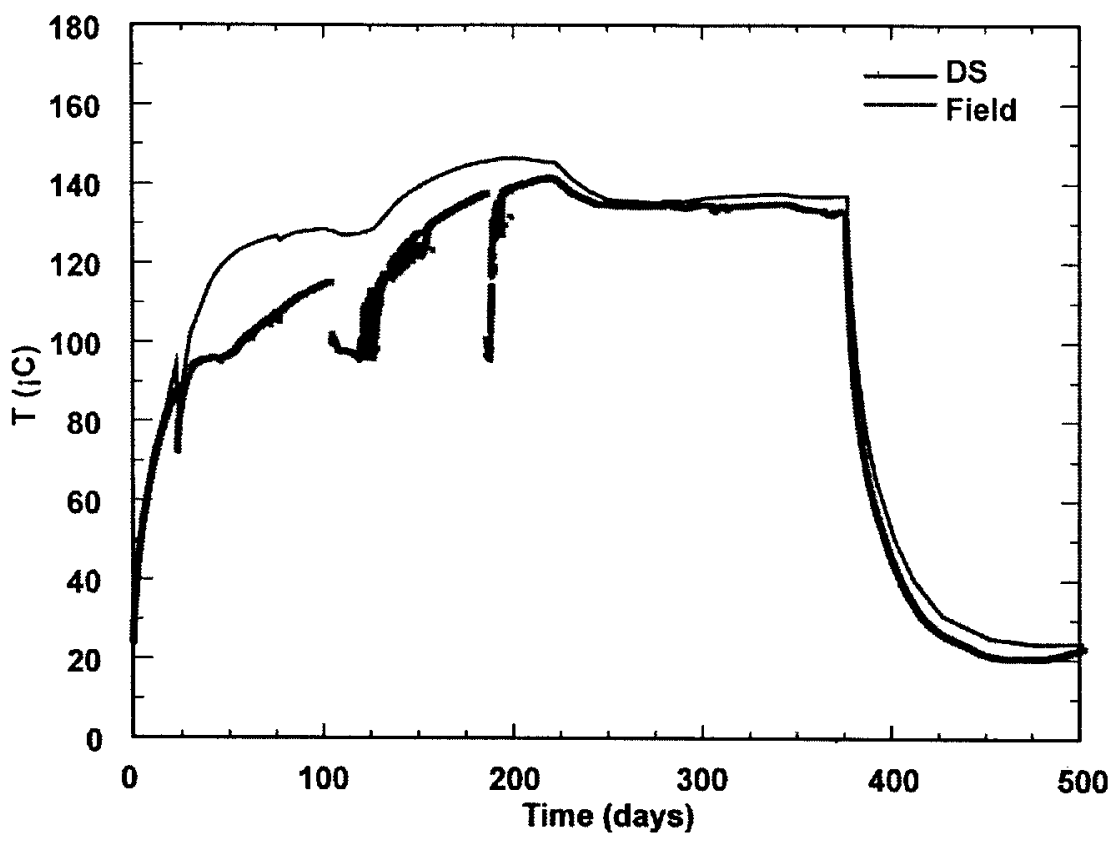

TT1-19

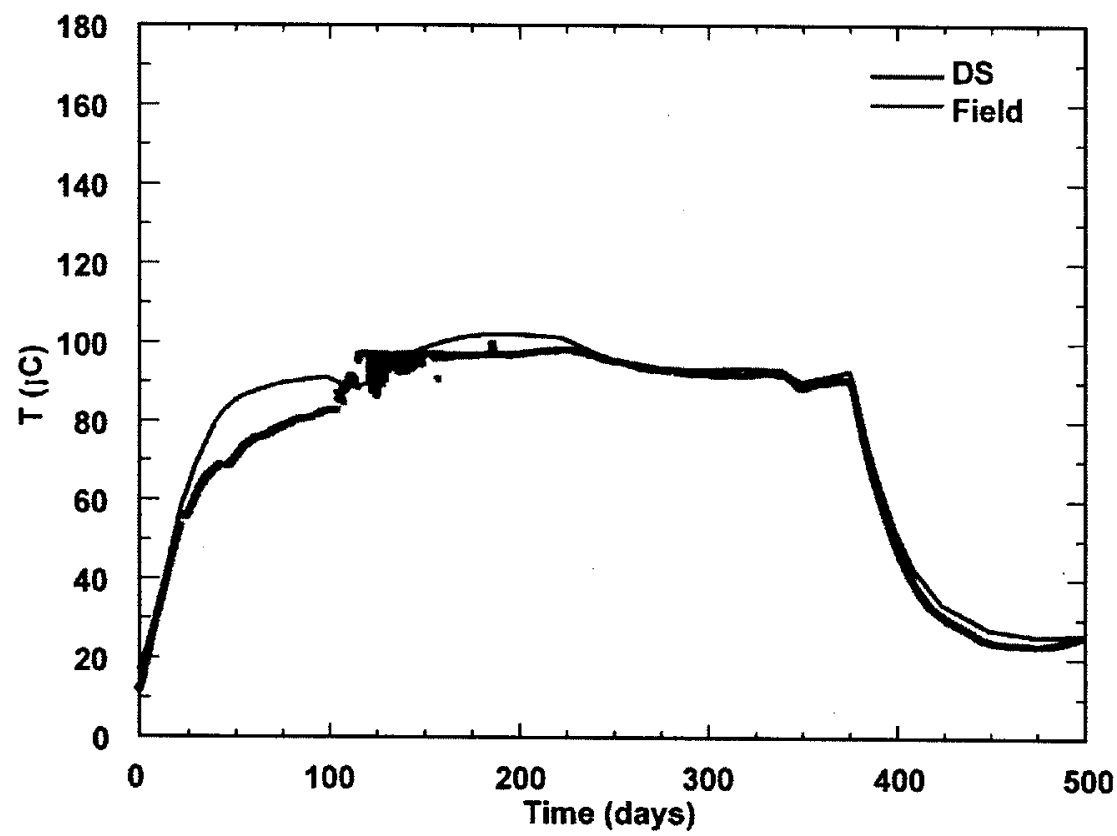

Figure 5-34. Comparison of the Measured Temperature at TT1-14 and TT1-19 with the Model Calculation Using the Drift-Scale Property Set 

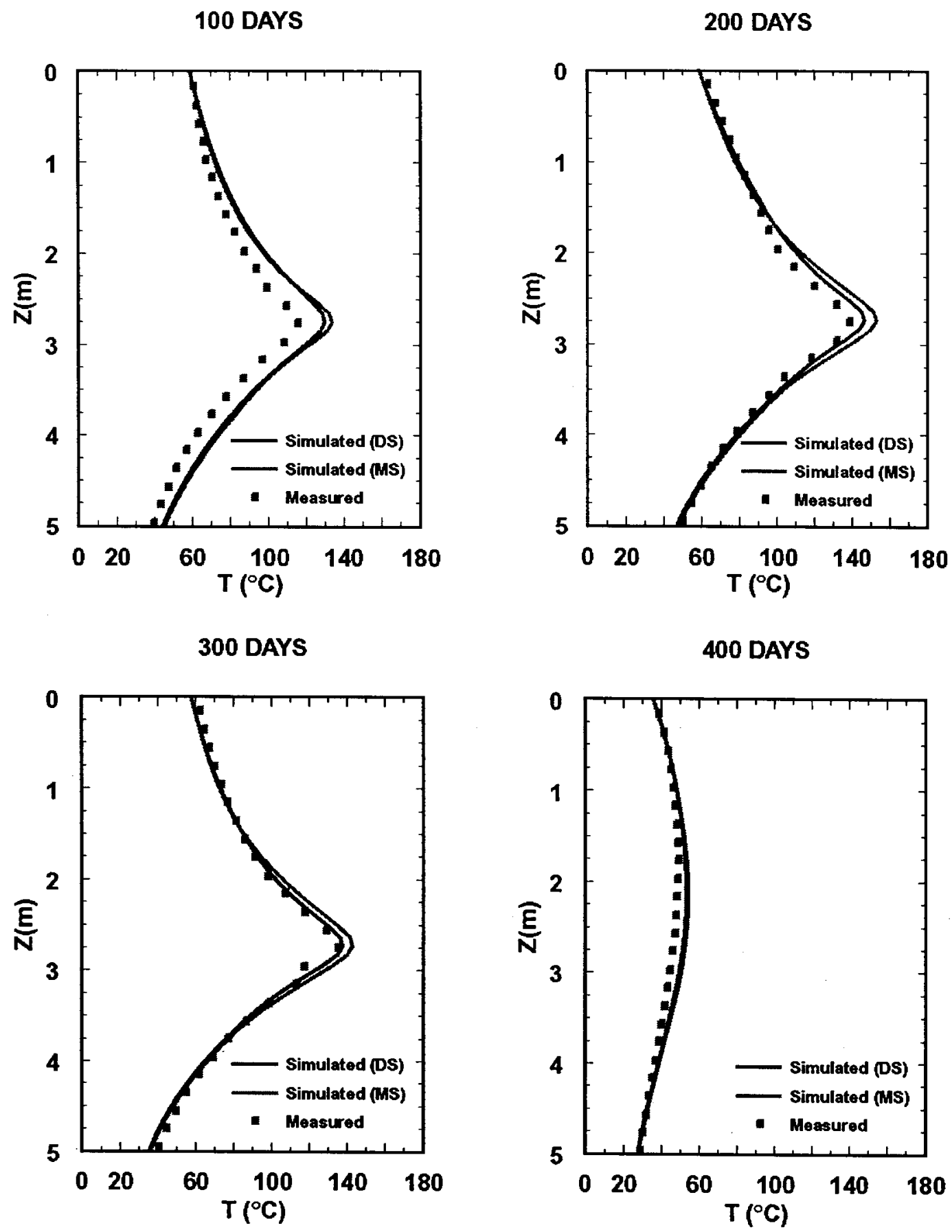

Figure 5-35. Predicted Temperature Profiles Along Hole \#TT1 of the LBT Using the DS and MS Property Sets, Compared with the Measured Temperature 


\section{THERMAL HYDROLOGIC MEASUREMENTS AND ANALYSIS}

\subsection{THERMAL HYDROLOGIC MEASUREMENTS}

The thermal hydrologic measurements include the determination of the spatial distribution and the temporal variation of the bulk air permeability and the moisture content in the block. Both single-hole injection and cross-hole flow measurements were used to determine the bulk air permeability. Tracer tests were also conducted to determine the flow paths in the block. The air permeability was determined before the heating and at the end of the heating phase. The moisture content was also determined before the heating, during the heating, and during the natural cooling phases. Both the pre-heat and in-heat measurements are presented in this section.

\subsubsection{Bulk Air Permeability and Flow Path Measurements}

\subsubsection{Pre-heating Permeability Measurements}

\subsection{LLNL Single Hole Permeability Test}

To estimate bulk permeability, air-injection tests were conducted after the first vertical borehole (N1, as shown in Figure 2-7 of Wilder, et al. [1997]) was drilled. (Note that N1 later became TH1, as shown in Figure 3-7, after the installation of instruments in the block.) Curve D in Figure 6-1 shows the air permeability as a function of depth. Most of the sampled depths have a permeability greater than $10^{-11} \mathrm{~cm}^{2}$. It should be noted that the permeability is dominated by the fractures that intersect the injection borehole. Because of the high fracture density in the block, the bulk permeability is likely to be more homogeneous.

Wang and Ahlers (1996) compared the results of the air-permeability tests with those conducted within the ESF for the Single Heater Test (SHT). They noted that the permeability results were more heterogeneous for the LBT than for the SHT (see Figure 2-18 of the LBT Status Report). As they noted, it is possible that the near-surface exposure does not generate sufficient stress to close up the larger fractures. In contrast, at the repository level, the stress may be sufficiently large that the apertures of the fractures would tend to be reduced. It is of interest to note that the LBT rock in general is much tighter than that of the SHT, but the LBT's more permeable zones are more permeable than those of the SHT. A possible implication is that after the air permeability tests were completed and the block was isolated by saw-cut and excavation, these fractures may have opened more, although the stresses near the surface may be so low that isolation of the block would have little effect.

As shown in Figure 6-1, permeability tests conducted in 1997 (curve B) prior to instrumentation is not much different from the pre-cut measurement in 1993 (curve D), and therefore do not support the hypothesis of further opening of fractures after the block was isolated.

\subsection{Lawrence Berkeley National Laboratory Cross Hole}

Air-injection tests were conducted, after all instruments were installed and the boreholes were sealed (except the heater boreholes and hydrology boreholes, which had packers in them), with injections of a gas tracer at a controlled flow rate into packed borehole intervals. Cross-hole transient pressure responses were simultaneously measured in surrounding boreholes. Figure 6-2 
shows the heterogeneous pneumatic permeability variation along five heater boreholes. High permeability zones were present near the block boundaries. In the block interior, two heater boreholes (EH-2 and EH-3) penetrated low-permeability zones in the first half of the boreholes. The heterogeneous permeability variation within the block indicates that the heater-induced vapor-flow processes can be very different in different zones in the block, with vapor flowing easily near the northern and eastern boundaries and in the back (western side) of the block away from the bottom of the boreholes. A "hard core" with low permeability and low vapor convection exists southeast, off center in the block.

Figure 6-3 illustrates the air-permeability variations along three horizontal hydrologic monitoring boreholes. Two hydrologic boreholes are located on the western side of the block with WH1 1.5 $\mathrm{m}(5 \mathrm{ft})$ and WH2 $0.5 \mathrm{~m}(1.5 \mathrm{ft})$ above the heater plane. Two sets of tests were conducted at WH1, as shown in Figure 6-3. The third hydrologic borehole NH1 from the north face is $0.3 \mathrm{~m}$ ( $1 \mathrm{ft})$ above the heater plane. The air-permeability variations along these three hydrologic monitoring boreholes do not exhibit the high permeability $\left(10^{-11}\right.$ to $10^{-10} \mathrm{~m}^{2}$ or 10 to 100 darcy) leaky boundary effects observed in some of the heater boreholes. Localized tight zones with airpermeability values one to two orders of magnitude lower than the average permeability in the $10^{-12} \mathrm{~m}^{2}$ (1 darcy) range were identified along the boreholes. The horizontal permeability profiles in Figure 6-3 supplement the vertical permeability profile previously measured along vertical borehole TH1 (Wilder et al. 1997).

\subsection{Lawrence Berkeley National Laboratory Trace Test}

Tracers were released in the heater boreholes at different intervals and detected at other locations above and below the heater plane. Figure 6-4 shows the tracer breakthrough detected at borehole WH1, $1.52 \mathrm{~m}$ above the heater plane and $3.35 \mathrm{~m}$ above the ground. The tracers were released from both the middle interval (interval 3) of borehole EH-2 south of the block center and borehole EH-5 near the northern boundary. Borehole EH-5-3 has the highest permeability measured in the block. Potential leakage through the north shield could have contributed to the high value. Compared to the tracers released at EH-2-3 near the center of the block, the tracer released at this interval moved easily to borehole WH1. Both breakthrough curves also had long tails, indicating that many other pathways in the block contributed to the tracer transport at different rates. The tracer breakthrough results substantiated the pneumatic test results with respect to high permeability zones enhancing flow and transport through heterogeneous, fractured rock media in the block. Comparison of TH analyses and heater test results can assess the importance of block-scale heterogeneity in determining near-field, heater-induced impacts.

\subsubsection{Post-Heating Air Permeability Measurement}

Air injection permeability measurements along Borehole TH1 were repeated in February 1998 while the block was still heated, shortly before the cool-down phase started. The purpose was to observe any effects of heating on rock fracture permeability in the block by comparing the hot permeability profile with the preheating profile measured after the block was constructed 1997. As with the previous tests, air was injected into packed-off sections of the borehole, and bulk permeability of the rock adjacent to the packed-off section was calculated from the gas pressure and temperature measured in the test zone, ambient pressure, and the mass flow rate of injected air. Air viscosity and density in the calculations were adjusted for temperature. The hot 
permeability profile was limited to a depth of $3.29 \mathrm{~m}$, because blockage in the borehole prevented deeper penetration by the packer probe. The preheating permeability profile went down to a depth of $4.72 \mathrm{~m}$.

Figure 6-5 compares the preheating and hot permeability profiles. The figure shows substantially higher permeability in the hot rock at depths above $1.83 \mathrm{~m}$. The greatest difference occurs at a depth of 1.22 to $1.3 \mathrm{~m}$, where the hot permeability is about two orders of magnitude greater. From $1.83 \mathrm{~m}$ to $3.17 \mathrm{~m}$, differences between the two permeability profiles are much smaller. Higher permeability values in the hot rock closer to the top of the block may suggest that movement along fractures, due to thermal expansion, was probably more pronounced in that region. Such movement could reopen healed fractures and cause aperture increases in existing fractures. This is in agreement with the deformation of the block observed during the test (see Section 7 for details).

\subsubsection{Moisture Content Measurements}

The moisture content in the block was determined by using ERT and neutron logging. Neutron logging provides very accurate determination of the moisture content within a region of about 10 $\mathrm{cm}$ radius distance from a borehole. The ERT provides 2-D distribution of the moisture content with less accuracy. The two methods were used to complement each other.

\subsubsection{Electrical Resistance Tomography}

\subsection{ERT Methodology}

ERT is a geophysical imaging technique that can be used to map subsurface resistivity (Daily and Owen 1991). The ERT measurements consist of a series of voltage and current measurements from buried electrodes using an automated data collection system. The data are then processed to produce electrical resistivity tomographs. ERT was proposed independently by Henderson and Webster (1978) as a medical imaging tool and by Lytle and Dines (1978) as a geophysical imaging tool. The technique has been actively developed for medical imaging (Isaacson 1986; Barber and Seager 1987; Yorkey et al. 1987). Early adaptations of the technique to the field of geophysics were by Pelton et al. (1978), Dines and Lytle (1981), Tripp et al. (1984), Wexler et al. (1985), Oldenburg and Li (1994), Sasaki (1992), Daily and Owen (1991), and $\mathrm{LaBrecque}$ et al. (1996a).

Here we describe briefly some of the important features of the 2-D algorithm used for ERT. The algorithm (see LaBrecque et al. 1996b) solves both the forward and inverse problems. The forward problem is solved using a finite element technique in two dimensions. The inverse problem implements a regularized solution that minimizes an objective function. The objective of the inverse routine is to minimize the misfit between the forward modeling data and the field data, and a stabilizing functional of the parameters. The stabilizing functional is the solution roughness. This means that the inverse procedure tries to find the smoothest resistivity model that fits the field data to a prescribed tolerance. Resistivity values assigned in this way to the finite element mesh constitute the ERT image. Although the mesh is of a large region around the electrode arrays, only the region inside the ERT electrode array is used in the calculations of 
moisture content and reported here, because the region outside the array is poorly constrained by the data.

To calculate the changes in the rock's electrical resistivity we compared a data set obtained after heating started, and a corresponding data set obtained prior to heating. One may consider subtracting, pixel by pixel, images from these two different conditions. However, this approach could not be used because the resistivity structure is three-dimensional. The finite element forward solver cannot generate a model that will fit the data, so the code chooses a solution with a poor fit. Our experience is that these effects can be reduced by inverting the quality:

$$
\frac{r_{a}}{r_{b}} \times r_{h}
$$

where:

$r_{a}$ is the measured transfer resistance after heating started,

$r_{b}$ is the transfer resistance before heating, and

$r_{h}$ is the calculated transfer resistance for a model of uniform resistivity.

This approach tends to reduce the effects of anomalies that do not satisfy the 2-D assumptions of the resistivity model because the 3-D effects tend to cancel in the ratio because they are contained in both terms $r_{\mathbf{a}}$ and $r_{\mathbf{b}}$.

\subsection{Changes in Moisture Content}

Resistivity of the rock is influenced by changes in moisture content, porosity, cation exchange capacity, solutes in the pore water, and temperature. In the following analysis we assume that only moisture content and temperature are important. An increase in temperature or moisture causes a resistivity decrease. However, near the heater there may be regions where the increasing temperature and decreasing pore water resistivity is opposed by the rockmass drying, which increases the resistivity. Our goal in this section is to use the images of resistivity change near the heater, along with the measured temperature field and what is known of initial conditions, in the rockmass to estimate moisture change during heating.

To estimate moisture content changes, we need to account for both the effects of temperature, measured at many points by temperature sensors, and resistivity changes, measured by ERT. This is possible by either using laboratory data establishing the relations among moisture, temperature, and resistivity or by using a suitable model of electrical conduction in porous media. Roberts and Lin (1997b) have published data on the resistivity of Topopah Spring tuff as a function of moisture content. There is, however, limited (unpublished) data on temperature dependence (only below $95^{\circ} \mathrm{C}$ ) so that direct use of this data is not possible.

On the other hand, Waxman and Thomas (1974a,b) describe a model for electrical conduction in partially saturated shaly sands typical of oil reservoirs (intended for oil field data), which accounts for conduction through the bulk pore water as well as conduction through the electrical double layer near the pore surface (see also Vinegar and Waxman 1984). This model can predict temperature dependence of the resistivity, but several of the model parameters are empirically 
determined and not available for tuff. Roberts and Lin (1997b) suggest that the Waxman model provides reasonably good estimates of resistivity for saturations greater than 20\%. For saturations less than 20\%, their data shows that the Waxman Smits model substantially underpredicts the resistivity. We will use this model to account for the temperature effects on the resistivity changes and to estimate changes in rock saturation.

Waxman and Thomas (1974a,b) begin with a parallel circuit model for conductance:

$$
C=\frac{1}{F^{*}}\left(C_{W}+B Q_{v}\right)
$$

where:

$C$ is the conductivity or $1 / R$ where $R$ is the resistivity,

$\mathrm{F}^{*}$ is the formation factor or $\mathrm{f}^{-\mathrm{m}}$ where $\mathrm{f}$ is the porosity and $\mathrm{m}$ the porosity exponent,

$\mathrm{C}_{\mathrm{W}}$ is the pore water conductivity,

$B$ is the equivalent conductance of counterions on the double layer, and

$Q_{V}$ is the effective concentration of exchange cations.

The first term represents conductance through the bulk pore water while the second term is the conductance along the double layer. This expression can be modified for partially saturated media by realizing that the first term is just Archie's equation and $Q / S=Q_{V}$ where $S$ is the fractional saturation. In terms of resistivity, Equation 6-2 can be re-written as:

$$
R=\frac{R_{W} \phi^{-m} S^{1-n}}{S+R_{W} B Q}
$$

where:

$n$ is approximately 2 , the saturation index in Archie's modified equation, and $R_{\mathrm{W}}$ is the water resistivity.

Waxman and Thomas (1974a,b) reported results that suggest that $m$ is approximately equal to $n$. When $R_{W} B Q \gg S$, the electrical double layer is the primary conduction pathway. When $R_{W} B Q$ $<S$, the primary conduction pathway is through the open pore space.

We can use Equation 6-3 in ratio form in order to calculate resistivity changes in the form of resistivity ratios. When the primary conduction pathway is the through the water in the open pore space, the resistivity ratio can be calculated as:

$$
\frac{R_{a}}{R_{b}}=\frac{R_{w, a}}{R_{w, b}}\left(\frac{S_{b}}{S_{a}}\right)^{2}
$$


where:

$\mathrm{R}_{\mathrm{b}}$ and $\mathrm{R}_{\mathrm{a}}$ are the resistivities before and after heating started,

$R_{W, b}$ and $R_{W, a}$ are the water resistivities before and after heating, and

$\mathrm{S}_{\mathrm{b}}$ and $\mathrm{S}_{\mathrm{a}}$ are the saturations before and after heating started; we will refer to this case as model 1.

This equation implies that the temperature dependence of the resistivity change is proportional to the change in water resistivity caused by temperature increases.

When the primary conduction pathway is through the electrical double layer, the ratio form of Equation 6-3 simplifies to:

$$
\frac{R_{a}}{R_{b}}=\frac{S_{b}}{S_{a}} \frac{B_{b}}{B_{a}}
$$

where:

$\mathrm{B}_{\mathrm{b}}$ and $\mathrm{B}_{\mathrm{a}}$ are the equivalent conductances of counter-ions in the electrical double layer.

We will refer to this case as model 2 . This equation implies that the temperature dependence of the resistivity ratio is caused by changes in counter-ion conductance due to temperature changes. Comparing Equations 6-4 and 6-5, we see that the resistivity changes caused by saturation changes are largest for model 1 where the primary conduction pathway is through the pore space. We note that neither of these two models accounts for changes in water resistivity caused by rock/water chemical interactions. If chemical reactions cause changes in the concentration or types of ions in the water, or change the porosity due to mineral precipitation or dissolution, the estimated saturation changes will be in error.

We used the available temperature data to construct temperature maps along each ERT image plane. It is necessary to have a reliable temperature measurement for each area (each tomograph pixel) where we wish to calculate the saturation change.

The ERT images provide a measure of change from baseline resistivity $R$ (through the resistivity ratio). Equations 6-4 and 6-5 can be used to relate electrical resistivity changes to changes in saturation when the temperatures are known and the temperature dependence of $R_{W}$ and $B$ can be calculated.

Calculation of changes in volumetric water content requires rock porosity and initial saturation values. Initial values of block saturation were calculated from neutron logs in four boreholes. The water saturation from all of these holes agrees and shows values ranging from about $60 \%$ to $90 \%$ by pore volume (Wilder et al. 1997). We assume a uniform initial saturation of $75 \%$ and a porosity of $12 \%$.

Since the magnitude of RwBQ (see Equation 6-3) is changing in space and time, we have chosen to estimate the changes in saturation by using both model 1 and 2 . This approach should provide bounds to the domain of possible saturations that may be present. However, there is some reason 
to believe that welded tuff should show behavior closer to model 2 than to model 1 . This can be seen by assuming average values of cation exchange capacity for welded tuff of about 3 meq/100 $\mathrm{g}$, porosity of 0.10 (porosity is used to calculate Q), and $\mathrm{Rw}=39 \mathrm{ohm} \mathrm{m}$ at $25^{\circ} \mathrm{C}$ (resistivity of $\mathrm{J}$ 13 water). For these values $\mathrm{RwBQ}$ is about 23 at $25^{\circ} \mathrm{C}$, and it increases with temperature. Since $\mathrm{S}$ is bounded by 0.0 and 1.0 , then RwBQ is $>\mathrm{S}$, and the primary pathway for conduction is the electrical double layer. However, if the cation exchange capacity, porosity, or water resistivity varied significantly across the ERT image plane, it is possible that model 1 results are closer to reality.

In fact, we believe that model 1 is more representative of the rock mass for two reasons. First, the saturation estimates based on this model are in better agreement with those of the neutron log where that data are available. Second, the saturation estimates based on model 2 occasionally predict $S>1.0$, which, of course, are nonphysical.

\subsection{ERT Results}

ERT data were taken from four planner arrays in the block before the heaters were turned on. Two horizontal planes of electrodes were arranged on the surface, azimuthally around the block. One plane was approximately $1.25 \mathrm{~m}$ above the heater plane and the other was approximately $1.25 \mathrm{~m}$ below the heater plane. The two other planes were vertical, dividing the block into four quadrants. The vertical planes were sampled from electrode arrays on the side of the block and a single vertical array at the center of the block. The electrode arrangement is shown in Figure 6-6.

At the intersection of these ERT planes, there should be agreement, and this is the case for the vertical planes because they share a common electrode array along that intersection. Such agreement is not very good for the intersection of the vertical and horizontal planes. Complete agreement cannot be expected in these cases for two reasons.

First, the spatial distribution of sensitivity and resolution is different for the vertical and horizontal planes because of the difference in how they are sampled by the electrodes. Therefore, the two planes will tend to resolve features differently. The common electrode array in the vertical planes produces good resolution where they intersect, and this is why they tend to agree. In general, two-sided sampling, as in the vertical planes, leaves a low-sensitivity region along the top and bottom. On the other hand, the all-around sampling of the horizontal planes leaves a lowsensitivity region in the center, where we shall see that agreement with the vertical planes is poorest.

Second, in all of these images we are trying to reconstruct a 3-D target using a model that is strictly 2-D. This means that the ERT algorithm finds the best finite element model for the resistivity structure of the block that fits the data within a given criterion. Unfortunately, it can only choose from models where the resistivity is constant orthoganal to the image plane. It picks the best model, but it cannot be the correct model. That model chosen for two different planes will be different, especially if the planes are perpendicular as the vertical and horizontal planes are here.

We note here that some of the resistivity images reconstructed late in the experiment (and the moisture changes inferred from them) are questionable because of the sparse data. As the 
rockmass dehydrated and the resistivity increased dramatically, the data quality declined. Fewer usable data results in a poorly constrained reconstruction that might look smeared or washed out. This is particularly noticeable in the vertical planes beginning early in 1998.

The results of interpreting the changes in the resistivity tomographs in terms of moisture content changes are shown in Figures 6-7 and 6-8. Blank image planes indicate data that did not converge to an ERT solution or where no data were collected. The 2-D orthogonal planes shown in Figure 6-7 and 6-8 don't provide a full description of the 3-D block, but they do show considerable detail that would be otherwise unavailable with only point temperature data or even with one-dimensional neutron data.

We will first discuss the results in terms of conceptual behavior-rock drying, condensate accumulation in fractures, and loss of condensate out of the block. The goal is to determine the impact that heterogeneieties (such as fractures) have on the distribution and fate of water in the block. We begin with a discussion of the horizontal planes.

\subsection{Horizontal Planes}

The obvious result shown in the horizontal planes (see Figure 6-7) is that changes in moisture content initially are very small and increase in magnitude and extent as the test proceeds. Notice, however, that there are some asymmetries between the two cases. Through June 25, 1997 (117 days into heating) the upper plane (plane above the heater elevation). shows significantly less change from initial conditions than the lower plane. As early as April 22, 1997 (53 days into heating), a strong and compact wetting anomaly appears below the heaters. It remains visible at May 22, 1997 (30 days later) but then disappears from subsequent images until November 19, 1997, when it reappears and persists to the end of the test on March 19, 1998. We believe that this feature resulted from a major fracture, or fracture system, intersecting the image plane and that in April and May condensate from the heated region found its way to this fracture and moved by gravity down the conduit and out of the block. Once the source is drained, the anomaly goes away. The source of the water from November 1997 through March 1998 is less certain but may be condensate from above the heaters that were turned off on March 10 . As the thermal field collapsed, condensate may have been able to drain through the heater plane and again into this conduit, reestablishing the anomaly late in the test.

There are only two other strong indications of saturation increasing in these planes. The one on August 26, 1997 (perhaps persisting to September 24, 1997) near the north edge of the lower image plane also behaves like a water-wet fracture - spatially compact, developing quickly and then going away. The other anomaly, on February 24, 1998, in the northwest corner of the upper image plane, does not look like the draining of water through a fracture. We do not know the cause of this feature.

The other characteristic of images in both planes are zones of drying that start to appear as early as May 27, 1997, in the lower plane. Clearly, this drying is a result of the high temperatures, but the effects recorded in these images appear different above and below the heater plane. Above the heater, drying appears later and appears to form anomalies with rounded outlines. Below the heater, the anomalies appear as early as May, are more localized, and are linear in shape. The linear shape may be caused by the matrix drying around a planar fracture that cuts through the 
image plane. A good example of this is the feature running diagonally from the southwest corner to the northeast corner between May 22, 1997, and March 19, 1998. Even though the anomaly changes character throughout this period, it probably arises from the same structure in the rockmass $-\mathbf{a}$ fracture or system of fractures.

Other anomalies of dehydration occur in both planes; some are quite prominent and some are minor. They all support the notion that the dehydration front is steadily advancing from the heater plane into both image planes but that the process is heavily controlled by rockmass heterogenieties.

\subsection{Vertical Planes}

The vertical image planes (see Figure 6-8) intersect the heater plane and even more clearly delineate the effects of heating the block. As expected, the most obvious feature is the drying zone surrounding the heaters. Although drying is not clearly associated with the heaters until May 22, 1997 (about 83 days into heating), once formed, the dry zone is the dominant feature in either image plane all the way through the last data of cool-down (March 19, 1998). Drying was not observed early in the test because the temperatures were not hot enough. It takes a lot of energy to remove the pore water from this rock because of the extremely low hydraulic permeability and high suction potential of the rock matrix. Initial evidence of drying can be observed adjacent to the heaters. On May 22 and June 25, 1997, in the south-to-north plane, zones of drier rock are centered on two heaters. After June 25 these individual zones have coalesced into a single continuous dehydrated zone that grows in size, with some locations eventually losing $85 \%$ of the original water content (saturation ratio 0.15 ). This large dry zone around the heater persists until late cool-down in March 1998, when it appears to be breaking up.

Once formed, the heater dry zone is not a smooth planer anomaly reflecting the heater geometry. Instead it is very irregular in shape with many appendages. There is also a tendency for the dry zone to be relatively flat on top and bottom early in the test but convex on top and concave on the bottom late in the test. We do not have a hypothesis for this behavior. We believe that this rugose image of the heated zone is the result of rock heterogeneities such as fractures. Consider, for example, the part of the block located above the heaters in the western half of the west-to-east plane. By May 22, 1997, there is a vertically oriented dry zone forming between the heater plane and the top of the block. Four months later (September 24, 1997) it is a very linear anomaly that looks a lot like dehydration along a vertical fracture intersecting both the heater plane and the image plane. In Figure 6-9, the interpolated temperature field is superimposed on the July 23, 1997, and the January 23, 1998, saturation images. Notice that this linear-looking zone in the ERT reconstruction corresponds to a high temperature anomaly—additional evidence for drying along a vertical fracture or fractured zone. This figure also demonstrates an approximate correspondence between the dehydration as defined by the ERT data and the $100^{\circ} \mathrm{C}$ isotherm.

During one month of high block temperatures-August 26, 1997, to September 24, 1997-there is a saturation anomaly adjacent to the dryer feature discussed above. We believe that this combination could be from drying along a nearly vertical fracture zone and wetting of adjacent rock that may be evidence for a heat pipe effect. 
Rain fell twice during the test: on June 12, 1997 (Day 104) and on September 2, 1997 (Day 186). The amount and duration of rain that fell in each of these events is unknown so that the more important data, how much rainwater got through the covering and onto the block itself, is also unknown. However, the temperature data offer good evidence that some rainwater reached the block during the June 12,1997, event because on that date a RTD $5 \mathrm{~cm}$ above the heater registered a sharp drop in temperature from about $120^{\circ} \mathrm{C}$ to $100^{\circ} \mathrm{C}$. No such temperature drop was observed below the heaters. This temperature data is consistent with rainwater moving quickly along a fracture from the top to deep within the block.

The RTD that experienced the temperature excursion possibly linked to the rain infiltration is only about $50 \mathrm{~cm}$ from the west-east ERT image plane and $25 \mathrm{~cm}$ from the south-north ERT plane. In the June 25, 1997, data there is no clear evidence of increased saturation at this location in the west-east plane. In the closer south-north plane, however, the projection of that RTD location onto the plane correlates precisely with the bottom of the strong moisture anomaly in the image (see Figure 6-8). Unfortunately, this identification is not so simple, because this same anomaly appears in the March 22,1997, image, before the rainfall, and appears to evolve in the July, August, and September images, after the rainfall. It is possible that these ERT anomalies represent a region of fractured rock where both condensate (in May) and meteoric water (in June) collected and that the July, August, and September images show this trapped moisture being driven out the top of the block (September 24, 1997 image).

\subsection{Comparison of ERT with Other Data Sets}

\subsection{Comparison of ERT and Fracture Distribution}

The ERT images show ample evidence that the block is behaving like a heterogeneous system and that the most obvious source of heterogeneity is fracturing. Of course, the block is heavily fractured, and those fractures were mapped at the five exposed surfaces. Unfortunately, a search for fractures that might be responsible for the ERT anomalies is complicated by two problems. First, fractures are not planar so that the surface expression may be only a guide to the fracture location inside the block. Second, the fracture density is so high that almost any anomaly can be matched with a fracture, making such an association of little value.

Undaunted by these facts, we made an attempt to see a correlation between fractures and anomalies in the ERT images. We chose ERT anomalies that were large in magnitude and persistent over several months and tried to match these with fractures that mapped continuously on two or more faces of the block and that appeared approximately planar. We could not see a consistently convincing correlation between the surface fractures and ERT image anomalies.

\subsection{Comparison of ERT and Neutron Logs}

Neutron logs were made in five vertical holes in the block, and from this data the moisture content was calculated along each borehole at 12 times during heating. In Figure 6-10 we compare the ERT moisture estimates with the neutron log data from the vertical borehole nearest to the west-to-east vertical ERT plane. Of course, the main feature in both data is the development of a large dehydrated zone around the heater that grows from $1 \mathrm{~m}$ to $2 \mathrm{~m}$ thick in the six months covered by the data. Notice that from both of these measurements a maximum 
change in water content near the heaters is calculated to be about $70 \%$. This close agreement is significant because the neutron log and ERT are two completely independent measurements.

There are also differences in the inferences about moisture content from the two methods. For example, the neutron data seems to indicate a slightly thicker dry zone around the heaters. Less subtle, however, are the comparisons outside the heated region. The neutron log does not indicate any significant wetting above the baseline condition anywhere in the block. On the other hand, ERT points to several zones of enhanced wetting. This difference implied by the two results is important because the fate of condensate water is important to understanding the water budget of the block during the test.

We believe that some condensate is stored in the block. However, while the neutron probe is insensitive to it, ERT is probably overly sensitive to its presence. This is because of how the water is distributed in the rockmass. As a matrix block dries, the steam moves into a fracture, then down the pressure gradient along the the fracture until it reaches the dew point, where it condenses. Since the matrix there is already nearly saturated (typically $80 \%$ ) and also has a very low permeability (typically a few $\mu$ Darcey), the condensate remains in the fracture aperture. Because the fracture porosity is small compared to the matrix porosity, the neutron probe correctly measures very little increase in moisture content at the location of this condensate. On the other hand, electric current can easily sample fracture networks because, when wet, they act as a network of highly conducting pathways. Therefore, ERT is overly sensitive to this small volume fraction of water and may overestimate the saturation when it is present in a fracture network.

Both methods are sensitive to dehydration of the block. The heat load drives large volumes of vapor from the pores of matrix blocks. This changes the amount of water in volumes comparable to the integration volume of the neutron probe so that this log is sensitive to the change. Matrix water loss also affects electrical current flow that happens along paths through the connected pore water in the matrix.

In Figure 6-10, the neutron log shows slight drying relative to baseline in the top two meters of the block. This may be a response to the vertically oriented drying zone seen in the ERT images only $50 \mathrm{~cm}$ from the neutron logging hole.

\subsection{Summary and Discussion}

We believe that the ERT images, along with the other data we have discussed, support a simple and physically realistic conceptual model for the TH behavior of the system during the LBT.

Dehydration around the heaters is progressive, producing first a small hot zone that grows larger and drier as time progresses. This is the principal process observed and is driven by the imposed heat load. This thermally driven dehydration is the central theme of Figure 6-8. The effect is approximately one dimensional, evolving with time along the vertical axis of the block. However, deviation from a uniformly one-dimensional moisture distribution is significant and appears to be controlled by heterogeneity in the block-probably fractures. For example, the heated zone is not imaged by ERT as a strictly planar anomaly. Even more obvious is the especially strong feature in the west-to-east plane forming as early as May 1997 and persisting to 
February 1998. This is a large, strong, and persistent dry zone extending from the heater plane upward to the top of the block.

Water in matrix pores vaporizes as temperature rises. Evaporation must occur even below boiling but becomes especially rapid at the boiling point that is about $96^{\circ} \mathrm{C}$.

Water vapor first leaves pores adjacent to fractures because the pressure gradient is steepest there. The drying front then progresses into the matrix block until the whole block is in equilibrium between the suction potential and the vapor pressure. ERT sees many linear features of high resistivity that are likely dehydration along fractures and systems.

Once in the fracture, the water vapor is highly mobile and moves quickly down the pressure gradient. Some of this vapor will exit the block, especially through the top because it is not sealed like the block sides.

That portion of the vapor that remains in the block will move down the pressure gradient, losing heat as it goes, until the dew point is reached, at which point it condenses. This condensate rapidly fills the fracture aperture. Such saturated fractures provide a network of conducting pathways for electrical current in the rock that weren't initially present, and ERT sees this network as an increase in electrical conductivity. Interpretation of this change results as an unnecessarily large moisture increase. This water is detected as a small perturbation by neutron probe because the fracture porosity accounts for a small part of the rock, and the tool is insensitive to it because of the volumetric averaging of the measurement.

The behavior and fate of this condensate that forms in fractures is the key to repository performance, because it is this water, if it can seep back into the emplacement drift, that is most likely to determine the useful lifetime of the canisters-the longer this water can be kept away from the canisters, the longer they will survive.

There are several potential fates for the condensate:

- This water may drain out of the system-through the bottom of the block. This is especially likely below the heaters. Notice the moisture anomaly below the heater near the center of the west-to-east plane in July 1997. It becomes weaker in January 1998, as though it is a fracture draining. However, drainage may also occur for water above the heaters, especially during the early part of the test before the boiling isotherms coalesce from individual heaters. In fact, even a well-developed boiling isotherm may be overwhelmed by large water volumes moving in fractures. This possibility is equivalent to the seepage of water back into the emplacement drift of the repository. Rainwater may have provided such an event on June 12, 1997, and August 2, 1997. Notice that in the July 1997 south-to-north plane there is a linear feature of high saturation-rain water and/or condensate - that is poised above a gap in the dehydration zone forming around the heated plane. The arrangement suggests fracture drainage may be keeping this zone near the heaters from drying. 
- This water may participate in a heat pipe. During August and September of 1997, directly above the heaters, there is a persistent wet anomaly adjacent to a persistent dry anomaly in the west-to-east plane. This may be the ERT signature for a heat pipe.

- This water may remain immobile, held by capillarity. There are several persistent wet zones imaged in Figure 6-8 that may exemplify this effect.

- This water may be imbibed into the matrix by the forces of capillary suction. (This seems unlikely to be an important fate of free water because of the short lifetime of this test because of the very small permeability of the matrix and the relatively low suction potential of the initially wet matrix.)

\subsubsection{Neutron Logging}

\subsection{Pre-Cut and Post-Cut Moisture Contents}

Scoping calculations determined that a minimum initial moisture content of about $50 \%$ was required for the block to be suitable for the test of coupled THMC processes. Neutron logging was conducted in four vertical boreholes E2, E3, E4, and E9 (as shown in Figure 2-7 of Wilder, et al. [1997]) before (in December 1993) and after (in March 1994) the sawing, but before the drilling of any horizontal holes. The neutron logging was conducted in bare holes without Teflon lining. The neutron tool was calibrated in a 3.81-cm-diameter hole without the Teflon liner and cement grout for the pre-cut and post-cut measurements. Figure 6-11 shows the water saturation as a function of depth, as determined by neutron logging, in hole E4 in December 1993 (pre-cut) and March 1994 (post-cut). The water saturation determined in other holes agrees well with the values shown here. The background moisture saturation levels were determined to be about 60 to $80 \%$, for a laboratory-determined porosity of about $11 \%$ (as shown in Figure 6-11). As shown in Figure 6-11, sawing of the block boundary using water was found to have no significant effect on the moisture content of the block (Lin et al. 1995).

Neutron logging was performed again to estimate the initial moisture content of the block after the installation of the instruments was completed, but before the heating was started. The preheat baseline neutron logging was conducted in all holes with the Teflon liner and the cement grout to seal the annular space between the borehole wall and the liner. The result of the pre-heat baseline moisture content will be presented in the following section.

\subsection{Pre-Heat Baseline Moisture Content}

As mentioned above, the pre-heat baseline moisture content in all neutron holes (both the five vertical and the ten horizontal holes) was measured after the completion of the installation of instruments (pre-heat) in February 1997. The pre-heat baseline moisture content was determined with both the Teflon liner and the cement grout in place. The pre-heat moisture content was determined to establish the baseline so that the effect on its moisture content of heating the block can be determined. The neutron tool was calibrated in a 3.81-cm-diameter hole with the Teflon liner/grout assembly exactly the same as in the neutron holes of the LBT. It was determined that the Teflon liner/grout assembly may have changed the moisture content by no more than 4 to $6 \%$ 
of the determined value. This is not surprising because the neutron holes in the LBT are designed in such a way that the thickness of the annular cement grout is minimal, only about $0.3 \mathrm{~cm}$.

Figures 6-12 through 6-16 show the pre-heat baseline fraction volume water content in the five vertical holes TN1 to TN5 as a function of depth from the top of the block, respectively. Generally, the initial moisture content in those holes increased with depth and ranged between 0.08 and 0.1 . A region at about $0.5 \mathrm{~m}$ depth in TN1 to TN3 had a fraction volume water content greater than 0.1 , which may be caused by a horizontal fracture intersecting those holes. There is a region at about $4 \mathrm{~m}$ depth in TN5 where the initial moisture content was greater than 0.14 . The average porosity of the core samples of the large block was determined to be $10.4 \pm 1.3 \%$, with a range of 8.2 to $13.6 \%$ (Table 3-4). The fraction volume water contents shown in Figures 6-12 through 6-16 correspond to a range of saturation levels between 77 and $96 \%$. The high moisture content at about 4-m depth in TN5 may be caused by a localized greater-porosity rock. As shown in Table $3-5$, the porosity determined by mercury porosimetry reached $20 \%$ at one location.

Figures 6-17 to 6-22 show the baseline moisture content in holes NN1 to NN6 as a function of depth from the north face of the block. Generally, the initial fraction volume water content in these holes ranged between 0.04 and 0.1 . The moisture content increased with respect to depth quickly in the first $0.5 \mathrm{~m}$ from the collar, then remained almost constant.

Figures 6-23 to 6-26 show the initial moisture content, in terms of fraction volume water, in holes WN1 to WN4 as a function of depth from the west face of the block. The initial fraction volume water content in these holes ranged from about 0.04 to about 0.11 . Similar to the north face horizontal holes, the baseline moisture content first quickly increased with depth, then remained almost constant in the deeper part of the holes.

As mentioned earlier, the purpose of conducting the neutron logging was to study the effect of heat on the moisture content in the block. The neutron counts of the pre-heat logging were subtracted from the in-heat neutron counts, and calibration data were used to convert the difference in the neutron counts into the difference in volume water content. The variation in the water saturation level can be determined by dividing the difference fraction volume water content by the porosity of the rock mass. However, for this report, the difference fraction volume water content, instead of water saturation, during the heating phase of the LBT is presented. The baseline fraction volume water presented so far will help determine the changes in the absolute water content, if necessary.

\subsection{Heating Phase and Cooling Phase Moisture Content}

The neutron logging results in both the heating phase and the cooling phase will be presented in this report. The neutron logging was conducted in the five vertical holes (TN1 to TN5), six horizontal holes from the north face (NN1 to NN6), and four horizontal holes from the west face (WN12 to WN4). The location of these neutron holes is given in Section 3.2 and in Appendix A of this report. The $x-y-z$ coordinates of the collar of those neutron holes are shown in Table 6-1 to facilitate the discussion of the moisture variation in each hole. The origin of the coordinates is the south-west corner of the top of the block, and $x$ and $y$ directions are east-west and northsouth respectively. For reference, the heater plane was at $\mathrm{z}=-2.743 \mathrm{~m}$. During each of the neutron logs, neutron counts were obtained in each hole at intervals of $10 \mathrm{~cm}$, starting from the bottom of 
the hole. The raw neutron counts, the location of measurements in each hole, and the converted difference fraction volume water content are all included in the spreadsheets in the data base at.

LLNL, as well as in the TDMS with DTN LL971204304244.047 (Table 1-1). The difference fraction volume water content in each hole during the test are presented in this section in graphical form, so that the process of the moisture movement can be analyzed. The difference fraction volume water was calculated by subtracting the baseline fraction volume water from that measured during the test. The baseline fraction volume water content in each hole was presented in the previous section. The fraction volume water content can be used to calculate the water saturation by dividing it with the porosity of the rock.

Table 6-1. X-Y-Z Coordinates of the Collar of the Neutron Holes

\begin{tabular}{|c|c|c|c|}
\hline Hole \# & $\mathbf{X}(\mathbf{m})$ & $\mathbf{Y}(\mathbf{m})$ & $\mathbf{Z}(\mathbf{m})$ \\
\hline TN1 & 1.219 & 0.61 & 0 \\
\hline TN2 & 1.829 & 0.61 & 0 \\
\hline TN3 & 1.219 & 1.2119 & 0 \\
\hline TN4 & 2.438 & 1.829 & 0 \\
\hline TN5 & 1.219 & 2.438 & 0 \\
\hline NN1 & 2.134 & 3.048 & -0.914 \\
\hline NN2 & 2.134 & 3.048 & -1.981 \\
\hline NN3 & 2.134 & 3.048 & -3.81 \\
\hline NN4 & 0.914 & 3.048 & -0.914 \\
\hline NN5 & 0.914 & 3.048 & -1.981 \\
\hline NN6 & 0.914 & 3.048 & -3.81 \\
\hline WN1 & 0 & 2.134 & -0.762 \\
\hline WN2 & 0 & 2.134 & -1.676 \\
\hline WN3 & 0 & 1.676 & -3.962 \\
\hline WN4 & 0 & 0.914 & -1.676 \\
\hline
\end{tabular}

NOTE: With respect to the southwest corner of the top of the block.

Figures 6-27 to 6-36 show the difference fraction volume water in holes TN1 to TN5 as a function of depth from the top of the block. The neutron results in each hole are divided into two figures so that the figures are not too crowded. The portion of TN2 below about $3.8 \mathrm{~m}$ from the top was not available for the logging (Figures 6-29 and 6-30). In these figures, the positive fraction volume water means gaining moisture content; the negative fraction volume water means losing moisture content. Generally speaking, these figures show that a well-defined dryout zone developed since the 48 days of heating at the heater plane, which was at about $2.74 \mathrm{~m}$ from the top of the block. One exception was TN4, in which the dryout zone did not develop until sometime between the 48 th day and the 60 th day of heating. The dryout zone widened with time, and the extent of the drying also increased with time, due to the continuous heating. The widths of the maximum dryout zones, as measured at the half of the extent of the dryness, were 1.66, $1.5,1.69,1.49$, and $1.59 \mathrm{~m}$ for TN1 to TN5 respectively. It is fair to say that the width of the dryout zone is quite uniform. There was not much change in the extent of the dryness since Day 361 of heating. In TN4, however, there was a decrease of the dryness by about 0.05 after Day 361 , and it remained unchanged subsequently. There were some variations in the shape of the tip 
of the dryout zone as shown in these figures. The dryness in those five vertical holes ranged from -0.07 to -0.09 fraction volume. Those variations among the five vertical holes illustrate the effect of heterogeneity in the block on the movement of moisture. Those figures do not show significant rewetting during the cool-down phase, i.e., after Day 376. The measured change in the moisture content agreed well with what had been predicted in the scoping TH calculations, as shown in Figure 3-3 of the Large Block Test Status Report (Wilder et al. 1997).

Figures 6-37 to 6-48 show the difference fraction volume water content in the six horizontal holes drilled from the north face of the block. Again, the neutron results in each hole are divided into two figures so that each figure is not too crowded. Generally, the variation of the moisture content was uniform across the block. The neutron results in those holes show decrease in moisture content with time, as expected. The variation of the moisture content in those holes depends on the vertical location of the hole. Holes NN1, NN3, and NN4 showed little change in the moisture content, about -0.02 in the entire test period. The moisture content in holes NN2 and NN5 showed the extent of dryness comparable to that near the heater plane in the vertical holes, to about -0.07 fraction volume. This is expected because, as shown in Table 6-1, NN2 and NN5 were the closest, among those six holes, to the heater plane, only about $0.76 \mathrm{~m}$ above the heater plane. The neutron results in NN6 showed some abnormal responses relative to the other holes. The measurements on July 8, 1997, November 6, 1997, and January 29, 1998, showed significant increase in the moisture content. This was probably related to the TH events shown by the temperature fluctuations, as discussed in Section 5.4.3. This seemed to be isolated to this hole; therefore, the moisture was probably related to a fracture zone in the block. Careful examination of Figures 6-40, 6-42, and 6-44 indicated that there were some indications of moisture refluxing. For example, the moisture content measured on July 8, 1997, was greater than that measured on the previous days in some locations in those holes. However, the amplitude of the variation was very small. Again, as mentioned above, there was no indication of re-wetting based on the moisture measured in those horizontal holes.

Figures 6-49 to 6-56 show the moisture content measured in the four horizontal holes drilled from the west face of the block, WN1 to WN4. Similar to the horizontal holes from the north face, the variation in the moisture content in these four holes from the west face showed uniform decrease of the moisture with time across the block. The extent of the moisture decrease depended on the vertical location of the hole. Generally, the vertical distance of these holes from the heater plane was greater than that of those holes from the north face. Therefore, it is expected that the variation in the moisture in the WN holes would be less than that in the NN holes. Figures 6-55 and 6-56 show an abnormal case. In this hole, WN4, there was a zone at about 1.3 $\mathrm{m}$ from the collar where significant gainings of moisture were measured on Days 74, 88, and 103 (May 13, 1997; May 27, 1997; and June 11, 1997). The moisture seemed to have refluxed during this period. This period was before the $\mathrm{TH}$ event as registered by the temperature measurement. The moisture might have been the condensed water channeled to the neutron hole by a fracture system. Drying began to develop on July 8, 1997 (Day 130) in a zone at about $0.8 \mathrm{~m}$ from the collar of this hole. This dryout zone continued to develop throughout the remainder of the test. This hole was in parallel with the heaters and was about $1.07 \mathrm{~m}$ above the heater plane. The distinguished dryout zone was probably caused by a fracture zone that intersected with the hole, which may have provided a flow path to facilitate the dryout. Those neutron results show no significant rewetting in the cool-down phase. 
In summary, the moisture content measured by neutron logging showed that the moisture movement in the block was almost one-dimensional. A well-defined dryout zone was developed at the heater plane. The neutron results did not show significant rewetting during the cool-down phase. Fractures have important roles in the localized movement of the moisture, and a discrete fracture model may be needed to analyze the process.

\subsection{THERMAL-HYDROLOGICAL ANALYSIS}

\subsubsection{In-Test Thermal-Hydrological Model}

Three-dimensional TH analysis of the LBT during the early stage of the test was reported in Section 3.1.3 of the LBT Status Report (Wilder et al. 1997). The calculations modeled the asbuilt block geometry. In the calculations, the block was heated at $450 \mathrm{~W}$ per heater for six months (182.5 days), after which the heaters were turned off, and the cool-down was simulated for an additional six months. Equivalent Continuum Model (ECM) was used in the model calculations, and the power outages were included. Seven model calculations were conducted for the in-test TH analysis. The first case was designated as Case A; the rest were called Case 1 to Case 6. Case A modeling used rock properties from the Reference Information Base (RIB) (DOE 1995), except that the bulk air-permeability was adjusted to approximate the median value obtained by the pre-heat single-hole air-injection (Figure 6-1). The Case A rock properties are shown in Table 6-2 as the TSw2 unit. The bulk permeability value of $9.87 \times 10^{-14} \mathrm{~m}^{2}$ is the value in the RIB; the value of $3.3 \times 10^{-15} \mathrm{~m}^{2}$ was the median value of the measured permeability (Figure 6-1). The initial moisture saturation for Case A was assumed to be $92 \%$. Case A did not include the power outage information.

Figure 6-57 shows one example of the calculated temperature and liquid saturation distributions on the block in Case A modeling. Figure 6-57 agrees well with the preheat predictions, as shown in Figure 3-26. As indicated in Section 5.3.2 and Section 6.1.2.2.3, the predictions agree well with the measured data.

Case 1 through Case 6 used the rock properties listed in Table 6-2, with various initial water saturation values. These modelings were to investigate the effects of rock property and the initial water saturation on the predicted temperature. Cases 1-3 used the TSw2 properties in Table 6-2 with initial water saturation of $92 \%, 70 \%$, and $65 \%$ respectively. Cases 1 and 2 used the greater bulk permeability in Table 6-2 for TSw2; Case 3 used the smaller bulk permeability value for TSw2 in Table 6-2. Cases 4-6 used the tsw34 rock properties in Table 6-2 with initial water saturation of $92 \%, 70 \%$, and $80 \%$ respectively. For a node at the heater level, adjacent to the heater midpoint and $35 \mathrm{~cm}$ from the center heater, the temperature history of those models show substantially higher temperatures for TSw2, compared with tsw34 (Figure 3-7 of Wilder et al. 1997). This difference may be partially explained by the lower permeability of TSw2, relative to that of tsw34, as shown in Table 6-2. The temperature for a smaller bulk permeability was greater than that for a model with greater bulk permeability; it was also true for the initial water saturation level. Cases 2 and 3 showed no sign of boiling due to the smaller level of initial water saturation. Case 1 showed some signature of rapid evaporation but not as rapid as boiling. Case 5 showed signatures of boiling. Case 6 showed prolonged boiling. The temperature in Case 4 stayed at boiling for the entire heating period of modeling, 180 days. The differences among Cases 4-6 were due to different initial water saturation. 
Table 6-2. Hydraulic and Thermal Properties of Rock Units

\begin{tabular}{|l|c|c|}
\hline \multicolumn{1}{|c|}{ Properties } & TSw2 & tsw34 \\
\hline Bulk permeability $\left(\mathrm{m}^{2}\right)$ & $9.87 \times 10^{-14}$ & $1.59 \times 10^{-12}$ \\
& $3.30 \times 10^{-15}$ & \\
\hline Matrix permeability $\left(\mathrm{m}^{2}\right)$ & $4.00 \times 10^{-18}$ & $1.01 \times 10^{-15}$ \\
\hline Fracture permeability $\left(\mathrm{m}^{2}\right)$ & $8.33 \times 10^{-10}$ & $6.55 \times 10^{-9}$ \\
\hline Matrix van Genuchten a (1/Pa) & $6.40 \times 10^{-7}$ & $7.72 \times 10^{-7}$ \\
\hline Fracture van Genuchten a (1/Pa) & $1.34 \times 10^{-3}$ & $6.86 \times 10^{-4}$ \\
\hline Matrix porosity & 0.11 & 0.11 \\
\hline Fracture porosity & $1.19 \times 10^{-4}$ & $2.34 \times 10^{-4}$ \\
\hline Matrix van Genuchten b (1/Pa) & $3.96 \times 10^{-6}$ & \\
\hline Fracture van Genuchten b (1/Pa) & 1.47 & 1.47 \\
\hline Wet thermal conductivity $(\mathrm{W} / \mathrm{m} \cdot \mathrm{C})$ & 3.00 & 1.48 \\
\hline Dry thermal conductivity $(\mathrm{W} / \mathrm{m} \cdot \mathrm{C})$ & 2.10 & 2.33 \\
\hline Specific heat $(\mathrm{J} / \mathrm{kg} \cdot \mathrm{C})$ & 2.10 & 1.56. \\
\hline
\end{tabular}

\subsubsection{Post-Test Analysis}

\subsubsection{Purpose}

The Thermal Test TH AMR (Section 6.2.4 of CRWMS M\&O 2000c) reported post-test modeling of the TH processes in the LBT with NUFT 3.0.1s (STN 10130-3.0.1s-00) code using the implicit dual-permeability model with the active fracture concept. Two TH property sets were used in the model analyses. Those are the drift-scale (DS) and the mountain-scale (MS) property sets, as shown in Table 5-1 (extracted from Table 7 of CRWMS M\&O 2000c), which includes the thermal properties of the insulation materials on the block sides. This section compares the results of TH calculations with the liquid-phase saturations measured in the block. Simulation results using the DS rock property set are first compared with field data to evaluate how well they match. Simulation results using the DS property set are then compared to results generated using the MS property set, and the two sets of results compared to field data. It is understood that the LBT was not designed to evaluate various TH property sets used in the YMP. However, it is interesting to see how well those property sets work.

\subsubsection{Numerical Model}

The model geometry, boundary and initial conditions, and heater power history are identical to those presented in Section 6.1.4 of the Thermal Test TH AMR for the DS property set. The DTN for heater power histories used in the analysis is LL980918904244.074.

\subsubsection{Rock Property Data Sets}

Hydraulic and thermal properties of the tsw34 unit of the DS base case property set (DTNLB990861233129.001) and the MS base case property set (DTN LB997141233129.001) were used in model calculations (Table 5-1). The tsw34 unit was used because the hydrogeologic 
unit of the LBT area is equivalent to the tsw34 model unit (Tptpmn) of the site-scale unsaturated zone (UZ) flow model. The only difference between the DS and MS properties for the tsw34 unit is a higher fracture permeability of $1.70 \sqcup 10^{-11} \mathrm{~m}^{2}$ for the MS versus $2.76 \square 10^{-13} \mathrm{~m}^{2}$ for the DS.

\subsubsection{Drift-scale Simulation Results Versus Field Data}

Figure 6-58 shows the liquid-phase saturation profile along TN3, a vertical borehole used for neutron probe measurements of water content. Model results are compared to liquid-phase saturations measured by neutron probe (DTN LL971204304244.047, LL980919304244.075). The field measurement times, 103, 361, and 501 days are compared at model times of 100, 365, and 500 days. The small differences between model and field times should have a negligible effect on the comparisons since saturation changes develop relatively slowly. The simulated dryout zone develops slowly and remains smaller than the field zone at all three times. At about 100 days, the model dryout zone is poorly developed, with no point on the profile having a liquid saturation less than 0.375 , half the initial saturation. Note that a locale where the liquid saturation is less than half the initial value is defined to be included in the dryout zone. In contrast, the field dryout zone is well developed at 100 days, showing a thickness of about $0.75 \mathrm{~m}$ and a minimum liquid saturation of about 0.12 . The field data also show a distinct recondensation zone approaching full saturation about $1.3 \mathrm{~m}$ below the heater horizon, and a small recondensation zone about $0.5 \mathrm{~m}$ below the upper surface of the block. The field data show sharp fluctuations in saturation not observed in the model results. This difference is probably due to heterogeneities in the TH properties of the fractured rock not incorporated into the property set. At about 365 days (10 days before power shutdown) the model dryout zone is fully developed, with a thickness of $1.4 \mathrm{~m}$, but continues to lag the field zone that now has a thickness of $1.9 \mathrm{~m}$. The recondensation zones above and below the heater horizon are still evident at 365 days, and the geometry is very similar to that observed at 100 days. At 500 days (125 days after power shutdown), the model dryout zone thickness is $1.25 \mathrm{~m}$, a reduction of only $11 \%$ from the 365-day thickness, and the field dryout zone thickness is $1.6 \mathrm{~m}$, a reduction of $16 \%$ from the 365 -day value. The field recondensation zones above and below the heater horizon persist at 500 days, while the model shows no significant recondensation.

\subsubsection{Drift-scale Versus Mountain-scale Simulation Results}

A comparison of the liquid saturation profiles for the two property sets, shown in Figure 6-59, will shed some light on the differences observed between the two temperature profiles, as shown in Figure 5-35. In Figure 6-59 liquid saturation profiles for the DS and the MS models are shown at 100,200 , and 300 days. The dominant feature of the profiles at all three times is a substantially larger dryout zone for the MS set. Note that the MS property set has a fracture permeability that is nearly two orders of magnitude greater than the fracture permeability for the DS property set. The higher permeability permits more rapid vapor flow from the hot zones to the cold zones, causing faster drying in the hot zones. The drier hot zones, with a lower thermal conductivity, then transfer heat by conduction at a slower rate and therefore experience a greater temperature rise. The higher fracture permeability therefore explains the reason why simulations with the MS property set consistently predict higher temperatures in and adjacent to the heater horizon, as shown in Figure 5-35. 


\subsubsection{Summary}

Water saturation, calculated in TH models using both DS and MS property sets, is compared with the LBT data measured by neutron logging. This comparative analysis is to assess the performance of the TH model in general, and the performance of the DS and MS property sets in particular. The results of the comparison are summarized as follows:

The movement of water in the LBT as predicted by the TH model is qualitatively consistent with the measured field data. In other words, both model predictions and field data show drying at the heater horizon. Quantitatively, the model-predicted dryout zone developed slower than the dryout zone in the field test, and the predicted dryout zone is smaller than the field data. The size of a dryout zone and the timing of developing a dryout zone are probably affected by rock properties, including heterogeneity. The dryout zone modeled using the DS property is significantly smaller than that modeled using the MS property. This is probably due to the greater fracture permeability in the MS property set. As mentioned earlier in this section, the LBT was not designed to test a rock property set, due to its size and the near-surface setting. Therefore, the effect of the property set on the predicted moisture movement as inferred from this comparison should be considered as for reference only. 


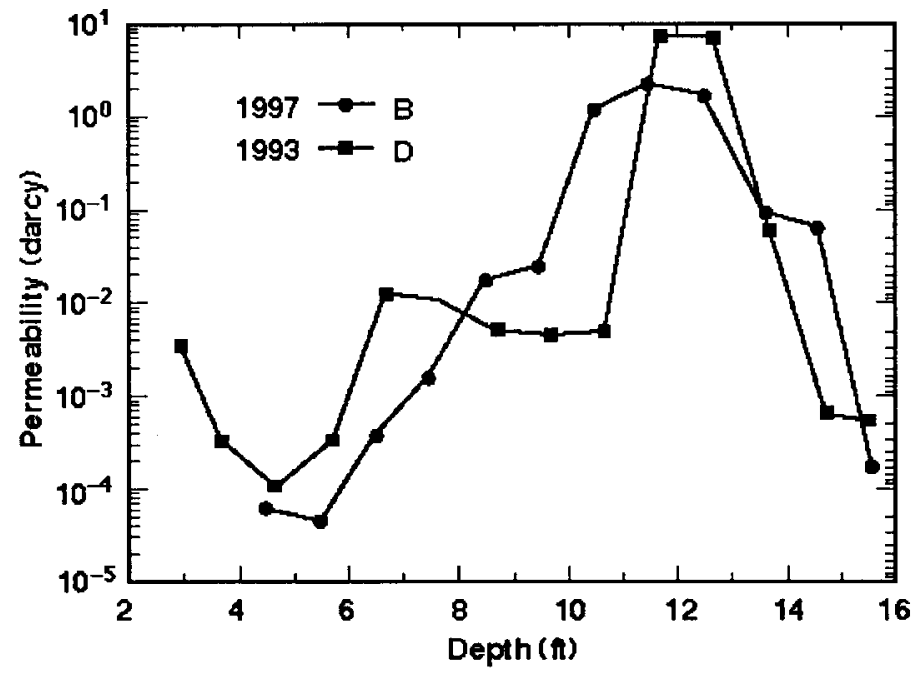

Figure 6-1. Air Permeability Measured in a Single Hole Before Cutting (D) and After Cutting (B) the Block as a Function of Depth.

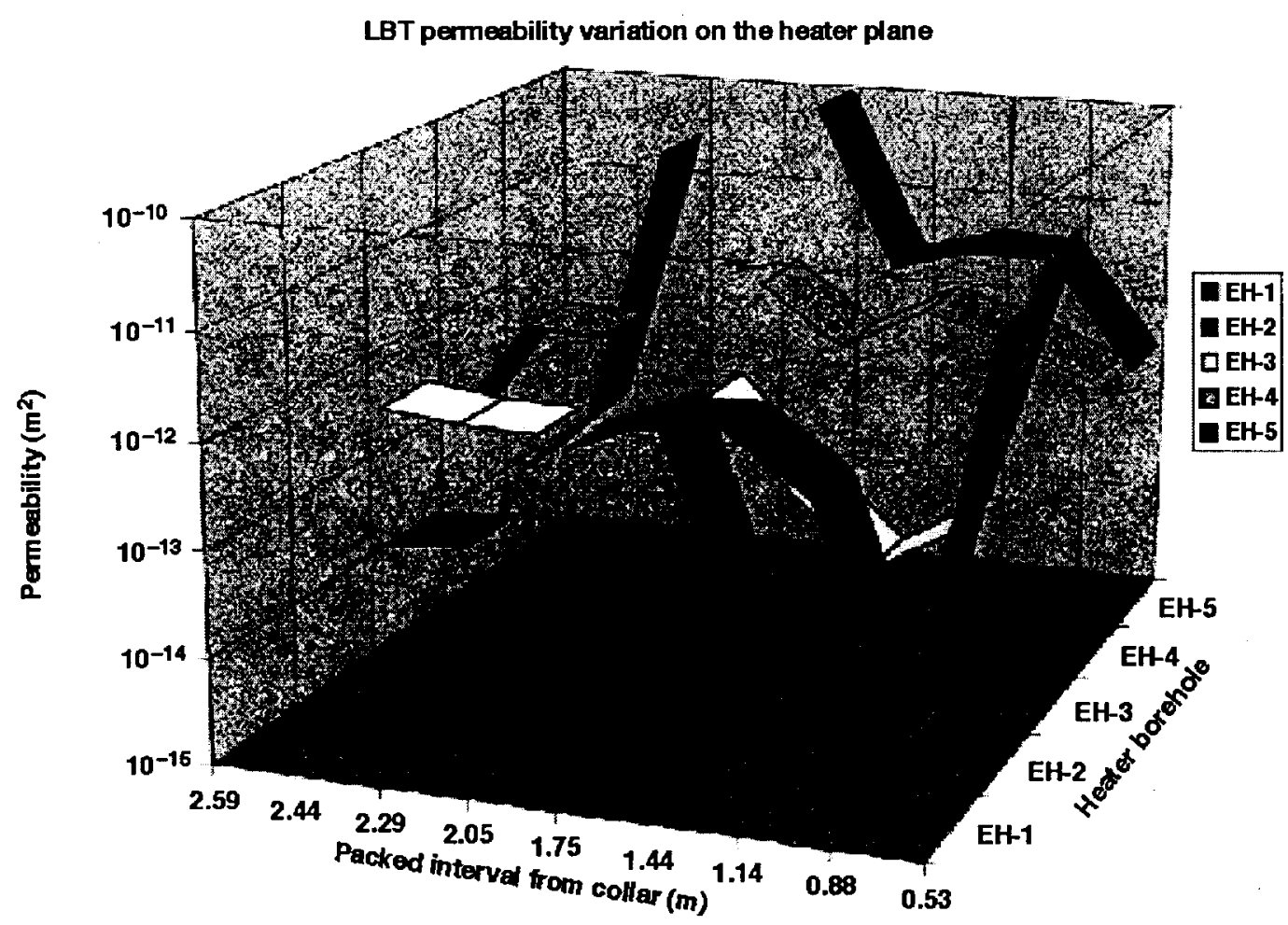

Figure 6-2. Air Permeability Variations Along Five Heater Boreholes at the LBT Site 


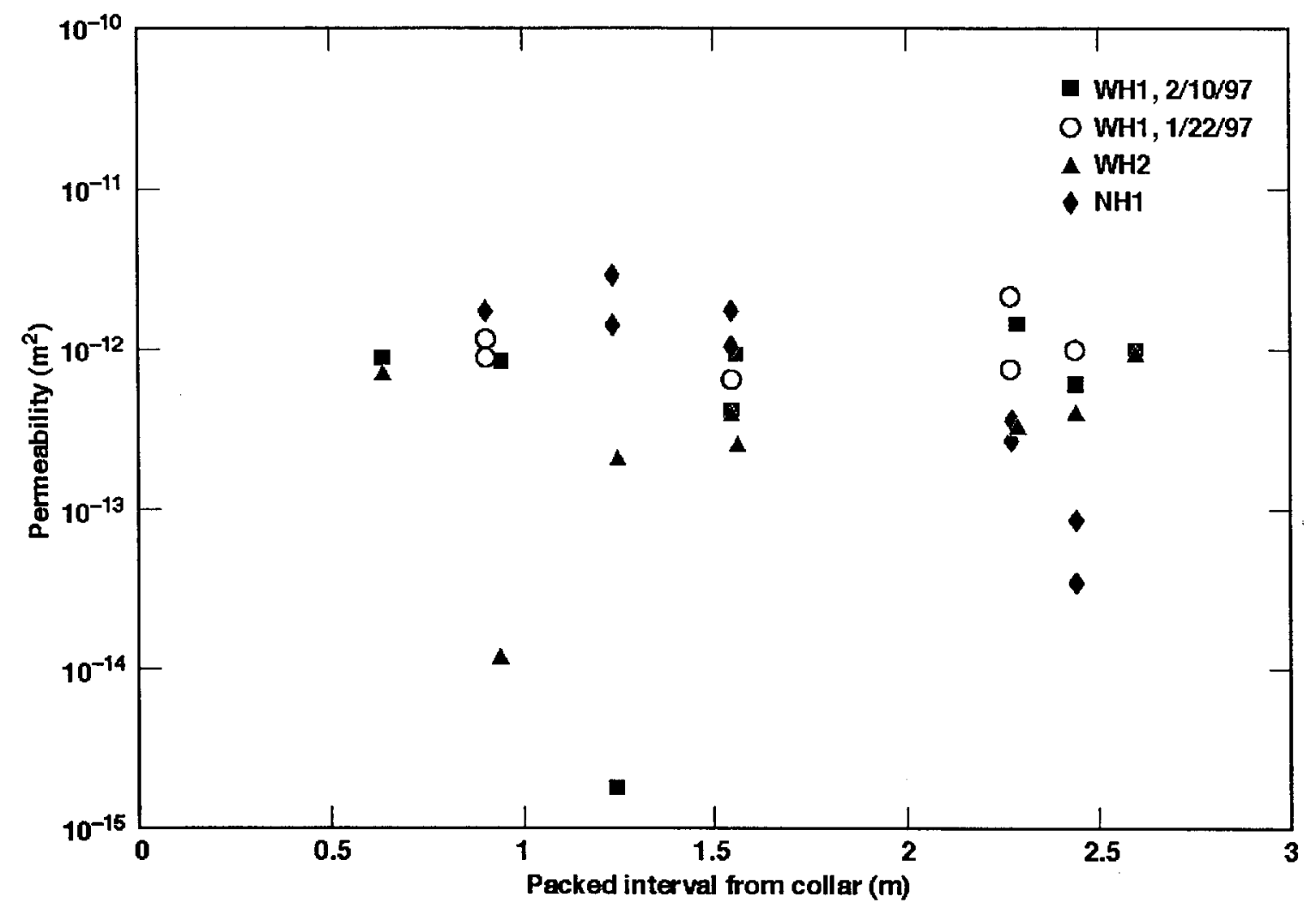

Figure 6-3. Air Permeability Variations Along Three Horizontal, Hydrologic, Monitoring Boreholes at the LBT Site

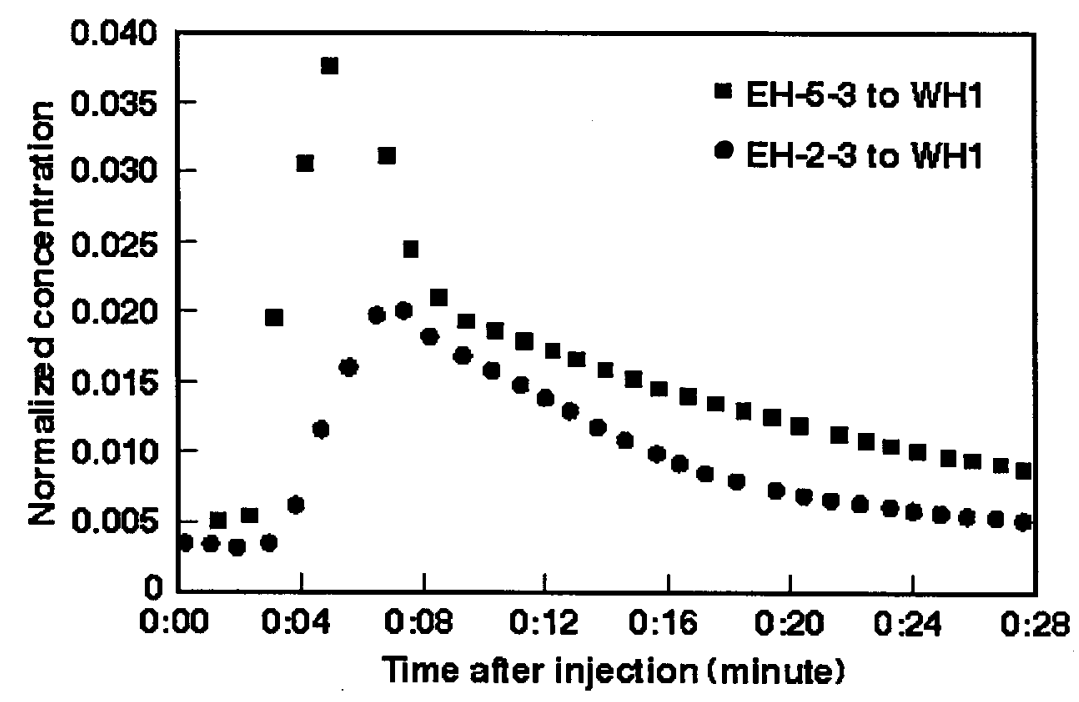

Figure 6-4. Tracer Breakthrough Curves from Heater Borehole Intervals to a Hydrologic, Monitoring Borehole WH1 at the LBT Site 


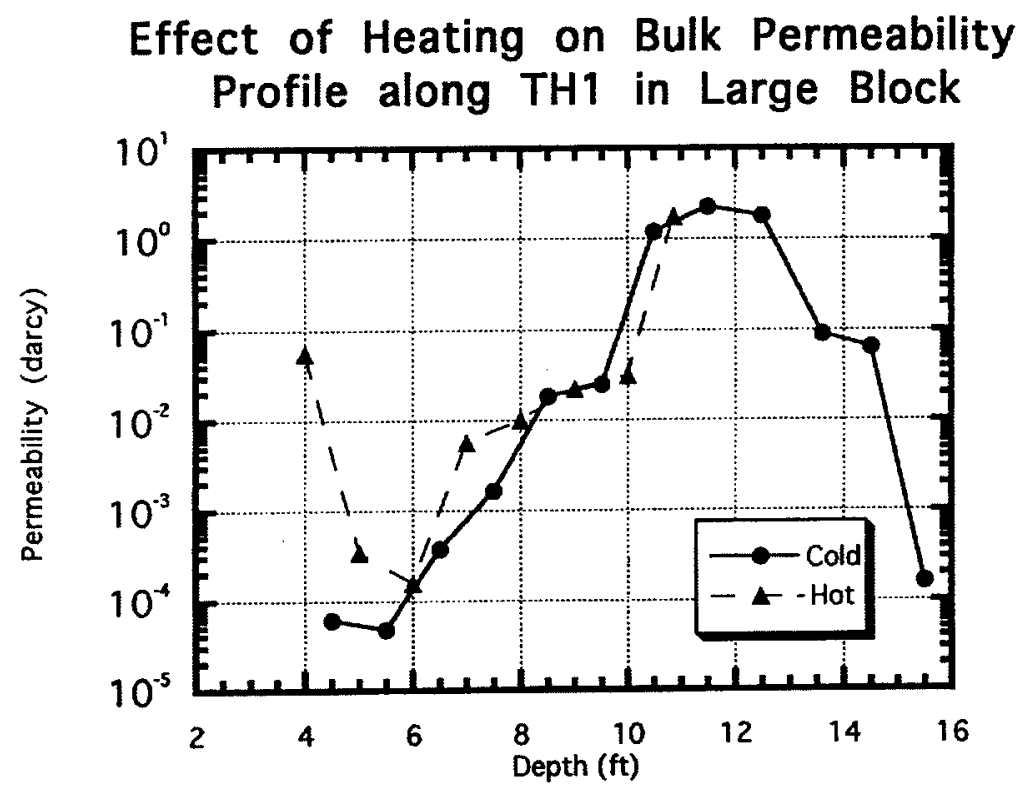

NOTE: The "cold" curve is the same as the "B" curve in Figure 6-1.

Figure 6-5. Air Permeability Measured by Single-hole Injection Along Hole TH1 in the Block Before Heating (Cold) and During Heating (Hot) 

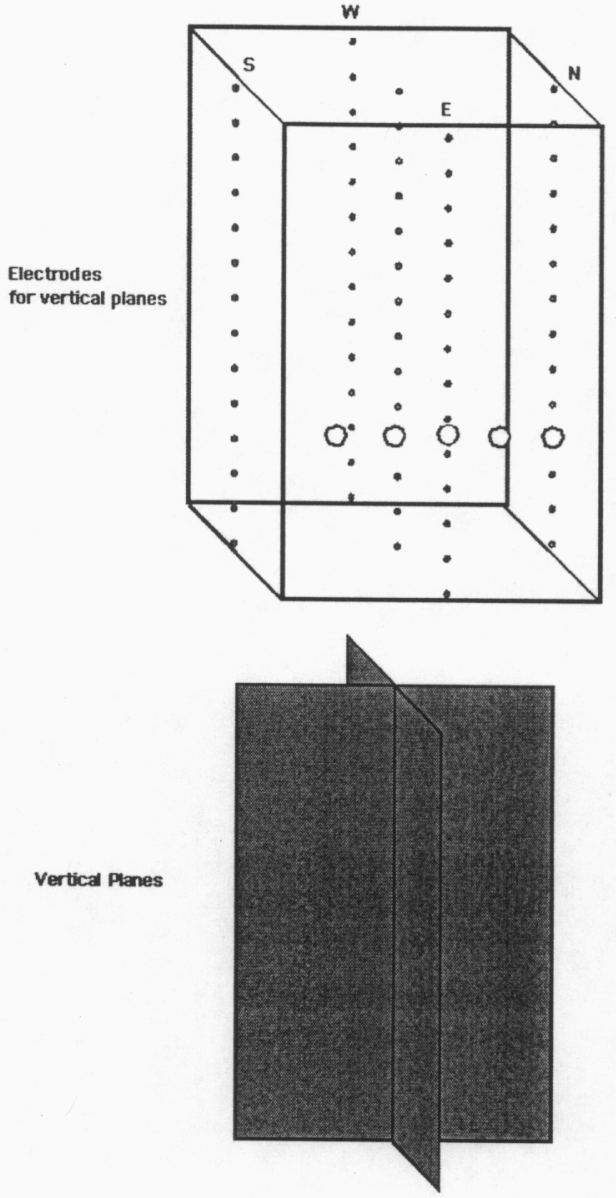

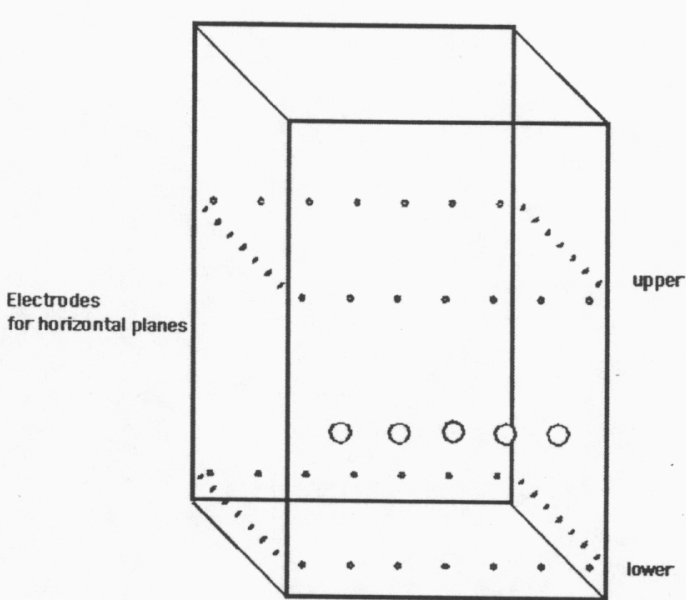

Horizontal Planes
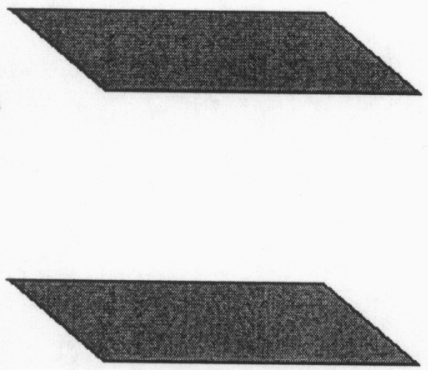

NOTE: All electrodes are on the surface of the block except the array in the center where the two vertical planes intersect. The heater hole locations are shown for reference.

Figure 6-6. Layout of ERT Electrodes for the Large Block Test 
(a) Upper Horizontal
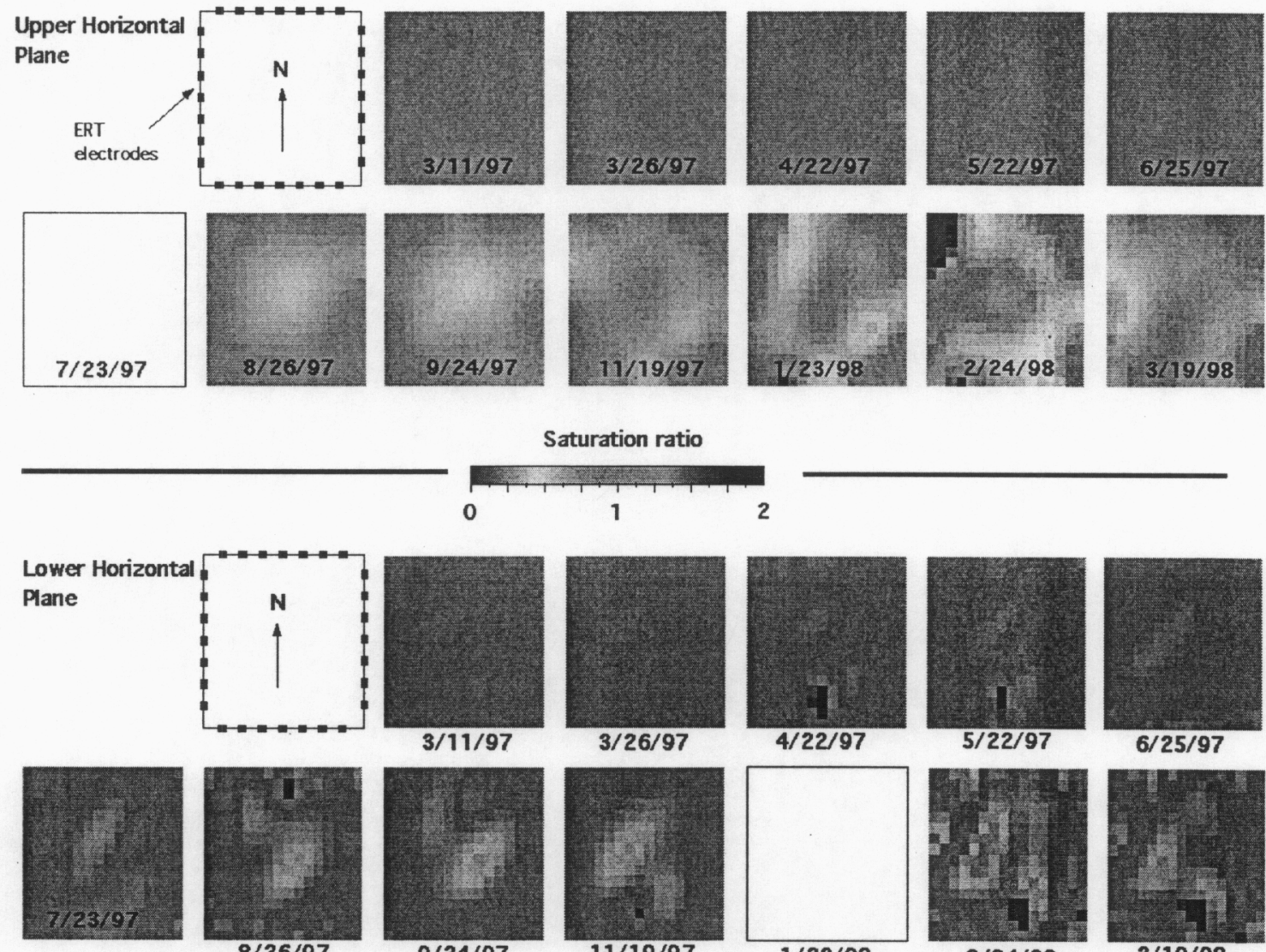

$8 / 26 / 97$

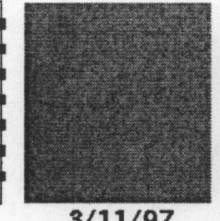

$3 / 11 / 97$

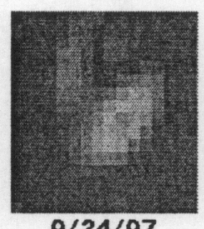

$9 / 24 / 97$

Saturation ratio

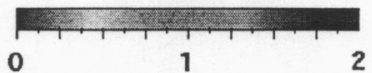

NOTE: Blank spaces indicate data sets that did not converge. The saturation ratio is (moisture content)/(initial moisture content). (a) The upper plane, (b) The lower plane.

Figure 6-7. Changes in the Distribution of Moisture Content in Two Horizontal Image Planes 
seueld әбещ! ןеэ!ฺә

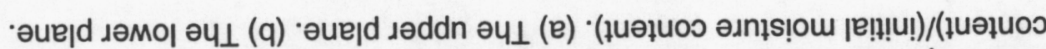

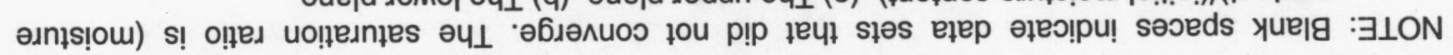
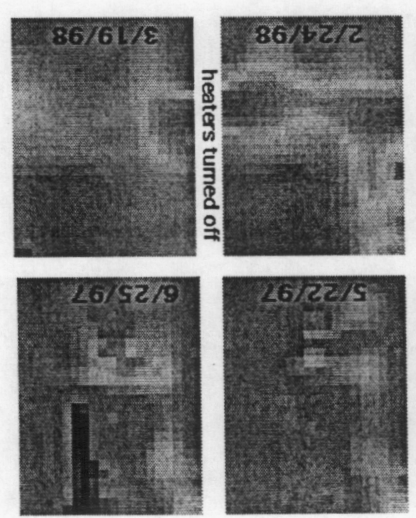

$$
\rightarrow \text { a.n6!y }
$$
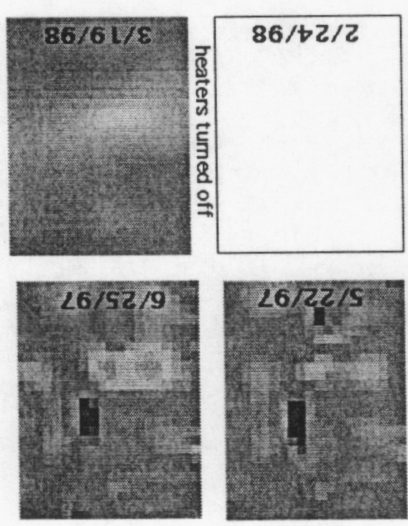
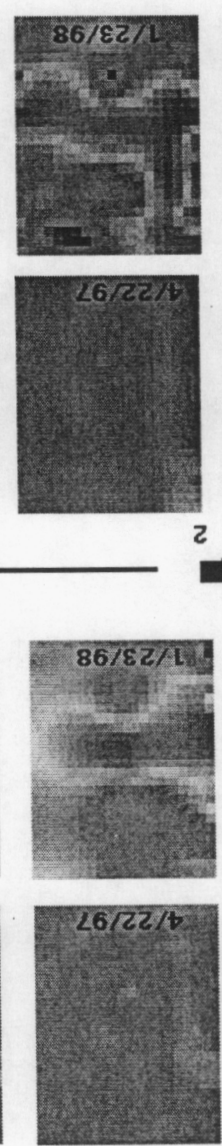
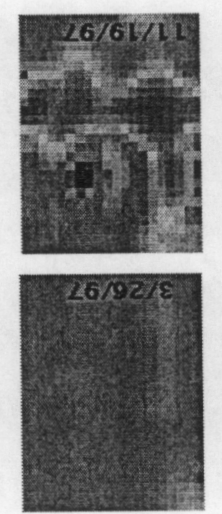

$\mathbf{L}$
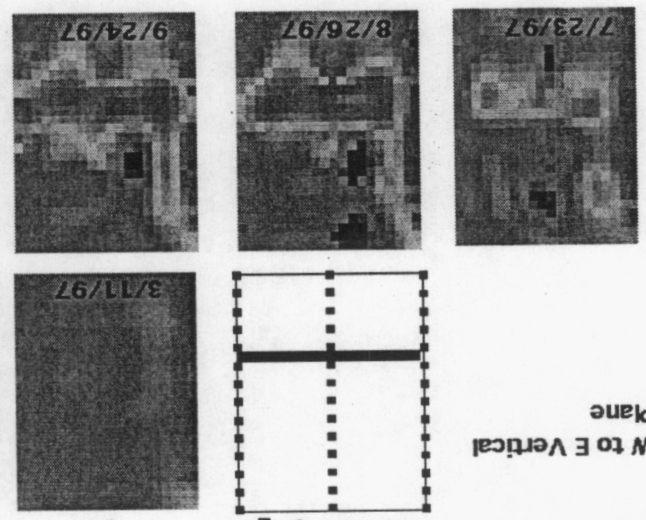

o!̣yed uọnednes
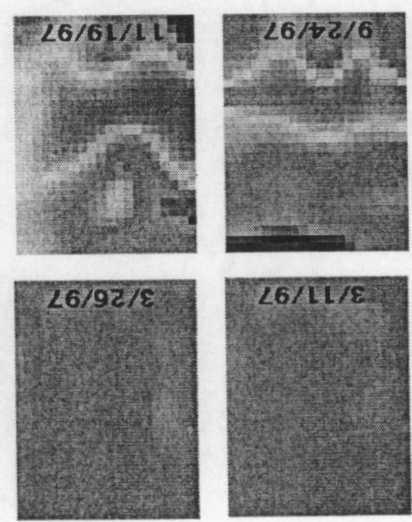

0
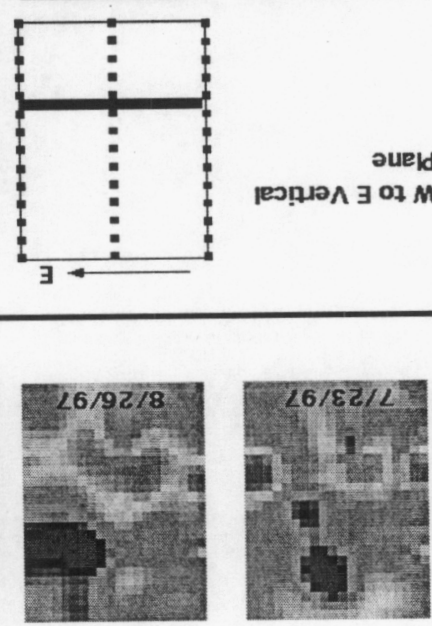

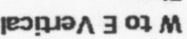
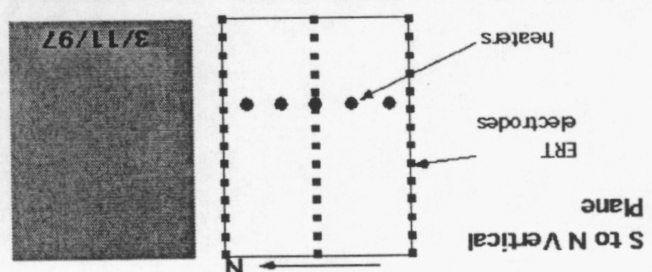


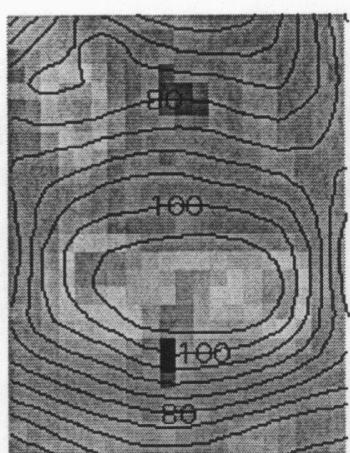

$7 / 23 / 97$

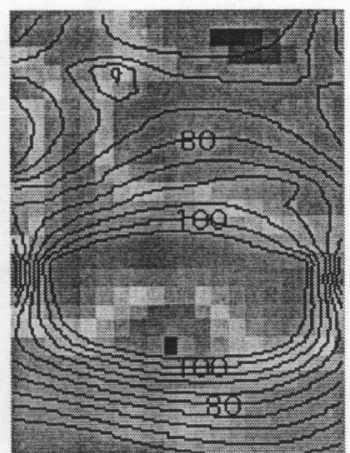

$1 / 23 / 98$

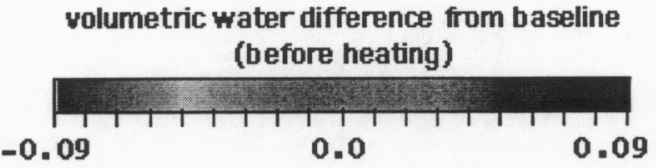

NOTE: The change in water content was calculated assuming a porosity of 0.12 and an initial saturation of 0.75 .

Figure 6-9. Interpolated Temperature Isotherms $\left({ }^{\circ} \mathrm{C}\right)$ Superimposed on ERT-Derived Images of Volumetric Water Change in the West-to-East Plane
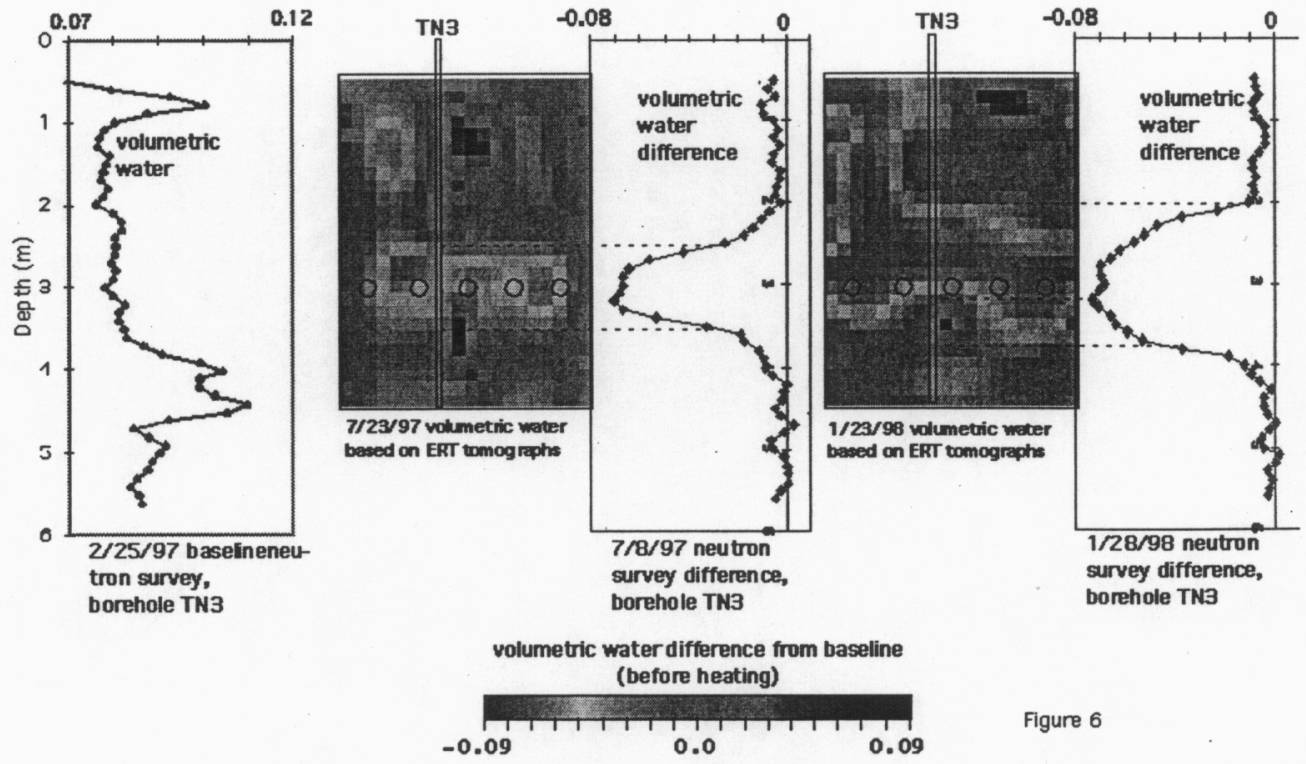

Figure 6

Figure 6-10. Comparison of Neutron Log and ERT Measurements of Changes in Moisture Content 


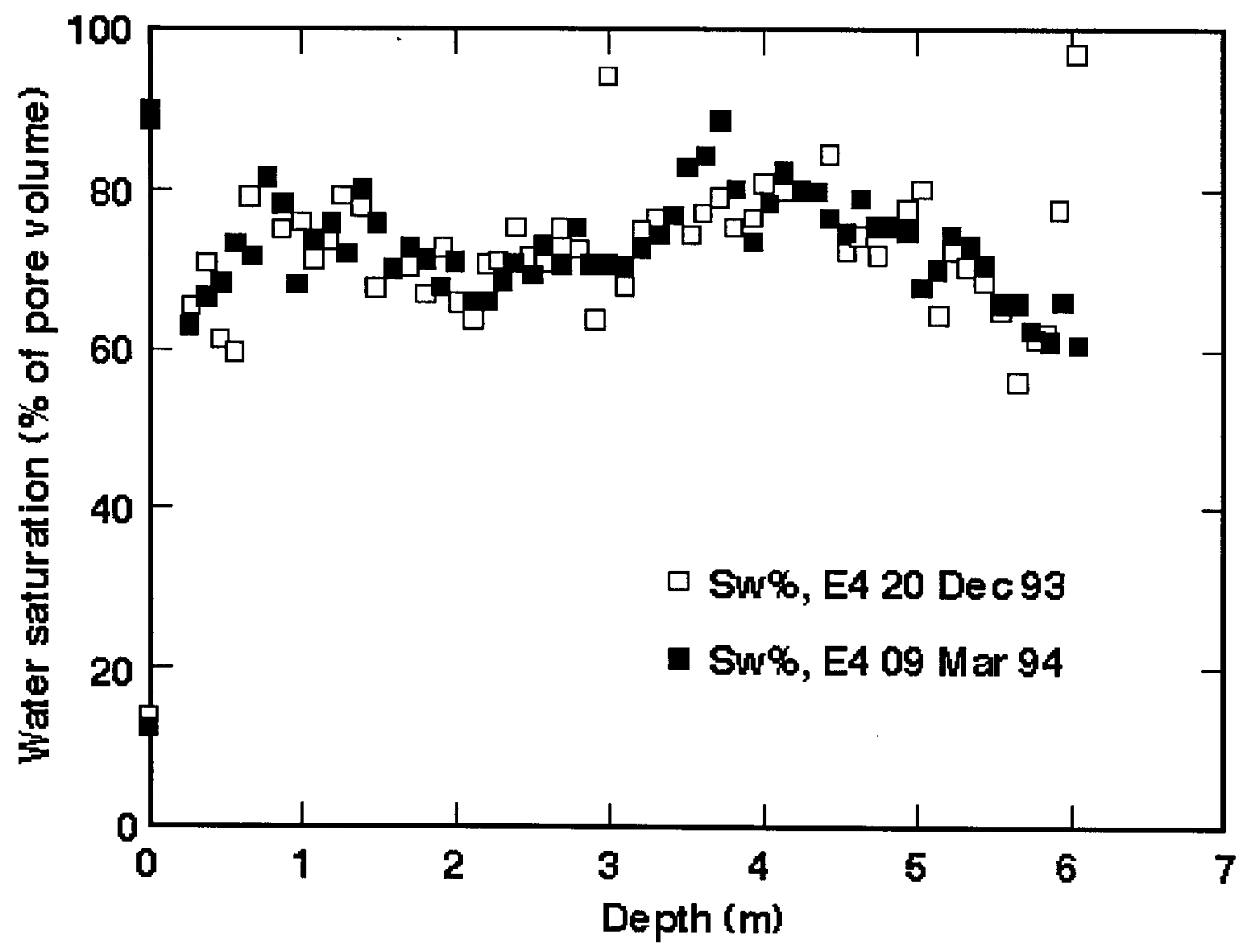

Figure 6-11. Pre-Cut and Post-Cut Water Saturation as a Function of Depth 


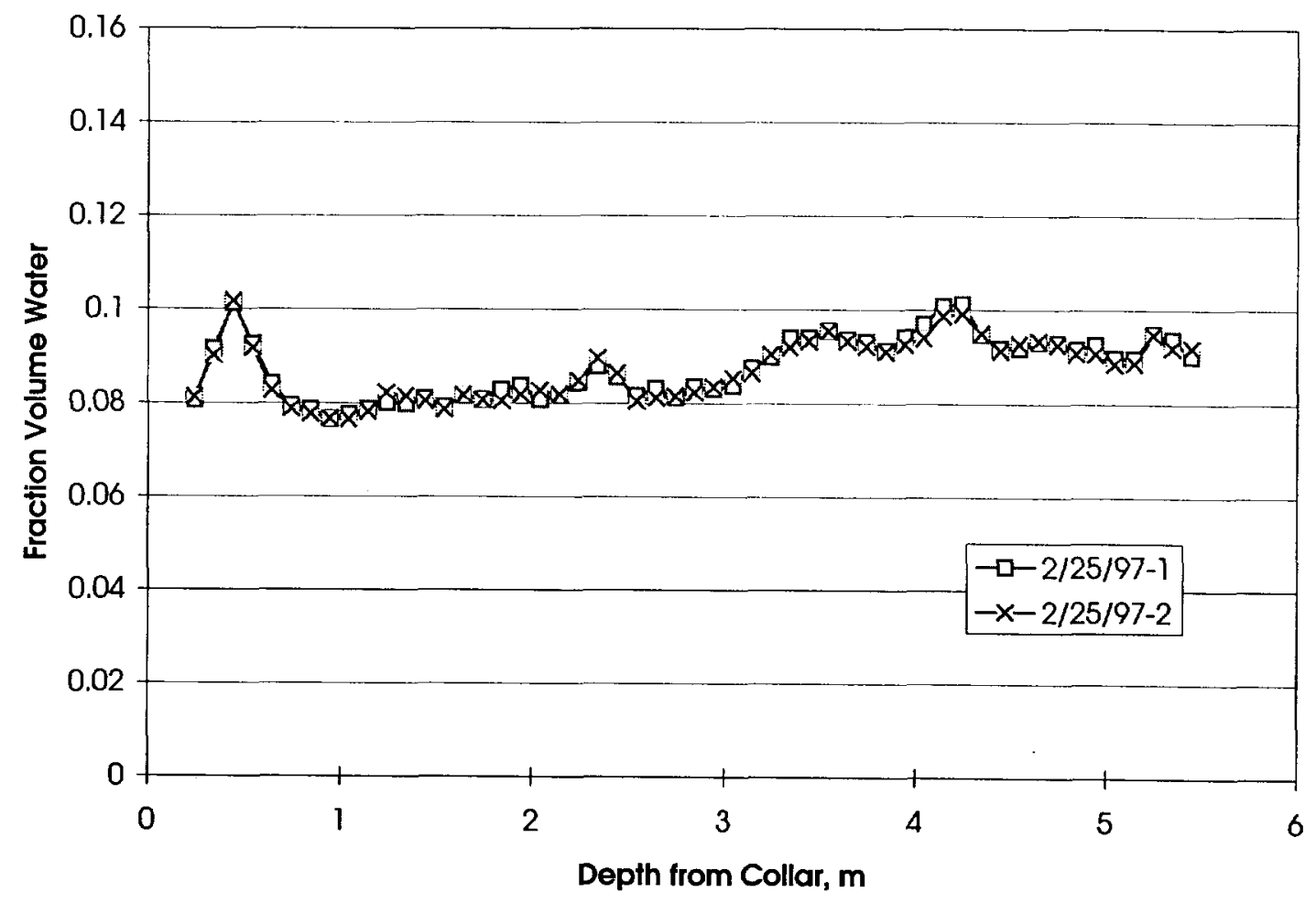

NOTE: As a function of depth from top of the block.

Figure 6-12. The Baseline Moisture Content Measured in Hole TN1 


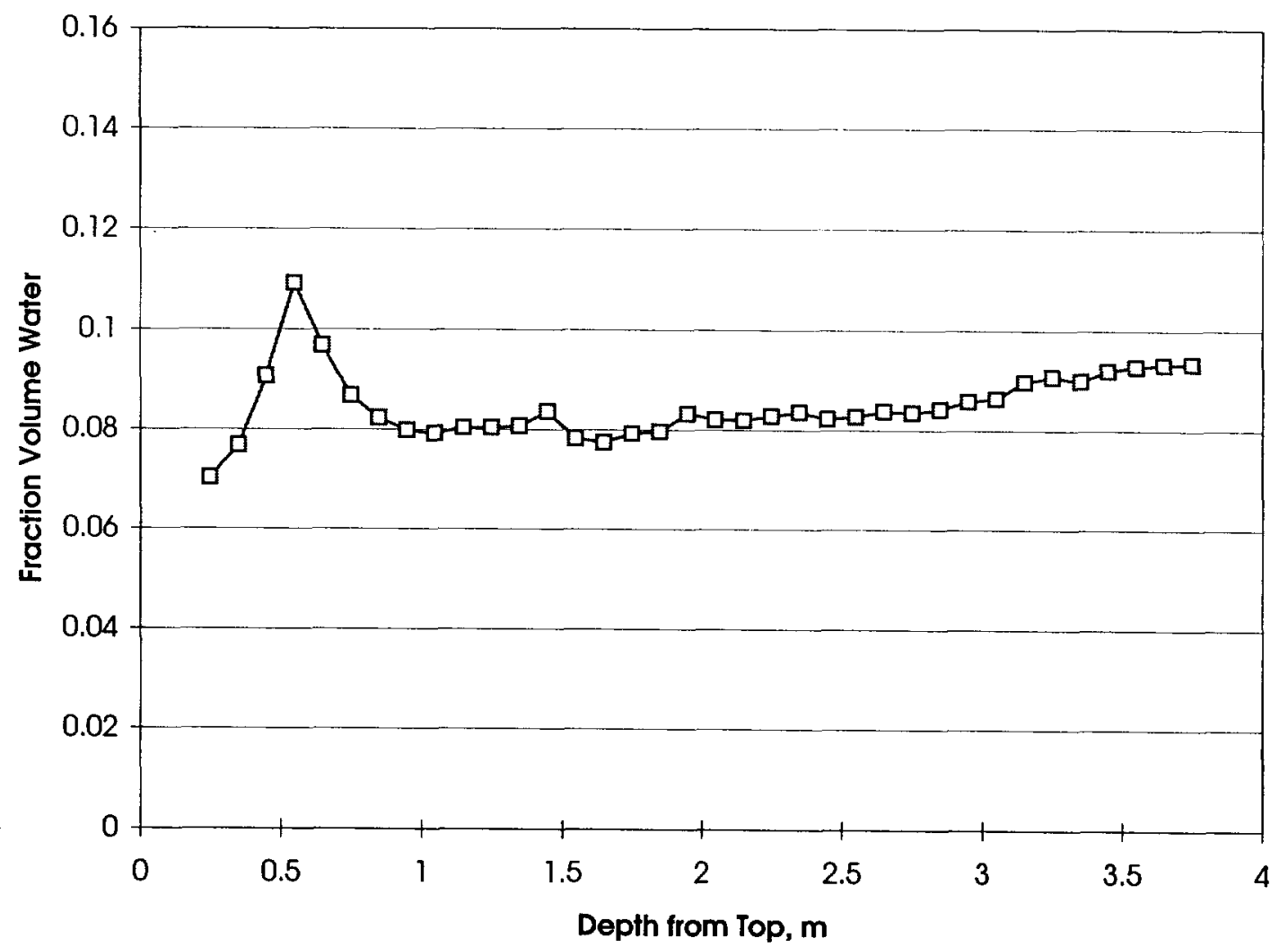

NOTE: As a function of depth from top of the block.

Figure 6-13. The Baseline Moisture Measured in Hole TN2 


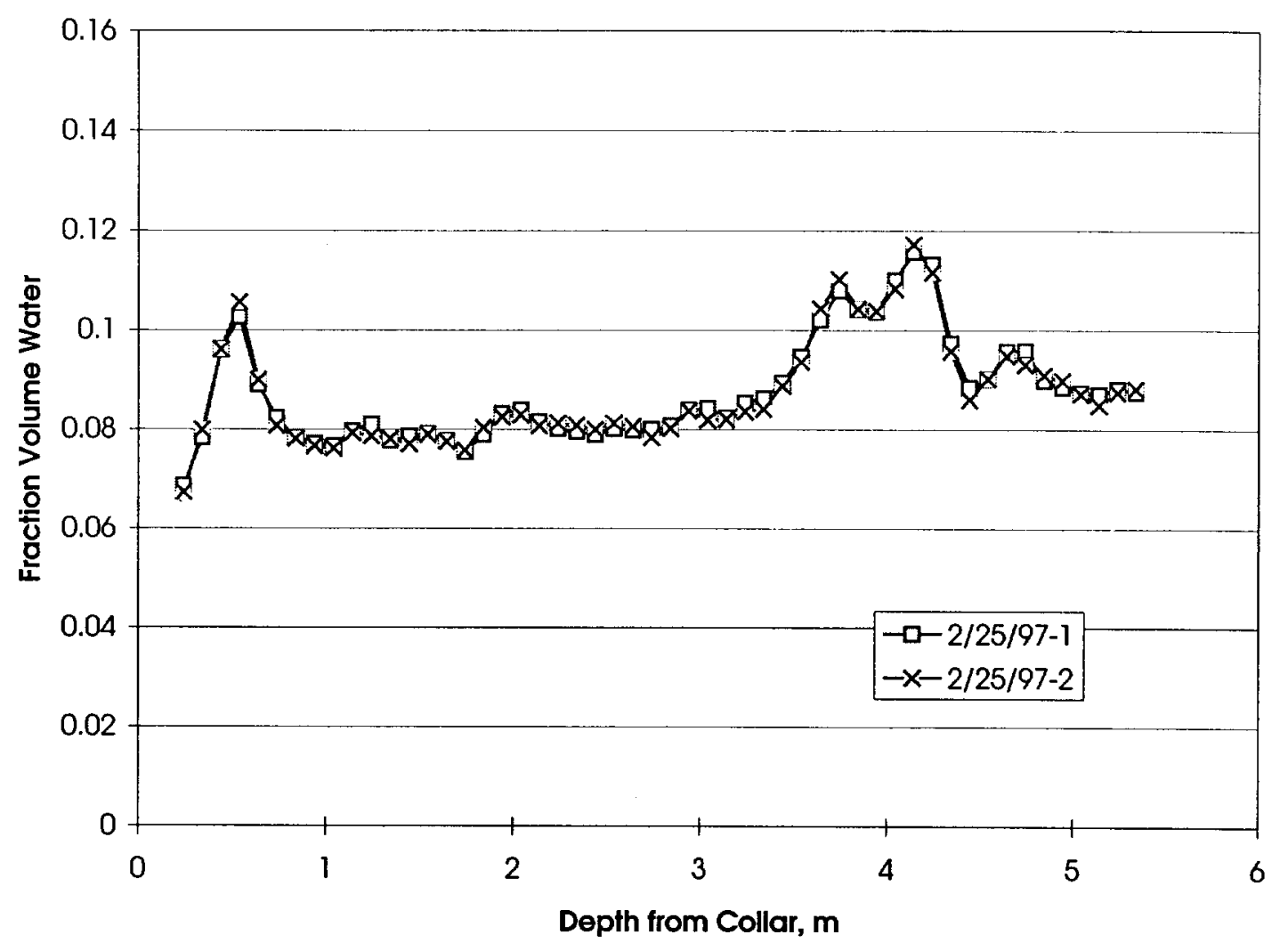

NOTE: As a function of depth from top of the block.

Figure 6-14. The Baseline Moisture Content Measured in Hole TN3 


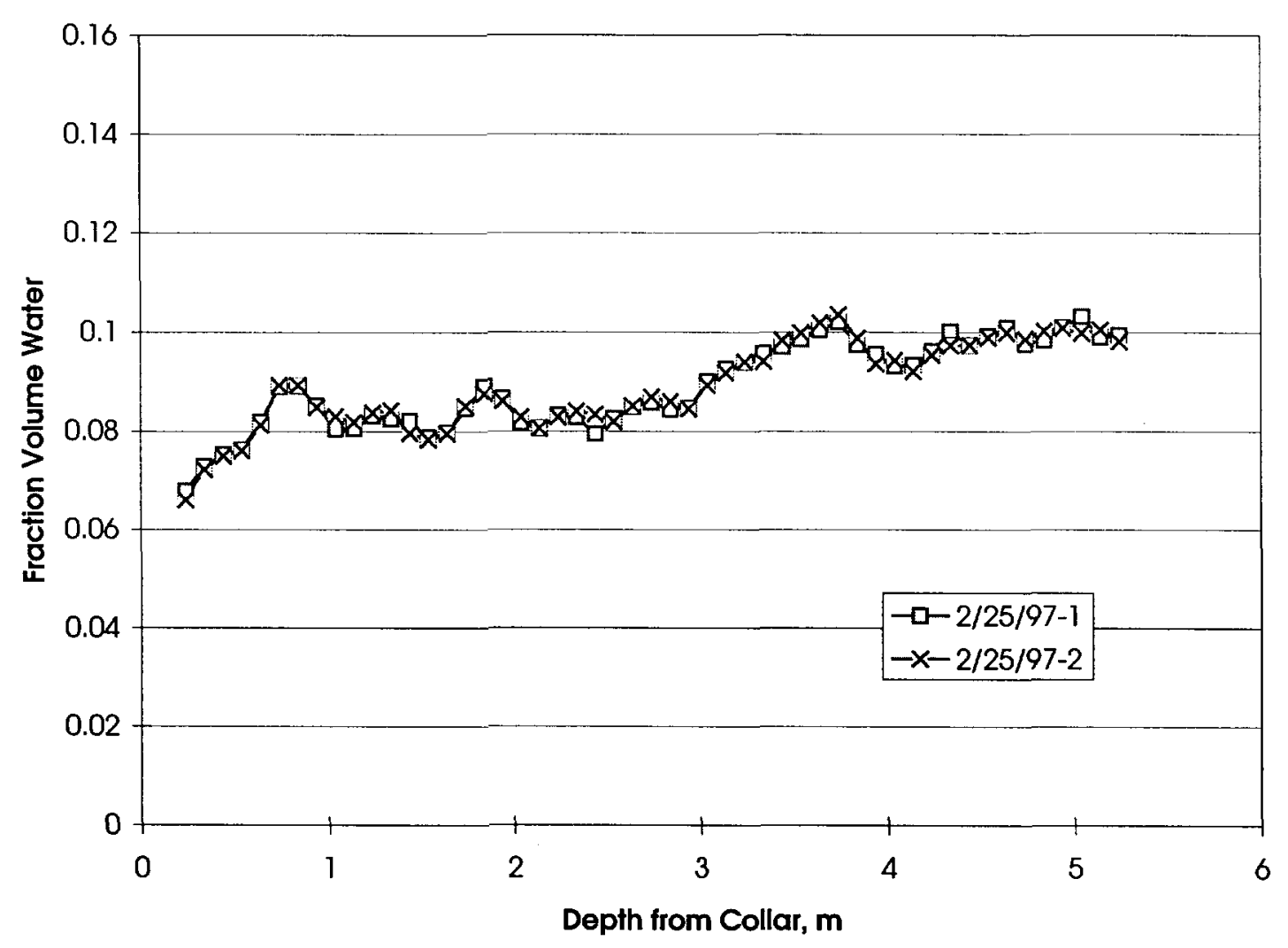

NOTE: As a function of depth from top of the block.

Figure 6-15. The Baseline Moisture Content Measured in Hole TN4 


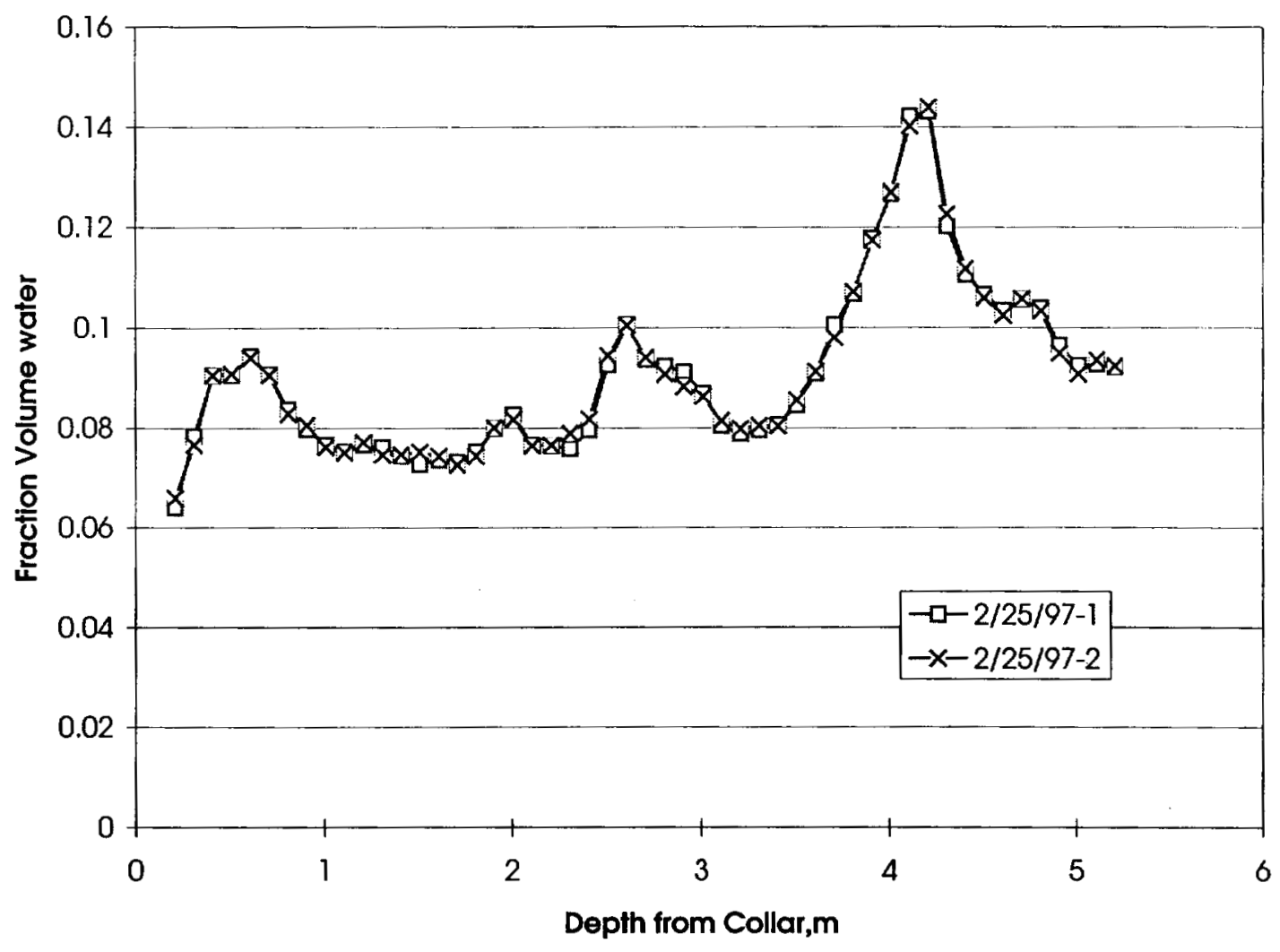

NOTE: As a function of depth from north face of the block.

Figure 6-16. The Baseline Moisture Content Measured in Hole TN1 
Baseline fraction volume water content in NN1.

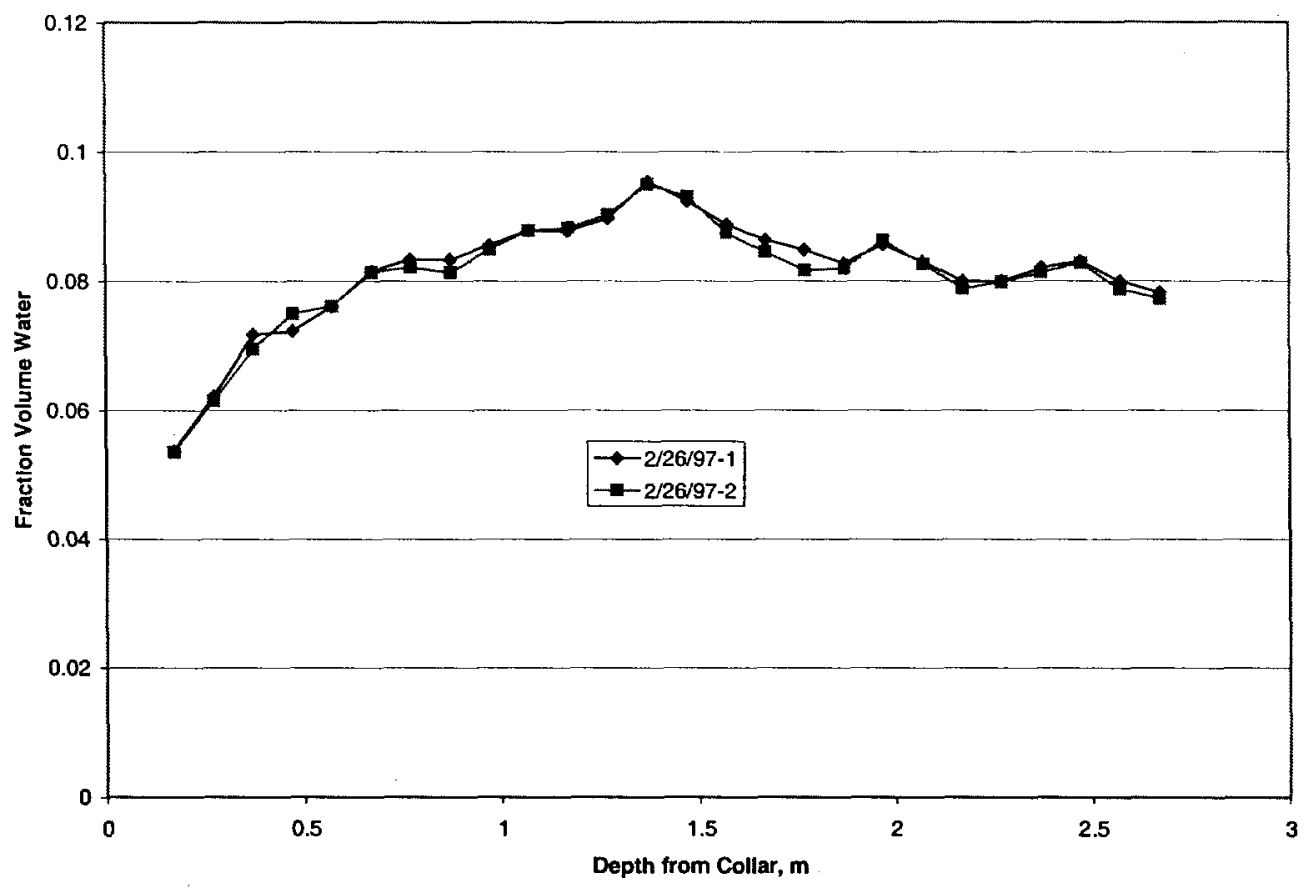

NOTE: As a function of depth from north face of the block.

Figure 6-17. The Baseline Moisture Content Measured in Hole NN1 
Baseline fraction volume water content in NN2.

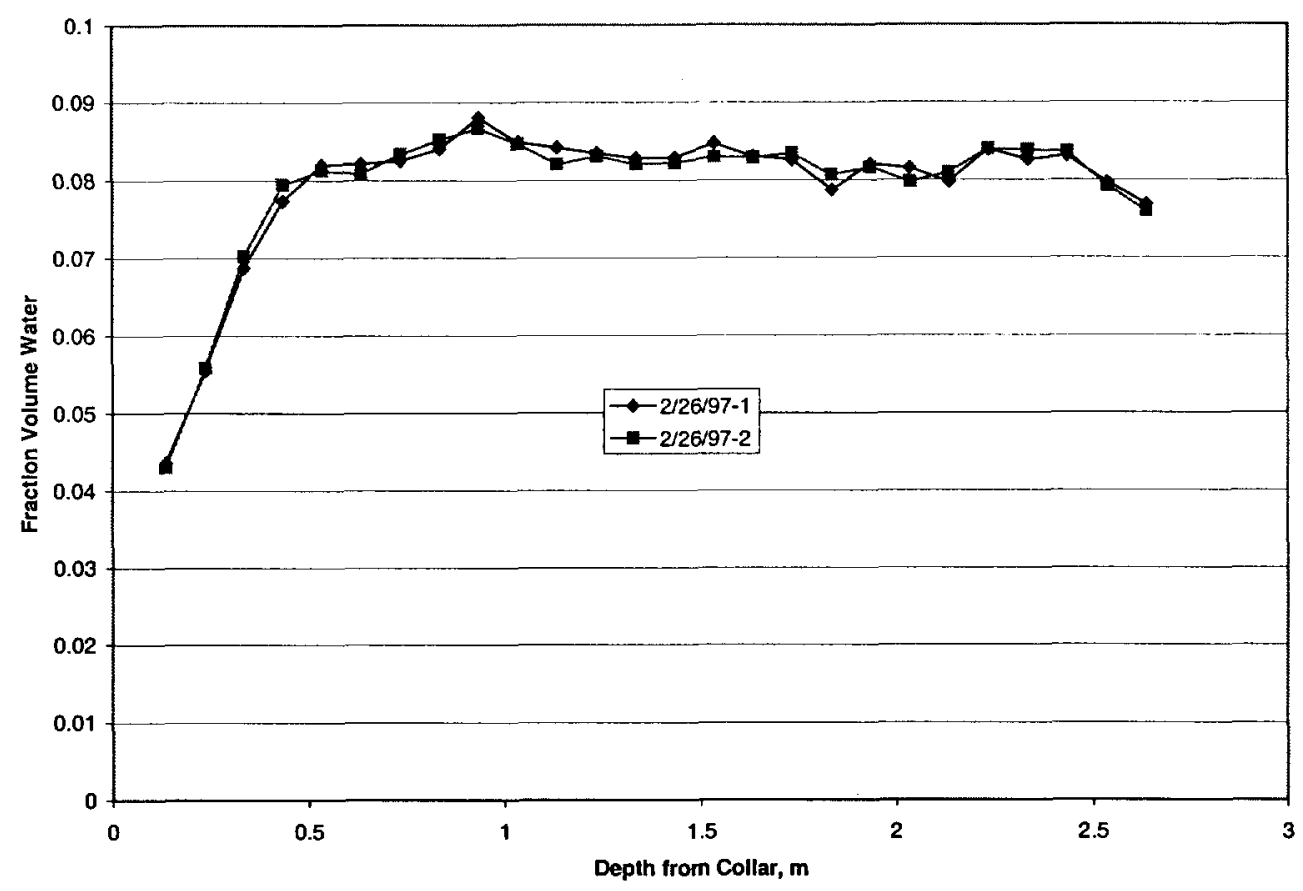

NOTE: As a function of depth from north face of the block.

Figure 6-18. The Baseline Moisture Content Measured in Hole NN2 
Baseline Fraction Volume Water in NN3.

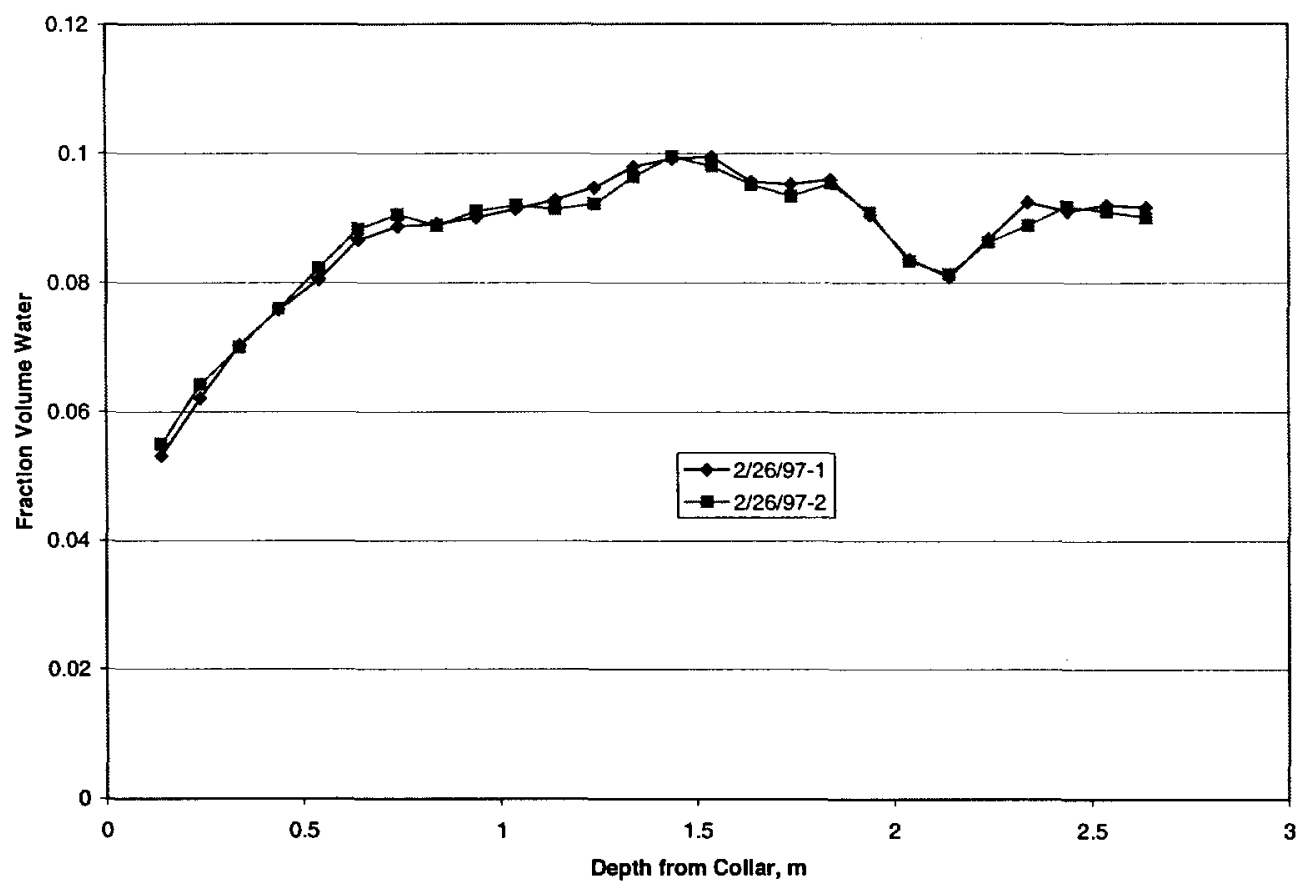

NOTE: As a function of depth from north face of the block.

Figure 6-19. The Baseline Moisture Content Measured in Hole NN3 
Baseline fraction volume water in NN4.

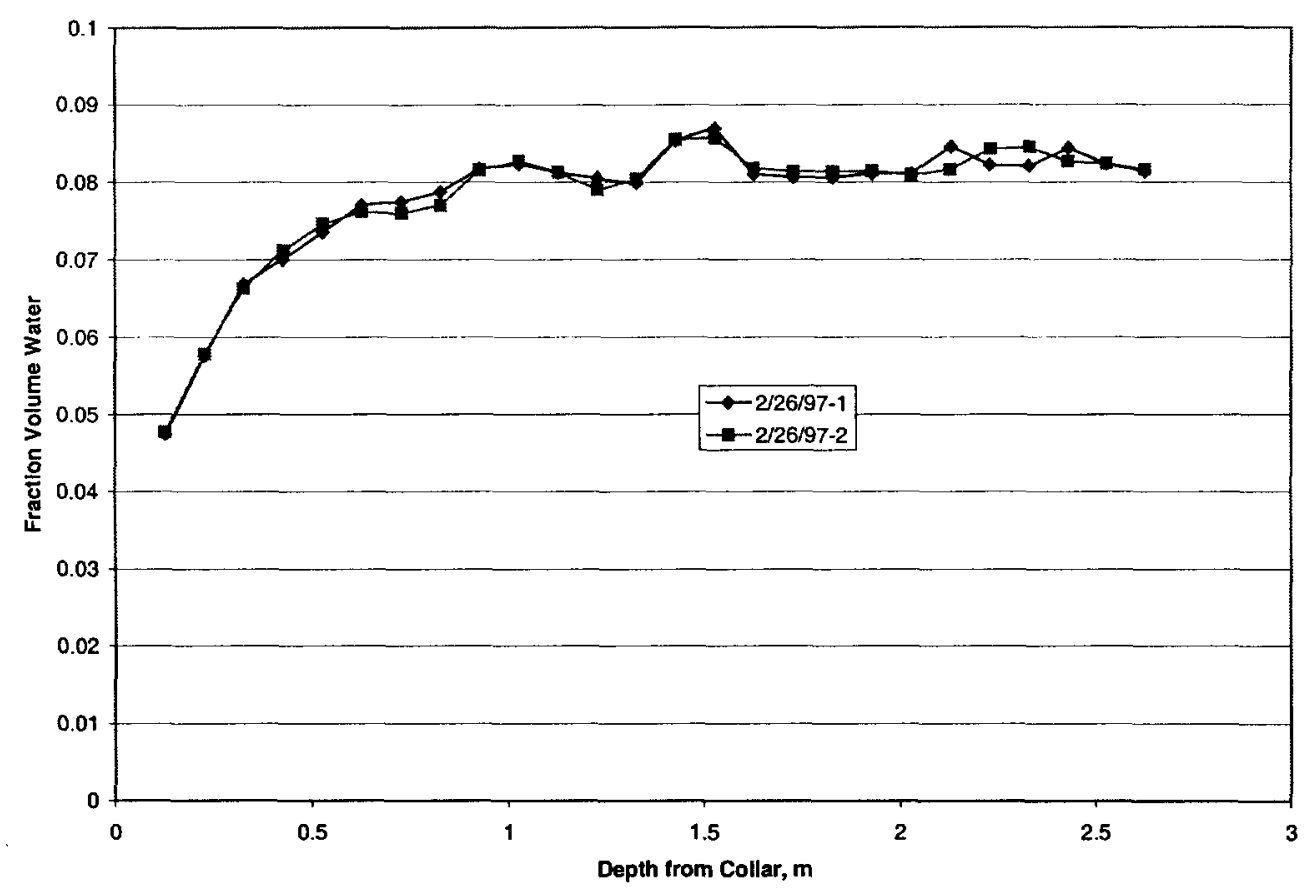

NOTE: As a function of depth from north face of the block.

Figure 6-20. The Baseline Moisture Content Measured in Hole NN4 
The baseline fraction volume water content in NN5.

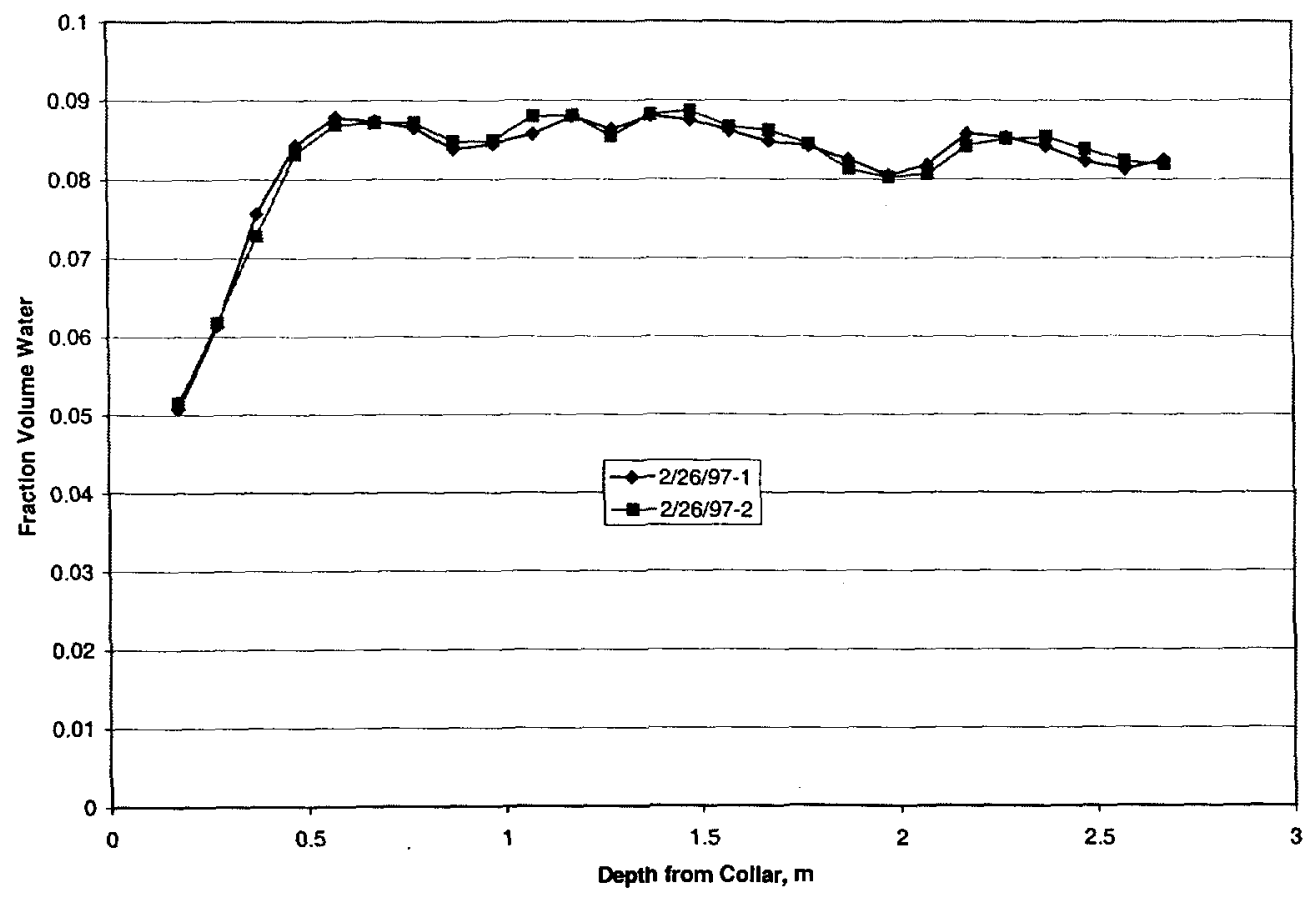

NOTE: As a function of depth from north face of the block.

Figure 6-21. The Baseline Moisture Content Measured in Hole NN5 
Baseline fraction volume water content in NN6

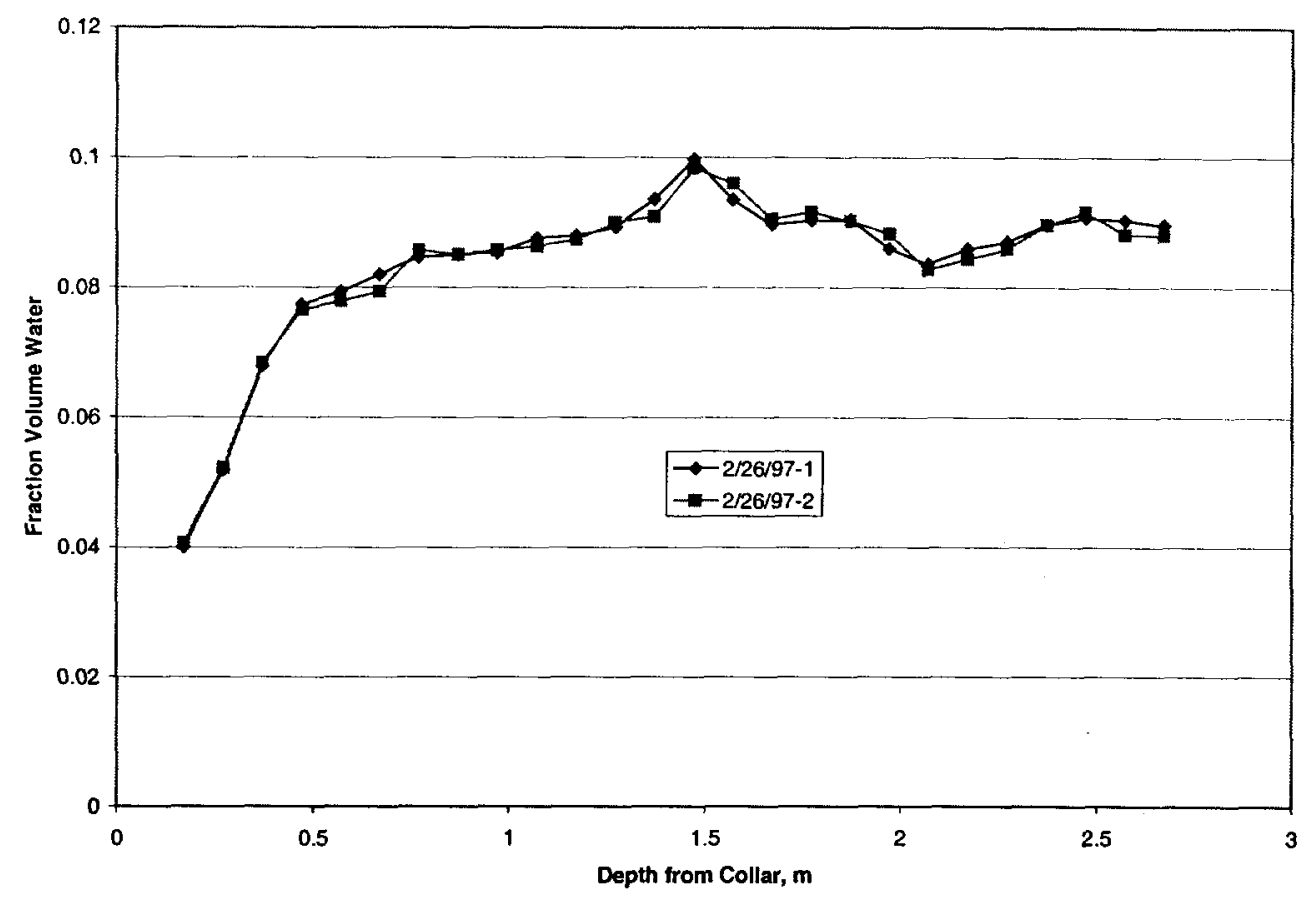

NOTE: As a function of depth from north face of the block.

Figure 6-22. The Baseline Moisture Content Measured in Hole NN6 
Baseline fraction volume water in WN1.

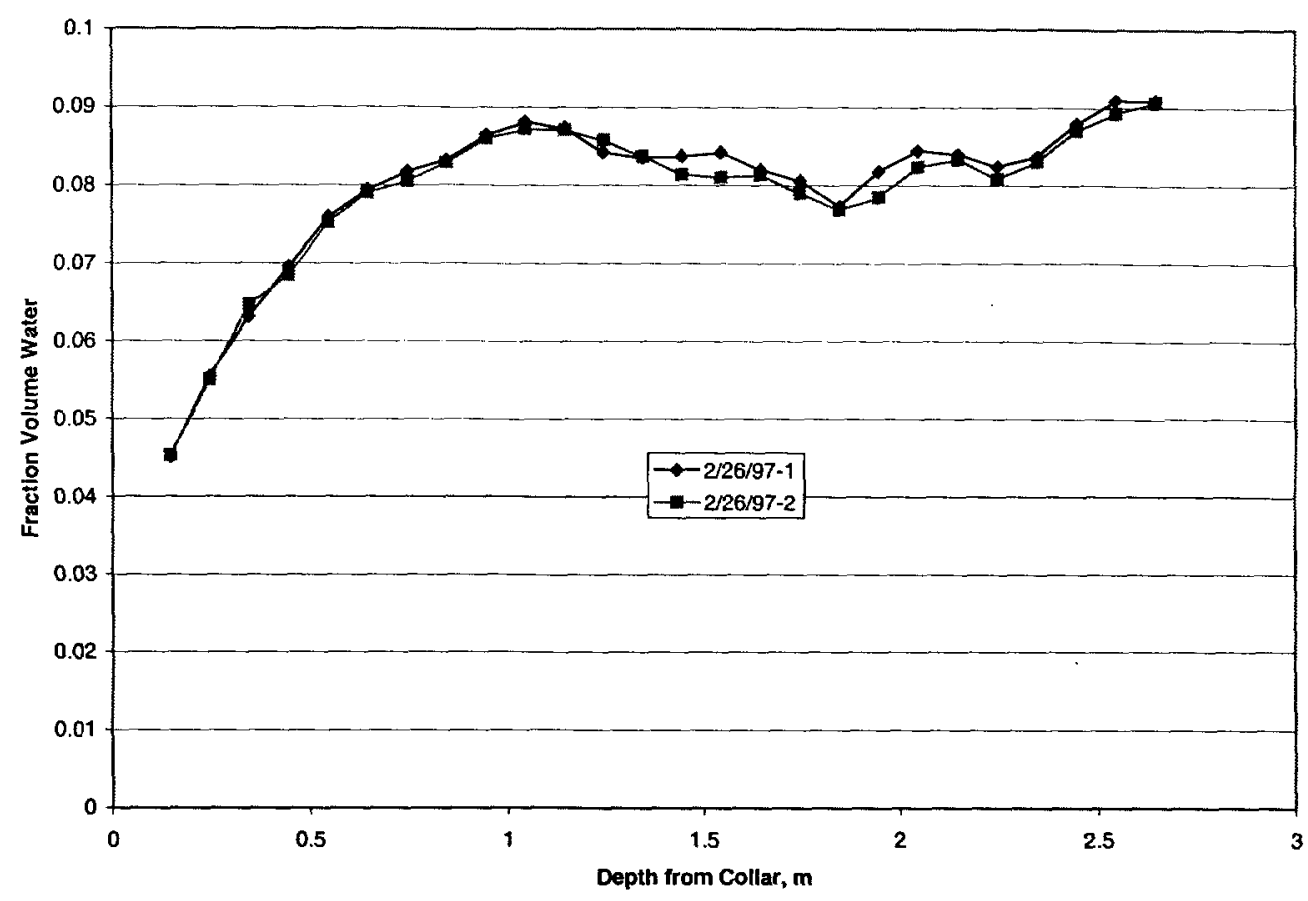

NOTE: As a function of depth from west face of the block.

Figure 6-23. The Baseline Moisture Content Measured in Hole WN1 
Baseline fraction volume water in WN2.

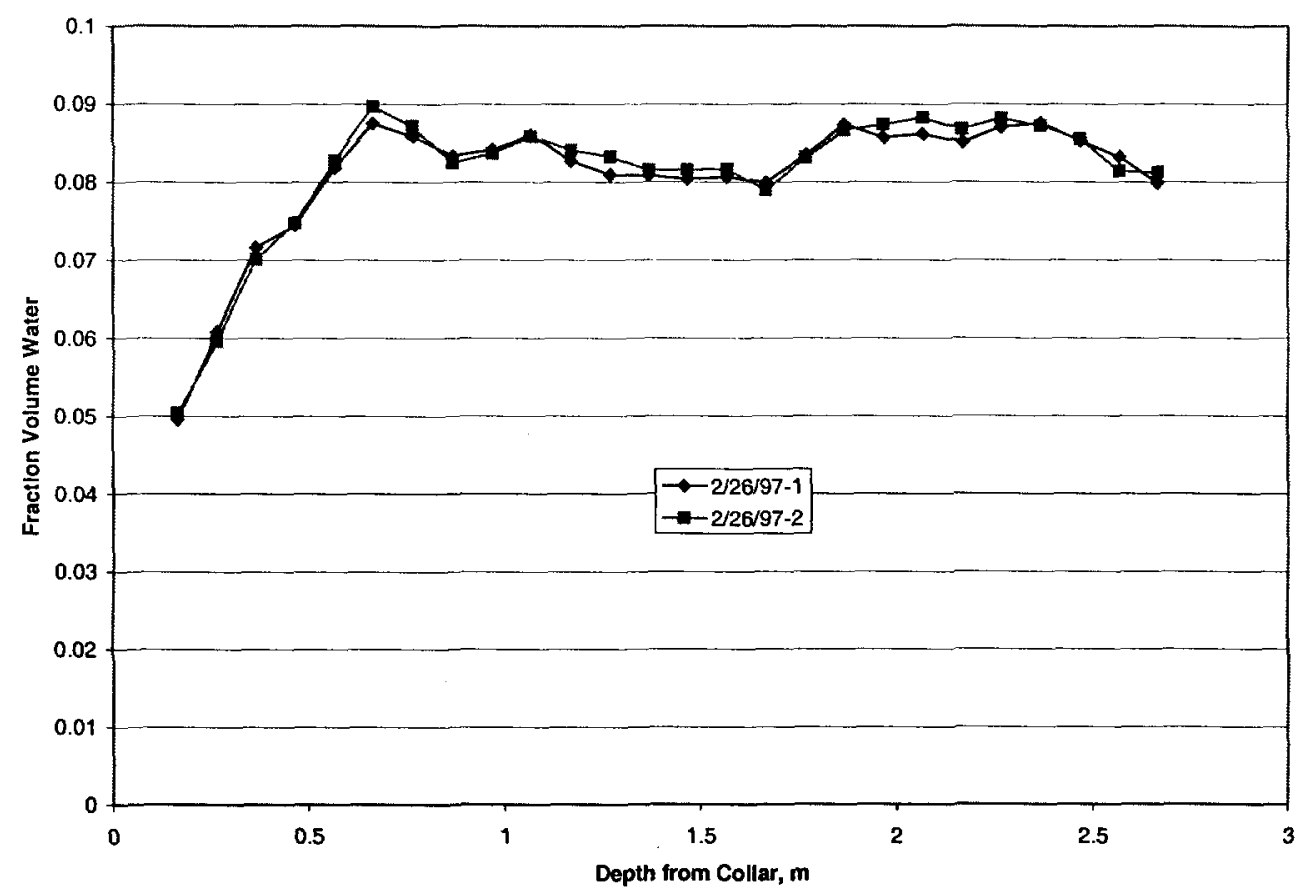

NOTE: As a function of depth from west face of the block.

Figure 6-24. The Baseline Moisture Content Measured in Hole WN2 
Baseline fraction volume water in WN3.

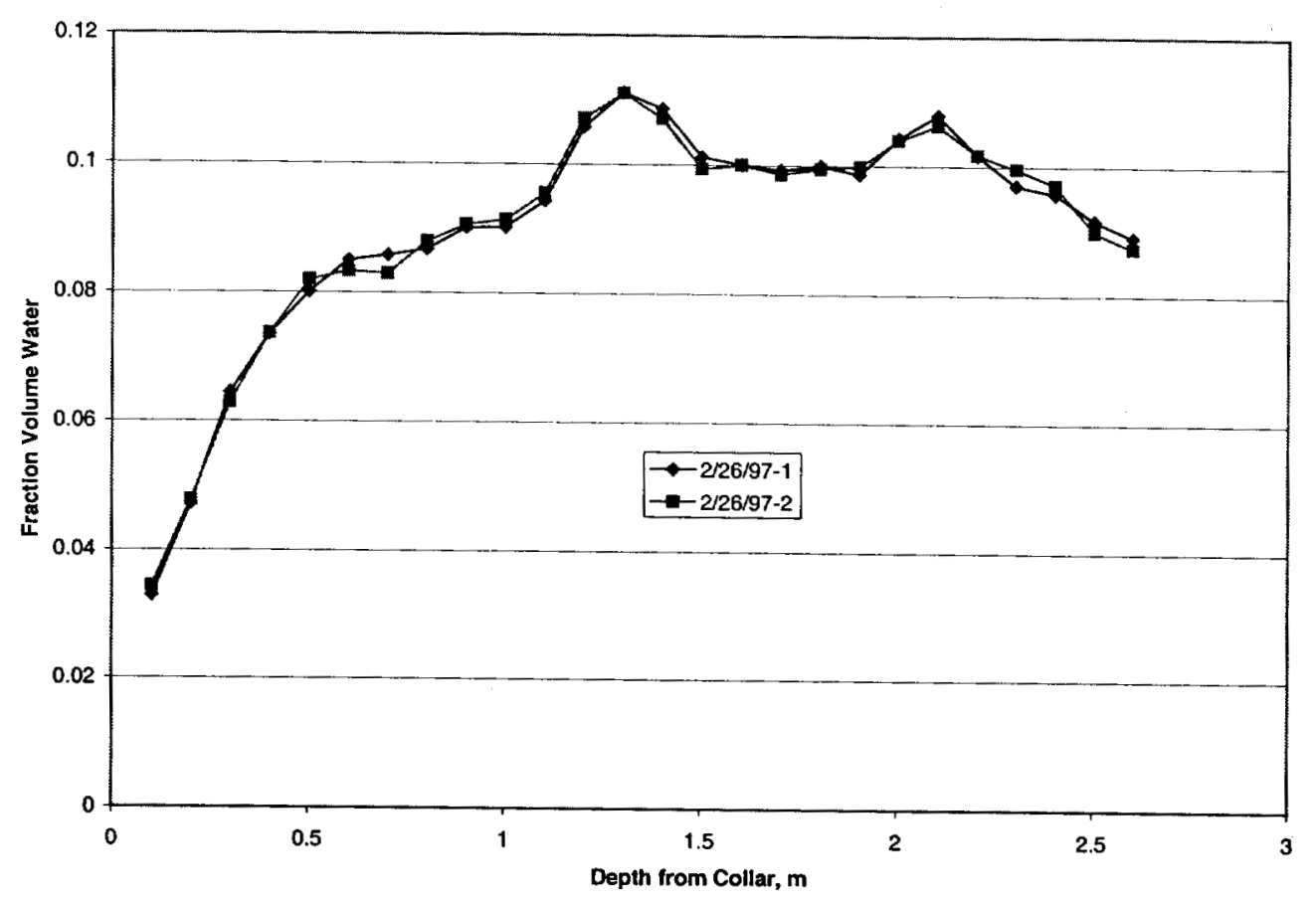

NOTE: As a function of depth from west face of the block.

Figure 6-25. The Baseline Moisture Content Measured in Hole WN3 
Baseline fraction volume water content in WN4.

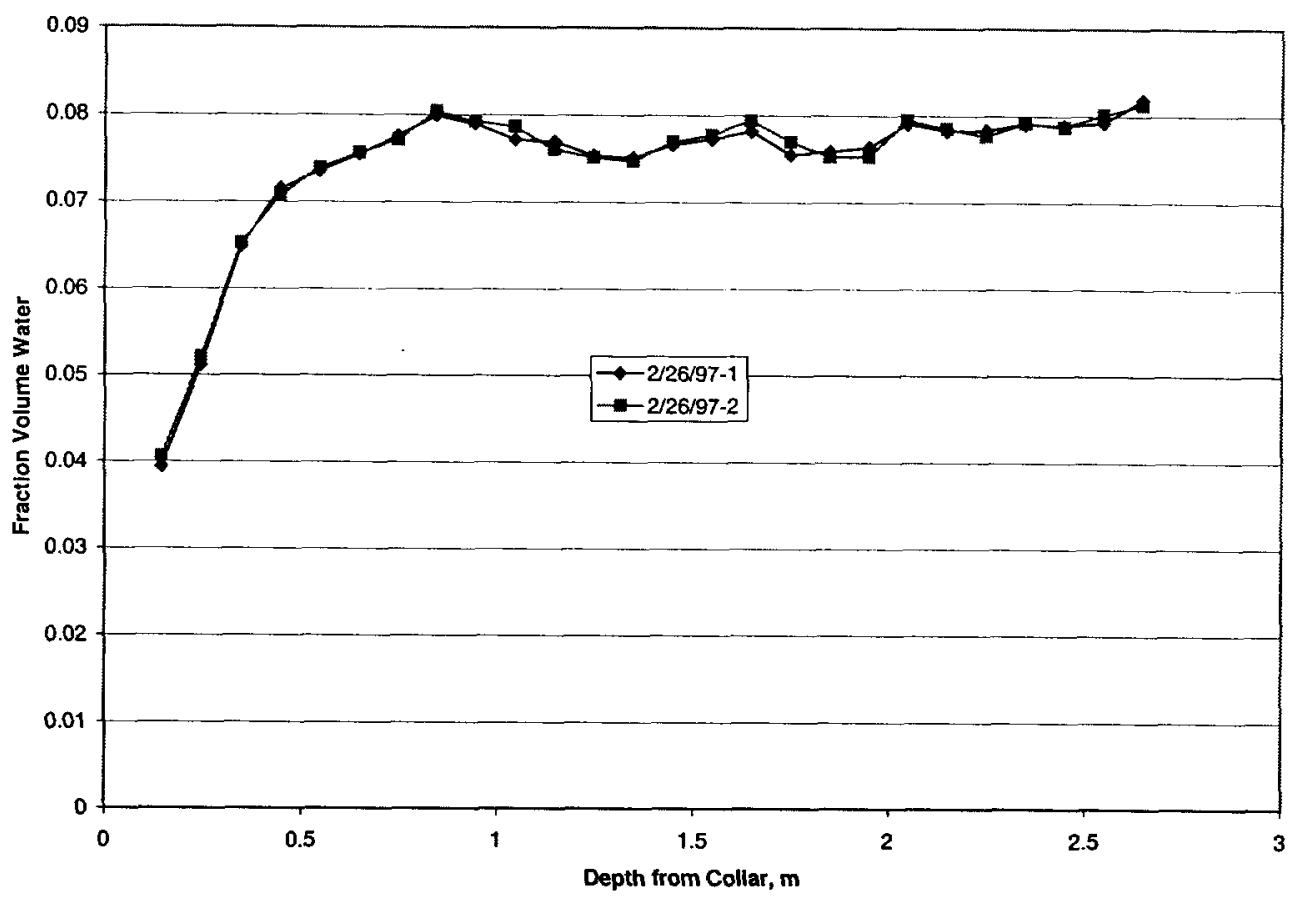

Figure 6-26. The Baseline Moisture Content Measured in Hole WN4 as a Function of Depth from the West Face of the Block 


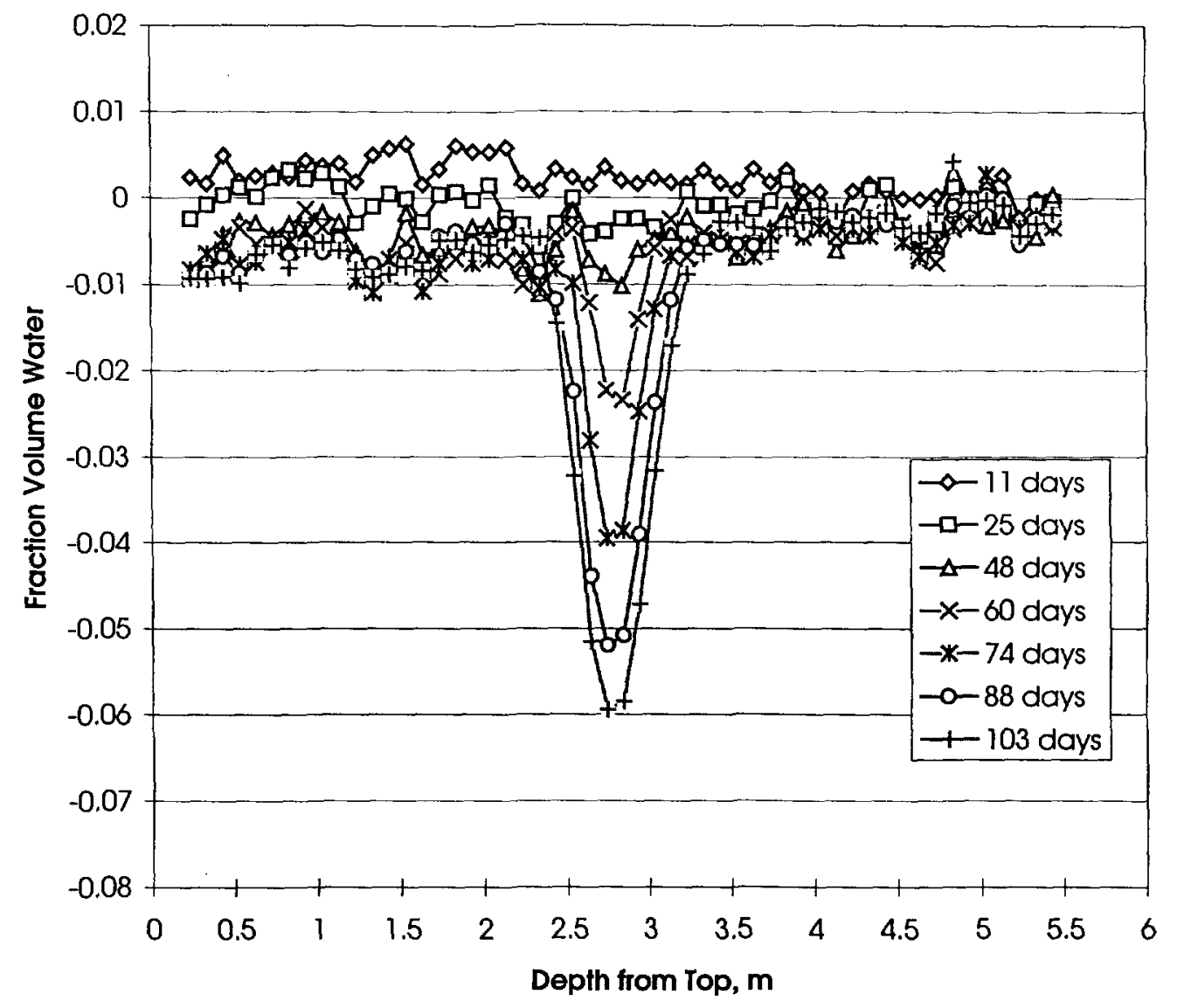

NOTE: Between the in-test and the baseline as a function of depth from the top of the block.

Figure 6-27. Difference Fraction Volume Water in Hole TN1 from March 11, 1997, to June 11, 1997 


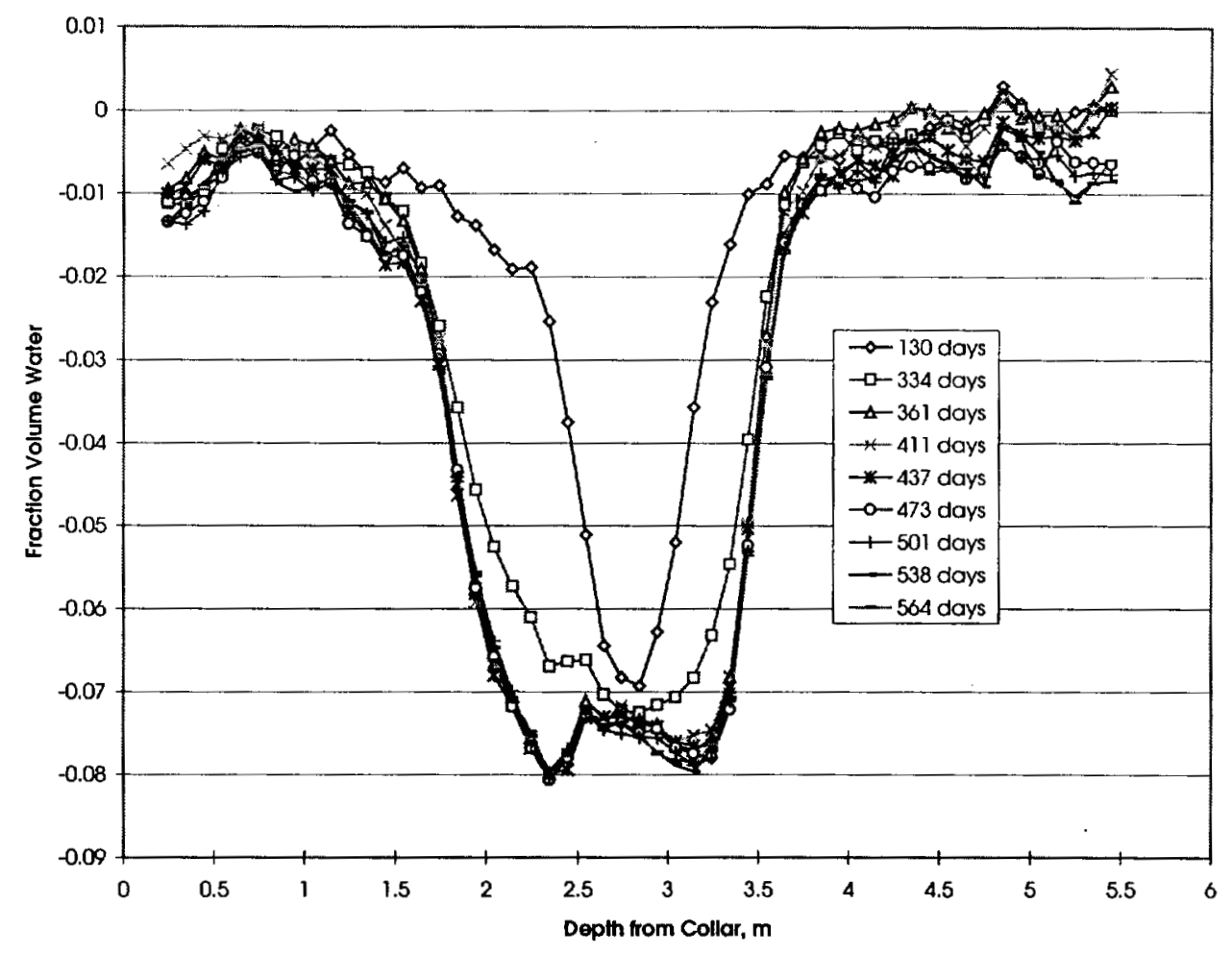

Figure 6-28. Difference Fraction Volume Water, Between the In-heat and the Baseline, in Hole TN1 as a Function of Depth from Top of the Block, from June 8, 1997, to September 15, 1998. 


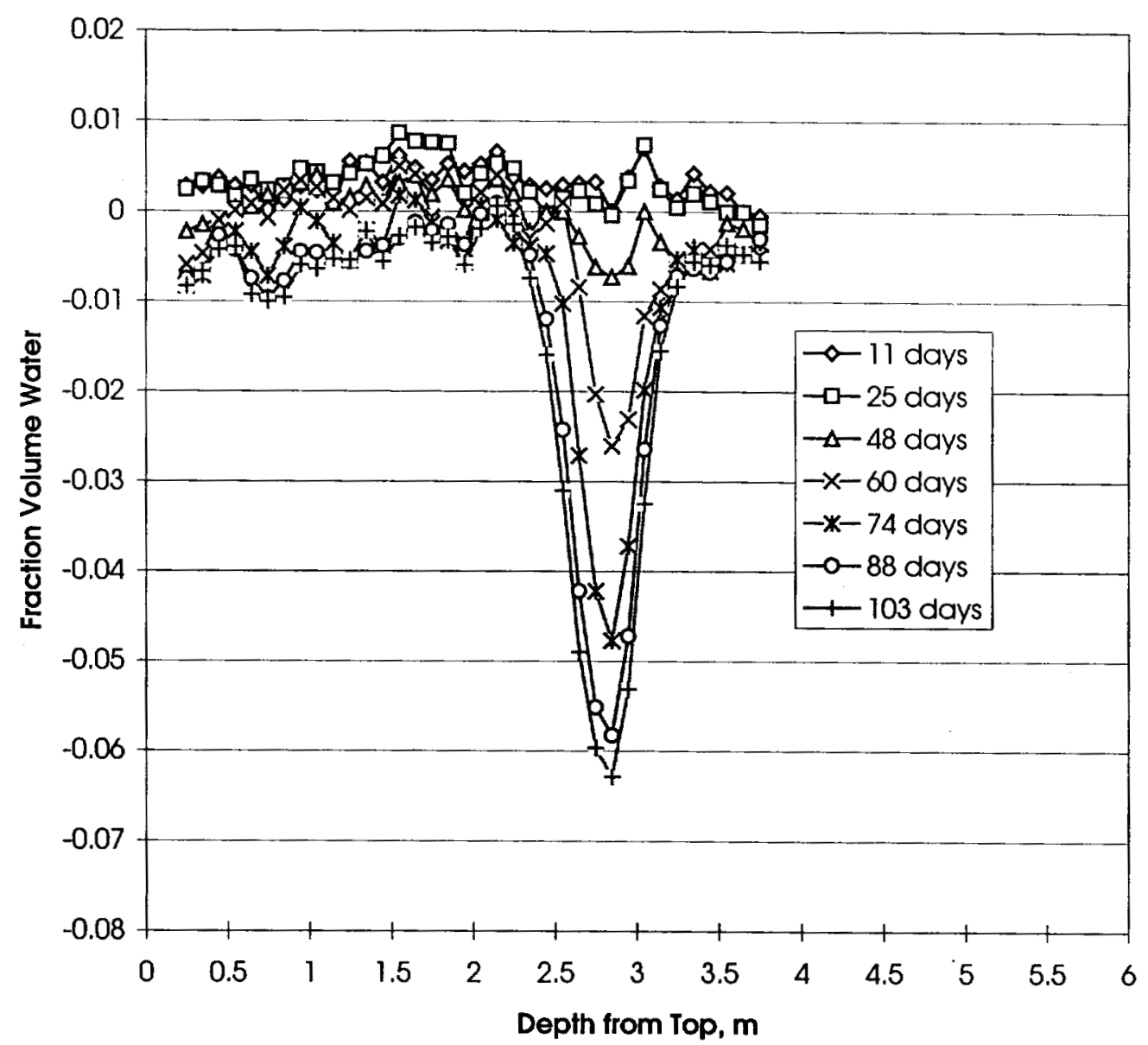

Figure 6-29. Difference Fraction Volume Water, Between the In-heat and the Baseline, in Hole TN2 as a Function of Depth from Top of the Block, from March 11, 1997, to June 11, 1997 


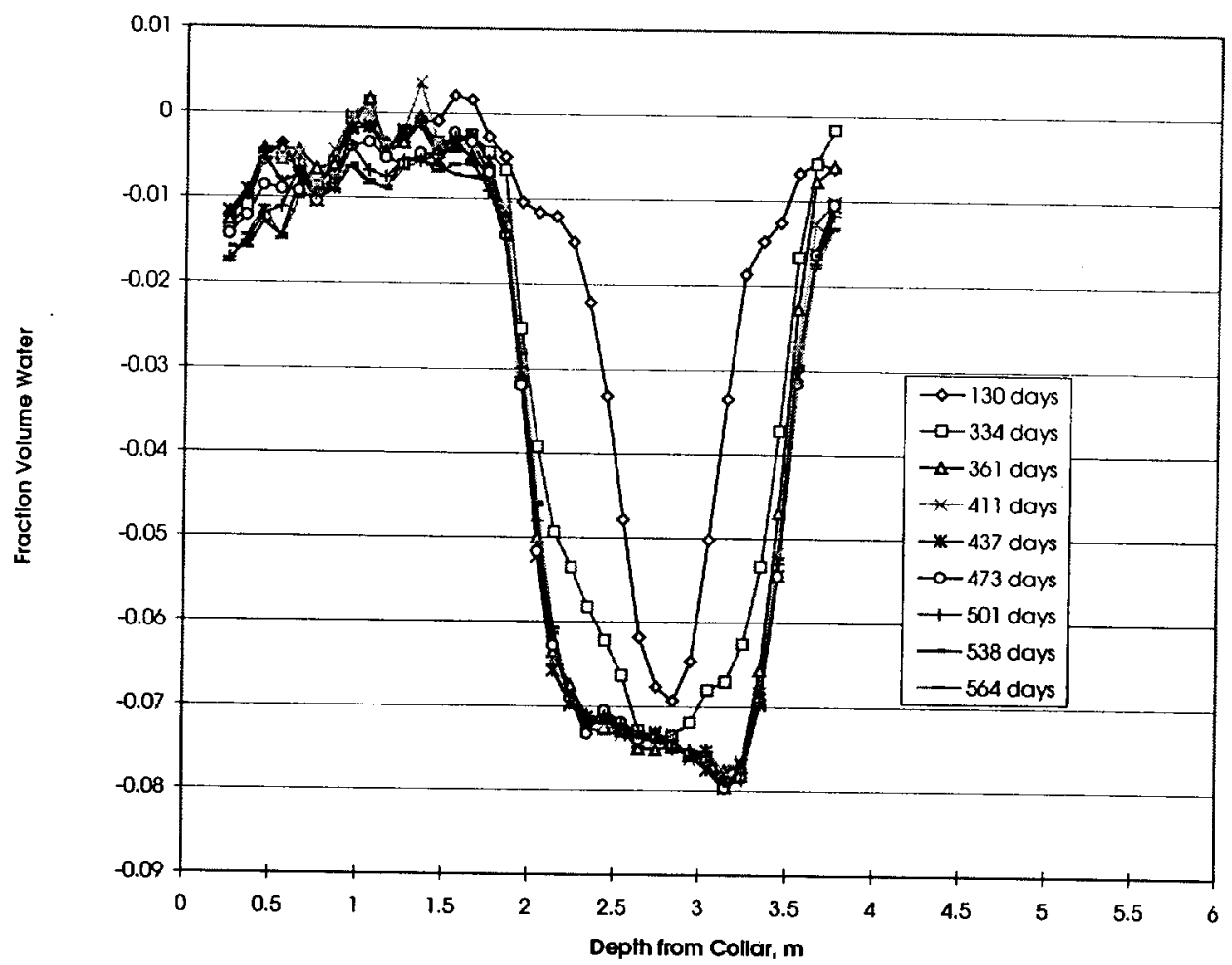

Figure 6-30. Difference Fraction Volume Water, Between the In-heat and the Baseline, in Hole TN2 as a Function of Depth from Top of the Block, from July 8, 1997, to September 15, 1998 


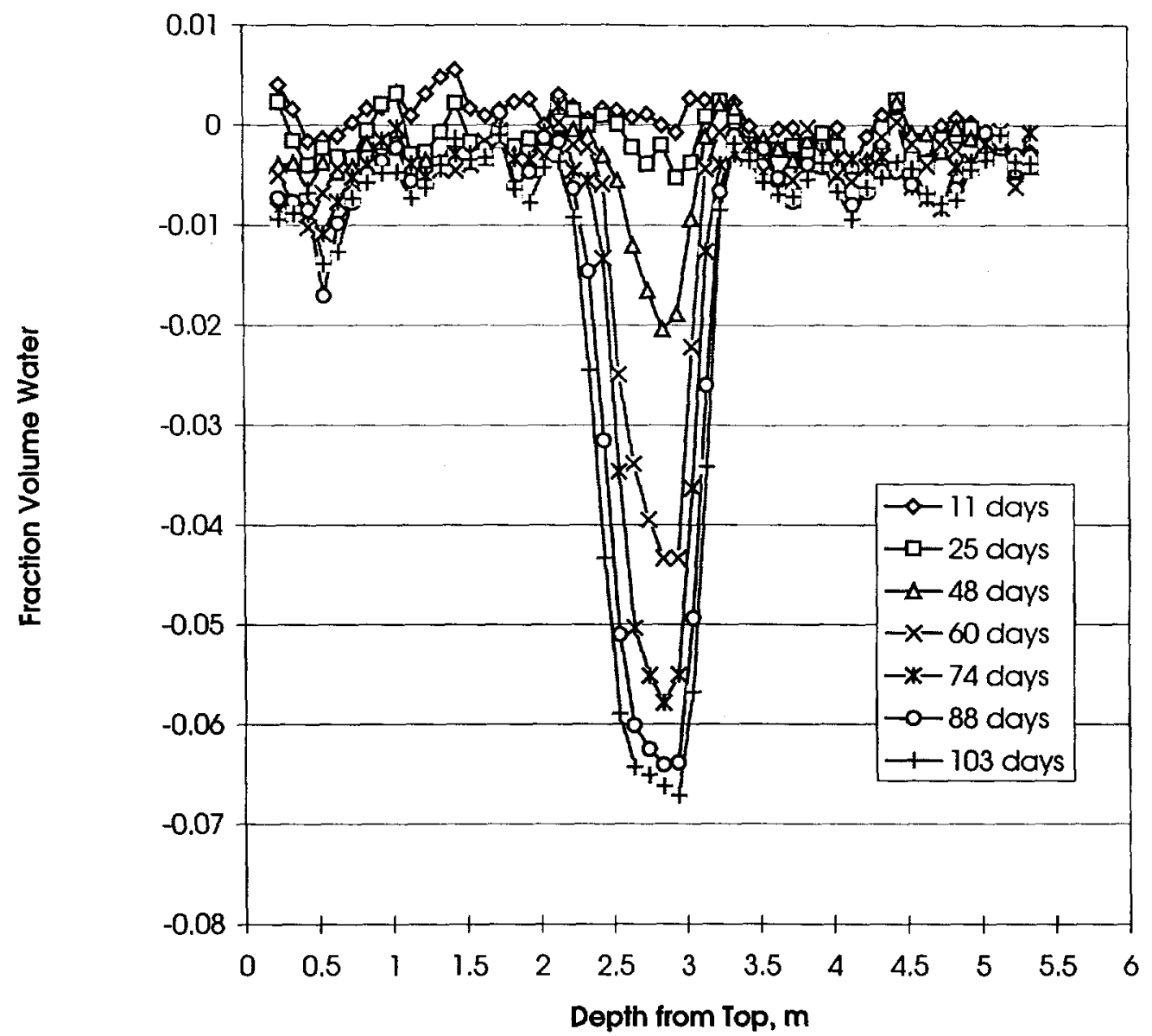

Figure 6-31. Difference Fraction Volume Water, Between the In-heat and the Baseline, in Hole TN3 as a Function of Depth from Top of the Block, from March 11, 1997, to June 11, 1997 


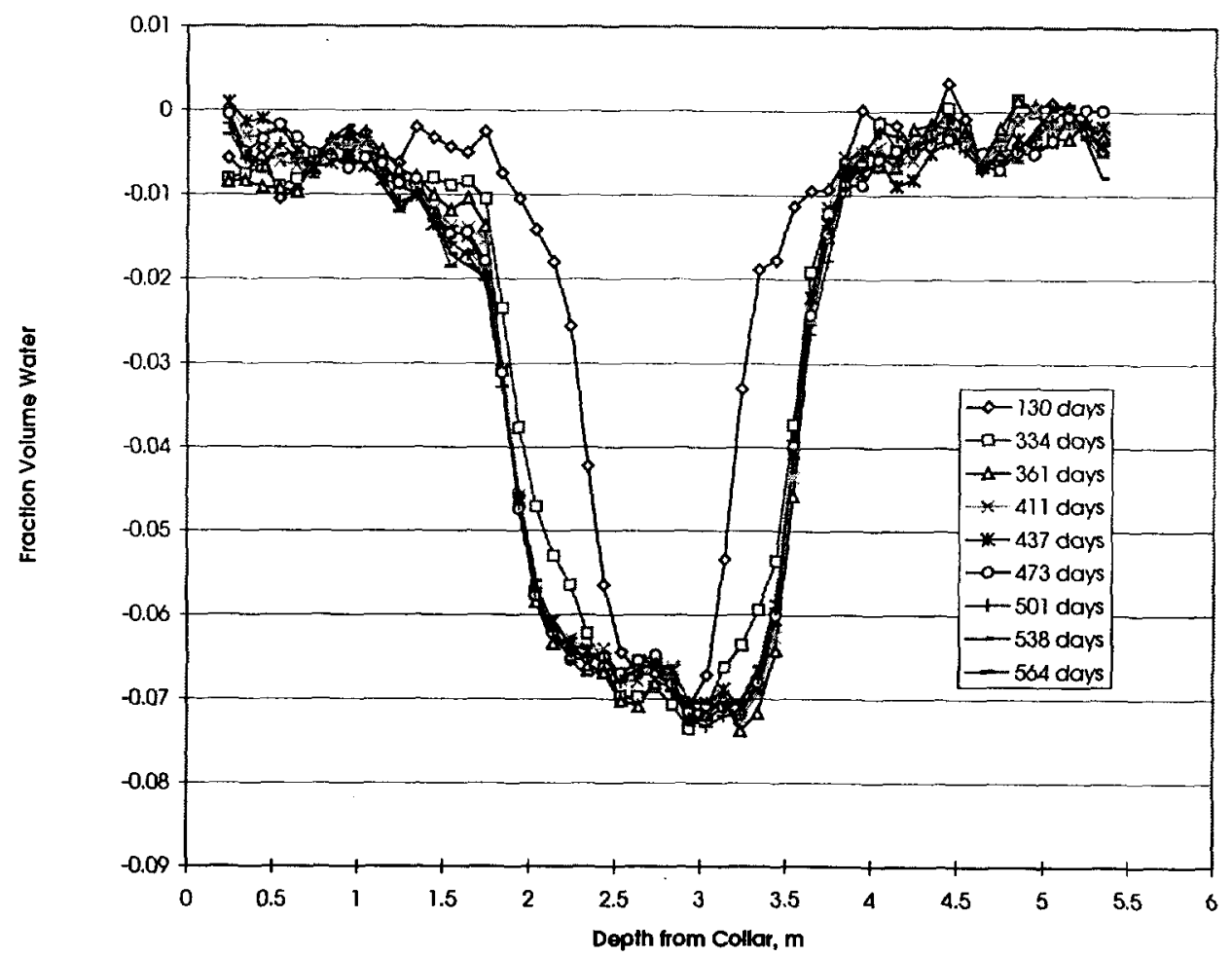

Figure 6-32. Difference Fraction Volume Water, Between the In-heat and the Baseline, in Hole TN3 as a Function of Depth from Top of the Block, from July 8, 1997, to September 15, 1998 


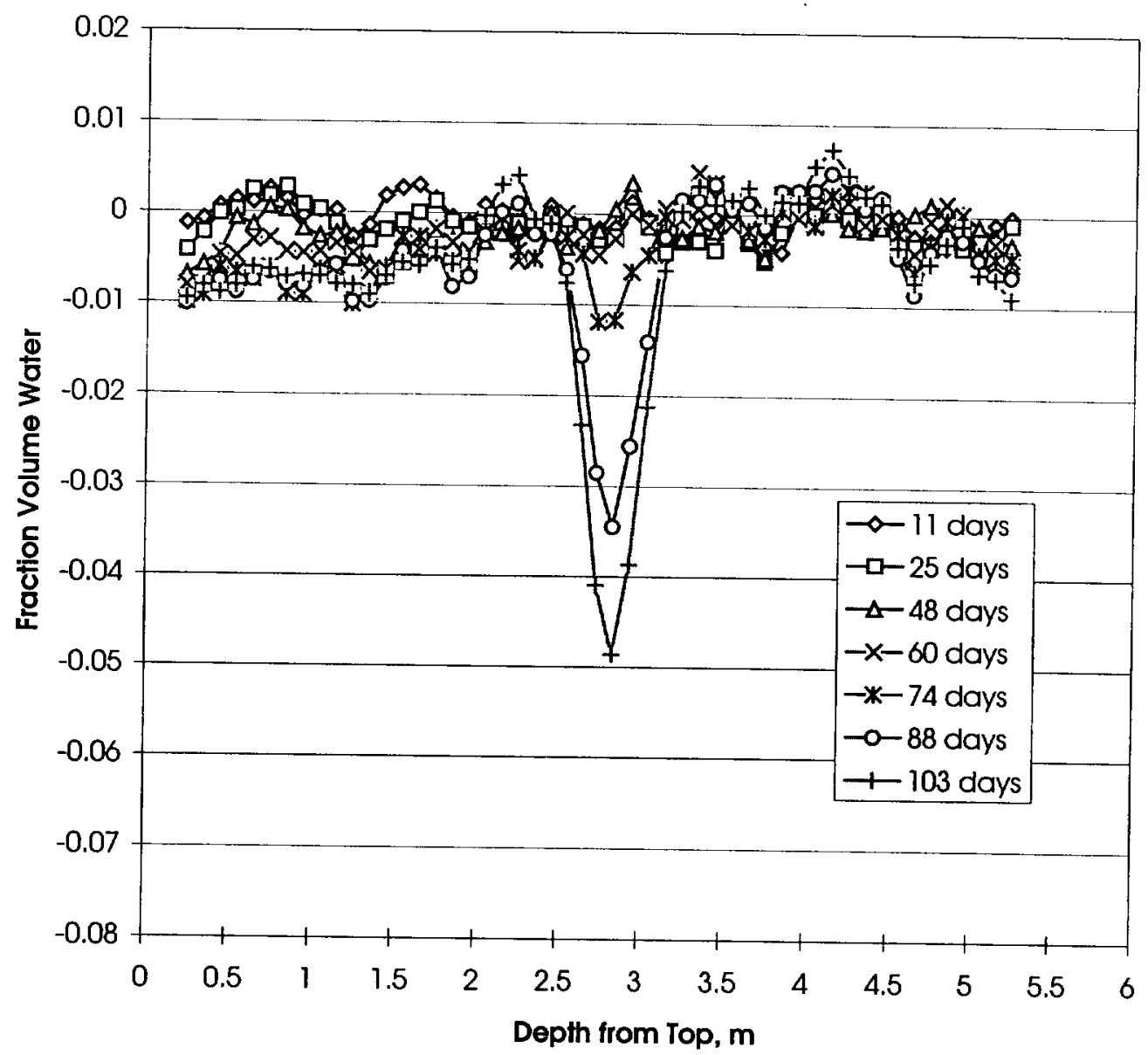

Figure 6-33. Difference Fraction Volume Water, Between the In-heat and the Baseline, in Hole TN4 as a Function of Depth from Top of the Block, from March 11, 1997, to June 11, 1997 


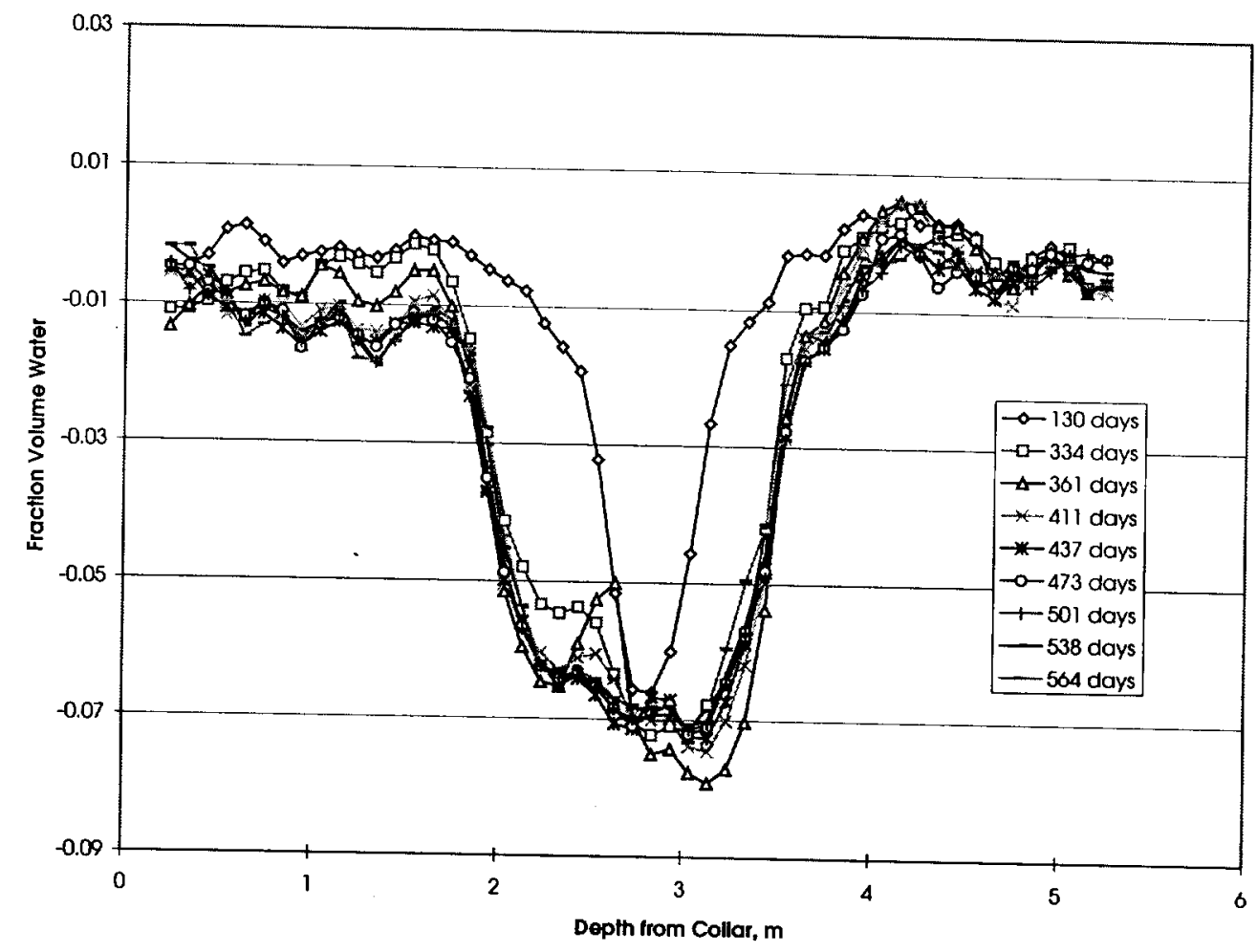

Figure 6-34. Difference Fraction Volume Water, Between the In-heat and the Baseline, in Hole TN4 as a Function of Depth from Top of the Block, from July 8, 1997, to September 15, 1998 


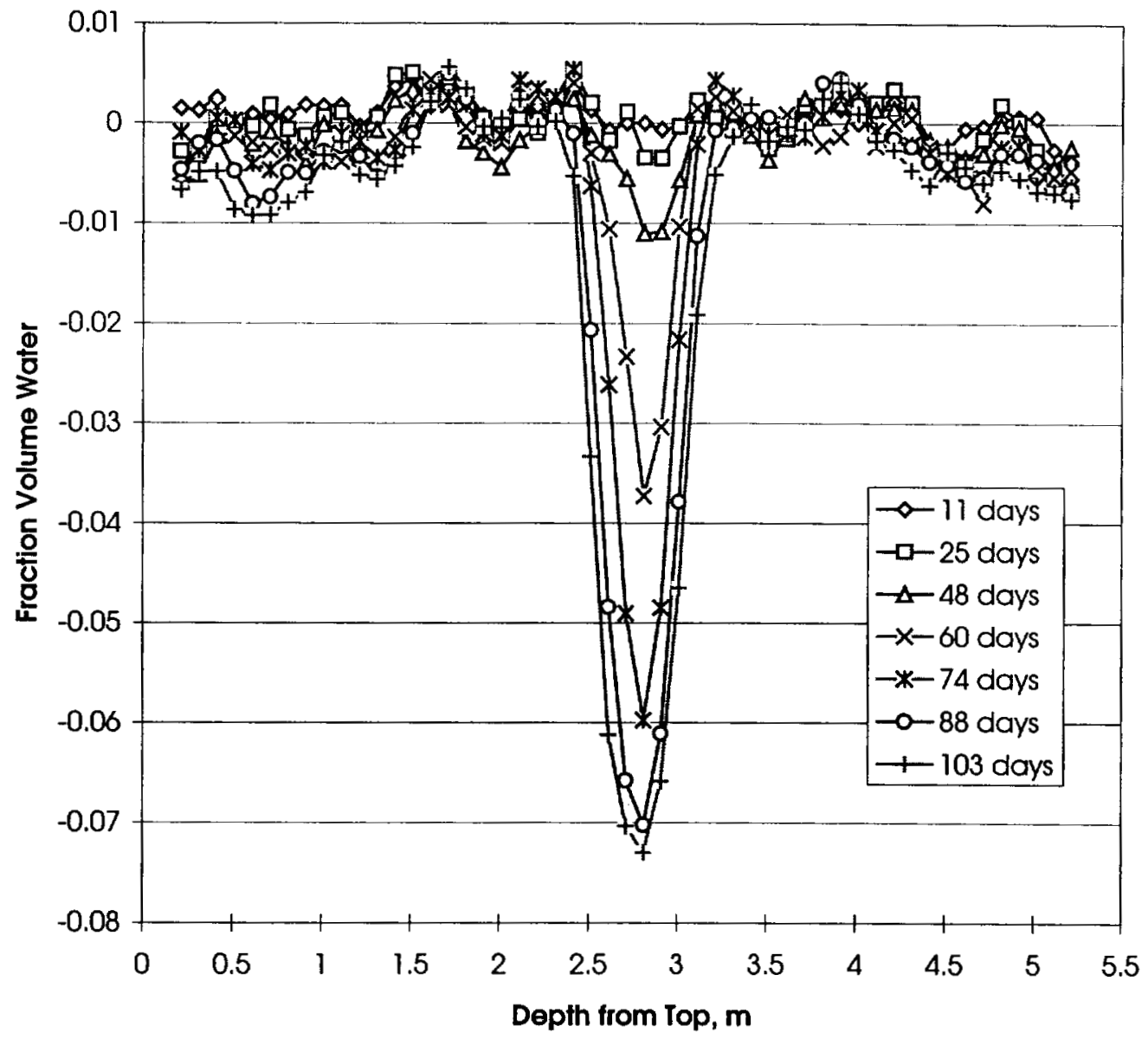

Figure 6-35. Difference Fraction Volume Water, Between the In-heat and the Baseline, in Hole TN5 as a Function of Depth from Top of the Block, from March 11, 1997 to June 11, 1997 


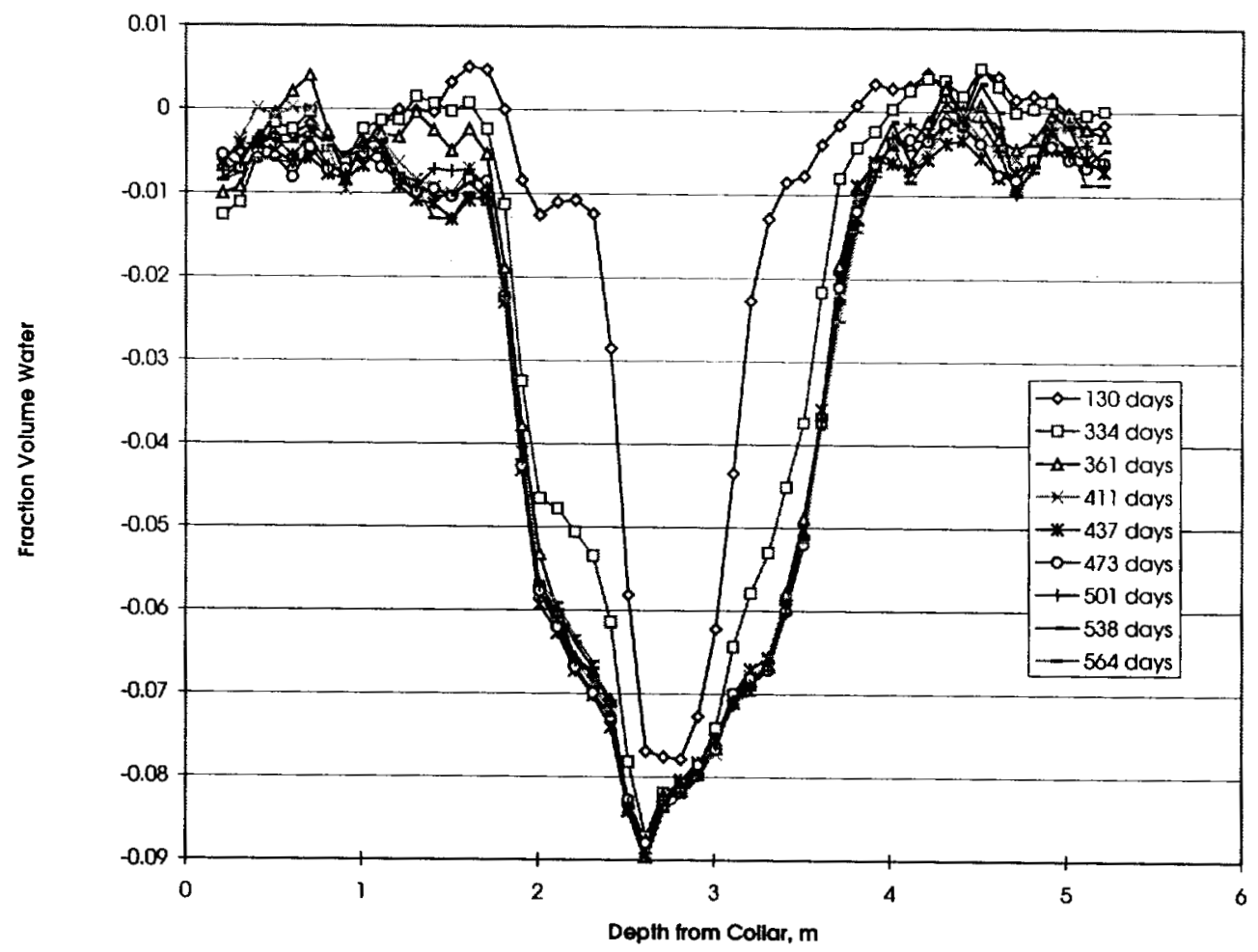

Figure 6-36. Difference Fraction Volume Water, Between the In-heat and the Baseline, in Hole TN5 as a Function of Depth from Top of the Block, from July 8,1997 , to September 15, 1998 


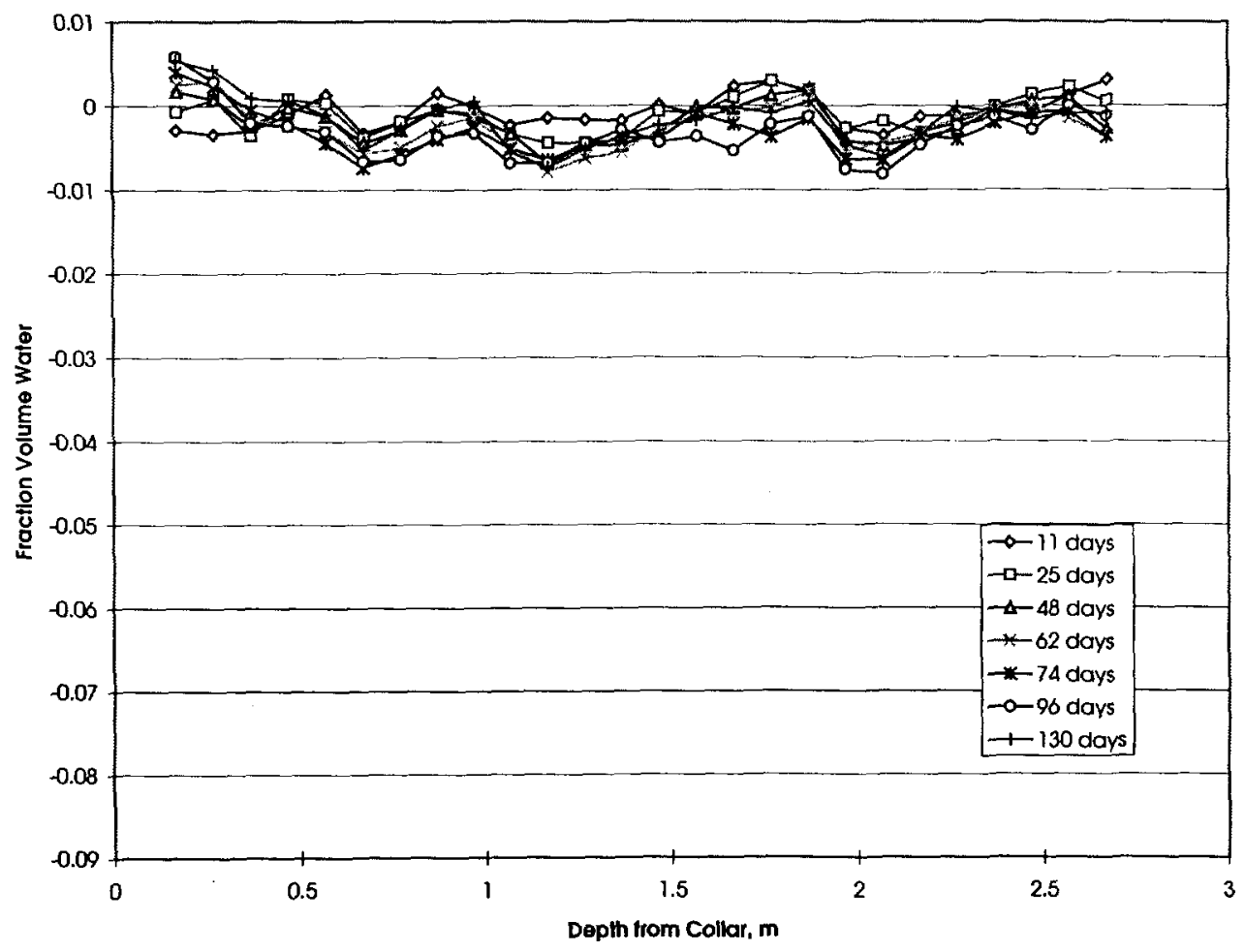

Figure 6-37. Difference Fraction Volume Water, Between the In-heat and the Baseline, in Hole NN1 as a Function of Depth from the North Face of the Block, from March 11, 1997, to July 8, 1997 


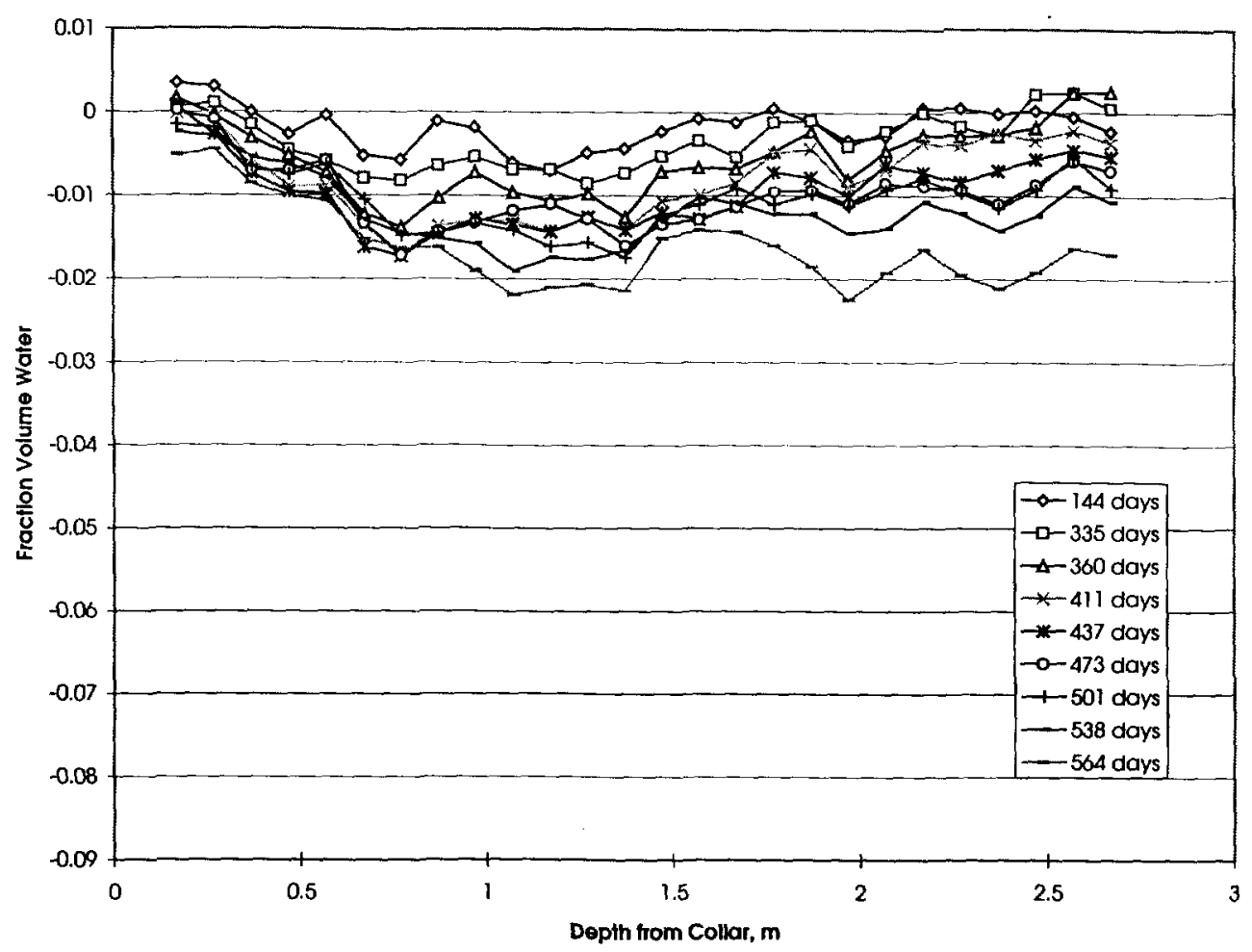

Figure 6-38. Difference Fraction Volume Water, Between the In-heat and the Baseline, in Hole NN1 as a Function of Depth from the North Face of the Block, from July 22, 1997, September 15, 1998 


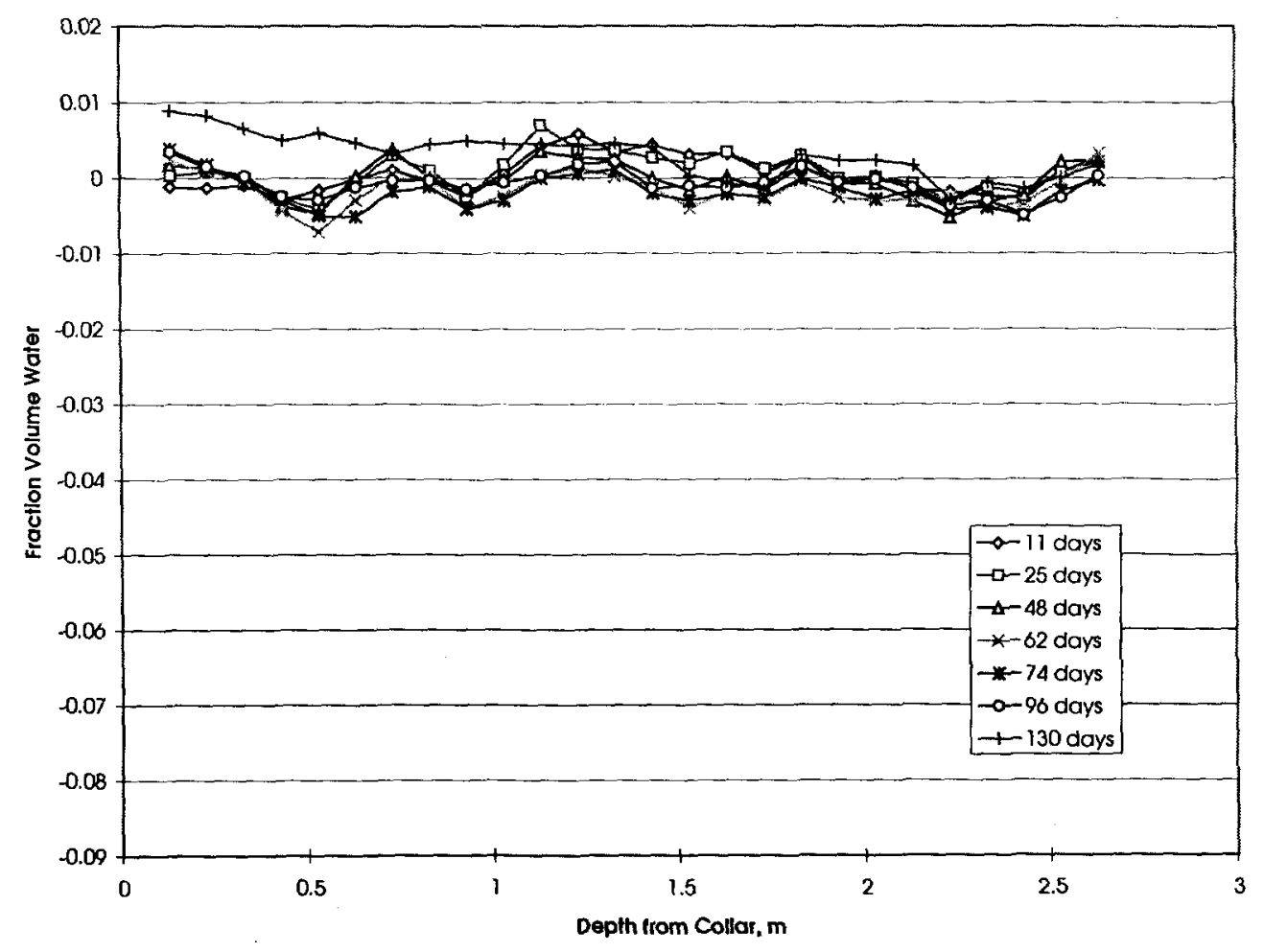

Figure 6-39. Difference Fraction Volume Water, Between the In-heat and the Baseline, in Hole NN2 as a Function of Depth from the North Face of the Block, from March 11, 1997, to July 8,1997 


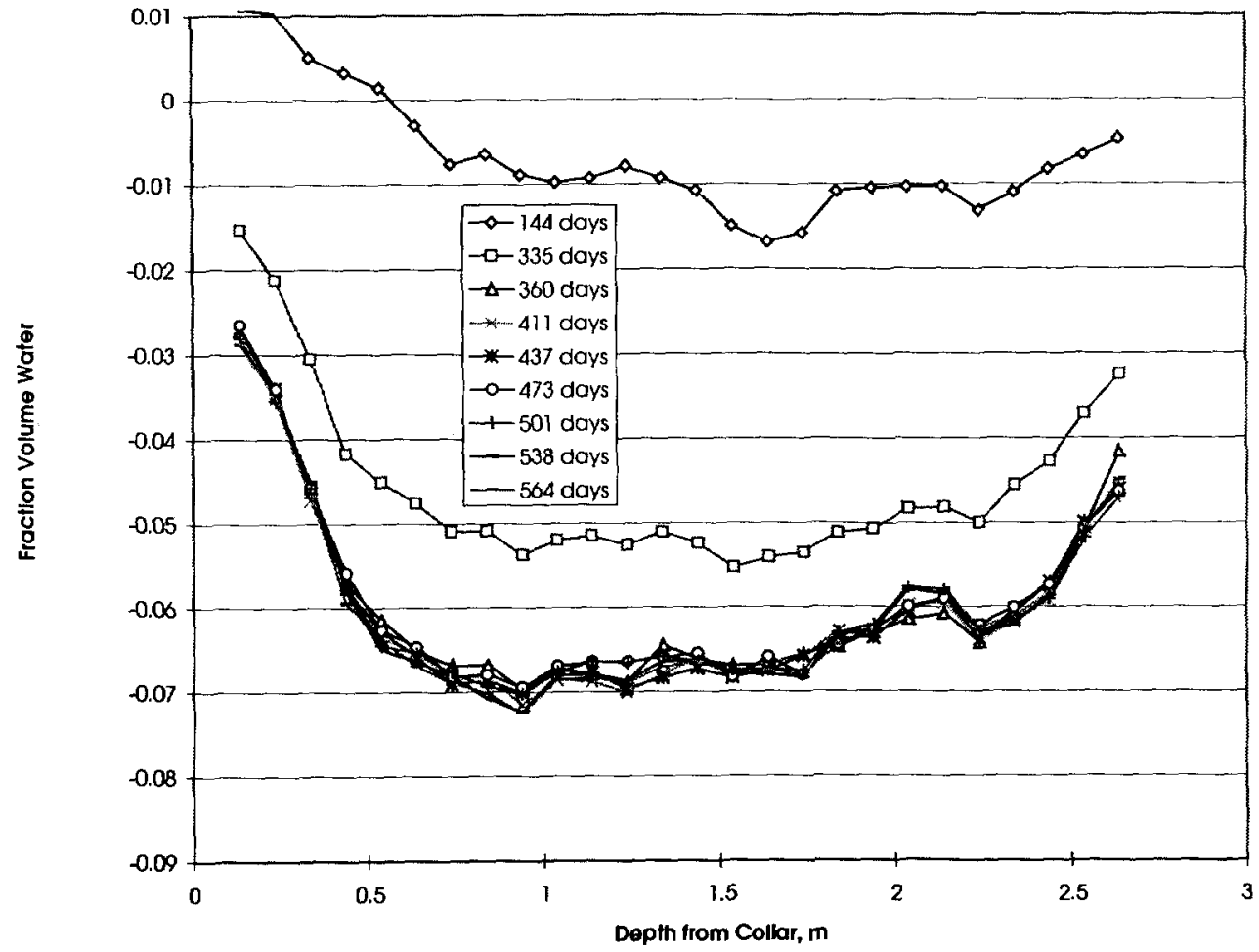

Figure 6-40. Difference Fraction Volume Water, Between the In-heat and the Baseline, in Hole NN2 as a Function of Depth from the North Face of the Block, from July 22, 1997, to September 15, 1998 


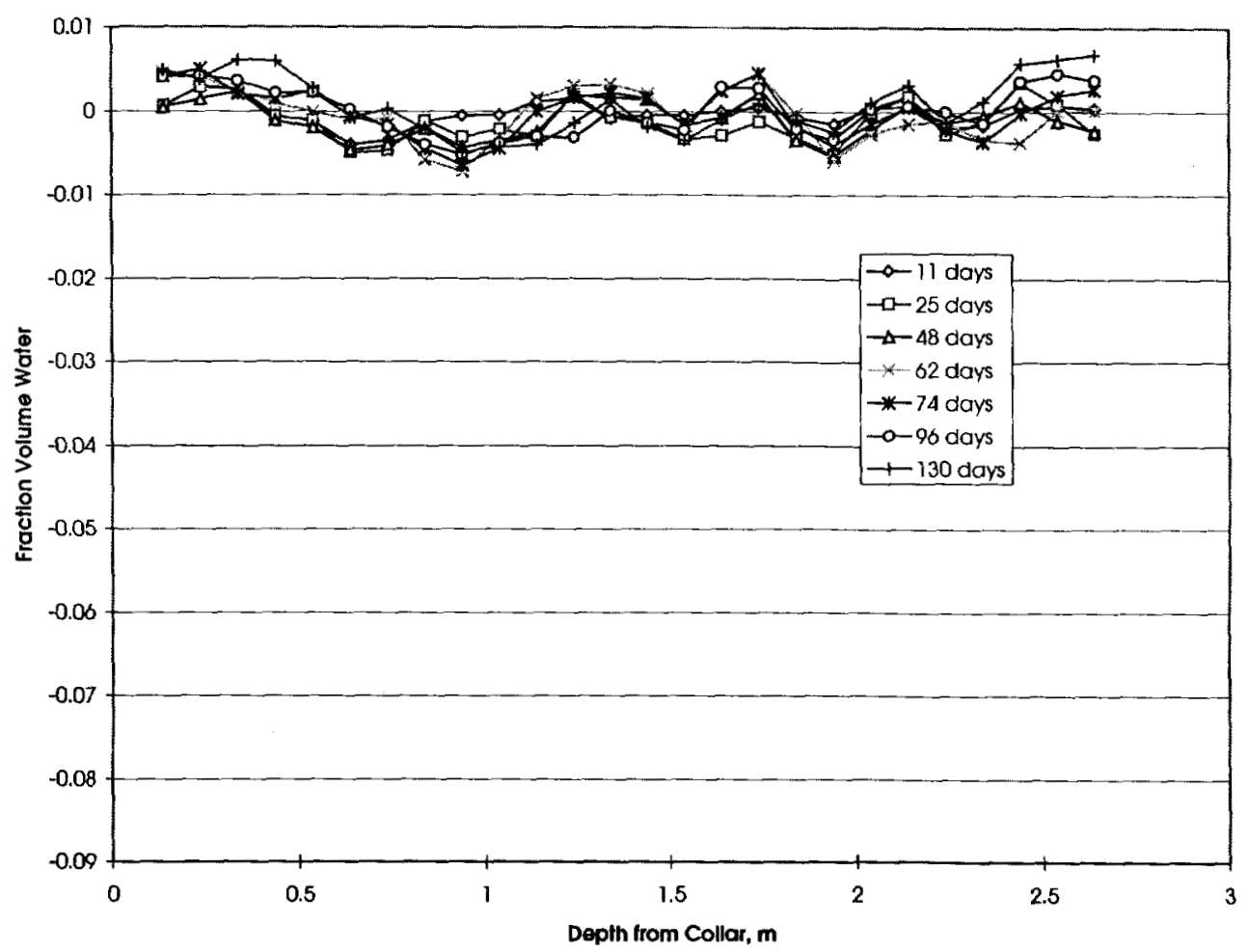

Figure 6-41. Difference Fraction Volume Water, Between the In-heat and the Baseline, in Hole NN3 as a Function of Depth from the North Face of the Block, from March 11, 1997, to July 8, 1997 


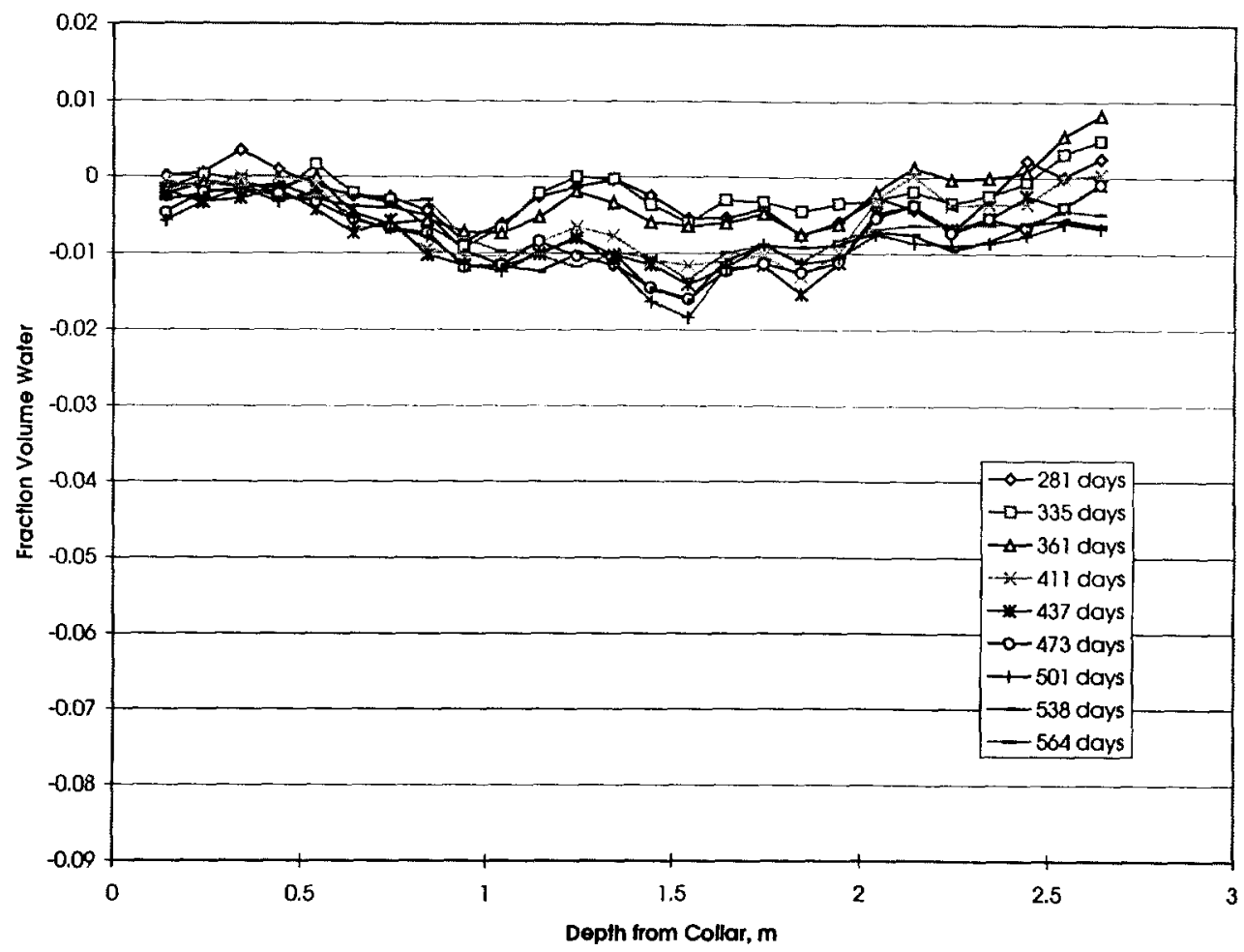

Figure 6-42. Difference Fraction Volume Water, Between the In-heat and the Baseline, in Hole NN3 as a Function of Depth from the North Face of the Block, from November 6, 1997, to September 15, 1998 


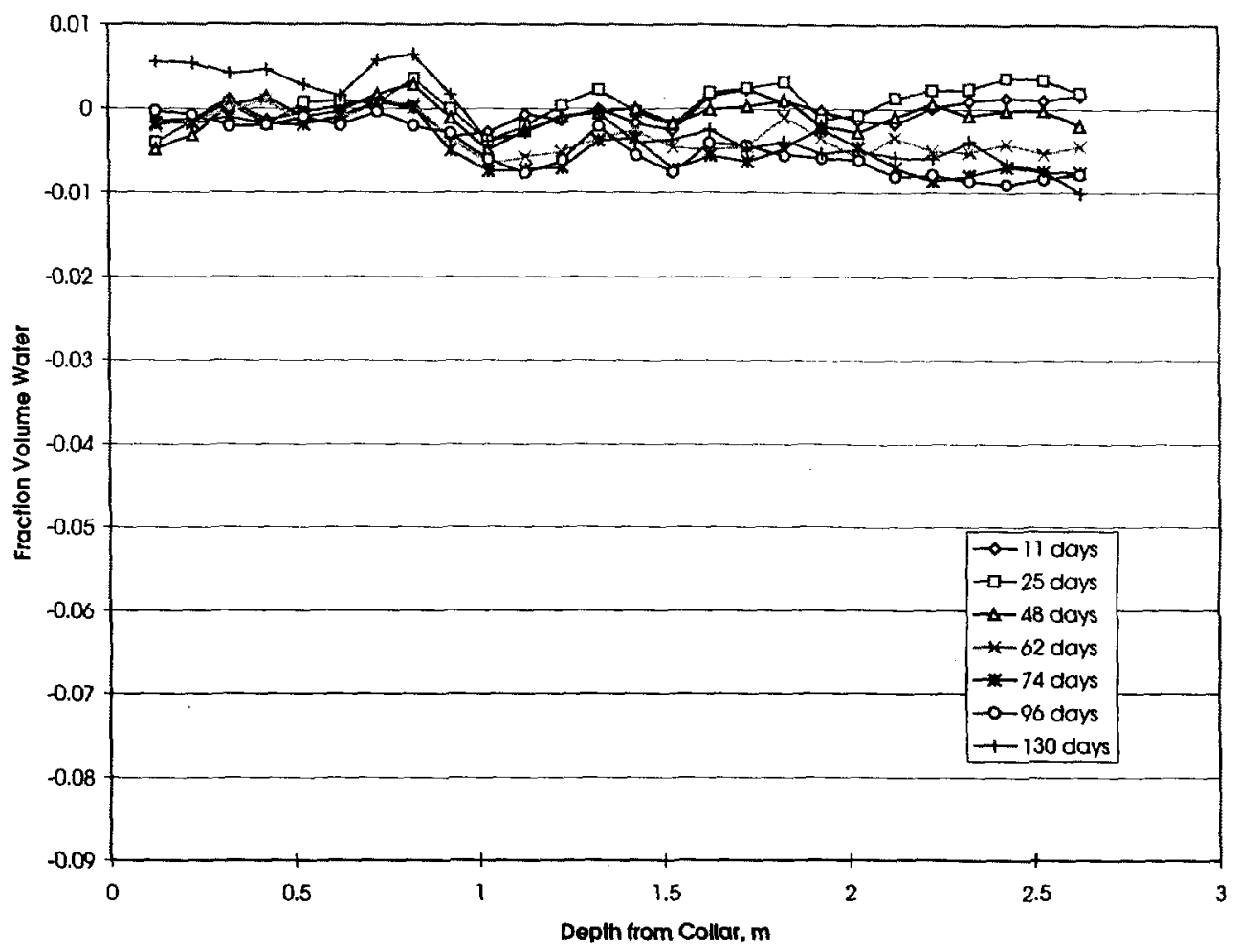

Figure 6-43. Difference Fraction Volume Water, Between the In-heat and the Baseline, in Hole NN4 as a Function of Depth from the North Face of the Block, from March 11, 1997, to July 8,1997 


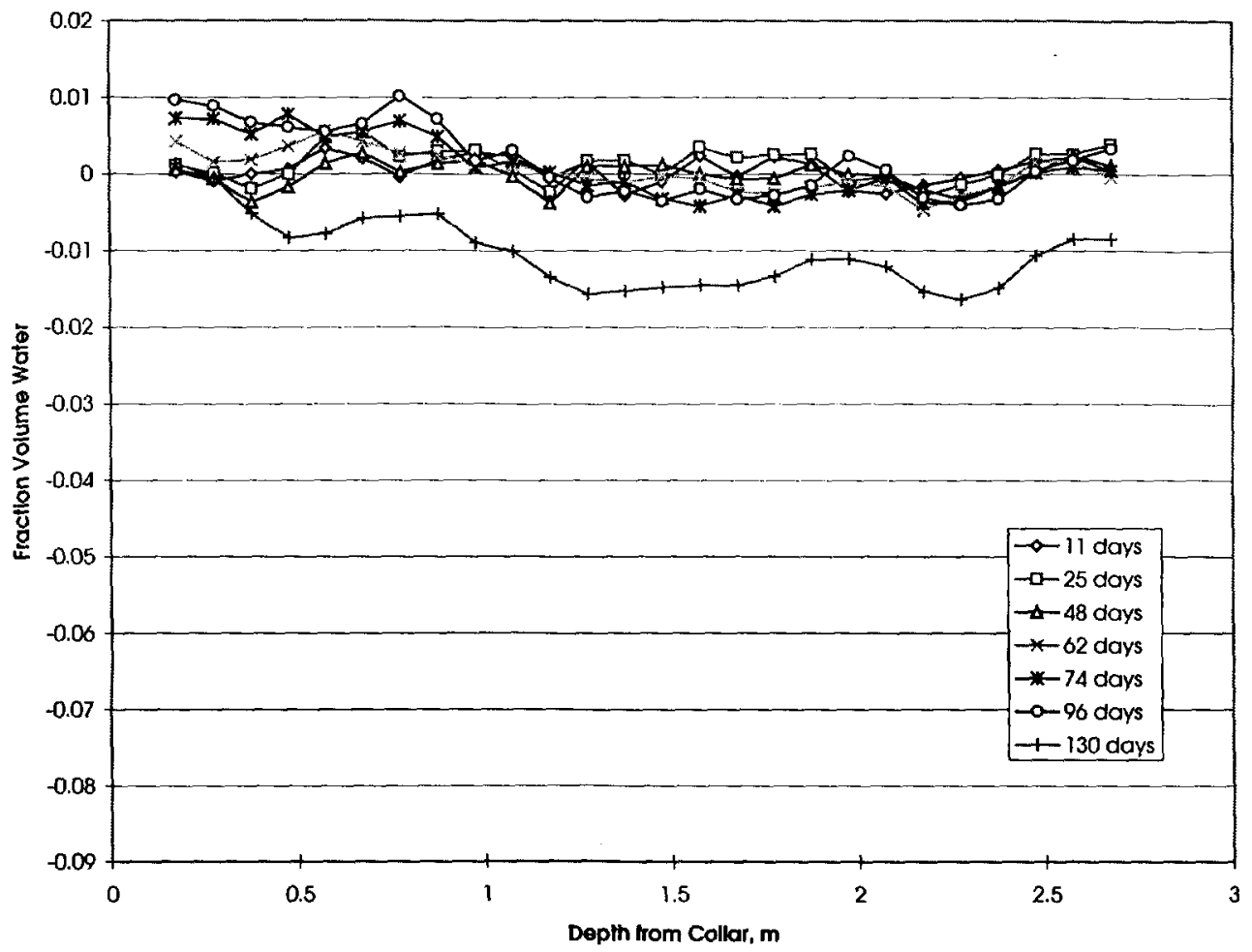

Figure 6-45. Difference Fraction Volume Water, Between the In-heat and the Baseline, in Hole NN5 as a Function of Depth from the North Face of the Block, from March 11, 1997, to July 8, 1997 


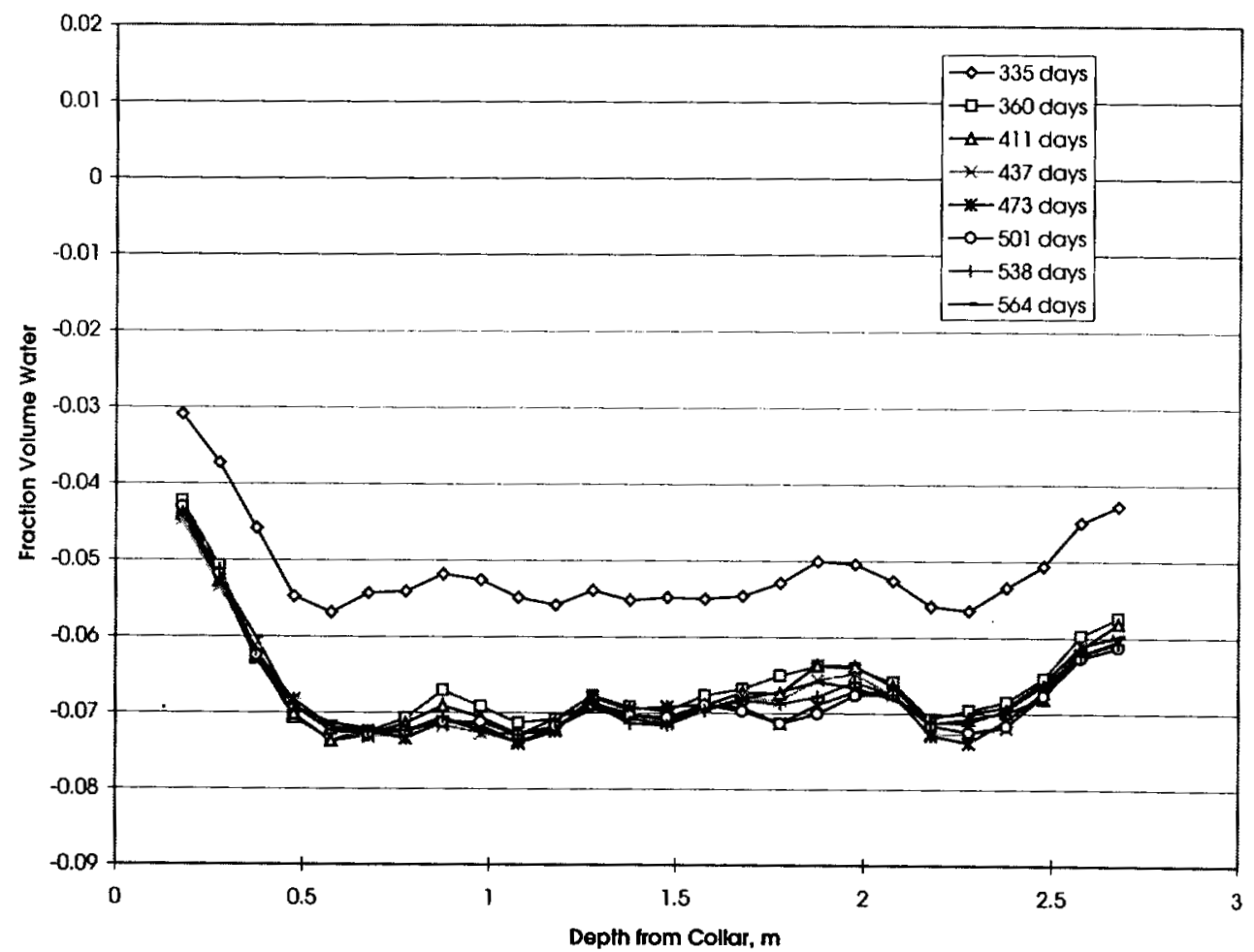

Figure 6-46. Difference Fraction Volume Water, Between the In-heat and the Baseline, in Hole NN5 as a Function of Depth from the North Face of the Block, from January 29, 1998, to September 15, 1998 


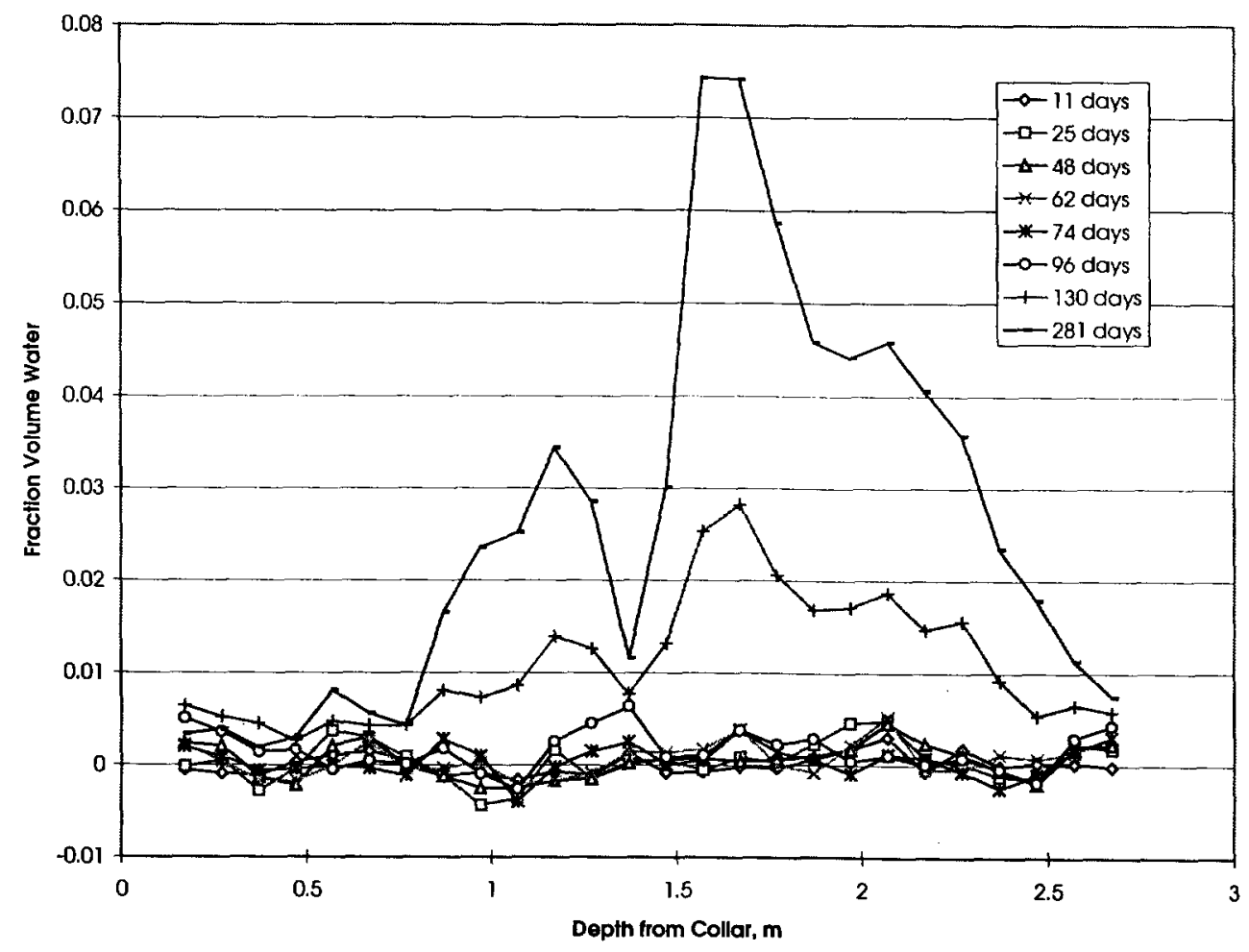

Figure 6-47. Difference Fraction Volume Water, Between the In-test and the Baseline as a Function of Depth from the North face of the Block, in Hole NN6 from March 11, 1997, to November 6, 1997 


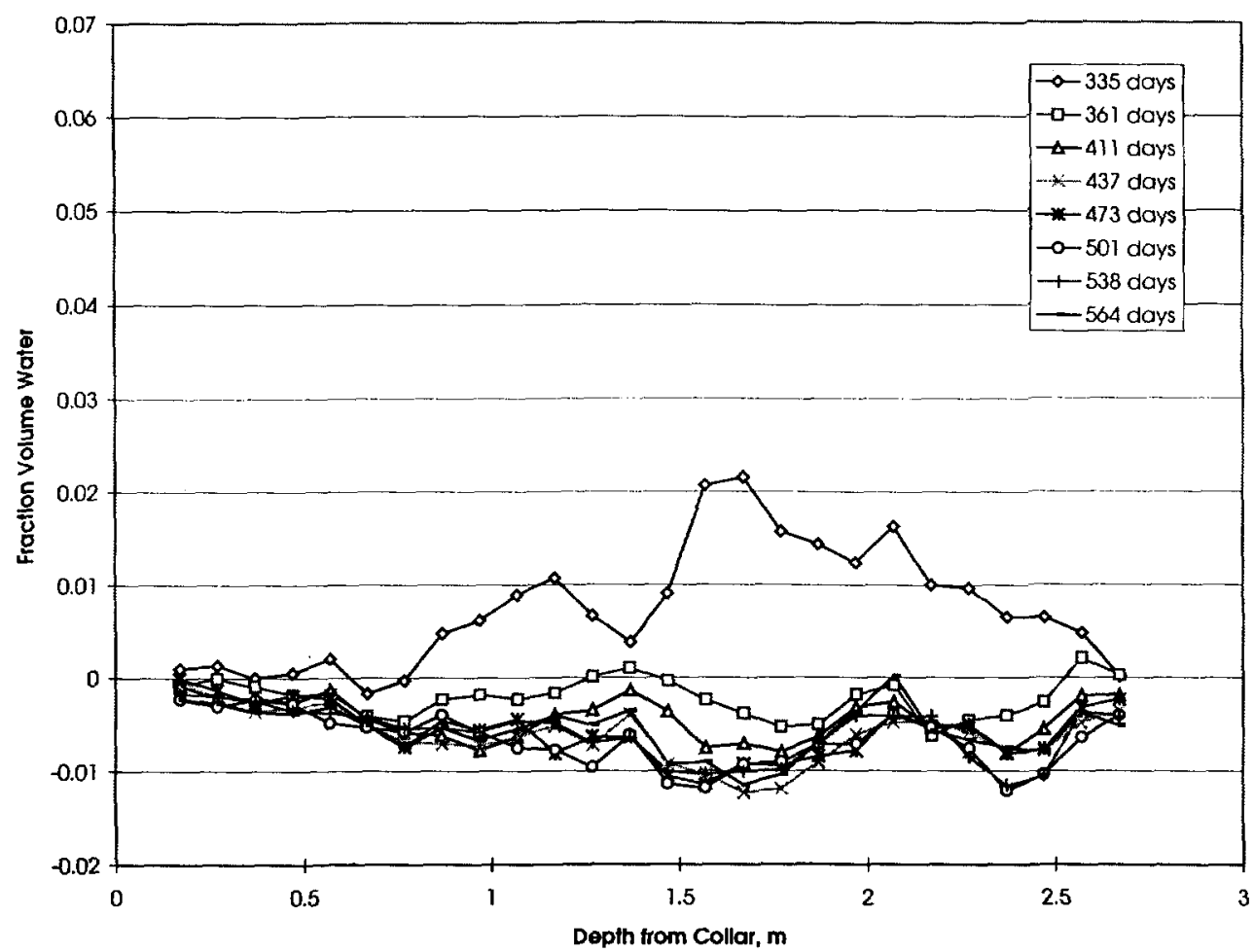

Figure 6-48. Difference Fraction Volume Water, Between the In-heat and the Baseline, in Hole NN6 as a Function of Depth from the North Face of the Block, from January 29, 1998, to September 15, 1998 


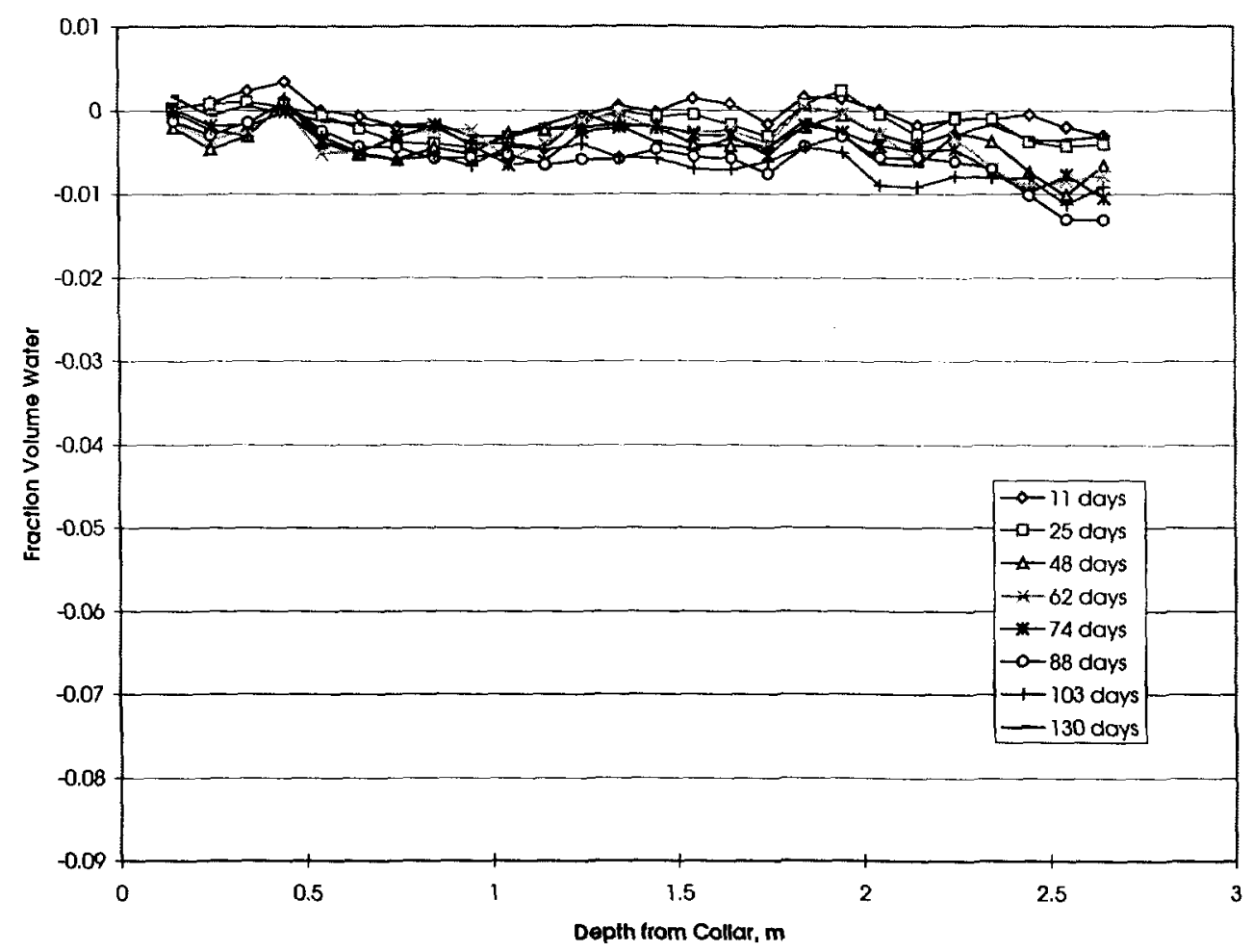

Figure 6-49. Difference Fraction Volume Water, Between the In-heat and the Baseline, in Hole WN1 as a Function of Depth from the West Face of the Block, from March 11, 1998, to July 8, 1997 


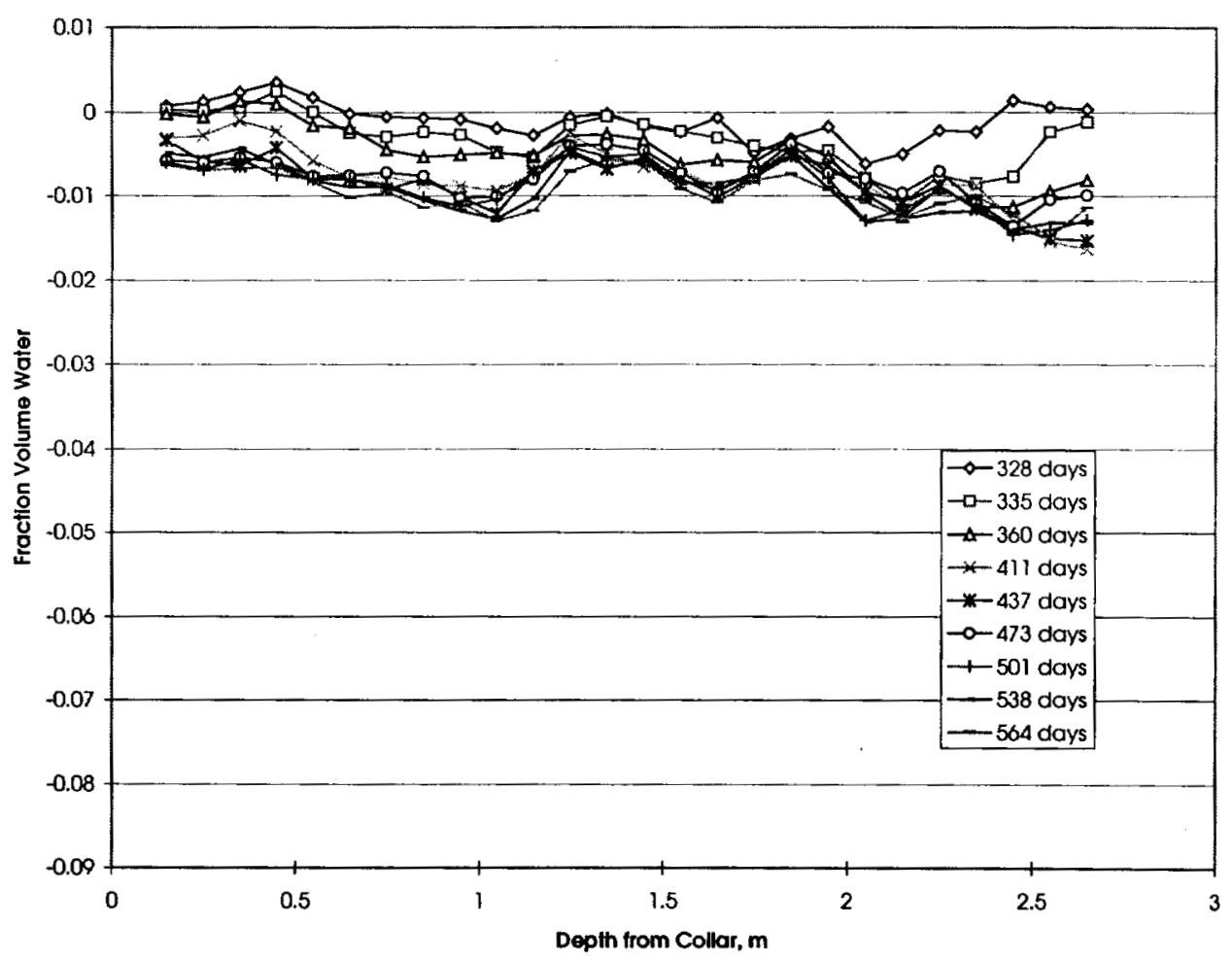

Figure 6-50. Difference Fraction Volume Water, Between the In-heat and the Baseline, in Hole WN1 as a Function of Depth from the West Face of the Block, from January 22, 1998, to September 15, 1998 


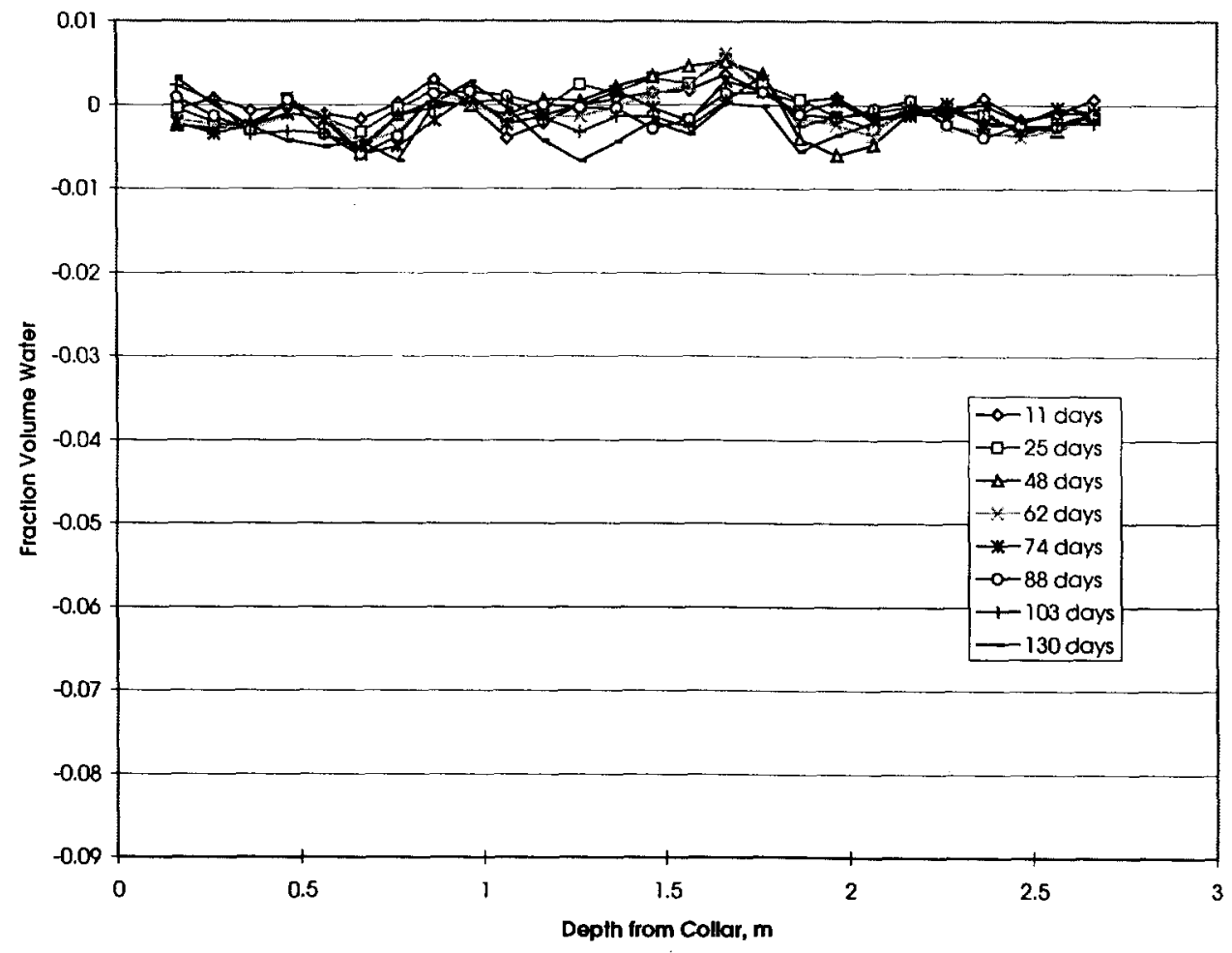

Figure 6-51. Difference Fraction Volume Water, Between the In-heat and the Baseline, in Hole WN2 as a Function of Depth from the West Face of the Block, from March 11, 1997, to July 8, 1997 


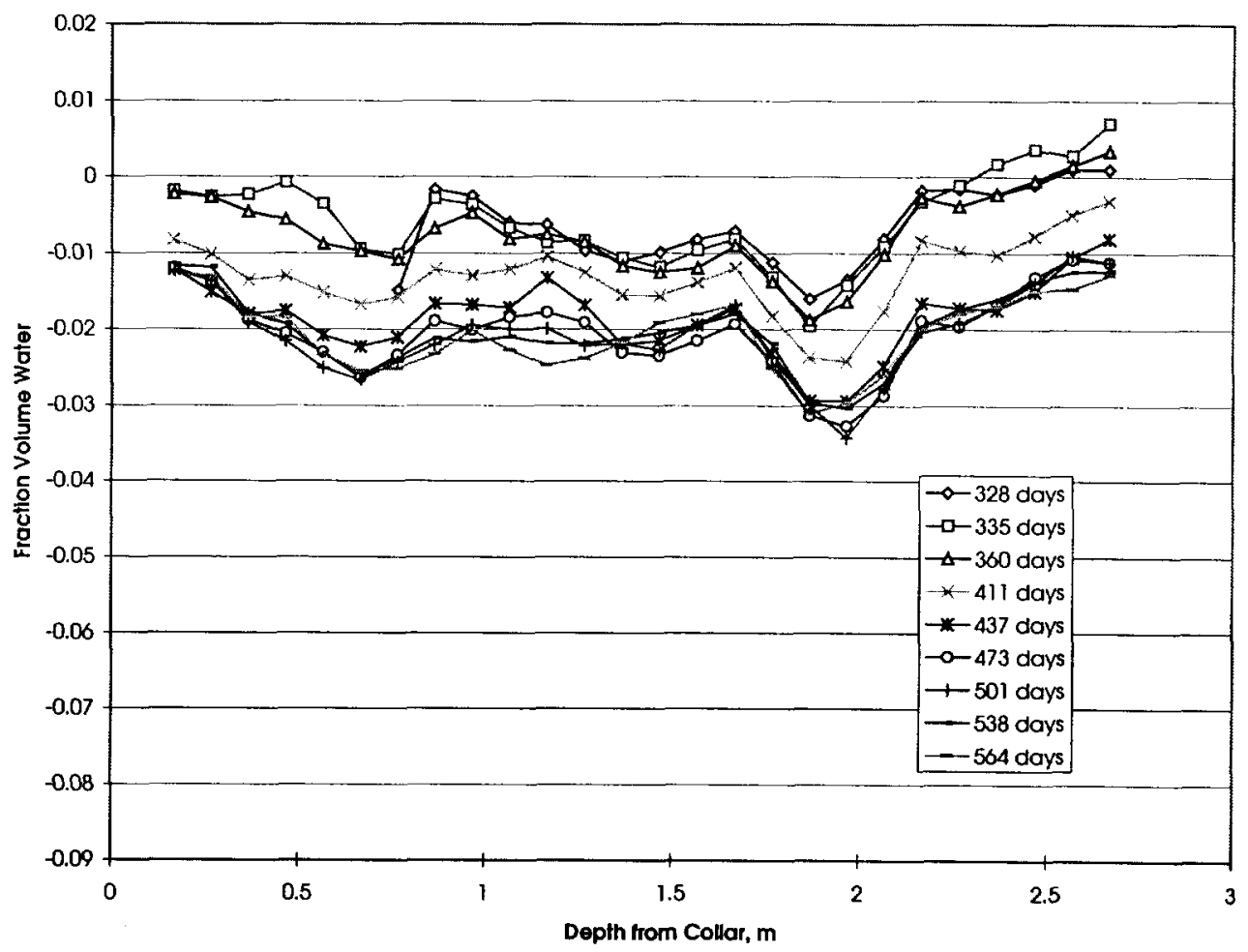

Figure 6-52. Difference Fracture Volume Water, Between the In-heat and the Baseline, in Hole WN2 as a Function of Depth from the West Face of the Block, from January 22, 1998, to September 15, 1998 


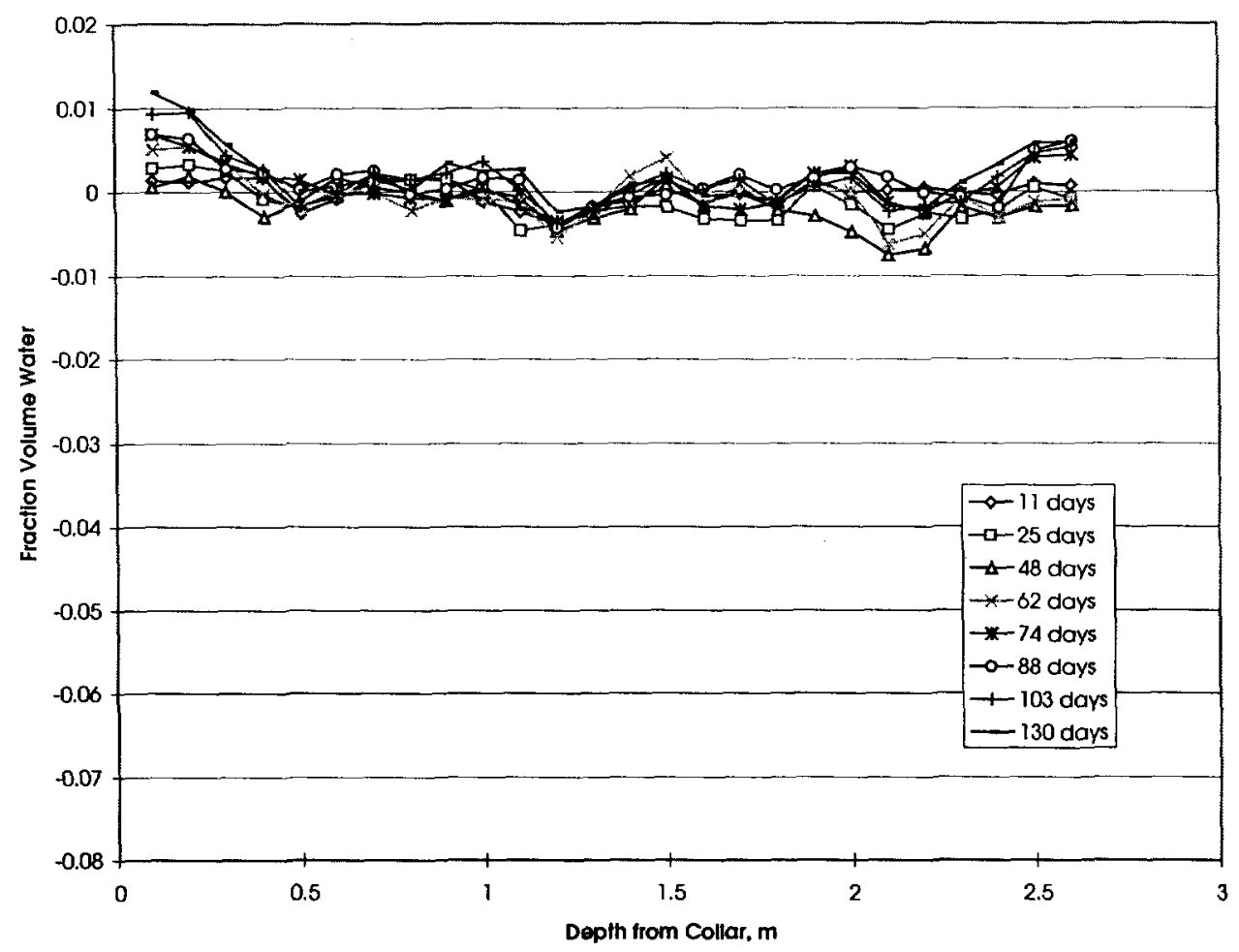

Figure 6-53. Difference Fraction Volume Water, Between the In-heat and the Baseline, in Hole WN3 as a Function of Depth from the West Face of the Block, from March 11, 1997, to July 8, 1997 


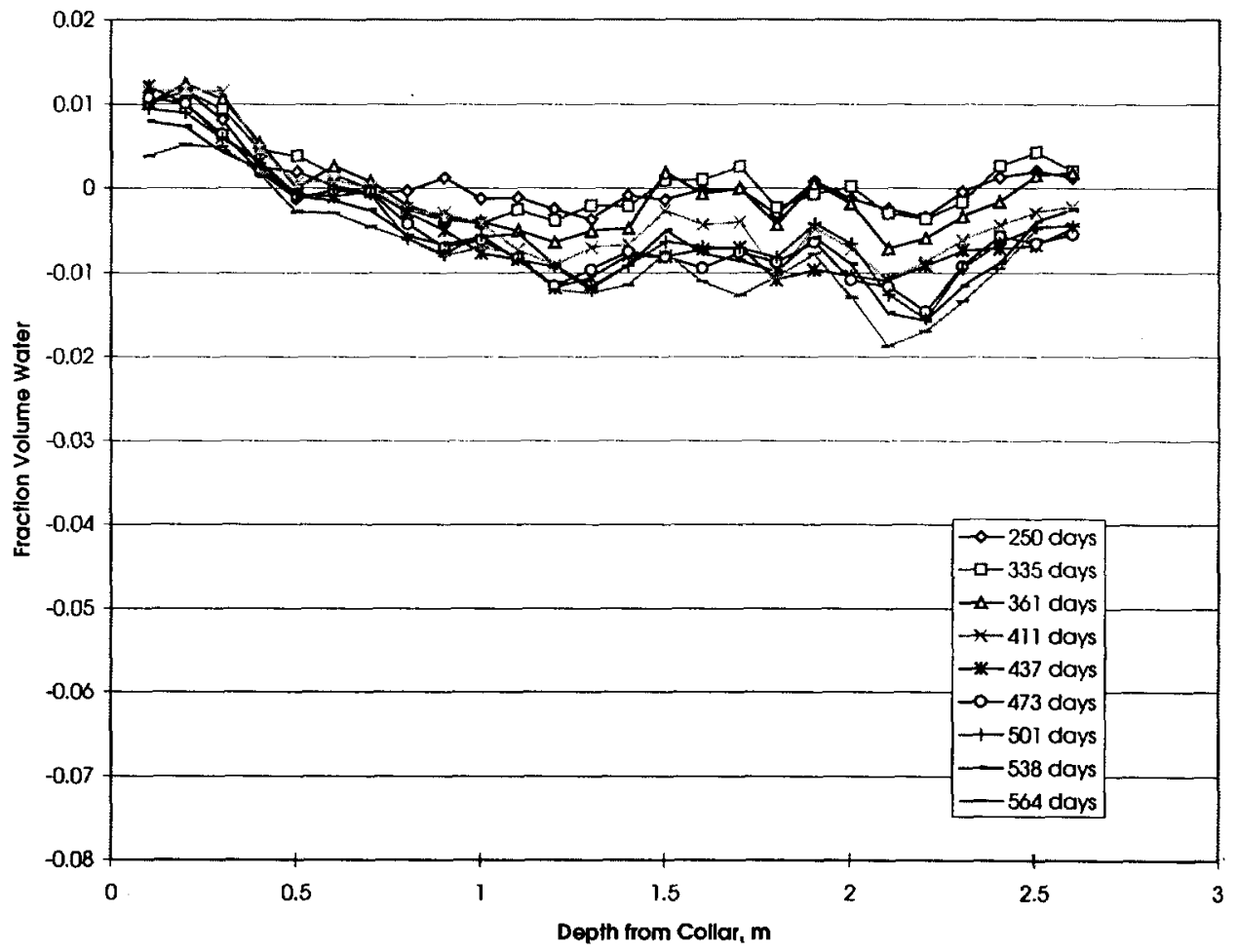

Figure 6-54. Difference Fraction Volume Water, Between the In-heat and the Baseline, in Hole WN3 as a Function of Depth from the West Face of the Block, from November 5, 1997, to September 15, 1998 


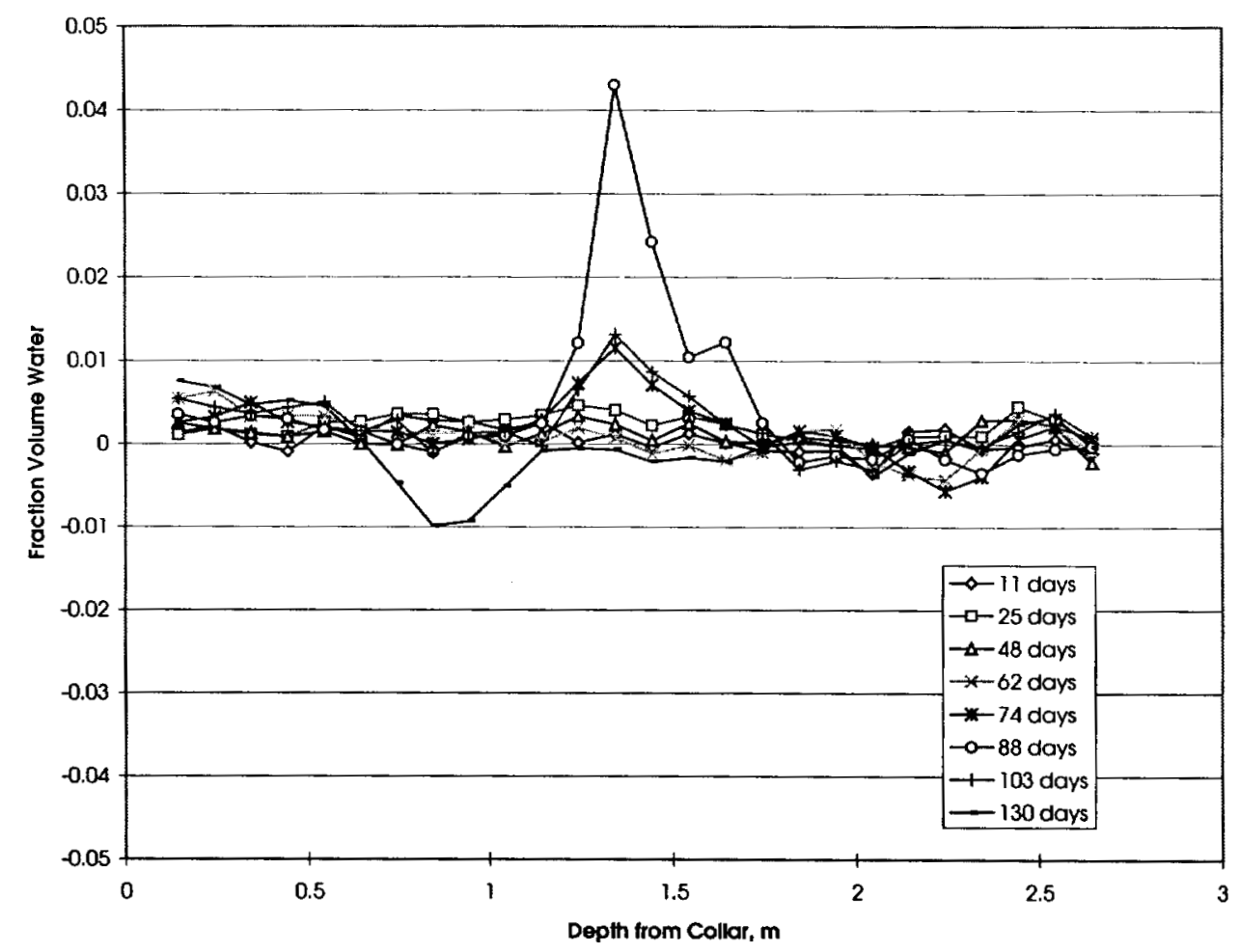

Figure 6-55. Difference Fraction Volume Water, Between the In-heat and the Baseline, in Hole WN4 as a Function of Depth from the West Face of the Block, from March 11, 1997, to July 8, 1997 


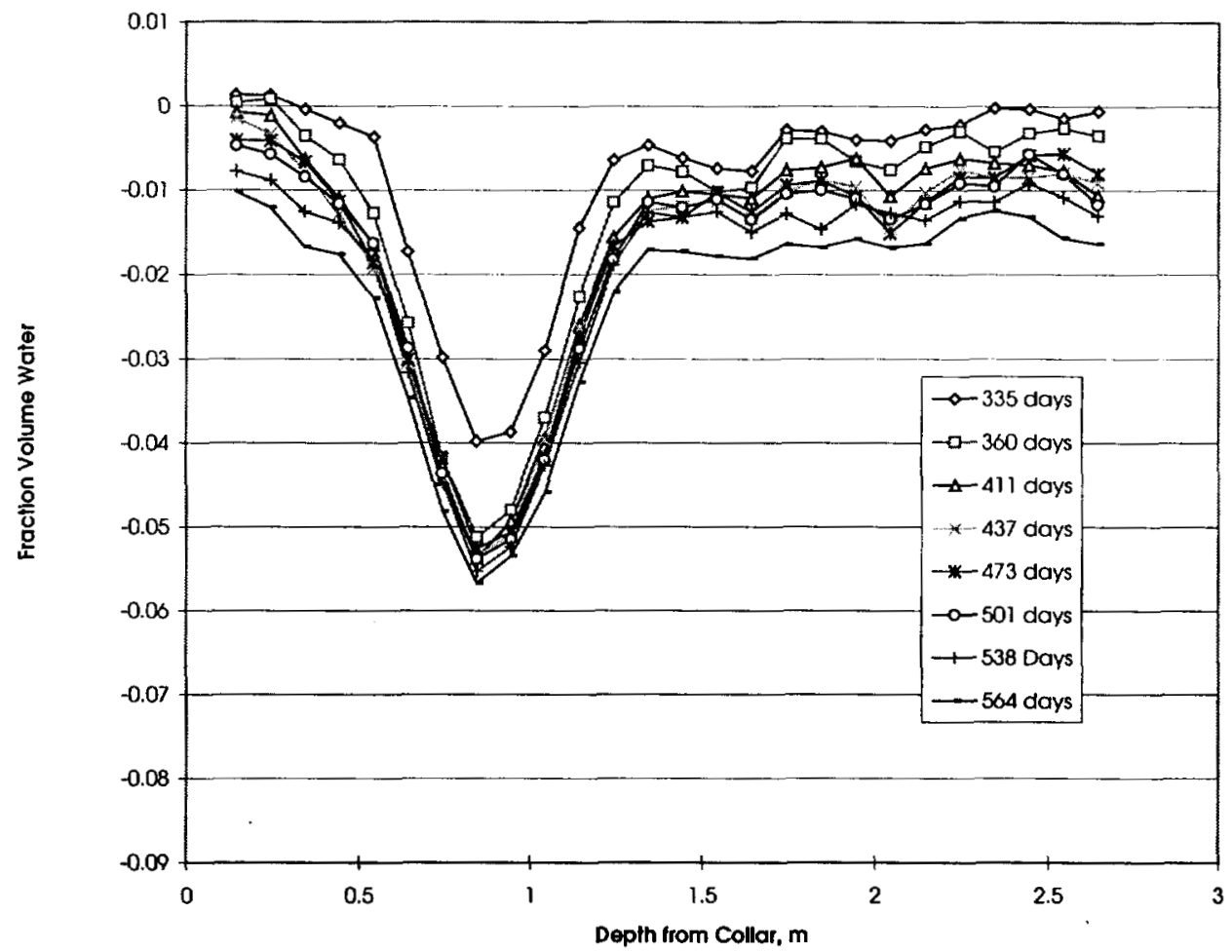

Figure 6-56. Difference Fraction Volume Water, Between the In-heat and the Baseline, in Hole WN4 as a Function of Depth from the West Face of the Block, from January 29, 1998, to September 15, 1998 


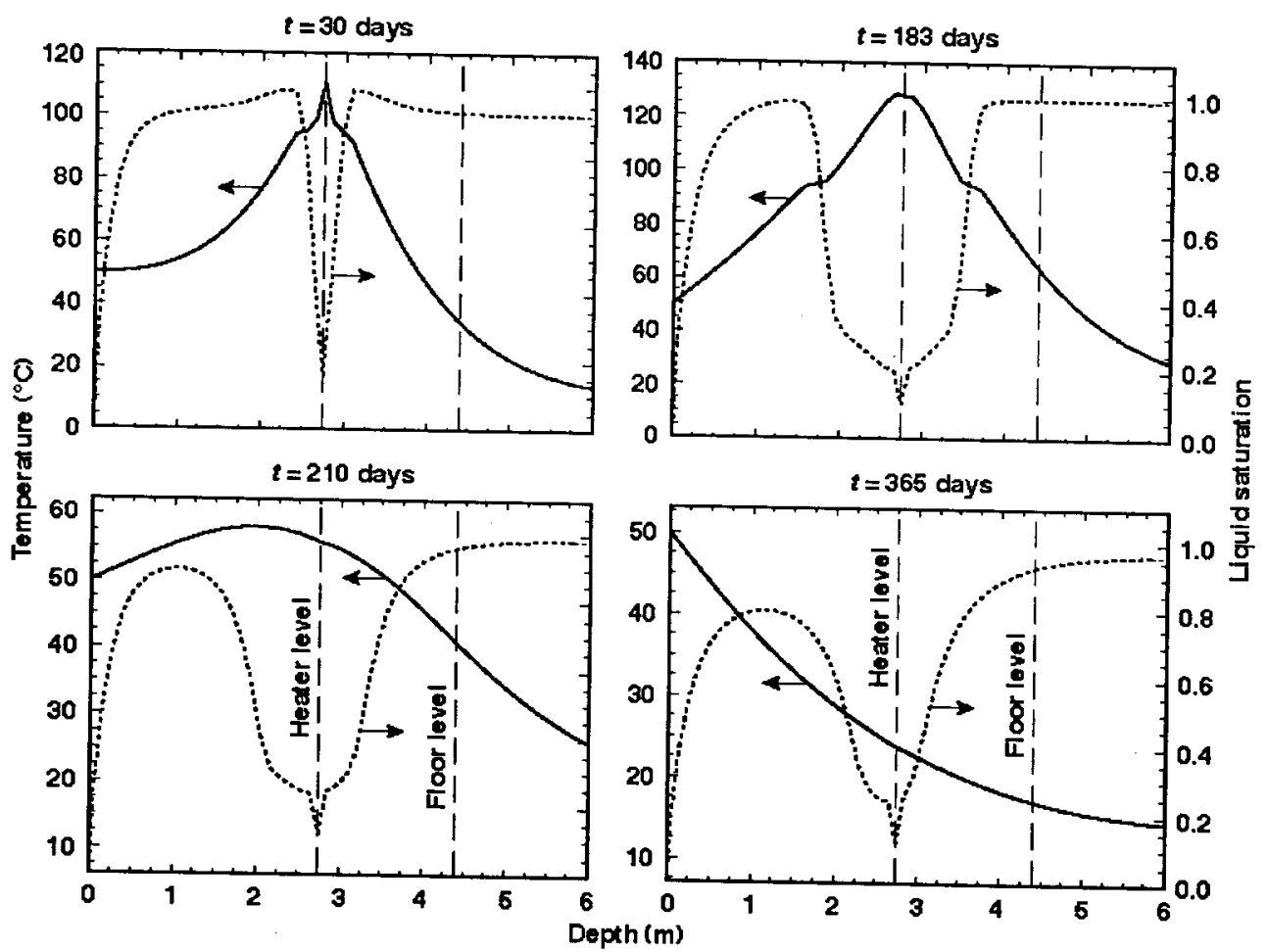

Figure 6-57. Pre-test Temperature and Liquid Saturation Calculated in Model Case A 
100 DAYS

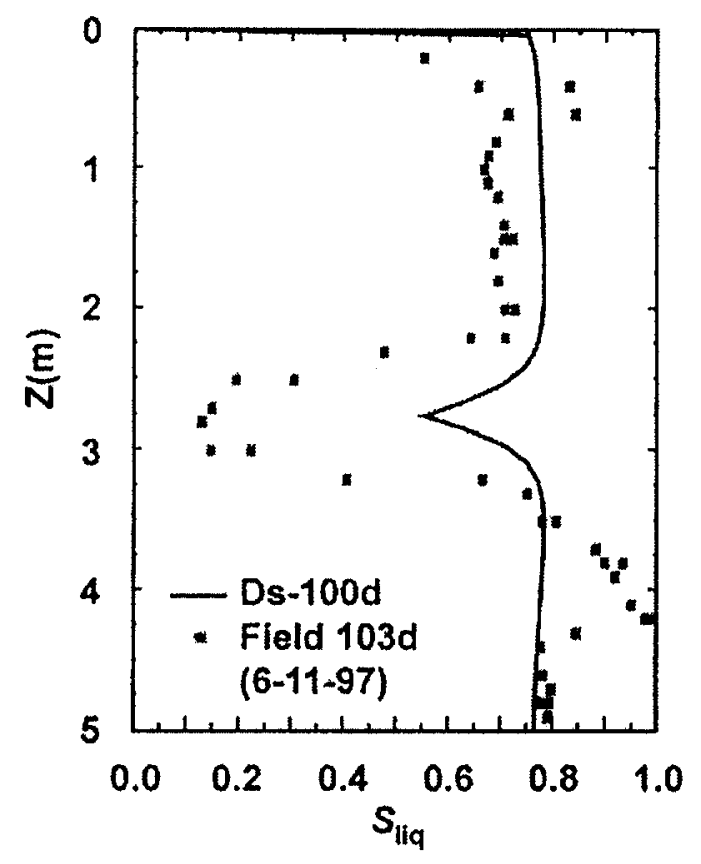

500 DAYS

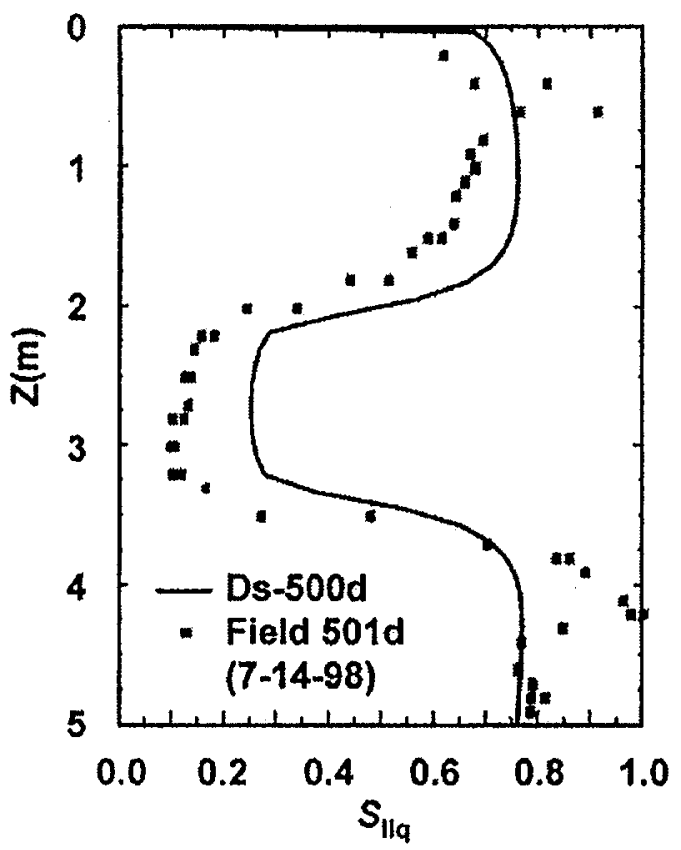

365 DAYS

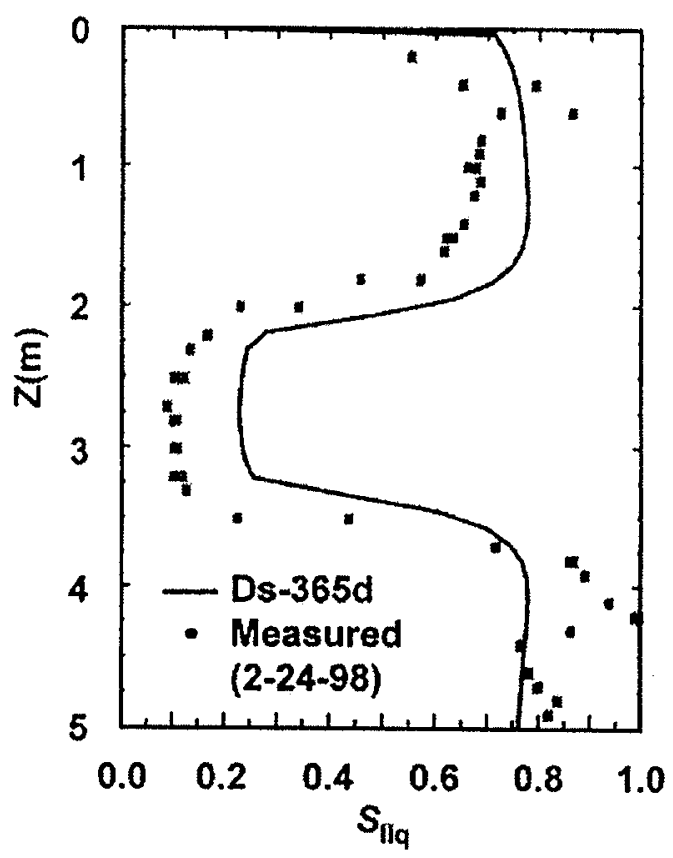

Figure 6-58. The Moisture Content Measured by Neutron in Hole TN3 Compared with the Model Calculations Using DS Property Set 
100 DAYS

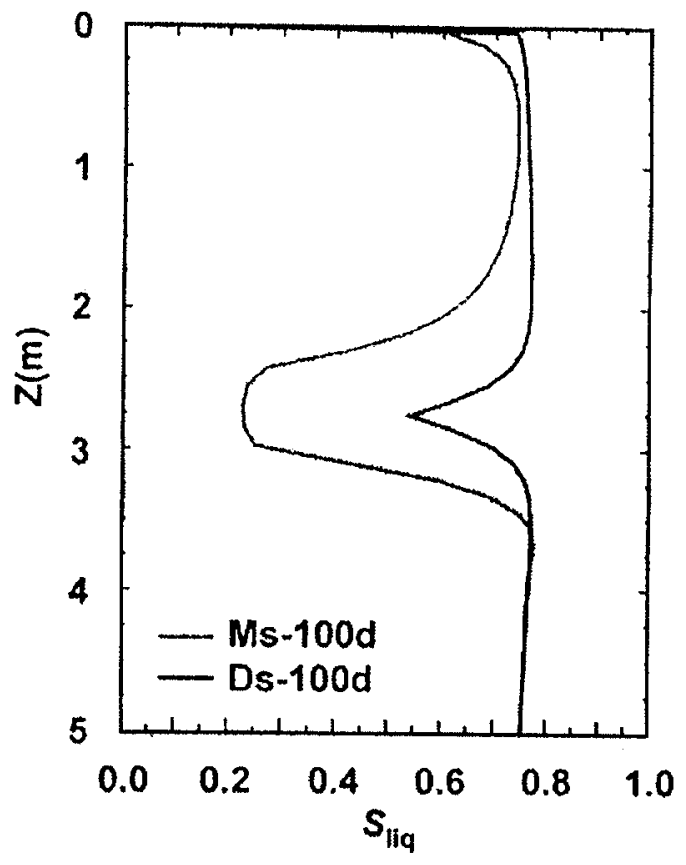

300 DAYS

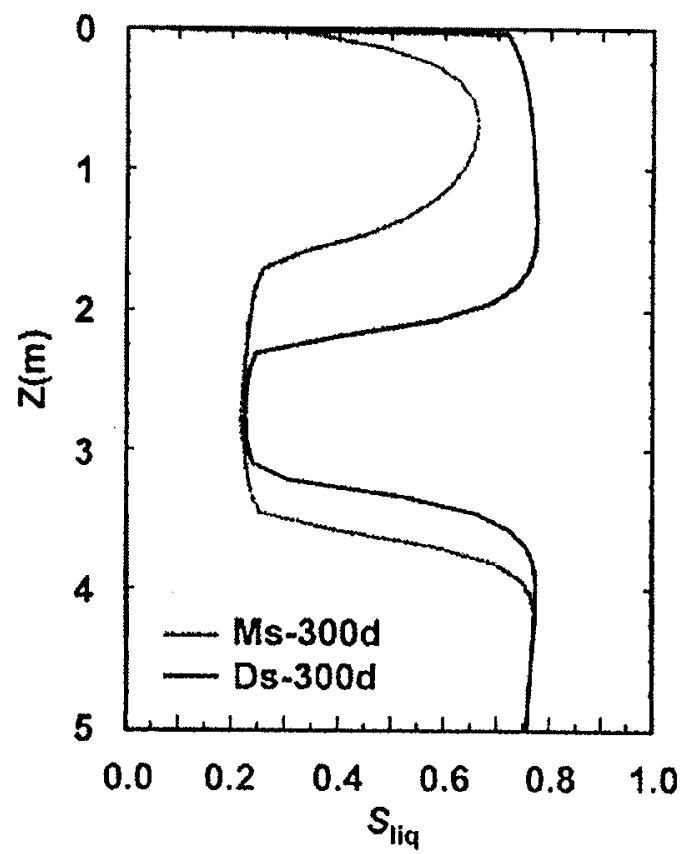

200 DAYS

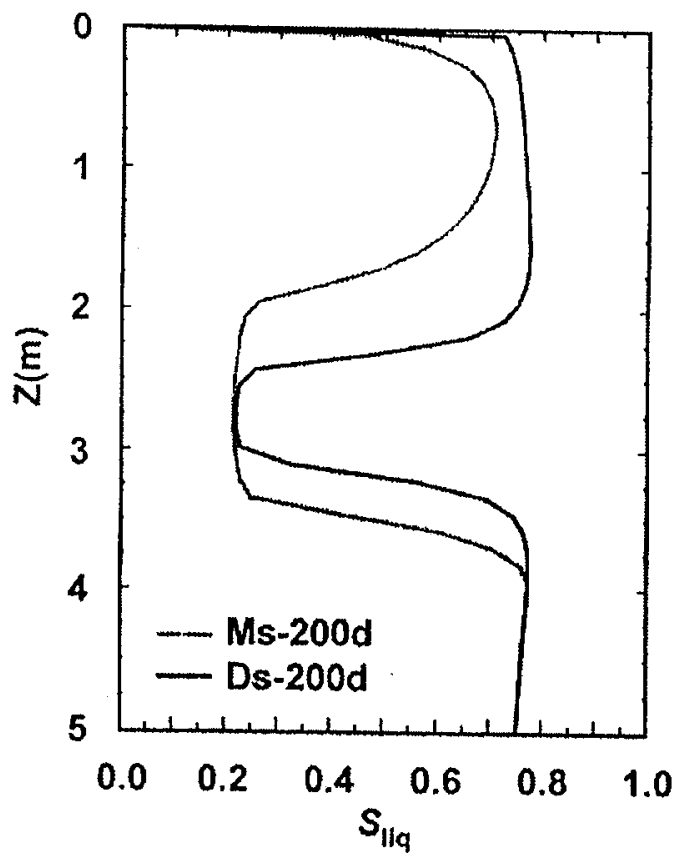
Figure 6-59. Comparison of Calculated Liquid Saturation of the LBT Along Hole TN3 Using MS Property
Set and DS Property Set 


\section{GEOMECHANICS OF THE LBT}

\subsection{DEFORMATION MEASUREMENTS}

\subsubsection{Introduction}

Six multiple-point borehole extensometers (MPBX) were deployed in the LBT. Each extensometer consisted of three or four borehole anchors connected to a surface collar by invar rods (Figure 7-1). The extensometers measured linear displacement relative to the surface collar. Linear variable differential transformers (LVDT) were used to measure the linear displacement. Extensometer TM-1 was emplaced in a vertical borehole, and the others were emplaced in horizontal boreholes, of which three were oriented north-south and two were oriented east-west (Figure 7-2, which is the same as Figure 3-13 but has been included again for easier reading.). The surface collars were located on the top, north and west faces. The first letter in the extensometer name denotes the face on which the surface collar is located. The anchors are numbered such that anchor 1 is nearest and anchor 4 is farthest from the collar. One of the northsouth MPBX systems, NM-2, was emplaced slightly above the heater plane. The other horizontal MPBX systems were deployed as two orthogonal pairs, one pair near the base of the large block (NM-1 and WM-1) and the other pair (NM-3 and WM-2) about $1 \mathrm{~m}$ below the top. The horizontal MPBX systems spanned baselines of about $2.6 \mathrm{~m}$. The vertical MPBX was a little more than twice as long. The extensometer collar and anchor coordinates are given in Table 7-1. The orientations, baseline lengths, and number of anchors of the MPBX systems are summarized in Table 7-2. An extensometer collar is shown in Figure 7-3.

Pre-heat MPBX measurements were conducted for several days before the heaters were energized on February 28, 1997. The LVDTs were zeroed before the heating was started. All of the extensometers performed well during the first few weeks, but problems developed over time beginning with NM-2, which is located near the heater plane. This extensometer failed early in the test and may have been adversely affected by high temperatures. Evidence of corrosion was noted on some of the invar rods in this hole at the conclusion of the LBT (Figure 7-4). The corrosion on the invar rods did not impact the mechanical integrity of the rods. Most of the extensometers performed well during the first 100 days of the test, during which temperatures rose rapidly and the largest thermal expansions occurred. The data from the first 100 days will therefore be examined in somewhat greater detail than those from the remainder of the test. Several extensometer transducers were replaced prior to cool-down, so that the large contractions that occurred after 375 days have also been captured. The data during the intermediate period, 100 to 375 days, are incomplete because of mechanical problems, but one extensometer, WM-2, performed well throughout the entire test, and its data provide a nearly continuous record of eastwest deformation in the upper portion of the block.

\subsubsection{The First 100 Days}

The MPBX data show that the large block began expanding within hours after heating began. Horizontal displacements at selected days during the first two weeks are shown as a function of depth in Figure 7-5. These displacements are measured in different directions, either east-west (WM-1, WM-2) or north-south (NM-1, NM-2, NM-3). The lines are drawn as an aid to the eye only. The expansion at the heater plane level exceeded that measured elsewhere in the block 
Table 7-1. MPBX Extensometer Collar and Anchor Coordinates. The Origin of the Coordinates Is the Southwestern Corner on Top of the Block

\begin{tabular}{|c|c|c|c|c|c|c|c|c|}
\hline MPBX & Anchor & Serial No. & $\begin{array}{c}\text { Calibration } \\
\text { factor }\end{array}$ & \begin{tabular}{|l|} 
Distance $(\mathrm{m})$ \\
\end{tabular} & $x(m)$ & $y(m)$ & $z(m)$ & Comment \\
\hline \multirow[t]{5}{*}{ NM-1 } & collar & -- & -- & 0.000 & 2.743 & 3.048 & -3.851 & \\
\hline & 1 & 10515 & 9.65E-05 & 0.832 & 2.743 & 2.216 & -3.851 & \\
\hline & 2 & 12217 & 9.72E-05 & 1.595 & 2.743 & 1.453 & -3.851 & \\
\hline & 3 & 12218 & 9.59E-05 & 2.159 & 2.743 & 0.889 & -3.851 & \\
\hline & 4 & 12219 & $9.46 \mathrm{E}-05$ & 2.616 & 2.743 & 0.432 & -3.851 & \\
\hline \multirow[t]{5}{*}{ NM-2 } & collar & -- & -- & 0.000 & 0.911 & 3.048 & -2.467 & \\
\hline & 1 & 10535 & $9.60 \mathrm{E}-05$ & 0.832 & 0.911 & 2.216 & -2.467 & removed \\
\hline & 2 & 10521 & 9.60E-05 & 1.595 & 0.911 & 1.453 & -2.467 & removed \\
\hline & 3 & 10526 & 9.62E-05 & 2.159 & 0.911 & 0.889 & -2.467 & removed \\
\hline & 4 & 10530 & $9.59 \mathrm{E}-05$ & 2.616 & 0.911 & 0.432 & -2.467 & removed \\
\hline \multirow[t]{5}{*}{ NM-3 } & collar & $-\cdot$ & -- & 0.000 & 0.314 & 3.048 & -0.930 & \\
\hline & 1 & 12224 & 9.61E-05 & 0.832 & 0.314 & 2.216 & -0.930 & \\
\hline & 2 & 12225 & $9.70 \mathrm{E}-05$ & 1.595 & 0.314 & 1.453 & -0.930 & \\
\hline & 3 & 12226 & $9.60 \mathrm{E}-05$ & 2.159 & 0.314 & 0.889 & -0.930 & \\
\hline & 4 & 12228 & $9.50 \mathrm{E}-05$ & 2.616 & 0.314 & 0.432 & -0.930 & \\
\hline \multirow[t]{4}{*}{ WM-1 } & collar & -- & -- & 0.000 & 0.000 & 2.172 & -3.985 & \\
\hline & 1 & 12227 & 9.61E-05 & 0.872 & 0.872 & 2.172 & -3.985 & \\
\hline & 3 & 10536 & 9.64E-05 & 2.086 & 2.086 & 2.172 & -3.985 & \\
\hline & 4 & 10517 & 9.51E-05 & 2.650 & 2.650 & 2.172 & -3.985 & \\
\hline \multirow[t]{5}{*}{ WM-2 } & collar & - & - & 0.000 & 0.000 & 0.619 & -1.235 & \\
\hline & 1 & 10519 & 9.63E-05 & 0.832 & 0.832 & 0.619 & -1.235 & \\
\hline & 2 & 10527 & 9.57E-05 & 1.595 & 1.595 & 0.619 & -1.235 & \\
\hline & 3 & 10538 & $9.65 \mathrm{E}-05$ & 2.159 & 2.159 & 0.619 & -1.235 & \\
\hline & 4 & 10534 & 9.52E-05 & 2.616 & 2.616 & 0.619 & -1.235 & \\
\hline \multirow[t]{5}{*}{ TM-1 } & collar & -- & - & 0.000 & 1.453 & 1.227 & 0.000 & \\
\hline & 1 & 10518 & $9.51 \mathrm{E}-05$ & 1.600 & 1.453 & 1.227 & -1.600 & \\
\hline & 2 & 10533 & $9.59 \mathrm{E}-05$ & 3.124 & 1.453 & 1.227 & -3.124 & \\
\hline & 3 & 10528 & $9.45 \mathrm{E}-05$ & 4.347 & 1.453 & 1.227 & -4.347 & \\
\hline & 4 & 10532 & $9.71 \mathrm{E}-05$ & 5.413 & 1.453 & 1.227 & -5.413 & \\
\hline
\end{tabular}

Table 7-2. MPBX Orientations, Baseline Lengths, and Number of Anchors

\begin{tabular}{|c|c|c|c|c|}
\hline Name & Orientation & Baseline length $(\mathbf{m})$ & Anchors & Comment \\
\hline NM-1 & North-south & 2.616 & 4 & Near east face \\
\hline NM-2 & North-south & 2.616 & 4 & Near heater plane \\
\hline NM-3 & North-south & 2.616 & 4 & Near west face \\
\hline WM-1 & East-west & 2.650 & 3 & Near north face \\
\hline WM-2 & East-west & 2.616 & 4 & Near south face \\
\hline TM-1 & Vertical & 5.413 & 4 & Near center \\
\hline
\end{tabular}


during only the first five days. By Day 6, the east-west expansion $1 \mathrm{~m}$ below the top of the block matched the expansion at the heater plane level $(0.7 \mathrm{~mm})$, and the north-south expansion was only about $0.1 \mathrm{~mm}$ less. Much less expansion occurred near the base of the block, which is attached to the ground. Expansion rates near the heater plane and near the top of the block were as high as $0.2 \mathrm{~mm} /$ day during the first couple of days of heating, and gradually diminished to under $0.05 \mathrm{~mm} /$ day within three weeks (Figure 7-6). Expansion rates near the base peaked at around $0.05 \mathrm{~mm} /$ day in the first week and gradually diminished. By the end of the first two weeks, both the north-south and east-west displacements near the top of the block exceeded the north-south displacement at the heater plane level. The larger displacements near the top of the block were unexpected because the highest temperatures occur at the level of the heater plane.

Displacement histories during the first 100 days are shown for each extensometer in Figures 7-7 through 7-12. The displacements are measured relative to February 28, 1997, the day heating began, and the plotted values are daily averages for selected days. Each of the displacements is measured relative to the borehole collar, so that the anchor 4 displacements are cumulative, in that they are measured over the entire baseline. The displacement data show a rapid expansion of the block in all three measured directions during the first 30 or 40 days. The expansions continue throughout the 100-day interval but at a gradually diminishing rate.

The displacements for the lowest extensometer from the top, WM-1, are shown in Figure 7-7. MPBX WM-1 measures the east-west displacements of three anchors relative to a collar located on the west face near the north side of the block, about $1.25-\mathrm{m}$ below the heater plane (See Table 7-1). The WM-1 displacements are relatively small; and east-west strains, calculated as the ratio of displacement to baseline length, are not uniform. The anchor 4 displacement at 100 days, of about $0.74 \mathrm{~mm}$, represents a strain of $0.28 \times 10^{-3}$ over the entire baseline of $2.65 \mathrm{~m}$. However, the anchor 3 displacement is only about $0.4 \mathrm{~mm}$ at 100 days; this is distributed over a baseline of $2.09 \mathrm{~m}$ and represents a strain of about $0.20 \times 10^{-3}$. The relative displacement between anchors 3 and 4 at 100 days is about $0.34 \mathrm{~mm}$ over a baseline of $0.56 \mathrm{~m}$, which yields a strain of about $0.60 \times 10^{-3}$. This strain, representing east-west deformation in the lower, northeast portion of the block, is about three times as large as the east-west strain along the rest of the WM-1 borehole and may indicate the opening of a fracture.

NM-1 is the other extensometer near the base of the block, and its record (Figure 7-8) also shows relatively small displacements at early times. NM-1 trends north-south about $1.1 \mathrm{~m}$ below the heater plane near the east face of the block. It is at about the same level as WM-1 and is orthogonal to it. The anchor 4 displacement of about $0.7 \mathrm{~mm}$ at 60 days was little changed 40 days later. Over the baseline of $2.616 \mathrm{~m}$, the $0.7-\mathrm{mm}$ displacement yields a strain of about $0.27 \times 10^{-3}$, nearly identical to the WM-1 anchor 4 strain. This suggests that the early deformation near the base of the block is essentially the same in the north-south and the east-west directions. An unexpected feature of the NM-1 record is the lack of relative displacement between two pairs of anchors. The anchor 1 and anchor 2 displacements are nearly identical, as are the anchor 3 and anchor 4 displacements. This suggests that no net strain occurred between anchors 1 and 2 or between anchors 3 and 4 , and is an unexpected result, because temperatures were rising rapidly early in the heating phase of the test. Thermal expansion should cause the entire block to expand, so that positive net strains would be expected between each pair of anchors. The lack of relative displacement may indicate a problem with two or more of the NM-1 anchors, or closure of open fractures between the two pairs of anchors may have taken up the thermal expansion. 
The large block contains many fractures and, because the overall displacements are so small, fracture closures of only about $0.1 \mathrm{~mm}$ would suffice to eliminate most of the relative anchor displacements.

MPBX NM-2 was located about $30 \mathrm{~cm}$ above the heater plane and experienced temperatures above $80^{\circ} \mathrm{C}$ a few days after heating began. The NM-2 displacement data are of low quality, and three of the four anchors ceased functioning entirely within the first 100 days (Figure 7-9). The anchor 4 data are probably the most reliable. They show a rapid expansion to about $1.3 \mathrm{~mm}$ within the first 40 days, more than twice that of the two lower extensometers (WM1 and NM1) at this time. Most of this displacement occurred in the first 10 days. The NM-2 anchor 4 displacement is about $0.8 \mathrm{~mm}$ at 10 days, compared to $0.25 \mathrm{~mm}$ for WM-1 or $0.3 \mathrm{~mm}$ for NM-1. The large and rapid expansion is in line with expectations because NM-2 is located close to the heater plane. Unfortunately, the anchor failed at Day 42.

MPBX WM-2 is located about $1.5 \mathrm{~m}$ above the heater plane near the south wall of the block, parallel and diagonally across from WM-1. The WM-2 data are of very good quality (Figure 710). The displacements increased rapidly during the first 40 days, then continued to rise at a much-reduced rate. The anchor displacements track each other well over time. The displacement data show expansion over each segment of the extensometer baseline. The displacements and strains are much larger than those recorded near the base of the block and, after six days, are even larger than those recorded by NM-2 near the heater plane. The WM-2 anchor 4 displacement at Day 100 is about $2.1 \mathrm{~mm}$, which translates to a strain of about $0.80 \times 10^{-3}$, nearly three times as large as the WM-1 anchor 4 strain. At Day 40 , the WM-2 anchor 4 displacement $(1.9 \mathrm{~mm})$ and strain $\left(0.74 \times 10^{-3}\right)$ are clearly larger than the NM-2 displacement $(1.3 \mathrm{~mm})$ and strain $\left(0.47 \times 10^{-3}\right)$. The WM-2 strains are relatively uniform, ranging from $0.7 \times 10^{-3}$ to $0.8 \times 10^{-3}$ over the entire baseline, except for anchor 1 , which is much larger at $1.4 \times 10^{-3}$. The large anchor 1 strain may indicate the opening of a fracture in the upper, southwest corner of the large block in the early days of the test.

MPBX NM-3 is located about $1.8 \mathrm{~m}$ above the heater plane and about $0.9 \mathrm{~m}$ below the top of the large block. The borehole collar is on the north face near the west wall, diagonally across from NM-1. The quality of the NM-3 data (Figure 7-11) is generally good, although inferior to WM2. The displacements measured over the longer baselines (anchors 3 and 4) are similar in magnitude to those measured by anchors 3 and 4 of WM-2, but the short baseline displacements are smaller. The anchor 2 displacements are even smaller than those for anchor 1, despite being made over a baseline about twice as long and, as such, are somewhat suspect. The other three anchors yield north-south strains ranging between $0.6 \times 10^{-3}$ for anchor 1 and $0.9 \times 10^{-3}$ for anchor 4. As the strain for WM-2 anchor 4 is about $0.8 \times 10^{-3}$, deformation near the top of the block during the first 100 days was essentially the same in the north-south and the east-west directions.

MPBX TM-1 is emplaced in a vertical borehole. The borehole collar is located near the center of the top face of the large block. MPBX TM-1 appears to have performed well for the first 80 days or so, but the anchors began to fail shortly thereafter, and only anchor 2 was functioning after Day 120 (Figure 7-12). The TM-1 record shows increasing displacements, until 80 days, as the rock heated up over time. Larger displacements were recorded for the longer baseline anchors. The anchor 4 strain at 100 days, which is measured over a $5.4 \mathrm{~m}$ baseline, is about $0.3 \times 10^{-3}$. This is considerably smaller than the horizontal strains measured near the top of the 
block and slightly larger than the horizontal strains measured by NM-1 and WM-1 near the bottom.

The anchor 4 strains over the first 100 days are shown for all MPBXs in Figure 7-13. The contractions that occurred at Days 24 and 41, most evident in the data for upper MPBXs, are probably due to brief cooling episodes that resulted from temporary power outages. The strains fall into three categories: small strains for the vertical and two lower MPBXs, large strains for the two upper MPBXs, and intermediate strain for NM-2, located near the heater plane. The base of the large block is still attached to the ground, which accounts, at least in part, for the relatively small strains near the base.

\subsubsection{Day 100 to Day 375}

Displacement records are incomplete during this interval because several MPBXs experienced mechanical problems or failed completely. However, enough data were gathered that general trends can be discerned, at least for the upper two extensometers, WM-2 and NM-3, and for one of the lower extensometers, WM-1. Overall, the MPBX displacements follow the temperature trend through time, as can be seen by comparing anchor 4 displacements for these extensometers with temperature records for RTDs at similar depths (Figures 7-14 through 7-16). The RTD temperatures rose rapidly during the first 50 days, then more gradually until Day 220 into the test, apart from dips on Day 104 and Day 186. Displacements increased rapidly during the first 50 days and peaked at about Day 220, when the heater power was reduced. The upper RTD (TT2-22) temperature then declined by about 10 degrees and remained relatively constant, except for a dip at Day 340, until cool-down began at Day 375. Temperatures fell rapidly during cool-down. The data for the upper extensometers follow a similar pattern: displacements drop slightly after Day 220, then remain nearly level until Day 375, and drop sharply afterward. The lower RTD (TT1-8) temperature appears to have fallen more or less continually during the Day 220 to Day 375 interval, and the lower MPBX (WM-1) also shows more or less continual contraction during this time, followed by a steep drop on cool-down.

The drop in temperature that began at Day 104 was probably related to reflux of steam/water along the TT1 RTD column (Section 5.4.3). Temperatures near the intersection of borehole TT1 and the heater plane dropped rapidly to the boiling point (Figure 7-17). The temperature response indicates that water came in contact with dry rock near the heater plane at this time. This water would have traveled along a fracture, because the matrix permeability of the tuff is too low to account for the sudden drop in temperature at the heater plane. The upper, east-west extensometer, WM-2, may have captured a transient signal related to the passage of fluids in a fracture at this time. A small double pulse can be seen at Day 105 in the WM-2 displacement records in Figure 7-17. The double pulse is present at Day 105 in the signals from all four anchors, which indicates that the displacement transient originated between the borehole collar on the west wall and the first anchor $0.83 \mathrm{~m}$ to the east. The borehole collar moved outward, away from the anchors. An enlarged image of the WM2 double pulse signal is shown in Figure $7-18$, along with temperature data from nearly the same depth $(1.2 \mathrm{~m})$ as the WM-2 borehole. The displacement transient occurred at about the same time as a brief drop in temperature at 1.2-m depth in borehole TT1. It seems unlikely that the small drop in temperature at this depth, or the large drop at the depth of the heater plane, explains the displacement transient, because the sense of the displacement (expansion initially) is opposite to that expected for the temperature 
change. The magnitude of the double pulse displacement is quite small, under $0.05 \mathrm{~mm}$, and it may represent a temporary opening or shear motion along a fracture, either of which would increase permeability. Presumably, this fracture intersected the surface of the large block near the upper southwest corner. The WM-2 borehole would then pass through it within $0.83 \mathrm{~m}$ of the west wall and would also likely intersect borehole TT1, so that water traveling downward through the fracture would come into contact with several of the TT1 RTDs.

The drop in temperature at Day 220 was due to a reduction in the heater power. A rainstorm triggered the temperature drop at Day 186. The drop in temperature at Day 186 is correlated with extensometer displacements in the upper portion of the large block (Figure 7-19). The correlation is especially good for extensometer NM-3, which passes closer to borehole TT1.

\subsubsection{Cool-down}

Repairs were made to the TM-1 and NM-1 extensometers shortly before the beginning of the cool-down phase. As a result, good displacement records exist over the cool-down for each of the MPBX systems, except NM-2, which wasn't repaired. The cool-down displacements show that the block contracted rapidly in all directions after the heaters were turned off. Records for individual extensometers, except NM-2, are given in Figures 7-20 through 7-24. Anchor 4 strains are shown in Figure 7-25 for comparison. The two upper MPBXs contracted more than the two lower MPBXs, as expected, since they lengthened considerably more during the heating phase. Contraction along the vertical extensometer was comparable to that of the upper horizontal extensometers, but the vertical extensometer has a longer baseline. The displacement during cool-down for the two lower extensometers was under $1 \mathrm{~mm}$.

The upper MPBXs did not contract as much during cool-down as they lengthened in the heating phase. NM-3 contracted about $1.5 \mathrm{~mm}$, and WM-2 contracted about $1.8 \mathrm{~mm}$, whereas both had expanded about $2.4 \mathrm{~mm}$ during the heating phase, so that there has been a net displacement of a few tenths of a millimeter in the upper portion of the block. In contrast, one of the lower MPBXs, WM-1, returned almost exactly to its starting length. It is not possible to determine if positive or negative net displacements occurred for the lower MPBX (NM-1) or the vertical MPBX (TM-1) because these extensometers were repaired during the test, and they were zeroed to the current position instead of the prerepair conditions. However, by comparing the anchor 4 strains during the first 100 days (Figure 7-13) to those during cool-down (Figure 7-25), it can be seen that NM-1 contracted at least as much or more during cool-down $\left(0.3 \times 10^{-3}\right)$ as it lengthened $\left(0.25 \times 10^{-3}\right)$ during the early portion of the heating phase. The vertical extensometer (TM-1) contracted slightly less, recording an extension of $0.33 \times 10^{-3}$ for anchor 4 during the first 100 days and a contraction of $0.22 \times 10^{-3}$ during cool-down. In summary, the horizontal strains near the base of the block were small, essentially the same in the two horizontal directions, and were recovered during cool-down. The horizontal strains near the top of the block were large, isotropic, and were only partially recovered. The vertical strain was fairly small but only partially recovered during cool-down. 


\subsubsection{Large Block Thermal Expansion}

\subsubsection{Introduction}

Rock thermal expansion coefficients are typically measured on laboratory specimens, which, because of their small size, usually exclude fractures and other heterogeneities that exist in situ. Brodsky et al. (1997) made a large number of such measurements on Topopah Spring tuff. Although laboratory-derived thermal expansion coefficients may accurately characterize the thermal expansivity of the rock matrix, they may not be representative of the rock mass. Fractures may close as a rock mass is heated and thereby lower bulk thermal expansion, so that laboratory-derived thermal expansion coefficients likely form an upper bound for in-situ thermal expansivity. Tighter constraints on in-situ thermal expansivity may be supplied by field measurements, but to date these are relatively rare. A few thermal expansion coefficients have been obtained from the Single Heater Test and are available in CRWMS M\&O (1999). Temperature and MPBX displacement data from the LBT provide a much-needed opportunity to estimate thermal expansion coefficients for a fractured block of Topopah Spring tuff.

\subsubsection{Data Reduction}

Twelve dates, mostly within the first 83 days of heating, were chosen for thermal expansion calculations. The initial temperature was taken to be $11.6^{\circ} \mathrm{C}$, based on the average of a large number of temperature measurements made prior to heating. Because temperatures were not measured in the MPBX boreholes, they had to be interpolated from temperatures measured at known RTD locations. Because the block was heated along a horizontal plane and insulated on its sides, the thermal gradients were primarily vertical, and the horizontal thermal gradients were small. Peak temperatures coincide with the heater plane, then fall off asymmetrically above and below, with the top surface temperature at $60^{\circ} \mathrm{C}$. The vertical thermal gradients were generally steeper in the lower portion of the block because the upper surface temperature was maintained at or near $60^{\circ} \mathrm{C}$ for much of the test. Because the vertical thermal gradients are relatively large, no attempt was made to calculate thermal expansion coefficients in the vertical direction.

Invar steel rod extensions were calculated from the interpolated temperatures and the linear thermal expansion coefficients for invar steel, given in SNL (1997). The invar rod extensions, $\delta$, are found as

$$
\delta=\alpha(T) \Delta T L
$$

where

$\alpha(\mathrm{T})$ is the invar thermal expansion coefficient,

$\Delta \mathrm{T}$ is the temperature change, and

$\mathrm{L}$ is the length of the invar rod.

Extension is positive. The total displacements for each anchor were corrected for the rod thermal extension. Because the invar rods expand during heating, the anchor displacements measured at the borehole collars are smaller than the actual rock mass displacements by the rod extensions. The anchor displacements were divided by distance to give strains. These distances vary from 
about $0.75 \mathrm{~m}$ for anchor 1 to about $2.6 \mathrm{~m}$ for anchor 4 . The thermal expansion coefficients for anchor 4 tend to be more consistent because of the longer baseline.

Thermal expansion coefficients were found by regressing cumulative strains, measured from anchor 4 to the borehole collar, against the average temperature change for each extensometer (Table 7-3). The temperature changes were calculated as the weighted-average of the interpolated temperature changes at each anchor location, using the anchor spacings as the weights. Temperature changes were fairly uniform along the horizontal extensometers, and the weighted-average technique was intended to lessen still further the effect of horizontal thermal gradients on the calculated thermal expansion coefficients. However, no corrections were applied for the effects of the vertical thermal gradients or for the fact that the base of the block is still attached to the outcrop.

Table 7-3. Interpolated Average Temperatures for Horizontal MPBX Extensometers

\begin{tabular}{|c|c|c|c|c|c|}
\hline & \multicolumn{5}{|c|}{ Interpolated temperatures $\left(^{\circ} \mathbf{C}\right)$} \\
\hline Date & NM1-4 & NM2-4 & NM3-4 & WM1-4 & WM2-4 \\
\hline $3 / 7 / 97$ & 10.7 & 31.5 & 7.6 & 12.4 & 10.4 \\
\hline $3 / 19 / 97$ & 27.5 & 53.4 & 29.2 & 30.0 & 31.1 \\
\hline $3 / 31 / 97$ & 33.0 & 63.7 & 40.7 & 36.6 & 43.9 \\
\hline $4 / 12 / 97$ & 35.4 & 69.3 & 44.4 & 38.9 & 48.4 \\
\hline $4 / 21 / 97$ & 39.4 & 71.9 & 47.4 & 42.4 & 51.6 \\
\hline $5 / 3 / 97$ & 41.7 & 75.4 & 49.3 & 45.1 & 54.4 \\
\hline $5 / 18 / 97$ & 47.3 & 79.6 & 53.2 & 49.2 & 58.2 \\
\hline $8 / 21 / 97$ & 69.3 & 98.7 & 66.6 & 69.3 & 74.9 \\
\hline $9 / 24 / 97$ & 68.7 & 105.1 & 65.9 & 71.8 & 76.1 \\
\hline $11 / 19 / 97$ & 61.0 & 100.1 & 63.0 & 65.2 & 70.0 \\
\hline $2 / 18 / 98$ & 56.2 & 98.8 & 55.4 & 58.1 & 65.4 \\
\hline $3 / 18 / 98$ & 44.4 & 62.1 & 48.8 & 44.2 & 55.7 \\
\hline
\end{tabular}

\subsubsection{Results}

Cumulative horizontal strains recorded by the MPBX extensometers during the early heating phase for the LBT are shown in Figure 7-26. The strains are plotted for the same dates used to estimate thermal expansion coefficients. The strains are quite isotropic-i.e., the north-south and east-west strains are nearly identical but vary by depth. Much larger horizontal displacements, and hence strains, were recorded near the top of the block than near the base, particularly during the first month of heating. The horizontal strains near the top of the block exceeded those recorded by extensometer NM-2 near the heating plane, even though the thermal pulse had not yet penetrated very far above or below the heater plane. The implication is that fractures in the relatively cool rock well above the heater plane were displaced more than in the hotter rock near the heater plane in the early portion of the test (See Section 7.2.2).

Anchor 4 strains are plotted against temperature changes in Figure 7-27, and lines are fit to the data using ordinary least squares. The anchor 4 strains were used to provide the longest possible baseline. The slopes of the fitted lines provide estimates of rock mass thermal expansion 
coefficients. The estimated slopes, correlation coefficients, and standard errors of the regression fits are given in Table 7-4.

Table 7-4. Thermal Expansion Coefficients (CTE) and Fitting Statistics

\begin{tabular}{|l|c|c|c|c|c|}
\hline \multicolumn{1}{|c|}{ MPBX } & NM1-4 & NM2-4 & NM3-4 & WM1-4 & WM2-4 \\
\hline Slope (CTE) & $6.586 \mathrm{E}-06$ & $7.214 \mathrm{E}-06$ & $1.420 \mathrm{E}-05$ & $7.135 \mathrm{E}-06$ & $1.085 \mathrm{E}-05$ \\
\hline Correlation & 0.999 & 0.996 & 0.989 & 0.998 & 0.990 \\
\hline Std. error & $8.466 \mathrm{E}-08$ & $6.669 \mathrm{E}-07$ & $7.479 \mathrm{E}-07$ & $1.391 \mathrm{E}-07$ & $5.338 \mathrm{E}-07$ \\
\hline
\end{tabular}

The measured MPBX displacements, and hence the calculated thermal expansion coefficients, were found to increase from the base to the top of the large block. The coefficient values approximately double, from about $7 \times 10^{-6}{ }^{\circ} \mathrm{C}^{-1}$ in the bottom third of the block to about $15 \times 10^{-6}$ ${ }^{\circ} \mathrm{C}^{-1}$ in the top third (Figure 7-28). The calculated thermal expansion coefficients in the two horizontal directions are fairly close, similar to the measured displacements.

\subsubsection{Discussion}

The overall horizontal deformation of the block revealed similar amounts of expansion in both the east-west and north-south directions. The expansion was essentially a positive, linear function of height above the base of the large block, independent of the temperature gradient above the heater plane. The latter result was unexpected because the hottest portion of the block is near the center. The MPBX extensometer data from the upper one-third of the block suggest that most of the deformation occurred in discrete, vertical zones, perhaps due to opening of vertical fractures in this region. Fracture gauge data indicate that several fractures opened more than $0.1 \mathrm{~mm}$ on the block surface during the test (Section 7.1.3). The fracture opening displacements were likely driven at least in part by block movement. Horizontal displacements in the lower portion of the block were restrained because the base of the block is attached to the ground, but the upper portion of the block was largely unconfined.

The thermal expansion coefficients calculated from the LBT extensometer data are somewhat higher than those for the Single Heater Test (CRWMS M\&O 1999a). Despite the vast difference in scale, the thermal expansion coefficients obtained for the lower half of the large block are similar to those measured for 19 saturated TSw2 laboratory specimens by Brodsky et al. (1997). Over the $25^{\circ}$ to $150^{\circ} \mathrm{C}$ temperature range, they found mean thermal expansion coefficients between $7.14 \times 10^{-6}{ }^{\circ} \mathrm{C}^{-1}$ and $9.98 \times 10^{-6}{ }^{\circ} \mathrm{C}^{-1}$. Thermal expansion coefficients of about $7 \times 10^{-6}$ ${ }^{\circ} \mathrm{C}^{-1}$ were calculated for the middle-to-lower portions of the large block for the first 83 days of heating (Table 7-4). The higher thermal expansion values obtained here for the upper portion of the large block are suspect, because a portion of the horizontal deformation recorded by the upper two MPBX extensometers may have been caused by opening of vertical fractures in the upper third of the block.

\subsubsection{Fracture Monitors}

Deformations of several major fractures that intersect the surface of the LBT block were monitored using three-component fracture monitors. The purpose of these gauges was to monitor the movement of fractures to gain information on the magnitude and direction of fracture 
deformation during the test, especially as it relates to TH behavior. A fracture monitor gauge is shown schematically in Figure 7-29. This gauge consists of two steel fixtures that are mounted on either side of a fracture. One of the fixtures serves as the mounting block for three linear variable displacement transducers (LVDTs) that are mounted orthogonal to each other. The other fixture serves as the reference block. The gauges were mounted in T-shaped slots cut into the block. The slots were cut so that one LVDT would measure aperture change or deformation across the fracture in the plane of the face, while the other two LVDTs would measure sliding in orthogonal directions, parallel and perpendicular to the face. These are approximate measures of in-plane and normal deformations for the fractures. The fractures chosen were oriented perpendicular to the face as much as possible; thus the information can be used to supply estimates of fracture deformation parameters, such as dilation with sliding. Fracture monitor (FM) locations are shown for each face in Figures 7-30 through 7-33, and a few of the fracture locations are visible as T-shaped grooves in Figure 7-34. One of the installed gauges is shown in Figure 7-35.

The fracture monitors were installed on the four vertical faces of the block as follows:

- On the east face (see Figure 7-30), three FM gauges (EF2, EF3, and EF4) were mounted along a prominent vertical fracture located near the center of the face. One FM (EF1) was used to monitor deformation on the large horizontal fracture near the top of the block.

- FM locations on the north face are shown in Figure 7-31. FMs on this side were used to detect motion on the major subhorizontal fracture near the top of the block (NF-5) and in a subvertical fracture zone that was exposed in the center of the north face (NF2, NF3, and NF4).

- On the west face, FMs were used to monitor the subhorizontal fracture near the top (WF5), a subvertical fracture on the northwest side (WF1, WF2, and WF4), and a subhorizontal fracture near the bottom (WF3) on the northwest side (see Figure 7-32). Data for several fractures on the west face were not collected during the first 20 days of the test because of an instrumentation problem. Data were set to zero at Day 20 for these transducers.

- On the south face of the block, three fractures were monitored (see Figure 7-33). Fracture monitors SF-1 and SF-2 monitored one subvertical fracture, while SF-3 monitored a subhorizontal fracture on the lower southwest part of the block, and SF-4 monitored a subhorizontal fracture near the top.

\subsubsection{Deformation on Vertical Fractures}

The fracture aperture data recorded for vertically oriented fractures are plotted in Figure 7-36, along with temperatures measured at RTD TT1-14. This figure shows that a fracture opening of between 0.1 and $0.36 \mathrm{~mm}$ occurred at several locations, and that fracture closing between 0.002 and $0.16 \mathrm{~mm}$ occurred on several others. Moreover, for fractures that show opening, a substantial portion of the opening occurs within the first 40 days of heating. Opening and closing are observed on all faces of the large block. The maximum apertures for four of the opening 
fractures occur near Day 300 at the end of the test. Most of these opening fractures show closing and reopening behavior associated with cool-down. However, one fracture, NF2, opens approximately $0.15 \mathrm{~mm}$ upon cool-down.

Approximately half of the fracture monitors also show fracture closing behavior during the heating period. As expected, the magnitude of closing is smaller and the nature of the closing signals is also different from opening fractures. Generally, closing behavior is smoother and less responsive to temperature. The fractures that close reach maximum closure sometime between Day 100 and 400 . The closing fractures show little change during cooling.

Figure 7-36 also shows that decreasing aperture was recorded by many of the fracture monitors on vertical fractures on about Day 125. Data for Days 100 to 140 are shown in more detail in Figure 7-37, along with temperatures recorded at TT1-14. This figure shows that the closing of vertical fractures between Day 125 and 130 occurred at several locations. This deformation can be associated with thermal recovery subsequent to the TH event that started on Day 105.

Slip on vertical fractures is shown in Figure 7-38, along with temperatures at TT1-14. This figure shows that slip on vertical fractures ranged from 0.07 to $0.58 \mathrm{~mm}$. For the FMs with the largest slip (NF-2, EF-3), slip followed the temperature quite closely, increasing rapidly upon initial heating and decreasing upon cooling. These two fracture monitors, along with EF2, also show opposite responses to the TH episode on Day 105. Slip increased on NF2 but decreased on EF3. This is associated with thermal recovery from the TH event. Fracture slip generally did not increase during the constant temperature portion of the test, except on EF4, where slip was observed at a steady rate until cool-down. Generally, slip is not recovered; however, the gauges with the most slip show some recovery.

Further analysis of the slip components shows that the largest slip deformations EF3, NF2, and EF-2 are associated with movement normal to the block face at each of these FM locations. For FMs showing total slip less than $2.5 \mathrm{~mm}$, both components perpendicular and parallel to the block face contributed.

It is important to note that this analysis computes slip as a vector sum of distance recorded in two perpendicular directions, and the computed slip is not cumulative. Thus, this analysis provides a minimum estimate of the amount of slip.

The maximum and residual aperture and slip data have been tabulated for the fractures and are presented in Table 7-5. Figure 7-39 shows values of maximum and residual aperture for just the vertical fractures, listed according to distance below the top of the block. This figure shows that the largest fracture aperture changes were due to opening in the lower portion of the block. Fracture aperture in the upper portion of the block (within $1.85 \mathrm{~m}$ of the top) generally remained constant or showed closure. This figure also shows that at most of the FM locations the residual values of fracture aperture are only slightly smaller than the maximum value, indicating that the fracture deformation did not recover upon cool-down.

Figure 7-40 presents a histogram of the maximum and residual values of slip on vertical fractures. This figure is consistent with Figure 7-39 in that the maximum values of slip occurred in the lower part of the block. The minimum slip displacements are at $1.2 \mathrm{~m}$ from the top, with 
Table 7-5. Maximum and Residual Fracture Slip and Aperture

\begin{tabular}{|l|c|c|c|c|c|c|c|}
\hline Face & $\begin{array}{c}\text { Fracture } \\
\text { monitor }\end{array}$ & $\begin{array}{c}\text { Fracture } \\
\text { orientation }\end{array}$ & $\begin{array}{c}\text { Depth } \\
(\mathbf{m})\end{array}$ & $\begin{array}{c}\text { Slip (max) } \\
(\mathbf{m m})\end{array}$ & $\begin{array}{c}\text { Slip (res) } \\
(\mathbf{m m})\end{array}$ & $\begin{array}{c}\text { Aperture } \\
(\mathbf{m a x}) \\
(\mathbf{m m})\end{array}$ & $\begin{array}{c}\text { Aperture } \\
(\mathbf{r e s}) \\
(\mathbf{m m})\end{array}$ \\
\hline $\mathrm{E}$ & $\mathrm{EF} 1$ & $\mathrm{H}$ & -0.68 & 0.320 & 0.245 & 0.226 & -0.045 \\
\hline $\mathrm{E}$ & $\mathrm{EF} 2$ & $\mathrm{~V}$ & -2.63 & 0.295 & 0.223 & 0.210 & 0.190 \\
\hline $\mathrm{E}$ & $\mathrm{EF3}$ & $\mathrm{V}$ & -3.37 & 0.575 & 0.260 & 0.350 & 0.275 \\
\hline $\mathrm{E}$ & $\mathrm{EF4}$ & $\mathrm{V}$ & -0.84 & 0.245 & 0.210 & 0.016 & 0.010 \\
\hline $\mathrm{N}$ & $\mathrm{NF} 2$ & $\mathrm{~V}$ & -2.70 & 0.570 & 0.230 & 0.360 & 0.310 \\
\hline $\mathrm{N}$ & $\mathrm{NF3}$ & $\mathrm{V}$ & -3.93 & 0.128 & 0.089 & -0.050 & -0.028 \\
\hline $\mathrm{N}$ & $\mathrm{NF4}$ & $\mathrm{V}$ & -0.87 & 0.143 & 0.117 & -0.158 & -0.146 \\
\hline $\mathrm{N}$ & $\mathrm{NF5}$ & $\mathrm{H}$ & -0.58 & 0.280 & 0.225 & 0.090 & 0.040 \\
\hline $\mathrm{S}$ & $\mathrm{SF} 1$ & $\mathrm{~V}$ & -1.18 & 0.122 & 0.120 & -0.036 & 0.010 \\
\hline $\mathrm{S}$ & $\mathrm{SF} 2$ & $\mathrm{~V}$ & -1.92 & 0.184 & 0.140 & 0.170 & 0.150 \\
\hline $\mathrm{S}$ & $\mathrm{SF} 3$ & $\mathrm{H}$ & -3.98 & 0.160 & 0.135 & -0.063 & 0.000 \\
\hline $\mathrm{S}$ & $\mathrm{SF} 4$ & $\mathrm{H}$ & -0.64 & 0.195 & 0.148 & 0.252 & 0.062 \\
\hline $\mathrm{W}$ & $\mathrm{WF1}$ & $\mathrm{V}$ & -1.85 & 0.109 & 0.109 & -0.090 & -0.072 \\
\hline $\mathrm{W}$ & $\mathrm{WF} 2$ & $\mathrm{~V}$ & -2.65 & 0.110 & 0.090 & 0.350 & 0.275 \\
\hline $\mathrm{W}$ & $\mathrm{WF3}$ & $\mathrm{H}$ & -3.95 & 0.050 & 0.030 & 0.050 & 0.050 \\
\hline $\mathrm{W}$ & $\mathrm{WF}$ & $\mathrm{V}$ & -1.21 & 0.073 & 0.073 & 0.055 & -0.040 \\
\hline $\mathrm{W}$ & $\mathrm{WF5}$ & $\mathrm{H}$ & -0.31 & 0.675 & -0.020 & 0.350 & -0.050 \\
\hline
\end{tabular}

slip generally increasing above and below this level. Exceptions are FMs WF2 and NF3 located 2.65 and $3.93 \mathrm{~m}$ below the top of the block. Both are in fracture zones. Also note that except for the largest slip, most of the slip is not recovered, and at least $0.2 \mathrm{~mm}$ of slip is not recovered at four locations.

The larger aperture values at the bottom of the block are associated with larger slip values, and this may provide information on fracture properties, as discussed later in this section. However, the trend of increased opening with distance below the top is inconsistent with the MPBX data.

\subsubsection{Deformation of Horizontal Fractures}

Fracture monitors were also deployed on three subhorizontal fractures in the block. The most prominent of these was the large subhorizontal fracture near the top of the block that intersected all four faces. Smaller subhorizontal fractures were also instrumented on the west and south sides of the block (see Figures 7-32 and 7-33).

The fracture aperture deformations for these horizontal fractures are plotted along with temperature at TT1-14 as a function of time in Figure 7-41. Overall, aperture changes are less than or equal to $0.3 \mathrm{~mm}$ and are similar to those on vertical fractures.

Fracture monitors WF5, SF4, EF1, and NF5 monitor the large subhorizontal fracture near the top of the block. Figure 7-41 shows that opening was observed for these four FMs. The response of SF4 during the first 40 days was similar to that shown for vertical fractures in that the initial deformation followed temperature near the heater. Fracture monitors EF1 and NF-5 do not show significant movement until after 30-40 days of heating. Data for fracture monitor WF-5 were not recorded during the initial 20 days of heating. However, after Day $20 \mathrm{WF}-5$ followed the temperature at TT1-14 until Day 220, after which the fracture continued to open while 
temperature remained constant. The most striking feature is that NF-5, EF-1, and WF-5 all show substantial spikes at the onset of the TH event at Day 105.

The association of fracture deformation with $\mathrm{TH}$ behavior is shown more clearly in Figure 7-42. This figure shows the aperture deformation of the large subhorizontal fracture, along with temperature of TT1-14 for 100-140 days. The figure shows that opening of the fracture at EF1 and NF5 preceded the thermal event by almost one day and that the fracture opened $0.1 \mathrm{~mm}$ at EF1 and 0.07 at NFS over a 2-3 day period and then closed back to original levels. Closing of fractures during initial thermal recovery is similar to behavior for the vertical fractures at this time.

Figure 7-41 shows that fracture monitors NF-5 and EF-1 also show similar behavior at Day 180 and at Day 340. The behavior is a spike associated with temperature drop followed by a closure. The spike at Day 180 can be associated with the TH event recorded by TT1-14. The aperture spikes at Day 340 are associated with at similar TH event recorded at RTD TT2-22, which is approximately $1.6 \mathrm{~m}$ above the heater plane.

Fracture monitors WF3 and NF3 recorded deformation of fractures lower in the block, and show overall fracture closing. These fractures show increased aperture closing during the thermal recovery at Day 125 (see Figure 7-42).

Finally spikes in aperture are associated with cool-down. In addition, fracture monitor data indicate that rock above the subhorizontal fracture near the top behaves as a unit; this may be because the heat exchanger and other hardware on top of the block serve as rigid connectors for rock blocks forming the top of the block.

Slip on horizontal fractures is shown in Figure 7-43 along with temperature for RTD TT1-14. This figure shows that slip on horizontal fractures lags the temperature at the heater plane. The values of slip are in the same range as for the vertical fractures, and for fracture \#LBT-1 (see Table 4-2) slip increases sharply up to Days 50-60 and then levels off, except for WF5, for which slip continues to increase until very late in the test. Figure 7-44 shows slip measurements for horizontal fractures for Day 100 to Day 140. Fracture monitors WF5, EF1, and NF5 show slip events at Days 104-105, but the slip events are not as dramatic as the changes in aperture. WF5 shows slip recovery between Day 125 and Day 130 that is opposite the behavior shown by vertical fractures. SF4 and EF1 show increased slip during this period, more like vertical fractures. Spikes in data are also found at Day 180 and Day 340 (EF1, WF5, and NF5). WF5 reacts to cooling on Day 220 but thereafter slip continues to increase. WF5 recovers on cooldown, while SF3 shows more than $1 \mathrm{~mm}$ of slip increase on cool-down.

Maximum and residual deformation across horizontal fractures have been tabulated (see Table 75) and is plotted in Figure 7-45. This figure shows that for three of the four vertical large-block faces, the large horizontal fracture near the top opened more than $0.2 \mathrm{~mm}$, but residual opening was much smaller. This is in contrast to the behavior of the vertical fractures (Figure 7-39), which show small opening or even closing motions. It is important to note that all vertical measurements were made below this fracture.

Figure 7-45 also shows that the aperture deformation of the two lower horizontal fractures was very different from that of Fracture LBT-1. Figure 7-46 presents maximum and residual slip 
Figure 7-45 also shows that the aperture deformation of the two lower horizontal fractures was very different from that of Fracture LBT-1. Figure 7-46 presents maximum and residual slip along all monitored horizontal fractures. This figure shows that slip on one fracture (WF5) was very large $(0.7 \mathrm{~mm})$ but was totally recovered upon cool-down. Slip of 0.2 to $0.3 \mathrm{~mm}$ was recorded on the other three sides, and most of this slip was irreversible. The least amounts of slip erew recorded on the lowest fractures in the block. This, again, is in contrast to the vertical fracture data, which show more fracture slip lower in the block.

\subsubsection{Summary of Fracture Monitor Results}

The FM data show that the vertical and horizontal fractures responded somewhat differently. Fracture \#LBT-1 opened coincidentally with the TH event at Day 105. Both vertical and horizontal fractures show closing during the thermal recovery from the TH events, that is, during periods of apparent refluxing.

Initial response for several of the FMs was associated with temperature at the heater plane. Overall FM deformations are consistent throughout the block, as indicated in Figure 7-47, which shows general block movements. FM data indicate that the top of the block moved to the east. Most of the FM deformation was not recoverable. The FM data are somewhat inconsistent with the MPBX data, as FMs indicate more deformation in lower portions of the block and less deformation the upper portions of the block.

\subsubsection{Discussion and Summary}

Analysis of the large block deformation has been conducted using data from six MPBX systems and approximately 20 fracture monitors. Results from both the MPBX systems (Figure 7-48) and the FMs (Figure 7-49) show that within a few hours of heater startup the block started expanding.

The MPBX data (Figure 7-48) show that most of the expansion has occurred in the upper third of the block and in the horizontal plane. In this region, horizontal strain of 0.0006 was observed through March 18, 1998. This is somewhat greater than the value of 0.0001 that is predicted in a TM simulation of the test (Blair, Berge, et al. 1996). Moreover, MPBX data from boreholes in this region show that most of the deformation occurred in discrete, vertical zones, which may be caused by the opening of vertical fractures in the upper portion of the block.

The central third of the block, although hotter than the upper third, shows less horizontal expansion. However, the observed strain of 0.0004 is in good agreement with the value of 0.0006 predicted for this zone after this period of heating. Finally, deformation in the lower third of the block was small, with total horizontal strain estimated as 0.0002 . This is consistent with the predicted thermal expansion. Data for one of the horizontal holes also indicate that deformation occurred in a discrete, vertically oriented zone. The data also indicate that vertical strains are smaller than horizontal strains, and that the portion of the block above the heaters is moving upward as a unit.

In summary, thermal expansion of the block was evident a few hours after the start of heating, as verified by the fracture monitors and MPBX systems. The MPBX data indicate that, during the first month of heating, the upper third of the block expanded horizontally more than predicted on 
expansions were more consistent with predicted values. In the vertical direction, the upper twothirds of the block (i.e., the portion above the heater horizon) extended as a unit.

\subsection{SIMULATIONS}

Three-dimensional distinct element analysis was performed to simulate MPBX deformations. The simulations will be discussed in chronological order. This work is documented in Blair, Berge, et al. (1996).

\subsubsection{Distinct Element Analysis}

A distinct element code (3DEC) (Itasca 1998) was used in this analysis to simulate TM behavior in a 3-D region of fractured rock forming the LBT. The distinct element method was chosen because it allows discrete fractures to be incorporated into the simulation. This is important because fracture movements can cause large local deformation and changes in permeability. Moreover, continuum approaches do not provide direct estimates for fracture deformation. The distinct element method also provides for calculation of joint behavior (normal and shear deformation) at specific points along a joint surface. Thus, this method can (a) handle redistribution of stress after shear slip occurs along a fracture, (b) provide insight into the primary mechanisms of fracture deformation over time, and (c) preserve the time history of fracture deformation. The 3DEC code can accommodate several constitutive material relations for blocks, and both Coulomb slip and continuous slip models for joints. The code can also be used to simulate an unfractured rock mass.

3DEC also has the capability to accept temperatures from external sources. This can be done by initializing the code for thermal analysis and then substituting temperatures from the external source into the 3DEC temperature array at each time step. Using this technique, the temperatures predicted using a robust $\mathrm{TH}$ code, such as NUFT, or a temperature field developed from field observations (e.g., the LBT) can be used to predict THM behavior.

\subsubsection{Model Development}

The spatial domain for the model is shown in Figure 7-50. This region has the same dimensions in cross section as the 2-D FLAC model discussed previously in Section 3.5.3. That is, the subsurface model domain extends $23 \mathrm{~m}$ beneath the large block and $23 \mathrm{~m}$ out from each vertical face beneath the surface.

\subsubsection{Boundary Conditions}

Roller boundary conditions were imposed on the four vertical sides and on the base of the subsurface region. These boundary conditions impose a zero displacement restriction on normal displacements along these surfaces but allow parallel (in plane) displacements. Thus, horizontal displacements are permitted along the base of the block, and vertical displacements are permitted along the sides. Fixed displacement boundary conditions provide an upper bound on thermal stresses because outward displacements of the model sides, which would relieve built-up stresses, are not allowed. 
The base of the block was fixed in the vertical direction to prevent the rock at $23 \mathrm{~m}$ below the LBT from moving vertically. The top of the block is allowed to move vertically. This is appropriate because the LBT column is unconfined, whereas the base of the model is supported by the underlying rock. A fixed stress boundary condition, equal to atmospheric pressure, is applied to the top and sides of the LBT columnar region and to the ground surface region in the model.

\subsubsection{Temperature Field and Calculation Times}

Deformation of the LBT was calculated at times of $0,10,25,55,85,115,145,182,200,275$, $340,350,375,385,395,410,430$, and 450 days after the start of heating. The temperatures in this analysis were derived from the TH analysis reported in Section 5.6. Files containing $x, y, z$ locations and temperatures for the model region simulated by NUFT were obtained at each time. The NUFT model assumes symmetry in the block; consequently, these files contained values for one quadrant of the region simulated in 3DEC. The 3DEC calculation includes the entire volume of the block, because the fractures are not symmetric. A 3-D temperature field for 3DEC was produced from the NUFT temperatures by reflecting the temperatures about the appropriate vertical planes. This was done as follows. Temperatures from the NUFT model and their coordinates were input into EarthVision, v.5.1 along with an array of grid points generated by 3DEC for the LBT model domain at each calculation time. EarthVision performed a 3-D interpolation of the 3-D NUFT model temperatures to provide an interpolated temperature for each calculation time at each of the $3 \mathrm{DEC}$ model grid points. The grid point temperatures were then input into $3 \mathrm{DEC}$ as a separate input file for each calculation time.

\subsubsection{Material Properties}

Input parameters for the LBT simulation are provided in Table 7-6. Calculated or assumed parameters are listed in Table 7-7. The input bulk and shear moduli were calculated with the equations given below using values of rock mass elasticity modulus (24.71 GPa) and Poisson's ratio (0.21) taken from CRWMS M\&O (1999b, Tables 10 and 11). The input values for joint normal stiffness and joint shear stiffness assume a joint spacing of $1 \mathrm{~m}$ and were calculated with a relationship given in the 3DEC User's Guide (Itasca 1998, Section 3, p. 94). The joint normal stiffness calculation used an intact rock elastic modulus of $33.03 \mathrm{GPa}$ (CRWMS M\&O 1999b, Table 8) and a rock mass elastic modulus of 24.71 GPa (CRWMS M\&O 1999b, Table 10). Shear moduli for the joint shear stiffness calculation were obtained from the above elastic moduli and a Poisson's ratio of 0.21 (CRWMS M\&O 1999b, Table 11). The other input parameter values were taken directly from the YMP Technical Data Management System. The input parameters are considered appropriate because they are derived from field and laboratory measurements of the host rock physical properties. 
Table 7-6. Input Parameters and Data Tracking Numbers

\begin{tabular}{|c|c|c|c|c|}
\hline $\begin{array}{l}\text { Item } \\
\text { No. }\end{array}$ & Description & Value & Units & Data Tracking Number \\
\hline \multicolumn{5}{|c|}{ Matrix properties } \\
\hline 1 & Dry bulk density & 2270 & $\mathrm{~kg} / \mathrm{m}^{3}$ & MO0003SEPDRDDA.000 \\
\hline 2 & Intact rock elasticity modulus & 33.03 & $\mathrm{GPa}$ & MO9911SEPGRP34.000 \\
\hline 3 & Rock mass elasticity modulus & 24.71 & $\mathrm{GPa}$ & MO9911SEPGRP34.000 \\
\hline 4 & Poisson's ratio & 0.21 & none & MO9911SEPGRP34.000 \\
\hline \multicolumn{5}{|c|}{ Joint properties } \\
\hline 5 & Joint friction & 41 & degree & MO0003SEPDADDA.000 \\
\hline 6 & Joint cohesion & 0.09 & $\mathrm{MPa}$ & MO9911SEPGRP34.000 \\
\hline 7 & Joint dilation angle & 29 & deg & MO9911SEPGRP34.000 \\
\hline 8 & Initial joint aperture & 0.098 & $\mathrm{~mm}$ & LB990501233129.001 \\
\hline \multicolumn{5}{|c|}{ Thermal properties } \\
\hline 9 & Thermal conductivity & 2.33 & $\mathrm{~W} / \mathrm{m}-\mathrm{K}$ & MO9911SEPGRP34.000 \\
\hline 10 & High thermal expansion coefficient & 9.73E-6 & $\operatorname{deg} \mathrm{C}^{-1}$ & MO0004RIB00035.001 \\
\hline 11 & Low thermal expansion coefficient & 5.27E-6 & $\operatorname{deg} \mathrm{C}^{-1}$ & SHT Final Report DTN \\
\hline \multicolumn{5}{|c|}{ Stress and stress gradient } \\
\hline 12 & In-situ stress & 0.1 & $\mathrm{MPa}$ & MO0007RIB00077.000 \\
\hline \multirow[t]{2}{*}{13} & Vertical stress gradient & 0.023 & $\mathrm{MPa} / \mathrm{m}$ & MO0007RIB00077.000 \\
\hline & Input temperatures & & & \\
\hline 14 & Input temperatures & various & $\operatorname{deg} C$ & LL000114004242.090 \\
\hline
\end{tabular}

Table 7-7. Calculated or Assumed Model Parameters

\begin{tabular}{|l|l|l|}
\hline Description & Value & Units \\
\hline Matrix properties & & \\
\hline Rock mass bulk modulus & 14.2 & $\mathrm{GPa}$ \\
\hline Rock mass shear modulus & 10.2 & $\mathrm{GPa}$ \\
\hline Joint properties & & \\
\hline Joint tensile strength & 0 & $\mathrm{MPa}$ \\
\hline Joint normal stiffness & 98.1 & $\mathrm{MPa} / \mathrm{mm}$ \\
\hline Joint shear stiffness & 40.5 & $\mathrm{MPa} / \mathrm{mm}$ \\
\hline
\end{tabular}

In addition, the following assumptions were made for the LBT simulations:

- First, nominal dimensions and approximations were used in developing the geometric model and used throughout the modeling instead of exact design or as-built dimensions. This is because the limited sensitivity of the numerical simulations to slight dimensional variations in no way merits the use of exact dimensions.

- The initial model displacement prior to heating was assumed to be zero, and the initial stresses throughout the model were assumed to be in equilibrium with the applied boundary stresses and the gravitational gradient.

- Stress and temperature dependence of rock mass material properties was neglected in assessing deformation. Except for temperature dependence of the thermal expansion 
coefficient, stress- and temperature-related changes to rock mass material properties are expected to have little impact on deformation in comparison to fracture displacements.

- Intact rock deformation is elastic and isotropic, such that bulk modulus, $\mathrm{K}$, and shear modulus, G, can be calculated from the elastic modulus, E, and Poisson's ratio, $v$, as:

$$
\begin{gathered}
K=E /\{3[1-(2 v)]\} \\
G=E /[2(1+v)]
\end{gathered}
$$

These relations are derived for linearly elastic, isotropic rock. Laboratory tests on intact rock samples from the repository horizon show them to behave as an elastic, isotropic material (Nimick et al. 1987, p. 32). The above equations of elasticity are from Jaeger and Cook (1979, p. 111). Moduli for blocks of rock forming the fractured rock mass may be lower and may generate lower stress levels than predicted.

Approximate normal and shear joint stiffness were calculated from information on joint spacing and elastic moduli for the fractured rock mass and intact portions of the rock. According to Itasca (1998, Section 3, p. 94), "If the jointed rock mass is assumed to have the same deformational response as an equivalent elastic continuum, then relations can be derived between jointed rock properties and equivalent continuum properties. For uniaxial loading of rock containing a single set of uniformly spaced joints oriented normal to the direction of loading, the following relation applies:

$$
1 / E_{m}=1 / E_{r}+1 / k_{n} s
$$

or

$$
k_{n}=E_{m} E_{r} / s\left(E_{r}-E_{m}\right)
$$

where $E_{m}=$ rock mass elastic modulus, $E_{r}=$ intact elastic modulus, $k_{n}=$ joint normal stiffness, and $s=$ joint spacing. A similar expression is used for joint shear stiffness:

$$
k_{s}=G_{m} G_{r} / s\left(G_{r}-G_{m}\right)
$$

where $G_{m}=$ rock mass shear modulus, $G_{r}=$ intact rock shear modulus, and $k_{s}=$ joint shear stiffness."

And "Joint shear deformation followed a Coulomb slip model. This model provides a linear representation of joint stiffness and yield limit and is based upon elastic stiffness; frictional, cohesive and tensile strength properties and dilation characteristics common to rock joints. The model simulates displacement-weakening of the joint by loss of cohesive and tensile strength at the onset of shear or tensile failure" (Itasca 1998, p. 73). 
Fractures used in the simulations were taken from the LBT fracture data set described in Section 4. Particular fractures used in the simulations are discussed below. The fractures were assumed to have no tensile strength. Fractures are very weak in tension, and any reasonable value for fracture tensile strength will be close to zero, which is the default value for the 3DEC program. Chen (1999, Table 3) used a fracture tensile strength of $0.04 \mathrm{MPa}$ in an UDEC analysis of drift stability at Yucca Mountain. This value was intended as a reasonable average value for fracture tensile strength (Chen 1999, p. 761). The minimum fracture tensile strength is assumed here to enhance changes in fracture aperture and permeability.

\subsubsection{Simulations}

A series of simulations was conducted to evaluate the effects of the number of fractures and the Coefficient of Thermal Expansion (CTE). The simulations are listed in Table 7-8, and the geometry of the model domain for the various simulations is shown in Figure 7-50.

Table 7-8. Summary of LBT TM Simulations

\begin{tabular}{|c|c|c|l|}
\hline Model \# & No. of Fractures & CTE $\left(\times 10^{-6} \% \mathbf{C}\right)$ & \multicolumn{1}{c|}{ Comment } \\
\hline 1 & 0 & 5.27 & Continuum model \\
\hline 2 & 6 & 9.73 & High CTE with six major fractures \\
\hline 3 & 6 & 5.27 & Low CTE with six major fractures \\
\hline 4 & 7 & 5.27 & Same as 3 with one additional fracture \\
\hline 5 & 28 & 5.27 & All fractures included in fracture analysis \\
\hline
\end{tabular}

\subsubsection{Results}

The 3DEC model was configured to produce displacement values at the locations of the MPBX anchors discussed in Section 7.1.1. Comparisons were made for most of the MPBX holes for the first 100 days of heating, as this is the period when MPBX results were most reliable. Comparisons were also made for the cool-down phase, for which new transducers were installed into some of the MPBX units. Data for the entire test are used for WM2 and NM3. Simulated deformation in the vertical direction is compared with observed displacement for anchor 4 in TM1 (TM1-4), as shown in Figure 7-51 for the first 100 days of heating. This figure plots results for each simulation along with the observed displacement and shows that while Model 2 (Table 7-8) with high CTE matches the early thermal deformation up to Day 20, it overpredicts the deformation at 100 days by more than a factor of 2 . Predictions produced by the other four models, with a lower CTE, are quite acceptable. The continuum and fractured models produce similar estimates, and the response of all of these models lags the observed deformation during the first 40 days. Thus, the number of fractures had very little effect on predicted deformation in the vertical direction.

Predicted deformation during cool-down is compared with field measurements at anchor TM1-4 in Figure 7-52. The predicted displacements were "corrected" to the observed value at Day 375. This plot shows that the continuum model (Model 1) fits both the shape and magnitude of the observations, while Model 3, with six fractures, also approximates the observations quite well. Model 2 (high CTE) overpredicts the contraction during cool-down, but not as badly as it overpredicted the expansion during the first 100 days. 
MPBX holes NM1 and WM1 were located close to the bottom of the block and in orthogonal directions. Measured and predicted displacements for Anchor NM1-4 are plotted in Figure 7-53. At this location, Model 3 provides the best match to the observations. Model 2 overpredicts displacement by nearly a factor of 2 , while the continuum model (Model 1) underpredicts the deformation. Models 1, 3, 4 and 5 bracket the observed values, with Model 1 underpredicting for the first 100 days and Models 4 and 5 overpredicting at Day 100. This plot indicates that adding one fracture (Model 4) caused more displacement at this location, but adding many fractures (Model 5) caused underprediction during the first 25 days and overprediction after Day 25.

Results for NM1-4 during cool-down are shown in Figure 7-54. Again, the data have been "corrected" to fit the observed value at Day 375. The correction for NM1-4 was different than the others because the field data were also shifted to avoid a negative displacement at the end of the test. At this location, Model 3 (low CTE) matches the magnitude of the displacement but does not accurately predict the cooling path. Model 2 (high CTE) overpredicts the displacement and best approximates the slope of the curve during the first 20 days of cooling. Models 1, 4, and 5 underpredict the amount of recovery during cool-down. The least contraction is predicted by Model 5 , the highly fractured rock mass.

Data and predictions for the first 100 days of heating for WM1 are shown in Figure 7-55. This plot shows similar results to Figure 7-53 in that Model 2 overpredicts by nearly a factor of 2, and Models 1, 3, 4 and 5 bracket the behavior. The highly fractured model (Model 5) best approximates the response during the first 20 days, Models 3 and 4 overpredict the displacement during the first 10 days, but are within about $0.05 \mathrm{~mm}$ of the observed displacement at Day 100 . Model 1 underpredicts the displacement by between 0.1 and $0.15 \mathrm{~mm}$ throughout this time interval.

Modeling results for WM1 during the cool-down period are shown in Figure 7-56. The cooldown simulations have again been "corrected" to the observed value at Day 375 , as only relative displacements can be determined. These results are similar to those for NM1-4 in that the continuum model (Model 1) most closely approximates the observed cool-down. The high CTE model (Model 2) overpredicts the deformation, while the other models underpredict the displacement. Interestingly, these results show that adding fractures to the model causes less recovery during cool-down. This suggests fracture slip is essentially unrecoverable under unconfined stress conditions.

Figure 7-57 presents simulated and observed displacements for borehole NM2 that was located near the heater plane. This figure shows that the continuum model (Model 1) underpredicts the displacement for anchor NM2-4, while the high CTE model (Model 2) predicts the deformation relatively well during the first 10 days, but overpredicts the magnitude of the total deformation at Day 40 by nearly a factor of 2 . Models 3, 4 and 5 produce similar results, and both Models 4 and 5 cross over the observed deformation at Day 40. Of these three models, Model 5 provides the best fit to the data for the first 40 days, indicating that rock in this region is highly fractured. It would be safer not to conclude anything from the NM2 data. Model 5 does a nice job of fitting the WM1-4 data during the first 25 days, but it could be argued that Model 1 fits the WM1 data just as well by Day 100 . 
The MPBX instrumentation in borehole WM2 functioned throughout the test, and data for anchor WM2-4 are shown along with simulated displacements for the entire test in Figure 7-58. This figure shows that, for this anchor, Models 3 and 4 did a very good job of predicting the deformation over much of the test duration. Model 3 predicts slightly less displacement than Model 4, and from Day 50 to Day 100 Model 4 is closer to the observations, while from Day 120 to Day 220 Model 3 fits slightly better. Models 3 and 4 also capture the cool-down relatively well. They underpredict the total amount of cool-down, by $0.4 \mathrm{~mm}$, and also show some contraction of the block about Day 270 that is not reflected by the observation. Models 1 and 5 both underpredict maximum deformation by significant amounts (1.6 and $1 \mathrm{~mm}$ respectively). Model 2 overpredicts the maximum deformation but does show the best fit to the displacements during the first 20 days of heating. Model 5 does not show contraction with cool-down, and Model 1 underpredicts the magnitude of the cool-down displacement. Model 2 correctly predicts the relative change in displacement during cool-down $(1.8 \mathrm{~mm})$, but the final value of $2.6 \mathrm{~mm}$ of displacement is too high.

Continuous data are also available for borehole NM3, anchor NM3-4, and these data are shown with the model predictions in Figure 7-59. It is important to note that the fracture intersected borehole NM3 at a high angle near the north face of the block. This borehole is the highest horizontal MPBX borehole in the block. This figure shows that while the measured displacement for NM3-4 is similar to that for WM2-4 (maximum between 2.5 and 3mm), all of the models underpredict the measurement. This is considerably different from the other comparisons. This figure show that adding one fracture to Model 3 to create Model 4 did increase the displacement at this location, but the increase was only a small fraction of the amount needed to compare well with the observed displacement. As expected, the continuum calculation Model 1 produced values similar to those predicted for WM2-4. The low displacement for this anchor indicates that Models 3 and 4 are missing one more key fractures. Moreover, the highly fractured Model 5 predicts less deformation than Model 4, indicating that different fractures make significantly different contributions to the deformation. This indicates that in the simulation, this portion of the block behaved more like a continuum, while the actual block response was that of a fractured medium. (While adding 21 fractures hurt the fit, having no fractures didn't help either.) The continuum model shows the least deformation.

The results discussed above indicate that Models 3 and 4 provide the best overall fits to the observations. Model 1 fits the results at the bottom of the block relatively well, and this is consistent with the results of Blair and Wood (1998), who compared observed MPBX displacements with the continuum model simulations discussed in Section 3.5.3. These authors concluded that the block could be modeled as a continuum below the heater plane, but not in the region above the heaters.

The difference between the measured and predicted deformation during the first 50 days of heating is of interest because it relates to the transient response of the rock to the temperature field. Figures 7-53 and 7-55 show that for horizontal boreholes near the base of the block, deformation in Models 3 and 4 lead the observed displacements during the first 20 days. Figures $7-57,7-58$, and 7-59 show that the observed deformation leads the predictions during the first 40 to 60 days of heating. Predicted and observed results for Anchor WM2-4 for the first 100 days of heating are replotted in Figure 7-60 along with temperature data for the plane of the heater (TT1-14) and the plane of WM2 (TT1-22) respectively. This figure shows that the observed 
deformation can be correlated with temperature at the heaters. However, Figure 7-60 also shows that the predicted deformation is correlated with temperature at the borehole location, at least for the first 30 days.

The correlation with heater temperature is consistent with the behavior of many of the fracture monitors discussed in Section 7.1.2. This indicates that movement of the rock above the heater plane is due to a far-field effect, and may imply that movement along fractures serves to propagate deformation.

\subsection{POST-TEST ANALYSIS}

\subsubsection{Introduction}

This section presents ultrasonic velocity, uniaxial compressive strength, and elastic moduli measurements on two suites of core samples (pre-test and post-test), taken from the large block. During field testing, the large block was heated internally to a peak temperature of $140^{\circ} \mathrm{C}$ over a period of several months ( $\mathrm{Lin}$ et al. 1997). The heating was accomplished with 2.4-m-long, $300-\mathrm{W}$ heaters emplaced in five 3.8-cm-diameter horizontal boreholes at the $2.75-\mathrm{m}$ level of the block. Elevated temperatures and high thermal gradients may have induced grain boundary scale microcracks in the tuff, particularly near the borehole heaters where conditions were most extreme. Thermal microcracking would contribute to the mechanical degradation of the rock, leading to a loss of strength and a reduction in elastic modulus. A loss of mechanical strength in the rock surrounding emplacement drifts would have adverse consequences for repository performance. The purpose of this work was to ascertain if measurable mechanical degradation did result around at least one of the heater boreholes during the LBT. Two suites of samples were collected. One suite, consisting of cores from one of the heater boreholes, was never exposed to elevated temperatures and will be termed the "pre-test" suite. The other suite, from overcore of the same borehole, experienced the highest temperatures and thermal gradients attained during the LBT and will be termed the "post-test" suite.

\subsubsection{Methods}

\subsubsection{Sample Selection and Preparation}

Two sample suites were collected from large block cores stored in the YMP Sample Management Facility at the Nevada Test Site. The pre-test suite consists of 11 cores taken from heater borehole UE-25 EH4. Borehole UE-25 EH4 was one of five horizontal heater holes emplaced at the 2.75-m level from the top of the large block. The second post-test suite consists of 12 subcores from UE25 FR PTC OC\#1, a 25.4-cm-diameter overcore of borehole UE-25 EH4. The overcore samples were taken within $80 \mathrm{~mm}$ of heater borehole UE-25 EH4, and each should have experienced essentially the same thermal history, including the peak temperature of $140^{\circ} \mathrm{C}$. All samples have the same axial orientation, since the overcore was taken parallel to the heater hole. Any preexisting anisotropy in rock strength or elastic properties should therefore be consistent for both sets of samples. Two views of the post-test coring activities are given in Figures 7-61 and 7-62.

The test specimens were prepared following ASTM standard procedures (ASTM 1997a). A 3:1 height-to-diameter ratio was chosen to be consistent with Blair, Berge et al. (1996). Because the 
heater core was only $21 \mathrm{~mm}$ in diameter, the pre-test specimens were cut to a length of $63.5 \mathrm{~mm}$. The post-test overcore specimens were prepared from 25 -mm-diameter cores and were therefore cut to a length of $76.2 \mathrm{~mm}$. The cores were visually inspected during specimen preparation, and all specimens having visible fractures were rejected. The specimen ends were ground flat and parallel to $0.02 \mathrm{~mm}$ using a precision grinder. The specimens were stored under a partial vacuum for over two weeks, then vented with dry nitrogen gas. The dry specimens were weighed with a calibrated Mettler AE240 mass balance, and their dimensions were measured with digital calipers. Densities were calculated by dividing mass by volume. The mean dry density for the pre-test and post-test samples are $2.281 \pm 0.014$ and $2.285 \pm 0.014 \mathrm{~g} / \mathrm{cc}$ respectively. They are statistically the same; no thermal fracturing effect is evident in the density data.

\subsubsection{Ultrasonic Velocity Measurements}

Travel time of ultrasonic waves were measured using standard through-transmission techniques consistent with ASTM guidelines (ASTM 1997b). The ultrasonic system was checked, prior to the measurements on the tuff specimens, by measuring compressional and shear wave travel times through an aluminum reference standard. Input signals were supplied by a Panametrics model 5058PR pulse-generator with the input voltage set to $200 \mathrm{~V}$. Matched pairs of Panasonics transducers, model V114 (1.0 MHz) for compressional and model V155 (5.0 MHz) for shear waves, served as transmitters and receivers. Honey was used as the transducer couplant. The received signals were amplified $40 \mathrm{~dB}$ with a Panametrics $5660 \mathrm{~B}$ pre-amplifier and digitized at 10-bit resolution with a LeCroy $9430150-\mathrm{MHz}$ digital oscilloscope. The travel times were manually picked on the oscilloscope screen. All of the travel-time measurements were performed twice as a simple check on data integrity. Three digitized waveforms, one compressional and two shear, were stored on the hard drive of a Mac Пcx for each specimen. Shear waveforms were recorded for two orientations, one parallel and the other orthogonal, to the sample scribe line.

The measured travel times were imported into an Excel spreadsheet, and velocities were calculated by dividing specimen lengths by travel time. Dynamic Young's modulus, E, was calculated as:

$$
E=\left[\rho V_{p}^{2}\left(3 V_{p}^{2}-4 V_{s}^{2}\right)\right] /\left(V_{p}^{2}-V_{s}^{2}\right)
$$

and dynamic Poisson's ratio, $v$, as:

$$
\mathrm{v}=\left(\mathrm{V}_{\mathrm{p}}^{2}-2 \mathrm{~V}_{\mathrm{s}}^{2}\right) /\left[2\left(\mathrm{~V}_{\mathrm{p}}^{2}-\mathrm{V}_{\mathrm{s}}^{2}\right)\right]
$$

where $\rho$ is density, $\mathrm{V}_{\mathrm{p}}$ is compressional velocity, and $\mathrm{V}_{\mathrm{s}}$ is shear velocity. Two moduli are sufficient to characterize fully the elastic properties of an isotropic material but are insufficient for an anisotropic material, for which additional velocity measurements at different angles are required. Because the degree of anisotropy is not known for the sample specimens, the calculated moduli should be regarded as a potential indicator of fracture damage in the specimens rather than as an estimate of the material elastic properties. 


\subsubsection{Compressive Strength Tests}

The compressive strength test procedures followed ASTM guidelines (ASTM 1997c). The specimens were tested in the same 100-ton-capacity loading frame used by Blair, Kelly, et al. (1996b). A 50-ton Enerpac hydraulic ram mounted at the base of the reaction frame supplied the axial load. Hydraulic oil was supplied to the ram through a metered valve to control the loading rate. Several trials were performed with an aluminum test piece to determine the proper valve setting for a nominal strain rate of $10^{-5} \mathrm{~s}^{-1}$. The test assembly consisted of a hemispherical swivel platen at the sample base, the test specimen, and two flat-top platens. The platens were $25.4 \mathrm{~mm}$ in diameter. Each test specimen was positioned in the loading column using two aluminum alignment fixtures. After a small load was applied, the operator checked the alignment of the loading column and repositioned the specimen if necessary. The fixtures were removed before each test.

The load was measured with a 55,000-lb spool type strain-gauged load cell for the first five tests (specimens OC-877-01 to OC-877-05). This load cell was damaged at the end of the fifth test and replaced with a similar, 80,000-lb load cell. The load cell sensitivities are $4.42 \mathrm{mV} / \mathrm{V} / \mathrm{lb}$ and $3.03 \mathrm{mV} / \mathrm{V} / \mathrm{lb}$, respectively. Both load cells were calibrated by Bechtel Nevada one month prior to the compressive strength tests. The difference in load cell sensitivity is of little significance, particularly in comparison to the heterogeneity of the sample materials. A constant 10-V DC excitation voltage was provided to the load cell by a Hewlett Packard 6205C power supply. Vertical displacement of the loading ram piston was measured with two Bourns model 80294 LVDTs mounted to the loading frame. The LVDTs were excited with constant 10-V DC power. The ambient temperature was measured during the tests with an Omega type $J$ thermocouple.

The data acquisition system was controlled by a National Instruments (NI) LabVIEW version 4.1 software program on a Dell XPS D266 personal computer. Data were recorded at one-second intervals during the tests and included room temperature, the load cell and LVDT excitation voltages, load cell output voltage, and LVDT output voltages. The signal lead wires were attached to NI model 1303 terminal boards, configured to read the input voltages in differential mode. Signal amplification and multiplexing were performed under software control by two NI SCXI 1100 modules in an NI 1000 chassis. The input signals were digitized with an NI PCIMIO-16XE-50 16-bit data acquisition board and stored along with a time stamp on the hard drive of the Dell computer.

After the tests, the time, ambient temperature and output voltages were imported into an Excel spreadsheet. The load cell output voltages were converted to axial load using sensitivity and voltage offset values given on the calibration sheets supplied by Bechtel Nevada. Axial stress was calculated by dividing axial load by the specimen's initial cross-sectional area. No correction was made for the very small change in specimen diameter with loading. The LVDT voltages were converted to centimeters of displacement using sensitivity factors derived from the LVDT calibrations, then converted to strains by dividing by the specimen length. Specimen strain rates were calculated by dividing incremental strains by the sampling interval. Young's modulus was calculated as the slope of the tangent line to the axial stress-strain curve at half the measured compressive strength, following standard ASTM procedures (ASTM 1997). A standard stress-strain plot was produced for each specimen. Two representative stress-strain plots are given in Figure 7-63. 


\subsubsection{Results}

Compressional and shear velocities and calculated dynamic moduli are given for pre-test and post-test specimens in Tables 7-9 and 7-10 and are plotted as histograms in Figures 7-64 and 7-65. The average compressional and shear velocities are nearly identical for both sample suites. The average compressional velocities of the post-test and pre-test cores are $4.86 \pm 0.05 \mathrm{~km} / \mathrm{s}$ and $4.87 \pm 0.04 \mathrm{~km} / \mathrm{s}$, respectively. The average shear velocities of the post-test and pre-test cores

Table 7-9 Pre-test Specimen Densities, Velocities, and Dynamic Moduli

\begin{tabular}{|l|c|c|c|c|c|}
\hline Specimen & Density & P Velocity & S Velocity & $\begin{array}{c}\text { Young's } \\
\text { modulus }\end{array}$ & $\begin{array}{c}\text { Poisson's } \\
\text { ratio }\end{array}$ \\
\hline & $\left(\mathbf{g} / \mathbf{c m}^{3}\right)$ & $(\mathbf{k m} / \mathbf{s})$ & $(\mathbf{k m} / \mathbf{s})$ & (GPa) & \\
\hline HC-071-05 & 2.290 & 4.83 & 3.27 & 52.7 & 0.08 \\
\hline HC-072-12 & 2.271 & 4.84 & 3.19 & 51.5 & 0.12 \\
\hline HC-074-26 & 2.308 & 4.88 & 3.25 & 53.6 & 0.10 \\
\hline HC-075-44 & 2.258 & 4.80 & 3.14 & 50.1 & 0.13 \\
\hline HC-075-47 & 2.274 & 4.80 & 3.23 & 51.5 & 0.09 \\
\hline HC-076-61 & 2.299 & 4.75 & 3.22 & 51.3 & 0.08 \\
\hline HC-076-65 & 2.255 & 4.80 & 3.18 & 50.5 & 0.11 \\
\hline HC-077-77 & 2.272 & 4.81 & 3.21 & 51.5 & 0.10 \\
\hline HC-077-80 & 2.290 & 4.87 & 3.21 & 52.7 & 0.11 \\
\hline HC-077-83 & 2.276 & 4.77 & 3.18 & 50.6 & 0.10 \\
\hline HC-077-87 & 2.272 & 4.81 & 3.23 & 51.6 & 0.09 \\
\hline HC-077-89 & 2.285 & 4.82 & 3.25 & 52.2 & 0.08 \\
\hline mean & 2.279 & 4.81 & 3.21 & 51.6 & 0.10 \\
\hline std. dev. & 0.015 & 0.04 & 0.04 & 0.97 & 0.02 \\
\hline
\end{tabular}

Table 7-10 Post-test Specimen Densities, Velocities, and Dynamic Moduli

\begin{tabular}{|l|c|c|c|c|c|}
\hline Specimen & Density & P Velocity & S Velocity & $\begin{array}{c}\text { Young's } \\
\text { modulus }\end{array}$ & $\begin{array}{c}\text { Poisson's } \\
\text { ratio }\end{array}$ \\
\hline & $\left(\mathbf{g} / \mathrm{cm}^{3}\right)$ & $(\mathbf{k m} / \mathbf{s})$ & $(\mathrm{km} / \mathbf{s})$ & (GPa) & \\
\hline OC-877-01 & 2.265 & 4.88 & 3.29 & 53.2 & 0.08 \\
\hline OC-877-02 & 2.287 & 4.84 & 3.21 & 52.1 & 0.11 \\
\hline OC-877-03 & 2.293 & 4.87 & 3.24 & 53.1 & 0.10 \\
\hline OC-877-04 & 2.307 & 4.83 & 3.20 & 52.4 & 0.11 \\
\hline OC-877-05 & 2.291 & 4.89 & 3.22 & 53.0 & 0.12 \\
\hline OC-877-06 & 2.269 & 4.84 & 3.15 & 50.9 & 0.13 \\
\hline OC-877-07 & 2.293 & 4.80 & 3.18 & 51.4 & 0.11 \\
\hline OC-877-08 & 2.284 & 4.81 & 3.20 & 51.6 & 0.10 \\
\hline OC-877-09 & 2.263 & 4.77 & 3.12 & 49.6 & 0.12 \\
\hline OC-874A-06 & 2.298 & 4.93 & 3.15 & 52.7 & 0.16 \\
\hline OC-874B-01 & 2.269 & 4.87 & 3.22 & 52.4 & 0.11 \\
\hline OC-874B-02 & 2.302 & 4.93 & 3.18 & 53.2 & 0.15 \\
\hline mean & 2.285 & 4.86 & 3.20 & 52.1 & 0.12 \\
\hline std. dev. & 0.014 & 0.05 & 0.05 & 01.05 & 0.02 \\
\hline
\end{tabular}


Table 7-11 Pre-test Specimen Strain Rates and Static Mechanical Properties

\begin{tabular}{|c|c|c|c|c|}
\hline Specimen & $\begin{array}{c}\text { Strain rate } \\
\left(\mathbf{\times 1 0 ^ { 6 }} \mathbf{s}^{-1}\right)\end{array}$ & $\begin{array}{c}\text { Compressive } \\
\text { strength }(\mathbf{M P a})\end{array}$ & $\begin{array}{c}\text { Axial strain } \\
\text { at failure }\left(\mathbf{x 1 0}^{\mathbf{3}}\right)\end{array}$ & $\begin{array}{c}\text { Young's modulus } \\
(\mathrm{GPa})\end{array}$ \\
\hline $\mathrm{HC}-071-05$ & 14.6 & 156.2 & 10.1 & 24.0 \\
\hline $\mathrm{HC}-072-12$ & 13.7 & 115.3 & 6.75 & 25.1 \\
\hline $\mathrm{HC}-074-26$ & 14.7 & 206.4 & 9.27 & 30.4 \\
\hline HC-075-44 & 13.5 & 132.5 & 4.83 & 26.7 \\
\hline HC-075-47 & 16.6 & 142.2 & 4.58 & 28.8 \\
\hline HC-076-61 & 15.7 & 200.4 & 7.93 & 25.0 \\
\hline HC-077-77 & 18.3 & 105.5 & 6.30 & 19.5 \\
\hline HC-077-80 & 12.1 & 149.3 & 7.79 & 24.9 \\
\hline HC-077-83 & 12.3 & 139.6 & 8.69 & 26.3 \\
\hline HC-077-87 & 21.2 & 198.0 & 8.38 & 25.4 \\
\hline HC-077-89 & 23.5 & 201.0 & 8.96 & 25.6 \\
\hline mean & 16.0 & 158.8 & 7.6 & 25.6 \\
\hline std. dev. & 3.48 & 36.71 & 1.71 & 2.75 \\
\hline
\end{tabular}

Table 7-12. Post-test Specimen Strain Rates and Static Mechanical Properties

\begin{tabular}{|c|c|c|c|c|}
\hline Specimen & $\begin{array}{c}\text { Strain rate } \\
\left(\times 10^{6} \mathbf{s}^{-1}\right)\end{array}$ & $\begin{array}{c}\text { Compressive } \\
\text { strength } \\
(\mathbf{M P a})\end{array}$ & $\begin{array}{c}\text { Axial strain } \\
\text { at failure } \\
\left(\mathbf{\times 1 0 ^ { 3 } )}\right.\end{array}$ & $\begin{array}{c}\text { Young's modulus } \\
(\mathrm{GPa})\end{array}$ \\
\hline OC-877-01 & 7.3 & 197.3 & 9.22 & 34.4 \\
\hline OC-877-02 & 7.3 & 223.6 & 1.4 & 30.4 \\
\hline OC-877-03 & 5.6 & 266.7 & 7.51 & 35.4 \\
\hline OC-877-04 & 9.5 & 198.0 & 7.37 & 35.4 \\
\hline OC-877-05 & 9.8 & 207.0 & 7.26 & 33.1 \\
\hline OC-877-06 & 12.0 & 114.0 & 4.83 & 26.3 \\
\hline OC-877-07 & 14.5 & 158.8 & 6.43 & 26.4 \\
\hline OC-877-08 & 11.1 & 150.5 & 5.75 & 27.8 \\
\hline OC-877-09 & 14.2 & 145.1 & 6.48 & 25.5 \\
\hline OC-874A-06 & 7.3 & 207.6 & 8.43 & 24.7 \\
\hline OC-874B-01 & 9.9 & 178.6 & 7.32 & 26.2 \\
\hline OC-874B-02 & 10.0 & 145.8 & 6.84 & 32.4 \\
\hline mean & 9.7 & 182.7 & 7.40 & 29.8 \\
\hline standard dev. & 2.6 & 42.11 & 1.63 & 3.94 \\
\hline
\end{tabular}

are $3.20 \pm 0.05 \mathrm{~km} / \mathrm{s}$ and $3.21 \pm 0.04 \mathrm{~km} / \mathrm{s}$, respectively. Neither velocity difference is statistically significant. Mean elastic moduli also differ by less than one standard deviation. The calculated average Young's moduli are 51.7 $\pm 0.97 \mathrm{GPa}$ and $52.15 \pm 1.05 \mathrm{GPa}$ for the pre-test and post-test cores respectively. The average Poisson's ratios are $0.10 \pm 0.02$ and $0.12 \pm 0.02$ for the pre-heat and post-test cores respectively. 
Most of the stress-strain plots were fairly linear to failure, and all but one of the test specimens failed at an axial stress over $100 \mathrm{MPa}$. Compressive strength results are given in Tables 7-11 and 7-12 and are plotted as histograms in Figure 7-66. Because of the failure of the 55,000-lb load cell, the data of the first five specimens in Table 7-12 should be treated as non-Q and, therefore, for reference only. The mean compressive strength for all 11 pre-test samples is $158.8 \pm 36.7 \mathrm{MPa}$. The mean compressive strength for the post-test samples is $182.8 \pm 42.1 \mathrm{MPa}$, or $157.1 \pm 29.4 \mathrm{MPa}$ if the first five samples in Table 7-12 are not included. The compressive strengths of the pre-test and the post-test cores are statistically the same.

Calculated static Young's moduli are included in Tables 7-11 and 7-12 and are plotted as histograms in Figure 7-67. Average static Young's moduli of $25.6 \pm 3.9 \mathrm{GPa}$ and $29.8 \pm 2.7 \mathrm{GPa}$ were calculated for the pre-test and post-test specimens, respectively. The average Young's modulus of the last seven post-test samples in Table 12 is $2.70 \pm 2.5 \mathrm{MPa}$. The Young's modulus of the pre-test and the post-test samples are statistically the same.

\subsubsection{Discussion}

Our results indicate no statistically significant differences in dry density, ultrasonic compressional and shear velocities, or compressive strength between the thermally cycled post-test and the nonthermally cycled pre-test cores. The post-test samples appear to be stiffer than the pre-test samples by about one standard deviation. However, thermally induced cracking is expected to make the rock more, rather than less, compliant, so the difference is likely the result of pre-existing sample heterogeneity. The dynamic Young's moduli calculated from the velocity measurements are considerably larger than the static Young's moduli determined from the stress-strain curves, as is generally the case for rocks under low confining pressures.

Uniaxial compressive strength and elastic wave velocities for Topopah Spring tuff have been measured previously by others. In general, our results are similar to those obtained in the earlier studies, which further suggests that the LBT thermal cycle did little to damage these rocks. Summing up a number of the earlier tests on tuff specimens from the proposed repository horizon, Wilder (1993) concluded that "these data indicate that the intact rock is quite strong, with a uniaxial strength of $155 \pm 59 \mathrm{MPa}$ and a high Young's modulus." Although there is clearly a lot of scatter in the data, our compressive strength results for the thermally cycled post-test specimens ( $183 \pm 42 \mathrm{MPa})$ are at least on the high side of the range. If the data of the first five specimens in Table 7-12 are not considered, our mean compressive strength of the overcore specimens $(157.1 \pm 29.4 \mathrm{MPa})$ agrees well with the previous results. More recently, Martin et al. (1993) have performed ultrasonic velocity and uniaxial compressive strength measurements on six Topopah Spring tuff specimens. Their strength data are not comparable to ours because they used a much slower loading rate $\left(10^{-9} / \mathrm{s}\right)$, but the velocity data are comparable. Their compressional velocities, measured on dry specimens, averaged $4.59 \mathrm{~km} / \mathrm{s}$, about $5 \%$ lower than our results.

Our compressive strength tests were designed to be similar to those of Blair, Kelly, et al. (1996). The same length-to-diameter ratio (3:1) and nominal loading rate were used. Specimen preparation procedures were similar. The loading frame and much of the other testing equipment were the same. Blair, Kelly, et al. (1996) tested two groups of 15 samples: an irradiated group and a control group. They found mean compressive strengths of $139 \pm 73 \mathrm{MPa}$ for the irradiated 
group and $154 \pm 36 \mathrm{MPa}$ for the control group. Our compressive strength values of $158 \pm 37$ $\mathrm{MPa}$ for the pre-test samples and $183 \pm 42 \mathrm{MPa}$ for post-test samples are in very good agreement with their results (Figure 7-68). Our Young's modulus values, $25 \pm 3 \mathrm{GPa}$ for the pre-test core and $30 \pm 5 \mathrm{GPa}$ for post-test core, are similar to values obtained by Blair, Kelly, et al. (1996) and to values contained in the YMP Reference Information Base (DOE 1990), as shown in Figure 7-69.

\subsubsection{Conclusions}

No statistically significant differences in dry density, ultrasonic velocity, compressive strength, or Young's modulus were observed between the thermally cycled post-test and nonthermally cycled pre-test suites. The dry densities, ultrasonic velocities, uniaxial compressive strengths, and elastic moduli obtained for these specimens are also similar to those obtained for Topopah Spring tuff in other laboratory studies. No evidence was found that the tuff specimens were damaged in the LBT. 


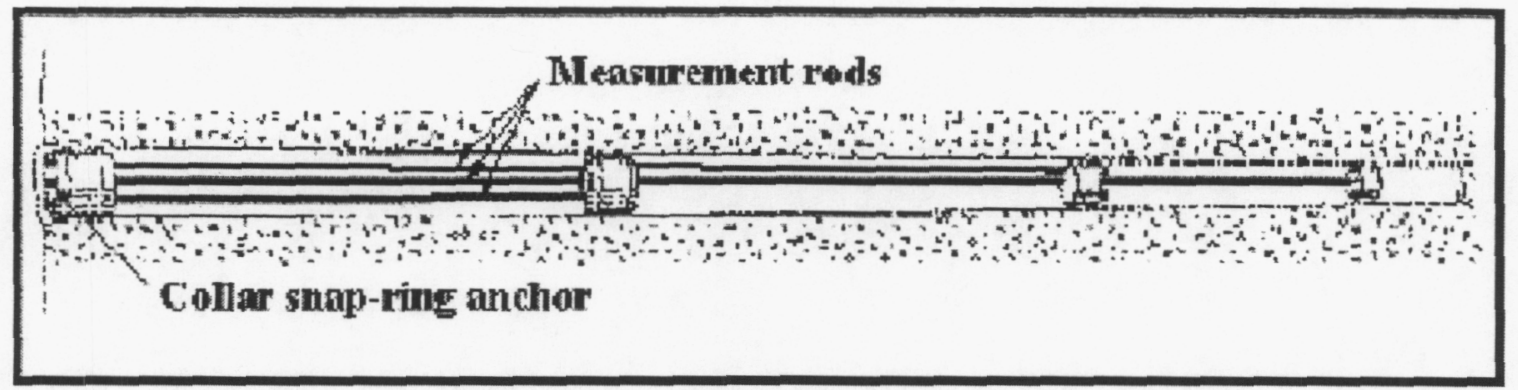

Figure 7-1. Multiple-Point Borehole Extensometer (MPBX)

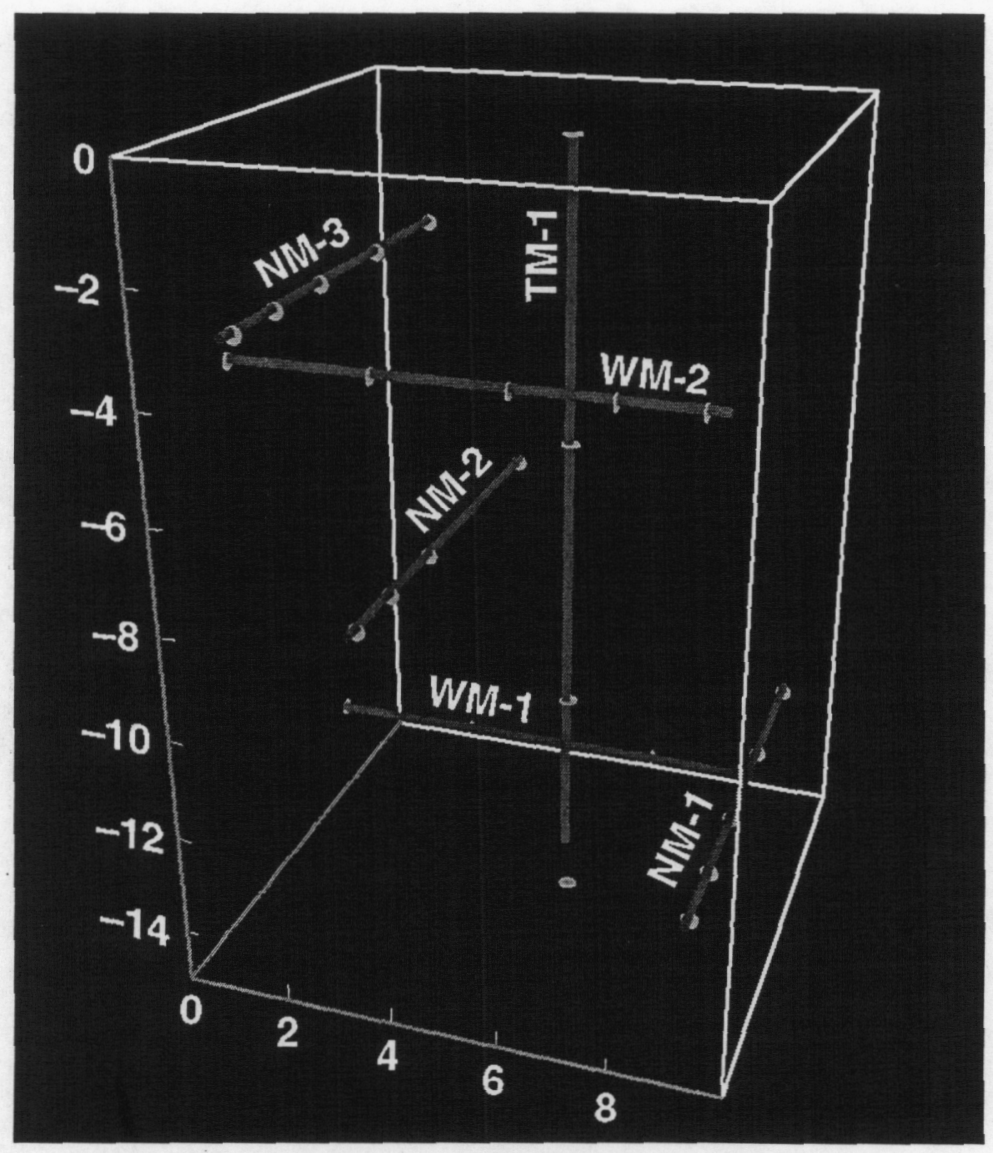

Figure 7-2. MPBX Borehole Locations, Viewed from the South Face 


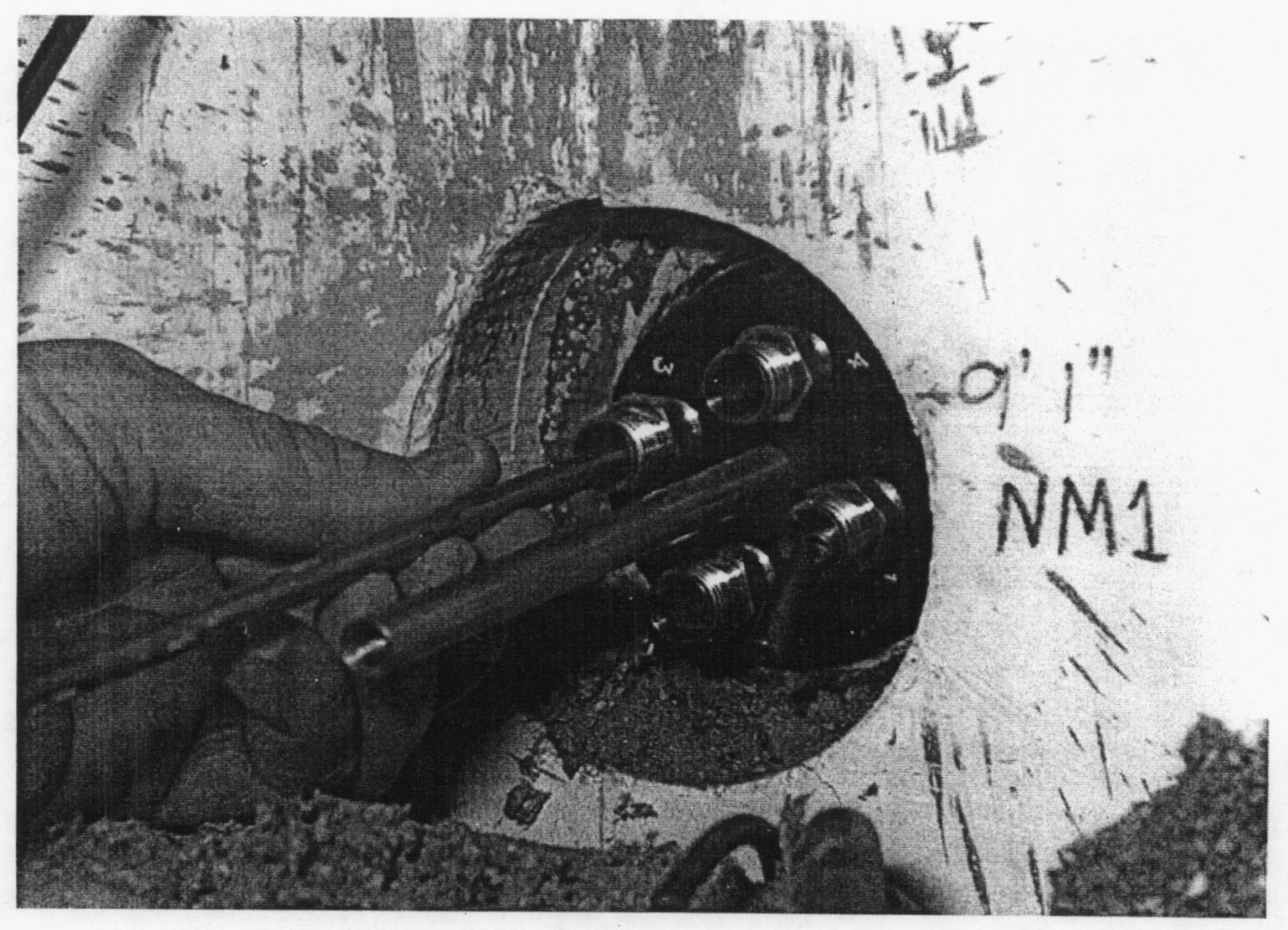

Figure 7-3. Extensometer NM-1 Collar and Invar Rod Installation 


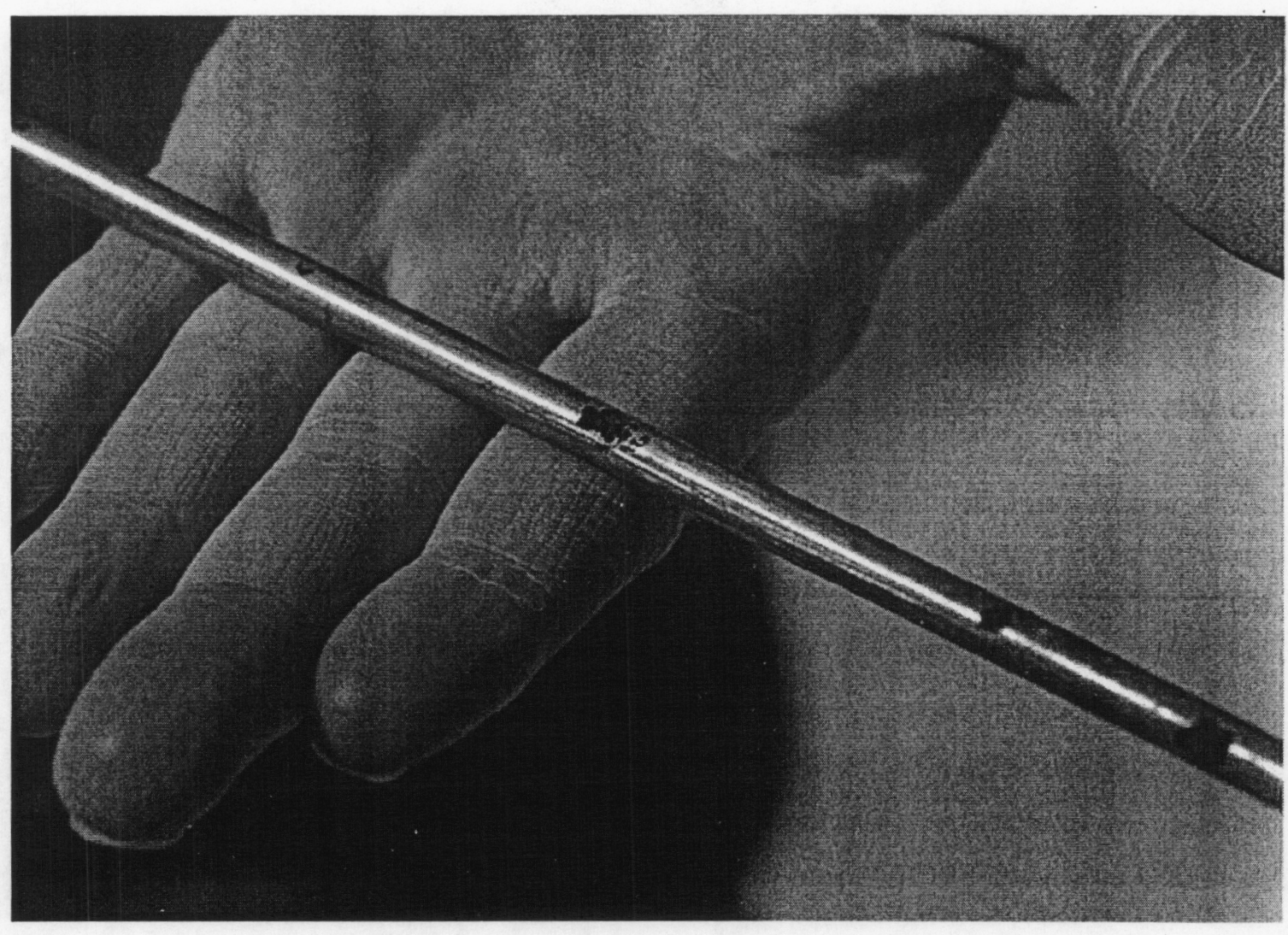

Figure 7-4. Invar Rod Corrosion 


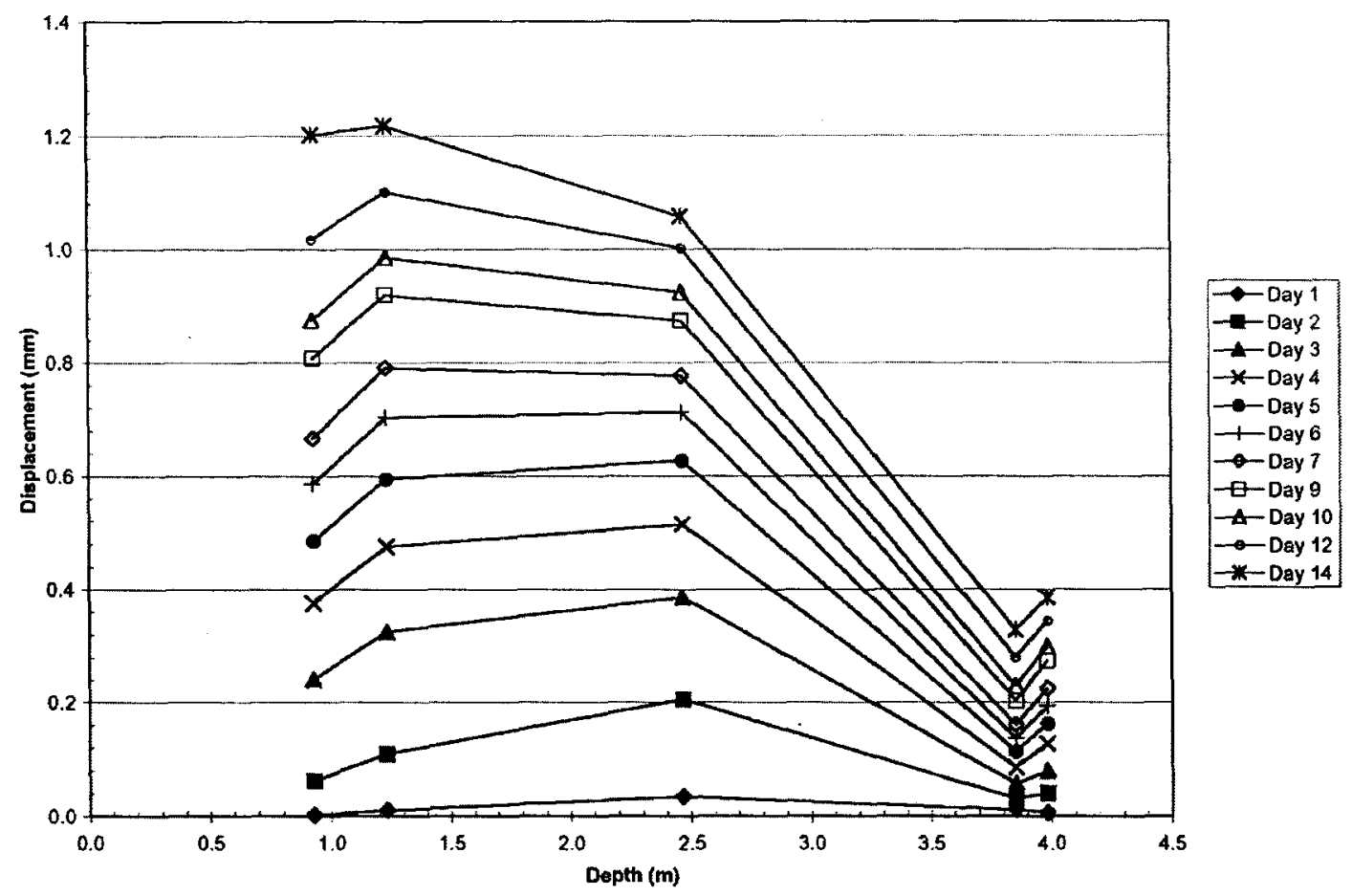

NOTE: Extensometers are oriented north-south (NM-1, NM-2, NM-3) or east-west (WM-1, WM-2). NM-2 is located slightly above the heater plane. Although NM-2 was in the hottest portion of the block, the upper extensometers show greater displacements by the second week of the test.

Figure 7-5. Horizontal Displacements During the First Two Weeks of the Large Block Test 


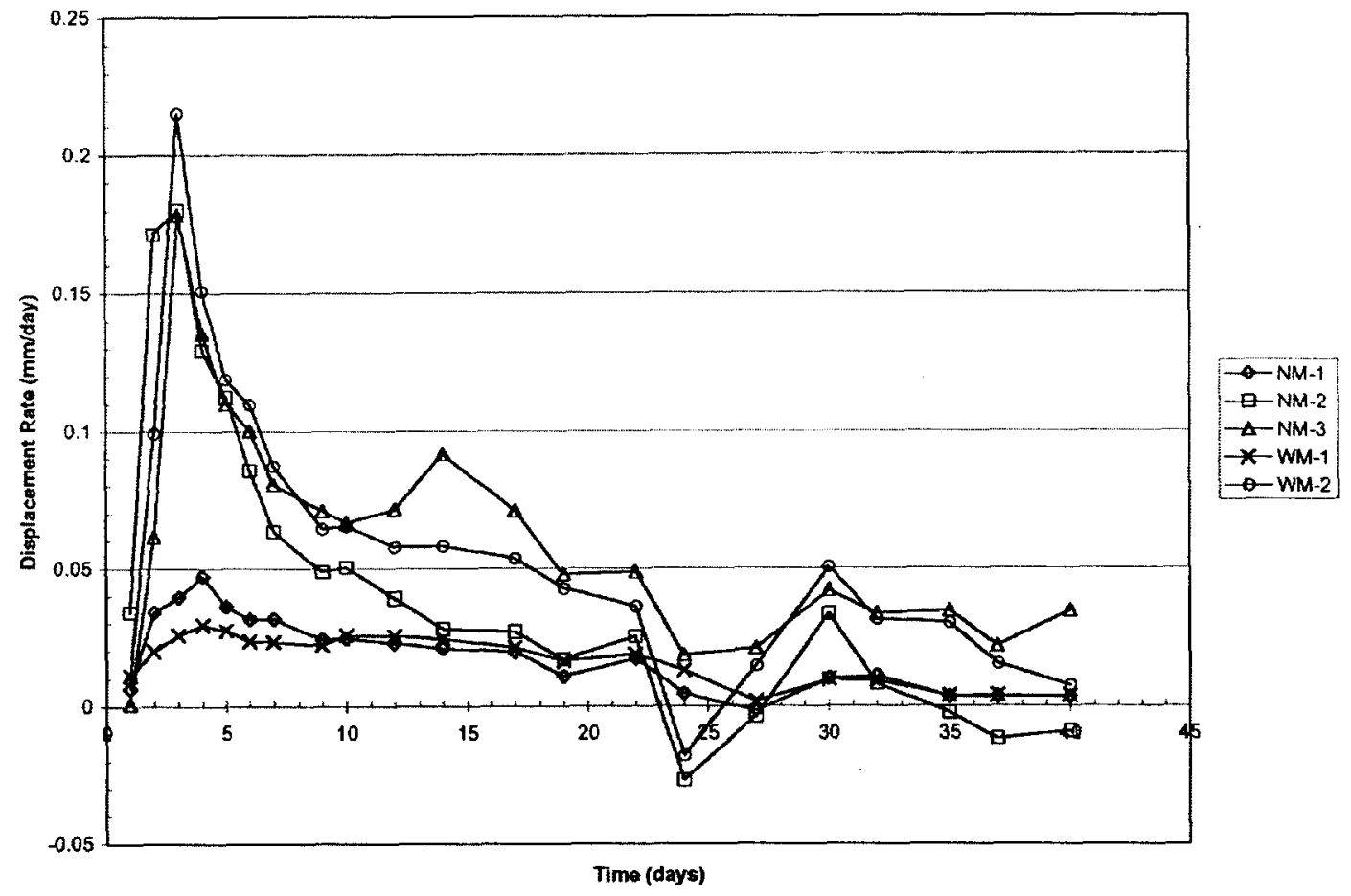

NOTE: The central and upper extensometers extended at a rapid rate during the first week of the test.

Figure 7-6. Displacement Rates for the Horizontal Extensometers During the First 40 Days of the LBT 
WM-1

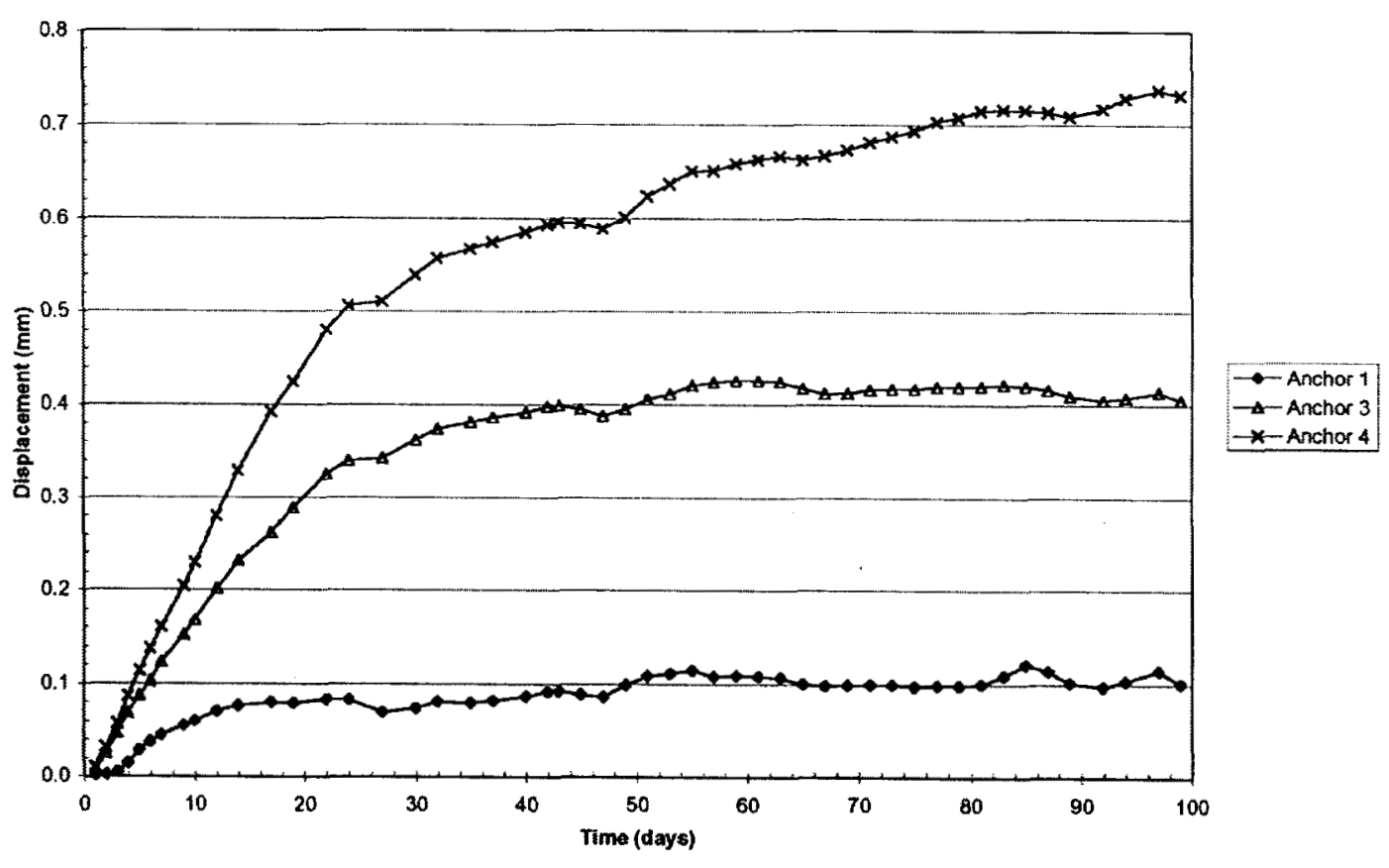

NOTE: Located near the north face at a depth of $3.98 \mathrm{~m}$. Only three anchors were installed.

Figure 7-7. East-West Anchor Displacements During the First 100 Days for WM-1 


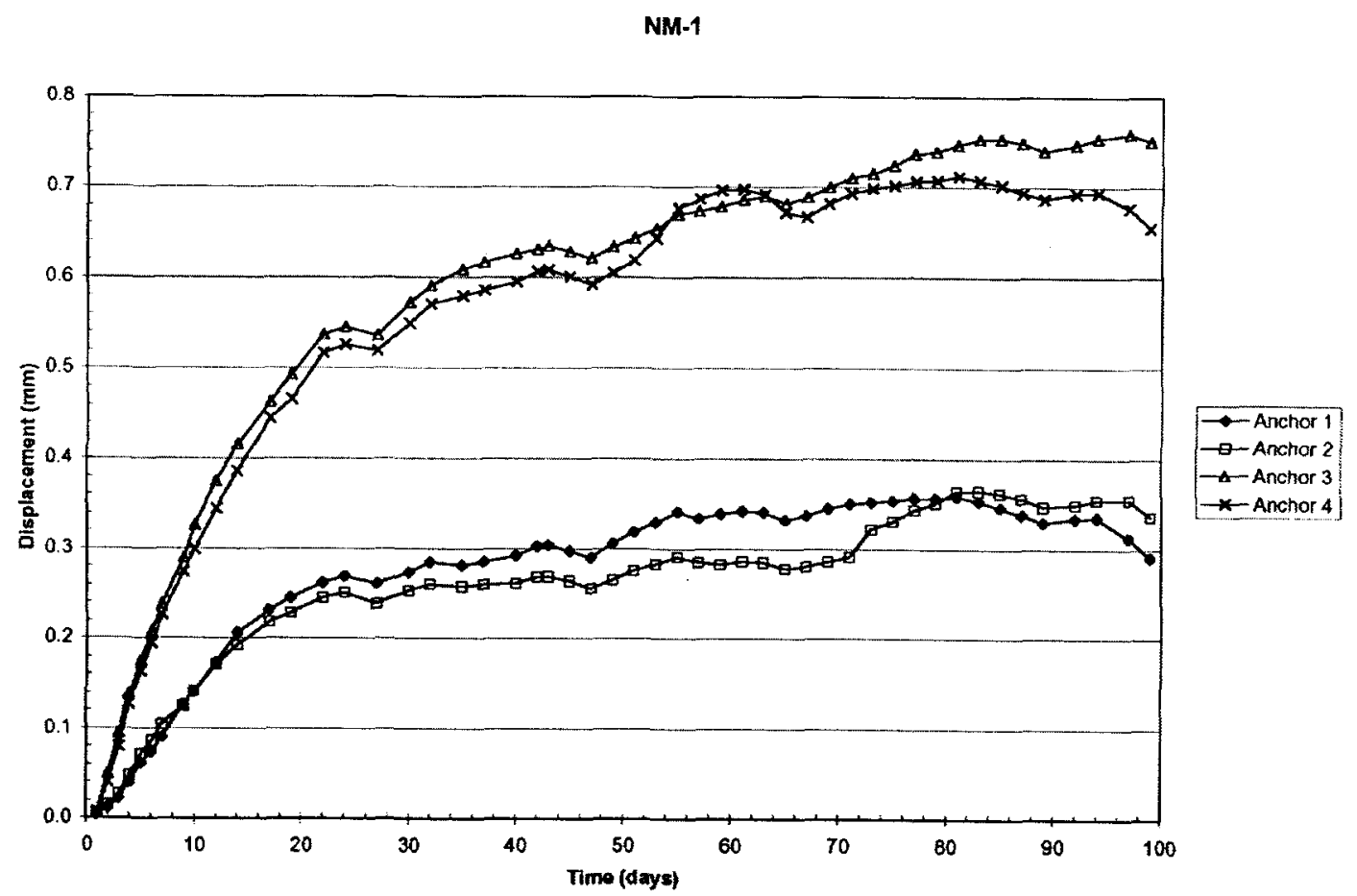

NOTE: Located near the east face at a depth of $3.85 \mathrm{~m}$.

Figure 7-8. North-South Anchor Displacements During the First 100 Days for NM-1 
NM-2

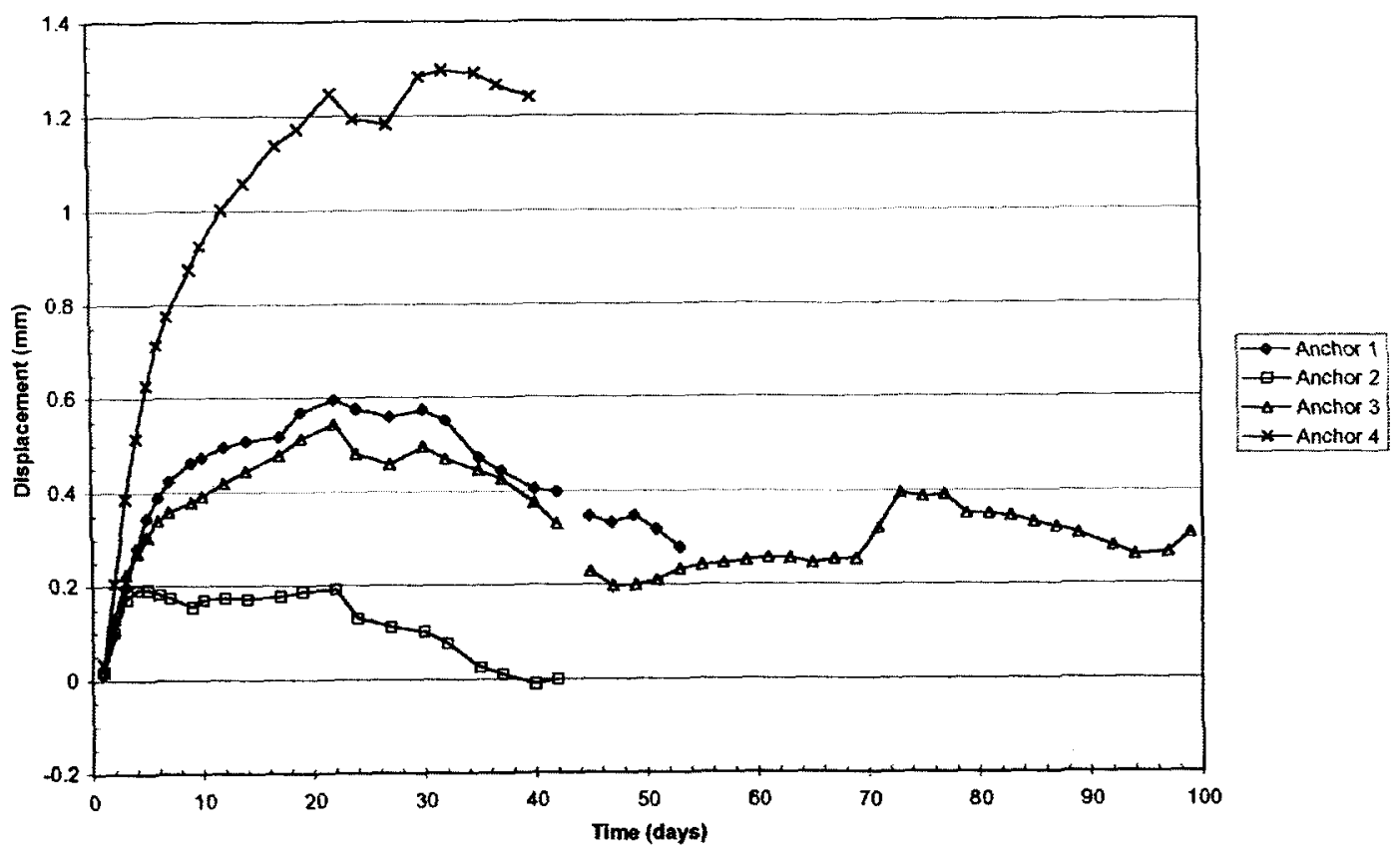

NOTE: Located at a depth of $2.47 \mathrm{~m}$, slightly above the heater plane.

Figure 7-9. North-South Anchor Displacements During the First 100 Days for NM-2 
WM-2

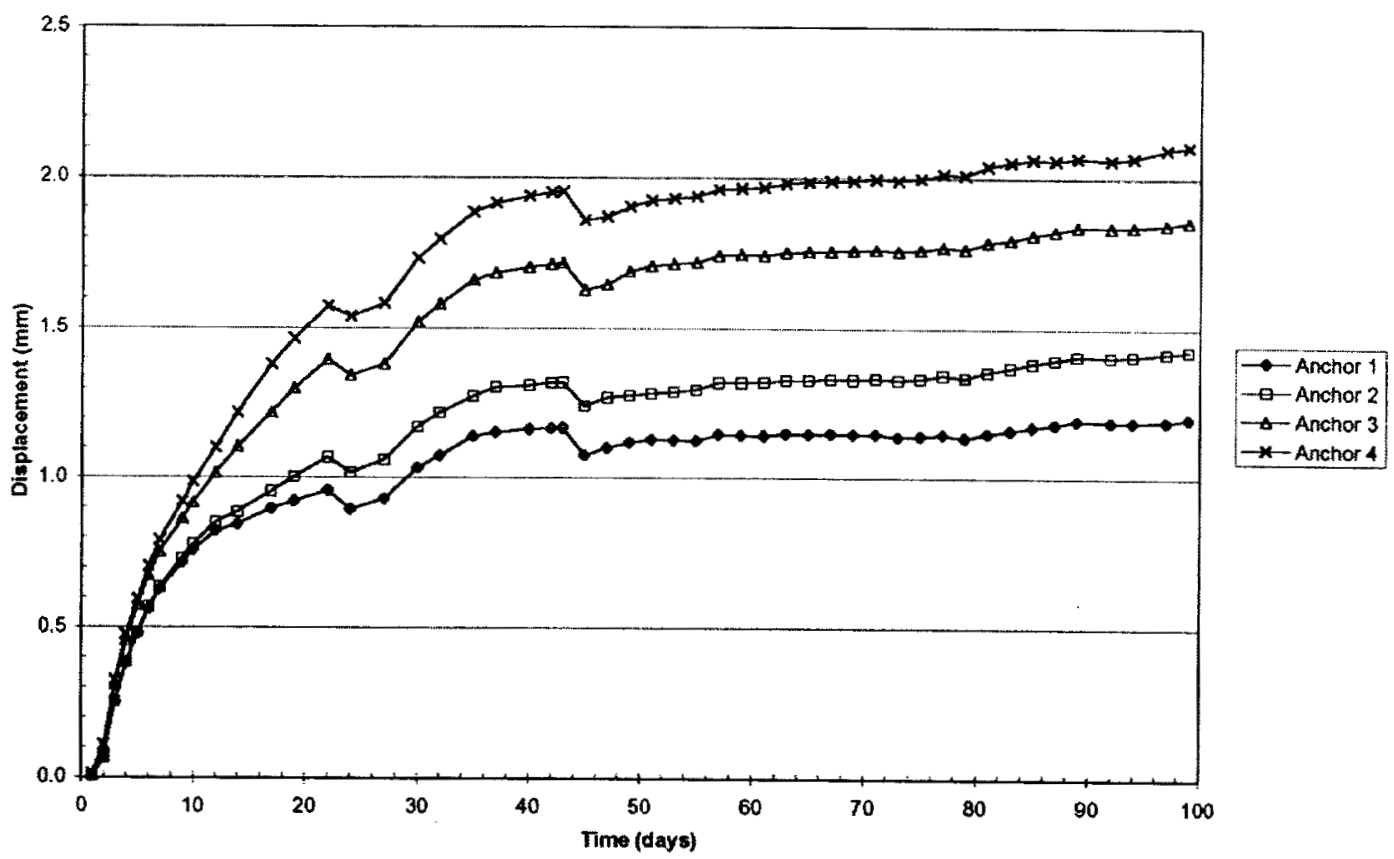

NOTE: Located near the south face at a depth of $1.23 \mathrm{~m}$

Figure 7-10. East-West Anchor Displacements During the First 100 Days for WM-2 
NAM-3

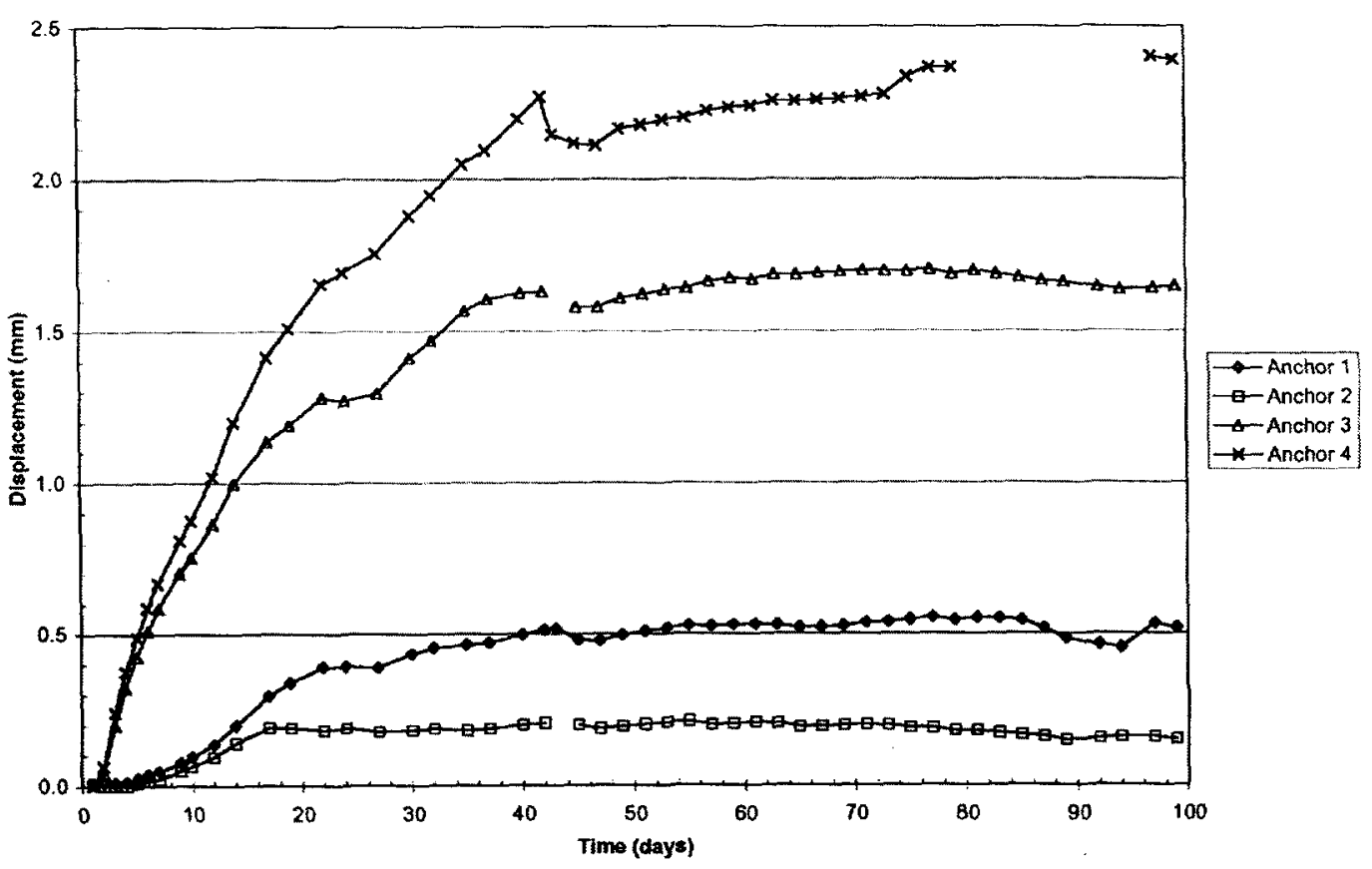

NOTE: Located near the west face at a depth of $0.93 \mathrm{~m}$

Figure 7-11. North-South Anchor Displacements During the First 100 Days for NM-3 
TM-1

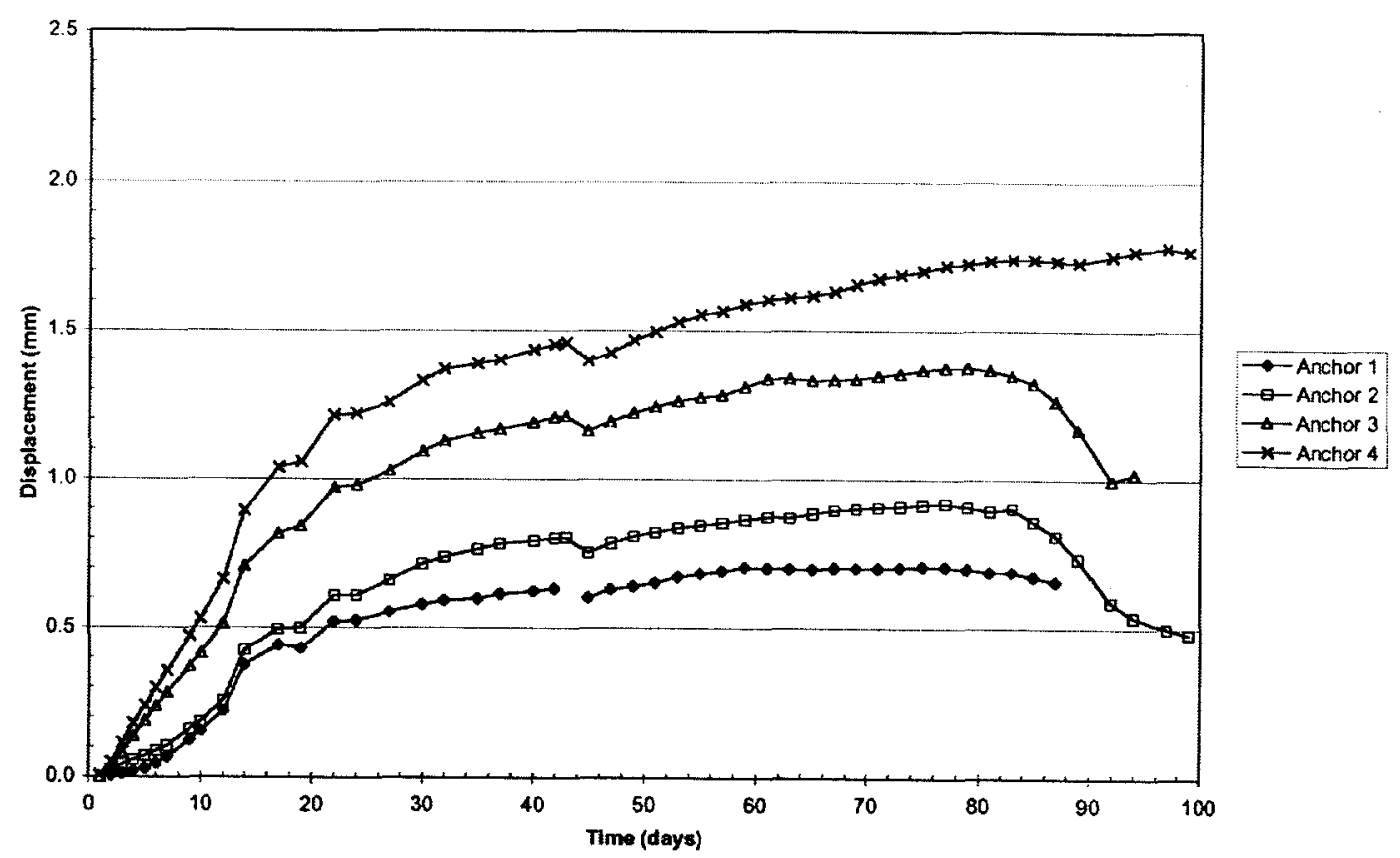

NOTE: Located near the center of the large block

Figure 7-12. Vertical Anchor Displacements During the First 100 Days for TM-1 


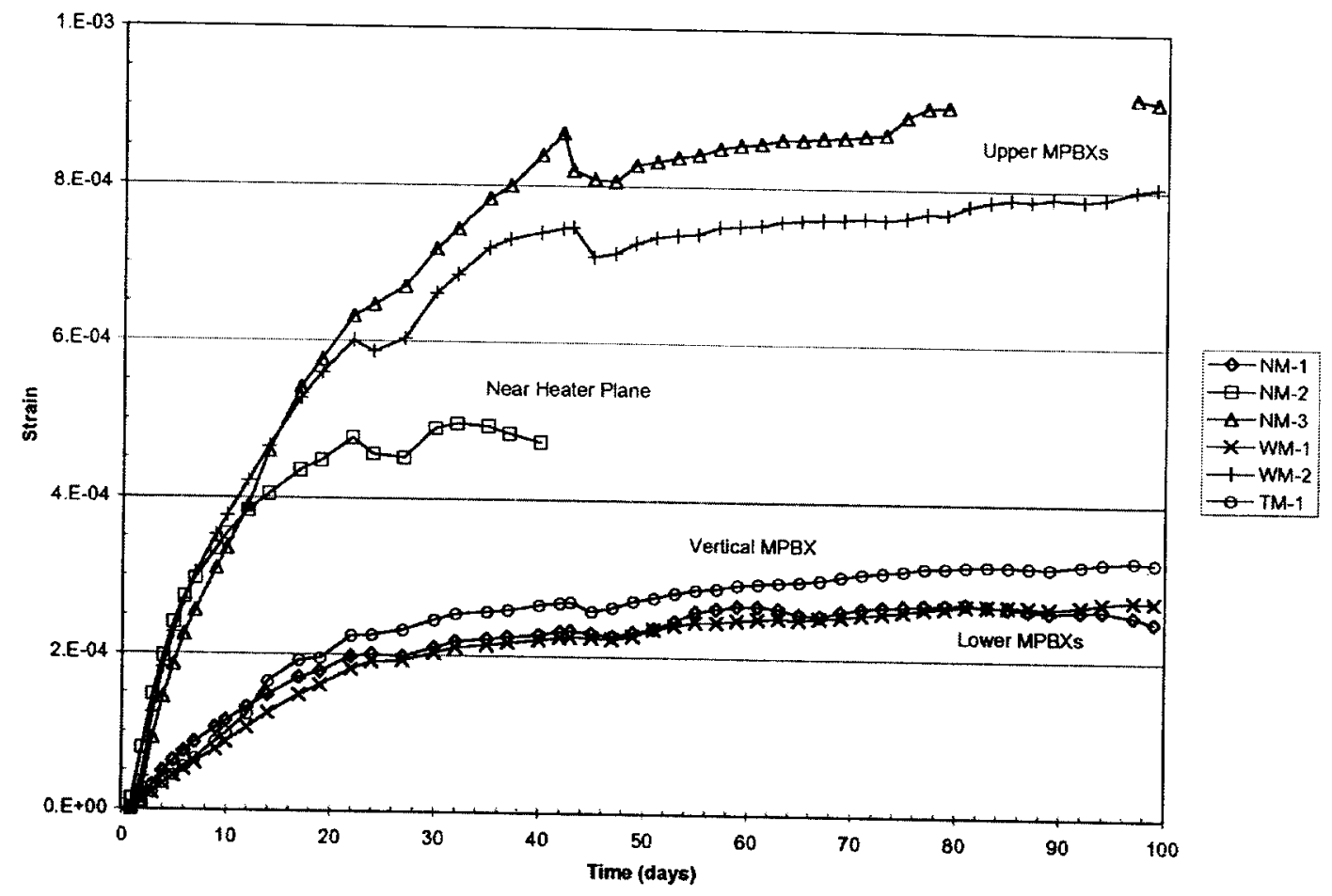

NOTE: The upper extensometers extended far more than the lower extensometers. The vertical strain was comparable to horizontal strains near the base of the block.:

Figure 7-13. Anchor 4 Strains for Each Extensometer During the First 100 Days 


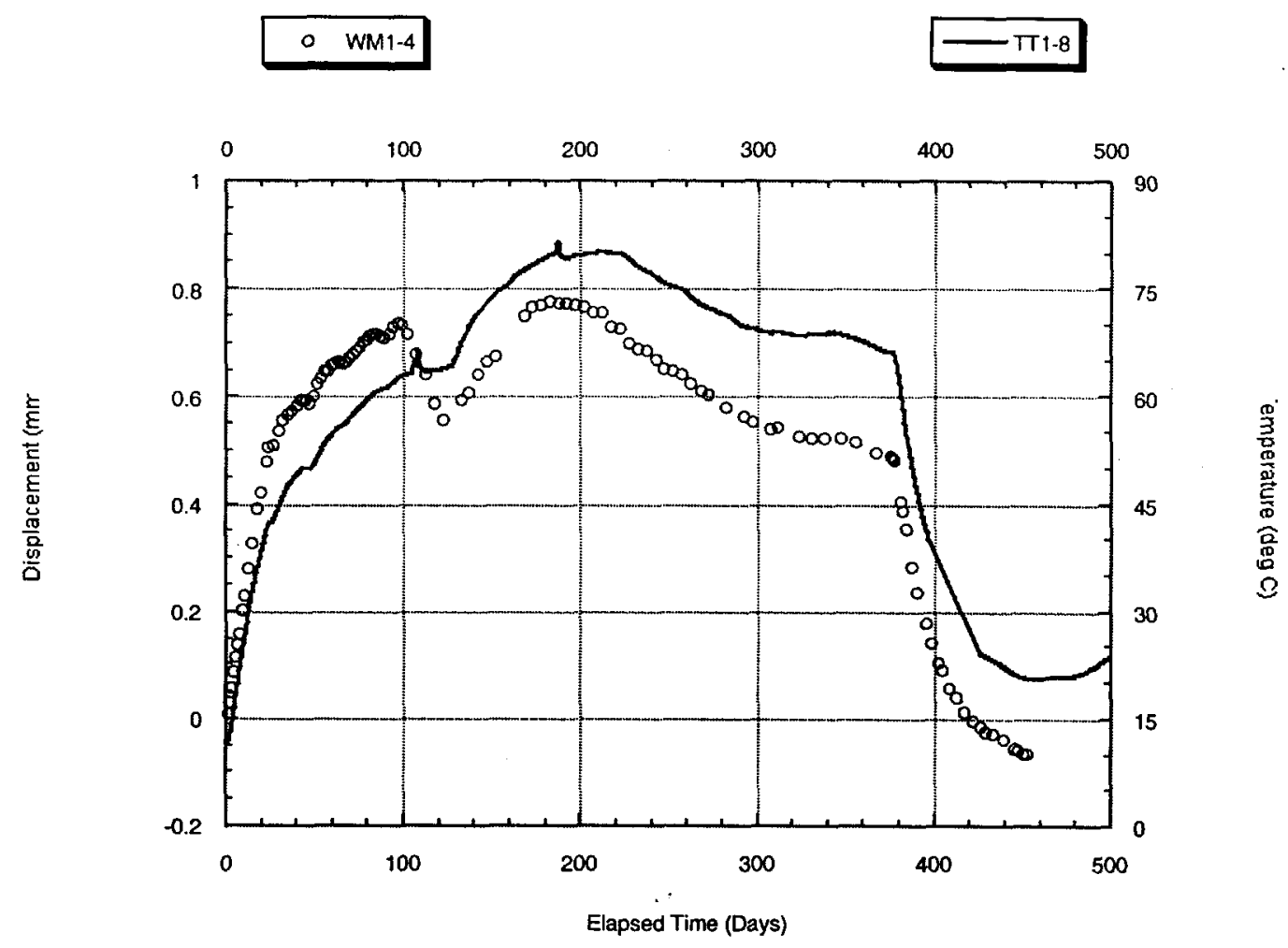

Figure 7-14. East-West Displacement for WM-1 Anchor 4 and Temperature at 4-m Depth

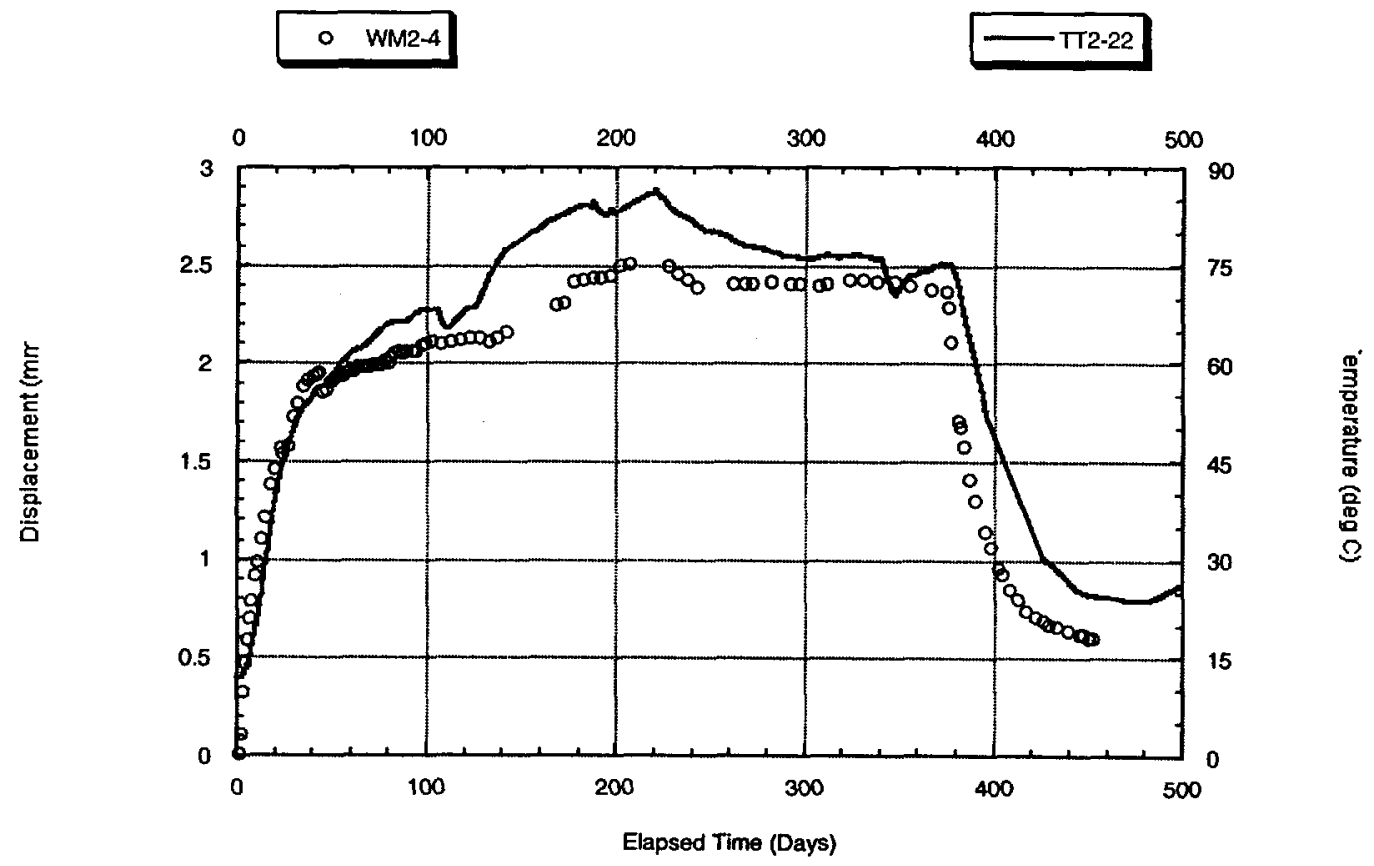

Figure 7-15. East-West Displacement for WM-2 Anchor 4 and Temperature at 1.2-m Depth 


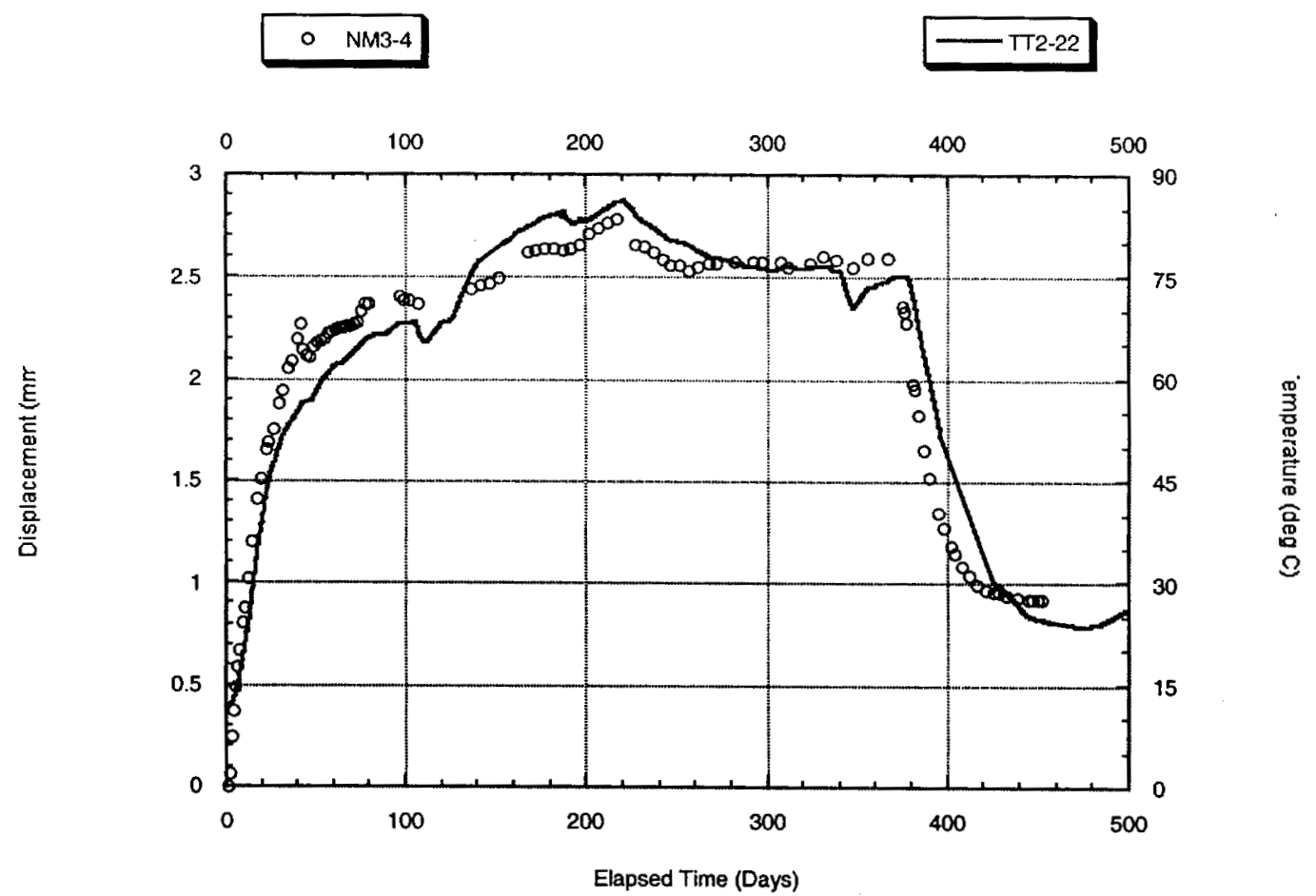

Figure 7-16. North-South Displacement for NM-3 Anchor 4 and Temperature at 1.2-m Depth 


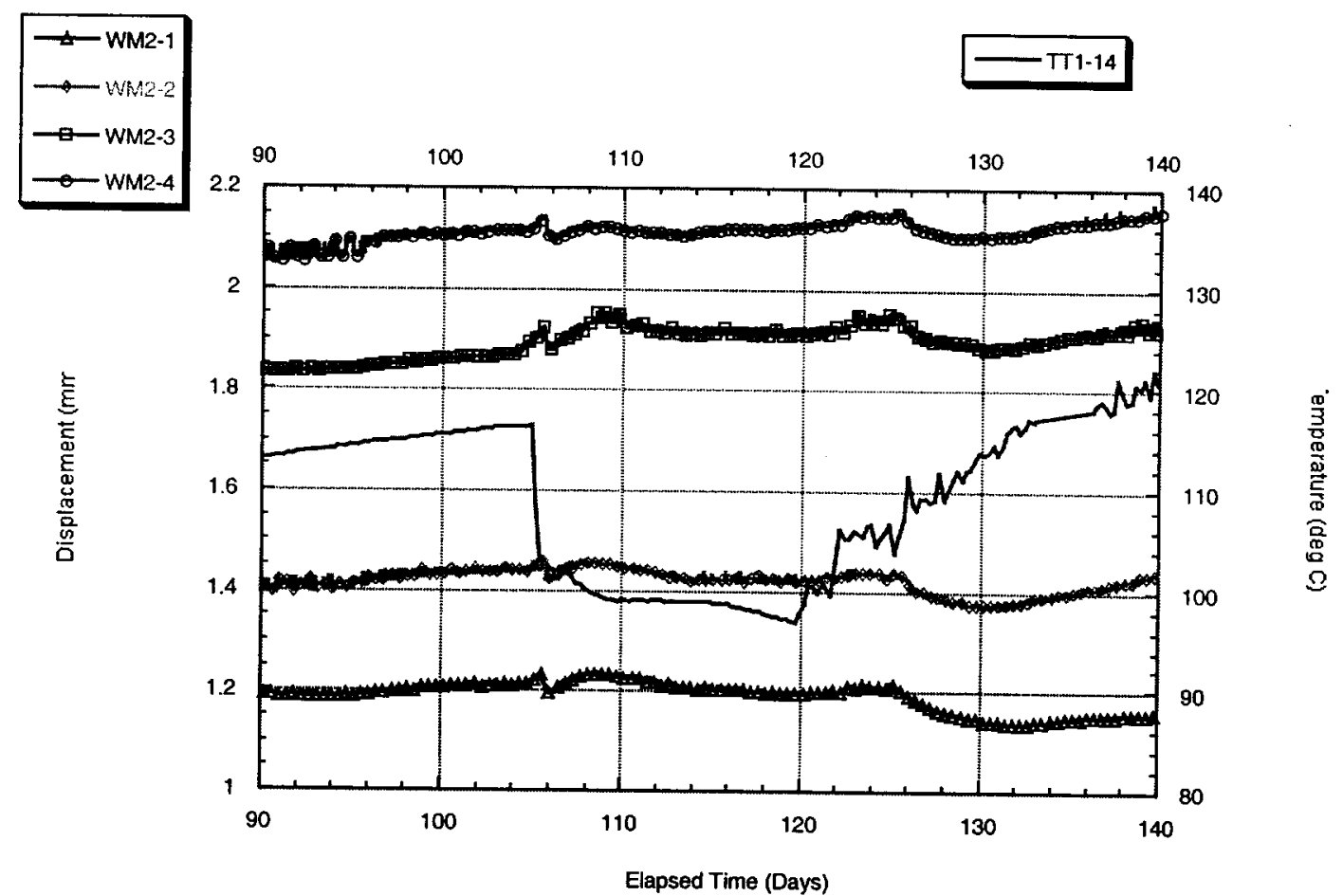

NOTE: Small, transient displacements for each anchor coincide with the sudden drop in temperature at the heater plane on Day 105.

Figure 7-17. WM-2 Anchor Displacements and Borehole TT1 Temperature Near the Heater Plane 


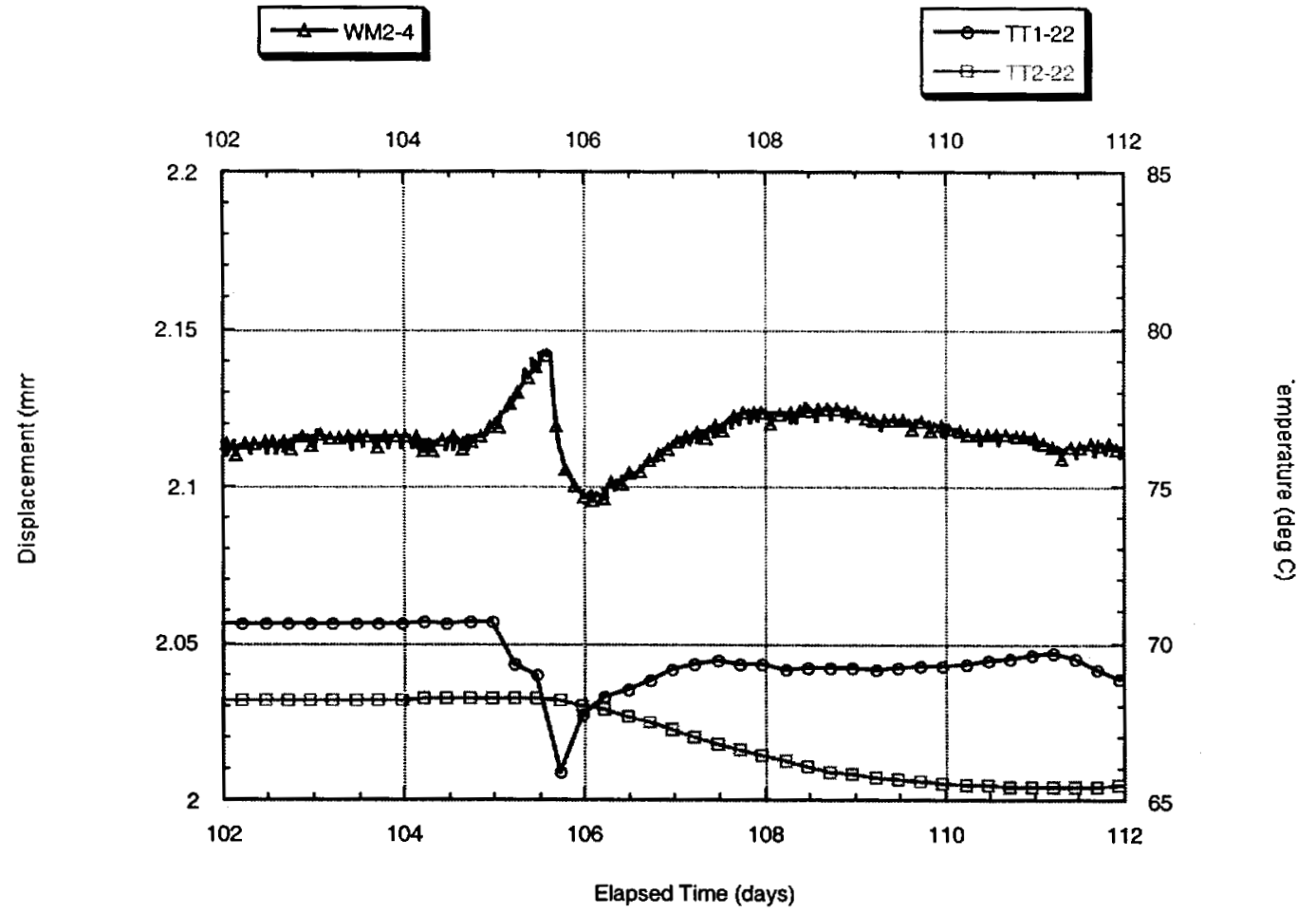

Figure 7-18. WM-2 Anchor 4 Displacements and Temperatures at $1.2 \mathrm{~m}$ Depth Around Day 105 


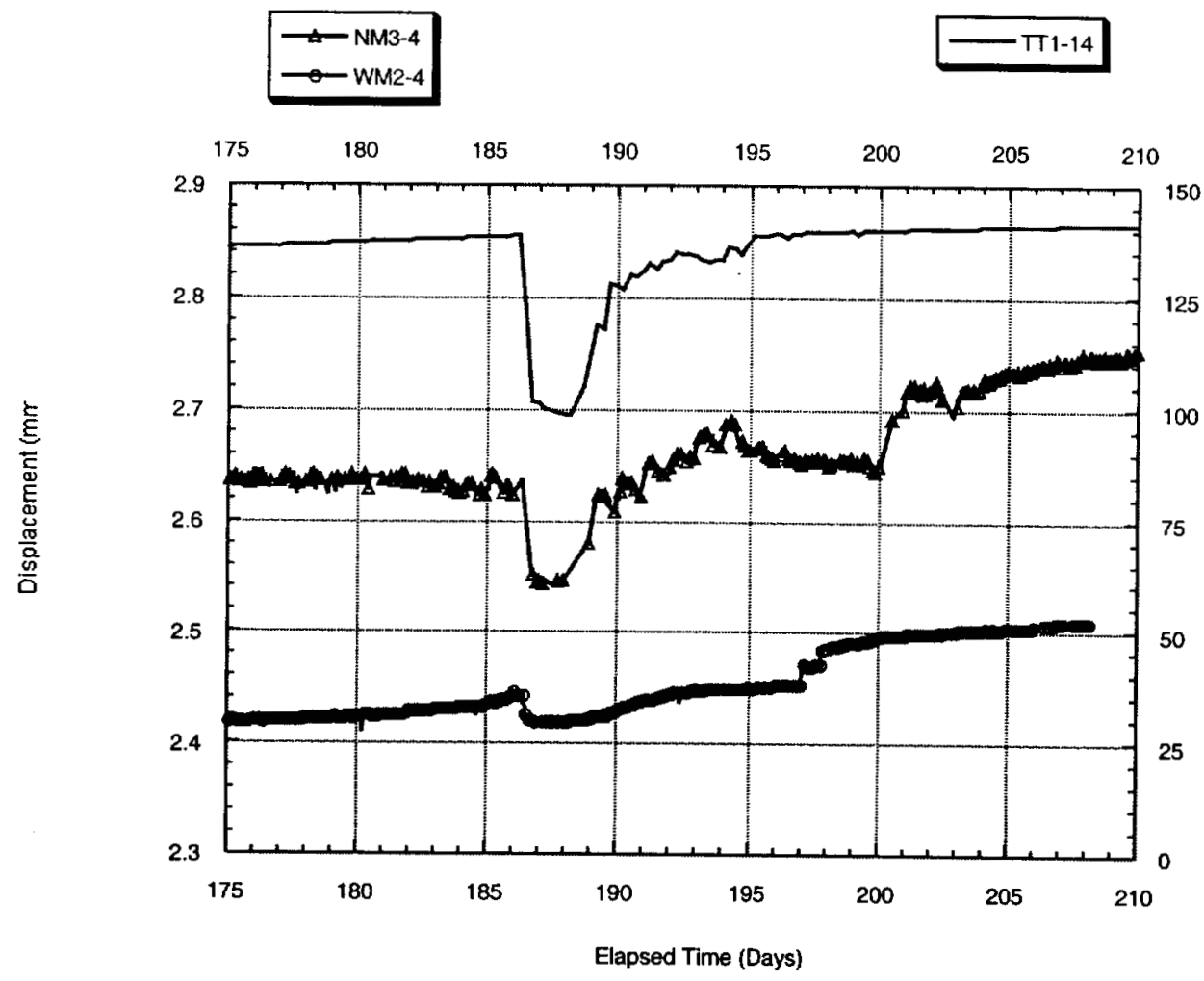

NOTE: A rainstorm at the Nevada Test Site preceded the sudden drop in temperature on Day 186. The cooling episode was accompanied by contractions in the two upper extensometers.

Figure 7-19. WM-2 and NM-3 Anchor Displacements and Borehole TT1 Temperature Near the Heater Plane 


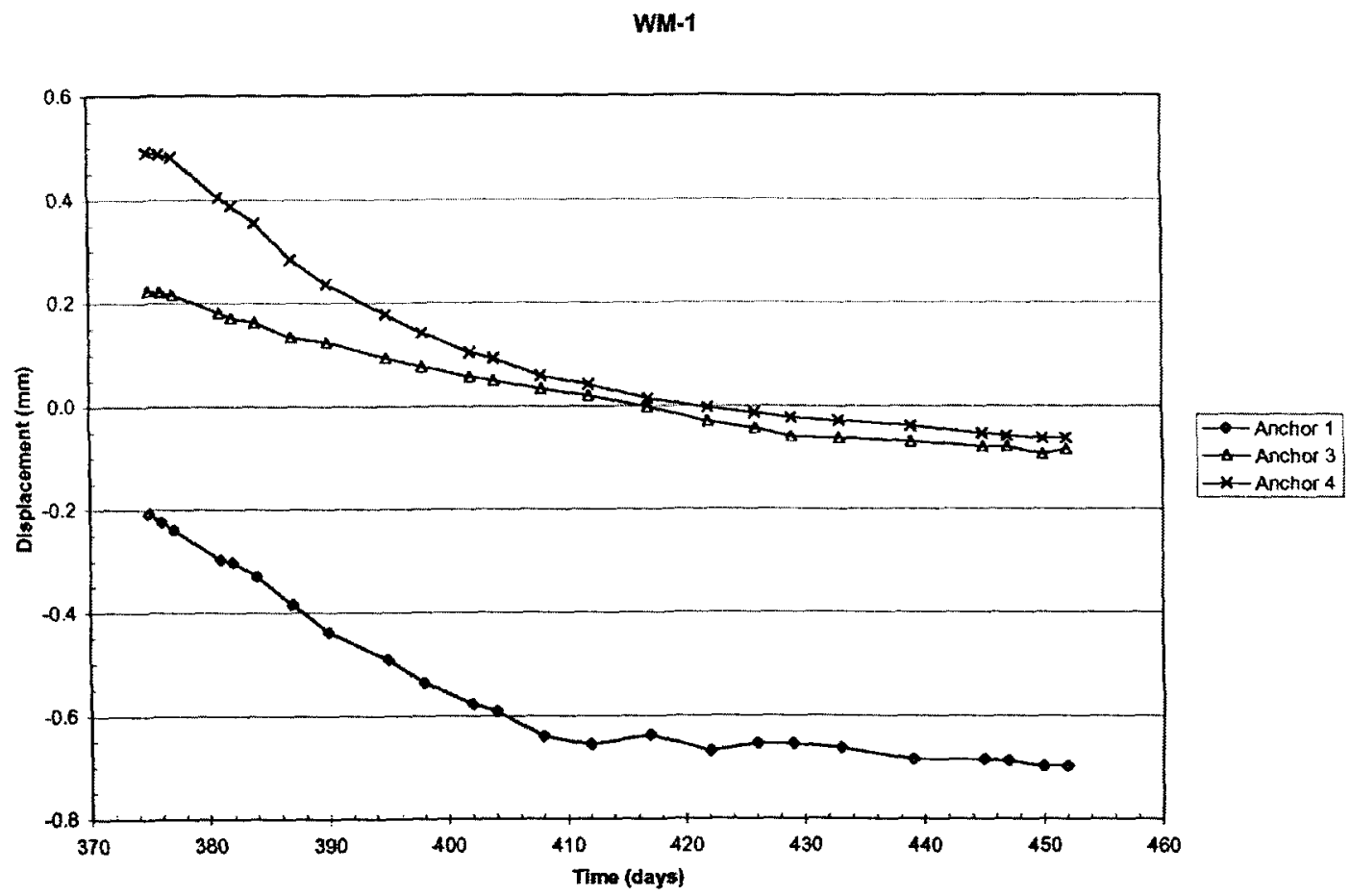

NOTE: Located near the north face at a depth of $3.98 \mathrm{~m}$. Only three anchors were installed.

Figure 7-20. WM-1 East-West Anchor Displacements During Cool-Down 
NM-1

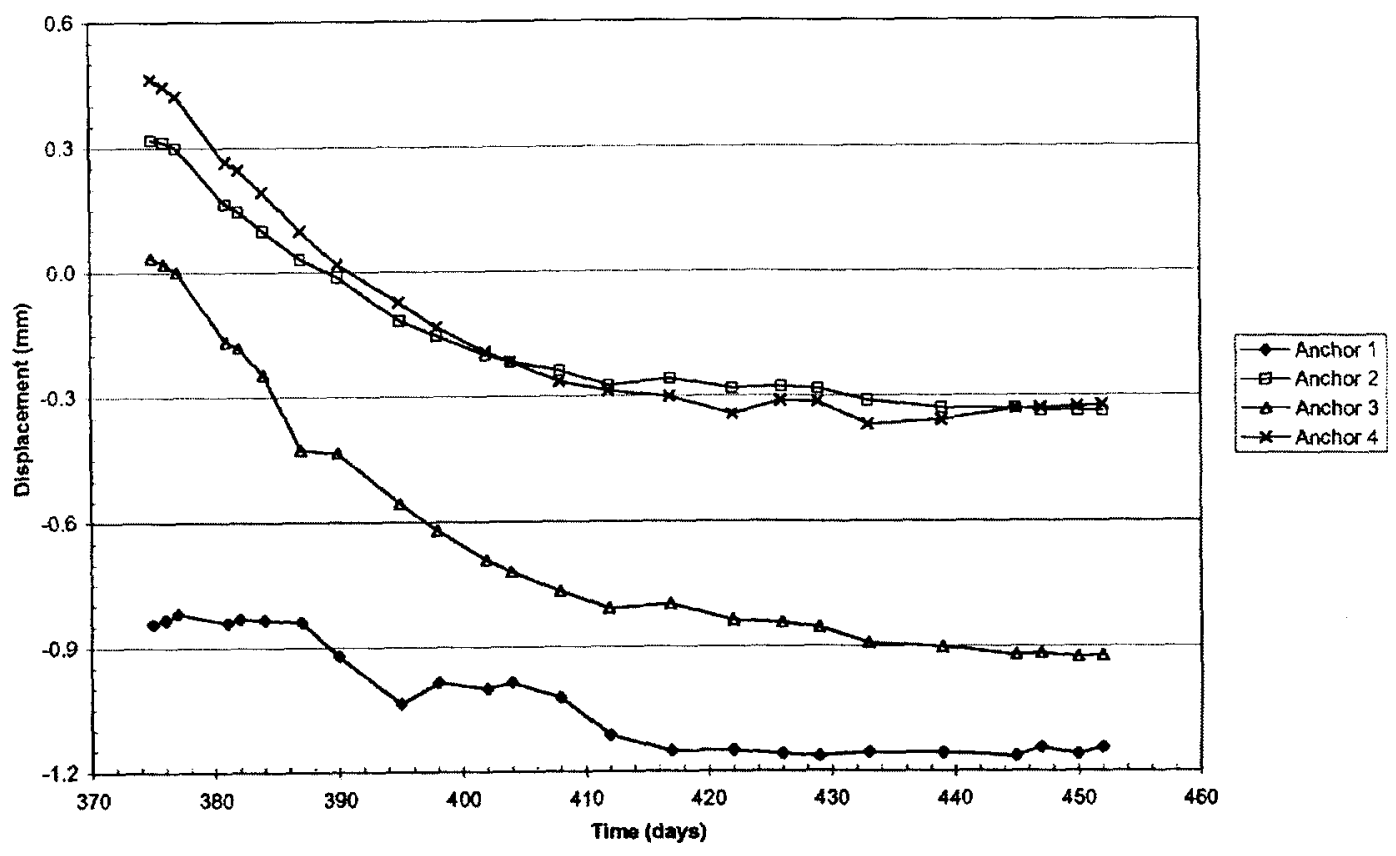

NOTE: Located near the east face at a depth of $3.85 \mathrm{~m}$

Figure 7-21. NM-1 North-South Anchor Displacements During Cool Down 
WM-2

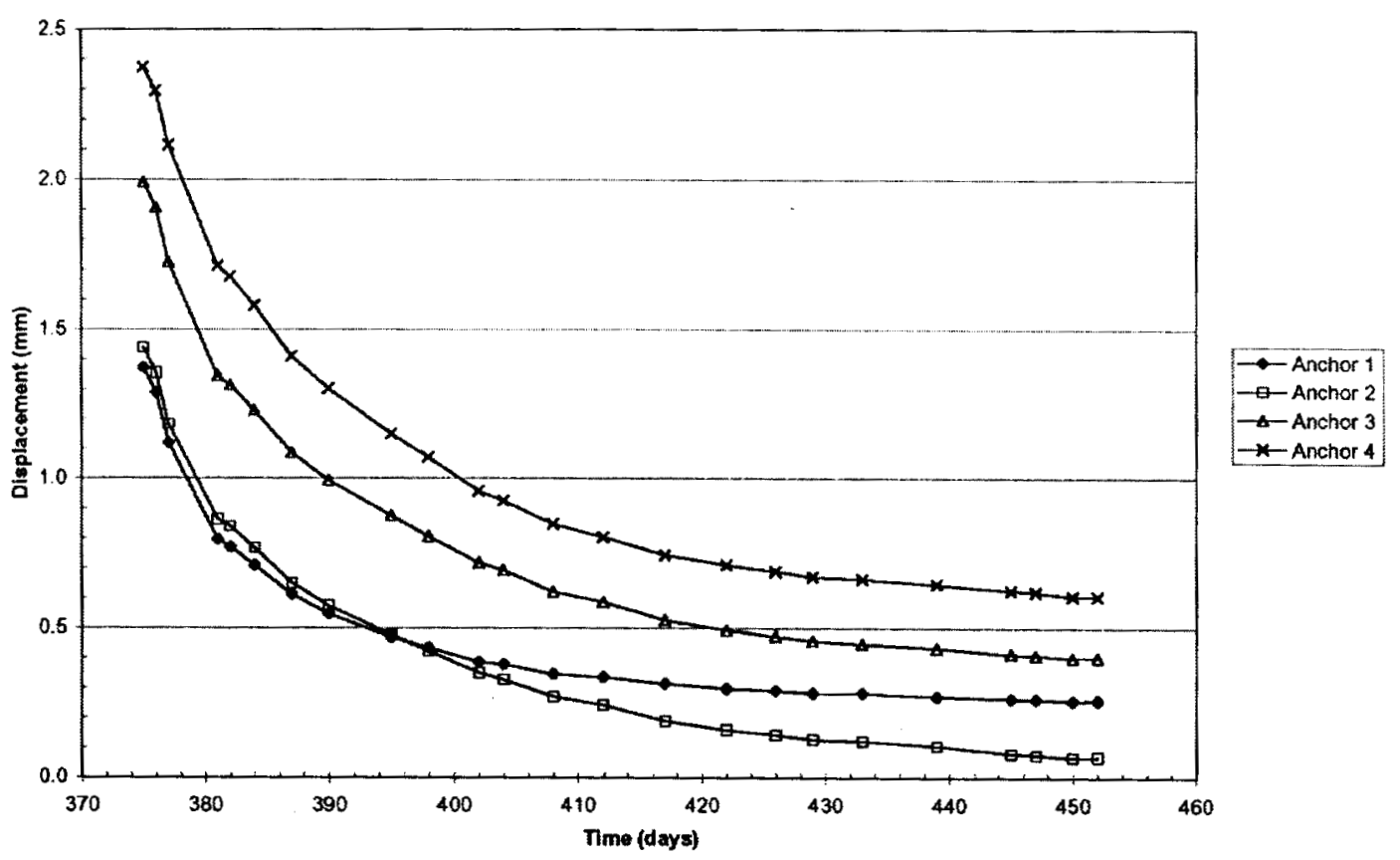

NOTE: Located near the south face at a depth of $1.23 \mathrm{~m}$

Figure 7-22. WM-2 East-West Anchor Displacements During Cool-Down 


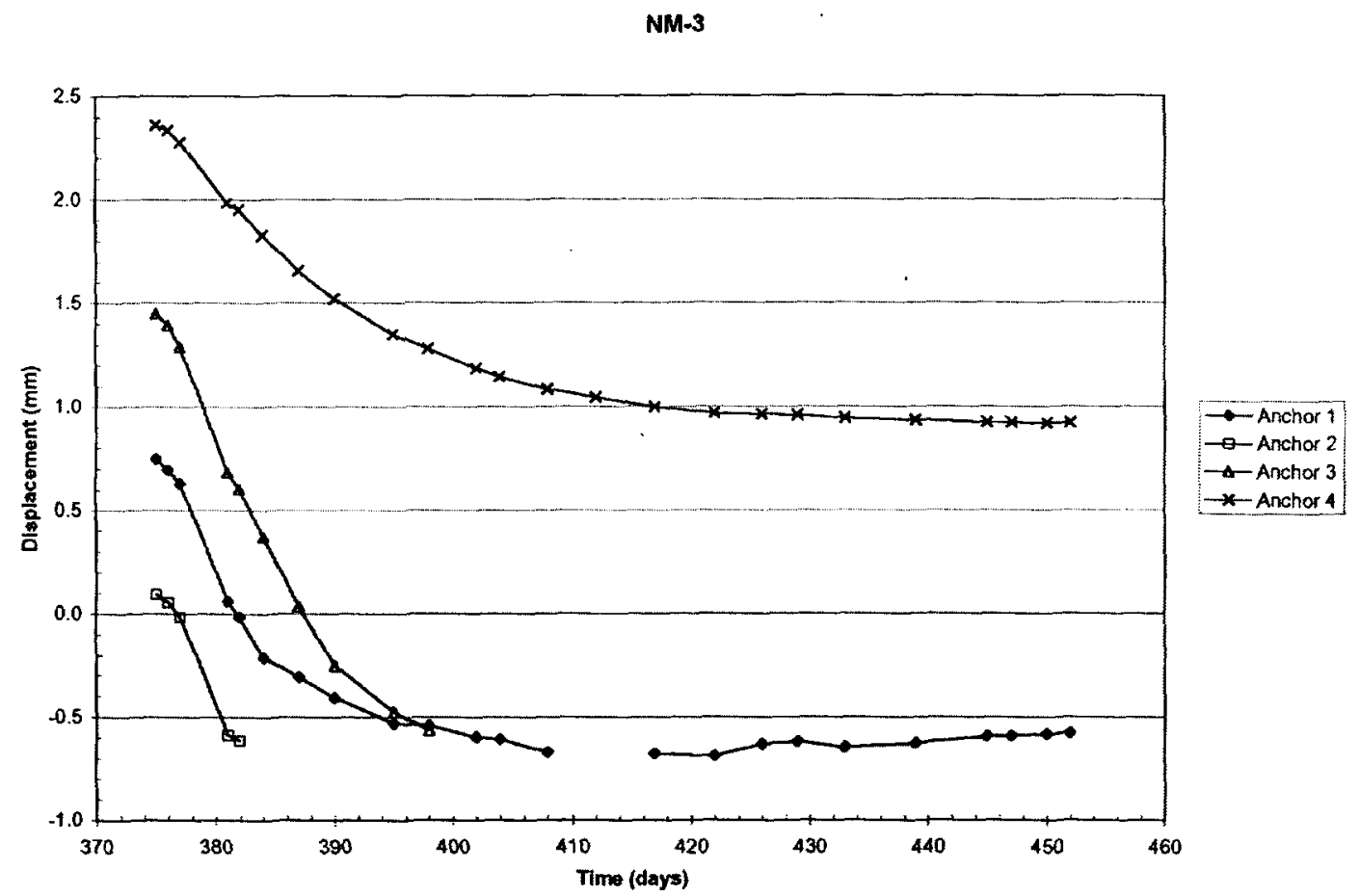

NOTE: Located near the west face at a depth of $0.93 \mathrm{~m}$

Figure 7-23. NM-3 North-South Anchor Displacements During Cool-Down 
TM-1

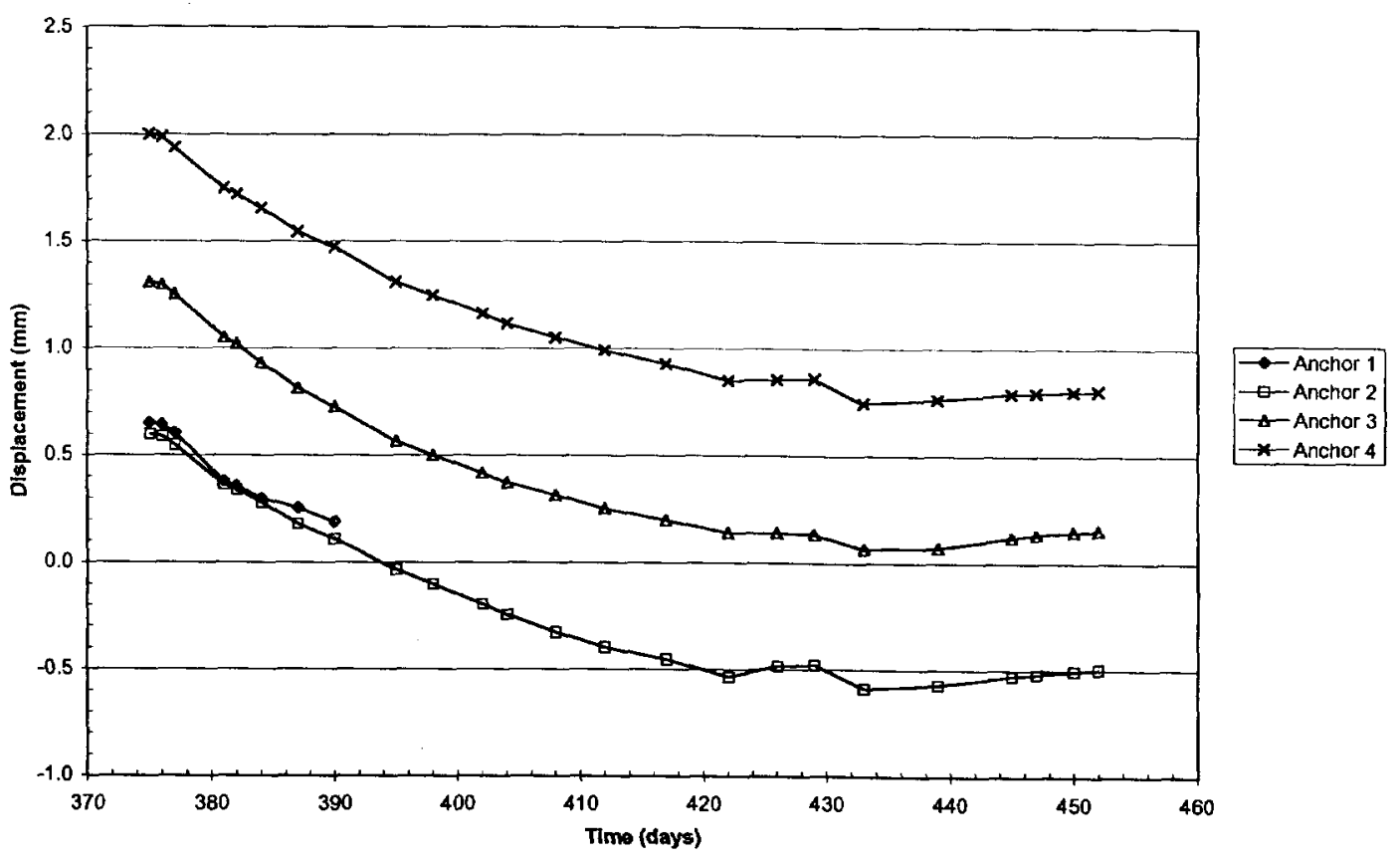

NOTE: Located Near the Center of the Large Block

Figure 7-24. TM-1 Vertical Anchor Displacements During Cool-Down 


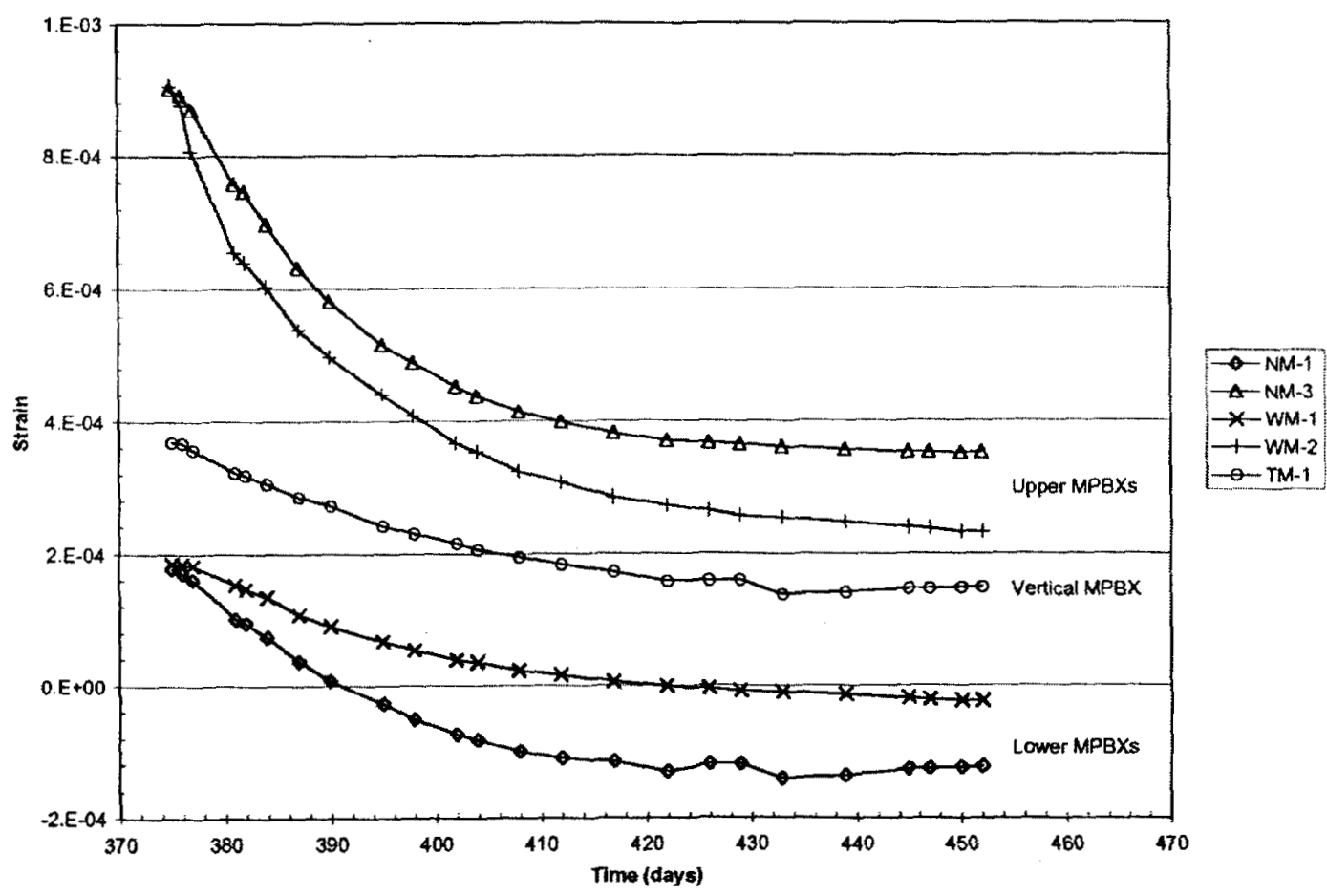

NOTE: No data are available for NM-2, which failed early in the test. The upper extensometers, which had extended more during the heating phase, contracted more on cool-down than the lower extensometers.

Figure 7-25. Anchor 4 Strains During Cool-Down 


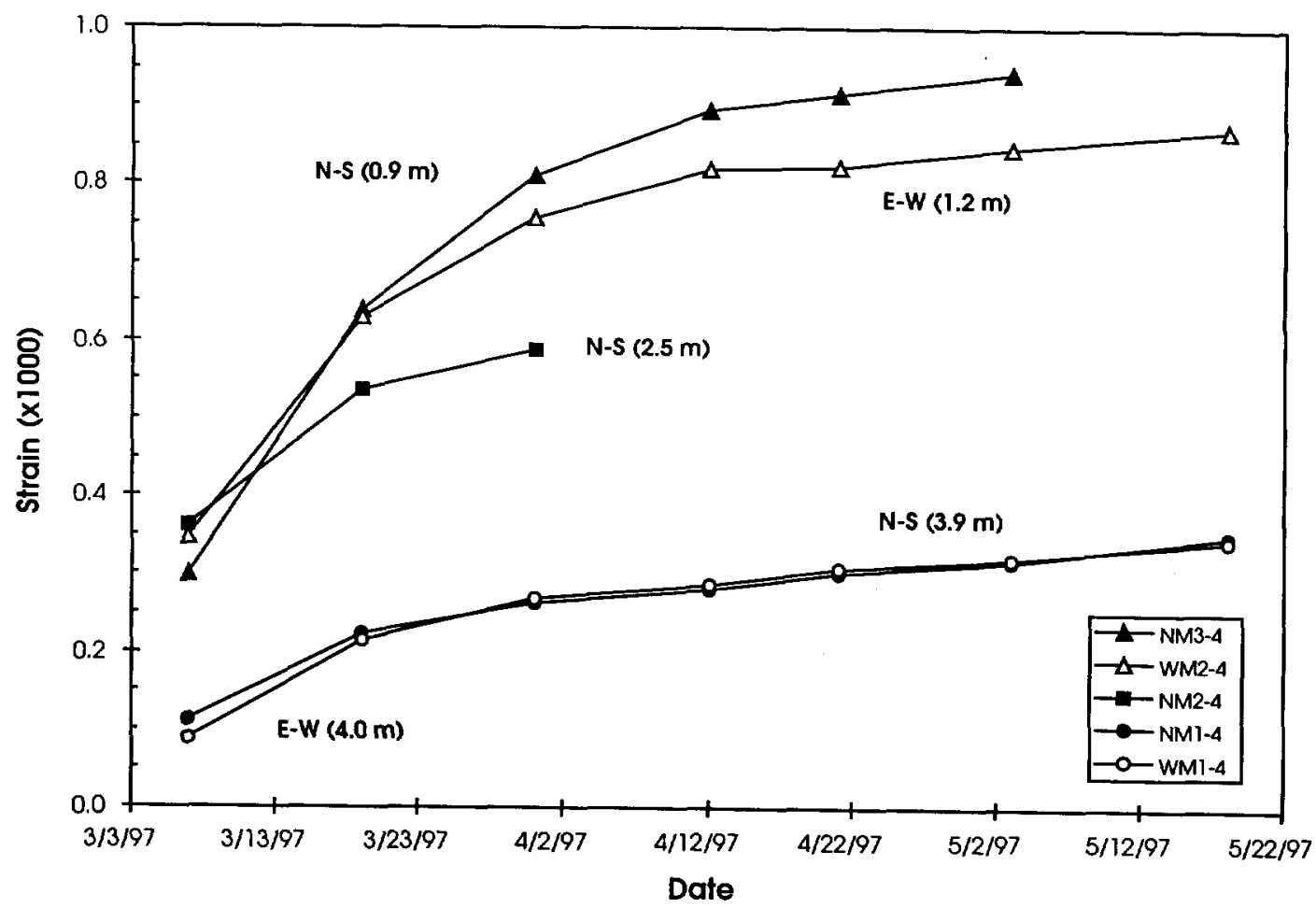

Figure 7-26. Strain Histories for Horizontal Extensometers 


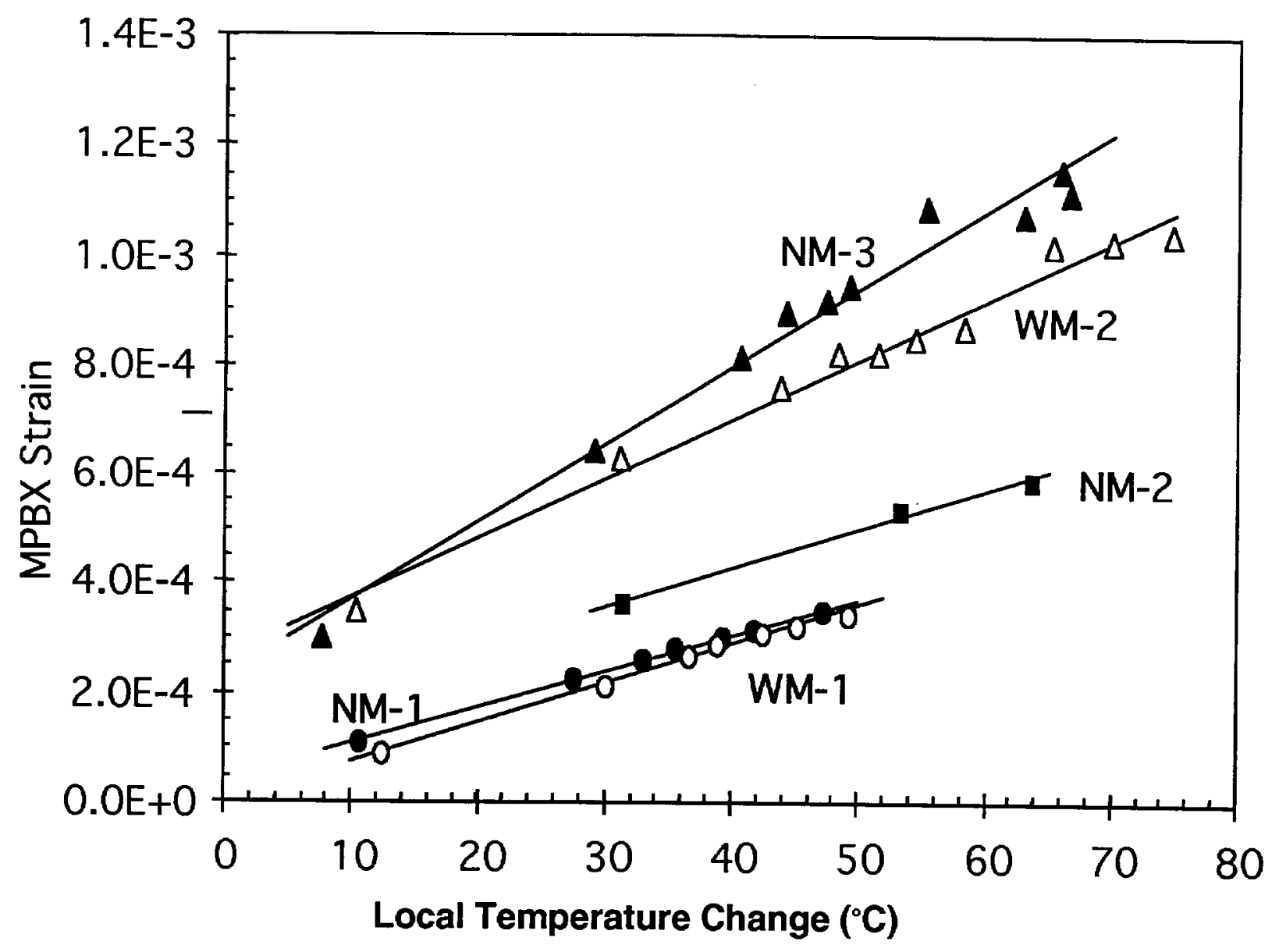

NOTE: The slopes of the fitted lines provide estimates of the rock mass thermal expansion coefficient.

Figure 7-27. Strains, Calculated from Displacements, Regressed Against Temperature Changes 


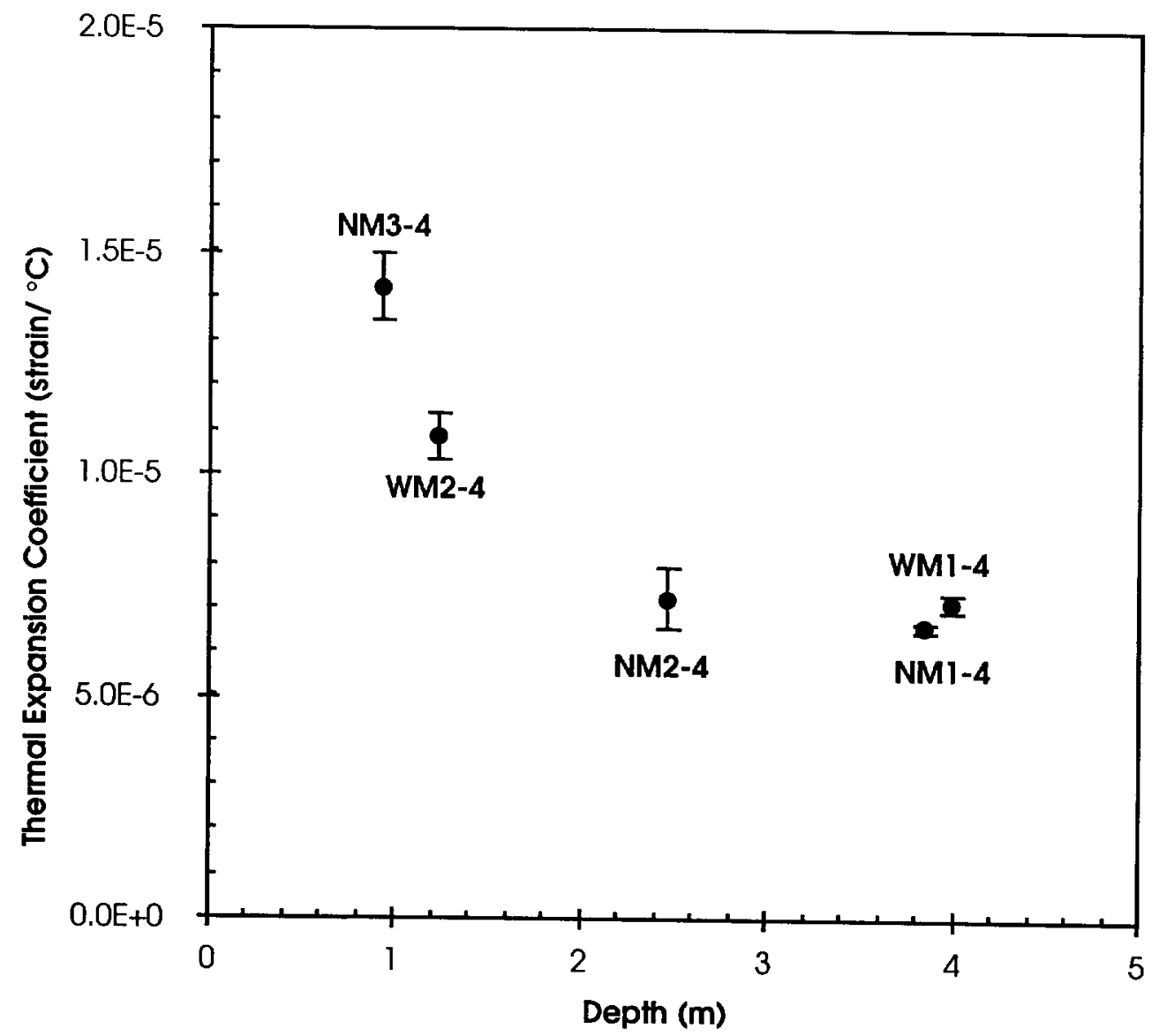

Figure 7-28. Linear Coefficients of Thermal Expansion with Depth into the Large Block 


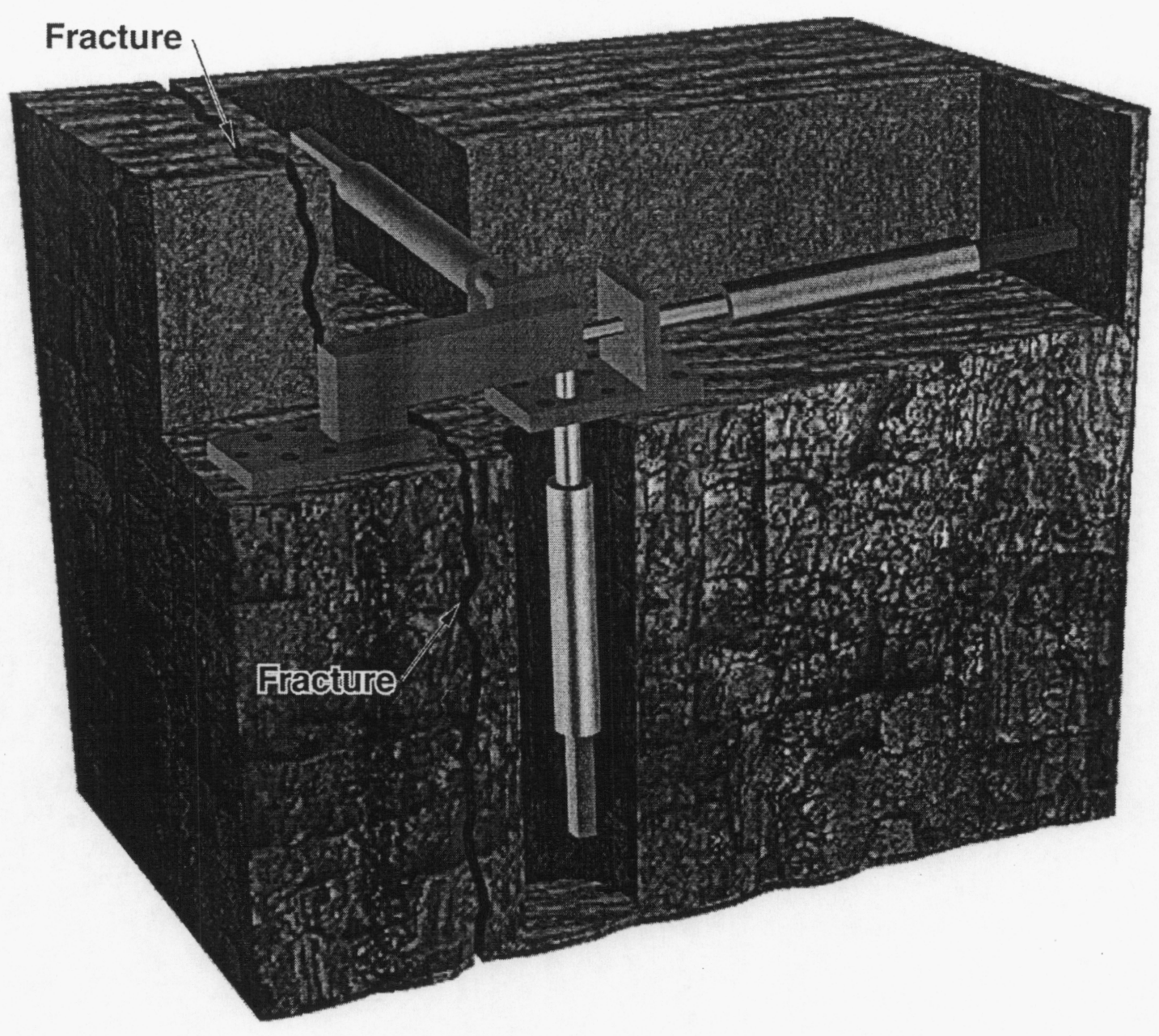

Figure 7-29. Cutaway View of LBT Surface Fracture Monitors 


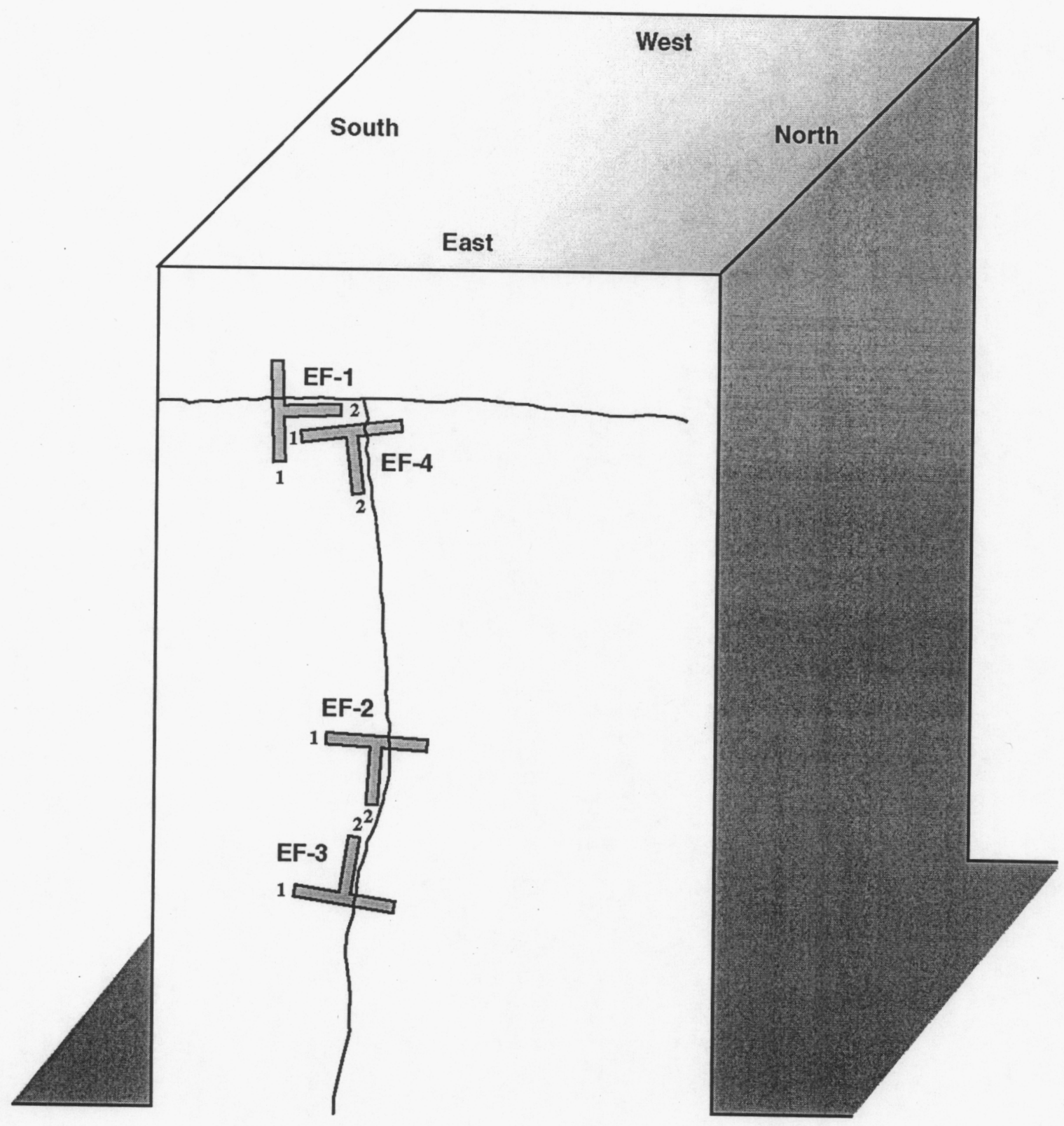

Figure 7-30. Fracture Monitor Locations on the East Side of the Large Block 


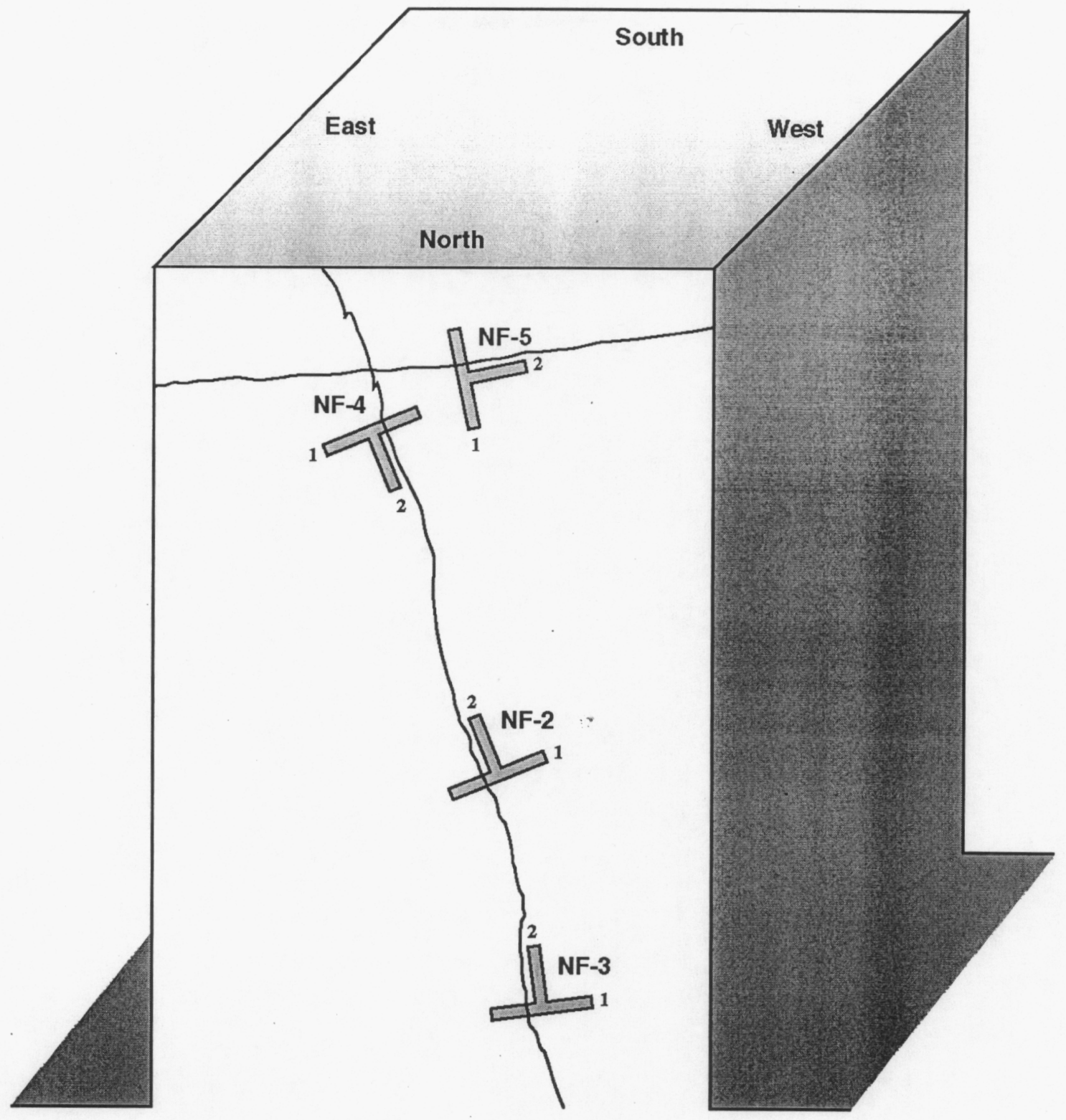

Figure 7-31. Fracture Monitor Locations on the North Side of the Large Block 


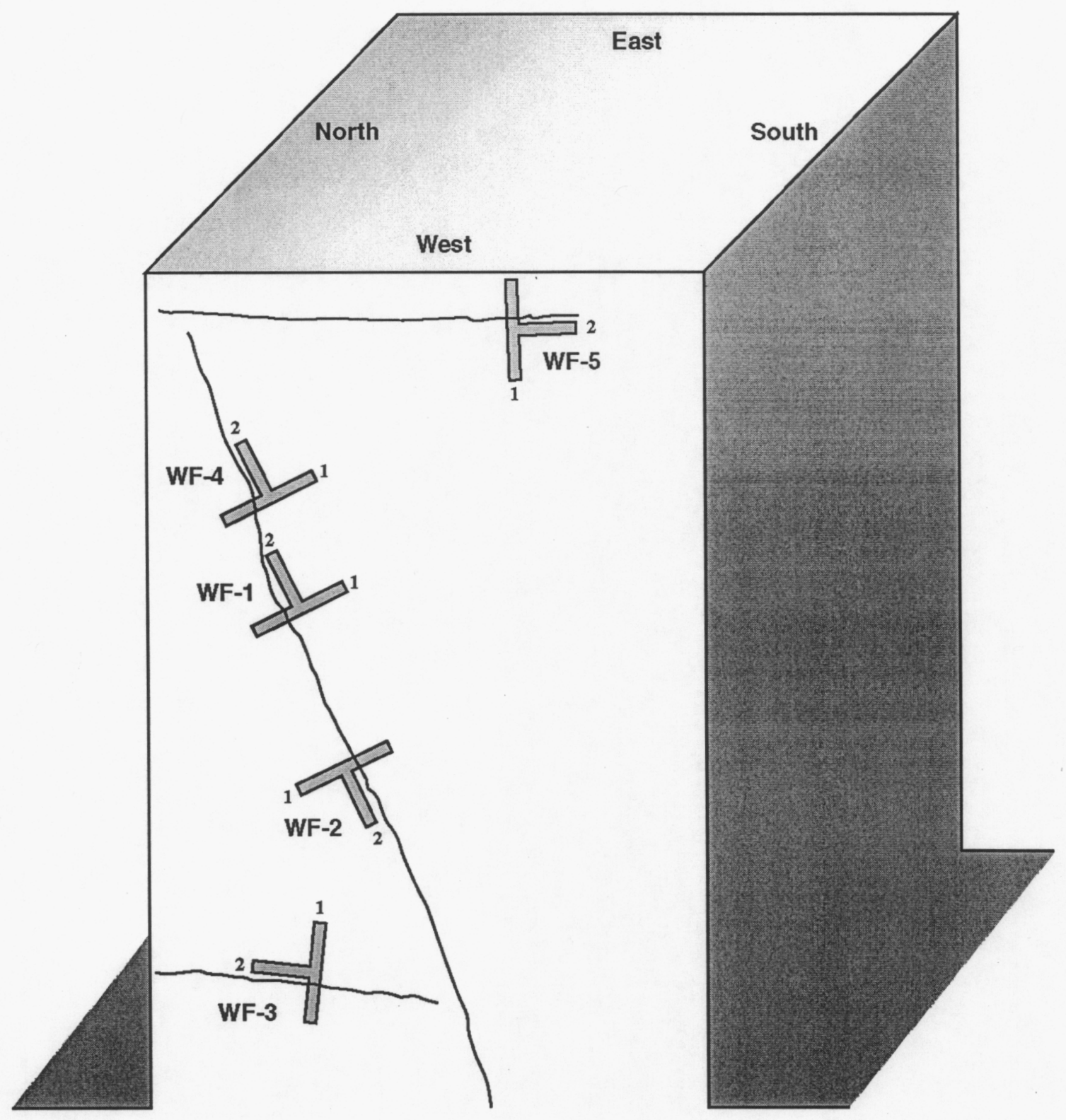

Figure 7-32. Fracture Monitor Locations on the West Side of the Large Block 


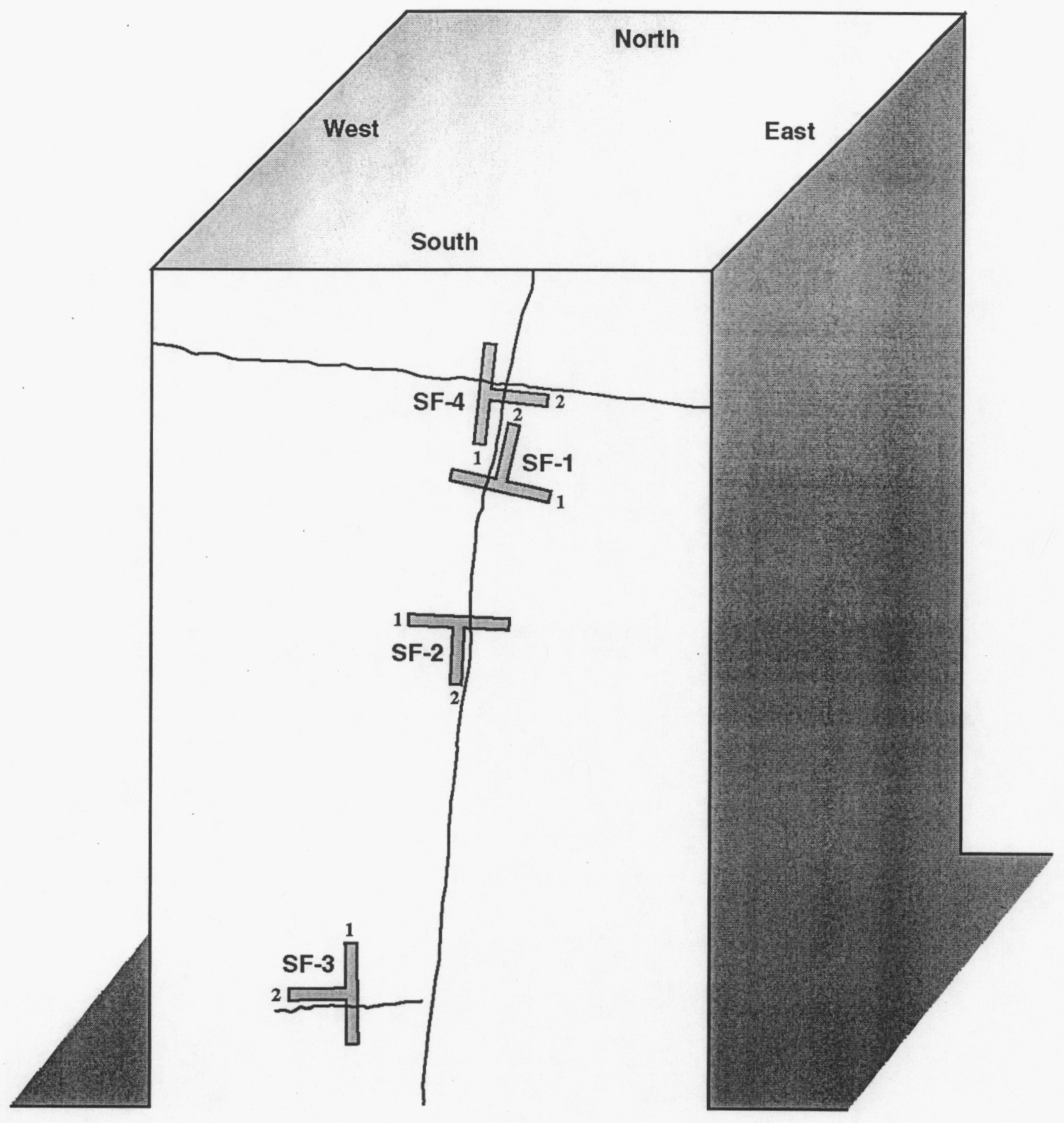

Figure 7-33. Fracture Monitor Locations on the South Side of the Large Block 


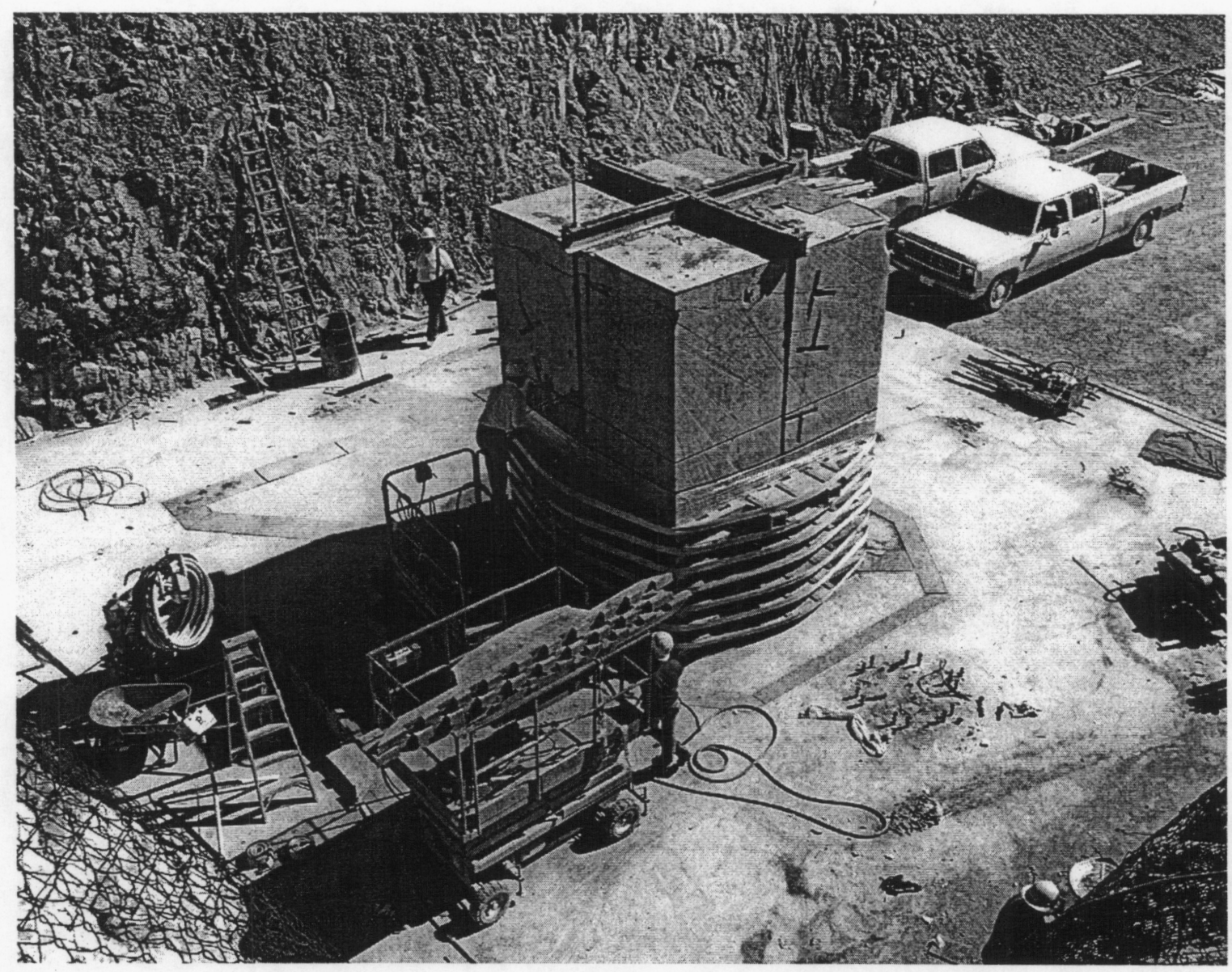

Figure 7-34. Photo of LBT with Fracture Monitor Grooves 


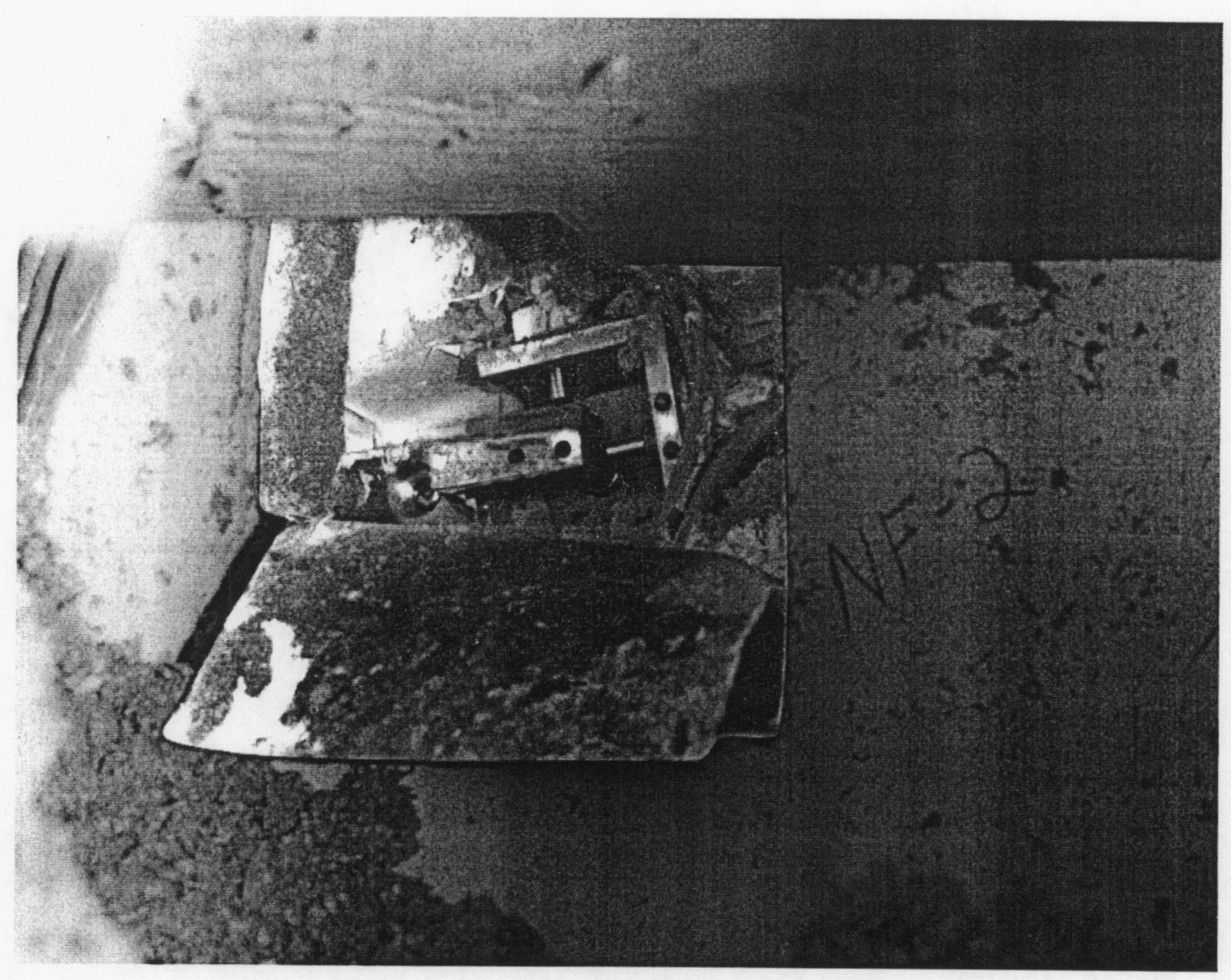

Figure 7-35. Photo of Installed Fracture Monitor 
Fracture Aperture Data for all Vertical Fracture Monitors

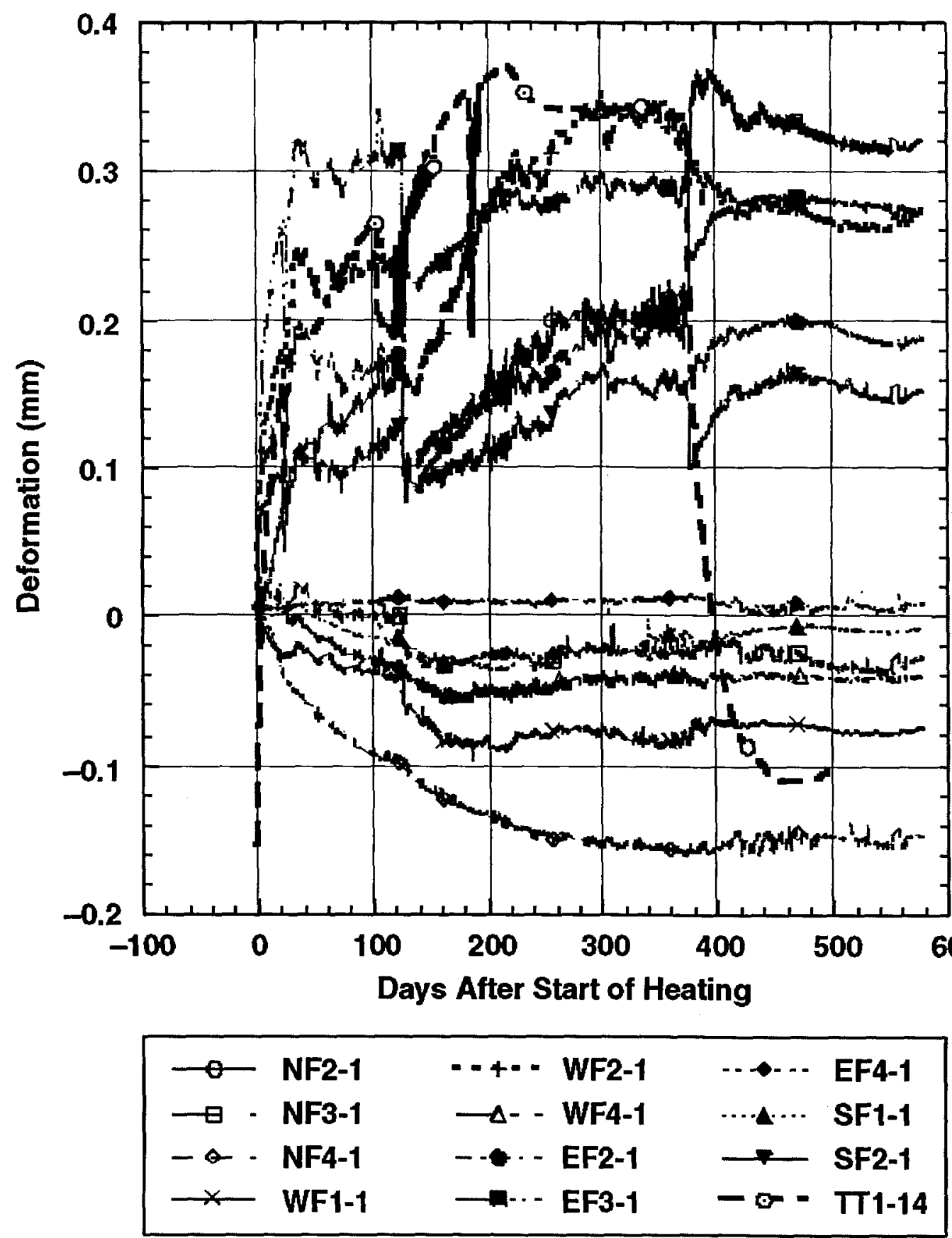

Figure 7-36. Fracture Aperture Data for All Vertical Fracture Monitors and Temperature Near the Heater Plane 
Fracture Aperture Data for all Vertical Fracture Monitors

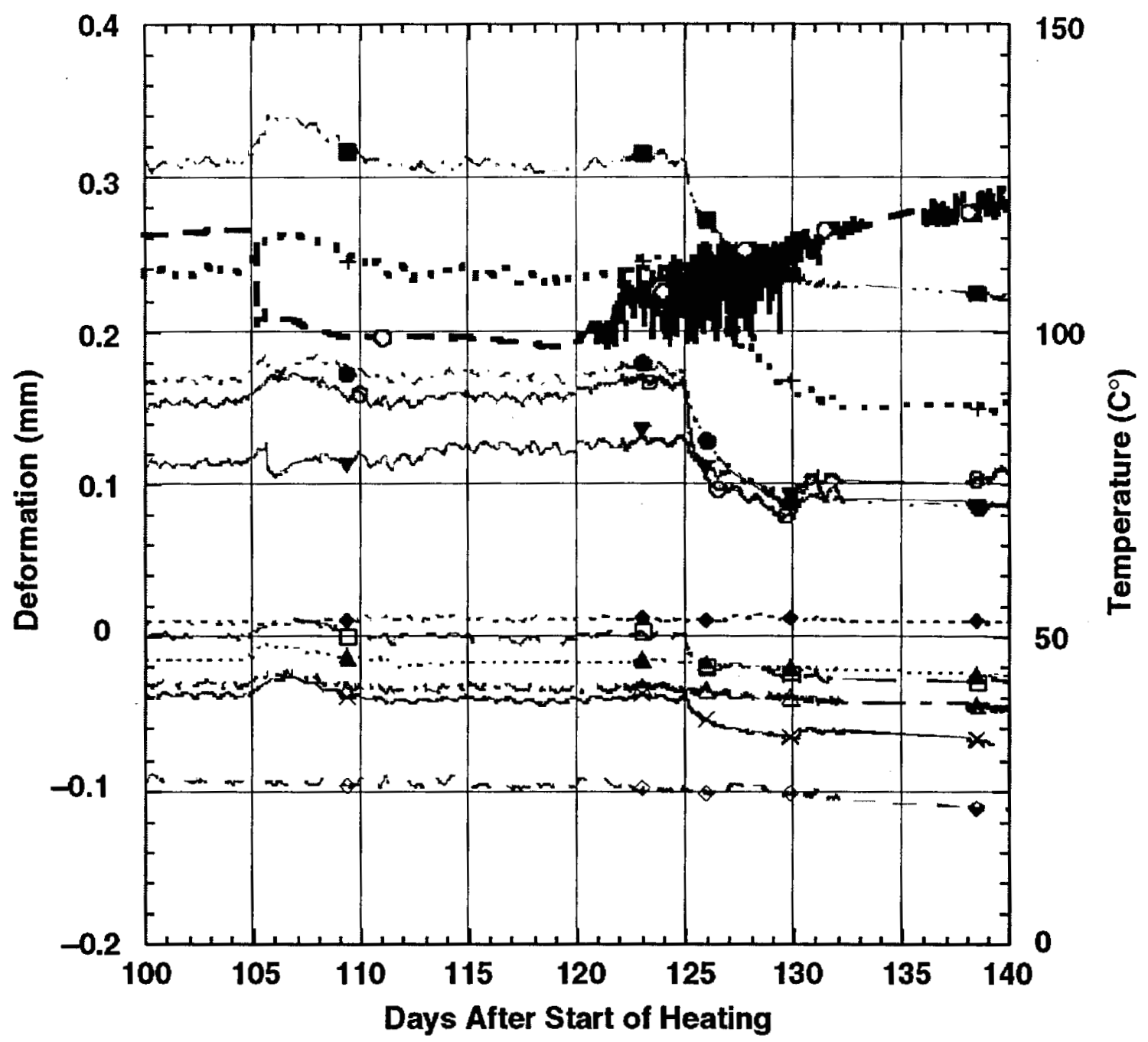

\begin{tabular}{|c|c|c|c|c|}
\hline 0 & NF2-1 & $\cdots+\cdots$ WF2-1 & $\cdots \leftrightarrow \cdots$ & EF4-1 \\
\hline$\rightarrow$ 曰 & NF3-1 & $-\Delta--$ WF4-1 & $\cdots \mathbf{a} \cdots$ & SF1-1 \\
\hline$-\infty$ & NF4-1 & $--*$ EF2-1 & $\rightarrow$ & SF2-1 \\
\hline$x$ & WF1-1 & $\rightarrow-\cdot$ EF3-1 & $-\sigma=$ & TT1-14 \\
\hline
\end{tabular}

Figure 7-37. Fracture Aperture Data Between Days 100 and 140 for All Vertical Fracture Monitors and Temperature Near the Heater Plane 
Fracture Slip for all Vertical Fractures

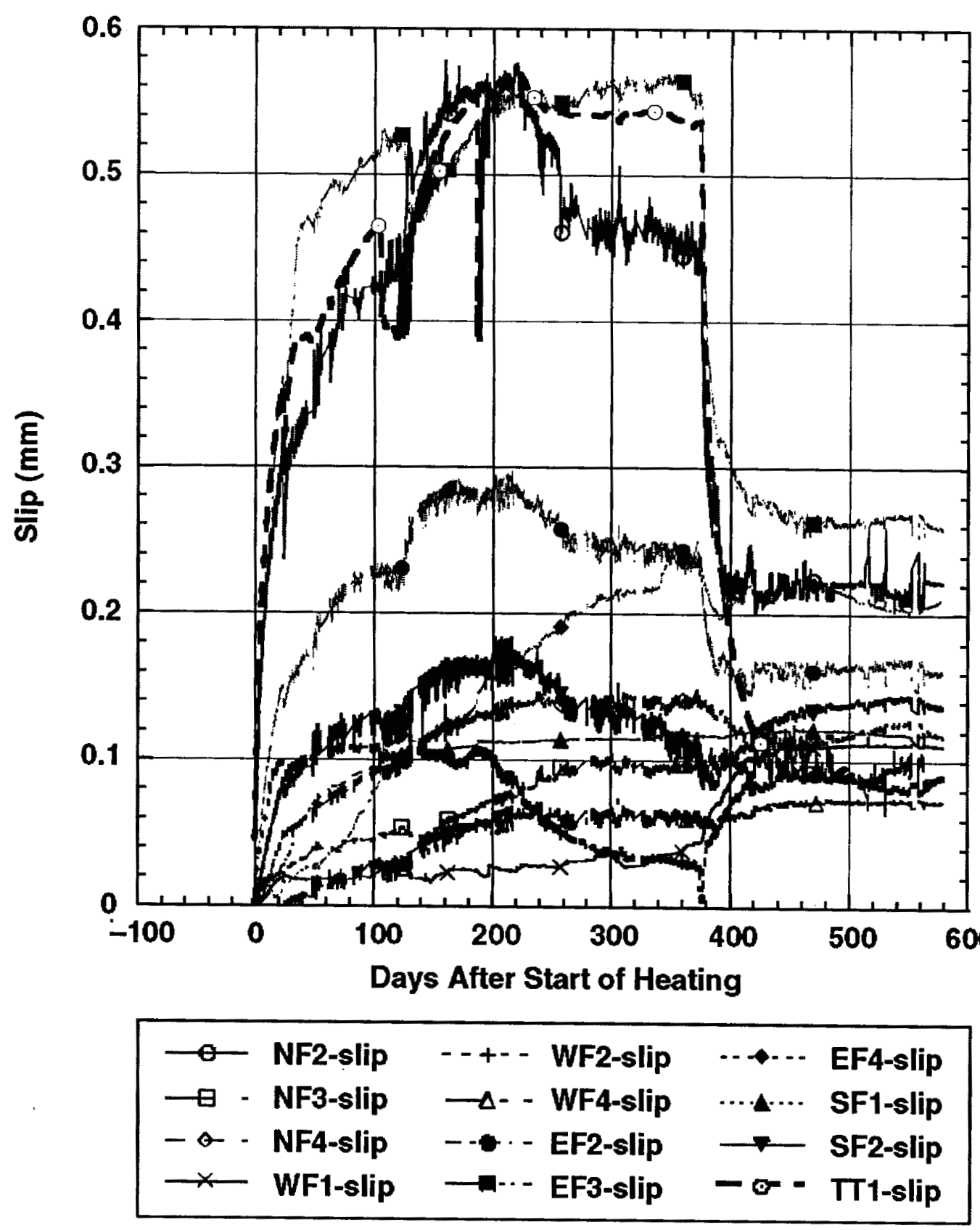

Figure 7-38. Fracture Slip for All Vertical Fractures and Temperature Near the Heater Plane 
Aperture Change For Vertical Fractures

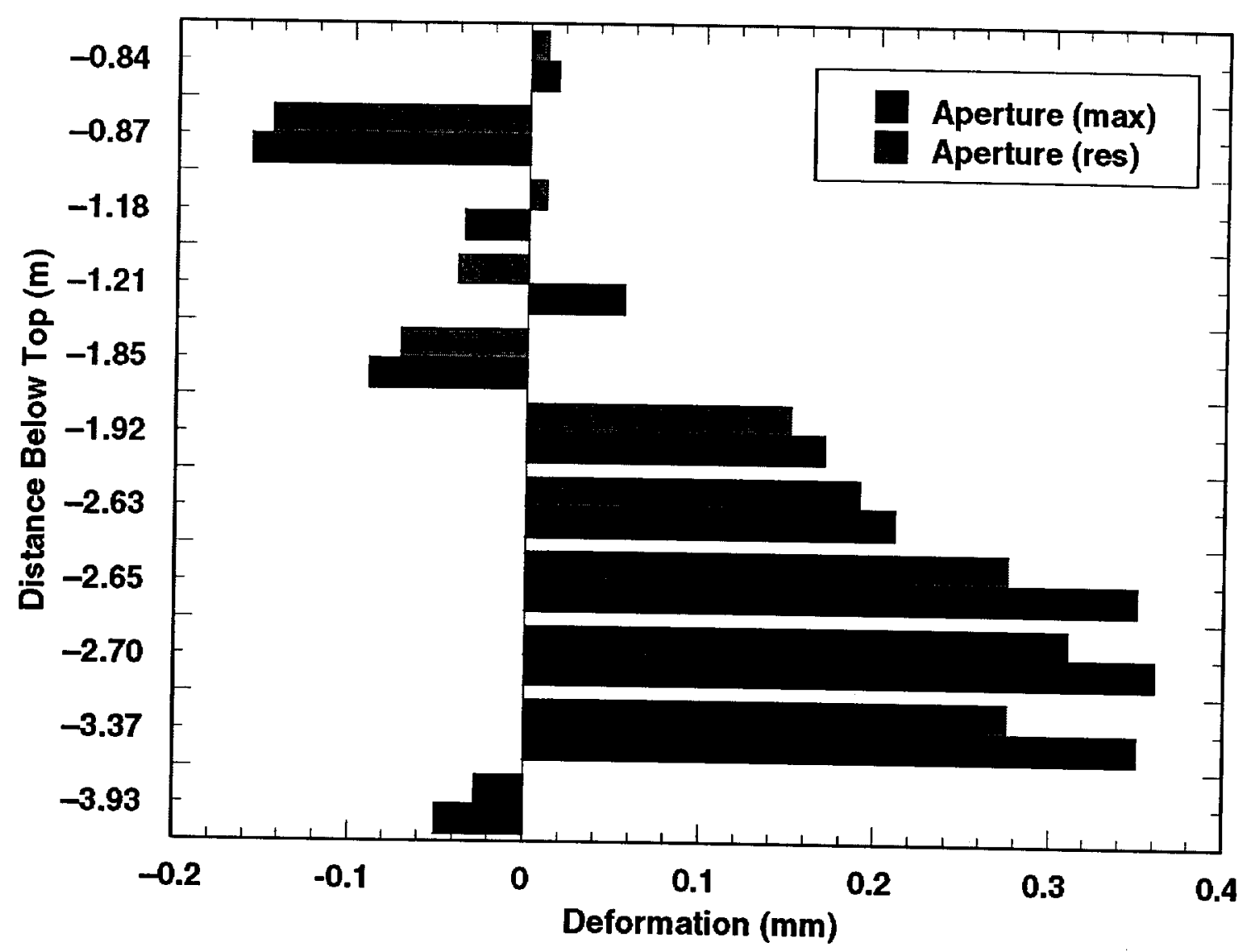

Figure 7-39. Aperture Change for Vertical Fractures 
Slip Along Vertical Fractures

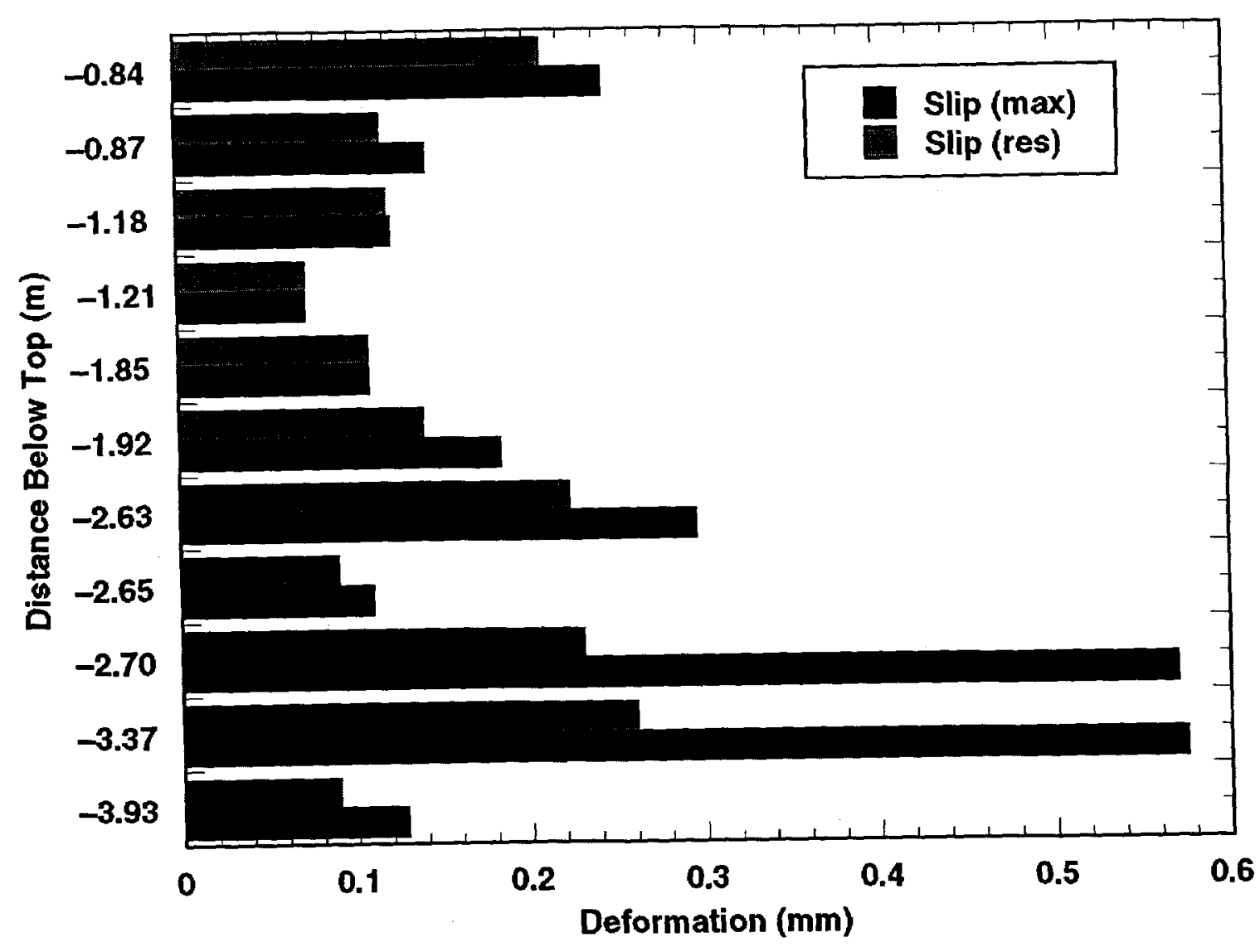

Figure 7-40. Slip Along Vertical Fractures 
Fracture Aperture Data for all Horizontal Fracture Monitors

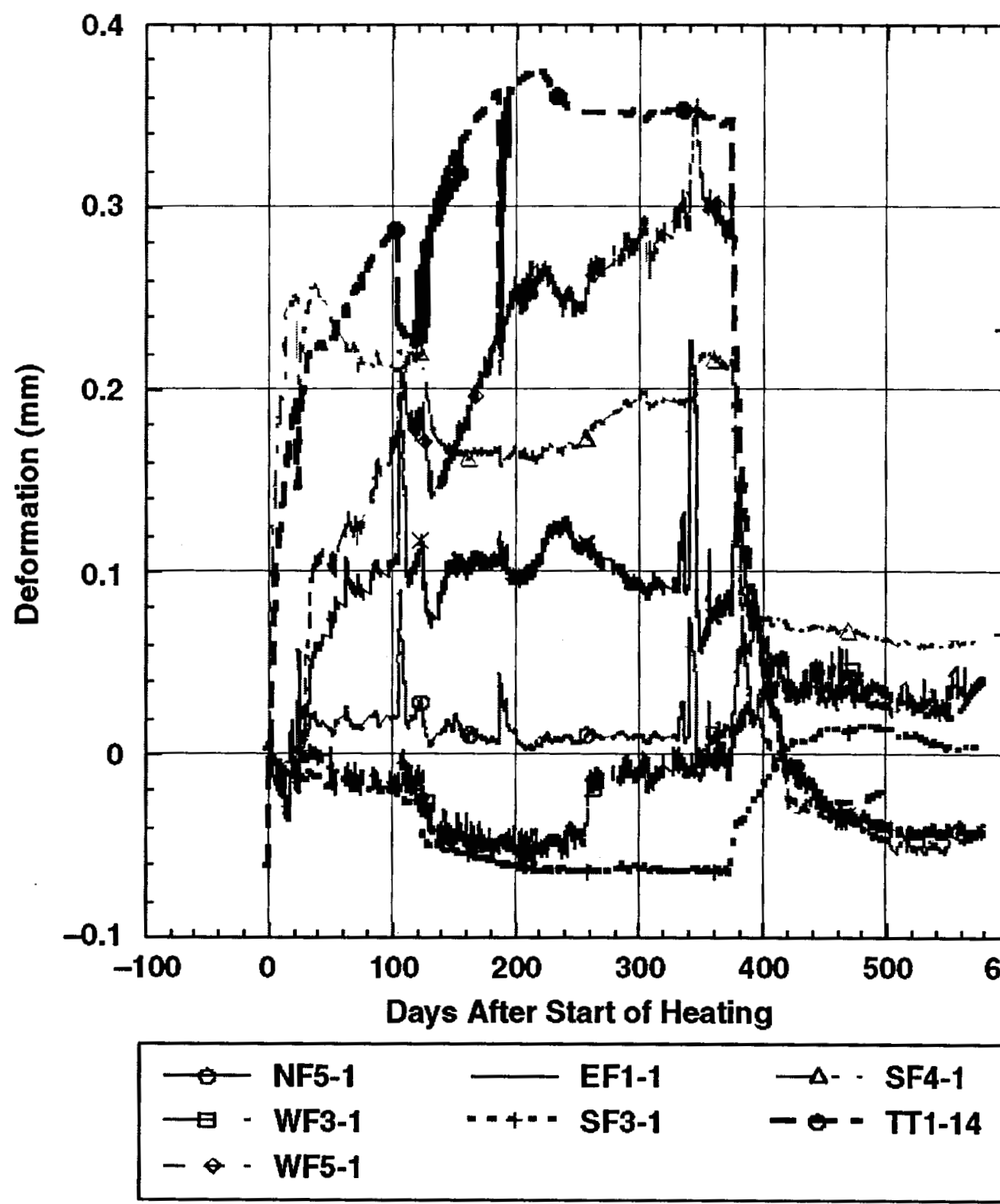

Figure 7-41. Fracture Aperture Data for All Horizontal Fracture Monitors and Temperature Near the Heater Plane 
Fracture Aperture Data for all Horizontal Fracture Monitors

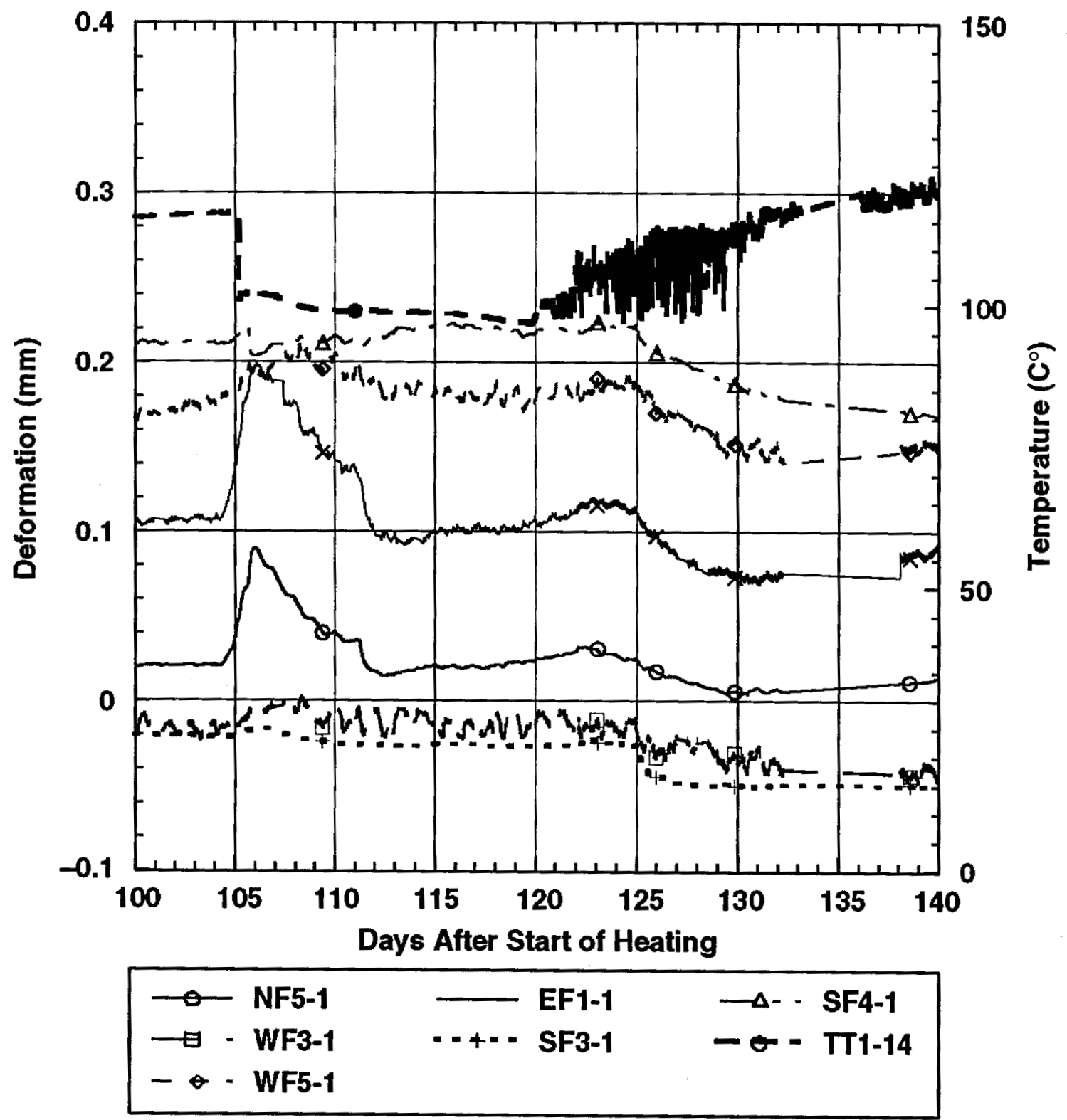

Figure 7-42. Temperature and Fracture Aperture Data for All Horizontal Fracture Monitors Between Day 100 and Day 140 
Fracture Slip for all Horizontal Fractures

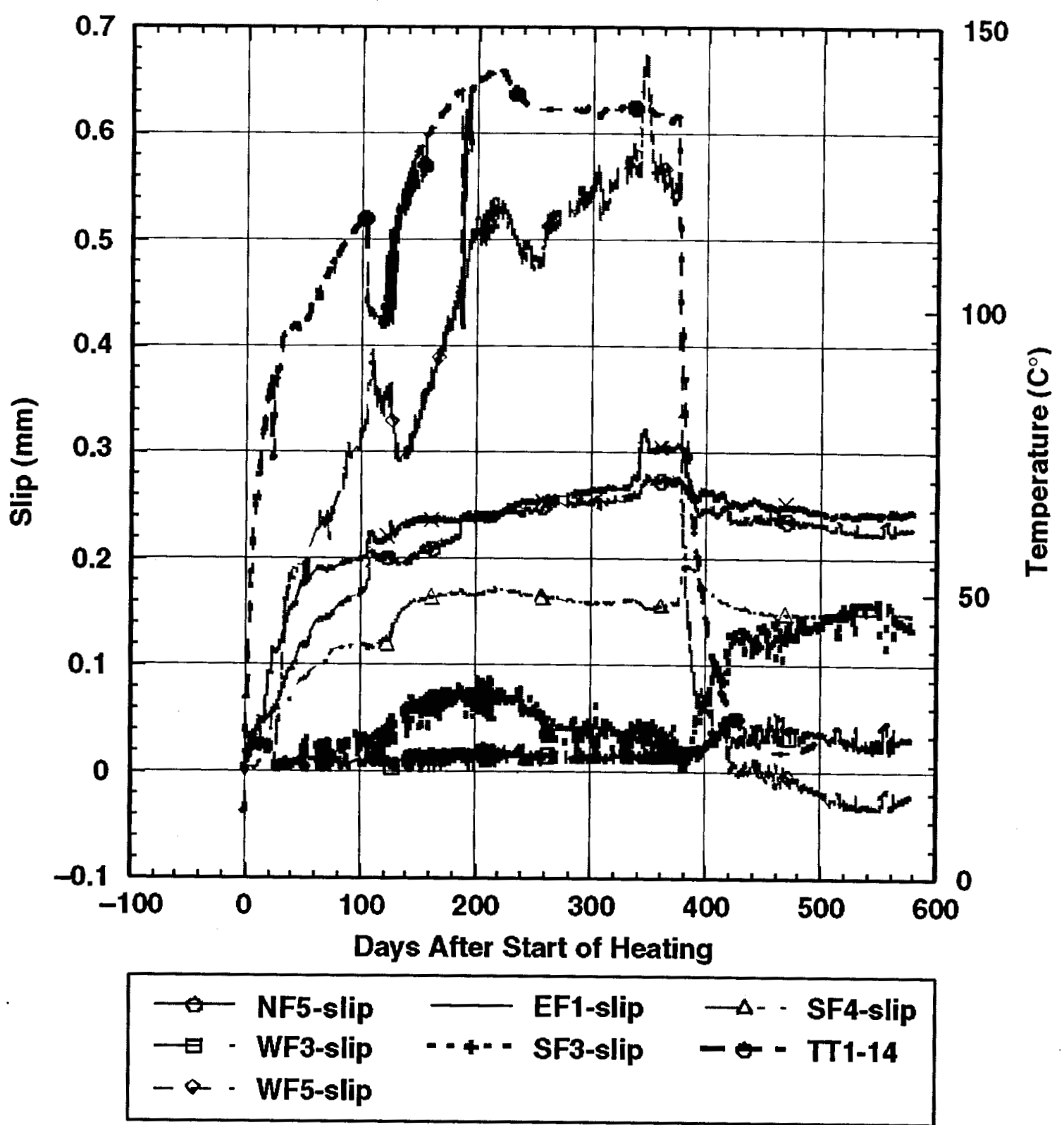

Figure 7-43. Fracture Slip for All Horizontal Fractures and Temperature Near the Heater Plane 
Fracture Slip for all Horizontal Fractures

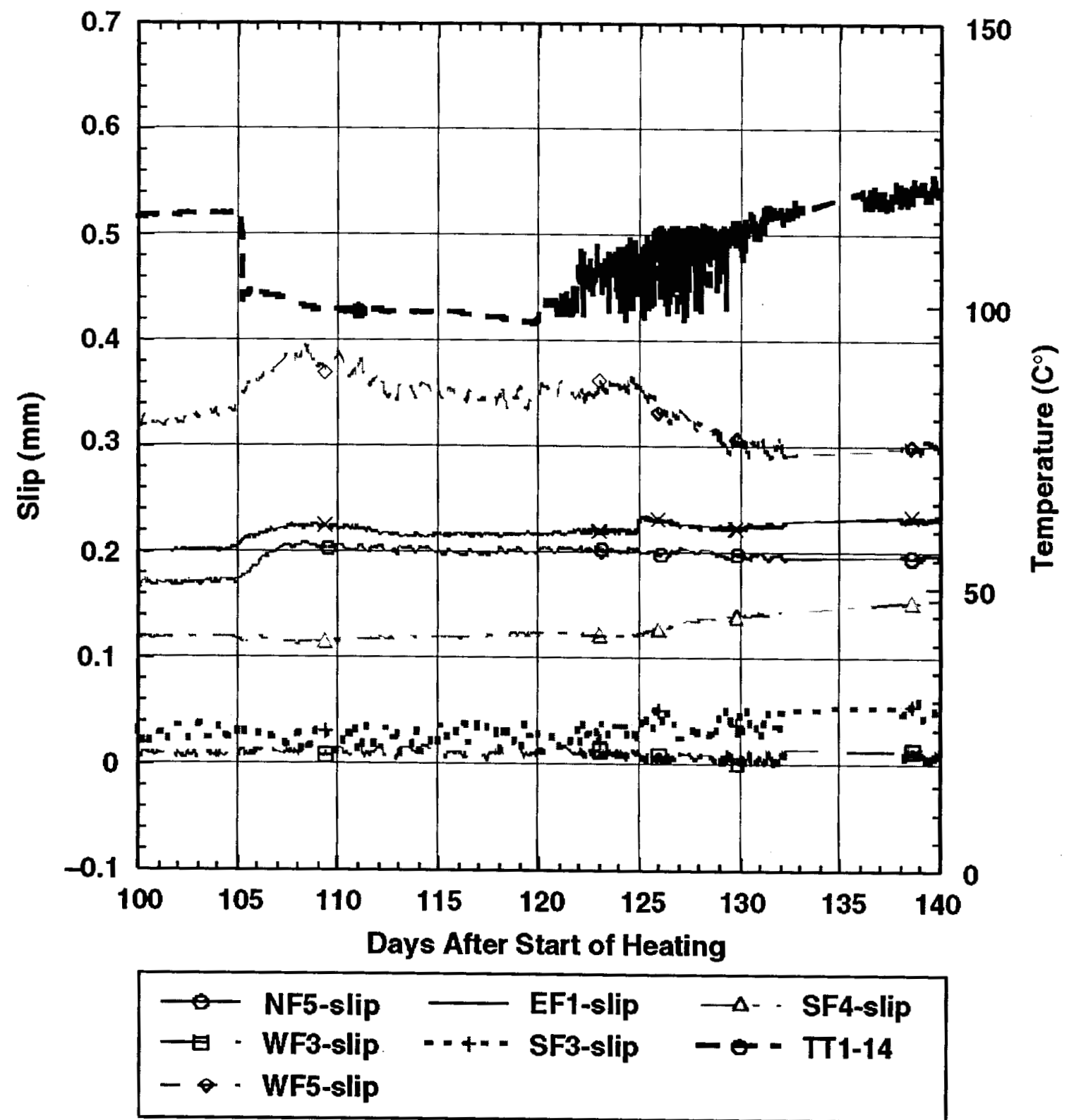

Figure 7-44. Fracture Slip for All Horizontal Fractures and Temperature Near the Heater Plane, Day 100 to Day 140 


\section{Deformation Across Horizontal Fractures}

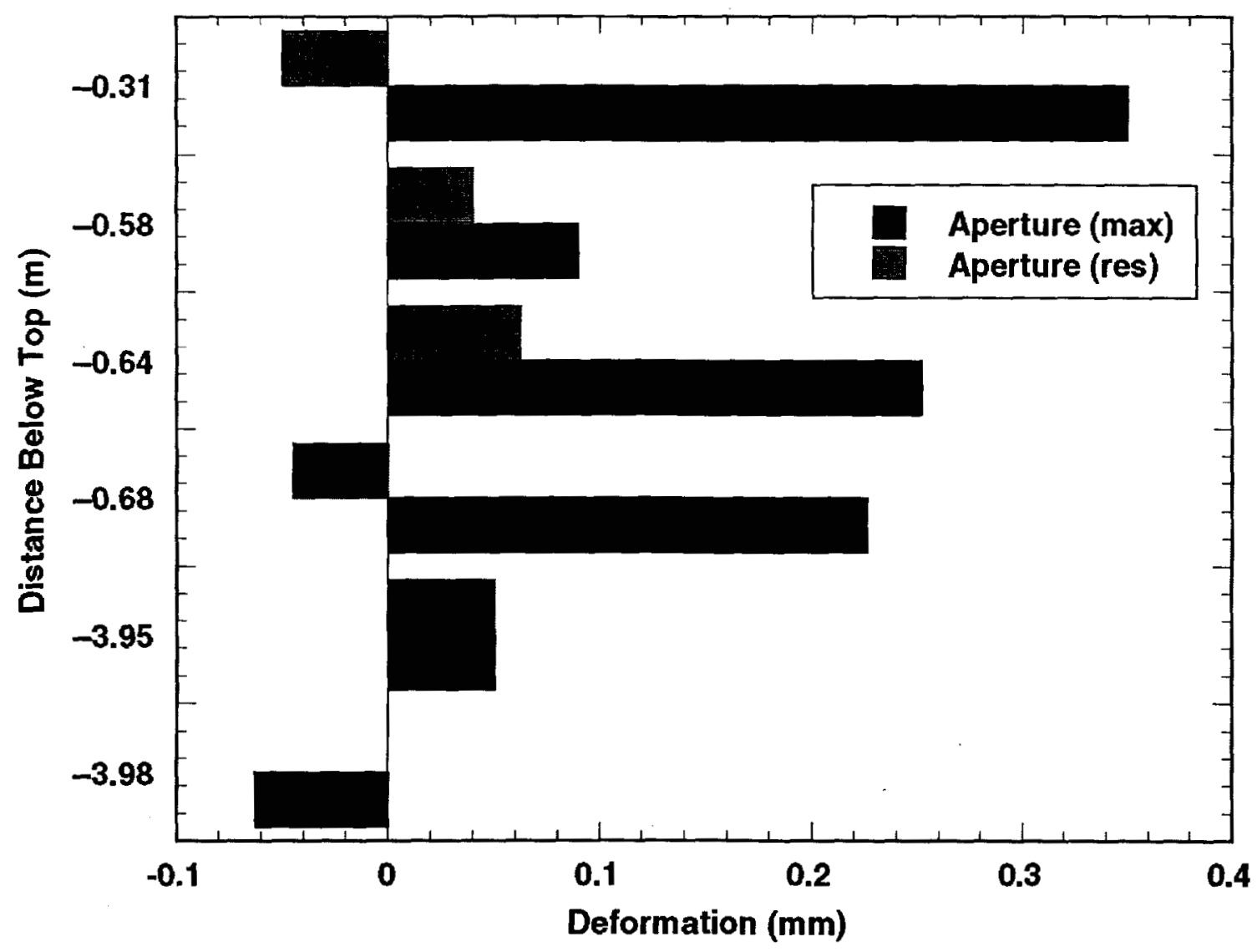

Figure 7-45. Deformation Across Horizontal Fractures 
Slip Along Horizontal Fractures

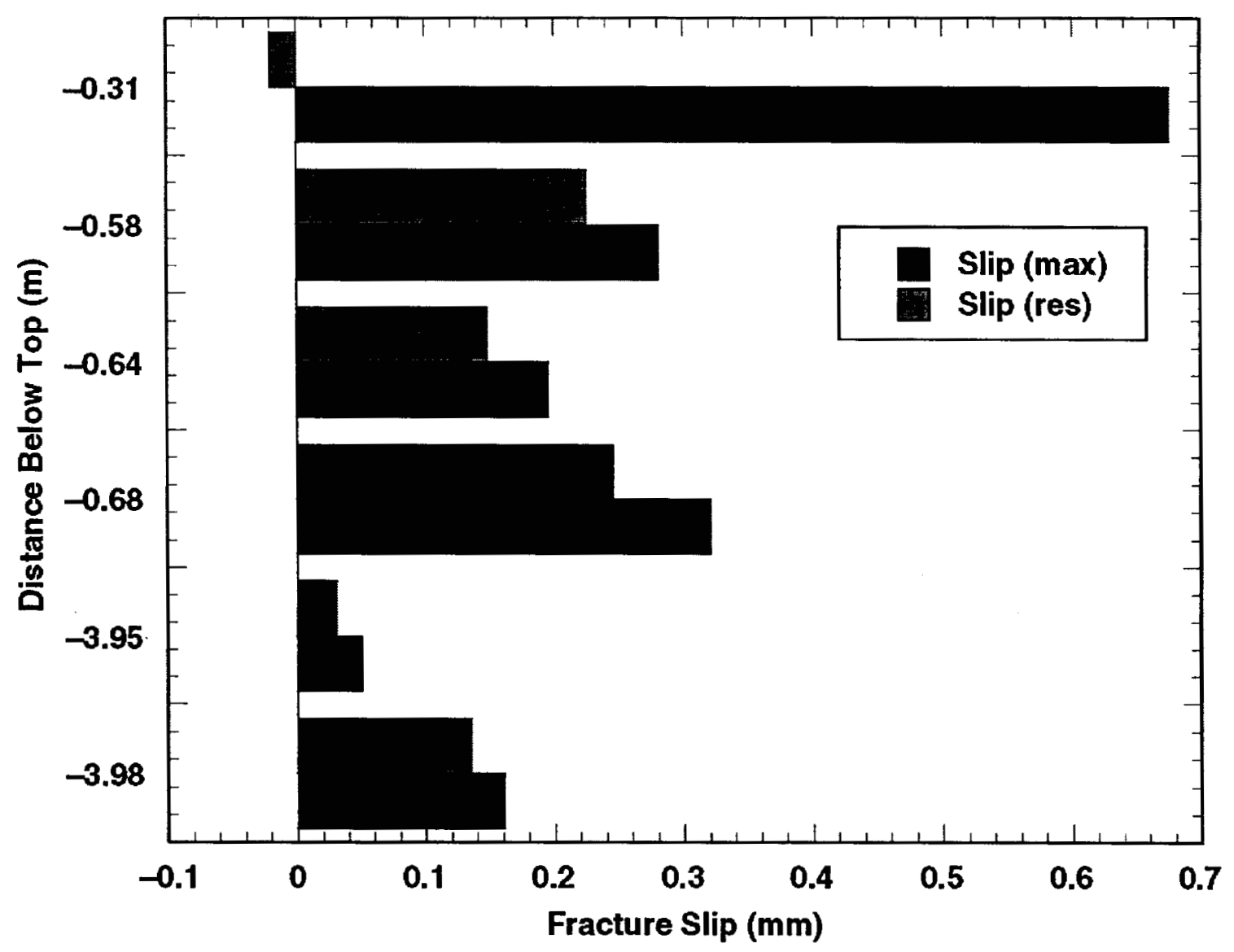

Figure 7-46. Slip Along Horizontal Fractures 


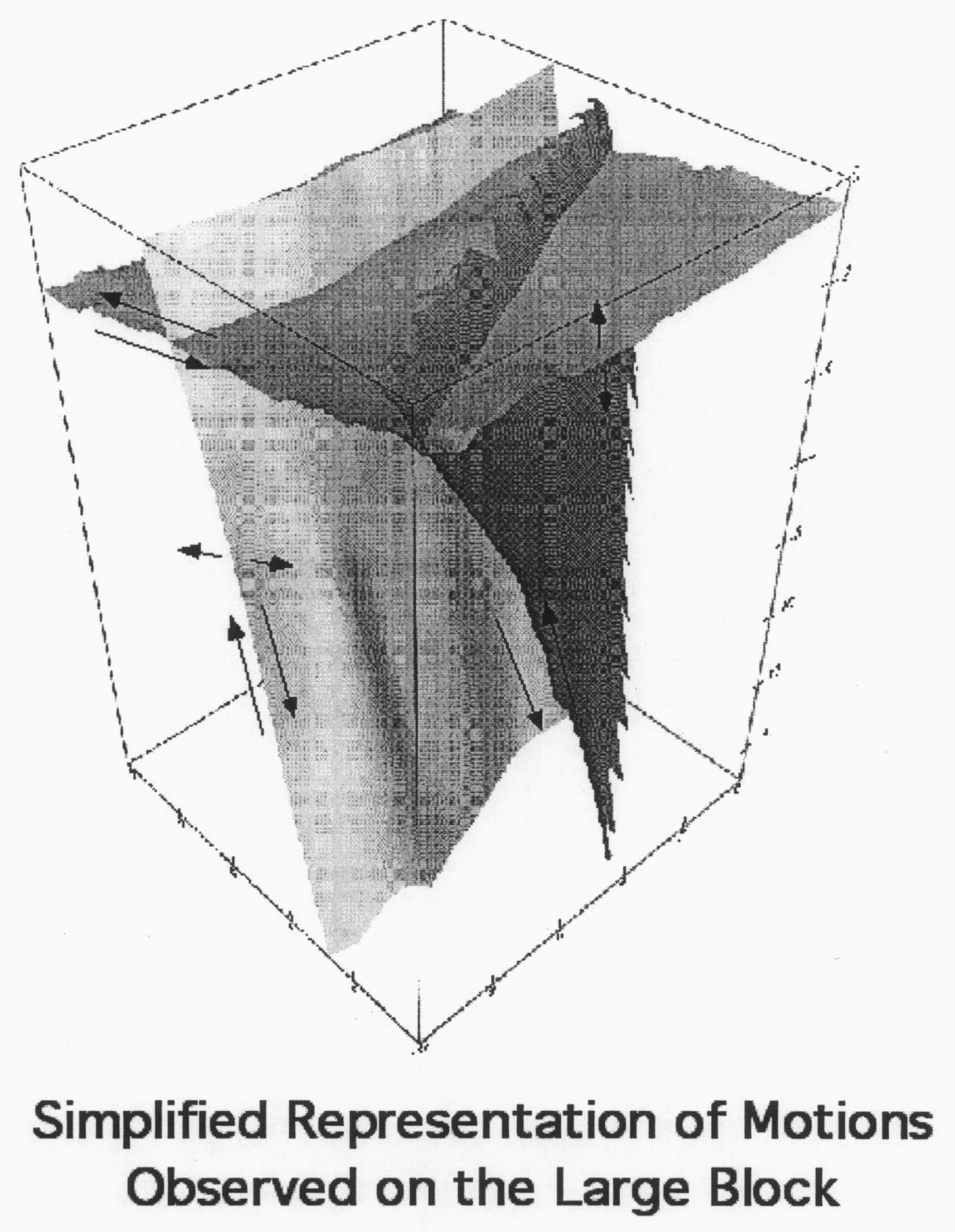

Figure 7-47. General Block Movement 


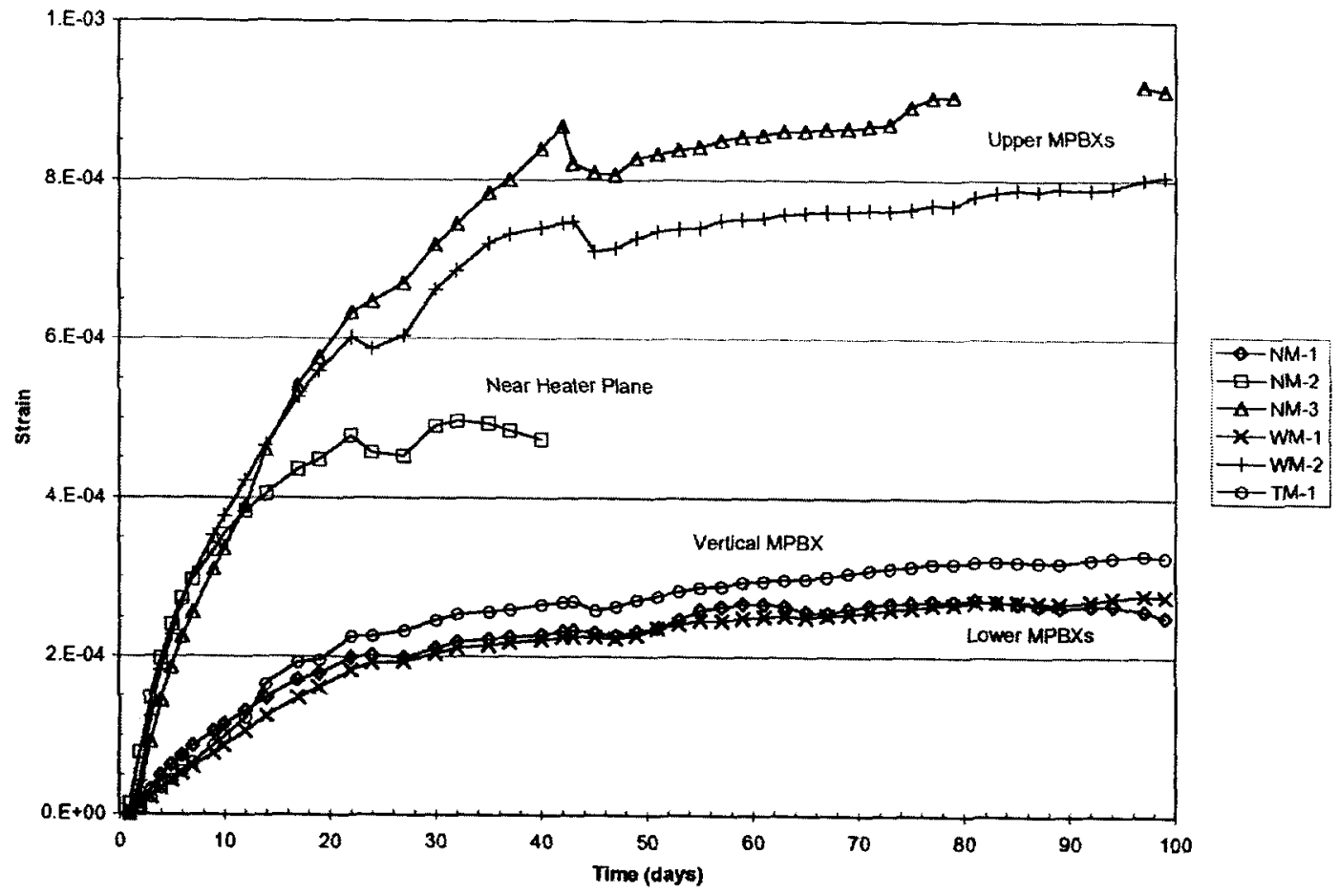

Figure 7-48. Anchor 4 Strains for Each Extensometer During the First 100 Days 
Fracture Aperture Data for all Vertical Fracture Monitors

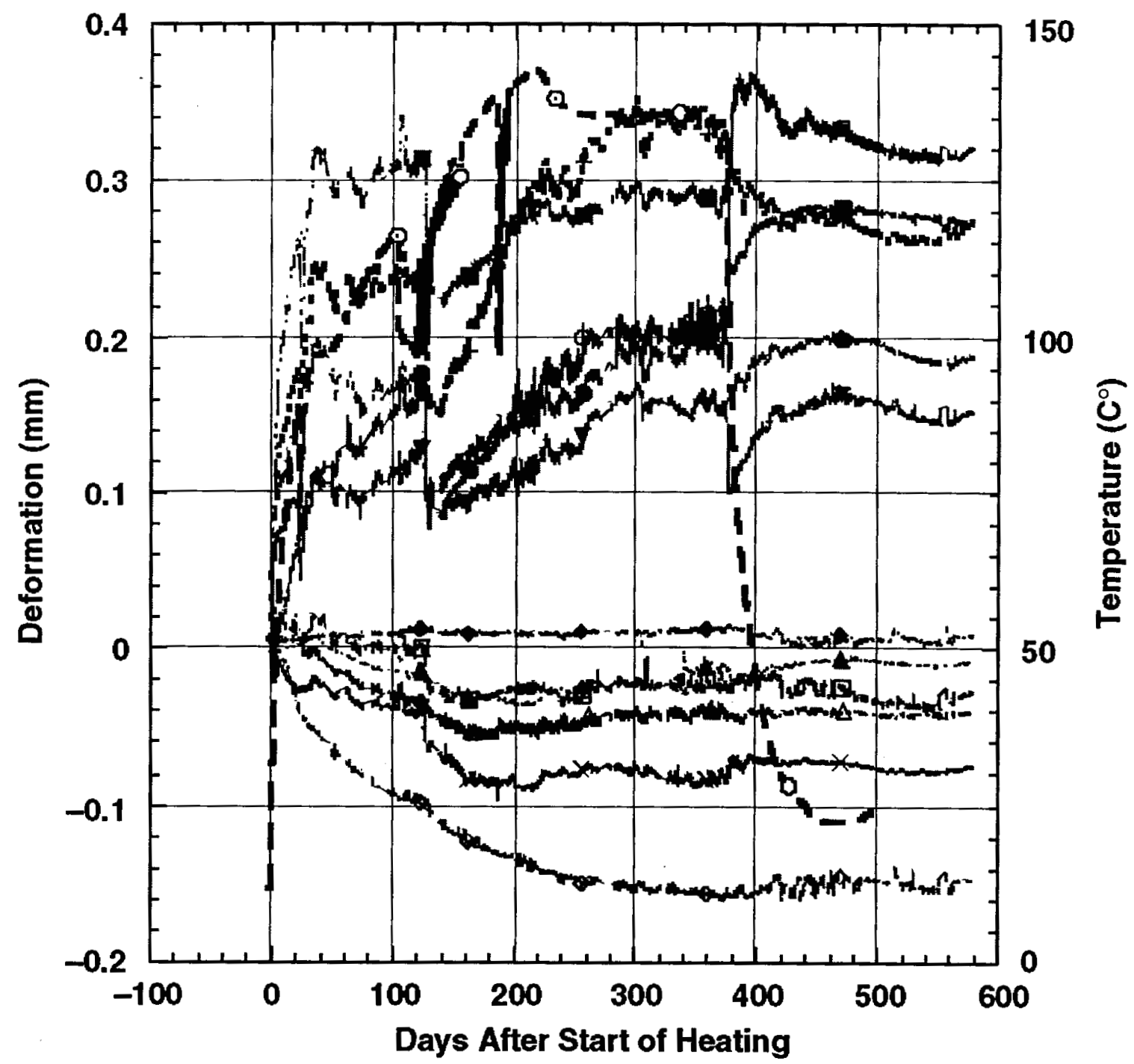

\begin{tabular}{|c|c|c|c|c|}
\hline$-\theta$ & NF2-1 & $--+-\cdot$ WF2-1 & $\cdots \leftrightarrow \cdots$ & EF4-1 \\
\hline$\rightarrow$ & NF3-1 & $-\Delta--$ WF4-1 & $\cdots+\cdots$ & SF1-1 \\
\hline$-\infty$ & NF4-1 & - EF2-1 & $\rightarrow$ & SF2-1 \\
\hline$x$ & WF1-1 & - & $-\sigma-$ & $\pi 1-14$ \\
\hline
\end{tabular}

Figure 7-49. Fracture Aperture Data for All Vertical Fracture Monitors 

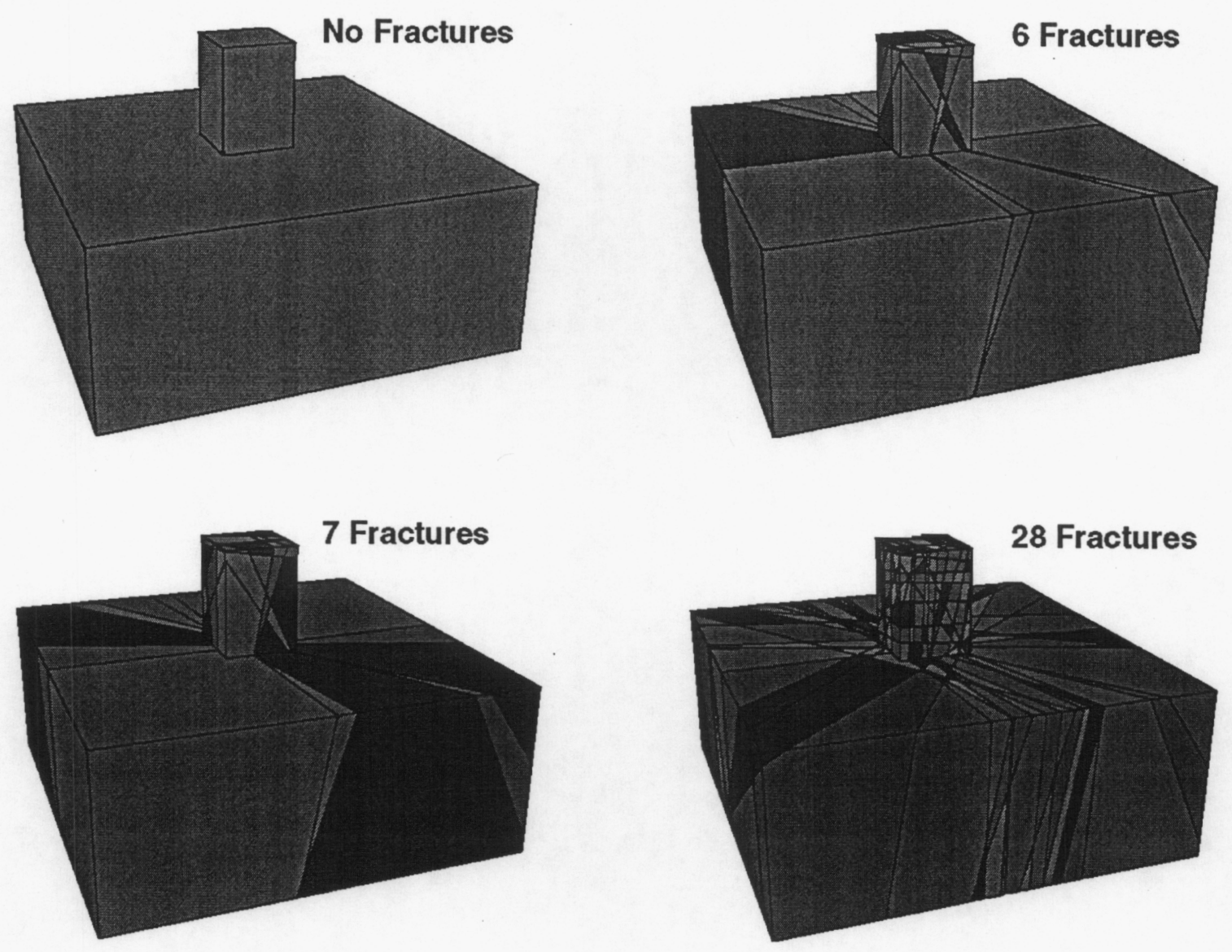

Figure 7-50. Large Block Test TM Model Domains 


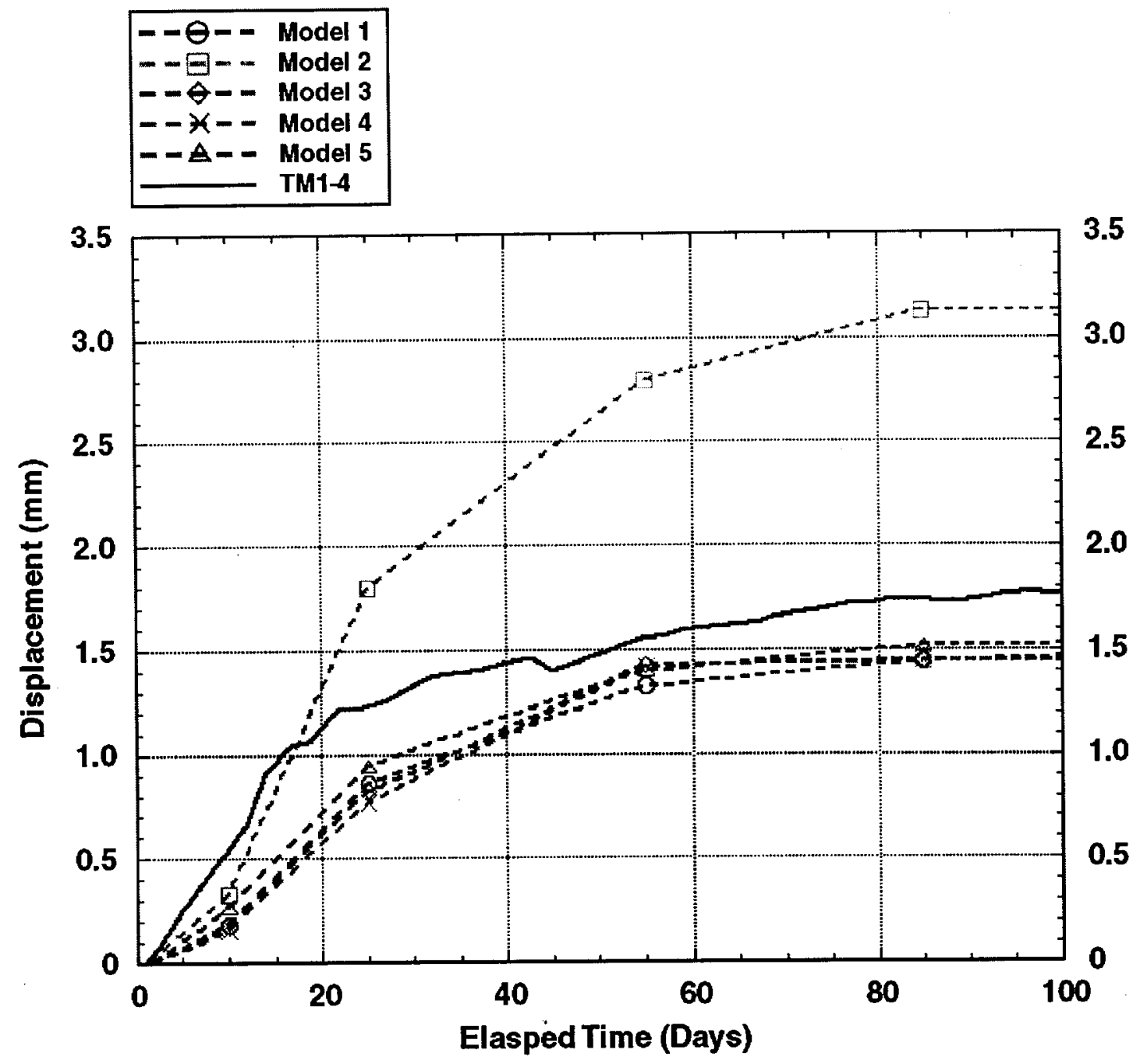

Figure 7-51. TM1 Anchor 4 Displacements and Model Results for the First 100 Days 


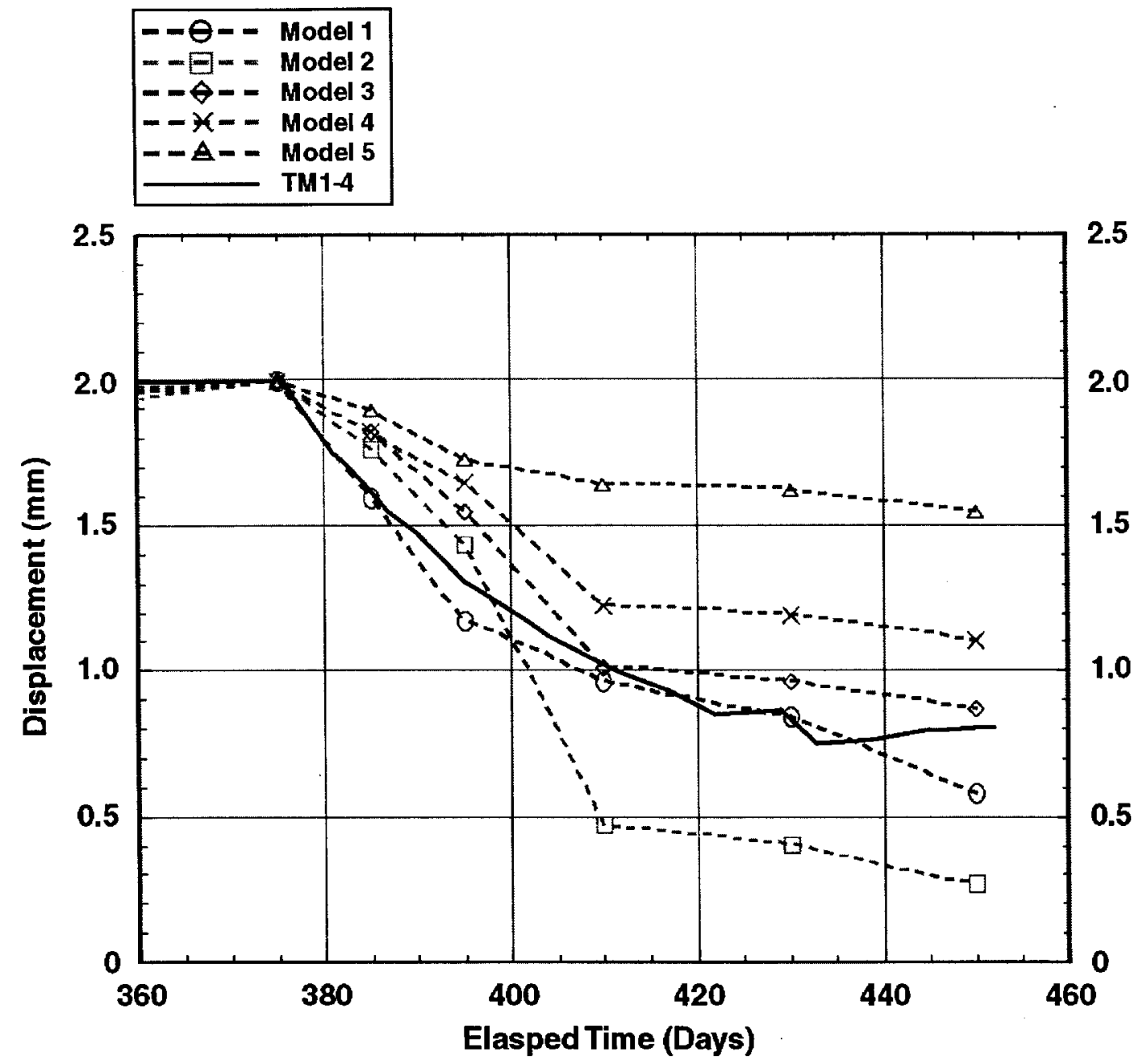

Figure 7-52. TM1 Anchor 4 Displacements and Model Results for the Cool-Down 


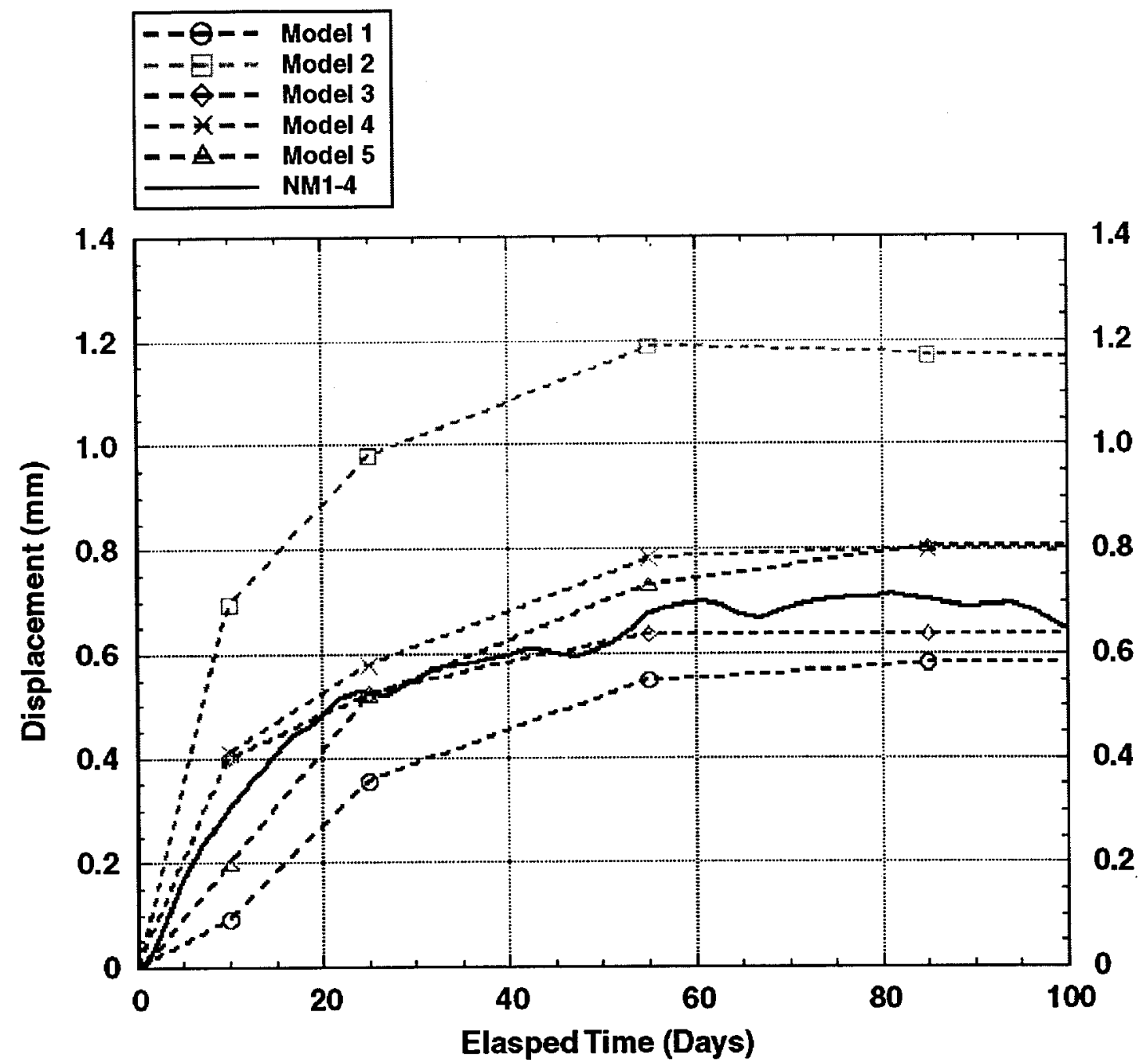

Figure 7-53. NM1 Anchor 4 Displacements and Model Results for the First 100 Days 


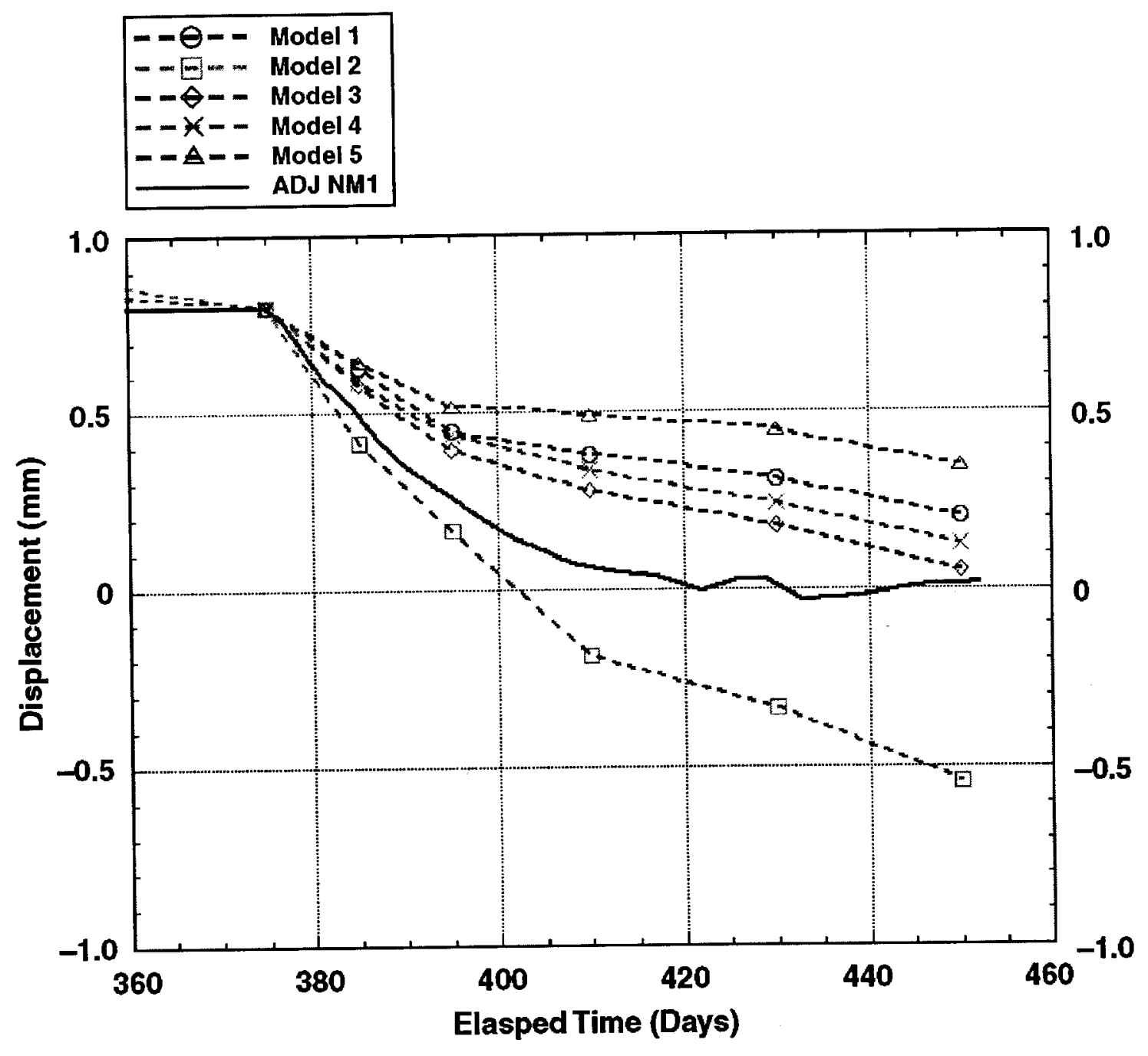

Figure 7-54. NM1 Anchor 4 Displacements and Model Results for the Cool-Down 


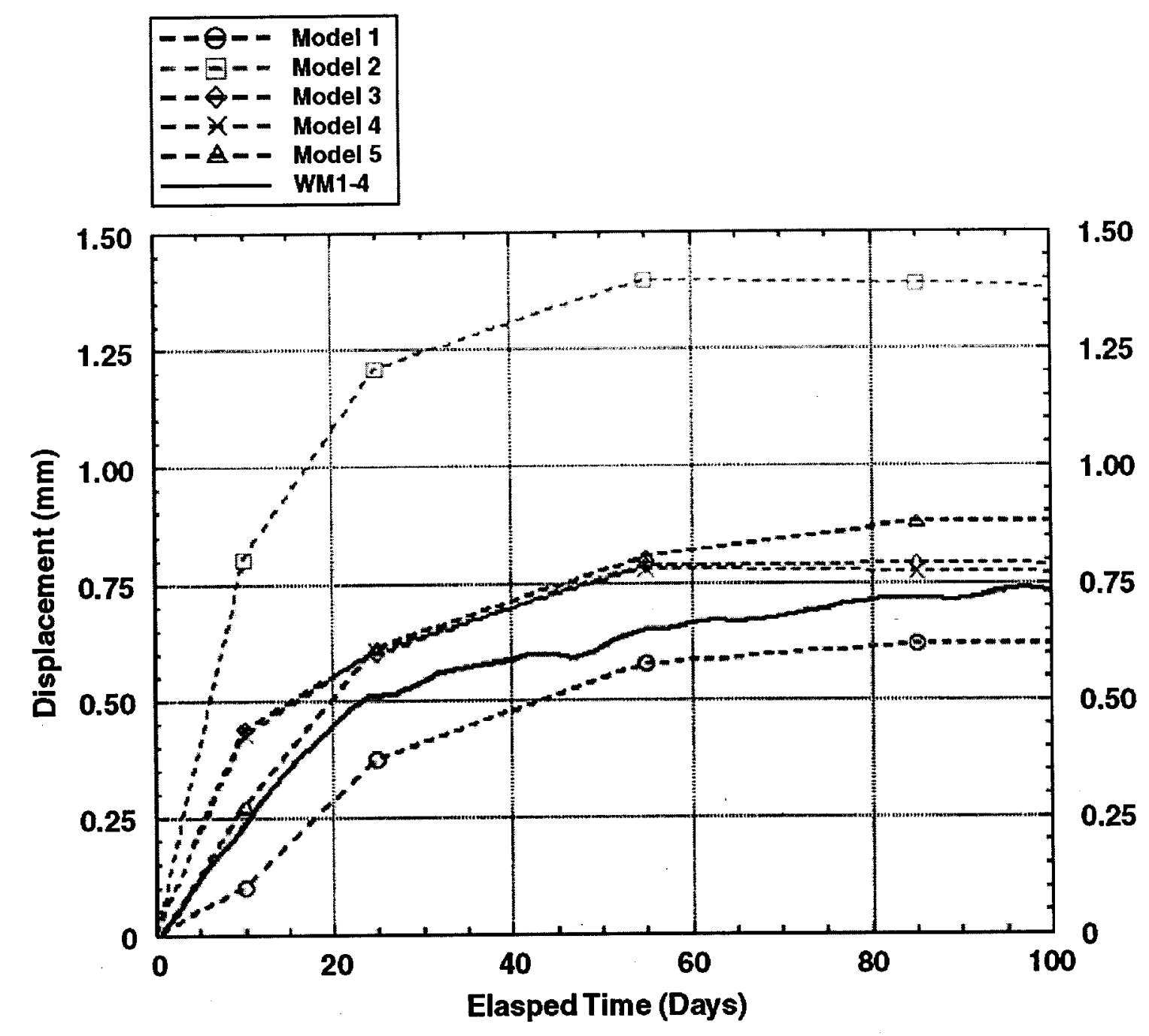

Figure 7-55. WM1 Anchor 4 Displacements and Model Results for the First 100 Days 


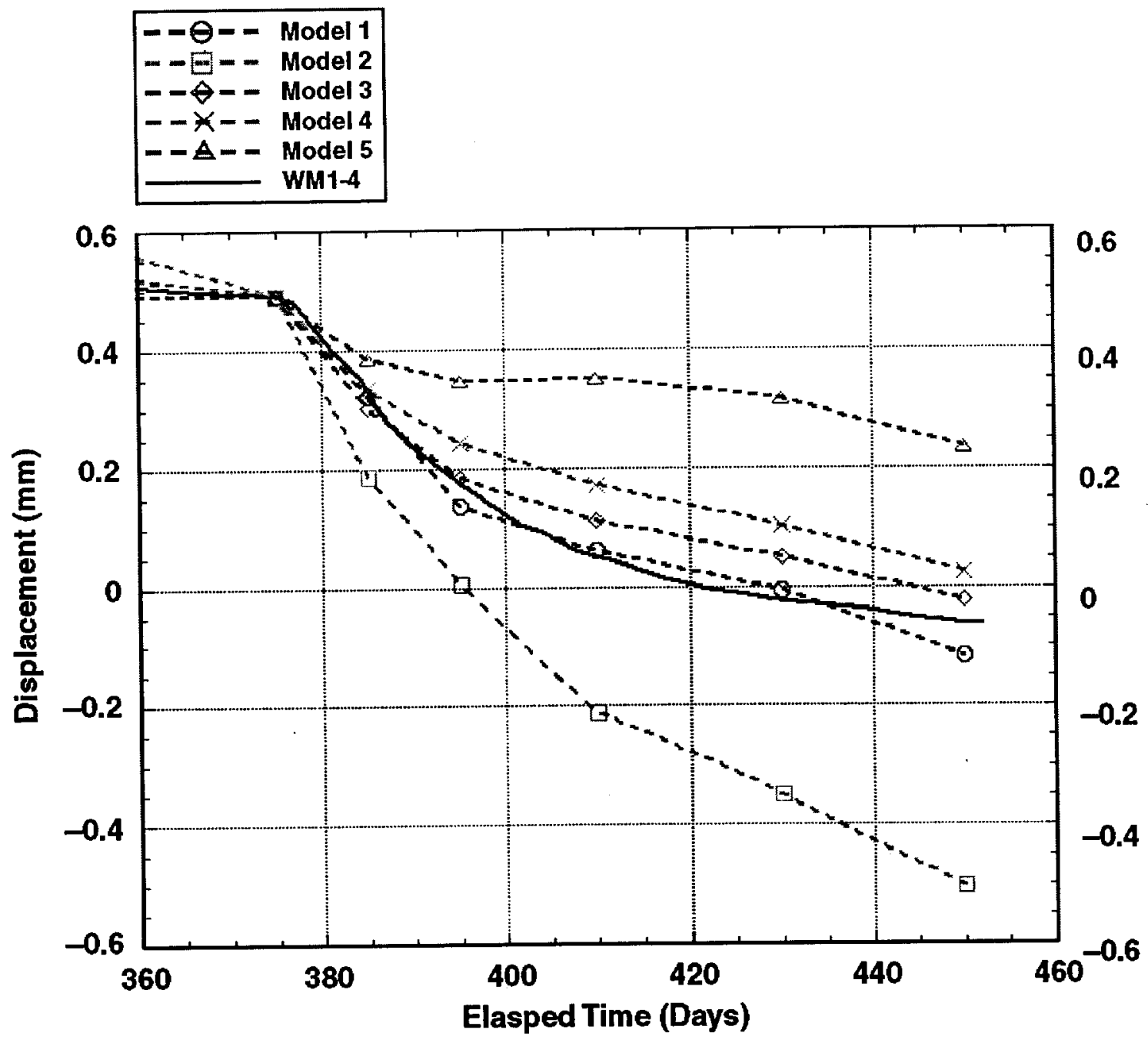

Figure 7-56. WM1 Anchor 4 Displacements and Model Results for the Cool-Down 


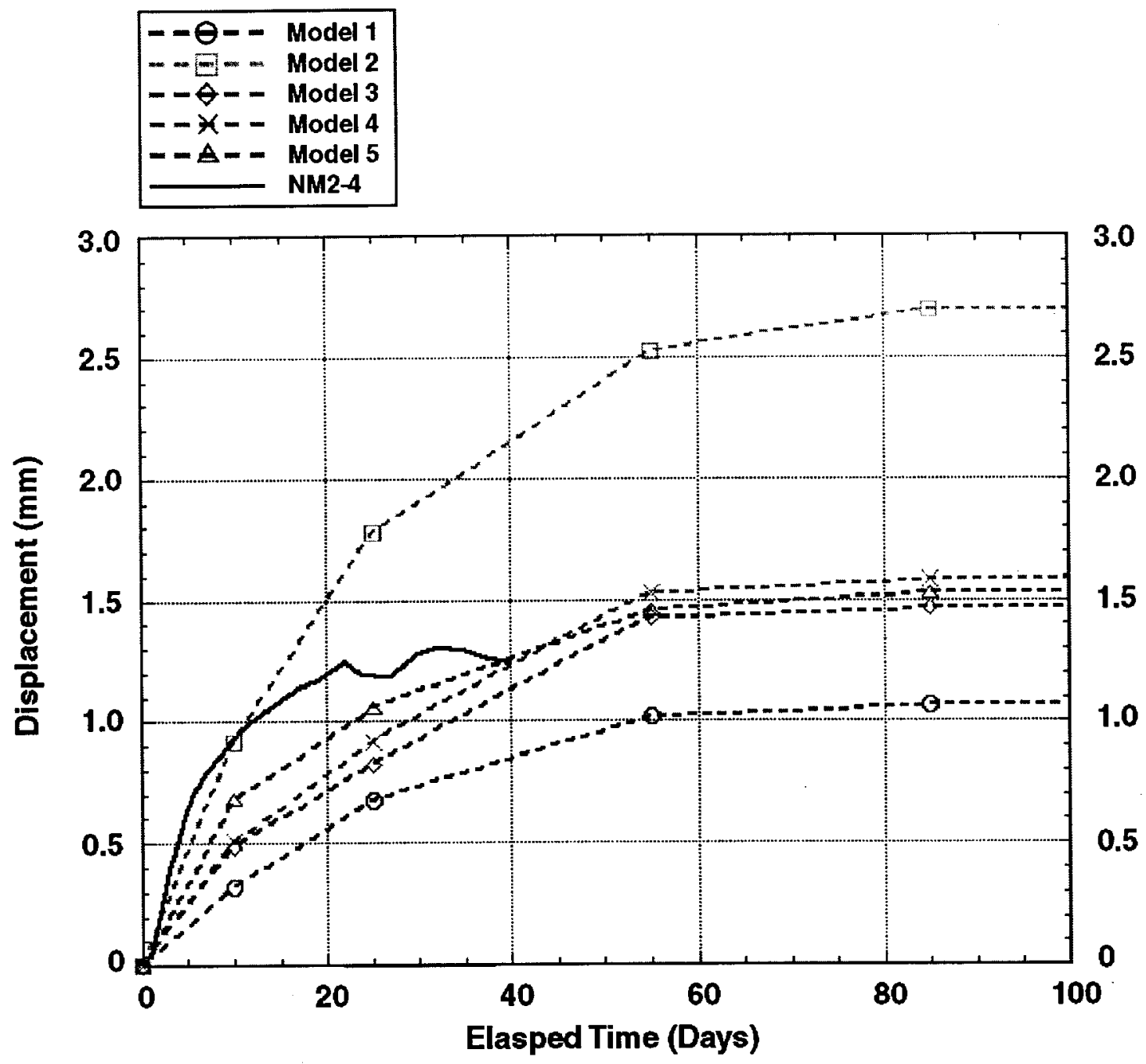

Figure 7-57. NM2 Anchor 4 Displacements and Model Results for the First 100 Days 


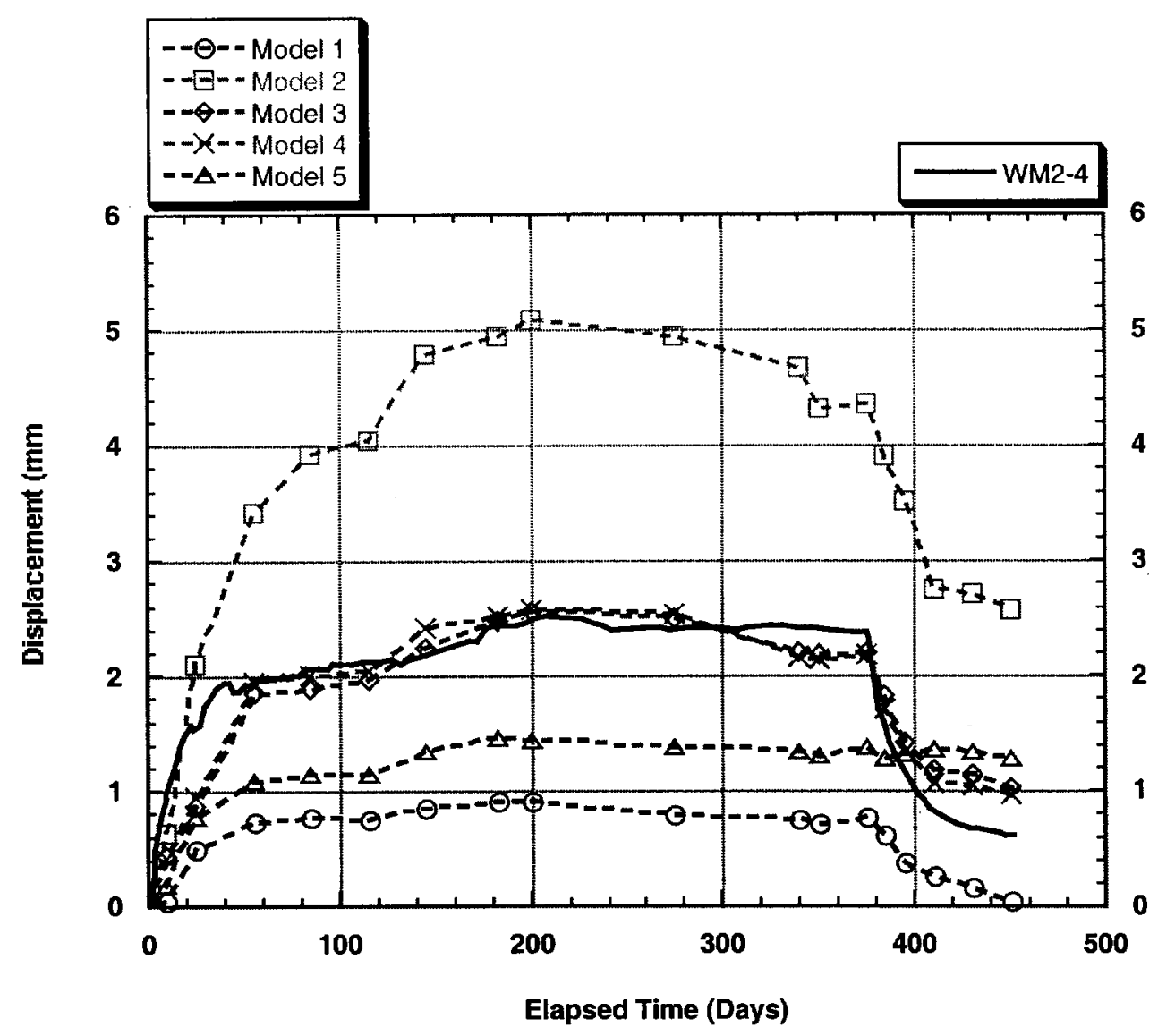

Figure 7-58. WM2 Anchor 4 Displacements and Model Results for the Entire Test 


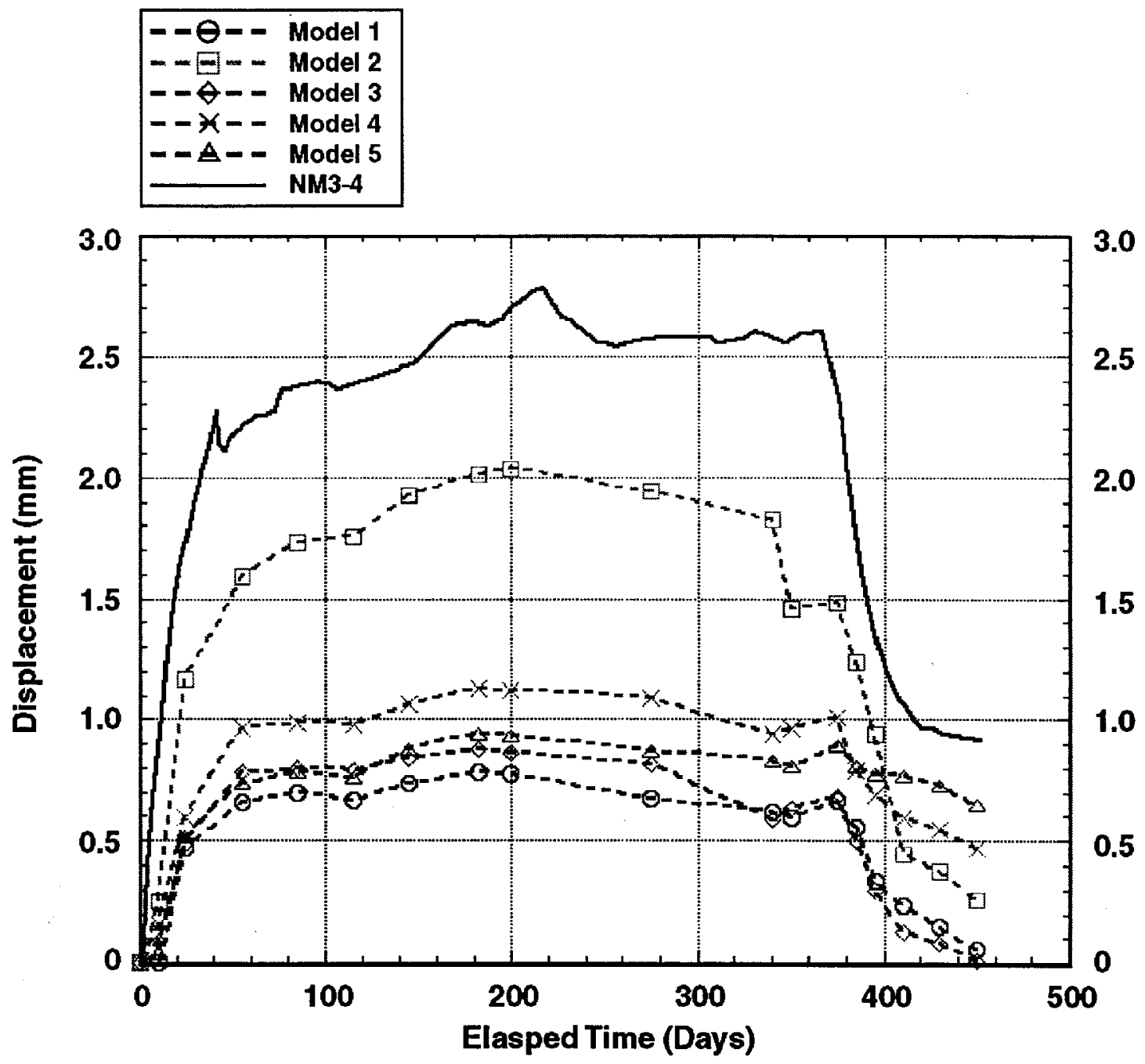

Figure 7-59. NM3 Anchor 4 Displacements and Model Results for the Entire Test 


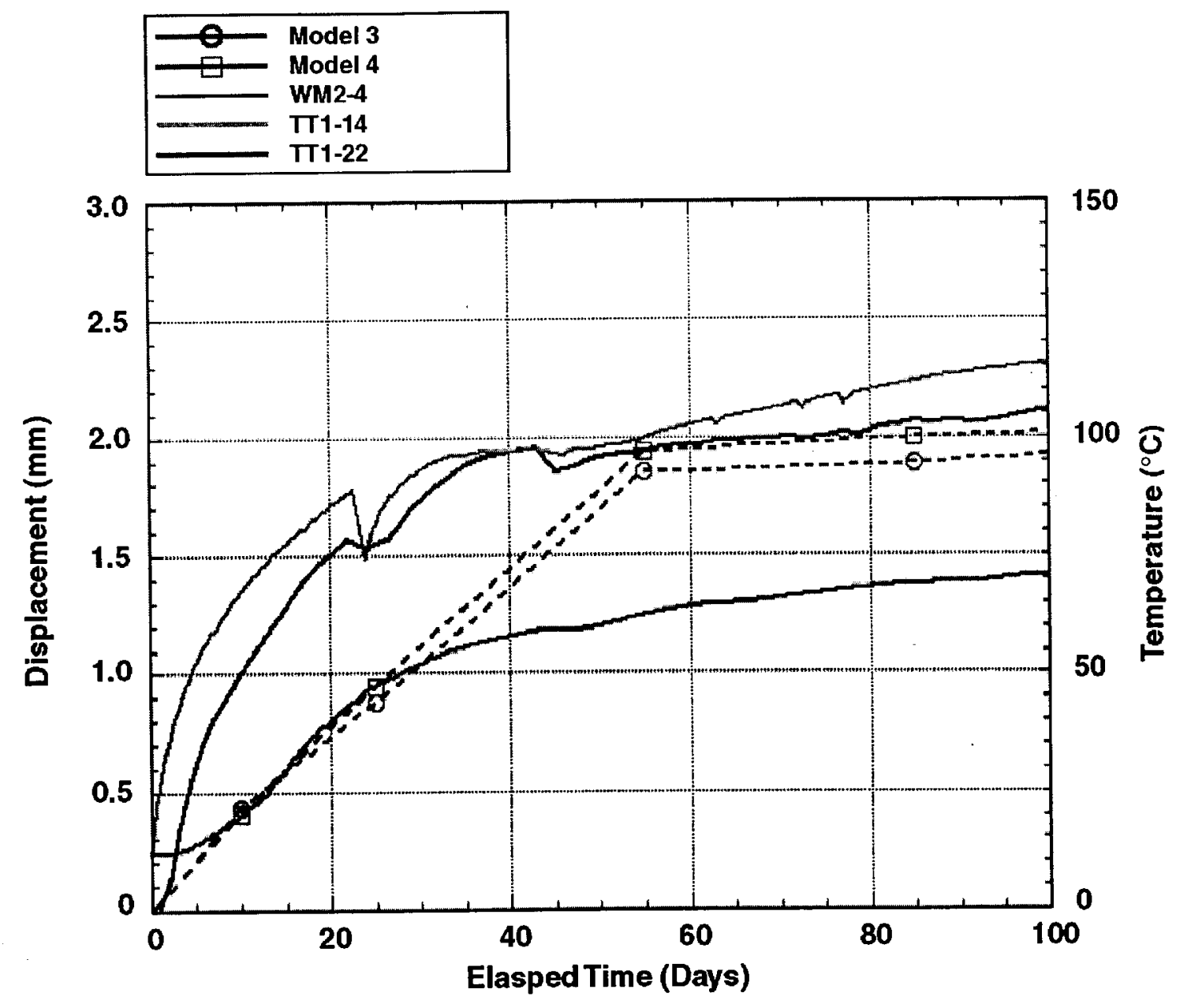

Figure 7-60. WM2 Anchor 4 Displacements, Heater Plane (TT1-14) and WM2-Level

(TT1-22) Temperatures and Results of Models 3 and 4 for the First 100 Days 


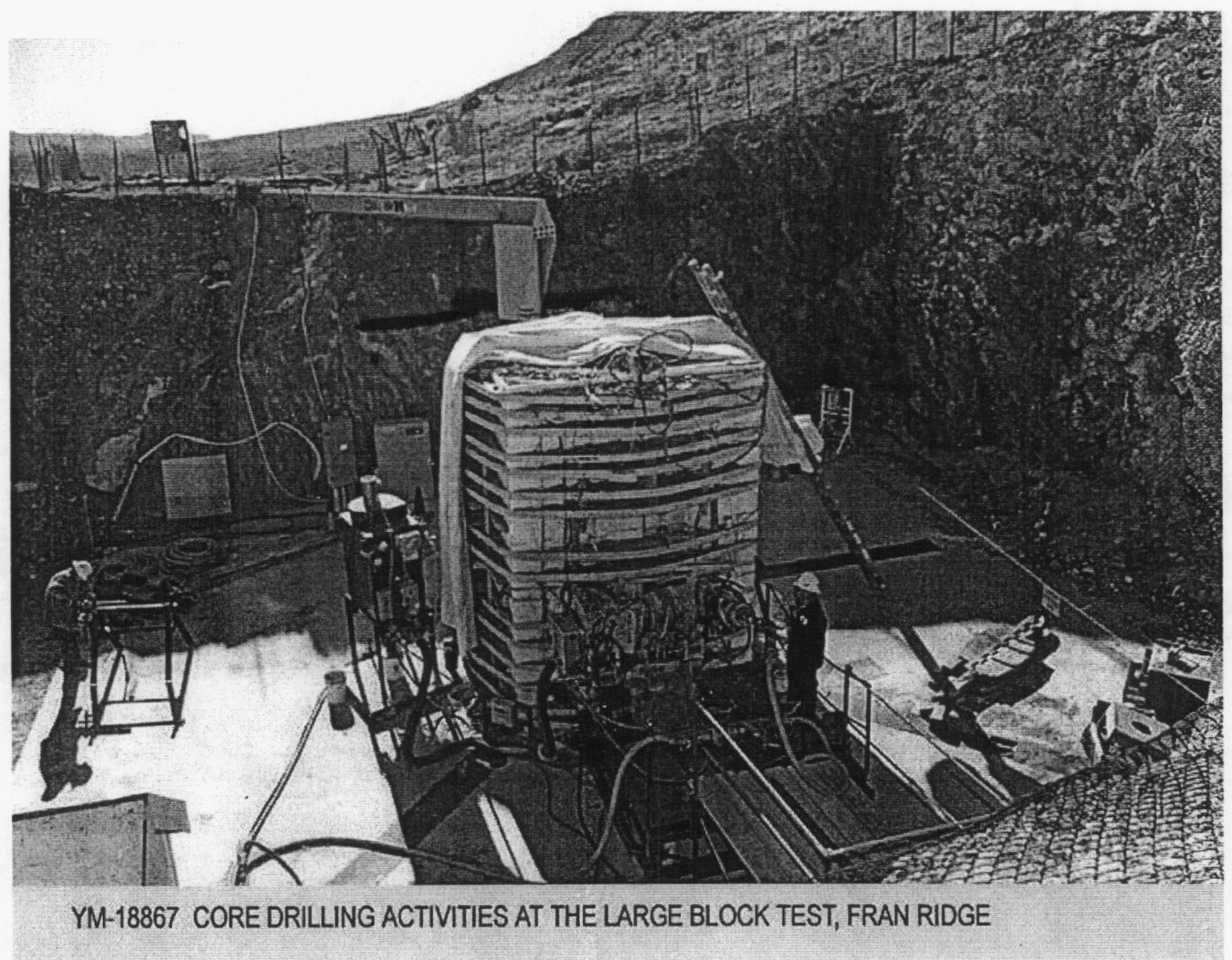

Figure 7-61. Post-Test Core Drilling Activities, Overview 


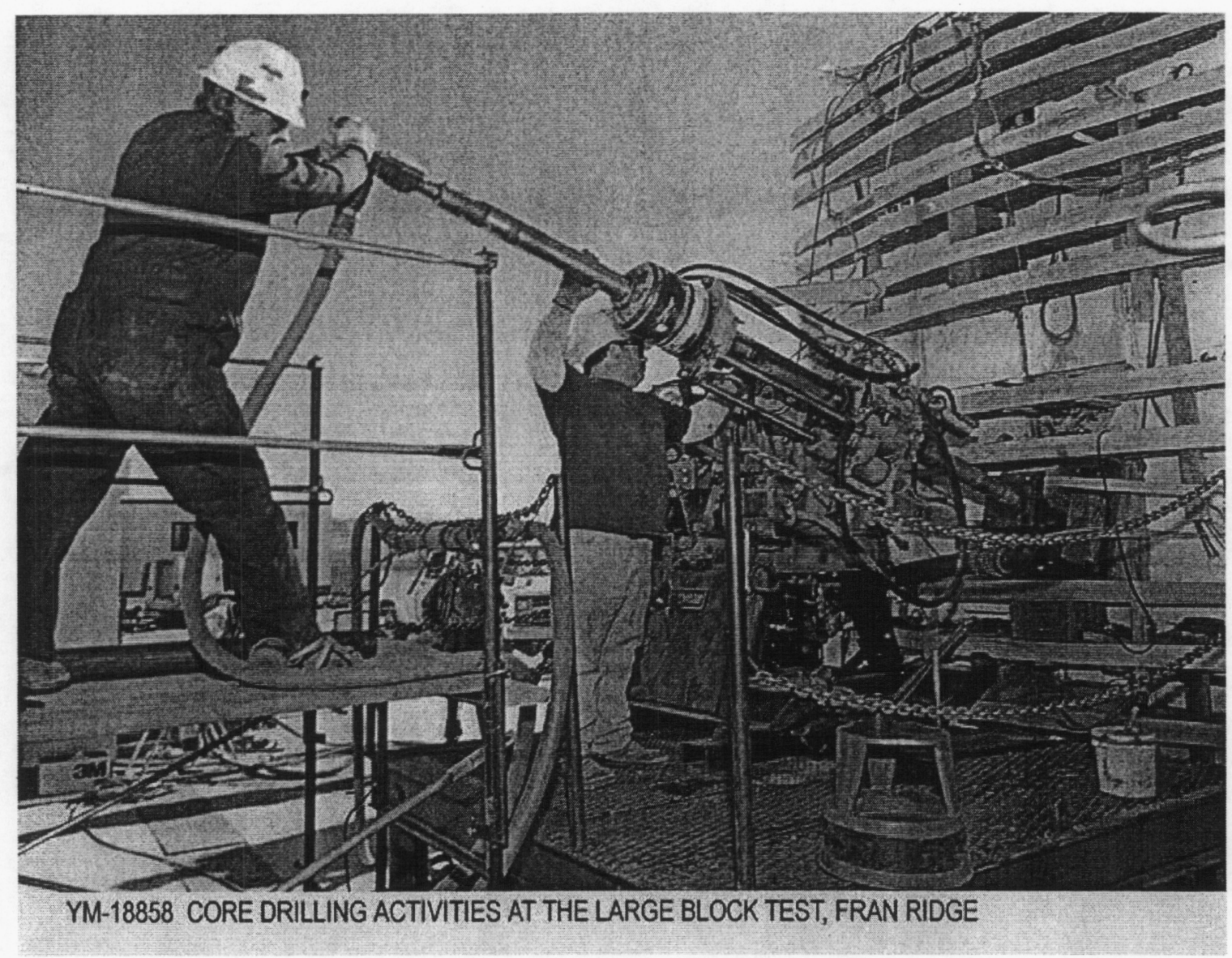

Figure 7-62. Post-Test Core Drilling Activities, Detail 

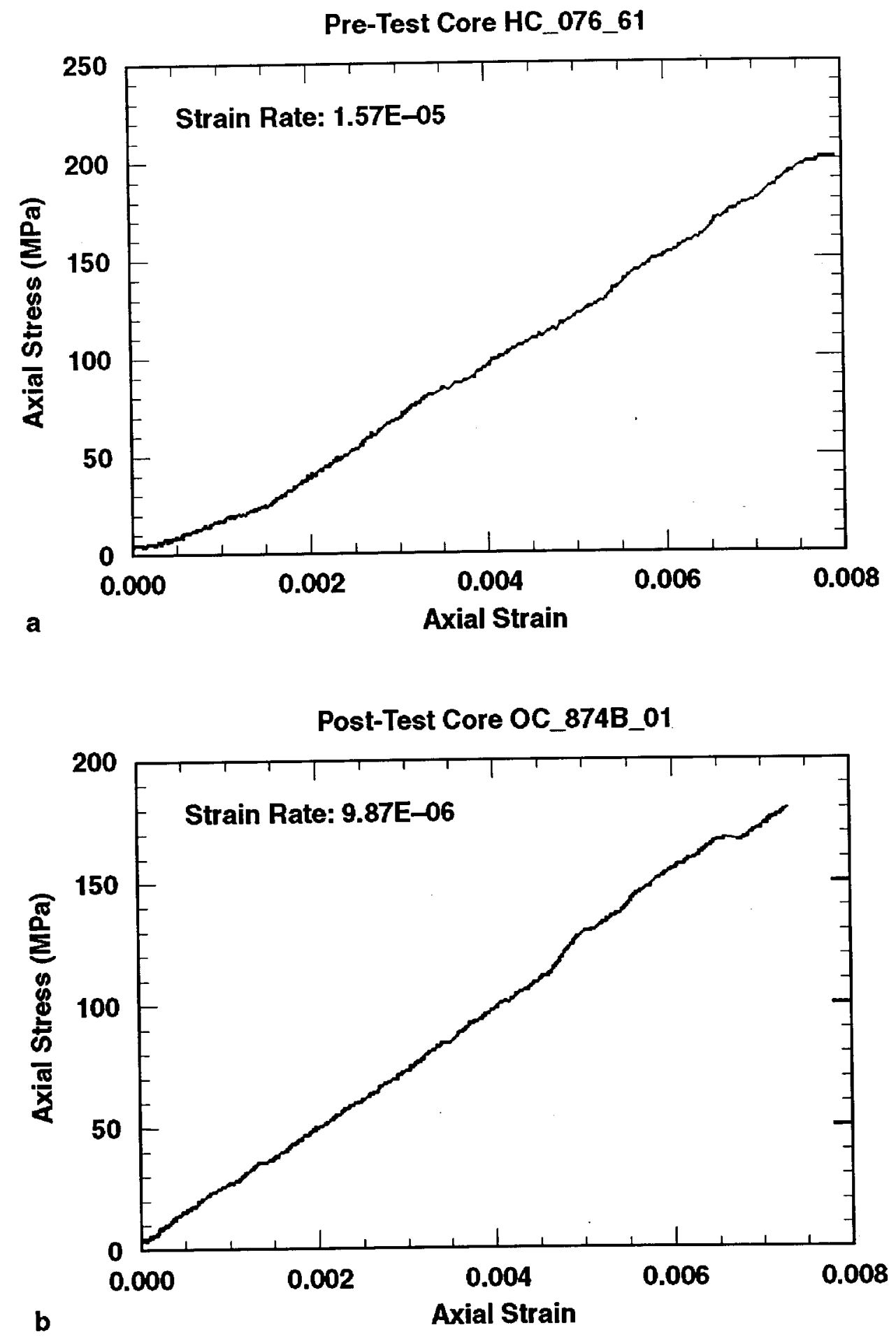

Figure 7-63. Representative Stress-Strain Plots for (a) Pre-test Core HC_076_61 and (b) Post-test Core OC_874B_01 


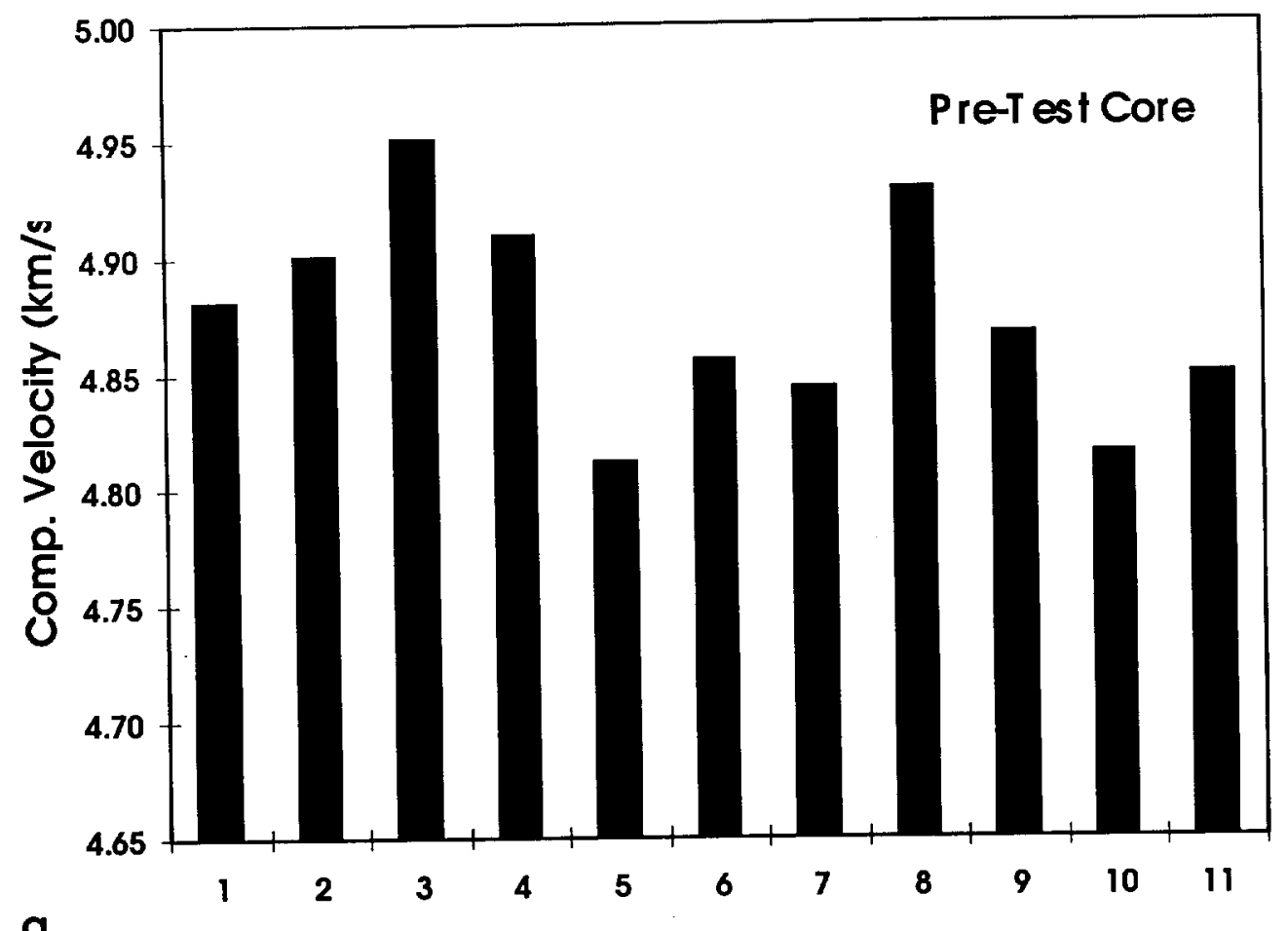

$a$

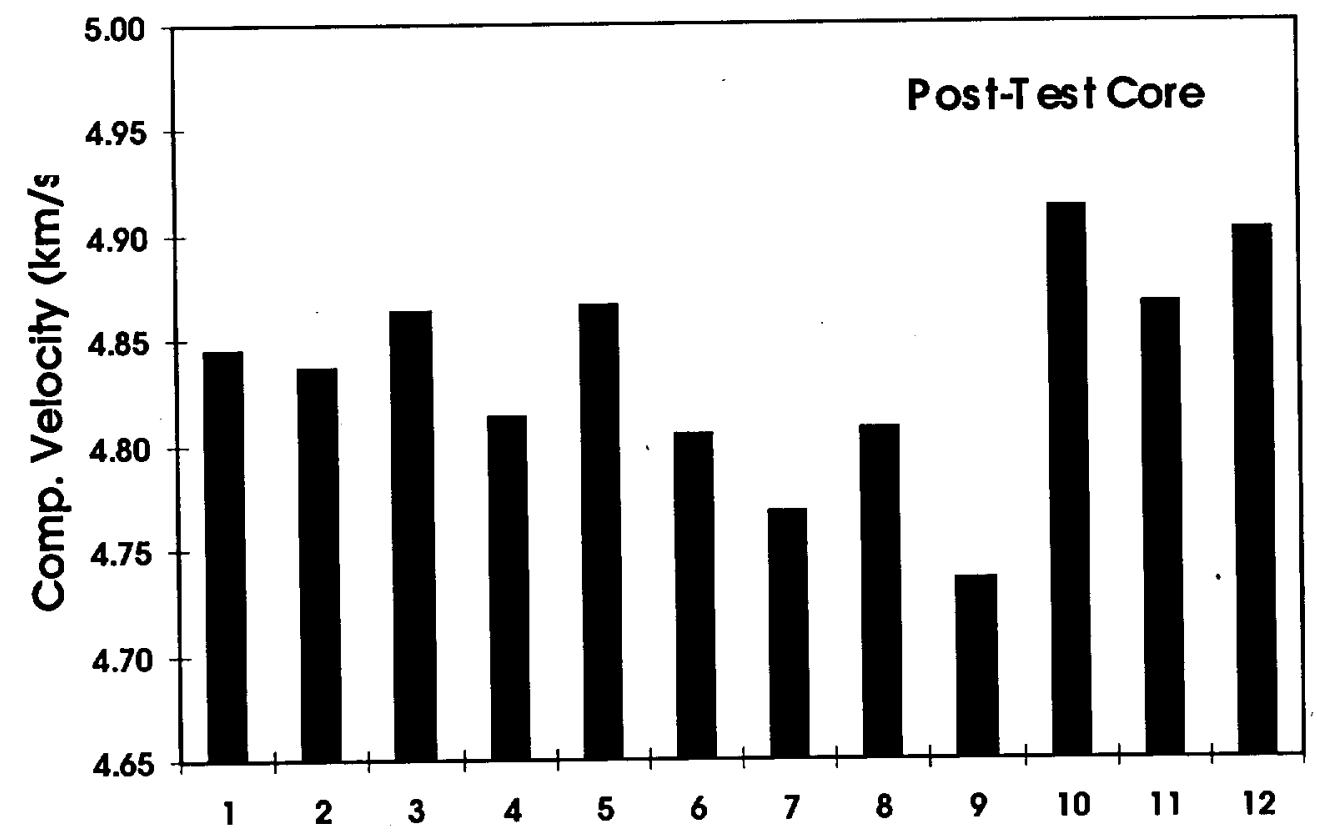

b.

Figure 7-64. Compressional Velocities for (a) Pre-test and (b) Post-test Specimens 

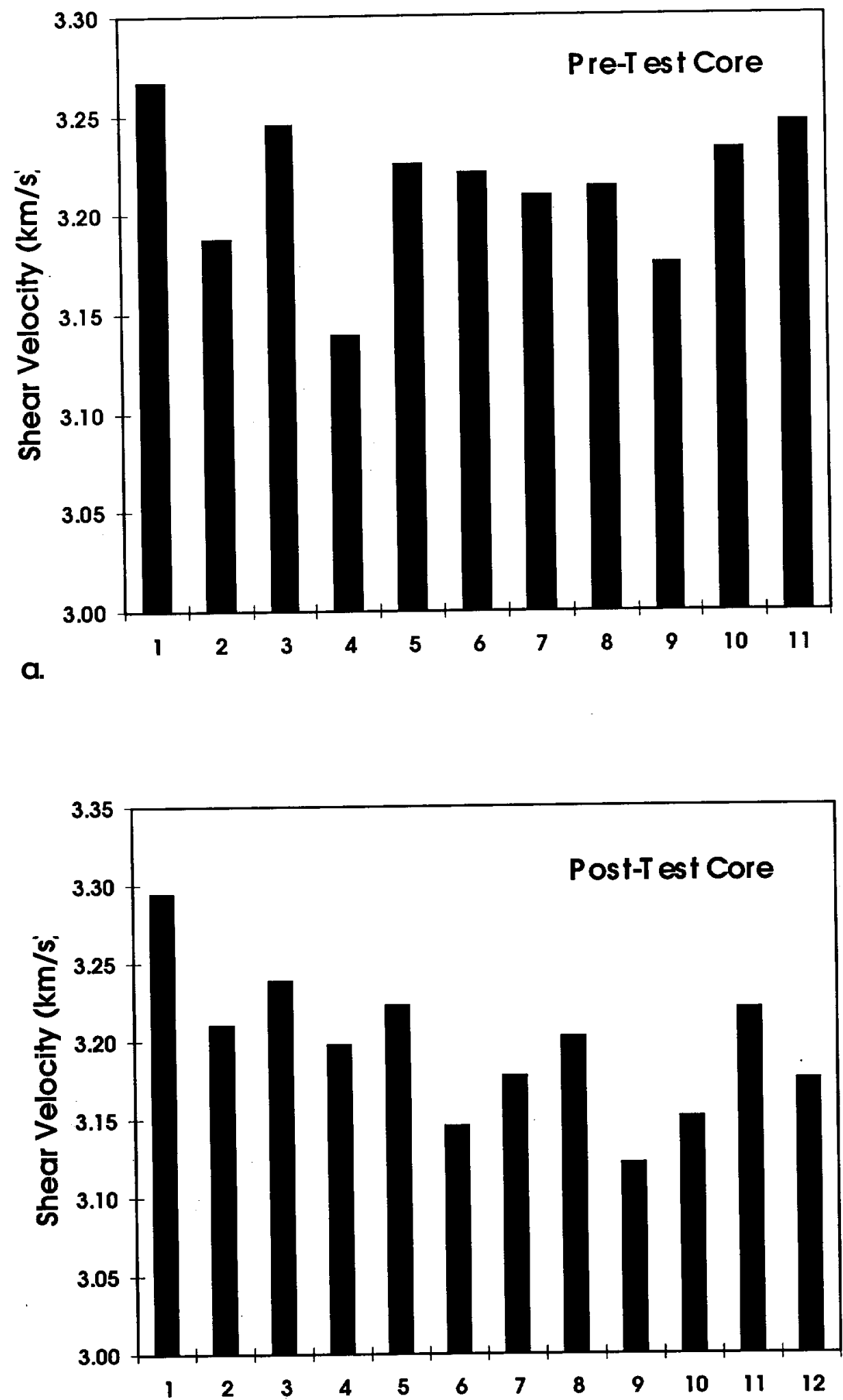

b.

Figure 7-65. Shear Velocities for (a) Pre-test and (b) Post-test Specimens 

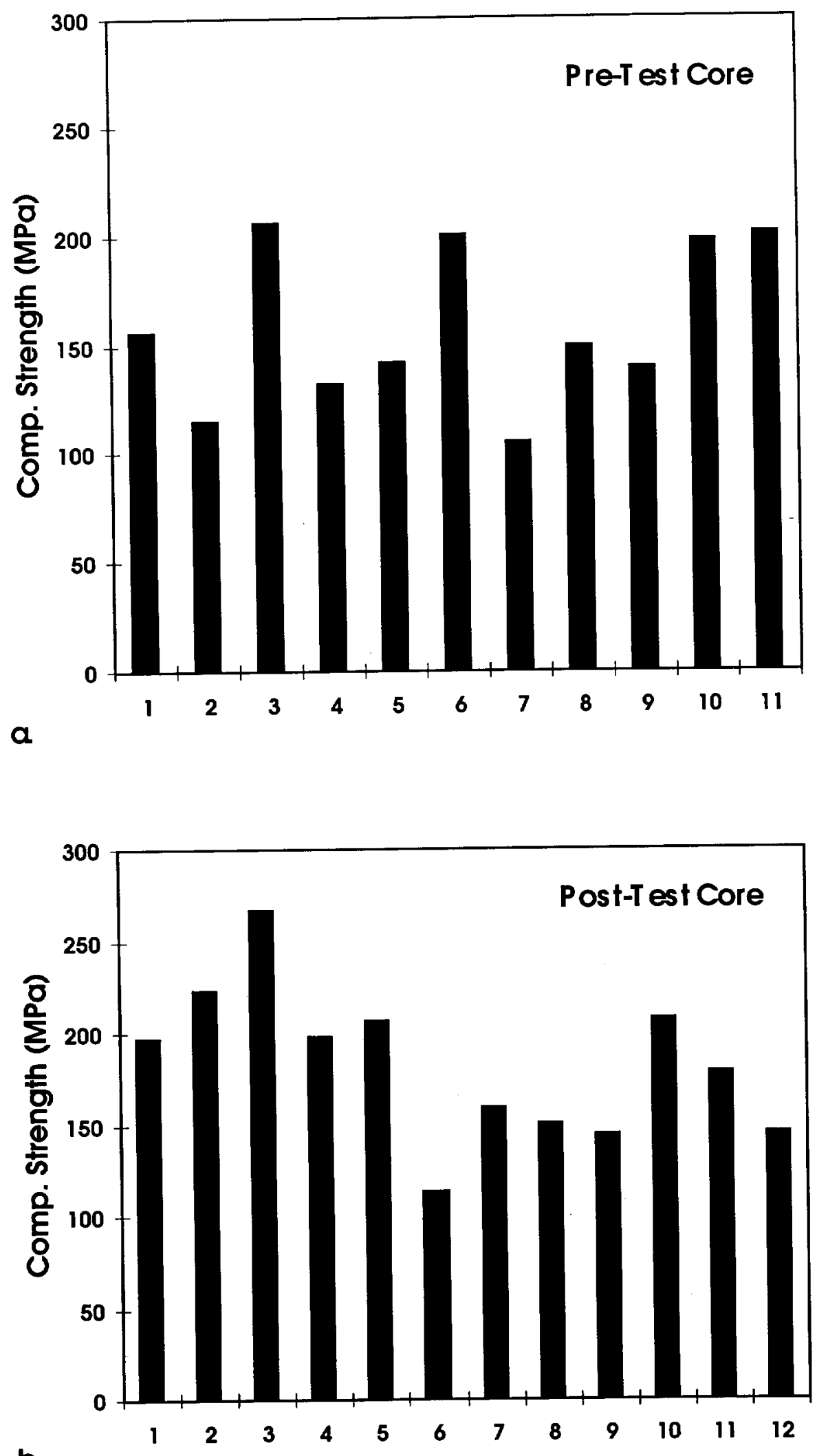

b.

Figure 7-66. Compressive Strength for (a) Pre-test and (b) Post-test Specimens 

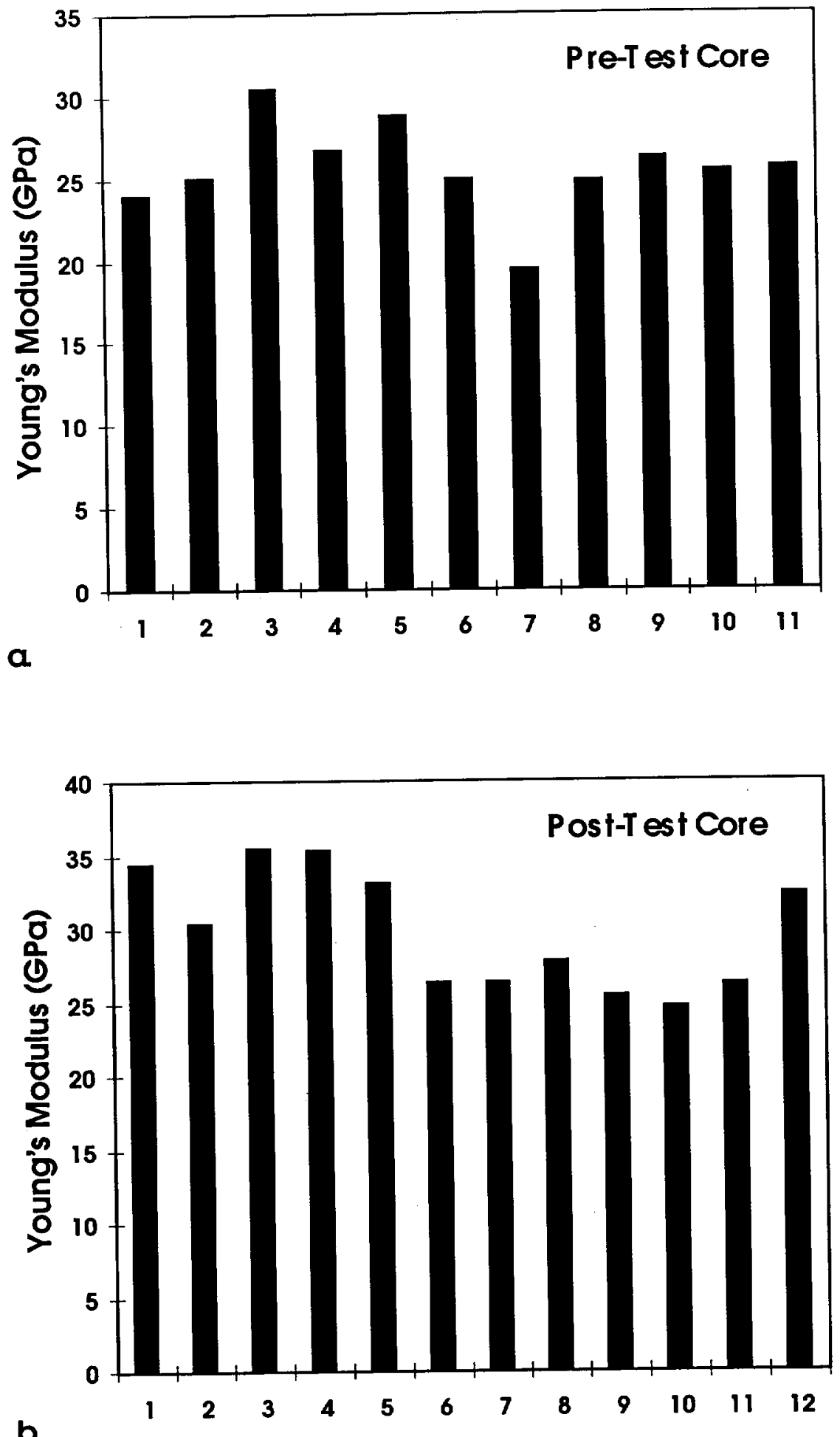

Figure 7-67. Static Young's Modulus for (a) Pre-test and (b) Post-test Specimens 
Topopah S pring T uff

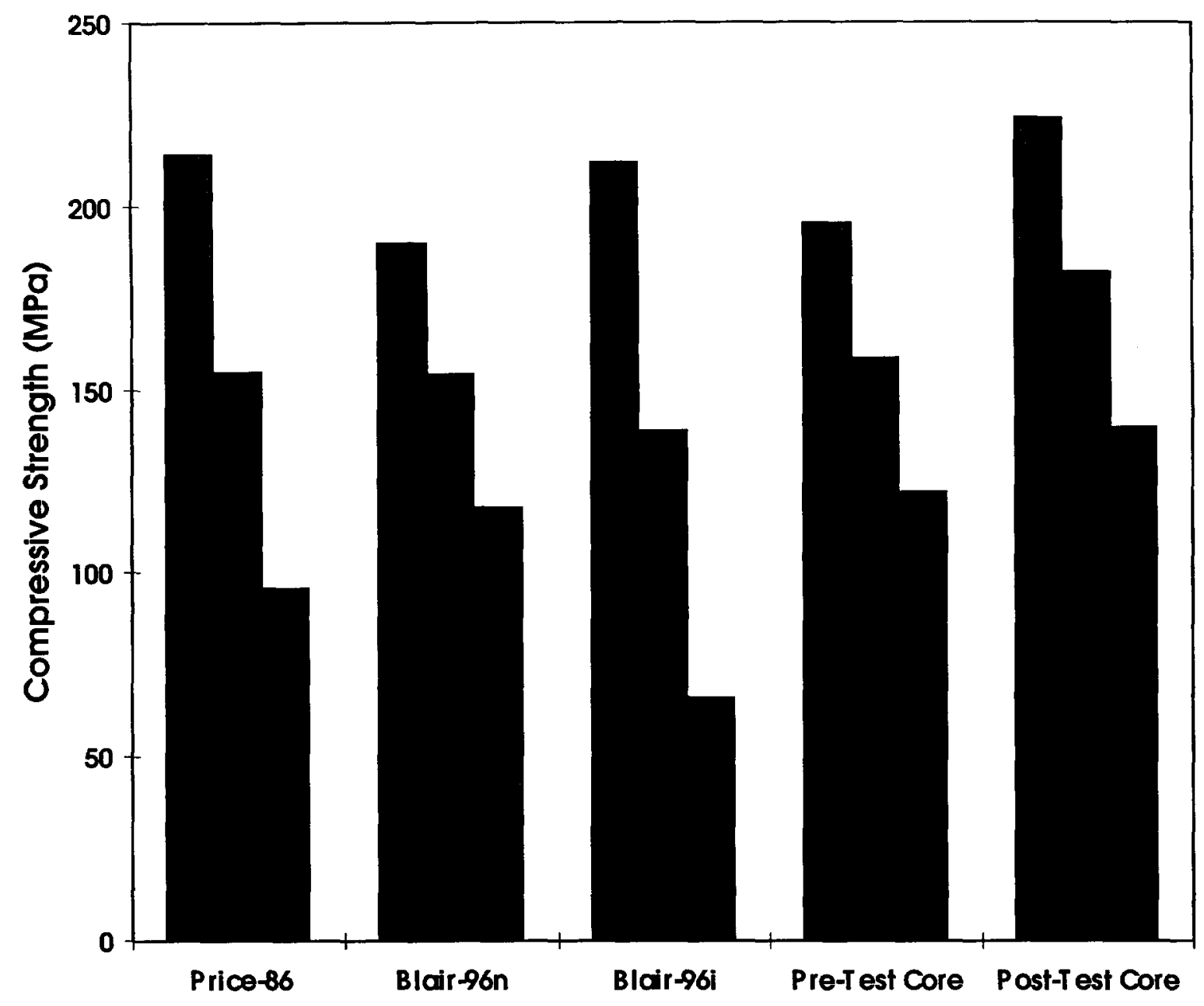

NOTE: Solid columns are mean values; stipled columns are mean values \pm one standard deviation. Price-86 data are from Price (1986). Blair-96n (nonirradiated) and Blair-96i (irradiated) data are from Blair, Kelly, et al. (1996).

Figure 7-68. Uniaxial Compressive Strength for Topopah Spring Tuff 


\section{Topopah S pring T uff}

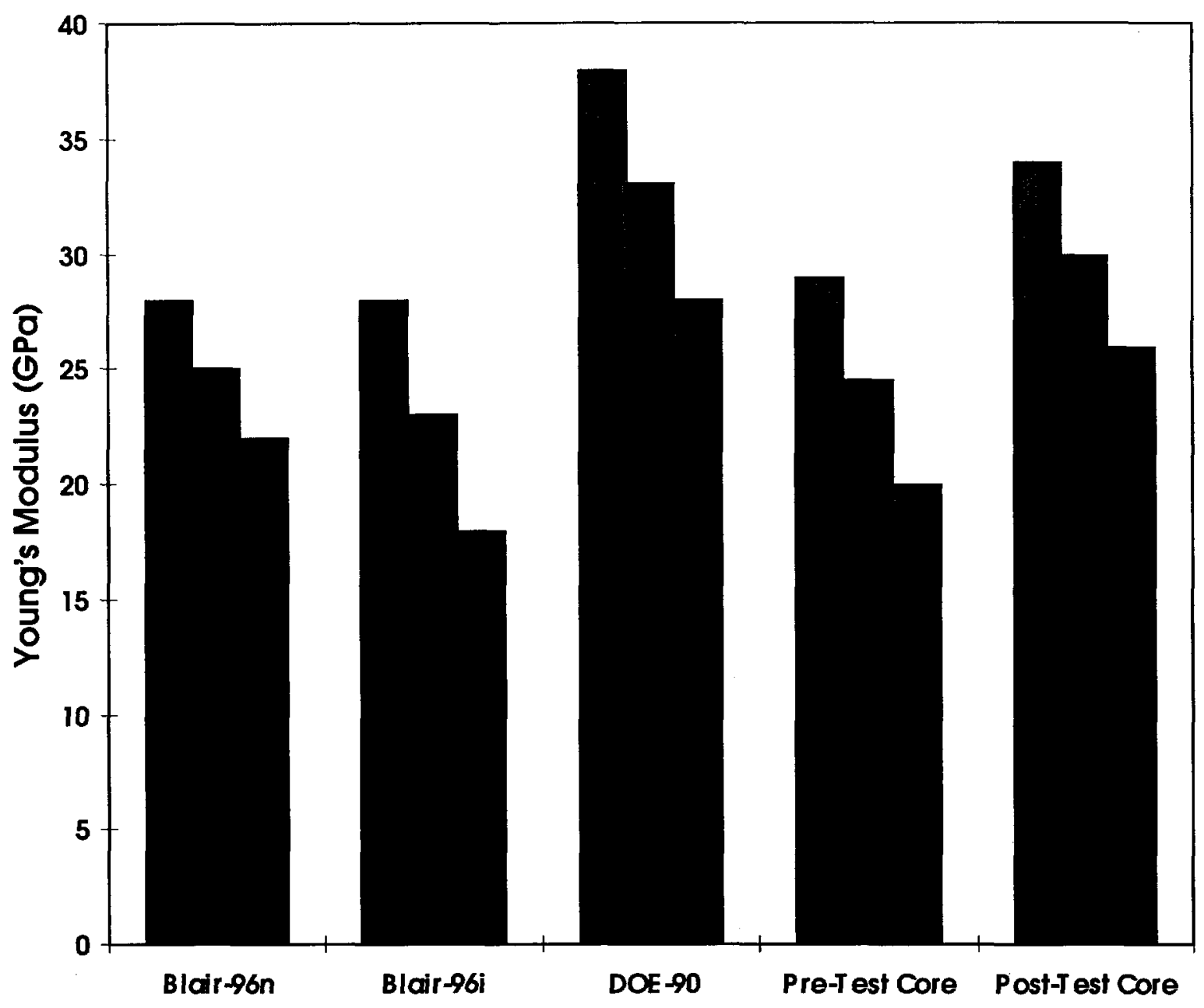

NOTE: Solid columns are mean values; stipled columns are mean values \pm one standard deviation. DOE-90 data are from Price (1986). Blair-96n (nonirradiated) and Blair,-96i (irradiated) data are from Blair, Kelly, et al. (1996).

Figure 7-69. Static Young's Modulus for Topopah Spring Tuff 
THIS PAGE INTENTIONALLY LEFT BLANK 


\section{ROCK-WATER INTERACTION IN THE LBT}

\subsection{INTRODUCTION}

The Topopah Springs tuff (Tptp) is actually one of several thick pyroclastic deposits within the unsaturated zone (UZ) beneath YM. Both its mineralogy and its alteration history have been studied as guides to the long-term physical and thermal stability of the site after possible waste emplacement. Useful summaries of the diagenesis of Yucca Mountain may be found in various project documents (for example, see the Yucca Mountain Site Description, TDR-CRW-GS000001 Rev.01, ICN.01, chp 5.2).

Compositionally, the Tptp unit (up to $380 \mathrm{~m}$ thick) is a compound volcanic series, variably zoned and graded from high silica rhyolite ascending to quartz latite. The interior is densely welded and would have developed in early stages of cooling-during viscous flow and glass compaction. Fracturing, faulting, and brecciation would also have developed while the tuff was cooling and also during tectonic events. After welding, the central core and vast majority of the unit devitrified into a fine-grained assemblage of alkali feldspar, silica polymorphs, and various accessory minerals. More rapid cooling of the upper and lower boundaries minimized compaction and devitrification processes to form instead thin glassy layers or vitrophyres at the transitions with over- and underlying units.

Extensive devitrification within the major portion of the Tptp layer was accompanied by localized alteration along fractures, within and below the devitrified zone. Identified in particular with the lower transitional boundary from basal vitrophyre to devitrified tuff, the alteration most likely resulted from fluid/mineral interactions at warm or near-ambient temperatures. The fluids were those present within the ash flow as well as percolating meteoric waters that would have preferentially flowed along the fractures and natural partings during the late stages of cooling (Levy and O'Neil 1989, p. 321). It is noteworthy that this syngenetic alteration, arising from the interaction of hot-rock and infiltrating waters, could be indicative of $\mathrm{TH}$ alterations that might develop in a hot repository environment. Presumably, the fracture system within the relatively nonporous rock of the Tptp had an important role in past (and present) fluid transport, and therefore in the geochemical changes that have occurred.

\subsubsection{Fracture Mineralogy in the Tptp}

The UZ fracture mineralogy of the Topopah Springs tuff reportedly comprises a diverse suite that reflects the nature and origin of geochemical variation. During cooling of the host pyroclastic flow, for example, vapor-phase minerals crystallized along the earliest formed fractures. Typical minerals to form were feldpars and silica polymorphs; but additionally amphibole, pseudobrookite, Mn-garnet, and andalusite developed in less abundance. Minerals that continued to form within the fractures long after cooling included zeolites, clays, Mn-oxides, opal, and calcite. Calcite and opal coatings reflect processes of deposition that have been nearly continuous along existing transport paths. The zeolite minerals, including mordenite, heulandite/clinoptilolite, and stellerite, are widespread in the UZ, although not particularly abundant in the fractures. Where stellerite $\left(\mathrm{CaAl}_{2} \mathrm{O}_{18} \bullet 7 \mathrm{H}_{2} \mathrm{O}\right)$ has been found, feldspar abundance is reduced, suggesting, at least in part, local alteration at the expense of feldspar. The upper and lower transition intervals between the devitrified Topopah Springs tuff and adjacent vitrophyres 
demonstrate an alteration transition from devitrification to glass dissolution and secondarymineral precipitation. In these intervals, significant zeolitization occurs along with clay and silica minerals.

Generally in the fractures of the volcanic tuffs under Yucca Mountain, the silica polymorphs, zeolites, and Mn-oxides are fairly ubiquitous; calcite, clay minerals, fluorite, and Fe-oxides occur with less frequency. For detailed studies of fracture minerals and their distributions, see examples of drill hole core characterizations as published in Carlos (1985) and Carlos et al. (1995).

\subsubsection{Test-Induced Geochemical Processes in the Large Block}

The late-stage syngenetic alteration localized along fractures in the lower intervals of the devitrified Tptp would presumably be useful as a self-analog to the thermally induced alteration of the LBT (and thermal field tests in general). The test conditions were intended to generate similar hydrothermal processes that might be expected from the emplacement of waste. Since evidence of these thermally induced processes would be concentrated where fluid/mineral interactions were greatest, the LBT postmortem characterizations focused primarily on samples collected from the existing fracture network. The test block as previously indicated was a relatively nonporous matrix of devitrified tuff with an extensive network of fractures.

Fluid and rock would interact within the fractures and openings as changing temperatures mobilized and/or redistributed pore fluids within the host block. During thermal testing, moisture redistribution was observed by various monitoring systems of the test as zones of dryout, condensation, and refluxing were formed within the block. Although matrix transport of the fluid is not altogether excluded, that process of fluid movement would presumably occur only in the dryout zone immediately surrounding the heaters, with much slower rates. Fluids would most likely have moved through the rock away from the heat source but eventually would have found fractures and migrated along openings in the cooler regions. In the zones of condensation and refluxing, more pronounced fluid/rock interactions would have been expected. Thermally induced geochemical processes occurring along fracture surfaces could have included dissolution and precipitation, hydration and dehydration, and phase transformations. Evidence of alteration would then be expected in extremely fine textures and volumetrically insignificant quantities of test product, both requiring microscopic methods of analysis and imaging.

\subsection{EVALUATION OF ALTERATION IN THE THERMAL TEST BLOCK}

\subsubsection{Large Block Mineralogical Characterization}

Because of the relatively short thermal periods of the test, evidence for geochemical alteration would most likely exist, for example, as finely textured surface pittings or deposits and minor quantities of alteration products. Sample characterizations would necessarily be over very small

surface areas, and analyses would be time-consuming. To augment the probability of observing such fine-scale geochemical changes, sample collection areas were prioritized and limited to fractures surfaces from zones where fluid/rock interactions were thought to be greatest.

Ideally one-to-one comparisons between pre-test and post-test samples would be studied, but sample acquisition of the pre-test pieces would exclude their comparison and use as post-test 
treated sample. A compromise and the best relative comparisons for before-and-after sampling were considered to be those made using archived, pre-test drilled core and post-test drilled overcore that could be aligned by position. The general idea would be to match the core and overcore along their lengths and circumference and look for microscopic changes between fracture surfaces that intersect both. Unfortunately, drilling restrictions placed on post-test coring and overcoring configurations effectively eliminated this sort of comparison. In addition, because of the highly fractured nature of the rock, the cores (and overcores that were collected) tended to break into multiple sections even over short archived segments, and alignments were difficult to ensure.

To proceed with mineralogical characterizations, representative fracture surfaces were collected from pre-test and post-test cores of the large block. Primary characterization was by the Scanning-Electron Microscope (SEM) to observe crystal morphology and to identify potential dissolution and/or precipitation features. An integrated Energy Dispersive X-ray (EDX) system was also employed to assess general chemical composition. Perhaps not surprisingly, conclusive evidence was not found to support mineralogical alteration attributed to thermal testing. Based on the suite of fracture samples studied, pre-test and post-test samples were similar to each other in mineralogy and textures. The dominant phases recognized included silica polymorphs, calcite, alkali feldspars, and Mn-oxide coatings. The findings were consistent with the Topopah Springs tuff fracture mineralogy reported throughout the literature.

\subsubsection{Sample Selection and Preparation}

Instead of the well-matched pre-test and post-test sample locations, comparison of representative before-and-after samples was conducted. Samples were selected from archived core of pre-testdrilled boreholes, those boreholes utilized for instrumentation and test monitoring. The thermally treated samples derived from post-test-drilled boreholes. All samples were requested through the Sample Management Facility (SMF) at the Nevada Test Site. Sampling locations were selected from areas reflecting higher moisture redistribution during the thermal test, and for which fracture mapping indicated prominent fracture intersections with post-test drilled holes. Finally, the remoteness of the post-test core was considered in regard to introduced and potentially chemically reactive materials-cementitious grout, for example.

\subsubsection{SEM and EDX Sample Preparation}

Core sections and sample designations are listed in Table 8-1 for the fracture filling minerals characterized using SEM. The first seven are pre-test samples and were selected from core sections having pronounced fractures, both well-sealed and open fractures. The last three samples listed in Table 8-1 were from post-test rock core. All samples listed were selected for identification of the fracture minerals only, and matrix was not considered. Samples were prepared in the same manner for both pre-test and post-test fractures: The core and fracture regions were surveyed using ultraviolet light, and most demonstrated some natural fluorescence. Although fluorescent colors and intensities may be characteristic of different minerals, the effects observed were generally weak; consequently, no attempt was made to develop this as a diagnostic tool. Certainly there were no clear observations made of post-test and pre-test differences. The selected core fractures were split using tungsten carbide hydraulic jaws, and then broken down again into approximately $1-\mathrm{cm}$ size chips, suitable for the SEM sample 
holders. In some samples in which fractures were more open, surface scrapings of the coatings were obtained for SEM study. Samples were mounted on SEM plugs using carbon tape, or simply held in a sample vice holder. Conductive carbon coatings were applied to reduce charge buildup that would otherwise interfere during imaging.

Table 8-1. Samples Selected for SEM Imaging and Analysis

\begin{tabular}{|c|c|c|c|}
\hline SMF sample no. & $\begin{array}{c}\text { Borehole } \\
\text { identification }\end{array}$ & Depth interval & Section examined \\
\hline SPC0039806 (pre) & UE25FR\#N6 & $19.4-20.5^{\prime}$ & $19.4^{\prime}$ \\
\hline SPC0039806 (pre) & UE25FR\#N6 & $19.4-20.5^{\prime}$ & $19.5^{\prime}$ \\
\hline SPC0039799 (pre) & UE25FR\#N4 & $6.8-8.4^{\prime}$ & $8^{\prime}$ \\
\hline SPC0039803 (pre) & UE25FR\#N7 & $19.3-20.6^{\prime}$ & $20.0^{\prime}$ \\
\hline SPC0039802 (pre) & UE25FR\#N4 & $8.1-9.8^{\prime}$ & $19.4^{\prime}$ \\
\hline SPC0032804 (pre) & UE25FR\#N1 & $19.4-21.0^{\prime}$ & $21.0^{\prime}$ \\
\hline SPC0039797 (pre) & UE25FR\#N5 & $18.0-19.8^{\prime}$ & $18.0^{\prime}$ \\
\hline SPC02015843 (pst) & UE25FRPTC\#18 & $5.0-6.1^{\prime}$ & $5.0^{\prime}$ \\
\hline SPC02015851 (pst) & UE25FRPTC\#17 & $1.1-1.8^{\prime}$ & $1.1^{\prime}$ \\
\hline SPC02015679 (pst) & UE25FRPTC\#11 & $3.7-4.5^{\prime}$ & $4.0^{\prime}$ \\
\hline
\end{tabular}

\subsubsection{SEM and EDX Results}

The SEM with integrated EDX system that has been used for this study is the field emission Hitachi S-4500. SEM photomicrographs were obtained using accelerating voltages down to $\sim 2$ $\mathrm{kV}$. X-ray voltages used for spectral analyses were $10-15 \mathrm{kV}$. At the accelerating voltages used for analysis, $\mathrm{X}$-ray signals are produced from silicates from a penetration depth of a few microns. Consequently, chemical analyses obtained from the $\mathrm{x}$-ray spectra are only qualitative for the identifications being made here, where fracture-coating minerals are in some cases a few microns down to submicron sizes. Where possible, mineral identification is based on both morphology and the qualitative analyses. The findings from the SEM imaging and the EDX analyses are all considered non-Q.

SEM results reveal that the typical fracture lining minerals in the large block are silica polymorphs, Mn-oxides, and calcite, and less commonly feldspars or some other more complex silicate. A fibrous, matted mineral coats the surfaces of grains, and a larger ribbon-like fiber mineral is seen bridging pore spaces. Both are found in pre-test and post-test samples and could possibly represent clay phases. Figures 8-1 through 8-8 are representative photomicrographs from various pre-test fracture sample surfaces. In each case, an x-ray spectrum generally corresponding to the imaged location is also displayed. In addition to the compositional information, visual examination of textural features was made. Photomicrographs were made of areas showing dissolution in both pre-test and post-test samples. Figures 8-9 through 8-11 appear to show dissolution features in various grains. Similar surfaces were observed in both pre-test samples and post-test samples, and no real distinctions were evident in the textural features to suggest alteration related to the thermal test. Figures 8-12 through 8-14 are the fibrous minerals that have been observed in before and after samples. Figure 8-14 is a post-test sample that also shows a variety of the fracture coating mineralogy in one small area. 


\subsubsection{X-Ray Diffraction Analyses}

\subsubsection{Introduction}

Geochemical process in the heated partially saturated rock mass is mainly due to the rock-water interaction at elevated temperatures. The results of the rock-water interaction include alteration of the minerals involved. In the LBT, the geochemical process was to be investigated by determining the changes in the mineralogy in the block due to heating. Post-test drilling was conducted to recover cores at locations where possible rock-water interactions might have occurred. X-ray diffraction (XRD) analyses were conducted on the pre-test and the post-test samples to assess the mineralogical alterations. It should be noted that pre-test and post-test samples cannot be obtained at the same location; therefore, the comparison is qualitative at best. Because the main purpose of the comparison is to assess the effect of the test on the alteration in the mineralogical composition of the block, only the qualitative XRD analysis, using Jade software, was performed. The XRD analysis is considered non-Q because the software used in the analyses, Jade v.3.1 is not qualified yet.

\subsubsection{Measurements}

A total of 19 LBT samples were $x$-rayed and analyzed. These 19 samples are listed in Table 8-2 with their computer filenames as well as their borehole and depth identifiers. The first eight samples were pre-test bulk samples. The next seven samples, 9-15, were post-test bulk samples. The final four samples, 16-19, were pre-test "fracture" samples. It was very difficult to obtain enough post-test fracture sample for $\mathrm{x}$-ray analysis. Sample \#10 in Table 8-1 was a post-test bulk sample with some fracture minerals. All samples were crushed in a tungsten carbide spexring-mill and then sieved to 100-200 mesh using a stainless steel sieve, then further pulverized to less than $10 \mu \mathrm{m}$ with a vibratory mill in a sintered corundum bowl and ball before being placed into a stainless steel holder for XRD analysis. (In a few of the resulting patterns, there is a diffraction peak that appears to be associated with the stainless steel holder used in these measurements.)

A qualitative characterization was done for all 19 LBT samples using Jade v.3.1 XRD patternprocessing and identification software. The powder pattern data for each sample was imported into Jade and corrected for peak positioning using a 2-theta calibration that resulted from the measurement of a National Institute of Standards and Technology (NIST) traceable standard made close in time to the unknown sample's measurement. The resulting pattern was then background subtracted before using the search/match function in Jade to identify possible mineral phases in the powder pattern. The resulting hit list was sorted by figure of merit. The first 10 or 20 patterns listed, those with the lowest figure-of-merit scores, were then visually compared with the pattern from the unknown sample. The fewest number of minerals that when combined best reproduced the sample pattern visually were then plotted and printed. Those minerals that visually reproduced the sample pattern the best are listed in Table 8-2 for each of the 19 LBT samples. The minerals identified are listed horizontally from the lowest to highest figure-of-merit score.

Generally, the "bulk" solids from both the pre- and post-test samples appear to be mixtures of $\mathrm{SiO}_{2}$ polymorphs-i.e., quartz and cristobalite-and various forms of feldspar minerals. The 
patterns of the final four samples, the "fracture" samples, were different from the first 15 LBT samples. All four appear to be mixtures of calcite and quartz. In one of the fracture samples, $\# 16$, there appears to be some feldspar that was best identified as sanidine. In another fracture sample, $\# 17$, there appears to be some corundum, but this may very well be a contamination that resulted from its being processed in the laboratory, because corundum was used as a quantification tracer for quantitative $x$-ray analyses. The results indicate that there was no significant change in the mineralogy of the matrix of the rock mass between the pre-test samples and the post-test samples. This is expected, because most of the rock-water interaction was expected to be along fractures. Due to sampling limitations, the change in the mineralogical composition in the fracture surface mineral was not conclusive.

Table 8-2. Qualitative Results for the 19 Solid Samples from the Large Block Test Resulting from Analyses using Jade Software Package.

\begin{tabular}{|c|c|c|c|c|c|c|c|c|c|c|}
\hline & & & & & \multicolumn{6}{|c|}{ In order of LOWEST "figure-of-merit" (FOM) } \\
\hline & & & & & 1 & 2 & 3 & 4 & 5 & 6 \\
\hline No. & $\begin{array}{l}\text { Computer } \\
\text { file name }\end{array}$ & Sample \# & Borehole \# & $\begin{array}{c}\text { Depth } \\
\text { from } \\
\text { collar (m) }\end{array}$ & & & & & & \\
\hline 1 & 39792 & SMF039792 & UE25FR\#E10 & 5.304 & quartz & cristobalite & anorthite & sanidine & albite & \\
\hline 2 & 39800 & SMF039800 & UE25FR\#N3 & 3.018 & quartz & microcline & anorthite & & & \\
\hline 3 & 39796 & SMF039796 & UE25FR\#N4 & 1.036 & cristobalite & sanidine & anorthite & quartz & & \\
\hline 4 & 39805 & SMF039805 & UE25FR\#N6 & 0.213 & quartz & anorthite & orthoclase & albite & & \\
\hline 5 & 39799 & SMF039799 & UE25FR\#N6 & 2.286 & cristobalite & quartz & microcline & anorthite & albite & \\
\hline 6 & 39797 & SMF039797 & UE25FR\#N6 & 5.608 & sanidine & cristobalite & orthoclase & anorthite & quartz & \\
\hline 7 & 39804 & SMF039804 & UE25FR\#N7 & $\overline{3.353}$ & quartz & anorthite & orthoclase & & & \\
\hline 8 & 39803 & SMF039803 & UE25FR\#N7 & 6.37 & crisobalite & quartz & orthoclase & anorthite & sanidine & albite \\
\hline 9 & $2679 \_1$ & SPC2015679 & UE25FRPTC\#11 & 1.128 & quartz & orthoclase & albite & anorthite & & \\
\hline 10 & SPC679 & SPC2015679 & UE25FRPTC\#11 & 1.219 & quartz & sanidine & orthoclase & albite & anorthite & \\
\hline 11 & $2679 \_2$ & SPC2015679 & UE25FRPTC\#11 & 1.372 & cristobalite & orthoclase & albite & quartz & anorthite & microcline \\
\hline 12 & $2851 \_1$ & SPC2015851 & UE25FRPTC\#17 & 0.335 & quartz & orthoclase & sanidine & anorthite & & \\
\hline 13 & $2851 \_2$ & SPC2015851 & UE25FRPTC\#17 & 0.549 & quartz & orthoclase & sanidine & anorthite & albite & \\
\hline 14 & $2843 \_1$ & SPC2015851 & UE25FRPTC\#17 & 1.524 & sanidine & cristobalite & albite & microcline & quartz & \\
\hline 15 & $2843 \_2$ & SPC2015843 & UE25FRPTC\#18 & 1.829 & quartz & anorthite & orthoclase & & & \\
\hline 16 & $39804 \mathrm{~A}$ & SMF039804 & UE25FR\#N1 & 5.913 & calcite & quartz & sanidine & & & \\
\hline 17 & $39802 \mathrm{~A}$ & SMF039802 & UE25FR\#N4 & 2.743 & calcite & corundum & quartz & & & \\
\hline 18 & $39799 A$ & SMF039799 & UE25FR\#N6 & 2.438 & calcite & quartz & & & & \\
\hline 19 & $39806 \mathrm{~A}$ & SMF039806 & UE25FR\#N6 & 5.913 & calcite & quartz & & & & \\
\hline
\end{tabular}




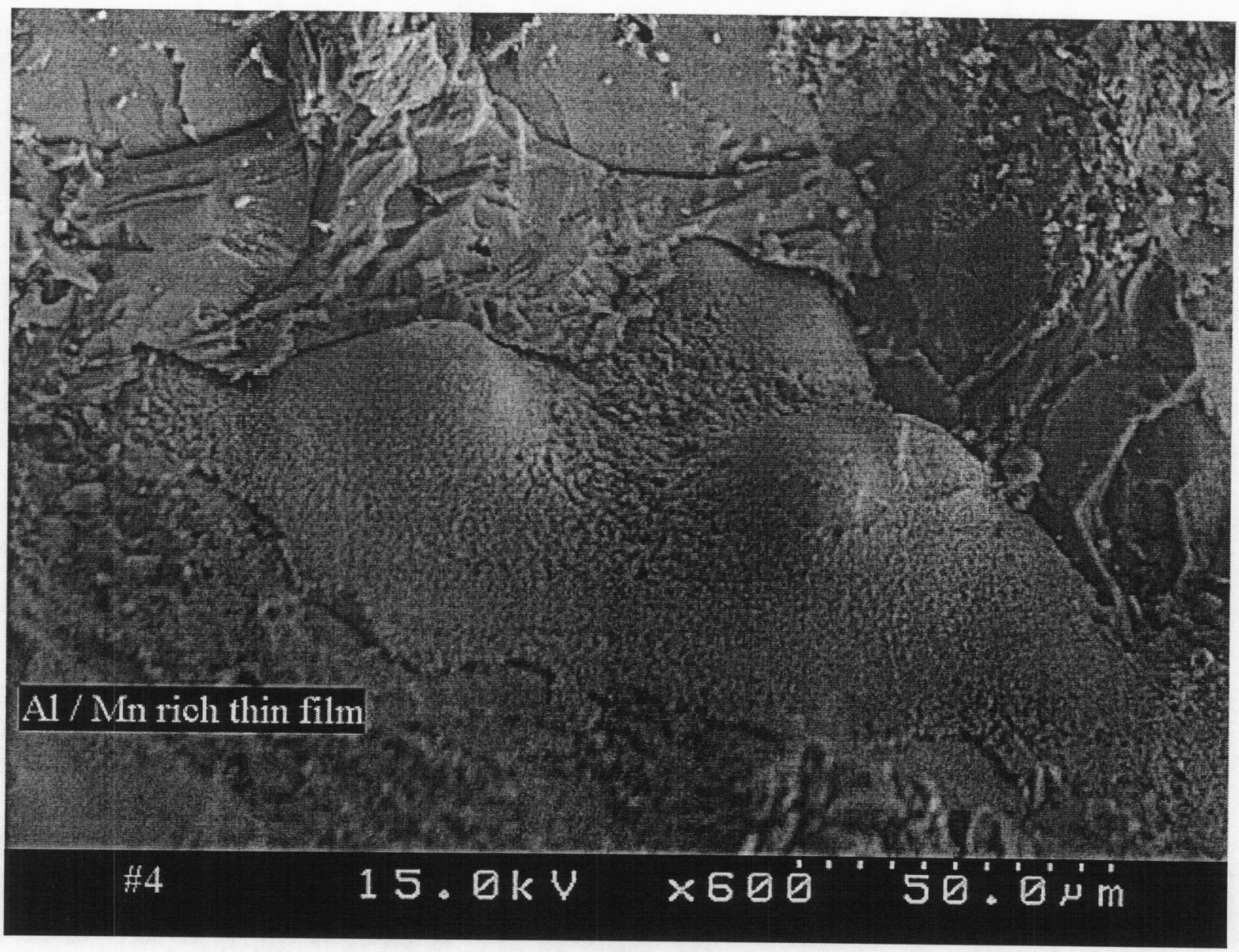

Figure 8-1. Sample SPC0039799: Pre-Test Fracture of a Crusty-Coating of Al, Mn-rich Composition. The Grain Appearance and Analysis (Figure 8-2) Are Consistent with the Layer-Structure Mn-oxides Lithiophorite. 


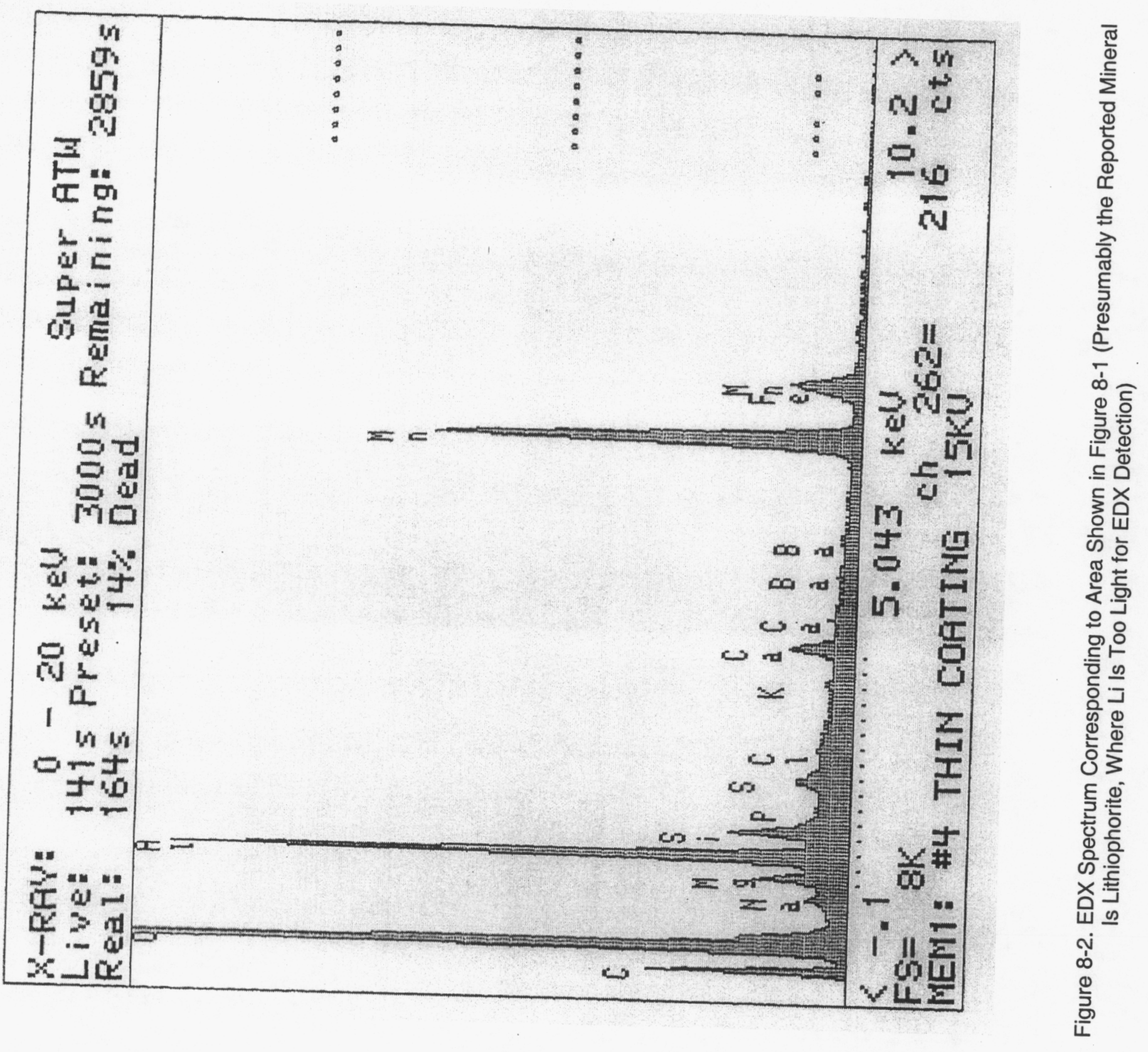




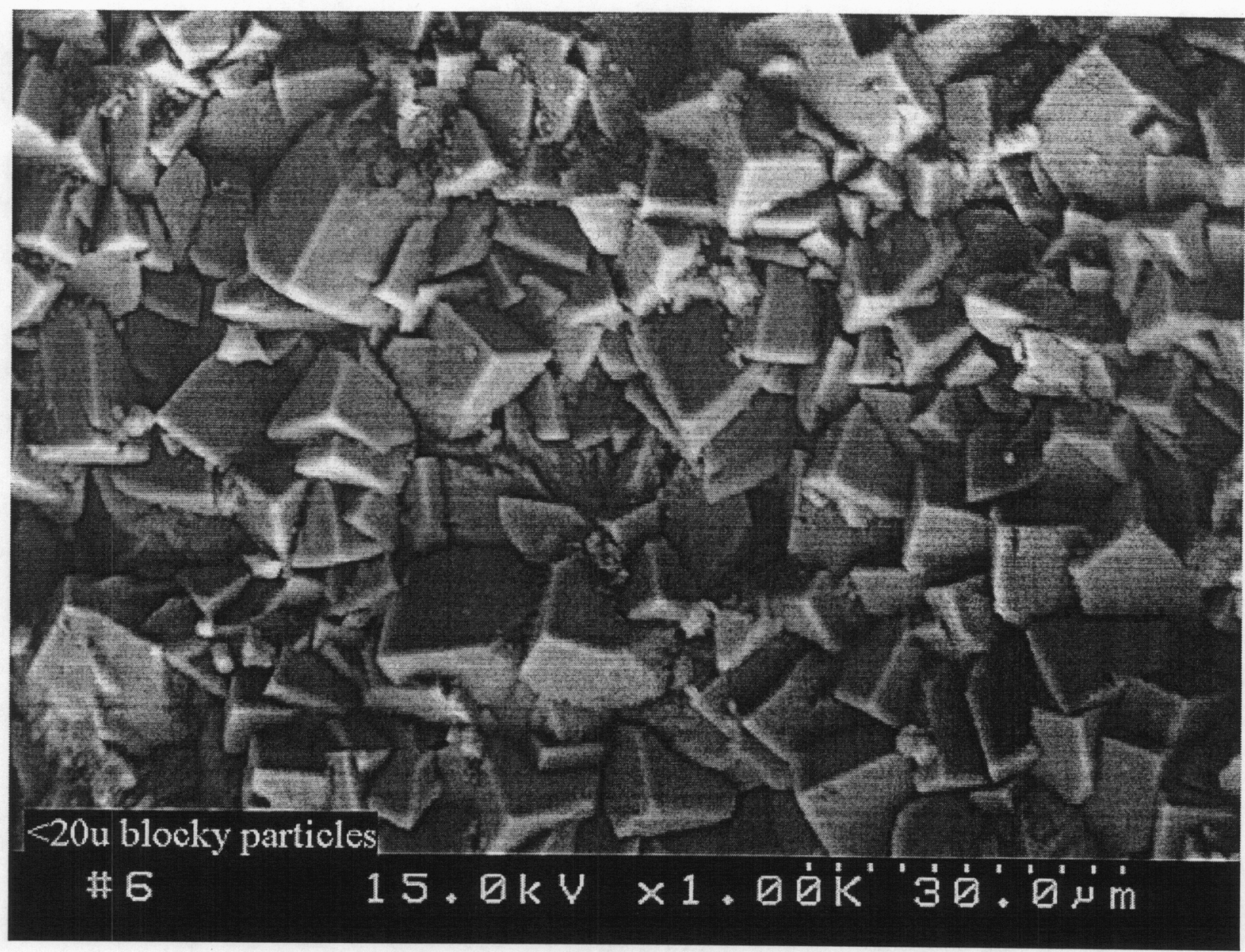

Figure 8-3. Sample SPC0039806: Pre-test Fracture Sample Exhibits Well-developed Crystalline Morphology. The Blocky Crystals Are Consistent with Calcite. Small Rounded Mounds, Lighter in Color and Scattered Around the Bases of the Calcite Are Determined to Be Silica Polymorph (See Figures 8-5 and 8-6) 


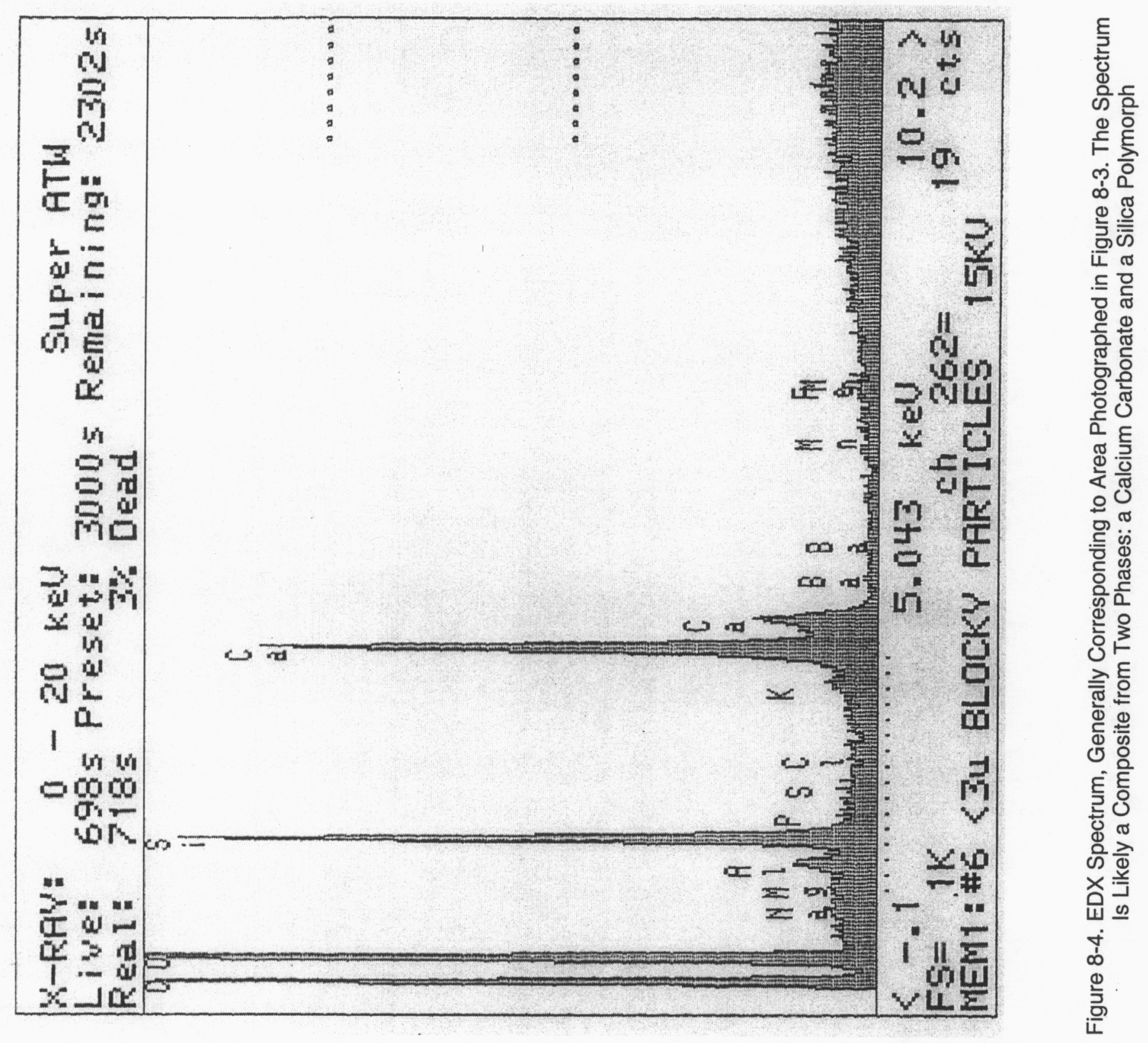

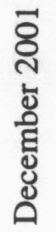




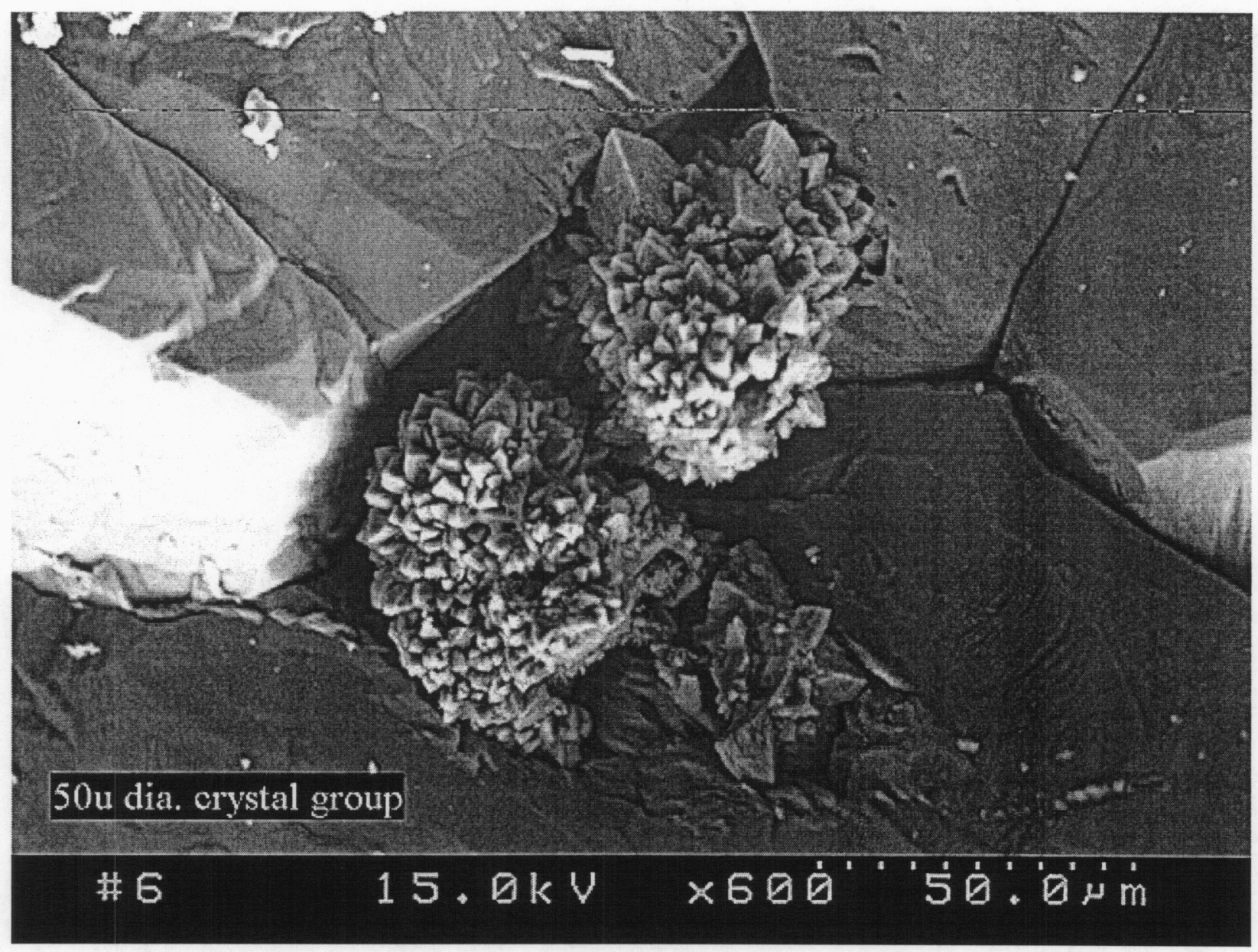

Figure 8-5. Sample SPC0039806: Pre-Test Fracture, Magnified View of Rounded Masses Similar to Those Shown in Figure 8-3 (Bases of Blocky Crystals). EDX Is Consistent with a Silica Polymorph 


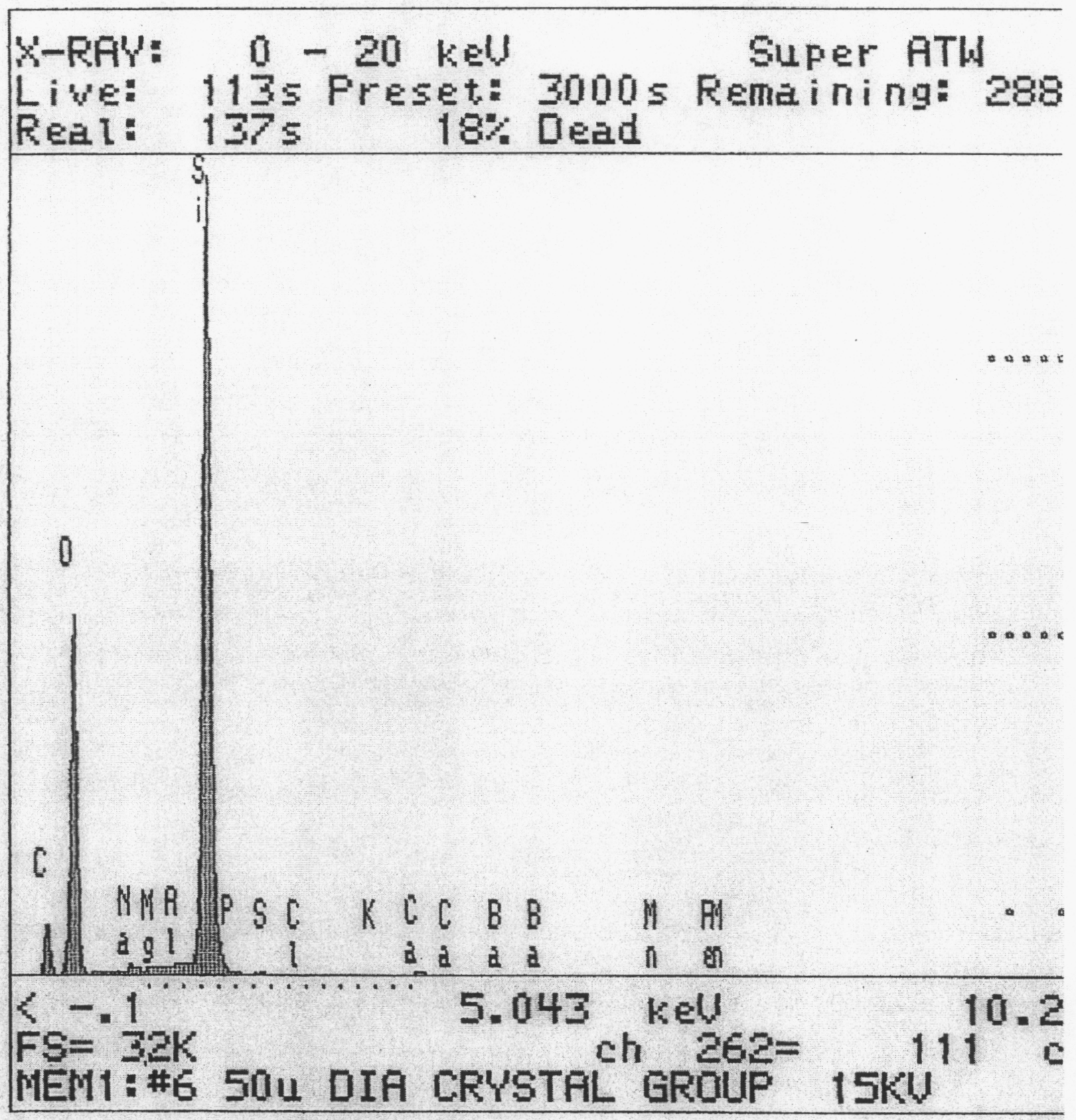

Figure 8-6. EDX of Rounded Nodular Crystal Mass. Spectrum is That of an $\mathrm{SiO}_{2}$ Phase 


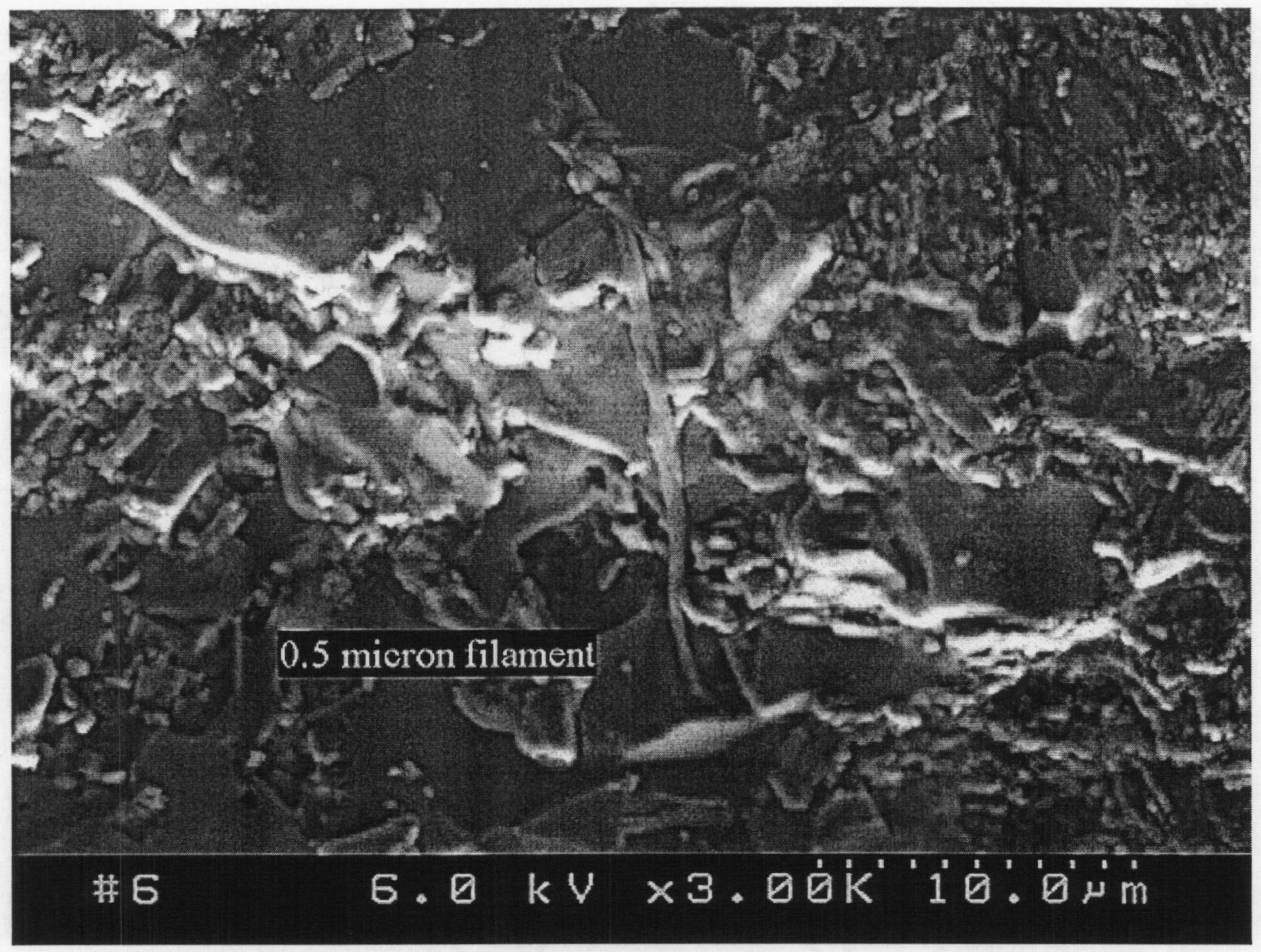

Figure 8-7. Sample SPC0039806: Strand Lying on the Surface of Other Minerals. The Morphology and Relative Chemistry Suggest a Clay Mineral 


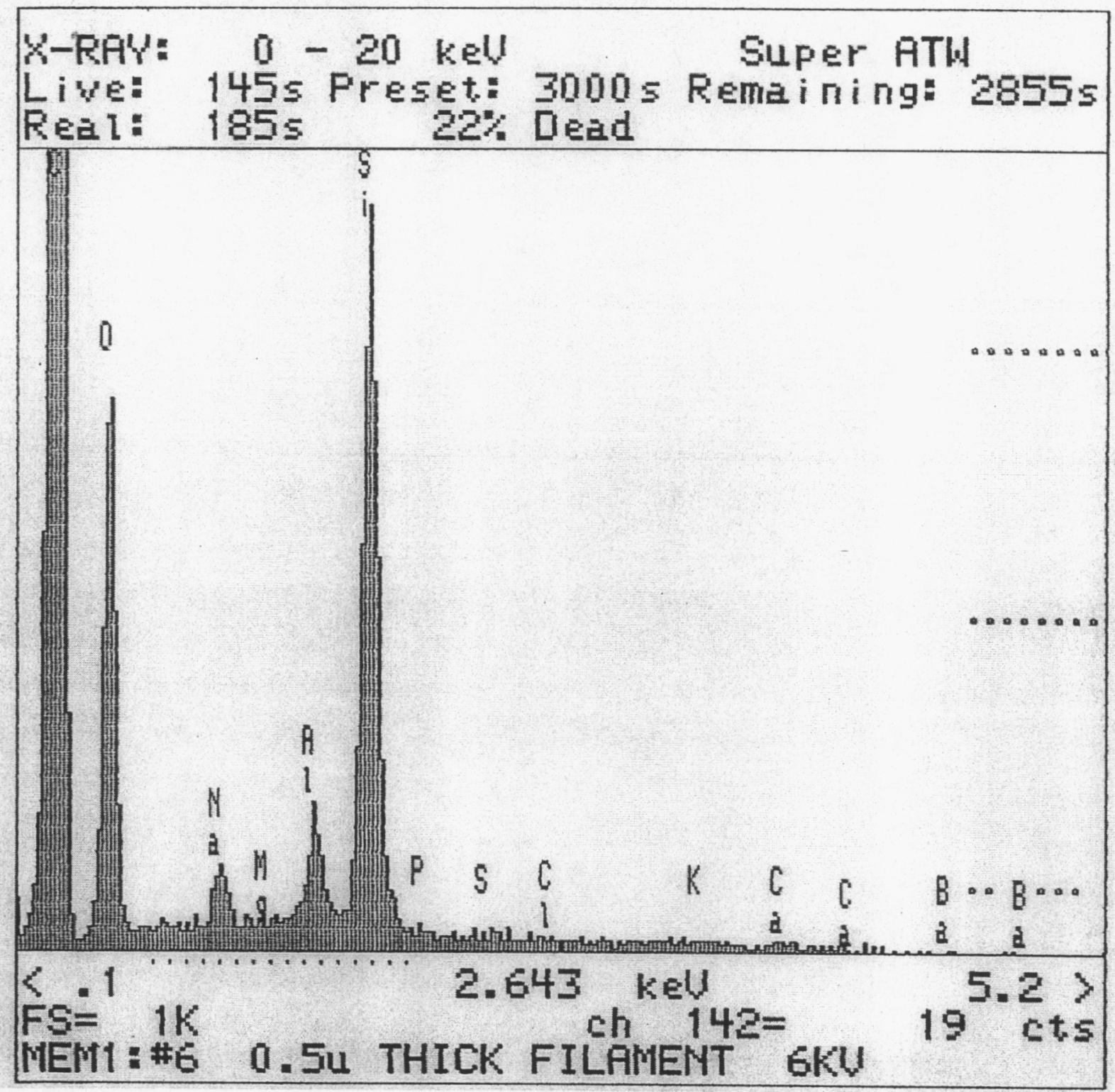

Figure 8-8. EDX Spectrum for Strand Shown in Figure 8-7, Possibly a Clay, Such as Smectite 


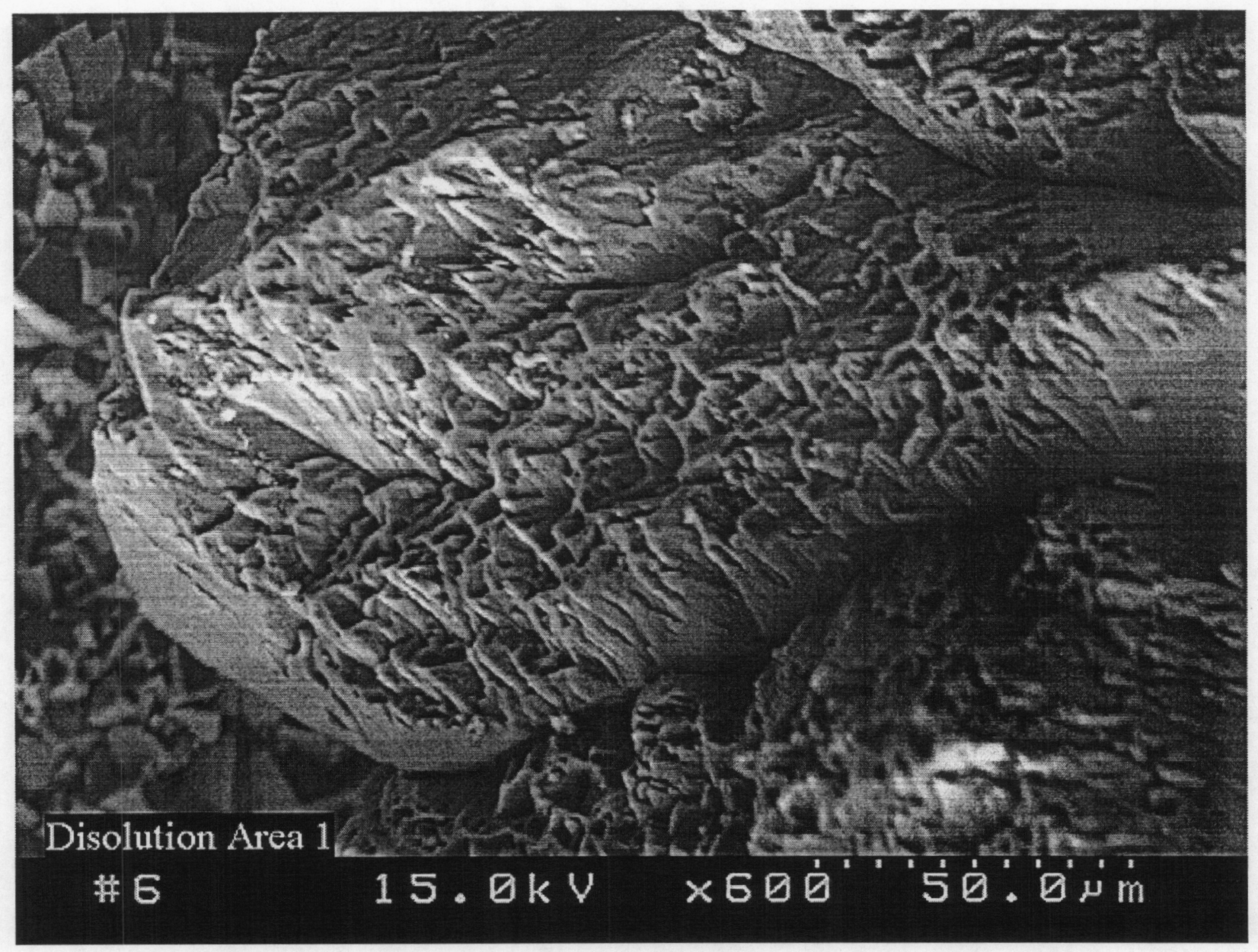

Figure 8-9. Sample SPC0039799 Pre-Test Sample with Calcite Grain (EDX Not Shown) Showing Pronounced Dissolution 


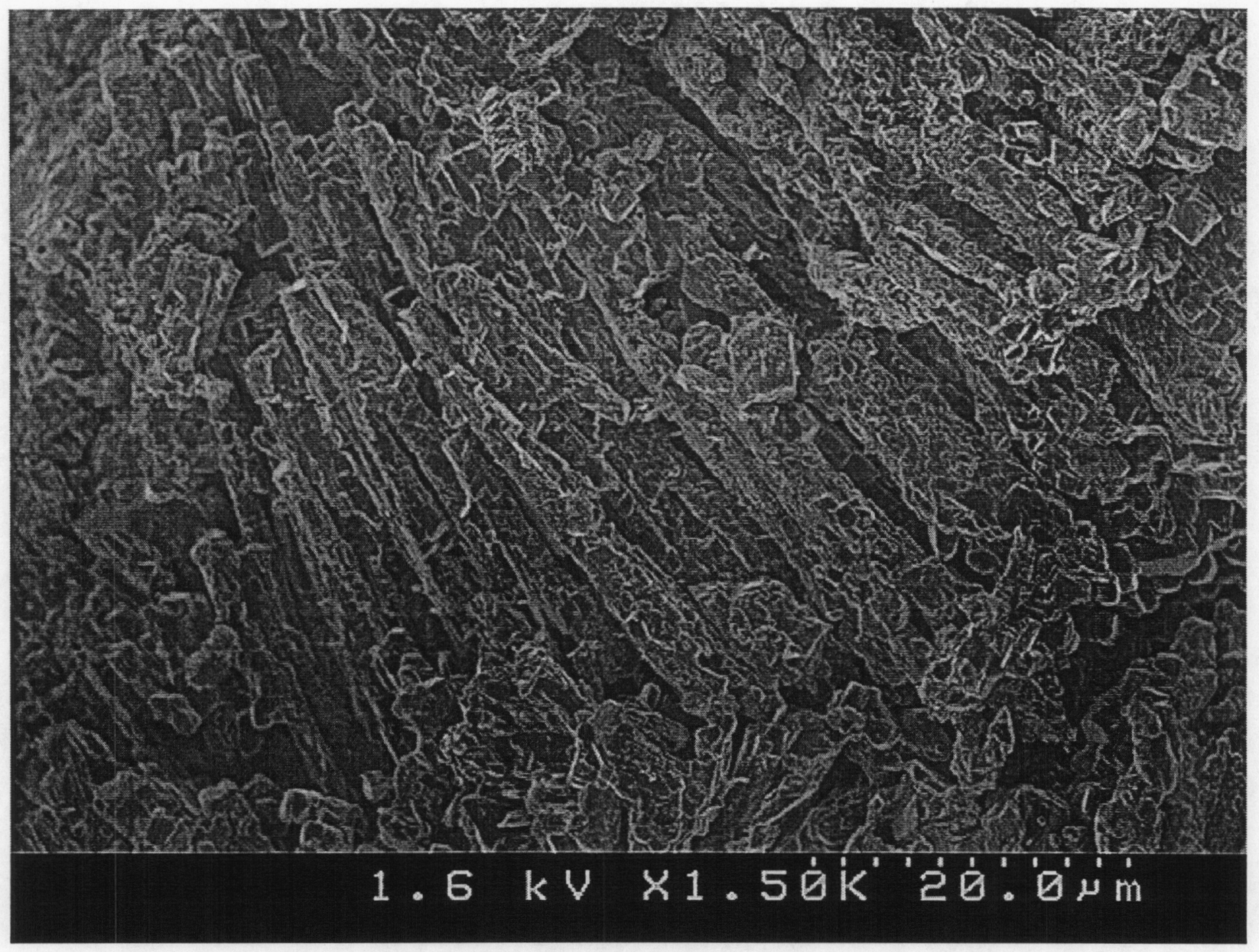

Figure 8-10. Sample SPC02015843 Post-Test Sample with Significant Dissolution of a K-Rich Feldspar (EDX Not Shown) 


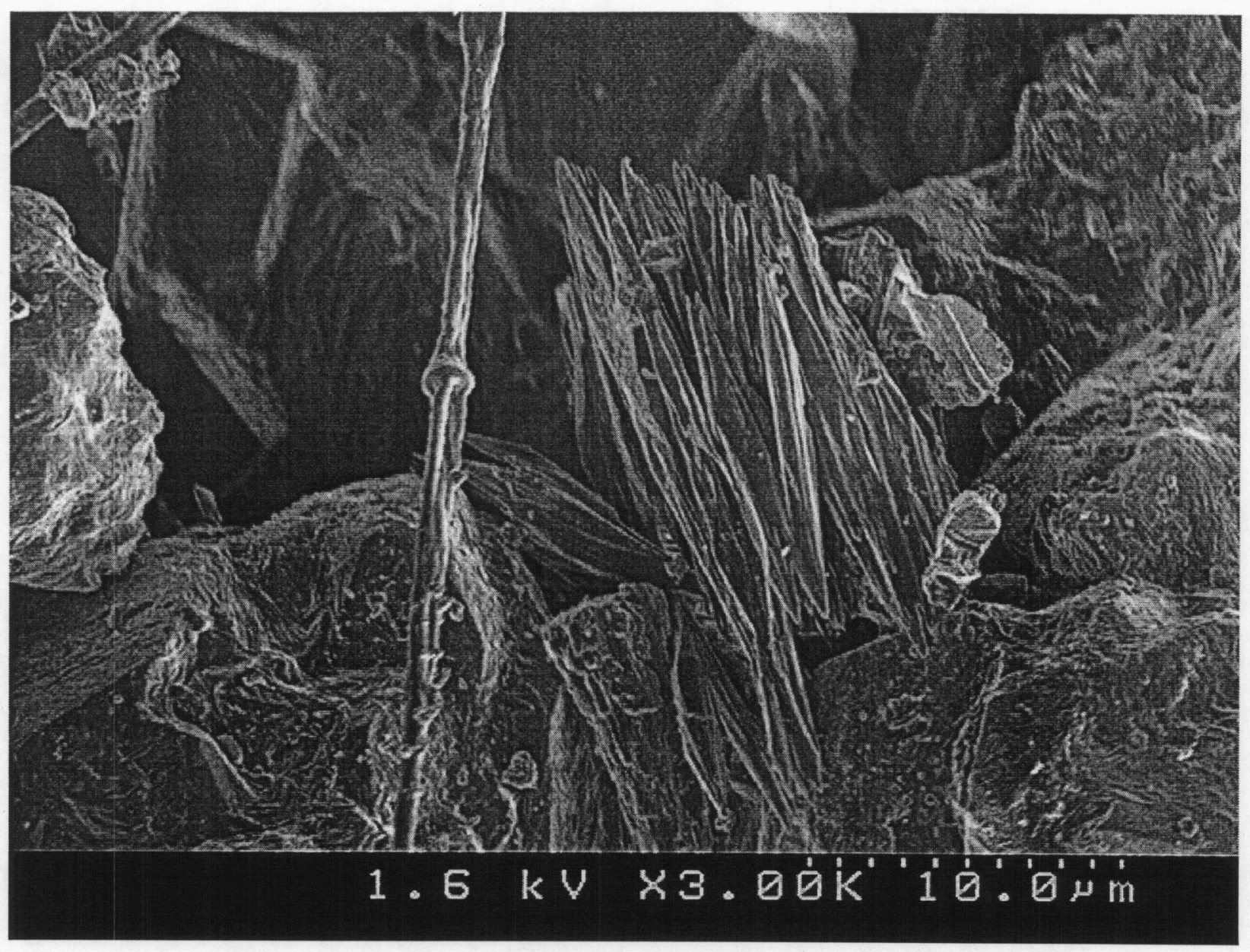

Figure 8-11. Sample SPC02015843 Post-Test Sample (Different Area Than Imaged in Figure 8-10): Chevron Features are Sub-Micron Sized, Appear to Be Further Evidence of Dissolution (Grain Not Identified by EDX) 


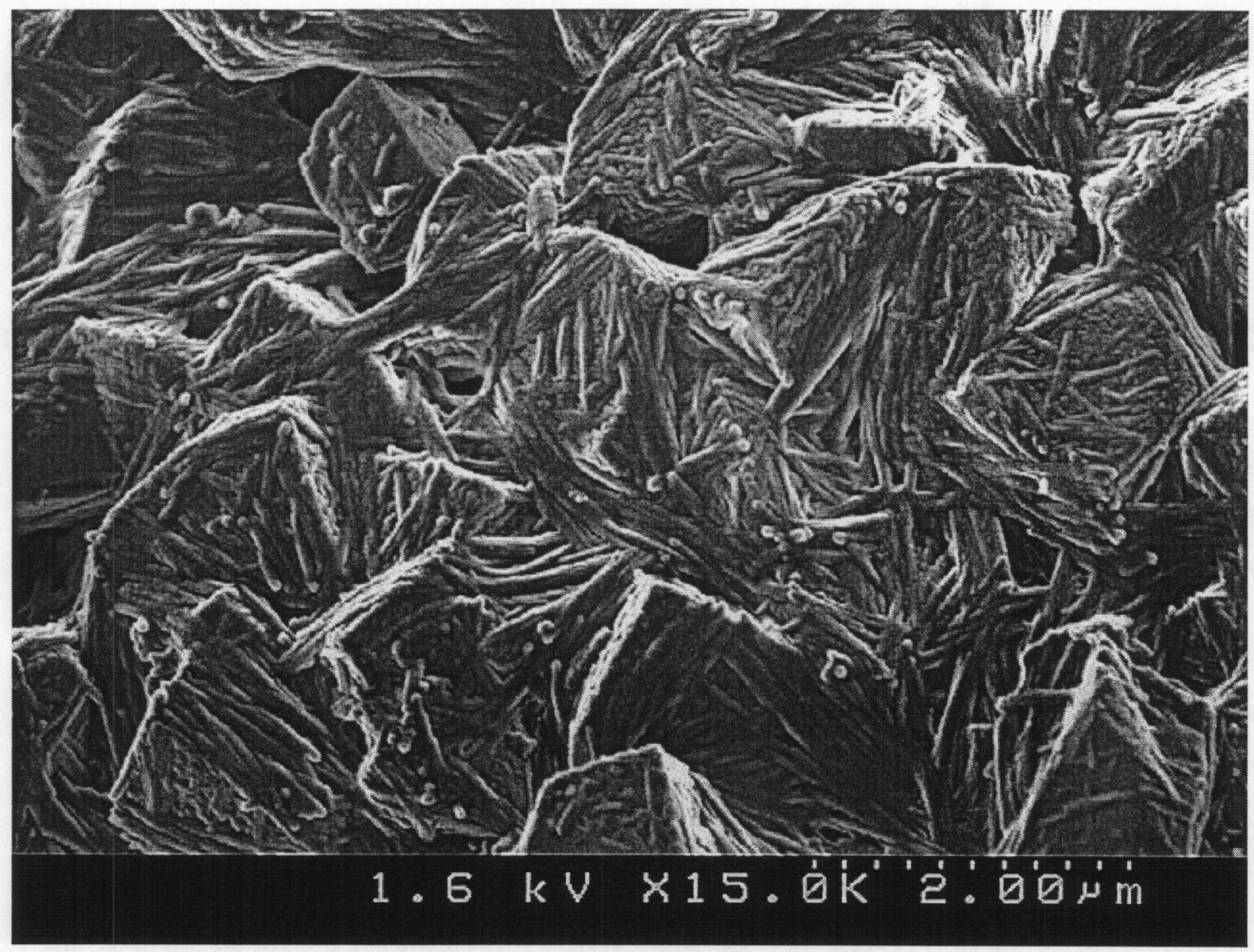

Figure 8-12. Sample SPC02015679 Post-Test Sample: an Abundance of the Matted Fibrous Material Covers Surfaces of Different Grains, Appears to Have a Morphology and Chemistry Consistent with a Clay 


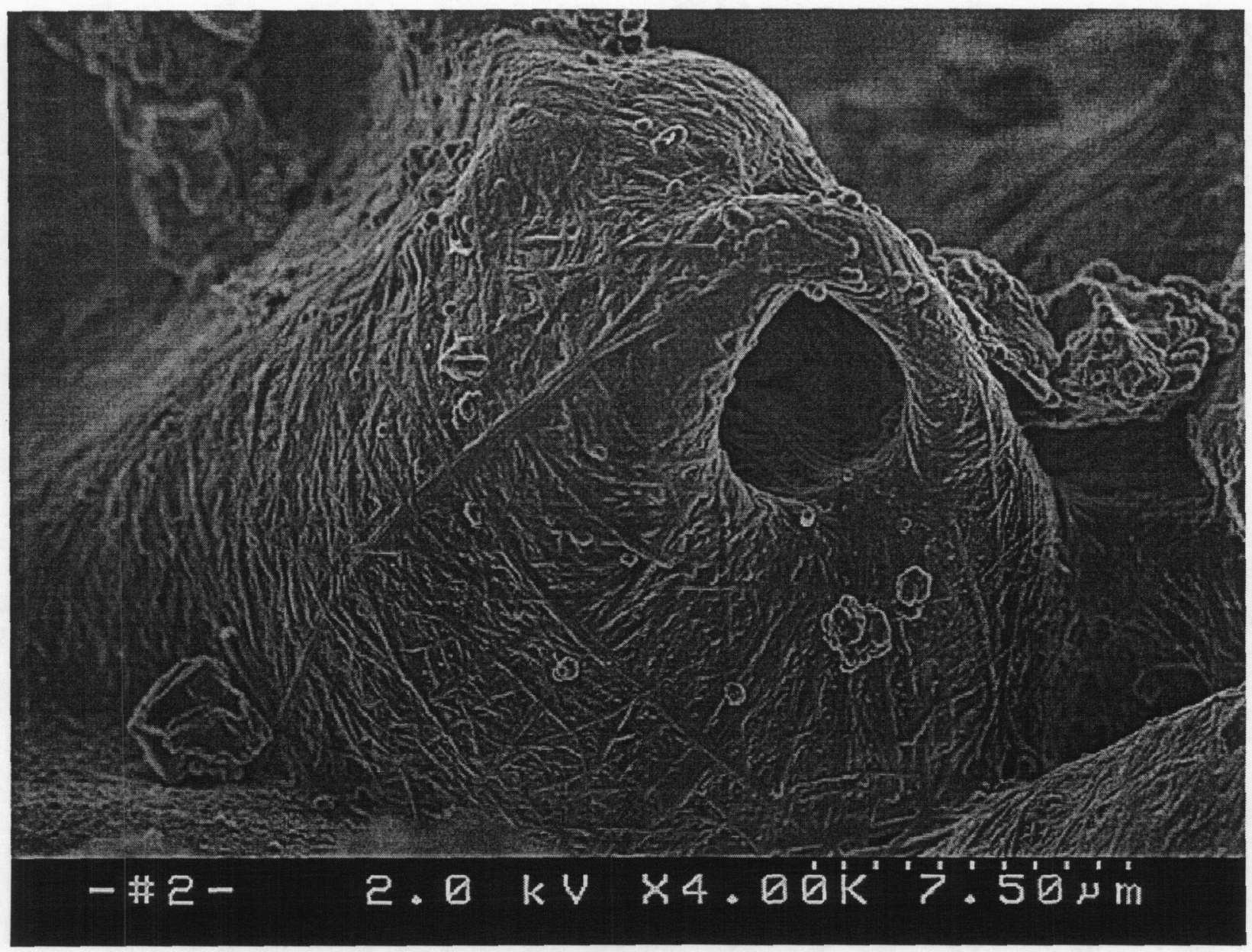

Figure 8-13. Sample SPC039806 Pre-Test Sample: the Same Fibrous Mineral Is Observed Coating Minerals of Fracture Surfaces Before Thermal Testing 


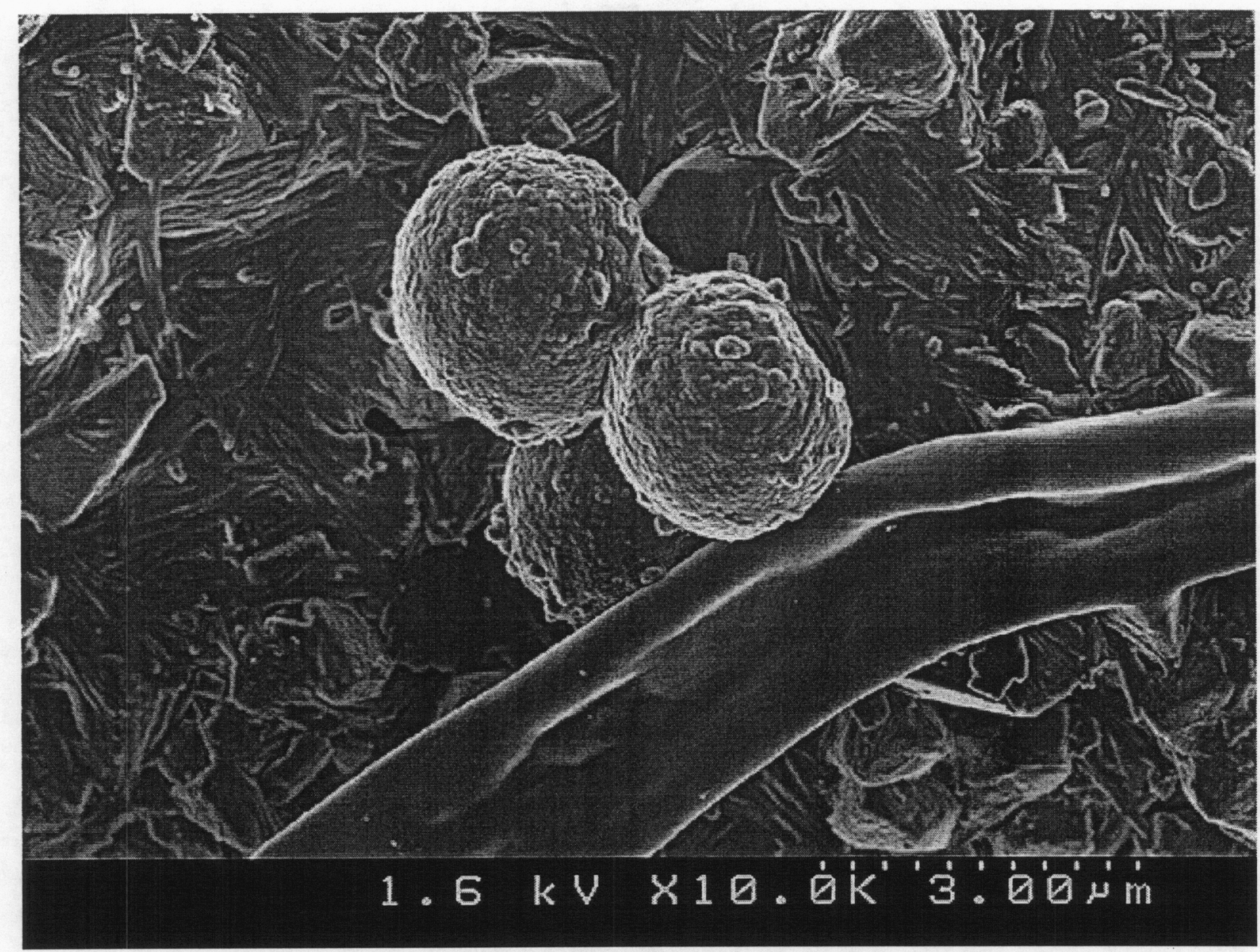

Figure 8-14. Sample SPC02015679 Post-Test Sample: Overview of Post-Test Mineral Surface Exhibits Several Minerals - the Fibrous Matted Mineral, the Spherical Silica Polymorphs, Possibly Ribbons of Pore-Bridging Illite 


\section{OTHER MEASUREMENTS AND OBSERVATIONS}

\subsection{OBSERVATION HOLES}

As mentioned in Section 3.3, four observation holes were drilled near the bottom of the block. Those were EO3, NO1, NO2, and WO5 (See Figures 3-3, 5-2, 5-3, and 5-4). The original purpose of those holes was to provide direct observation of the drainage of water in the block during the test. Later, those holes became the sampling spots for the microbial investigation (See Section 9.3). The $x-y-z$ coordinates of those holes are shown in Table 9-1 below. The observation was considered scoping in nature, therefore was a non-quality-affecting activity. It did not produce Q-results. There was no measurement and test equipment involved.

Table 9-1, X-Y-Z Coordinates of the Collar, C, and Bottom, TD, of the Observation Holes of the LBT

\begin{tabular}{|l|c|c|c|}
\hline \multicolumn{1}{|c|}{ Hole\# } & $\mathbf{x}(\mathrm{m})$ & $\mathbf{y}(\mathbf{m})$ & $\mathbf{z}(\mathbf{m})$ \\
\hline EO3-C & 3.048 & 2.743 & -3.962 \\
\hline EO3-TD & 0.305 & 2.743 & -3.962 \\
\hline NO1-C & 2.134 & 3.048 & -4.115 \\
\hline NO1-T & 2.134 & 0.305 & -4.115 \\
\hline NO2-C & 0.914 & 3.048 & -4.115 \\
\hline NO2-TD & 0.914 & 0.305 & -4.115 \\
\hline WO5-C & 0 & 0.914 & -3.962 \\
\hline WO5-TD & 2.743 & 0.914 & -3.962 \\
\hline
\end{tabular}

NOTE: The origin of the coordinates is the southwest corner of the block top.

EO3 was drilled from the east face, at about $0.305 \mathrm{~m}$ from the northern edge and $3.962 \mathrm{~m}$ below the top (about $1.22 \mathrm{~m}$ below the heater plane). NO1 was drilled from the north face at about $0.914 \mathrm{~m}$ from the east edge and $4.115 \mathrm{~m}$ below the top (about $1.37 \mathrm{~m}$ below the heater plane). NO2 was drilled from the north face at about $0.914 \mathrm{~m}$ from the west edge and $4.115 \mathrm{~m}$ below the top (about $1.37 \mathrm{~m}$ below the heater plane). WO5 was drilled from the west face at about $0.914 \mathrm{~m}$ from the south edge and $3.962 \mathrm{~m}$ below the top (about $1.22 \mathrm{~m}$ below the heater plane).

\subsubsection{Construction of the Observation Assembly}

One observation assembly set was inserted into each of the observation holes. The observation assembly consisted of one Pyrex ${ }^{\mathrm{TM}}$ tube of about $1.9 \mathrm{~cm}$ inside diameter and $2.769 \mathrm{~m}$ in length, one piece of white cloth strip about $3 \mathrm{~m}$ in length and about $5 \mathrm{~cm}$ in width, and one half polyvinyl chloride (PVC) pipe, which was split longitudinally. The inside diameter of the PVC pipe was about the same as the outside diameter of the Pyrex ${ }^{\text {TM }}$ tube. The Pyrex ${ }^{\text {TM }}$ tube was placed within the half PVC pipe, and the white cloth was placed between the Pyrex ${ }^{\mathrm{TM}}$ tube and the PVC pipe. Lines of water-soluble marker (commercial black ink and blue powder tempera paint were used) were drawn on top of the Pyrex ${ }^{\text {TM }}$ tube longitudinally. Tape was used to hold the assembly together. The assembly was placed in the observation holes with the open end of the Pyrex ${ }^{\mathrm{TM}}$ tube facing the collar of the hole, and the closed end of the Pyrex ${ }^{\mathrm{TM}}$ tube touched the bottom of the hole. The Pyrex ${ }^{\text {TM }}$ tube was to allow a borehole video camera to be used to view the inside of the borehole wall. The lines of water-soluble marker were used to register water 
dripping on the Pyrex ${ }^{\mathrm{TM}}$ tube (be washed away by water), and the dissolved marker might leave stains on the white cloth.

\subsubsection{Observations}

The observation assembly was satisfactory tested outside of the hole, but it was soon found that fog in the Pyrex ${ }^{\mathrm{TM}}$ tube (the tube) made the video viewing not very successful. It was decided to periodically remove the observation assembly. The moisture on the tube and the white cloth were sampled for the microbial investigation when the observation assembly was removed and reconditioned. The observation assemblies were removed for inspection and reconditioning on the following dates: April 16, 1997 (day 47 since the heating started), August 4, 1997 (day 157 since the heating started), November 12, 1997 (day 257 since the heating started), February 3, 1998 (day 340 since the heating started), March 25, 1998 (390th day since the heating started, and the 15th day since the heaters were turned off), July 9, 1998 (121st day since the heating ended), and October 2, 1998 (206th day since the heating ended). The observations were focused on the evidence of discrete flow of water. The observations are recorded below.

\section{- April 16, 1997 (47 days of heating)}

E03: The tube was not warm to touch. Water drops were continuously on the inner half of the tube ( 0 to $1.37 \mathrm{~m}$ from the bottom). The black ink was gone. There were stain spots at the following distances from the bottom of the hole: $0.11-0.356,0.89,1.65,1.69$, and $1.77 \mathrm{~m}$. There was a black spot at the collar ( $2.74 \mathrm{~m}$ from the bottom). Right after the assembly was reemplaced in the hole, video image showed that moisture appeared on the section of the tube from $1.22 \mathrm{~m}$ from the collar to the end of the tube.

NO1: The tube was not warm to touch. Water condensation on the entire length of the tube. The black ink was gone. One black spot on the white cloth at $0.83 \mathrm{~m}$ from the bottom. The cloth was damp to touch; water was seen on the PVC pipe.

NO2: The tube was not warm to touch. Water condensation on the entire length of the tube. The black ink was gone. There was one black spot on the cloth at $0.1 \mathrm{~m}$ from the collar. Black spots on the cloth at the following distances from the bottom: $0.25,0.46,0.48,0.51,0.53$, and $0.91 \mathrm{~m}$. The cloth was damp in the inner two-thirds of the hole.

WO5: The tube could not be removed, probably due to the deformation of the block.

\section{- August 4, 1997 (157 days of heating)}

E03: The tube was warm to touch. All markers were gone. No water condensation was seen. Stains covered the cloth. The cloth was not wet.

N01: The tube was hot to the touch. There was water condensation on the entire tube. The cloth was damp. There were no discrete marks on the cloth.

NO2: The tube was hot to the touch (similar to NO1). There was water condensation on the inner $2.44 \mathrm{~m}$ of the tube. There was no discrete mark on the cloth. The cloth was damp, but not as damp as that of NO1. 
WO5: The tube could not be removed. This was the same for all of the following observations.

\section{- November 12, 1997 (257 days of heating)}

EO3: The tube was warm to the touch. There was water condensation on the outer $1.043 \mathrm{~m}$ of the tube. The blue paint on the tube was gone at the follow distances from the bottom: $1-1.15$, $1.25-1.36,1.4-1.5$, and $1.72-2.47 \mathrm{~m}$. There was a stain spot on the cloth at $0.78-0.92 \mathrm{~m}$ from the bottom. The cloth was dry.

NO1: The tube was warm to the the touch. There were spotty water condensations on the tube for the entire length. There was no discrete mark on the cloth. The cloth was wet.

NO2: The tube was warmer than that in EO3 and NO1. There was no water condensation on the tube, but the tube was wet at the bottom end. There was no discrete mark on the cloth. The cloth was wet to touch. There was water in the PVC pipe.

\section{- February 3, 1998 (340 days of heating)}

EO3: The tube was warm to the touch. The blue paint was intact. There were black spots on the cloth at the following distances from the bottom: $0.57-0.7,0.77,0.78,0.8,0.83-1.07 \mathrm{~m}$. This was an area with blue color on top; there were seven black spots, each $0.5-1 \mathrm{~cm}$ in diameter. Those spots were at $0.9,0.94,0.96,0.98,1.02,0.13,1.05,1.15$, and $1.41 \mathrm{~m}$. There was no water condensation on the tube. The cloth was wet from $0-0.5 \mathrm{~m}$, and $1.4-2.76 \mathrm{~m}$ from the bottom. There were blue stains in the cloth at $2-2.76 \mathrm{~m}$ from the bottom. There was a big black spot on the cloth near the collar.

NO1: The tube was warm to the touch. There was water condensation in the entire length of the tube. There was black stain on the cloth at the collar. The blue paint was washed away at the follow distances from the bottom: $0.4,0.5,0.51,0.52,0.53,0.54,0.55,0.56,0.58,0.59,0.6$, $0.69,0.88,0.89,0.92,0.94,0.99,1.16,1.37,1.57,2.31,2.32$, and $2.33 \mathrm{~m}$. The cloth was wet in its entire length. There were blue spots on the cloth at the following distances from the bottom: $0.08,0.43,0.83-0.9,1.3-1.4,2.05$, and $2.3 \mathrm{~m}$.

NO2: The tube was warm to the touch; it was warmer than NO1 and EO3. There was no water condensation on the tube. There was no obvious spot on the cloth. The cloth was wet.

\section{- March 25, 1998 (15 days since cooling)}

EO3: The tube was warm to touch. There was no water condensation. The black ink was gone. The blue paint disappeared at the following distances from the bottom: $0-0.1,0.95-1.2,2.12$ 2.32, and $2.33-2.75 \mathrm{~m}$. There were black stain spots on the cloth at the following distances from the bottom: $0-0.17,0.18-0.56,0.65,0.69,0.72,0.88-1.0$, and $2,75 \mathrm{~m}$. There was a water stain at $1-1.85 \mathrm{~m}$ from the bottom. There was a big blue/black spot at $1.13-1.6 \mathrm{~m}$ from the bottom. There were blue spots on the cloth at the following distances from the bottom: $2.11,2.14,2.15$, $2.17,2.2,2.23,2.33,2.34,2.35,2.36,2.39,2.4,2.44,2.45,2.59,2.61$, and $2.62 \mathrm{~m}$. The cloth was not wet. 
NO1: The tube was slightly warm to the touch. There was water condensation on the entire length of the tube. The black ink was gone; the blue paint was intact. The blue paint was washed away at $1.33-1.42 \mathrm{~m}$ from the bottom. The cloth was damp. There was no obvious stain spot.

NO2: The tube was slightly warm. There were some water condensations but less than in NO1. The black ink was gone; the blue paint was intact. There was no stain spot. The cloth was damp.

\section{- July 9, 1998 (121 days since cooling)}

E03: The tube was not warm. There was no water condensation. The black ink was gone; the blue paint was intact. There were black marks on the cloth at the following distances from the bottom: $0.33,0.5-0.58,1.5$, and $2.5-2.74 \mathrm{~m}$. There were drip marks on the tube at $1.83-2.2 \mathrm{~m}$ from the bottom. The cloth was wet.

NO1: The tube was not warm. There was no water condensation. The black ink was gone. The blue paint had gaps at the follow distances from the bottom: $0.43,0.57,0.72,0.85$, and $0.93 \mathrm{~m}$. There were five flow marks on the tube at $1.03-1.12 \mathrm{~m}$ from the bottom, and there was one flow mark at 2.17-2.22 $\mathrm{m}$ from the bottom. The cloth was wet.

NO2: The tube was not warm. There was no water condensation. The black ink was gone; the blue paint was intact. There was a gap of the blue paint at $0.4 \mathrm{~m}$ from the bottom. There were black spots in the cloth at the following distances from the bottom: $2.23,2.25,2.27,2.28,2.34$, 2.37 , and $2.38 \mathrm{~m}$. The cloth was damp.

\section{- October 2, 1998 (206 days since cooling)}

E03: There was no water condensation. Most of the black ink was gone; the blue paint was intact. There was no sign of drips. There was a big blue/black stain on the cloth at $0.5-0.67 \mathrm{~m}$ from the bottom, and there were some spots at $1.6 \mathrm{~m}$ and $2.1-2.4 \mathrm{~m}$ from the bottom. The cloth felt wet.

N01: There was no water condensation. The black ink was gone; the blue paint was intact. There were black spots on the cloth at the following distances from the bottom: $0.2,0.23,0.25$, $0.27,0.3,0.45,0.47,0.53,0.8,0.87,0.89,0.91,0.92,0.93-1.0$, and $1.69 \mathrm{~m}$. There were stains at 1.4-1.6 $\mathrm{m}$ and $2.3-2.75 \mathrm{~m}$ form the bottom. The cloth felt wet.

NO2: There was no water condensation. The black ink was gone; the blue paint was intact. There was stain on the cloth throughout the entire length. There were some spots at the following distances from the bottom: $0.53-0.59,1.32,1.35,1.39,1.8,1.91$, and $1.97 \mathrm{~m}$. The cloth felt wetter than in NO1 and EO3.

\subsubsection{Conclusions}

The observations near the bottom of the block indicate that water did flow away and downward from the heater plane. It is possible that the in-situ moisture content of the rock mass caused the observed water condensation on the tube. However, the water condensation did not occur in all 
holes, nor did it occur all the time in each hole. Therefore, the observed water condensation may shed some light about the hydrological processes below the heater plane. The observation assemblies did provide discrete traces of water flow, but it is difficult to distinguish the marks caused by condensation dripping from those caused by discrete fracture flow.

\subsection{RELATIVE HUMIDITY AND TEMPERATURE RESULTS FROM HYDROLOGY BOREHOLES}

Four hydrology boreholes were emplaced in the large block and instrumented with Humicap sensors that provide temperature and relative humidity data. One hydrology borehole was vertical, and the other three were horizontal: two east-west and one north-south. The vertical borehole, TH1, was bored from the top surface. Two hydrology boreholes were bored from the west face of the large block: WH1, at $1.5 \mathrm{~m}$, and $\mathrm{WH} 2$, at $0.5 \mathrm{~m}$ above the heater plane respectively. The north-south hydrology borehole, $\mathrm{NH} 1$, was bored from the north face at $0.3 \mathrm{~m}$ above the heater plane. A single Humicap was installed in each of the horizontal hydrology boreholes, and three were installed in the vertical hydrology borehole. Markers were installed in those hydrology holes to seal the holes. One Humicap was installed in a pack-off zone between two markers. The pack-off zone was about $0.46 \mathrm{~m}$ in length. The location of the sensors in each hole is shown in Table 9-2. The Humicap sensors in the first two zones of the vertical borehole performed unreliably during the LBT, and the discussion below, therefore, focuses on the third zone of the vertical hole and the horizontal boreholes.

Table 9-2. The Location of Humicap in the Hydrology Holes

\begin{tabular}{|c|c|c|}
\hline Borehole \# & Zone & Depth from collar (m) \\
\hline NH1 & 1 & 1.78 \\
\hline WH1 & 1 & 1.76 \\
\hline WH2 & 1 & 1.76 \\
\hline TH1 & 1 & 3.57 \\
\hline TH1 & 2 & 2.57 \\
\hline TH1 & 3 & 1.56 \\
\hline
\end{tabular}

Of the horizontal hydrology boreholes, $\mathrm{NH} 1$ is the closest to the heater plane $(0.305 \mathrm{~m}$ above the heater plane). This distance from the heater plane is in between that of RTD \#15 and \#16 in the temperature hole TT1. The NH1 Humicap sensor provided usable temperature and humidity data for approximately 135 days, measured from the onset of heating. All of the Humicap sensors experienced a problem at about 135 days, but those in the other horizontal boreholes recovered after a few days. Temperatures recorded by $\mathrm{NH1}$ rose over the entire 135-day interval, except for a couple of minor dips related to heater power outages (Figure 9-1). Most of the temperature increase occurred in the first 30 days, and the expected boiling point of water was reached at approximately 70 days. The maximum temperature recorded, just before failure, was about $110^{\circ} \mathrm{C}$. This temperature was very close to what was measured at TT1-15, but it was greater than that at TT1-16, TT2-15, and TT2-16, because those RTDs were engaged in a TH event as described in Section 5. The initial relative humidity $(\mathrm{RH})$ in borehole NH1 was about $90 \%$, and high $\mathrm{RH}$ values were recorded throughout the lifetime of the sensor. Relative humidity values of about $90 \%$ were recorded until about 85 days into heating, after which the humidity level appears to have gradually declined to just under $80 \%$ by 125 days (Figure $9-2$ ). This high humidity is 
expected because the moisture content in the proximity of this Humicap had decreased by only about $2.5 \%$ fraction volume, as determined by neutron logging.

Borehole WH2 is located $0.5 \mathrm{~m}$ above the heater plane and about $0.2 \mathrm{~m}$ above borehole NH1. The WH2 Humicap sensor provided somewhat noisy, but usable, data for nearly 600 days. Temperature and $\mathrm{RH}$ data recorded by $\mathrm{WH} 2$ during the heating phase and early cooling phase of the LBT (327 days) are shown in Figures 9-3 and 9-4. The WH2 temperature (Figure 9-3) results during the first 125 days are similar to those for NH1. Most of the temperature increase occurred in the first 30 days. The expected boiling point of water was reached by 105 days, a little later than for $\mathrm{NH} 1$, as expected, since $\mathrm{WH} 2$ is an additional $20 \mathrm{~cm}$ above the heater plane. Temperatures rose until about 230 days, then declined slowly, due to the power reduction in order to maintain a steady-state temperature. As with $\mathrm{NH} 1$, the initial $\mathrm{RH}$ was about $90 \%$ and remained high until slightly past 125 days (Figure 9-4). Temperatures remained near $100^{\circ} \mathrm{C}$ between 105 and 125 days. Data were lost at about 135 days, as with NH1, but data acquisition was restored within a few days. Relative humidity values slightly under $80 \%$ were recorded upon recovery and were followed by a large and sustained drop, falling to about $25 \%$ by about 230 days. This decrease in the humidity was sooner than expected, because the rock at this distance from the heater plane did not lose 7\% of its total moisture loss until Day 330 (January 28,1998 ). During the same time interval, temperatures rose to slightly above $120^{\circ} \mathrm{C}$. Coincident with the drop in temperatures, $\mathrm{RH}$ recovered somewhat, and $\mathrm{RH}$ values of about $40 \%$ were recorded at 270 days. Later, the temperature showed a rapid drop at approximately 375 days, due to the turning off of the heaters, and a decline in $\mathrm{RH}$ to $20 \%$ at 380 days, followed by a gradual recovery to just over $30 \%$ at the end of the record. Approximately 20 days of data were lost at about the time that temperatures at WH2 began to fall sharply.

Borehole WH1 is located $1.5 \mathrm{~m}$ above the heater plane and $1 \mathrm{~m}$ above borehole WH2. The temperature and humidity data for the WH1 Humicap are noisy, but usable data are provided for nearly 600 days. Temperature and RH data from WH1 are shown for the first 327 days in Figures 9-5 and 9-6. Reliable data are again missing for the interval between 135 and 145 days, as with WH2. The WH1 temperature history (Figure 9-5) shows that the expected boiling point of water was never attained in borehole WH1. Temperatures increased throughout the LBT heating phase and reached a peak of about $90^{\circ} \mathrm{C}$ near 230 days, then declined to about $80^{\circ} \mathrm{C}$ by 290 days. This temperature history agreed well with that measured by RTD of TT1-21 and TT122, which are at comparable distances from the heater plane. Relative humidity (Figure 9-4) remained at, or above, $90 \%$ throughout the entire 327-day record. This is expected because the rock at this distance from the heater plane had only lost about $1 \%$ of the fraction volume. As with WH-2, approximately 20 days of data were lost beginning around Day 375 .

The temperature and the RH measured in zone 3 in TH1 are presented in Figures 9-7 and 9-8 respectively. As shown in Table 9-2, this zone was at about $1.18 \mathrm{~m}$ from the heater plane. TH1 was only about $0.61 \mathrm{~m}$ due east from TT1. The distance from the heater plane of zone 3 in TH1 was similar to that of TT1-20. The temperature measurement of the Humicap stopped functioning at about Day 365, and the humidity measurement stopped functioning at Day 200. The measured temperature (Figure 9-7) agreed well with that measured by RTD at TT1-20. The RH remained at about $90 \%$ until about Day 107, then engaged in fluctuations until Day 200 , when it ceased functioning. This fluctuation in the humidity coincided with the TH event on 
June 13, 1997 (Day 107). The high RH before Day 107 was expected because the rock at this distance from the heater plane had not yet lost much moisture.

The small number of humidity sensors deployed in the LBT limits the conclusions that can be drawn. However, the temperature and $\mathrm{RH}$ records from borehole $\mathrm{WH} 2$ clearly show that a dry zone extended $0.5 \mathrm{~m}$ or more above the heater plane after 135 days. Higher in the block, at 1.5 $\mathrm{m}$ above the heater plane (borehole WH-1), boiling conditions were never reached, and $\mathrm{RH}$ remained high throughout the LBT.

\subsection{MICROBIAL SURVIVABILITY AND MIGRATION}

Bacteria may affect the performance of a potential nuclear waste repository. Bacteria can carry out reduction-oxidation reactions that may change the waste package environment. The waste package environment may affect the integrity of waste package materials and the transport of radionuclides. The survivability of bacteria in the conditions of heat and radiation produced by waste packages is of interest to the performance assessment of a repository. The migration of bacteria is also of interest to the understanding of the near-field environment. Qualitative observations of the survivability and migration of bacteria in a heated rock mass were conducted in the LBT. It should be stressed that those observations were scoping in nature; therefore they should be treated as non-Q, in accordance with the LLNL YMP Quality Assurance Program. The summary of the microbial work presented below is extracted from a report by Chen et al. (1998).

For the investigation of the survival and migration of bacteria in the LBT, double-drug-resistant mutants of microbes collected from the large block as markers of bacterial migration were used. Native double-drug-resistant mutants are rare. Therefore, if a large number of cultured doubledrug-resistant microbes are injected into the block, there is a high probability that any doubledrug-resistant microbes that are recovered were derived from the injected microbes. Bacillus subtilis and Arthrobacter oxydans were isolated from Topopah Spring tuff collected adjacent to the large block. These selected microbial species were labeled to be resistant to two drugs: rifampicin and streptomicin. The double-drug-resistant strains were resuspended in $1.5 \%$ agar. The inoculated agar, with a bacteria population in the order of about $10^{10}$, was extruded into each of the five horizontal heater holes (EH-1 through EH-5) on February 14, 1997, just prior to the insertion of the heaters. It was distributed along the length of the heater holes by lateral extrusion from the end of a syringe fabricated from a 9-foot length of PVC tubing.

Samples were collected at Days 172, 271, 354, 404, 510, and 595 after the initiation of the heating phase and through the cooling phase in the observation holes (NO1, NO2, and EO3). The heaters were turned off on Day 389. The construction of the observation holes is described in Section 9.1. These horizontal holes were located approximately $1.5 \mathrm{~m}$ below the injection (heater) holes at the base of the block. At each sampling, the white cloth strips were removed and replaced. Bacterial cells were also collected with a moistened sterile swab (glass fiber or filter paper) from the top of the Pyrex ${ }^{\mathrm{TM}}$ glass tube. Samples were also collected from the heater holes two times: once during the heating phase, when a single heater was removed for repair, and a second sampling was conducted after the LBT was completed. For the sampling in the heater hole, the heaters were removed from the block, and their surfaces were swiped with moistened sterile filter paper. 
The sampling results showed that double-drug-resistant bacteria were found in the observation holes. The number of double-drug-resistant bacteria that were identified in each borehole increased with time up to Day 404, and no double-drug-resistant bacteria were observed at Day 510 and Day 595, reflecting, in general, the thermal history of the block. There appears to be consistency among the three holes of cell number versus time; all have the highest cell number observed at Day 404. However, more cells were retrieved from NO1 than from any other boreholes. Sampling in the heater holes showed no double-drug-resistant bacteria during the heating phase. However, positive indications did result from the sample collected after the cooling phase. These results indicate that double-drug-labeled microbes migrated from the heater holes to the observation holes within nine months, possibly assisted by water flow. Culturable cells reappeared on the heaters after the test, indicating that microbial activity in the local environment of the heaters was only inactive during the thermal pulse. 


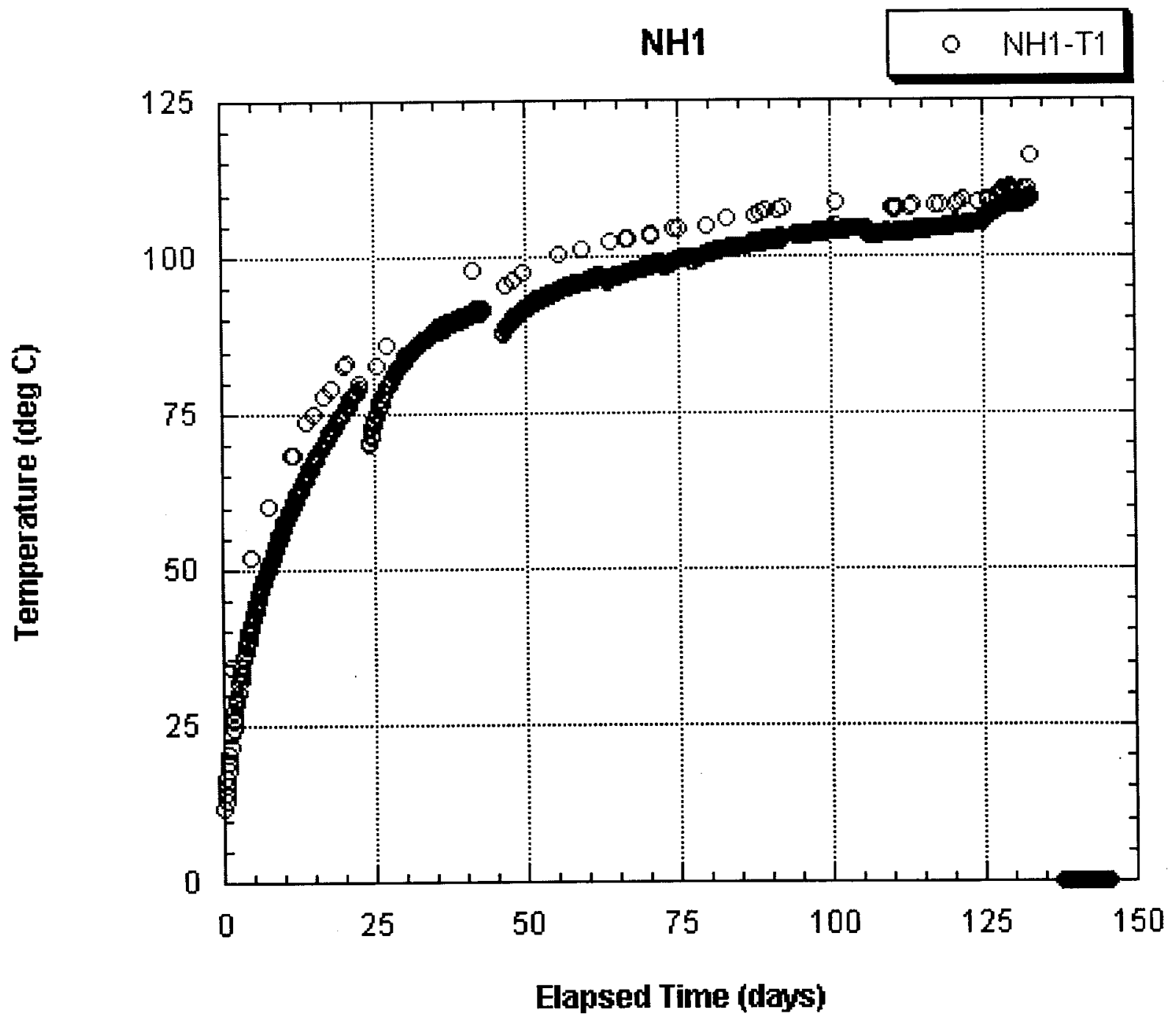

Figure 9-1. Temperature Measured by the Humicap in Hole NH-1 as a Function of Time 


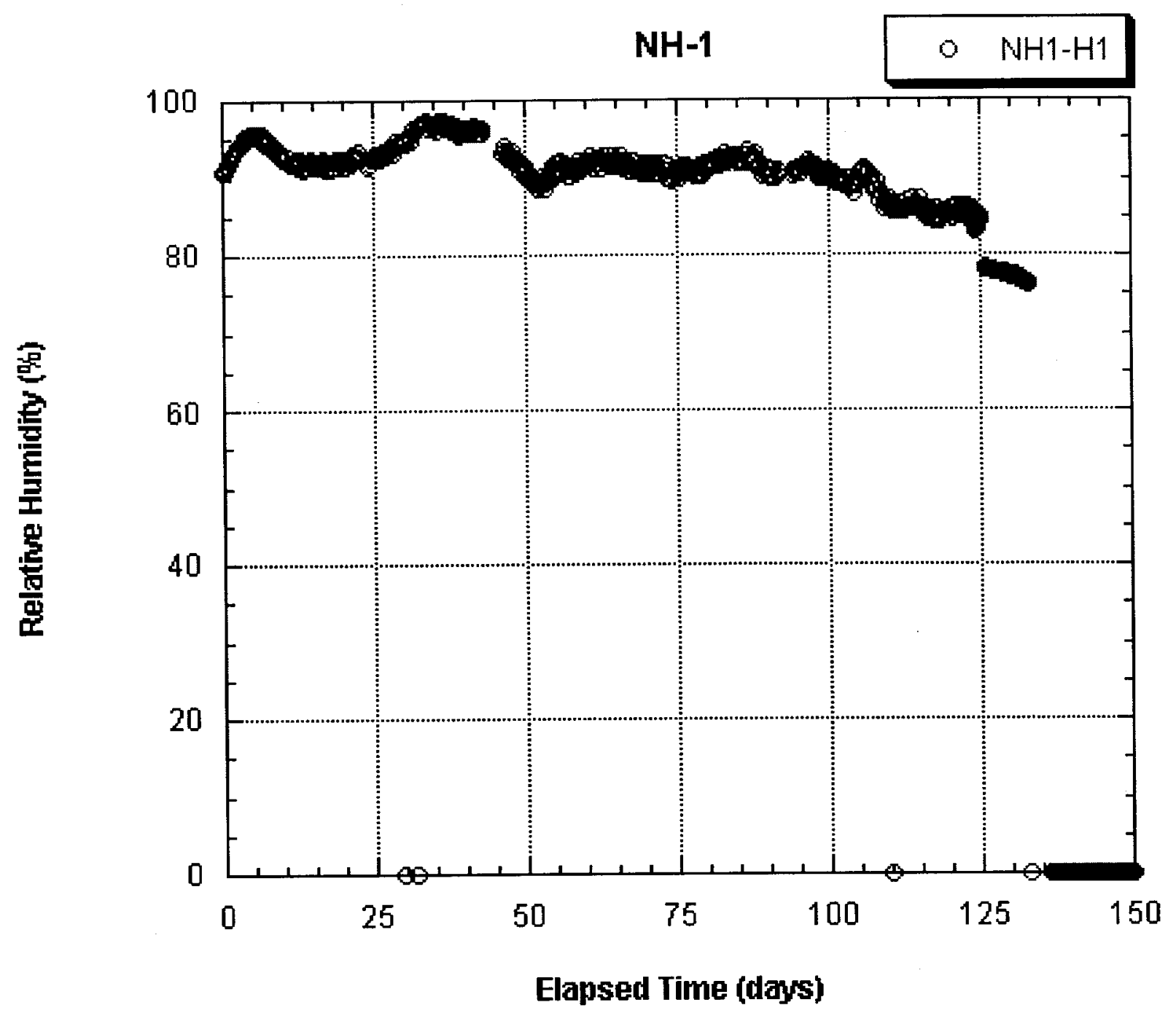

Figure 9-2. Relative Humidity Measured by the Humicap in Hole NH-1 as a Function of Time 


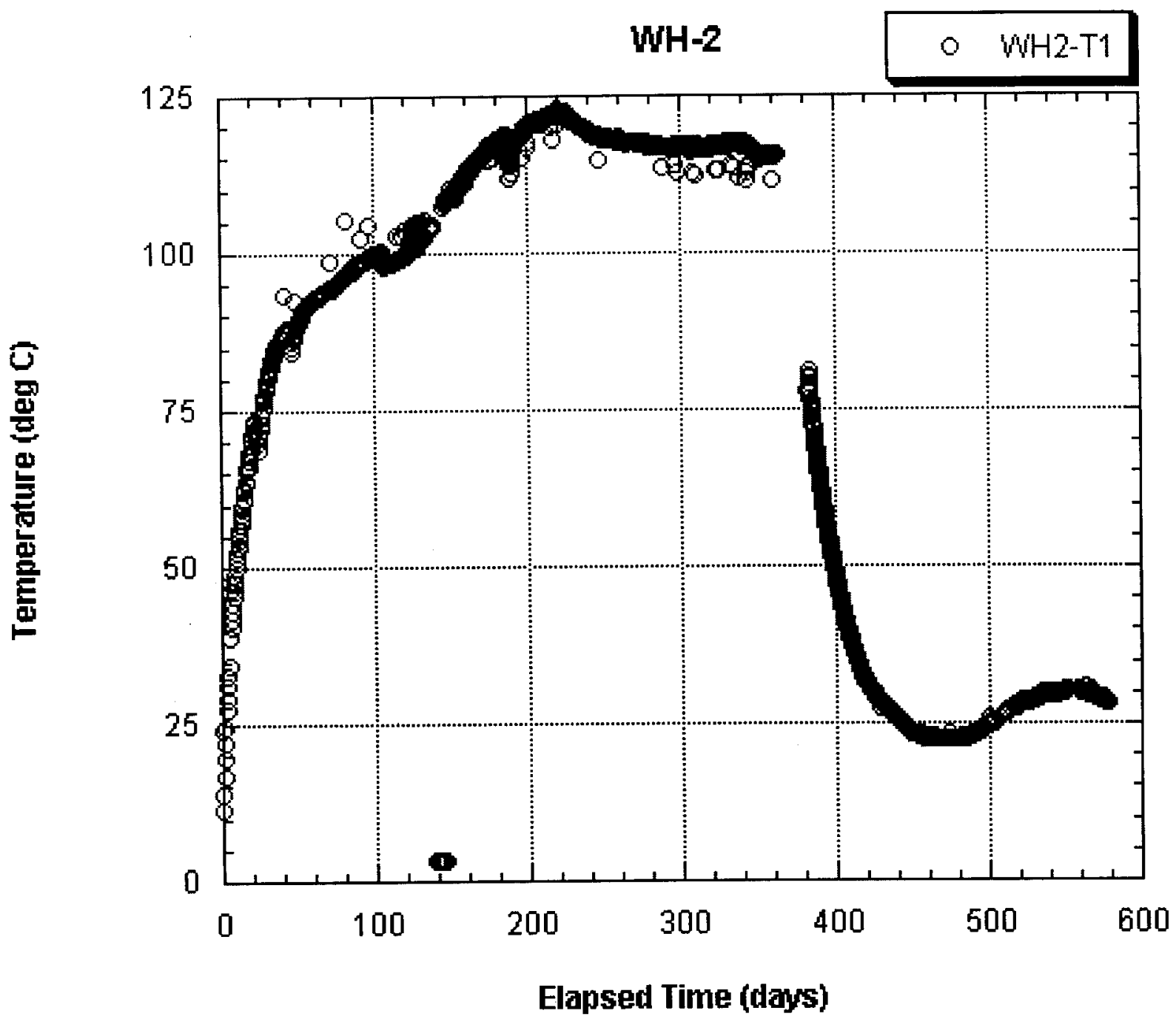

Figure 9-3. Temperature Measured by the Humicap in Hole WH-2 as a Function of Time 


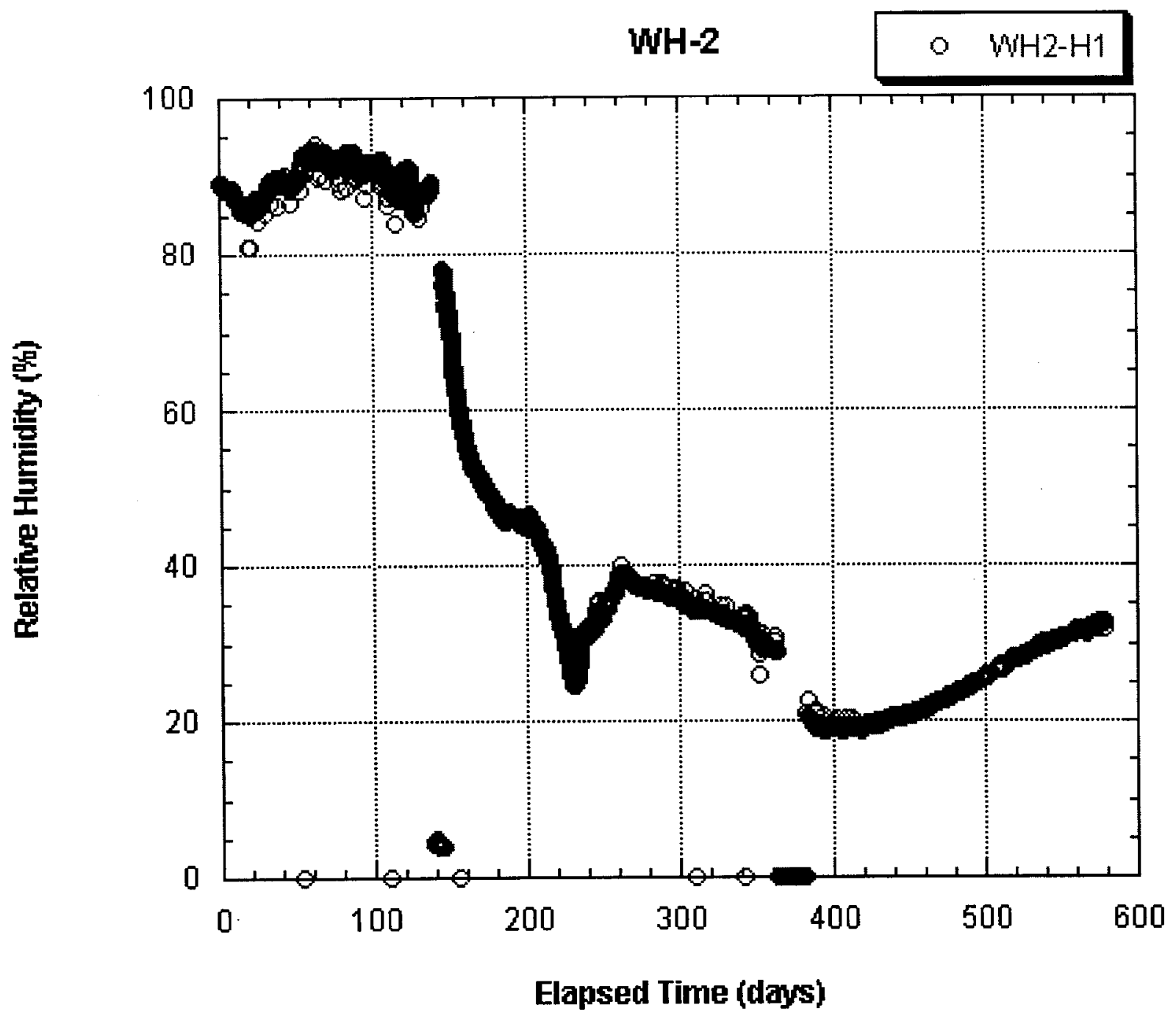

Figure 9-4. Relative Humidity Measured by the Humicap in Hole WH-2 as a Function of Time 


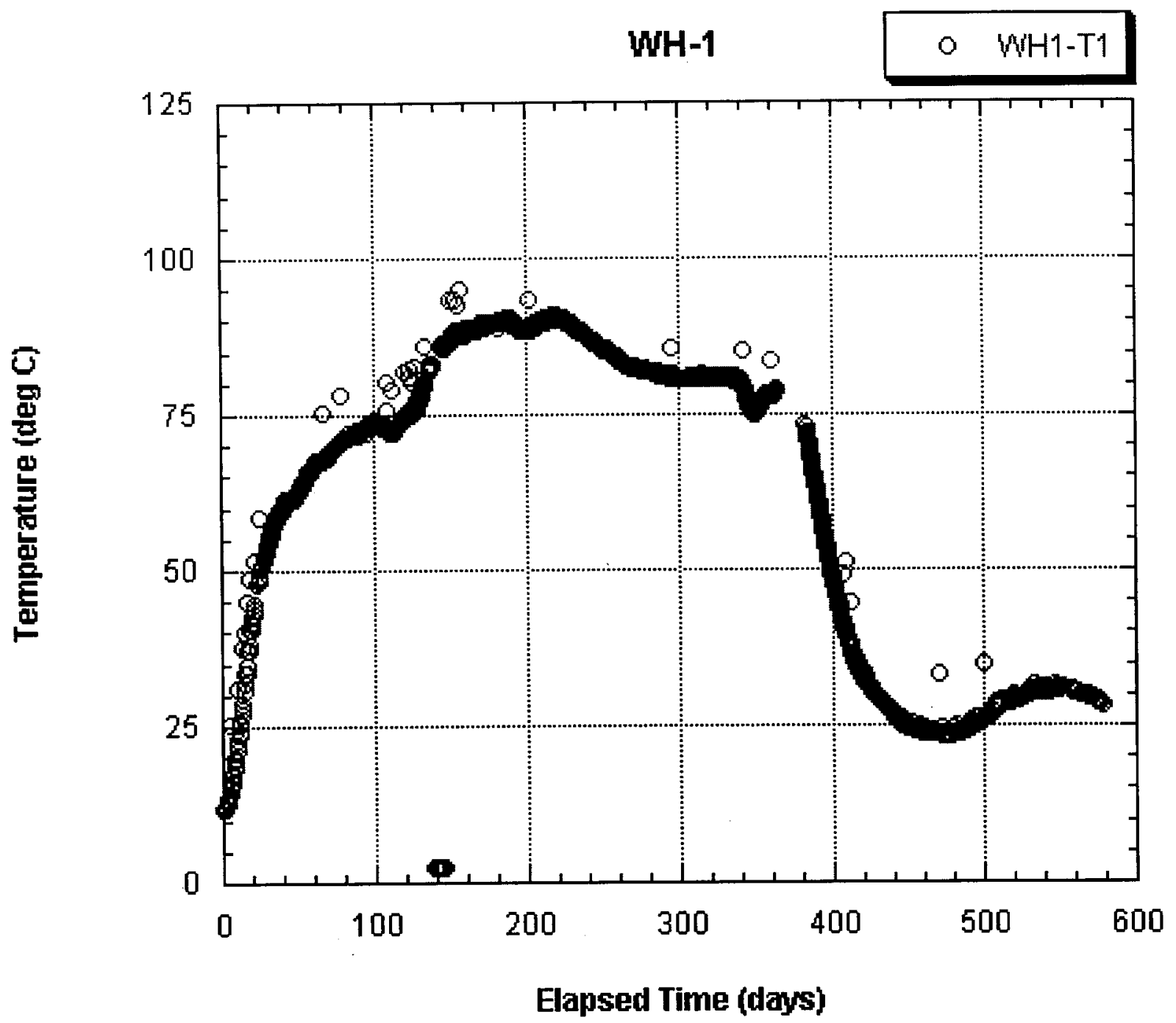

Figure 9-5. Temperature Measured by the Humicap in Hole WH-1 as a Function of Time 


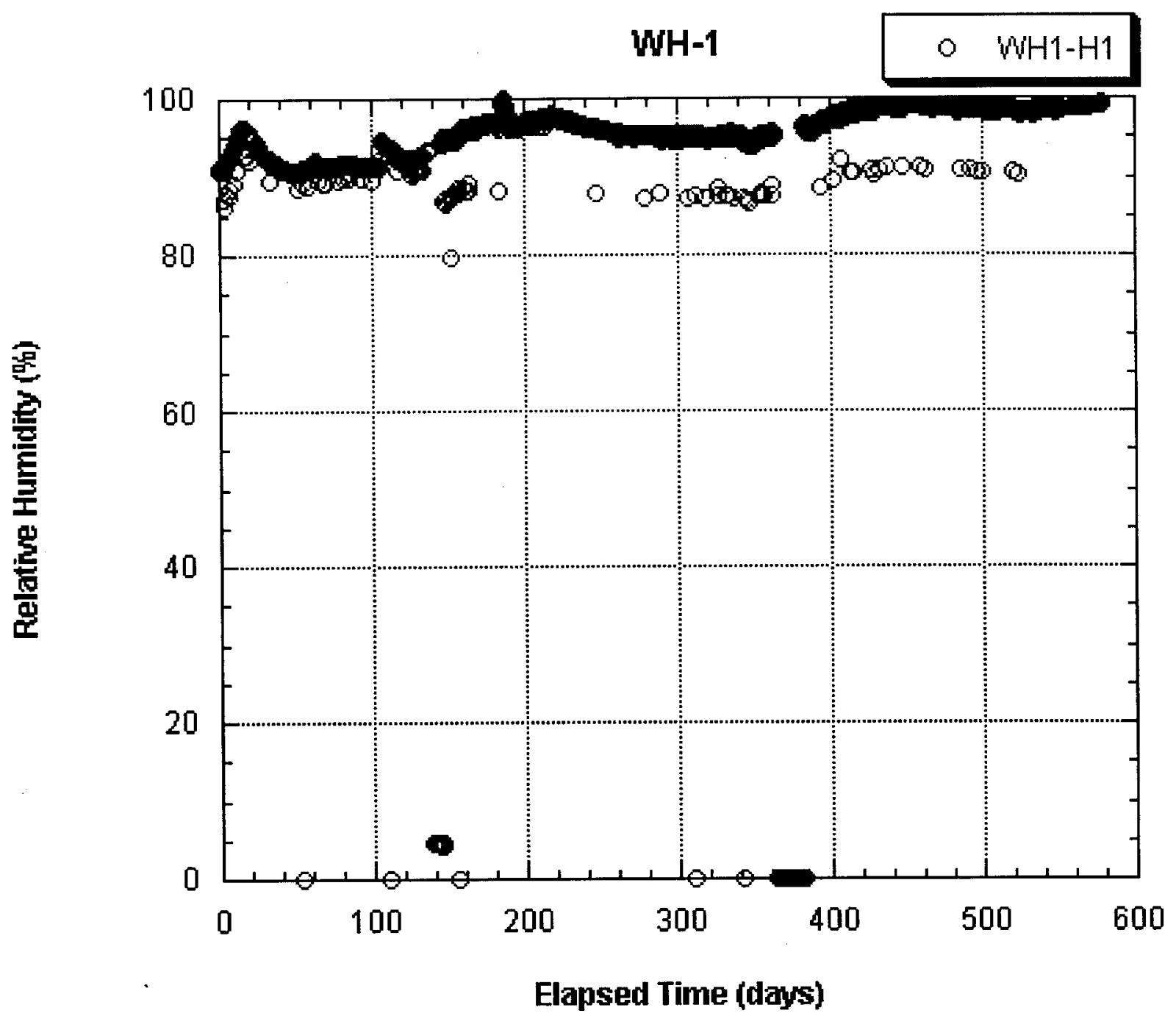

Figure 9-6. Relative Humidity Measured by the Humicap in Hole WH-1 as a Function of Time 


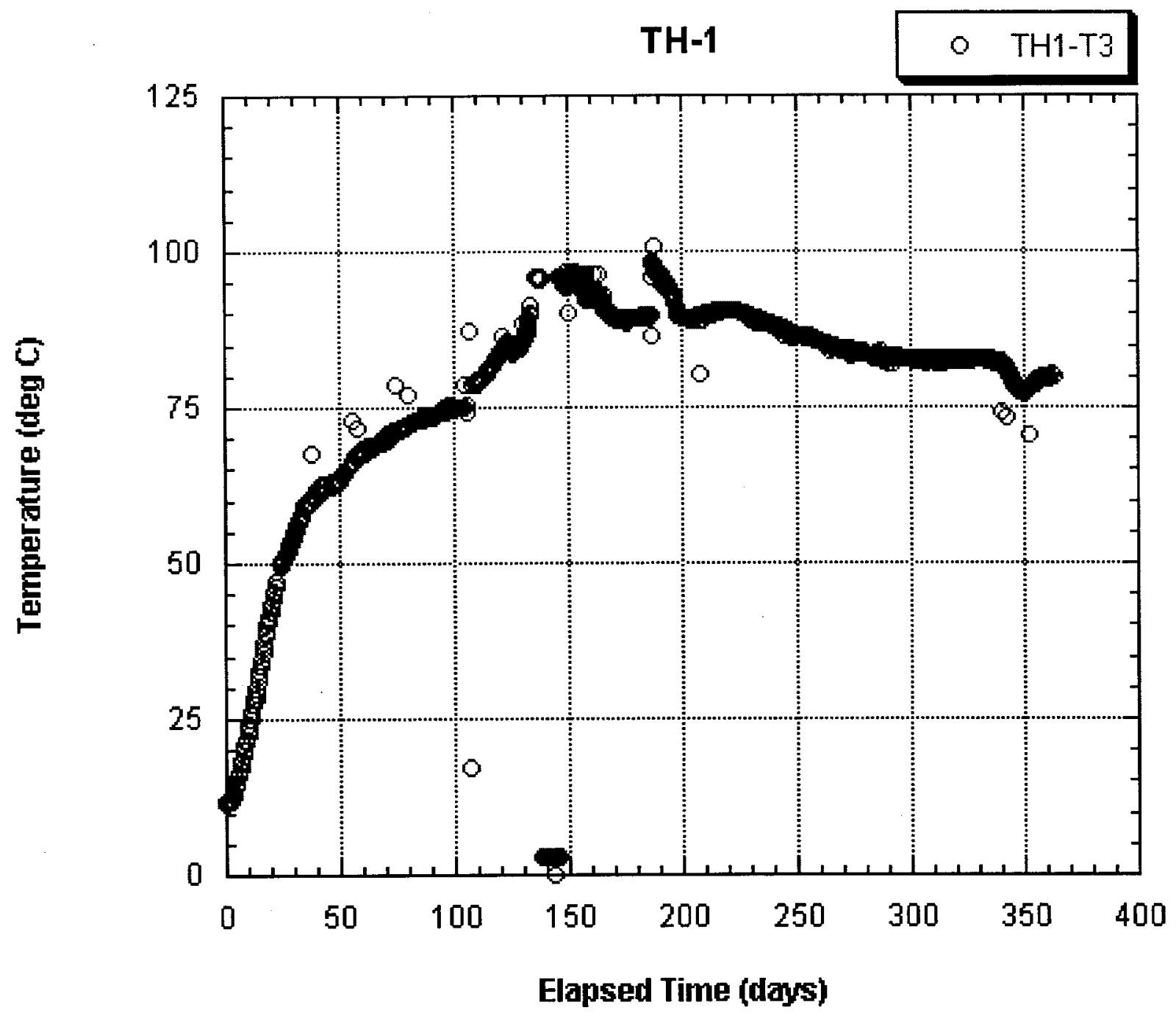

Figure 9-7. Temperature Measured by the Humicap in the Third Zone of Hole TH-1 as a Function of Time 


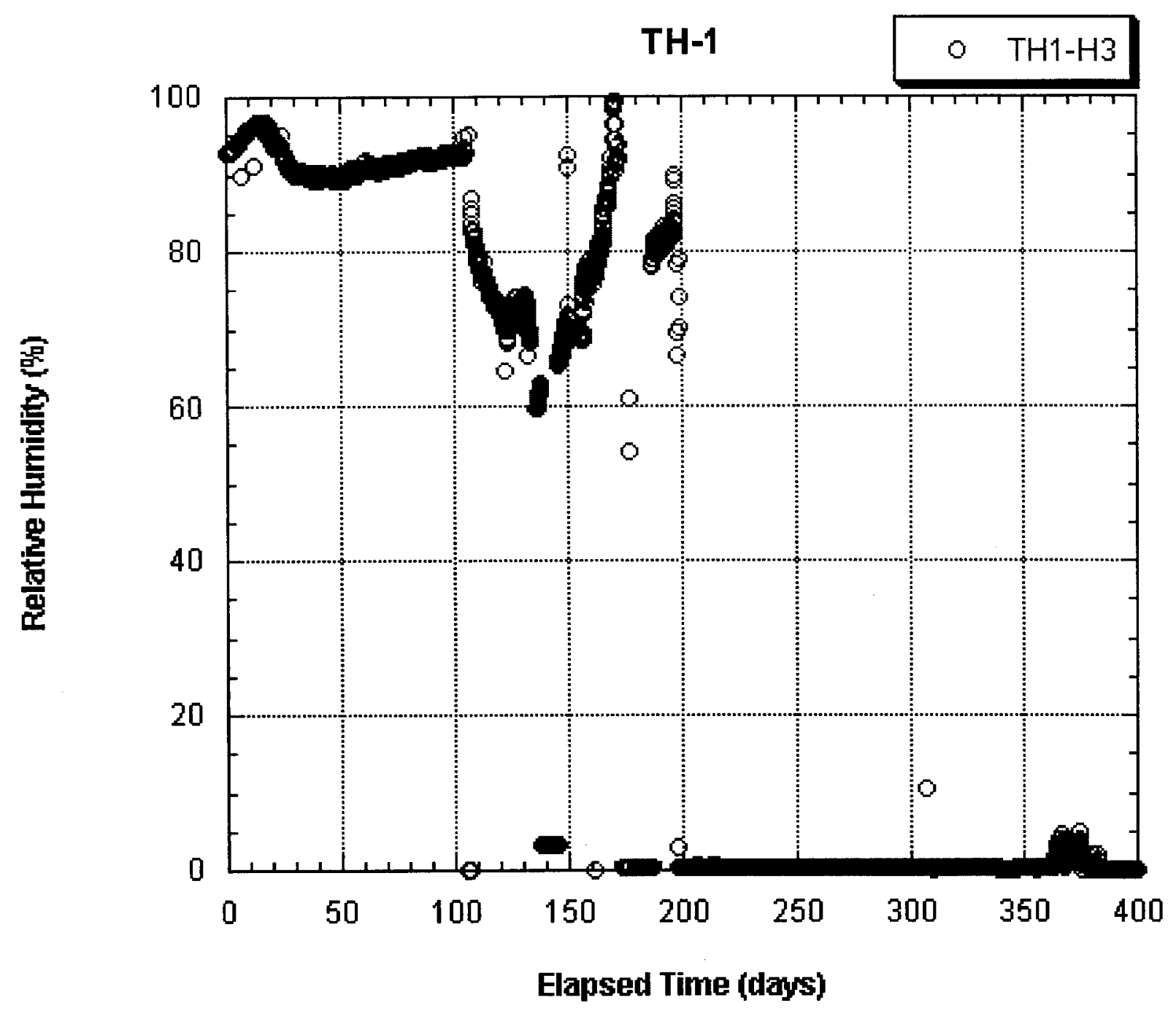

Figure 9-8. Relative Humidity Measured by the Humicap in the Third Zone of Hole TH-1 as a Function of Time 


\section{LARGE BLOCK TEST CONCLUSION AND FINDINGS}

\subsection{GENERAL CONCLUSION}

In general, the LBT performed as intended. Block heating was fairly one-dimensional. The boundary conditions of the LBT were controlled as planned: (1) the top temperature was maintained at about $60^{\circ} \mathrm{C}$, and (2) the four vertical surfaces were not adiabatic, but they were insulated and the thermal gradient at the block surface was measured, so that the heat flux away from the block can be calculated.

The TH processes in the LBT agreed well with the conceptual model of heating a partially saturated rock mass. Both the temperature and the moisture content measured in the LBT agreed well with model predictions. The LBT showed that a well-defined dryout zone was generated at the heater plane, as expected. The water in the block moved away from the heater plane, both upwardly and downwardly. The LBT showed, via temperature measurements, that condensate could reflux. The water involved in the condensation refluxing may not have come entirely from the original pore water in the block. In at least one of the two TH events (the September 2, 1997 event), rainwater assisted in trigging the event. There is no evidence that external water was involved in the first TH event (June 13, 1997). The LBT also showed that the refluxing condensate could penetrate the boiling zone, such that temperatures measured below the boiling zone were affected. The water that moved downward from the heater plane drained away due to gravity.

The moisture content of the dryout zone reached a minimum late in the heating phase, with a residual moisture content of about 1-2\%. It appears that the thermal energy was being expended in drying out additional rock rather than inducing additional moisture loss in the dryout zone. Based on the neutron log data, there appeared to be no rewetting of the rock mass in the dryout zone within the 6-month natural cool-down period. Given more time, some of the moisture may rewet the rock mass. However, it is very unlikely that the rock would be rewetted to near its original status; some of the water left the system for good.

The heating and the associated TH processes did not change the rock matrix very much. Based on the post-test core analyses, heating did not significantly alter the mechanical and the mineralogical properties of the rock matrix. On the other hand, fracture deformations were affected by the heating. The block was so deformed that the observation assembly in WO5 became permanently stuck in the hole.

\subsection{SPECIFIC FINDINGS}

1. Deviation from a uniformly one-dimensional moisture distribution is significant and appears to be controlled by heterogeneity in the block, probably fractures. For example, the heated zone was not imaged by ERT as a strictly planar anomaly. Even more obvious was a large, strong, and persistent dry zone that extended from the heater plane upward to the top of the block, near the western side of the ERT east-west plane. The dry zone formed as early as May 1997 and persisted to February 1998. The neutron results in borehole WN4 showed a similar dryout feature at about $0.8 \mathrm{~m}$ from the west face, starting in early July 1997 and lasting throughout the remainder of the test. 
2. Water drainage below the heater plane was observed through ERT as a wet zone near the center of the east-west plane in July 1997. The wet zone became drier in January 1998. Water drainage was also observed by neutron logging as two relatively wet zones along borehole NN6 from early July 1997 until February 1998.

3. A heat-pipe event may have been observed by ERT. During August and September 1997, a persistent wet anomaly was observed in the east-west ERT plane directly above the heaters and adjacent to a persistent dry anomaly. This may be the ERT signature of a heat pipe.

4. The results of the microbial investigation indicate that double-drug-labeled microbes migrated from the heater holes to the observation holes during the test. This finding means that the microbes survived the thermal environment and were able to migrate toward the observation holes, possibly assisted by the drainage water. Culturable cells reappeared on the heaters after the test, indicating that microbial activity near the heaters was only inactive during the thermal pulse. 


\section{REFERENCES}

American Society for Testing and Materials 1995, "Standard Test Method for Unconfined Compressive Strength of Intact Rock Core Specimens." (D 2938-95). Annual Book of ASTM Standards, v. 04.08, Soil and Rock, pp. 279-281. West Conshoshocken, PA: ASTM. TIC: 241090.

American Society for Testing and Materials 1997a, "Standard Practice for Preparing Rock Core Specimens and Determining Dimensional and Shape Tolerances." (D 4543-85). Annual Book of ASTM Standards, v. 04.08, Soil and Rock, 660-663. West Conshoshocken, PA: ASTM.

American Society for Testing and Materials 1997b, "Standard Test Method for Laboratory Determination of Pulse Velocities and Ultrasonic Elastic Constants of Rock." (D 2845-95). Annual Book of ASTM Standards, v. 04.08, Soil and Rock, pp. 245-259. West Conshoshocken, PA: ASTM.

American Society for Testing and Materials 1997c. "Standard Test Method for Elastic Moduli of Intact Rock Core Specimens in Uniaxial Compression." (D 3148-96). Annual Book of ASTM Standards, v. 04.08, Soil and Rock, pp. 306-310. West Conshoshocken, PA: ASTM. TIC: 247128.

Arulmoli, K. and St. John, C. M. 1987. Analysis of Horizontal Waste Emplacement Boreholes of a Nuclear Waste Repository in Tuff. SAND86-7133. Albuquerque, NM: Sandia National Laboratories. TIC: 202838.

Barber, D. C. and Seagar, A. D. 1987. "Fast Reconstruction of Resistance Images." Clinical Physics and Physiological Measurement, 8, Supplement A, 47-55.

Bevington, P. R. and Robinson, D. K. 1992. Data Reduction and Error Analysis for the Physical Sciences, $2^{\text {nd }}$ Edition. New York: McGraw-Hill, Inc. TIC: 243514.

Blair, S. C. 1999. Thermal Test Progress Report \#2. Section 3, "Large Block Test." BABEAF000-01717-5700-0001 REV 00.

Blair, S. C. and Berge, P. A. 1996. "Uniaxial Compression Behavior of Small Blocks of Welded Tuff." Proceedings 1996 International High Level Radioactive Waste Management Conference, April 29-May 3, 1996. Las Vegas, NV: American Nuclear Society and American Society of Civil Engineers, pp. 409-411.

Blair, S. C. and Wood, S. A. 1998. "Geomechanical Observations during the Large Block Test." Proceedings 1998 International High-Level Radioactive Waste Management Conference, May 11-14, 1998. Las Vegas, NV: American Nuclear Society and American Society of Civil Engineers.

Blair, S. C.; Berge, P. A. and Wang, H. F. 1996. Geomechanical Analysis of the Large Block Test. UCRL-ID-122898. Livermore, CA: Lawrence Livermore National Laboratory. ACC: MOL.19970506.0117. 
Blair, S. C; Kelly, J. M.; Pine, O.; Pletcher, R.; and Berge, P. A. 1996. Effect of Radiation on the Mechanical Properties of Topopah Spring Tuff. UCRL-ID-122899. Livermore, CA: Lawrence Livermore National Laboratory. ACC: MOL.19961021.0132.

Bodvarsson, G. S. and Bandurraga, T. M. 1996. Development and Calibration of the ThreeDimensional Site-Scale Unsaturated Zone Model of Yucca Mountain, Nevada. Berkeley, CA: Lawrence Berkeley National Laboratory.

Brodsky, N.S.; Riggins, M; Connolly, J.; and P. Ricci 1997. Thermal Expansion, Thermal Conductivity, and Heat Capacity Measurements for Boreholes UE25 NRG-4, UE25 NRG-5, USW NRG-6, and USW NRG-7/7A. SAND95-1955. Albuquerque, New Mexico: Sandia National Laboratories. ACC: MOL.19961029.0115.

Buscheck, T. A. and Nitao, J. J. 1995. Thermal-Hydrological Analysis of Large-Scale Thermal Tests in the Exploratory Studies Facility at Yucca Mountain. UCRL-ID-121791. Livermore, CA: Lawrence Livermore National Laboratory. ACC: MOL.19960501.0392.

Buscheck, T. A.; Shaffer, R. J.; Lee, K. H.; and Nitao, J. J. 1997. Analysis of ThermalHydrological Behavior during the Heating Phase of the Single Heater Test at Yucca Mountain. Livermore, CA: Lawrence Livermore National Laboratory. SP9266M4. ACC: MOL19980109.0262

Buscheck, T. A.; Wilder, D. G.; and Nitao, J. J. 1993. Large-Scale In Situ Heater Tests for Hydrothermal Characterization at Yucca Mountain. UCRL-JC-112445. Livermore, CA: Lawrence Livermore National Laboratory. ACC: NNA.19930331.0009.

Carlberg, E. D. and Roberts, J. J. 2000. Microstructural Analyses of Topopah Spring Tuff from the Large Block Test at Fran Ridge, Nevada. UCRL-ID-137110. Livermore, CA: Lawrence Livermore National Laboratory.

Carlos, B. A. 1985. Minerals in Fractures of the Unsaturated Zone from Drill Core USW G-4, Yucca Mountain, Nye County, Nevada. LA-10415-MS, Los Alamos, New Mexico: Los Alamos National Laboratory. ACC: NNA.19920506.0037.

Carlos, B. A.; Chipera, S. J., and Bish, D. L. 1995. Distribution and Chemistry of FractureLining Minerals at Yucca Mountain, Nevada. LA-12977-MS. Los Alamos, New Mexico: Los Alamos National Laboratory. ACC: MOL.19960306.050X.

Chen, R. 1999. "Analyses of Drift Stability and Rockfall Due to Earthquake Ground Motion at Yucca Mountain, Nevada." Rock Mechanics for Industry, Proceedings of the $37^{\text {th }}$ U. S. Rock Mechanics Symposium, Vail, Colorado, USA, 6-9 June 1999. B. Amadei, R. L. Kranz, G. A. Scott, and P. H. Smeallie, eds. 2, 743-750. Rotterdam, The Netherlands: A. A. Balkema. TIC: 245246. 
Chen, C-I.; Meike, A.; Chuu, Y-J.; Sawvel, A.; and Lin, W. 1998. "Investigation of Bacterial Transport in the Large Block Test, A Thermally Perturbed Block of Topopah Spring Tuff." Proceedings of the MRS '98 Fall Meeting, Scientific Basis for Nuclear Waste Management XXII, Boston, MA, November 30-December 4, 1998, 556, 1151-1158. Also available as Lawrence Livermore National Laboratory Report UCRL-JC-130811, Revision 1, November 6, 1998.

CRWMS M\&O (Civilian Radioactive Waste Management System Management and Operating Contractor) 1997. Drift Scale Test Design and Forecast Results. BAB000000-01717-4600-00007 REV 01. Las Vegas, Nevada: CRWMS M\&O. ACC: MOL.19980710.0155.

CRWMS M\&O 1998. Total System Performance Assessment-Viability Assessment (TSPA-VA) Analyses Technical Basis Document. B00000000-01717-4301-00003 REV 01. Las Vegas, Nevada: CRWMS M\&O. ACC: MOL.19981008.0003.

CRWMS M\&O 1999a. Single Heater Test Final Report. BAB000000-01717-5700-00005 REV 00. Las Vegas, Nevada: CRWMS M\&O. ACC: MOL.20000103.0634.

CRWMS M\&O 1999b. License Application Design Selection Report. B00000000-01717-460000123 REV 01 ICN 01. Las Vegas, Nevada: CRWMS M\&O. ACC: MOL.19990908.0319.

CRWMS M\&O 2000a. Engineered Barrier System Features, Events, and Processes and Degradation Modes Analysis. ANL-EBS-MD-000035 REV 00 ICN 01. Las Vegas, Nevada: CRWMS M\&O. ACC: MOL.20000324.0558.

CRWMS M\&O 2000b. Near-Field Environment Process Model Report. TDR-NBS-MD-000001 REV 00 ICN 01. Las Vegas, Nevada: CRWMS M\&O. ACC: MOL.20000421.0034.

CRWMS M\&O 2000c. Thermal Tests Thermal-Hydrological Analysis/Model Report. ANLNBS-TH-000001 REV 00A. Las Vegas, Nevada: CRWMS M\&O. ACC: MOL.20000505.0231.

CRWMS M\&O 2000d. Technical Work Plan for Nearfield Environment Thermal Analyses and Testing. TWP-NBS-TH-000001 REV 00. Las Vegas, Nevada: CRWMS M\&O. ACC: MOL.20001201.0026.

Daily, W. and Lin, W. 1991. "Laboratory Determined Suction Potential of Topopah Spring Tuff at High Temperatures." Proceedings of the Second Annual International Conference on High Level Radioactive Waste Management, Las Vegas, NV, April 28-May 3, 1991, 583-588. La Grange Park, IL: American Nuclear Society. ACC:NNA19910304.0097.

Daily, W. and Owen, E. 1991. "Cross-Borehole Resistivity Tomography." Geophysics, 56, 1228-1235. Tulsa, OK: Society of Exploration Geophysicists.

Danko, G. and Buscheck, T.A., 1993. "Single-hole In Situ Heater Tests for Hydrothermal Characterization at Yucca Mountain." Proceedings, Fourth International High Level Nuclear Waste Conference, April, Las Vegas, Nevada, pp. 975-985. 
Danko, G.; Creech, H.; Phillips, J.; and Tippabhatla, S. 1998. "In Situ Reka Probe Measurements at Fran Ridge and in the ESF at Yucca Mountain." Proceedings, Eighth International High-Level Radioactive Waste Management Conference, Las Vegas, NV, 54-56. La Grange Park, IL: American Nuclear Society.

Danko, G. and Mousset-Jones, P. F. 1991. "A Probe Method for Measuring In Situ Thermophysical Properties." Proceedings, 2nd International High Level Radioactive Waste Management Conference, Las Vegas, NV, April 28-May 3, 1991, 555-563. La Grange Park, IL: American Nuclear Society.

Dines, K. A. and Lytle, R. J. 1981. "Analysis of Electrical Conductivity Imaging." Geophysics, 46, 1025-1036. Tulsa, OK: Society of Exploration Geophysicists.

DOE (U.S. Department of Energy) 1988. Site Characterization Plan, Yucca Mountain Site, Nevada Research and Development Area, Nevada, Office of Civilian Radioactive Waste Management, Washington, DC, DOE/RW-0199. ACC: HQO.881201.0002.

DOE 1990. Yucca Mountain Project Reference Information Base, Version 4. Las Vegas, NV: Yucca Mountain Site Characterization Project Office, U.S. Department of Energy. ACC: NNA.19890330.0077.

DOE 1995. Yucca Mountain Project Reference Information Base, YMP/CC-0002, Version 4.002, Yucca Mountain Site Characterization Project Office, Las Vegas, NV, YMP/CC-0002. ACC: NNA.910129.0066.

Eisenberg, D. and Kauzmann, W. 1969. The Structure and Properties of Water. New York, NY: Oxford University Press.

Hardin, E. L. 1998. Near-Field/Altered-Zone Models Report. UCRL-ID-129179. Livermore, CA: Lawrence Livermore National Laboratory.

Henderson, R. P. and Webster, J. G. 1978. "An Impedance Camera for Spatially Specific Measurements of Thorax." IEEE Transactions Biomedical Engineering, BME-25, 250-254.

Isaacson, D., 1986. "Distinguishability of Conductivities by Electric Current Computed Tomography." IEEE Transactions on Medical Imaging, MI-5, (2), 91-95.

Itasca Consulting Group, Inc., 1993. FLAC User's Manual, v. I-III. Minneapolis, MN: Itasca Consulting Group, Inc. TIC: 232823.

Jaeger, J. C. and Cook, N. G. W. 1979. Fundamentals of Rock Mechanics. $3^{\text {rd }}$ ed. London: Chapman and Hall.

Kim, K. and McCage, W. M. 1984. "Geomechanics Characterization of the Candidate Nuclear Waste Repository Site in Basalt." Proceedings 25th U.S. Symposium on Rock Mechanics, 874 882. ACC: NNA.891212.0018. 
Klavetter, E. A., and Peters, R. R. 1986. Estimation of Hydrologic Properties of an Unsaturated, Fractured Rock Mass. SAND84-2642. Albuquerque, NM: Sandia National Laboratories. ACC: NNA.870317.0738.

Konikow, L. F. and Bredehoeft, J. D. 1992. "Ground-Water Models Cannot Be Validated." Advances in Water Resources, 15, 75-83. U.K.: Elsevier Science. TIC: 239876.

Krueger, S.; Olson, G. J.; Johnsonbaugh, D.; and Beveridge, T. J. 1993. Applied Environmental Microbiology, 59, 4056-4064. Washington, DC: American Society for Microbiology.

LaBrecque, D. J.; Miletto, M.; Daily, W.; Ramirez, A.; and Owen, E., 1996a. "The Effects of Noise on Occam's Inversion of Resistivity Tomography Data." Geophysics, 61, 538-548. Tulsa, OK: Society of Exploration Geophysicists. TIC: 247174.

LaBrecque, D. J.; Ramirez, A.; Daily, W.; Binley, A.; and Schima, S. 1996b. "ERT Monitoring of Environmental Remediation Processes." Measurement Science Technology, 7, 375-383. IOP Publications, Ltd: U.K. TIC: 236228.

Lee, K. H. 1995a. Progress Report on Pre-Test Calculations for the Large Block Test. UCRLID-118699. Livermore, CA: Lawrence Livermore National Laboratory. ACC: MOL.19950406.0143.

Lee, K. H. 1995b. Second Progress Report on Pre-Test Calculations for the Large Block Test. UCRL-ID-122300. Livermore, CA: Lawrence Livermore National Làboratory. ACC: MOL.19960417.0218.

Levy, S. S. and O'Neil, J. R. 1989, "Moderate-Temperature Zeolitic Alteration in a Cooling Pyroclastic Deposit." Chemical Geology, 76, (3/4), 321-326. Amsterdam, The Netherlands: Elsevier Science. TIC 237819.

Lin, W. 1994. Activity Plan, AP-LBT-01, Rev. 0, for Large Block Tests of Coupled ThermalHydrological-Chemical Processes (YMP WBS Element 1.2.3.12.4). Livermore, CA: Lawrence Livermore National Laboratory. ACC: MOL.19940908.0093.

Lin, W. 1999. Activity Plan AP-LBT-10-0-4 for Large Block Tests of Coupled ThermalMechanical-Hydrological-Chemical Processes. Livermore, CA: Lawrence Livermore National Laboratory.

Lin, W. and Daily, W. 1984. Transport Properties of Topopah Spring Tuff. UCRL-53602. Livermore, CA: Lawrence Livermore National Laboratory.

Lin, W.; Wilder, D. G.; Blair, S. C.; Buscheck, T. A.; Daily, W.; Gdowski, G.; Grassley, W.; Lee, K. H.; Meike, A.; Ramirirez, A.; Roberts, J. J.; Ruddle, D.; Wagoner, J.; Watwood, D.; Williams, T.; Carlson, R.C.; and Neubauer, D. 1997. An Overview of Progress on the Large Block Test. UCRL-JC-128796. Livermore, CA: Lawrence Livermore National Laboratory. TIC 237082. 
Lin, W.; Wilder, D. G.; Blink, J.; Berge, P. A.; Blair, S. C.; Brugman, V.; Carlson, R. C.; Lee, K. H.; Owens, M.; Pletcher, R.; Rector, N.; Roberts, J. J.; Ruddle, D.; Sommer, S.; Ueng, T.; and Wagoner, J. 1995. "The Large Block Test, A Progress Report." Proceedings Sixth Annual International Conference on High-Level Radioactive Waste Management, Las Vegas, NV, April 30-May 5, 1995, 46-47. La Grange Park, IL: American Nuclear Society, Inc. TIC: 240140.

Lin, W., Wilder, D. G.; Blink, J. A.; Blair, S. C.; Buscheck, T. A.; Glassley, W. E.; Lee, K. H.; Owens, M. W.; and Roberts, J. J. 1995. “A Heated Large Block Test for High Level Nuclear Waste Management." Proceedings Second International Conference on the Mechanics of Jointed and Faulted Rock, Vienna, Austria, April 1995. Brookfield, VT: A.A. Balkema. TIC: 247051.

Lovley, D.R.; Widman, P. K.; Woodward, J. C.; and Phillips, E. J. P. 1993. "Reduction of Uranium by Cytochrome C3 of Desulfovibrio vulgaris." Applied Environmental Microbiology, 59 (11), 3572-3576. Washington, DC: American Society for Microbiology. TIC: 24486.

Lytle, R. J. and Dines, K. A. 1978. An Impedance Camera: A System for Determining the Spatial Variation of Electrical Conductivity. UCRL-52413. Livermore, CA: Lawrence Livermore Laboratory.

Martin, III, R. J.; Price, R. H.; Boyd, P. J.; and Noel, J. S. 1993. Unconfined Compression Experiments on Topopah Spring Member Tuff at $22^{\circ} \mathrm{C}$ and a Strain Rate of $10^{-9} \mathrm{~s}^{-1}$ : Data Report. SAND92-1810. Albuquerque, NM: Sandia National Laboratories. ACC: NNA.19930728.0088.

Meike, A. and Horn, J. 1997. "Installation of Labeled Microbe Samples into Large Block." Letter Report (SPLG1BM4). Livermore, CA: Lawrence Livermore National Laboratory.

Morelli, G. and LaBrecque, D. 1996. "Robust Scheme for ERT Inverse Modeling," Proceedings of Symposium on Applications of Geophysics to Engineering and Environmental Problems, Keystone, CO, April 28-May 1, 1996. Wheat Ridge, CO: Environmental and Engineering Geophysical Society.

Nimick, F.B. 1990. The Thermal Conductivity of the Topopah Spring Member at Yucca Mountain, Nevada. SAND86-0090. Albuquerque, NM: Sandia National Laboratories. ACC: NNA.890516.0183.

Nimick, F. B.; Van Buskirk, R. G.; and McFarland, A.F. 1987. Uniaxial and Triaxial Compression Test Series on the Topopah Spring Member form USW G-2, Yucca Mountain, Nevada. SAND85-0703. Albuquerque, New Mexico: Sandia National Laboratories. TIC: 202747.

Oldenburg, D. W. and Li, Y. 1994. "Inversion of Induced Polarization Data." Geophysics, 59 (9), 1327-1341. Tulsa, OK: Society of Exploration Geophysicists.

Pelton, W. H., Rijo, L.; and Swift, C. M., Jr. 1978. "Inversion of Two-Dimensional Resistivity and Induced-Polarization Data." Geophysics, 43 (4), 788-803. Tulsa, OK: Society of Exploration Geophysicists. TIC: 239348. 
Price, R. H. 1986. Effects of Sample Size on the Mechanical Behavior of Topopah Spring Tuff. SAND85-0709. Albuquerque, NM: Sandia National Laboratories. ACC: NNA.891 106.0125.

Price, R. H. et al., 1993. Laboratory Determination of the Mechanical Properties of Fractures. YMP Study Plan 8.3.1.15.1.4 RO. Washington, DC: U.S. Department of Energy.

Roberts, J. J. and Lin, W. 1996a. Report on Laboratory Tests of Drying and Wetting of Intact Rocks. UCRL-ID-121513. Livermore, CA: Lawrence Livermore National Laboratory.

Roberts, J. J. and Lin, W. 1996b. "X-Ray Radiography of Fracture Flow and Matrix Imbibition." Proceedings of the Seventh Annual International Conference on High Level Radioactive Waste Management, Las Vegas, Nevada, April 29-May 3, 1996. La Grange Park, IL: American Nuclear Society, Inc.

Roberts, J. J. and Lin, W. 1997a. "X-Ray Radiography of Fracture Flow and Matrix Imbibition in Topopah Spring Tuff under a Thermal Gradient." International Journal of Rock Mechanics and Mining Sciences, 34, (3/4), 482. London, U.K.: Elsevier Science Ltd. TIC: 237400.

Roberts, J. J. and Lin, W. 1997b. "Electrical Properties of Partially Saturated Topopah Spring Tuff: Water Distribution as a Function of Saturation." Water Resources Research, 33 (4), $577-$ 587. Washington, DC: American Geophysical Union. TIC: 239736.

Sandia National Laboratories 1997. Thermal Expansion of Carbon Fiber and Invar Rods. Albuquerque, NM: Sandia National Laboratories. ACC: MOL.19980210.0196.

Sasaki, Y. 1992. "Resolution of Resistivity Tomography Inferred from Numerical Simulation." Geophysical Prospecting, 40, 453-463. Oxford, U.K.: Blackwell Science Ltd. TIC: 247151.

Sweetkind, D. S.; Verbeck, E. R.; Singer, F. R.; Byers, F. M., Jr.; and Martin, L. G. 1995. Surface Fracture Network at Pavement P2001, Fran Ridge, Near Yucca Mountain, Nye County, Nevada. Denver, CO: U. S. Geological Survey. MOL. 19960213.0253.

Thorpe, R. and Springer, J. 1981. Fracture Mapping for Radionuclide Migration Studies in the Climax Granite. UCID-19081. Livermore, CA: Lawrence Livermore National Laboratory.

Tripp, A. C.; Hohmann, G. W.; and Swift, C. M. 1984. "Two-Dimensional Resistivity Inversion." Geophysics, 49 (10), 1708-1717. Tulsa, OK: Society of Exploration Engineers. TIC: 239779.

Vinegar, H. J. and Waxman, M. H. 1984. "Induced Polarization of Shaly Sands." Geophysics, 49 (8), 1267-1287. Tulsa, OK: Society of Exploration Geophysicists.

Wagoner, J. L. 1999. Fracture Characterization of the Large-Block Test, Fran Ridge, Yucca Mountain, Nevada. UCRL-ID-133846. Livermore, CA: Lawrence Livermore National Laboratory. 
Wagoner, J. L. 2000. Surface Fracture Data Report for the Large-Block Test, Fran Ridge, Yucca Mountain, Nevada. UCRL-ID-40784. Livermore, CA: Lawrence Livermore National Laboratory.

Wang, J. S. Y. and Ahlers, C. F. 1996. Pre-Test Analysis of the Large Block Test-Air Flow and Gas Tracer Transport for Thermal-Hydrological Evolution. Berkeley, CA: Lawrence Berkeley Laboratory. SP9012M5.

Waxman, M. H. and Thomas, E. C. 1974a. "Electrical Conductivities in Shaly Sands-I. The Relation Between Hydrocarbon Saturation and Resistivity Index." Journal of Petroleum Technology, 26, 213-218. Dallas, TX: Society of Petroleum Engineers. TIC: 239699.

Waxman, M. H. and Thomas, E. C. 1974b. "Electrical Conductivities in Shaly Sands-II. The Temperature Coefficient of Electrical Conductivity." Journal of Petroleum Technology, 26, 218225. Dallas, TX: Society of Petroleum Engineers. TIC: 239699.

Wexler, A.; Fry, B.; and Neuman, M. R. 1985. "Impedance-Computed Tomography Algorithm and System." Applied Optics, 24, (23), 3985-3992.

Wildenschild, D.; Costantino, M. S.; Roberts, J. J.; Kneafsey, T. J.; and Lin, W. 1998.

Fracture/Matrix Flow Experiments Results. UCRL-ID-131775. Livermore, CA: Lawrence Livermore National Laboratory.

Wilder, D. G. 1993. Preliminary Near-Field Environment Report, Volume I: Technical Basis for EBS Design. UCRL-LR-107476. Livermore, CA: Lawrence Livermore National Laboratory. ACC: NNA.19920501.0002.

Wilder, D. G. 1996. Near-Field and Altered-Zone Environment Report: Volume II. UCRL-LR124998. Livermore, CA: Lawrence Livermore National Laboratory. ACC: MOL.19961212.0122.

Wilder, D. G. 1997. Near-Field and Altered-Zone Environment Report: Volume I, Technical Basis for EBS Design. UCRL-LR-107476. Livermore, CA: Lawrence Livermore National Laboratory. ACC: MOL.19961212.0121.

Wilder, D. G.; Lin, W.; Blair, S. C.; Buscheck, T. A.; Carlson, R. C.; Lee, K. H.; Meike, A.; Ramirez, A. L.; Wagoner, J. L.; and Wang, J. 1997. Large-Block Test Status Report. UCRL-ID128776. Livermore, CA: Lawrence Livermore National Laboratory. ACC: MOL.19980508.0727.

Yorkey, T. J.; Webster J. G.; and Tompkins, W. J. 1987. "Comparing Reconstruction Algorithms for Electrical Impedance Tomography." IEEE Transactions Biomedical Engineering, BME-34, (11), 843-852.

Yow, J. L. Jr. and Wilder, D. G. 1983. "Planning Exploratory Drilling: The Effect of Blind Zones and Level of Logging Effort." Proceedings of the 24th U.S. Symposium on Rock Mechanics, 807-812. Washington, DC: National Academy of Sciences. TIC: 237604. 
Zimmerman, R. M.; Schuch, M; Mason, R. C.; and Donald, S. 1986. Final Report: G-Tunnel Heated Block Experiment. SAND84-2620. Albuquerque, NM: Sandia National Laboratories. TIC: 202721.

Zimmerman, R. M. and Blanford, M. L. 1986. "Expected Thermal and Hydrothermal Environments for Waste Emplacement Holes Based on G-Tunnel Heater Experiments." Proceedings of the 27th U.S. Symposium on Rock Mechanics, 874-882. Littleton, CO: Society of Mining Engineers, Inc. ACC: NNA.891212.001 
THIS PAGE INTENTIONALLY LEFT BLANK 


\section{APPENDIX A: \\ BOREHOLE LISTING AND INSTRUMENT TYPE BY BLOCK SURFACE}

\begin{tabular}{|c|c|c|c|}
\hline $\begin{array}{c}\text { Hole or } \\
\text { instrument } \\
\text { number }\end{array}$ & Description & Installation method & $\begin{array}{l}\text { Borehole } \\
\text { size }(\mathrm{cm})\end{array}$ \\
\hline \multicolumn{4}{|c|}{ Top of block } \\
\hline RTD1 & Surface mounted on Al plate & clamp or epoxy after coils emplaced & \\
\hline RTD2 & Surface mounted on Al plate & & \\
\hline RTD3 & Surface mounted on Al plate & & \\
\hline RTD4 & Surface mounted on Al plate & & \\
\hline RTD5 & Surface mounted on Al plate & & \\
\hline TN1 & Neutron liner grouted in place & & 3.81 \\
\hline TN2 & Neutron liner grouted in place & . & 3.81 \\
\hline TN3 & Neutron liner grouted in place & & 3.81 \\
\hline TN4 & Neutron liner grouted in place & & 3.81 \\
\hline TN5 & Neutron liner grouted in place & & 3.81 \\
\hline TM1 & Mech MPBX grouted in & & 7.62 \\
\hline TM2 & Optical extens with liner & & 7.62 \\
\hline$\overline{\mathrm{TH} 1}$ & Hydrol with packer & LBNL will use for $\mathrm{K}$ test, then install & 7.62 \\
\hline$\pi 1$ & RTDs grouted & 30 RTD bundle & 3.81 \\
\hline$\pi 2$ & RTDs grouted & 30 RTD bundle & 3.81 \\
\hline TR1 & Reka probe & 4-ft deep hole grouted after installed & 1.27 \\
\hline LBL1 & Open hole & Used for $\mathrm{K}$ test and then grouted in & 3.81 \\
\hline LBL2 & Open hole & Used for $K$ test and then grouted in & 3.81 \\
\hline \multicolumn{4}{|c|}{ North side } \\
\hline NN1 & Neutron liner grouted in place & & 3.81 \\
\hline NN2 & Neutron liner grouted in place & & 3.81 \\
\hline NN3 & Neutron liner grouted in place & & 3.81 \\
\hline$\overline{\mathrm{NN} 4}$ & Neutron liner grouted in place & & 3.81 \\
\hline NN5 & Neutron liner grouted in place & & 3.81 \\
\hline NN6 & Neutron liner grouted in place & & 3.81 \\
\hline NT1 & RTDs grouted & 14 RTD bundle & 3.81 \\
\hline NT2 & RTDs grouted & 14 RTDs-larger borehole to grout & 7.62 \\
\hline NT3 & RTDs grouted & 14 RTDs-larger borehole to grout & 7.62 \\
\hline NT4 & RTDs grouted & 14 RTD bundle & 3.81 \\
\hline NM1 & Mech MPBX & Grouted after install & 7.62 \\
\hline NM2 & Mech MPBX & Grouted after install & 7.62 \\
\hline NM3 & Mech MPBX & Grouted after install & 7.62 \\
\hline $\mathrm{NH} 1$ & Hydrol with packer & LBNL may use for K test, then install & 7.62 \\
\hline N01 & Observation hole & pyrex liner w/crape paper-pvc support & 3.81 \\
\hline NO2 & Observation hole & pyrex liner w/crape paper-pvc support & 3.81 \\
\hline \multicolumn{4}{|c|}{ West side } \\
\hline WN1 & Neutron liner grouted in place & & 3.81 \\
\hline WN2 & Neutron liner grouted in place & & 3.81 \\
\hline WN3 & Neutron liner grouted in place & & 3.81 \\
\hline WN4 & Neutron liner grouted in place & & 3.81 \\
\hline
\end{tabular}




\begin{tabular}{|l|l|l|c|}
\hline WM1 & Mech MPBX & grouted after emplacement & 7.62 \\
\hline WM2 & Mech MPBX & grouted after emplacement & 7.62 \\
\hline WM3 & Mech MPBX & grouted after emplacement & 7.62 \\
\hline WT1 & RTDs grouted & 14 RTD bundle & 3.81 \\
\hline WT2 & RTDs grouted & 14 RTD bundle & 3.81 \\
\hline WT3 & RTDs grouted & 14 RTD bundle & 3.81 \\
\hline WH1 & Hydrol w/ packer & LBNL may use for K test, then install & 7.62 \\
\hline WH2 & Hydrol w/ packer & LBNL may use for K test, then install & 7.62 \\
\hline WR1 & Reka Probe & Grout after install & 1.27 \\
\hline WH2 & Reka Probe & Grout after install & 1.27 \\
\hline WO5 & Observation hole & pyrex liner w/crape paper-pvc support & 3.81 \\
\hline & & East side & \\
\hline & South side: no boreholes & 3.81 \\
\hline EH1 & heater & Centralizers, RTDs and heaters & 3.81 \\
\hline EH2 & heater & Centralizers, RTDs and heaters & 3.81 \\
\hline EH3 & heater & Centralizers, RTDs and heaters & 3.81 \\
\hline EH4 & heater & Centralizers, RTDs and heaters & 3.81 \\
\hline EH5 & heater & Centralizers, RTDs and heaters & 3.81 \\
\hline EO3 & Observation hole & pyrex liner w/crape paper-pvc support & \\
\hline
\end{tabular}




\section{APPENDIX B:}

THE X-Y-Z COORDINATES (IN CM), WITH RESPECT TO THE SOUTHWESTERN CORNER ON TOP OF THE BLOCK, OF THE COLLAR (THE FIRST LINE) AND THE TOTAL DEPTH (THE SECOND LINE) OF ALL OF THE HOLES IN THE LBT. THE HOLE I.D. IN PARENTHESES IS THE CORRESPONDING INSTRUMENTATION HOLE AS LISTED IN APPENDIX A.

\begin{tabular}{|c|c|c|c|}
\hline$x$ & $y$ & $z$ & Hole I.D. \\
\hline 1.22 & 3.05 & -2.29 & UE25FRPTC\#1 \\
\hline 1.22 & 0.00 & -2.29 & UE25FRPTC\# 1 \\
\hline 1.22 & 3.05 & -2.02 & UE25FRPTC\#2 \\
\hline 1.22 & 0.00 & -1.48 & UE25FRPTC\#2 \\
\hline 1.22 & 3.05 & -2.56 & UE25FRPTC\#3 \\
\hline 1.22 & 0.00 & -3.09 & UE25FRPTC\#3 \\
\hline 1.22 & 3.05 & -1.73 & UE25FRPTC\#4 \\
\hline 1.22 & 0.00 & -0.62 & UE25FRPTC\#4 \\
\hline 1.22 & 3.05 & -2.84 & UE25FRPTC\#5 \\
\hline 1.22 & 0.00 & -3.95 & UE25FRPTC\#5 \\
\hline 1.22 & 3.05 & -1.41 & UE25FRPTC\#6 \\
\hline 1.22 & 0.00 & 0.35 & UE25FRPTC\#6 \\
\hline 1.22 & 3.05 & -3.76 & UE25FRPTC\#7 \\
\hline 1.22 & 0.00 & -6.70 & UE25FRPTC\#7 \\
\hline 1.22 & 3.05 & -1.01 & UE25FRPTC\#8 \\
\hline 1.22 & 0.00 & 1.55 & UE25FRPTC\#8 \\
\hline 1.22 & 3.05 & -2.29 & UE25FRPTC\#9 \\
\hline 2.53 & 0.00 & -2.29 & UE25FRPTC\#9 \\
\hline 0.00 & 1.83 & -1.09 & UE25FRPTC\#10 \\
\hline 3.05 & 1.83 & 1.29 & UE25FRPTC\#10 \\
\hline 0.00 & 1.83 & -1.30 & UE25FRPTC\#11 \\
\hline 3.05 & 1.83 & 0.68 & UE25FRPTC\# 11 \\
\hline 0.00 & 1.83 & -1.51 & UE25FRPTC\#12 \\
\hline 3.05 & 1.83 & 0.04 & UE25FRPTC\#12 \\
\hline 0.00 & 1.83 & -1.73 & UE25FRPTC\#13 \\
\hline 3.05 & 1.83 & -0.62 & UE25FRPTC\#13 \\
\hline 0.00 & 1.83 & -1.91 & UE25FRPTC\#14 \\
\hline 3.05 & 1.83 & -1.15 & UE25FRPTC\#14 \\
\hline 0.00 & 1.83 & -2.26 & UE25FRPTC\#15 \\
\hline 3.05 & 1.83 & -2.21 & UE25FRPTC\#15 \\
\hline 0.00 & 1.83 & -2.55 & UE25FRPTC\#16 \\
\hline 3.05 & 1.83 & -3.09 & UE25FRPTC\#16 \\
\hline 0.00 & 1.83 & -2.84 & UE25FRPTC\# 17 \\
\hline 3.05 & 1.83 & -3.95 & UE25FRPTC\#17 \\
\hline 0.00 & 1.83 & -3.35 & UE25FRPTC\#18 \\
\hline 3.05 & 1.83 & -5.49 & UE25FRPTC\#18 \\
\hline 0.00 & 1.83 & -2.18 & UE25FRPTC\#19 \\
\hline 3.05 & 1.83 & -1.97 & UE25FRPTC\#19 \\
\hline 0.00 & 1.83 & -2.29 & UE25FRPTC\#19a \\
\hline 3.05 & 0.52 & -2.29 & UE25FRPTC\#19a \\
\hline 2.44 & 1.22 & 0.00 & el (T2) \\
\hline 2.40 & 1.19 & -4.04 & el (T2) \\
\hline
\end{tabular}




\begin{tabular}{|c|c|c|c|}
\hline 1.52 & 1.37 & 0.00 & tel (ERT) \\
\hline 1.52 & 1.37 & -3.96 & tel (ERT) \\
\hline 1.27 & 1.32 & 0.00 & trl (TR1) \\
\hline 1.27 & 1.32 & -1.42 & $\operatorname{trl}(\operatorname{TR} 1)$ \\
\hline 0.61 & 1.83 & 0.00 & e10 (LBLI) \\
\hline 0.55 & 1.81 & -4.00 & el0 (LBLI) \\
\hline 2.44 & 1.83 & 0.00 & $\mathrm{e} 2(\mathrm{TN} 4)$ \\
\hline 2.41 & 1.82 & -4.09 & e2 (TN4) \\
\hline 1.83 & 0.61 & 0.00 & e3 (TN2) \\
\hline 1.83 & 0.62 & -4.04 & e3 (TN2) \\
\hline 1.22 & 0.61 & 0.00 & e5 (TNI) \\
\hline 1.18 & 0.57 & -3.99 & e5 (TN 1$)$ \\
\hline 1.22 & 1.22 & 0.00 & e6 (TN3) \\
\hline 1.21 & 1.16 & -4.02 & e6 (TN3) \\
\hline 1.22 & 1.83 & 0.00 & e7 (Tा) \\
\hline 1.22 & 1.83 & -3.90 & e7 (ा1) \\
\hline 1.22 & 2.44 & 0.00 & e8 (TN5) \\
\hline 1.21 & 2.45 & -3.99 & e8 (TN5) \\
\hline 0.61 & 1.22 & 0.00 & e9 (LBL2) \\
\hline 0.61 & 1.22 & -3.98 & e9 (LBL2) \\
\hline 3.05 & 0.30 & -2.74 & ehl (EHI) \\
\hline 0.30 & 0.30 & -2.74 & ehl (EHI) \\
\hline 3.05 & 0.91 & -2.74 & eh2 (EH2) \\
\hline 0.30 & 0.91 & -2.74 & eh2 (EH2) \\
\hline 3.05 & 1.52 & -2.74 & eh3 (EH3) \\
\hline 0.30 & 1.52 & -2.74 & eh3 (EH3) \\
\hline 3.05 & 2.13 & -2.74 & eh4 (EH4) \\
\hline 0.30 & 2.13 & -2.74 & eh4 (EH4) \\
\hline 3.05 & 2.74 & -2.74 & eh5 (EH5) \\
\hline 0.30 & 2.74 & -2.74 & eh5 (EH5) \\
\hline 3.05 & 2.74 & -3.96 & eo3 (EO3) \\
\hline 0.30 & 2.74 & -3.96 & eo3 (EO3) \\
\hline 1.83 & 1.83 & 0.00 & $\mathrm{nl}(\mathrm{TH} \mathrm{I})$ \\
\hline 1.81 & 1.76 & -4.01 & $\mathrm{nl}(\mathrm{THI})$ \\
\hline 1.83 & 1.22 & 0.00 & $\mathrm{n} 2(\mathrm{TM} 1)$ \\
\hline 1.82 & 1.21 & -4.05 & $\mathrm{n} 2(\mathrm{TM} 1)$ \\
\hline 1.83 & 2.44 & 0.00 & n3 (TM2) \\
\hline 1.78 & 2.42 & -4.09 & n3 (TM2) \\
\hline 1.52 & 3.05 & 0.00 & $\mathrm{n} 4$ \\
\hline 1.52 & 3.05 & -6.4 & $\mathrm{n} 4$ \\
\hline 0 & 1.52 & 0 & n5 \\
\hline 0 & 1.52 & -6.4 & n5 \\
\hline 1.52 & 0 & 0 & no \\
\hline 1.52 & 0 & -6.4 & no \\
\hline 3.05 & 1.52 & 0 & $\mathrm{n} 7$ \\
\hline 3.05 & 1.52 & -6.4 & $\mathrm{n} 7$ \\
\hline 0.00 & 3.05 & -2.44 & $\mathrm{nhl}(\mathrm{NHI})$ \\
\hline 2.13 & 0.30 & -2.44 & $\mathrm{nhl}(\mathrm{NHI})$ \\
\hline 2.74 & 3.05 & -3.81 & $\mathrm{nml}(\mathrm{NMI})$ \\
\hline 2.74 & 0.30 & -3.81 & $\mathrm{nml}(\mathrm{NMI})$ \\
\hline
\end{tabular}




\begin{tabular}{|c|c|c|c|}
\hline 0.91 & 3.05 & -2.44 & $\mathrm{~nm} 2(\mathrm{NM} 2)$ \\
\hline 0.91 & 0.30 & -2.44 & $\mathrm{~nm} 2(\mathrm{NM} 2)$ \\
\hline 0.30 & 3.05 & -0.91 & $\mathrm{~nm} 3(\mathrm{NM} 3)$ \\
\hline 0.30 & 0.30 & -0.91 & $\mathrm{~nm} 3(\mathrm{NM} 3)$ \\
\hline 2.13 & 3.05 & -0.91 & $\mathrm{nnl}(\mathrm{NNI})$ \\
\hline 2.13 & 0.30 & -0.91 & $\mathrm{nnl}(\mathrm{NNI})$ \\
\hline 2.13 & 3.05 & -1.98 & $\mathrm{n} n 2$ (NN2) \\
\hline 2.13 & 0.30 & -1.98 & $\mathrm{nn} 2(\mathrm{NN} 2)$ \\
\hline 2.13 & 3.05 & -3.81 & $\mathrm{nn} 3$ (NN3) \\
\hline 2.13 & 0.30 & -3.81 & $\mathrm{nn} 3(\mathrm{NN} 3)$ \\
\hline 0.91 & 3.05 & -0.91 & $\mathrm{nn} 4$ (NN4) \\
\hline 0.91 & 0.30 & -0.91 & $\mathrm{nn} 4$ (NN4) \\
\hline 0.91 & 3.05 & -1.98 & nn5 (NN5) \\
\hline 0.91 & 0.30 & -1.98 & $\mathrm{nn} 5$ (NN5) \\
\hline 0.91 & 3.05 & -3.81 & nn6 (NN6) \\
\hline 0.91 & 0.30 & -3.81 & nnb (NN6) \\
\hline 2.13 & 3.05 & -4.11 & nol (NOI) \\
\hline 2.13 & 0.30 & -4.11 & nol (NOl) \\
\hline 0.91 & 3.05 & -4.11 & no2 (NO2) \\
\hline 0.91 & 0.30 & -4.11 & no2 (NO2) \\
\hline 2.74 & 3.05 & -2.44 & ntl (NTI) \\
\hline 2.74 & 0.30 & -2.44 & ntl (NTI) \\
\hline 0.91 & 3.05 & -0.61 & $\mathrm{n}+2$ (NT2) \\
\hline 0.91 & 0.30 & -0.61 & $\mathrm{n}+2$ (NT2) \\
\hline 0.91 & 3.05 & -3.20 & $n+3$ (NT3) \\
\hline 0.91 & 0.30 & -3.20 & $n+3$ (NT3) \\
\hline 0.30 & 3.05 & -2.44 & nt4 (NT4) \\
\hline 0.30 & 0.30 & -2.44 & $n+4$ (NT4) \\
\hline 0.00 & 1.68 & -1.22 & Whl (WHI) \\
\hline 2.74 & 1.68 & -1.22 & Whl (WHI) \\
\hline 0.00 & 1.68 & -2.29 & wh2 (WH2) \\
\hline 2.74 & 1.68 & -2.29 & wh2 (WH2) \\
\hline 0.00 & 2.13 & -3.96 & Wml (WMI) \\
\hline 2.74 & 2.13 & -3.96 & wml (WMI) \\
\hline 0.00 & 0.91 & -1.22 & wm2 (WM2) \\
\hline 2.74 & 0.91 & -1.22 & wm2 (WM2) \\
\hline 0.00 & 0.91 & -3.05 & wm3 (WM3) \\
\hline 2.74 & 0.91 & -3.05 & wm3 (WM3) \\
\hline 0.00 & 2.13 & -0.76 & Wnl (WNI) \\
\hline 2.74 & 2.13 & -0.76 & wnl (WNI) \\
\hline 0.00 & 2.13 & -1.68 & wn2 (WN2) \\
\hline 2.74 & 2.13 & -1.68 & wn2 (WN2) \\
\hline 0.00 & 1.68 & -3.96 & wn3 (WN3) \\
\hline 2.74 & 1.68 & -3.96 & wn3 (WN3) \\
\hline 0.00 & 0.91 & -1.68 & wn4 (WN4) \\
\hline 2.74 & 0.91 & -1.68 & WnA (WN4) \\
\hline 0.00 & 0.91 & -3.96 & wo5 (WO5) \\
\hline 2.74 & 0.91 & -3.96 & wo5 (WO5) \\
\hline 0.00 & 1.68 & -0.76 & wtl (WTI) \\
\hline 2.74 & 1.68 & -0.76 & $w+1$ (WTI) \\
\hline
\end{tabular}




$\begin{array}{llll}0.00 & 1.68 & -1.68 & w+2 \text { (WT2) } \\ 2.74 & 1.68 & -1.68 & w+2 \text { (WT2) } \\ 0.00 & 1.68 & -3.05 & w+3 \text { (WT3) } \\ 2.74 & 1.68 & -3.05 & w+3 \text { (WT3) } \\ 0.00 & 0.63 & -1.86 & w r 1 \text { (WR1) } \\ 1.75 & 0.63 & -1.86 & w r 1 \text { (WR1) } \\ 0.00 & 0.91 & -3.32 & w r 2 \text { (WR2) } \\ 1.75 & 0.91 & -3.32 & w r 2 \text { (WR2) }\end{array}$




\section{APPENDIX C: \\ STRUCTURES DOCUMENTED IN THE BOREHOLE VIDEOS}

\begin{tabular}{|c|c|c|c|c|c|c|c|c|}
\hline Hole & $\begin{array}{l}\text { Surface } \\
\text { feature }\end{array}$ & $\begin{array}{c}\text { Model } \\
\text { east }\end{array}$ & $\begin{array}{l}\text { Model } \\
\text { north }\end{array}$ & $\begin{array}{l}\text { Model } \\
\text { depth }\end{array}$ & $\begin{array}{l}\text { Strike } \\
\text { direct }\end{array}$ & $\begin{array}{c}\text { Dip } \\
\text { direct }\end{array}$ & $\begin{array}{c}\text { TRUE } \\
\text { dip }\end{array}$ & Comments \\
\hline \multicolumn{9}{|l|}{ N1 } \\
\hline & & 1.83 & 1.83 & -0.14 & $\mathrm{~N}-\mathrm{S}$ & W & 77 & top minor subvertical \\
\hline & & 1.83 & 1.83 & -0.47 & $\mathrm{~N}-\mathrm{S}$ & $W$ & 77 & bottom minor subvertical \\
\hline & LBT1 & 1.83 & 1.83 & -0.53 & & & 18 & top large subhorizontal \\
\hline & LBTI & 1.83 & 1.83 & -0.55 & & & 18 & base large subhorizontal \\
\hline & & 1.83 & 1.83 & -0.55 & NW-SE & SW & 72 & top minor subvertical \\
\hline & & 1.83 & 1.83 & -0.78 & NW-SE & SW & 72 & bottom minor subvertical \\
\hline & & 1.83 & 1.83 & -0.93 & $E-W$ & $N$ & & top subhorizontal \\
\hline & & 1.83 & 1.83 & -0.96 & $E-W$ & $N$ & & bottom subhorizontal \\
\hline & & 1.83 & 1.83 & -1.59 & $E-W$ & $S$ & 84 & tight fracture \\
\hline & & 1.83 & 1.83 & -2.28 & $E-W$ & $S$ & 84 & tight fracture \\
\hline & & 1.83 & 1.83 & -2.47 & & & & horizontal fracture \\
\hline & & 1.83 & 1.83 & -2.68 & SW-NE & $\mathrm{SE}$ & 72 & tight fracture \\
\hline & & 1.83 & 1.83 & -2.91 & SW-NE & SE & 72 & tight fracture \\
\hline & & 1.83 & 1.83 & -3.37 & SW-NE & SE & 34 & open subhorizontal \\
\hline & & 1.83 & 1.83 & -3.42 & SW-NE & SE & 34 & open subhorizontal \\
\hline & & 1.83 & 1.83 & -3.37 & SW-NE & SE & 72 & tight fracture \\
\hline & & 1.83 & 1.83 & -3.60 & SW-NE & $\mathrm{SE}$ & 72 & tight fracture \\
\hline & & 1.83 & 1.83 & -3.78 & & & & horizontal fracture \\
\hline & & 1.83 & 1.83 & -4.08 & & & & horizontal fracture \\
\hline & & 1.83 & 1.83 & -4.26 & & & & horizontal fracture \\
\hline & & 1.83 & 1.83 & -4.36 & & & & large horizontal fracture \\
\hline & & 1.83 & 1.83 & -4.69 & & & & subhorizontal \\
\hline & & 1.83 & 1.83 & -5.10 & & & & horizontal partly open \\
\hline & & 1.83 & 1.83 & -5.28 & & & & horizontal fracture \\
\hline & & 1.83 & 1.83 & -5.30 & NW-SE & SW & 34 & top subhorizontal \\
\hline & & 1.83 & 1.83 & -5.35 & NW-SE & SW & & bottom subhorizontal \\
\hline & & 1.83 & 1.83 & -5.45 & $E-W$ & $\mathrm{NE}$ & 59 & major open fracture \\
\hline & & 1.83 & 1.83 & -5.58 & $E-W$ & $\mathrm{NE}$ & 59 & major open fracture \\
\hline \multicolumn{9}{|l|}{ N2 } \\
\hline & & 1.83 & 1.22 & -0.18 & $E-W$ & $S$ & 63 & minor fracture \\
\hline & & 1.83 & 1.22 & -0.34 & $E-W$ & $\mathrm{~s}$ & 63 & minor fracture \\
\hline & LBTI & 1.83 & 1.22 & -0.49 & & & & top large subhorizontal \\
\hline & LBT1 & 1.83 & 1.22 & -0.52 & & & & base large subhorizontal \\
\hline & & 1.83 & 1.22 & -0.93 & & & & horizontal partly open \\
\hline & LBT17 & 1.83 & 1.22 & -0.93 & $\mathrm{~N}-\mathrm{S}$ & $W$ & & may have alteration halo \\
\hline & LBT17 & 1.83 & 1.22 & -1.35 & $\mathrm{~N}-\mathrm{S}$ & $W$ & & may have alteration halo \\
\hline & & 1.83 & 1.22 & -1.61 & $E-W$ & $N$ & 53 & tight fracture \\
\hline & & 1.83 & 1.22 & -1.71 & $E-W$ & $\mathrm{~N}$ & 53 & tight fracture \\
\hline & & 1.83 & 1.22 & -2.95 & $E-W$ & $N$ & & top subvertical \\
\hline & & 1.83 & 1.22 & -3.00 & $\mathrm{E}-\mathrm{W}$ & $\mathrm{N}$ & & bottom subvertical \\
\hline & & 1.83 & 1.22 & -3.69 & $E-W$ & $N$ & & top subhorizontal \\
\hline
\end{tabular}




\begin{tabular}{|c|c|c|c|c|c|c|c|c|}
\hline Hole & $\begin{array}{l}\text { Surface } \\
\text { feature }\end{array}$ & $\begin{array}{l}\text { Model } \\
\text { east }\end{array}$ & $\begin{array}{l}\text { Model } \\
\text { north }\end{array}$ & $\begin{array}{l}\text { Model } \\
\text { depth }\end{array}$ & $\begin{array}{l}\text { Strike } \\
\text { direct }\end{array}$ & $\begin{array}{l}\text { Dip } \\
\text { direct }\end{array}$ & $\begin{array}{c}\text { TRUE } \\
\text { dip }\end{array}$ & Comments \\
\hline & & 1.83 & 1.22 & -3.72 & $E-W$ & $\mathrm{~N}$ & & bottom subhorizontal \\
\hline & & 1.83 & 1.22 & -3.94 & $E-W$ & $N$ & & top subhorizontal \\
\hline & & 1.83 & 1.22 & -3.97 & $E-W$ & $\mathrm{~N}$ & & bottom subhorizontal \\
\hline & & 1.83 & 1.22 & -4.30 & NW-SE & $\mathrm{NE}$ & & top subhorizontal \\
\hline & & 1.83 & 1.22 & -4.35 & NW-SE & $\mathrm{NE}$ & & bottom subhorizontal \\
\hline & & 1.83 & 1.22 & -5.29 & NW-SE & SW & & top subvertical \\
\hline & & 1.83 & 1.22 & -5.44 & NW-SE & SW & & bottom subvertical \\
\hline \multicolumn{9}{|l|}{ N3 } \\
\hline & & 1.83 & 2.44 & 0.00 & & & & open horizontal fracture \\
\hline & LBT8 & 1.83 & 2.44 & 0.08 & NW-SE & SW & & top subvertical \\
\hline & LBT8 & 1.83 & 2.44 & -0.25 & NW-SE & SW & & bottom subvertical \\
\hline & LBT1 & 1.83 & 2.44 & -0.51 & & & 27 & top large subhorizontal \\
\hline & LBT1 & 1.83 & 2.44 & -0.55 & & & 27 & base large subhorizontal \\
\hline & & 1.83 & 2.44 & -0.99 & SW-NE & NW & & top subvertical \\
\hline & & 1.83 & 2.44 & -1.19 & SW-NE & NW & & bottom subvertical \\
\hline & & 1.83 & 2.44 & -1.02 & SW-NE & NW & 72 & $\begin{array}{l}\text { top moderate/major tight } \\
\text { subvertical }\end{array}$ \\
\hline & & 1.83 & 2.44 & -1.24 & SW-NE & NW & 72 & $\begin{array}{l}\text { bottom moderate/major } \\
\text { tight subvertical }\end{array}$ \\
\hline & & 1.83 & 2.44 & -2.11 & & & & horizontal fracture \\
\hline & & 1.83 & 2.44 & -2.79 & $E-W$ & $S$ & 34 & major subhorizontal \\
\hline & & 1.83 & 2.44 & -2.84 & $E-W$ & $\mathrm{~S}$ & 34 & major subhorizontal \\
\hline & LBT22 & 1.83 & 2.44 & -3.06 & SW-NE & NW & 71 & major open subvertical \\
\hline & LBT22 & 1.83 & 2.44 & -3.28 & $S W-N E$ & NW & 71 & major open subvertical \\
\hline & LBT14 & 1.83 & 2.44 & -3.40 & & & 79 & horizontal \\
\hline & LBTI4 & 1.83 & 2.44 & -3.81 & & & 79 & horizontal \\
\hline & & 1.83 & 2.44 & -4.17 & & & & horizontal fracture \\
\hline & & 1.83 & 2.44 & -4.27 & & & & horizontal fracture \\
\hline & & 1.83 & 2.44 & -4.32 & SW-NE & SE & 82 & minor fracture \\
\hline & & 1.83 & 2.44 & -4.34 & & & & horizontal fracture \\
\hline & & 1.83 & 2.44 & -4.52 & & & & horizontal fracture \\
\hline & & 1.83 & 2.44 & -4.75 & & & & horizontal fracture \\
\hline & & 1.83 & 2.44 & -4.88 & SW-NE & $\mathrm{SE}$ & & minor \\
\hline & & 1.83 & 2.44 & -5.03 & $E-W$ & & & horizontal fracture \\
\hline & & 1.83 & 2.44 & -5.31 & & & & horizontal fracture \\
\hline \multicolumn{9}{|l|}{ N4 } \\
\hline & LBTI & 1.52 & 3.05 & -0.43 & & & 18 & top large subhorizontal \\
\hline & LBTI & 1.52 & 3.05 & -0.46 & & & 18 & base large subhorizontal \\
\hline & & 1.52 & 3.05 & -0.56 & & & 84 & subhorizontal \\
\hline & & 1.52 & 3.05 & -1.23 & & & 84 & subhorizontal \\
\hline & LBT 12 & 1.52 & 3.05 & -1.27 & SW-NE & NW & 78 & major subvertical \\
\hline & LBT 12 & 1.52 & 3.05 & -1.62 & SW-NE & NW & 78 & major subvertical \\
\hline & & 1.52 & 3.05 & -1.98 & & & & horizontal fracture \\
\hline & LBTI3 & 1.52 & 3.05 & -1.98 & $E-W$ & & & $\begin{array}{l}\text { top highly fractured zone- } \\
\text { open major }\end{array}$ \\
\hline
\end{tabular}




\begin{tabular}{|c|c|c|c|c|c|c|c|c|}
\hline Hole & $\begin{array}{l}\text { Surface } \\
\text { feature }\end{array}$ & $\begin{array}{c}\text { Model } \\
\text { east }\end{array}$ & $\begin{array}{l}\text { Model } \\
\text { north }\end{array}$ & $\begin{array}{l}\text { Model } \\
\text { depth }\end{array}$ & $\begin{array}{l}\text { Strike } \\
\text { direct }\end{array}$ & $\begin{array}{l}\text { Dip } \\
\text { direct }\end{array}$ & $\begin{array}{c}\text { TRUE } \\
\text { dip }\end{array}$ & Comments \\
\hline & LBT13 & 1.52 & 3.05 & -3.07 & $E-W$ & & & $\begin{array}{l}\text { base highly fractured zone- } \\
\text { open major }\end{array}$ \\
\hline & & 1.52 & 3.05 & -3.43 & SW-NE & NW & 59 & top subvertical \\
\hline & & 1.52 & 3.05 & -3.55 & SW-NE & NW & 59 & base subvertical \\
\hline & & 1.52 & 3.05 & -3.45 & & & & horizontal fracture \\
\hline & & 1.52 & 3.05 & -3.48 & & & & horizontal fracture \\
\hline & & 1.52 & 3.05 & -3.76 & & & & horizontal fracture \\
\hline & & 1.52 & 3.05 & -3.73 & & & & horizontal fracture \\
\hline & & 1.52 & 3.05 & -3.99 & & & & horizontal fracture \\
\hline & LBT22 & 1.52 & 3.05 & -4.09 & $\mathrm{~N}-\mathrm{S}$ & $W$ & 53 & top major subvertical-open \\
\hline & LBT22 & 1.52 & 3.05 & -4.19 & $N-S$ & $W$ & 53 & base major subvertical-open \\
\hline & & 1.52 & 3.05 & -4.32 & NW-SE & & 69 & top major open fracture \\
\hline & & 1.52 & 3.05 & -4.52 & NW-SE & & 69 & base major open fracture \\
\hline & & 1.52 & 3.05 & -4.60 & & & & horizontal fracture \\
\hline & & 1.52 & 3.05 & -4.98 & & & & horizontal fracture \\
\hline & & 1.52 & 3.05 & -5.03 & $E-W$ & $N$ & 53 & tight fracture \\
\hline & & 1.52 & 3.05 & -5.13 & $E-W$ & $\mathrm{~N}$ & 53 & tight fracture \\
\hline & & 1.52 & 3.05 & -4.80 & NW-SE & SW & 83 & tight fracture \\
\hline & & 1.52 & 3.05 & -5.38 & NW-SE & SW & 83 & tight fracture \\
\hline & & 1.52 & 3.05 & -5.41 & SW-NE & $\mathrm{SE}$ & 53 & major open fracture \\
\hline & & 1.52 & 3.05 & -5.51 & SW-NE & SE & 53 & major open fracture \\
\hline \multicolumn{9}{|l|}{ N5 } \\
\hline & LBT1 & 0.00 & 1.52 & -0.17 & & & & top large subhorizontal \\
\hline & LBT] & 0.00 & 1.52 & -0.51 & & & & base large subhorizontal \\
\hline & & 0.00 & 1.52 & -0.29 & $\mathrm{~N}-\mathrm{S}$ & $E$ & 90 & base vertical fracture \\
\hline & LBT 2 & 0.00 & 1.52 & -0.24 & $\mathrm{~N}-\mathrm{S}$ & $E$ & 77 & top major subvertical \\
\hline & LBT 2 & 0.00 & 1.52 & -0.57 & $\mathrm{~N}-\mathrm{S}$ & $E$ & 77 & base major subvertical \\
\hline & & 0.00 & 1.52 & -0.60 & SW-NE & NW & & fracture \\
\hline & & 0.00 & 1.52 & -0.93 & & & & base subvertical \\
\hline & & 0.00 & 1.52 & -1.03 & & & & horizontal fracture \\
\hline & & 0.00 & 1.52 & -1.51 & & & & horizontal fracture \\
\hline & & 0.00 & 1.52 & -1.89 & & & & horizontal fracture \\
\hline & & 0.00 & 1.52 & -2.60 & NW-SE & NE & & $\begin{array}{l}\text { top sharp, open minor } \\
\text { subvertical }\end{array}$ \\
\hline & & 0.00 & 1.52 & -2.76 & NW-SE & NE & & $\begin{array}{l}\text { bottom sharp, open minor } \\
\text { subvertical }\end{array}$ \\
\hline & & 0.00 & 1.52 & -2.86 & & & & horizontal fracture \\
\hline & & 0.00 & 1.52 & -3.16 & & & & horizontal fracture \\
\hline & & 0.00 & 1.52 & -3.47 & & & & horizontal fracture \\
\hline & LBT20 & 0.00 & 1.52 & -3.47 & $E-W$ & $S$ & 78 & $\begin{array}{l}\text { top major subvertical open } \\
\text { fracture }\end{array}$ \\
\hline & LBT2O & 0.00 & 1.52 & -3.82 & $E-W$ & $s$ & 78 & $\begin{array}{l}\text { base major subvertical open } \\
\text { fracture }\end{array}$ \\
\hline & & 0.00 & 1.52 & -3.70 & & & & horizontal fracture \\
\hline & & 0.00 & 1.52 & -3.87 & & & & horizontal fracture \\
\hline & & 0.00 & 1.52 & -4.69 & $\mathrm{~N}-\mathrm{S}$ & 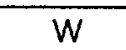 & 81 & top open fracture \\
\hline
\end{tabular}




\begin{tabular}{|c|c|c|c|c|c|c|c|c|}
\hline Hole & $\begin{array}{l}\text { Surface } \\
\text { feature }\end{array}$ & $\begin{array}{c}\text { Model } \\
\text { east }\end{array}$ & $\begin{array}{l}\text { Model } \\
\text { north }\end{array}$ & $\begin{array}{l}\text { Model } \\
\text { depth }\end{array}$ & $\begin{array}{l}\text { Strike } \\
\text { direct }\end{array}$ & $\begin{array}{l}\text { Dip } \\
\text { direct }\end{array}$ & $\begin{array}{c}\text { TRUE } \\
\text { dip }\end{array}$ & Comments \\
\hline & & 0.00 & 1.52 & -4.74 & & & & horizontal fracture \\
\hline & & 0.00 & 1.52 & -4.89 & & & & horizontal fracture \\
\hline & & 0.00 & 1.52 & -5.17 & $\mathrm{~N}-\mathrm{S}$ & $w$ & 81 & base open fracture \\
\hline \multicolumn{9}{|l|}{ No } \\
\hline & & 1.52 & 0.00 & 0.05 & & & & horizontal fracture \\
\hline & & 1.52 & 0.00 & -0.15 & & & & horizontal fracture \\
\hline & LBT1 & 1.52 & 0.00 & -0.38 & & & 18 & top large subhorizontal \\
\hline & LBT I & 1.52 & 0.00 & -0.40 & & & 18 & base large subhorizontal \\
\hline & & 1.52 & 0.00 & -0.66 & $\mathrm{~N}-\mathrm{S}$ & $w$ & 89 & $\begin{array}{l}\text { top subvertical, cannot see } \\
\text { base }\end{array}$ \\
\hline & & 1.52 & 0.00 & -0.73 & & & 89 & horizontal fracture \\
\hline & & 1.52 & 0.00 & -0.81 & & & & horizontal fracture \\
\hline & & 1.52 & 0.00 & -1.22 & & & & horizontal fracture \\
\hline & & 1.52 & 0.00 & -1.57 & & & & horizontal fracture \\
\hline & & 1.52 & 0.00 & -1.67 & & & & horizontal fracture \\
\hline & & 1.52 & 0.00 & 0.00 & $\mathrm{~N}-\mathrm{S}$ & $W$ & & base subvertical \\
\hline & LBT2 & 1.52 & 0.00 & -1.90 & $\mathrm{~N} 45 \mathrm{~W}$ & $\mathrm{NE}$ & 76 & top significant subvertical \\
\hline & LBT2 & 1.52 & 0.00 & -2.21 & N45W & $\mathrm{NE}$ & 76 & base significant subvertical \\
\hline & & 1.52 & 0.00 & -2.10 & & & & horizontal fracture \\
\hline & & 1.52 & 0.00 & -2.66 & & & & horizontal fracture \\
\hline & LBT17 & 1.52 & 0.00 & -2.92 & N3OW & SW & 73 & $\begin{array}{l}\text { top major subvertical } \\
\text { (alteration halo) }\end{array}$ \\
\hline & LBT17 & 1.52 & 0.00 & -3.17 & N3OW & SW & 73 & $\begin{array}{l}\text { base major subvertical } \\
\text { (alteration halo) }\end{array}$ \\
\hline & & 1.52 & 0.00 & -3.65 & & & & horizontal fracture \\
\hline & & 1.52 & 0.00 & -4.49 & $\mathrm{~N}-\mathrm{S}$ & $W$ & 81 & top subvertical \\
\hline & & 1.52 & 0.00 & -4.95 & $\mathrm{~N}-\mathrm{S}$ & $W$ & 81 & base subvertical \\
\hline & & 1.52 & 0.00 & -4.95 & $\mathrm{~N}-\mathrm{S}$ & $w$ & 53 & top subvertical \\
\hline & & 1.52 & 0.00 & -5.05 & $\mathrm{~N}-\mathrm{S}$ & $W$ & 53 & base subvertical \\
\hline & & 1.52 & 0.00 & -5.10 & & & & horizontal fracture \\
\hline & & 1.52 & 0.00 & -5.25 & N3OW & $\mathrm{NE}$ & 82 & major sharp open fracture \\
\hline \multicolumn{9}{|l|}{ N7 } \\
\hline & & 3.05 & 1.52 & -0.24 & & & & horizontal fracture \\
\hline & LBTI & 3.05 & 1.52 & -0.63 & & & & top large subhorizontal \\
\hline & LBT1 & 3.05 & 1.52 & -0.65 & & & & base large subhorizontal \\
\hline & & 3.05 & 1.52 & -1.06 & & & & horizontal fracture \\
\hline & LBT21 & 3.05 & 1.52 & -1.26 & $\mathrm{~N}-\mathrm{S}$ & $w$ & 76 & top minor subvertical \\
\hline & LBT21 & 3.05 & 1.52 & -1.57 & $\mathrm{~N}-\mathrm{S}$ & $W$ & 76 & base minor subvertical \\
\hline & & 3.05 & 1.52 & -1.49 & & & & horizontal fracture \\
\hline & & 3.05 & 1.52 & -1.92 & & & & horizontal fracture \\
\hline & & 3.05 & 1.52 & -3.27 & & & & horizontal fracture \\
\hline & & 3.05 & 1.52 & -3.88 & & & & horizontal fracture \\
\hline & & 3.05 & 1.52 & -4.41 & $E-W$ & $\mathrm{~N}$ & & top subvertical \\
\hline & & 3.05 & 1.52 & -4.46 & $E-W$ & $\mathrm{~N}$ & & base subvertical \\
\hline
\end{tabular}




\begin{tabular}{|c|c|c|c|c|c|c|c|c|}
\hline Hole & $\begin{array}{l}\text { Surface } \\
\text { feature }\end{array}$ & $\begin{array}{c}\text { Model } \\
\text { east }\end{array}$ & $\begin{array}{l}\text { Model } \\
\text { north }\end{array}$ & $\begin{array}{l}\text { Model } \\
\text { depth }\end{array}$ & $\begin{array}{l}\text { Strike } \\
\text { direct }\end{array}$ & $\begin{array}{l}\text { Dip } \\
\text { direct }\end{array}$ & $\begin{array}{c}\text { TRUE } \\
\text { dip }\end{array}$ & Comments \\
\hline & & 3.05 & 1.52 & -4.72 & & & 90 & several vertical cooling joints \\
\hline & & 3.05 & 1.52 & -5.02 & & & & horizontal fracture \\
\hline & & 3.05 & 1.52 & -5.15 & & & & horizontal fracture \\
\hline \multicolumn{9}{|l|}{ El } \\
\hline & & 2.44 & 1.22 & -0.09 & & & & horizontal fracture \\
\hline & LBT1 & 2.44 & 1.22 & -0.57 & & & 34 & top large subhorizontal \\
\hline & LBTT & 2.44 & 1.22 & -0.59 & & & 34 & base large subhorizontal \\
\hline & & 2.44 & 1.22 & -0.92 & $\sim \mathrm{N} 45 \mathrm{~W}$ & & & goes out same side of hole \\
\hline & & 2.44 & 1.22 & -1.03 & & & & horizontal fracture \\
\hline & & 2.44 & 1.22 & -1.10 & $\sim N 45 W$ & & & goes out same side of hole \\
\hline & & 2.44 & 1.22 & -1.23 & & & & horizontal fracture \\
\hline & & 2.44 & 1.22 & -1.38 & & & & horizontal fracture \\
\hline & & 2.44 & 1.22 & -1.43 & $\sim \mathrm{N} 45 \mathrm{~W}$ & & & fracture \\
\hline & & 2.44 & 1.22 & -1.79 & & & & horizontal fracture \\
\hline & & 2.44 & 1.22 & -2.78 & & & & base of open fracture \\
\hline & & 2.44 & 1.22 & -3.08 & NW & SW & & significant fracture \\
\hline & & 2.44 & 1.22 & -3.29 & NW & SW & & significant fracture \\
\hline & & 2.44 & 1.22 & -3.34 & $\mathrm{~N}-\mathrm{S}$ & $E$ & & $\begin{array}{l}\text { top open dipping } \\
\text { subhorizontal fracture }\end{array}$ \\
\hline & & 2.44 & 1.22 & -3.36 & $\mathrm{~N}-\mathrm{S}$ & $\bar{E}$ & & $\begin{array}{l}\text { bottom open dipping } \\
\text { subhorizontal fracture }\end{array}$ \\
\hline & & 2.44 & 1.22 & -3.46 & & & & top rubble continuous \\
\hline & & 2.44 & 1.22 & -3.72 & & & & base rubble zone \\
\hline & & 2.44 & 1.22 & -3.57 & & & & $\begin{array}{l}\text { gaping fracture (cooling } \\
\text { joint) }\end{array}$ \\
\hline & & 2.44 & 1.22 & -3.72 & & & & leaves same side of hole \\
\hline & & 2.44 & 1.22 & -4.05 & $E-W$ & $S$ & 79 & top subvertical \\
\hline & & 2.44 & 1.22 & -4.25 & $E-W$ & $S$ & 79 & base subvertical \\
\hline & & 2.44 & 1.22 & -4.28 & $E-W$ & $S$ & 78 & top subvertical \\
\hline & & 2.44 & 1.22 & -4.38 & & & & horizontal fracture \\
\hline & & 2.44 & 1.22 & -4.45 & $E-W$ & $S$ & 78 & base subvertical \\
\hline & & 2.44 & 1.22 & -4.53 & & & & horizontal fracture \\
\hline & & 2.44 & 1.22 & -4.61 & & & & top subhorizontal \\
\hline & & 2.44 & 1.22 & -4.66 & & & & bottom subhorizontal \\
\hline & & 2.44 & 1.22 & -4.81 & $E-W$ & $S$ & 69 & top subvertical \\
\hline & & 2.44 & 1.22 & -4.91 & $E-W$ & $S$ & 69 & base subvertical \\
\hline & & 2.44 & 1.22 & -5.17 & $\mathrm{~N}-\mathrm{S}$ & $w$ & 84 & top major open subvertical \\
\hline & & 2.44 & 1.22 & -5.22 & & & & horizontal fracture \\
\hline & & 2.44 & 1.22 & -5.52 & $\mathrm{~N}-\mathrm{S}$ & $W$ & 84 & $\begin{array}{l}\text { bottom major open } \\
\text { subvertical }\end{array}$ \\
\hline \multicolumn{9}{|r|}{ ton } \\
\hline & & 2.44 & 1.83 & -0.16 & & & & horizontal fracture \\
\hline & LBTI & 2.44 & 1.83 & -0.59 & & & 34 & top large subhorizontal \\
\hline & $\overline{\text { LBTI }}$ & 2.44 & 1.83 & -0.62 & & & 34 & base large subhorizontal \\
\hline & & 2.44 & 1.83 & -1.02 & & & & horizontal fracture \\
\hline
\end{tabular}




\begin{tabular}{|c|c|c|c|c|c|c|c|c|}
\hline Hole & $\begin{array}{l}\text { Surface } \\
\text { feature }\end{array}$ & $\begin{array}{c}\text { Model } \\
\text { east }\end{array}$ & $\begin{array}{l}\text { Model } \\
\text { north }\end{array}$ & $\begin{array}{l}\text { Model } \\
\text { depth }\end{array}$ & $\begin{array}{l}\text { Strike } \\
\text { direct }\end{array}$ & $\begin{array}{l}\text { Dip } \\
\text { direct }\end{array}$ & $\begin{array}{l}\text { TRUE } \\
\text { dip }\end{array}$ & Comments \\
\hline & & 2.44 & 1.83 & -1.40 & & & & horizontal fracture \\
\hline & & 2.44 & 1.83 & -1.68 & $E-W$ & $S$ & & minor fracture \\
\hline & & 2.44 & 1.83 & -1.86 & $\mathrm{E}-\mathrm{W}$ & $S$ & & minor fracture \\
\hline & & 2.44 & 1.83 & -1.86 & & & & horizontal fracture \\
\hline & & 2.44 & 1.83 & -1.99 & & & & horizontal fracture \\
\hline & & 2.44 & 1.83 & -2.85 & & & & horizontal fracture \\
\hline & & 2.44 & 1.83 & -3.16 & $\mathrm{~N}-\mathrm{S}$ & & & major open cooling joint \\
\hline & & 2.44 & 1.83 & -3.46 & $\mathrm{~N}-\mathrm{S}$ & & & major open cooling joint \\
\hline & & 2.44 & 1.83 & -3.40 & $E-W$ & $\mathrm{~S}$ & & top minor subhorizontal \\
\hline & & 2.44 & 1.83 & -3.44 & $E-W$ & $S$ & & bottom minor subhorizontal \\
\hline & & 2.44 & 1.83 & -3.56 & $E-W$ & $E$ & & $\begin{array}{l}\text { top minor/moderate } \\
\text { subhorizontal }\end{array}$ \\
\hline & & 2.44 & 1.83 & -3.59 & $E-W$ & $E$ & & $\begin{array}{l}\text { bottom minor/moderate } \\
\text { subhorizontal }\end{array}$ \\
\hline & & 2.44 & 1.83 & -4.02 & & & & horizontal fracture \\
\hline & & 2.44 & 1.83 & -4.07 & & & & horizontal fracture \\
\hline & & 2.44 & 1.83 & -4.15 & $\mathrm{~N}-\mathrm{S}$ & $W$ & & top minor subhorizontal \\
\hline & & 2.44 & 1.83 & -4.20 & $\mathrm{~N}-\mathrm{S}$ & $W$ & & bottom minor subhorizontal \\
\hline & & 2.44 & 1.83 & -4.30 & & & & horizontal fracture \\
\hline & & 2.44 & 1.83 & -4.43 & NW-SE & NE & & $\begin{array}{l}\text { top open moderate sharp } \\
\text { subhorizontal }\end{array}$ \\
\hline & & 2.44 & 1.83 & -4.45 & NW-SE & NE & & $\begin{array}{l}\text { bottom moderate open } \\
\text { sharp subhorizontal }\end{array}$ \\
\hline & & 2.44 & 1.83 & -4.76 & & & & horizontal fracture \\
\hline & & 2.44 & 1.83 & -4.83 & $\mathrm{~N}-\mathrm{S}$ & $E$ & 53 & minor fracture \\
\hline & & 2.44 & 1.83 & -4.88 & $\mathrm{~N}-\mathrm{S}$ & $E$ & 53 & minor fracture \\
\hline & & 2.44 & 1.83 & -4.91 & $\mathrm{~N}-\mathrm{S}$ & & & $\begin{array}{l}\text { major open curving cooling } \\
\text { joint }\end{array}$ \\
\hline & & 2.44 & 1.83 & -5.09 & $\mathrm{~N}-\mathrm{S}$ & & & $\begin{array}{l}\text { major open curving cooling } \\
\text { joint }\end{array}$ \\
\hline & & 2.44 & 1.83 & -5.14 & & & & horizontal fracture \\
\hline & & 2.44 & 1.83 & -5.19 & & & & open joint \\
\hline & & 2.44 & 1.83 & -5.37 & & & & open joint \\
\hline & & 2.44 & 1.83 & -5.26 & $\mathrm{~N}-\mathrm{S}$ & $W$ & 90 & major open vertical joint \\
\hline \multicolumn{9}{|l|}{ E3 } \\
\hline & & 1.83 & 0.61 & 0.03 & $\mathrm{~N}-\mathrm{S}$ & W & & $\begin{array}{l}\text { top subvertical (can't see } \\
\text { base) }\end{array}$ \\
\hline & LBTI & 1.83 & 0.61 & -0.37 & & & 34 & top large subhorizontal \\
\hline & LBTI & 1.83 & 0.61 & -0.39 & & & 34 & base large subhorizontal \\
\hline & & 1.83 & 0.61 & -0.79 & & & & horizontal fracture \\
\hline & LBTI7 & 1.83 & 0.61 & -0.89 & $\mathrm{~N}-\mathrm{S}$ & $W$ & 79 & major subvertical (halo) \\
\hline & LBT17 & 1.83 & 0.61 & -1.09 & $\mathrm{~N}-\mathrm{S}$ & $W$ & 79 & major subvertical (halo) \\
\hline & & 1.83 & 0.61 & -1.09 & NE-SW & & 69 & minor fracture \\
\hline & & 1.83 & 0.61 & -1.19 & NE-SW & & 69 & minor fracture \\
\hline & & 1.83 & 0.61 & -1.24 & & & & horizontal fracture \\
\hline & & 1.83 & 0.61 & -1.80 & & & & horizontal fracture \\
\hline
\end{tabular}




\begin{tabular}{|c|c|c|c|c|c|c|c|c|}
\hline Hole & $\begin{array}{l}\text { Surface } \\
\text { feature }\end{array}$ & $\begin{array}{l}\text { Model } \\
\text { east }\end{array}$ & $\begin{array}{l}\text { Model } \\
\text { north }\end{array}$ & $\begin{array}{l}\text { Model } \\
\text { depth }\end{array}$ & $\begin{array}{l}\text { Strike } \\
\text { direct }\end{array}$ & $\begin{array}{l}\text { Dip } \\
\text { direct }\end{array}$ & $\begin{array}{l}\text { TRUE } \\
\text { dip }\end{array}$ & Comments \\
\hline & & 1.83 & 0.61 & -2.77 & & & & horizontal fracture \\
\hline & & 1.83 & 0.61 & -3.07 & & & & horizontal fracture \\
\hline & & 1.83 & 0.61 & -3.68 & & & & horizontal fracture \\
\hline & & 1.83 & 0.61 & -4.34 & & & & horizontal fracture \\
\hline & & 1.83 & 0.61 & 0.00 & $\mathrm{~N}-\mathrm{S}$ & $W$ & & minor fracture \\
\hline & & 1.83 & 0.61 & -4.42 & $\mathrm{~N}-\mathrm{S}$ & $w$ & & minor fracture \\
\hline & & 1.83 & 0.61 & -4.90 & NE-SW & NW & 76 & moderate fracture \\
\hline & & 1.83 & 0.61 & -5.05 & NE-SW & NW & 76 & moderate fracture \\
\hline & & 1.83 & 0.61 & -5.28 & & & & horizontal fracture \\
\hline \multicolumn{9}{|l|}{ E4 } \\
\hline & & 1.52 & 1.37 & -0.18 & & & & horizontal fracture \\
\hline & LBTI & 1.52 & 1.37 & -0.38 & & & 34 & top large subhorizontal \\
\hline & LBTI & 1.52 & 1.37 & -0.41 & & & 34 & base large subhorizontal \\
\hline & & 1.52 & 1.37 & -0.48 & & & & horizontal fracture \\
\hline & & 1.52 & 1.37 & -0.89 & & & & horizontal fracture \\
\hline & & 1.52 & 1.37 & -1.25 & & & & horizontal fracture \\
\hline & & 1.52 & 1.37 & -1.42 & $\mathrm{~N}-\mathrm{S}$ & $W$ & 69 & minor fracture \\
\hline & & 1.52 & 1.37 & -1.53 & $\mathrm{~N}-\mathrm{S}$ & $W$ & 69 & minor fracture \\
\hline & & 1.52 & 1.37 & -1.58 & $\mathrm{~N}-\mathrm{S}$ & $W$ & & $\begin{array}{l}\text { top open major/moderate } \\
\text { subhorizontal }\end{array}$ \\
\hline & & 1.52 & 1.37 & -1.65 & $\mathrm{~N}-\mathrm{S}$ & W & & $\begin{array}{l}\text { base open major/moderate } \\
\text { subhorizontal }\end{array}$ \\
\hline & & 1.52 & 1.37 & -2.29 & $E-W$ & $S$ & 69 & minor fracture \\
\hline & & 1.52 & 1.37 & -2.39 & $E-W$ & $S$ & 69 & minor fracture \\
\hline & & 1.52 & 1.37 & -2.42 & & & & horizontal fracture \\
\hline & & 1.52 & 1.37 & -3.25 & & & & horizontal fracture \\
\hline & & 1.52 & 1.37 & -3.46 & & & & horizontal fracture \\
\hline & & 1.52 & 1.37 & -3.58 & & & & horizontal fracture \\
\hline & & 1.52 & 1.37 & -3.63 & & & & horizontal fracture \\
\hline & & 1.52 & 1.37 & -3.86 & $E-W$ & $\mathrm{~S}$ & 63 & minor fracture \\
\hline & & 1.52 & 1.37 & -3.94 & $\mathrm{E}-\mathrm{W}$ & $\mathrm{s}$ & 63 & minor fracture \\
\hline & & 1.52 & 1.37 & -3.89 & $\mathrm{~N}-\mathrm{S}$ & $W$ & 63 & minor fracture \\
\hline & & 1.52 & 1.37 & -3.96 & $\mathrm{~N}-\mathrm{S}$ & $W$ & 63 & minor fracture \\
\hline & & 1.52 & 1.37 & -4.12 & & & & horizontal fracture \\
\hline & & 1.52 & 1.37 & -5.13 & & & & horizontal fracture \\
\hline \multicolumn{9}{|l|}{ E5 } \\
\hline & & 1.22 & 0.61 & 0.13 & & & & horizontal fracture \\
\hline & LBTI & 1.22 & 0.61 & -0.30 & & & & top large subhorizontal \\
\hline & LBT1 & 1.22 & 0.61 & -0.34 & & & & base large subhorizontal \\
\hline & LBT16 & 1.22 & 0.61 & -0.13 & $\mathrm{~N}-\mathrm{S}$ & $w$ & 34 & moderate fracture \\
\hline & LBT16 & 1.22 & 0.61 & -0.15 & $\mathrm{~N}-\mathrm{S}$ & $W$ & 34 & moderate fracture \\
\hline & & 1.22 & 0.61 & -0.20 & $E-W$ & $\mathrm{~S}$ & 81 & minor subvertical \\
\hline & & 1.22 & 0.61 & -0.43 & $E-W$ & $\mathrm{~S}$ & 81 & minor subvertical \\
\hline & & 1.22 & 0.61 & -0.74 & & & & horizontal fracture \\
\hline & & 1.22 & 0.61 & -1.14 & & & & horizontal fracture \\
\hline
\end{tabular}




\begin{tabular}{|c|c|c|c|c|c|c|c|c|}
\hline Hole & $\begin{array}{l}\text { Surface } \\
\text { feature }\end{array}$ & $\begin{array}{l}\text { Model } \\
\text { east }\end{array}$ & $\begin{array}{l}\text { Model } \\
\text { north }\end{array}$ & $\begin{array}{l}\text { Model } \\
\text { depth }\end{array}$ & $\begin{array}{l}\text { Strike } \\
\text { direct }\end{array}$ & $\begin{array}{c}\text { Dip } \\
\text { direct }\end{array}$ & $\begin{array}{c}\text { TRUE } \\
\text { dip }\end{array}$ & Comments \\
\hline & & 1.22 & 0.61 & -1.55 & & & & horizontal fracture \\
\hline & & 1.22 & 0.61 & -1.57 & & & & horizontal fracture \\
\hline & LBT2 & 1.22 & 0.61 & -2.18 & $\mathrm{~N}-\mathrm{S}$ & $E$ & & top open subvertical \\
\hline & LBT2 & 1.22 & 0.61 & -2.23 & $\mathrm{~N}-\mathrm{S}$ & $E$ & & base open subvertical \\
\hline & & 1.22 & 0.61 & -3.43 & $E-W$ & $S$ & 89 & minor fracture \\
\hline & & 1.22 & 0.61 & 0.00 & $E-W$ & $\mathrm{~s}$ & 89 & minor fracture \\
\hline & & 1.22 & 0.61 & -3.61 & & & & horizontal fracture \\
\hline & & 1.22 & 0.61 & -4.09 & & & & open horizontal fracture \\
\hline & & 1.22 & 0.61 & -4.72 & & & & horizontal fracture \\
\hline & & 1.22 & 0.61 & -4.77 & & & & horizontal fracture \\
\hline & & 1.22 & 0.61 & -4.88 & $E-W$ & $\mathrm{~S}$ & & minor fracture \\
\hline & & 1.22 & 0.61 & -4.95 & NE-SW & SE & 79 & moderate fracture \\
\hline & & 1.22 & 0.61 & -5.16 & NE-SW & SE & 79 & moderate fracture \\
\hline \multicolumn{9}{|l|}{ E6 } \\
\hline & & & & & & & & horizontal fracture \\
\hline & LBTI & 1.22 & 1.22 & -0.32 & & & 34 & top large subhorizontal \\
\hline & LBT1 & 1.22 & 1.22 & -0.34 & & & 34 & base large subhorizontal \\
\hline & LBT16 & 1.22 & 1.22 & -0.22 & $\mathrm{~N}-\mathrm{S}$ & $W$ & 63 & moderate open subvertical \\
\hline & LBTI6 & 1.22 & 1.22 & -0.29 & $\mathrm{~N}-\mathrm{S}$ & $W$ & 63 & moderate open subvertical \\
\hline & & 1.22 & 1.22 & -1.16 & & & & horizontal fracture \\
\hline & & 1.22 & 1.22 & -1.59 & & & & horizontal fracture \\
\hline & & 1.22 & 1.22 & -2.63 & & & 34 & minor subvertical \\
\hline & & 1.22 & 1.22 & -2.65 & & & 34 & minor subvertical \\
\hline & LBT 2 & 1.22 & 1.22 & -3.52 & $\mathrm{~N}-\mathrm{S}$ & $E$ & & highly fractured zone \\
\hline & & 1.22 & 1.22 & -4.05 & $\mathrm{~N}-\mathrm{S}$ & $E$ & & $\begin{array}{l}\text { probably more than one } \\
\text { fracture }\end{array}$ \\
\hline \multicolumn{9}{|r|}{ th } \\
\hline & & 1.22 & 1.83 & 0.00 & & & & horizontal fracture \\
\hline & & 1.22 & 1.83 & -0.38 & & & & horizontal fracture \\
\hline & LBTI & 1.22 & 1.83 & -0.46 & & & 34 & top large subhorizontal \\
\hline & LBTI & 1.22 & 1.83 & -0.48 & & & 34 & base large subhorizontal \\
\hline & LBT16 & 1.22 & 1.83 & -0.25 & $\mathrm{~N}-\mathrm{S}$ & $W$ & 73 & moderate open fracture \\
\hline & LBT16 & 1.22 & 1.83 & -0.38 & $\mathrm{~N}-\mathrm{S}$ & $W$ & 73 & moderate open fracture \\
\hline & & 1.22 & 1.83 & -0.86 & $\mathrm{~N}-\mathrm{S}$ & $W$ & 69 & minor fracture \\
\hline & & 1.22 & 1.83 & -0.89 & & & 69 & horizontal fracture \\
\hline & & 1.22 & 1.83 & -0.96 & $\mathrm{~N}-\mathrm{S}$ & $w$ & & minor fracture \\
\hline & & 1.22 & 1.83 & -1.09 & & & & $\begin{array}{l}\text { wandering partly open } \\
\text { fracture }\end{array}$ \\
\hline & & 1.22 & 1.83 & -1.55 & & & & $\begin{array}{l}\text { wandering partly open } \\
\text { fracture }\end{array}$ \\
\hline & & 1.22 & 1.83 & -2.16 & $E-W$ & $\mathrm{~s}$ & & top minor fracture \\
\hline & & 1.22 & 1.83 & -2.39 & $E-W$ & $\mathrm{~S}$ & & base minor fracture \\
\hline & & 1.22 & 1.83 & -2.62 & & & & horizontal fracture \\
\hline & & 1.22 & 1.83 & -3.17 & & & & horizontal fracture \\
\hline & & 1.22 & 1.83 & -3.61 & & & & horizontal fracture \\
\hline
\end{tabular}




\begin{tabular}{|c|c|c|c|c|c|c|c|c|}
\hline Hole & $\begin{array}{l}\text { Surface } \\
\text { feature }\end{array}$ & $\begin{array}{c}\text { Model } \\
\text { east }\end{array}$ & $\begin{array}{l}\text { Model } \\
\text { north }\end{array}$ & $\begin{array}{l}\text { Model } \\
\text { depth }\end{array}$ & $\begin{array}{l}\text { Strike } \\
\text { direct }\end{array}$ & $\begin{array}{l}\text { Dip } \\
\text { direct }\end{array}$ & $\begin{array}{l}\text { TRUE } \\
\text { dip }\end{array}$ & Comments \\
\hline & & 1.22 & 1.83 & -3.71 & & & & horizontal fracture \\
\hline & & 1.22 & 1.83 & -3.83 & & & & horizontal fracture \\
\hline \multicolumn{9}{|l|}{ E8 } \\
\hline & & 1.22 & 2.44 & -0.01 & & & & horizontal fracture \\
\hline & LBTI & 1.22 & 2.44 & -0.50 & & & & top large subhorizontal \\
\hline & LBTI & 1.22 & 2.44 & -0.55 & & & & base large subhorizontal \\
\hline & LBTI6 & 1.22 & 2.44 & -0.06 & $\mathrm{~N}-\mathrm{S}$ & $W$ & & $\begin{array}{l}\text { moderate/major open } \\
\text { subvertical }\end{array}$ \\
\hline & LBTI6 & 1.22 & 2.44 & -0.11 & $\mathrm{~N}-\mathrm{S}$ & $W$ & & $\begin{array}{l}\text { moderate/major open } \\
\text { subvertical }\end{array}$ \\
\hline & & 1.22 & 2.44 & -0.27 & & & 34 & open subhorizontal fracture \\
\hline & & 1.22 & 2.44 & -0.29 & & & 34 & open subhorizontal fracture \\
\hline & & 1.22 & 2.44 & -1.36 & $E-W$ & $S$ & & minor subvertical \\
\hline & & 1.22 & 2.44 & -1.44 & $E-W$ & $\mathrm{~s}$ & & minor subvertical \\
\hline & & 1.22 & 2.44 & -1.87 & & & & horizontal fracture \\
\hline & LBT 12 & 1.22 & 2.44 & -2.40 & $E-W$ & $\mathrm{~s}$ & & top major open subvertical \\
\hline & LBT 12 & 1.22 & 2.44 & -2.48 & $E-W$ & $S$ & & $\begin{array}{l}\text { bottom major open } \\
\text { subvertical }\end{array}$ \\
\hline & & 1.22 & 2.44 & -2.73 & $E-W$ & $S$ & & \begin{tabular}{|l|}
$\begin{array}{l}\text { bottom major open } \\
\text { subvertical }\end{array}$ \\
\end{tabular} \\
\hline & & 1.22 & 2.44 & -2.65 & $\mathrm{~N}-\mathrm{S}$ & $W$ & 83 & top major open subvertical \\
\hline & & 1.22 & 2.44 & -2.96 & $\mathrm{~N}-\mathrm{S}$ & $W$ & 83 & $\begin{array}{l}\text { bottom major open } \\
\text { subvertical }\end{array}$ \\
\hline & & 1.22 & 2.44 & -2.65 & $E-W$ & $\mathrm{~N}$ & 77 & top major open subvertical \\
\hline & & 1.22 & 2.44 & -2.82 & $E-W$ & $N$ & 77 & $\begin{array}{l}\text { bottom major open } \\
\text { subvertical }\end{array}$ \\
\hline & & 1.22 & 2.44 & -3.04 & NE-SW & SE & & major open fracture \\
\hline & & 1.22 & 2.44 & -3.09 & NE-SW & SE & & major open fracture \\
\hline & LBT22 & 1.22 & 2.44 & -3.11 & NE-SW & NW & & major open fracture \\
\hline & LBT22 & 1.22 & 2.44 & -3.19 & NE-SW & NW & & major open fracture \\
\hline & & 1.22 & 2.44 & -3.44 & $E-W$ & $\mathrm{~s}$ & 79 & minor fracture \\
\hline & & 1.22 & 2.44 & -3.65 & $E-W$ & $S$ & 79 & minor fracture \\
\hline & & 1.22 & 2.44 & -3.54 & & & & horizontal fracture \\
\hline & & 1.22 & 2.44 & -3.65 & & & & horizontal fracture \\
\hline & & 1.22 & 2.44 & -3.67 & $\mathrm{~N}-\mathrm{S}$ & $W$ & 84 & top major open subvertical \\
\hline & & 1.22 & 2.44 & -4.05 & $\mathrm{~N}-\mathrm{S}$ & $W$ & 84 & $\begin{array}{l}\text { bottom major open } \\
\text { subvertical }\end{array}$ \\
\hline & & 1.22 & 2.44 & -4.26 & NE-SW & SE & 73 & minor fracture \\
\hline & & 1.22 & 2.44 & -4.38 & NE-SW & $\mathrm{SE}$ & 73 & minor fracture \\
\hline & & 1.22 & 2.44 & -4.51 & & & 90 & vertical open joint \\
\hline & & 1.22 & 2.44 & -4.71 & & & 90 & vertical open joint \\
\hline & & 1.22 & 2.44 & -4.76 & & & & subhorizontal fracture \\
\hline & & 1.22 & 2.44 & -4.94 & & & & horizontal fracture \\
\hline & & 1.22 & 2.44 & -5.07 & $\mathrm{~N}-\mathrm{S}$ & $W$ & & tight, minor fracture \\
\hline & & 1.22 & 2.44 & -5.17 & $\mathrm{~N}-\mathrm{S}$ & $W$ & & tight, minor fracture \\
\hline E9 & & & & & & & & \\
\hline
\end{tabular}




\begin{tabular}{|c|c|c|c|c|c|c|c|c|}
\hline Hole & $\begin{array}{l}\text { Surface } \\
\text { feature }\end{array}$ & $\begin{array}{l}\text { Model } \\
\text { east }\end{array}$ & $\begin{array}{l}\text { Model } \\
\text { north }\end{array}$ & $\begin{array}{l}\text { Model } \\
\text { depth }\end{array}$ & $\begin{array}{l}\text { Strike } \\
\text { direct }\end{array}$ & $\begin{array}{c}\text { Dip } \\
\text { direct }\end{array}$ & $\begin{array}{c}\text { TRUE } \\
\text { dip }\end{array}$ & Comments \\
\hline & & 0.61 & 1.22 & 0.04 & & & & horizontal fracture \\
\hline & LBTI & 0.61 & 1.22 & -0.29 & & & 34 & top large subhorizontal \\
\hline & LBT1 & 0.61 & 1.22 & -0.32 & & & 34 & base large subhorizontal \\
\hline & & 0.61 & 1.22 & -0.32 & $\mathrm{~N}-\mathrm{S}$ & $W$ & 63 & tight, minor fracture \\
\hline & & 0.61 & 1.22 & -0.40 & $\mathrm{~N}-\mathrm{S}$ & $w$ & 63 & tight, minor fracture \\
\hline & & 0.61 & 1.22 & -0.73 & & & & horizontal fracture \\
\hline & & 0.61 & 1.22 & -0.95 & & & & horizontal fracture \\
\hline & & 0.61 & 1.22 & -0.95 & SE-NW & SW & 63 & tight, minor fracture \\
\hline & & 0.61 & 1.22 & -1.03 & SE-NW & SW & 63 & tight, minor fracture \\
\hline & & 0.61 & 1.22 & -1.13 & & & & horizontal fracture \\
\hline & LBT16 & 0.61 & 1.22 & -1.13 & $\mathrm{~N}-\mathrm{S}$ & $W$ & & open fracture \\
\hline & & 0.61 & 1.22 & -1.26 & $E-W$ & $S$ & 63 & moderate fracture \\
\hline & & 0.61 & 1.22 & -1.34 & $E-W$ & $S$ & 63 & moderate fracture \\
\hline & & 0.61 & 1.22 & -1.44 & $\mathrm{~N}-\mathrm{S}$ & $W$ & 73 & moderate fracture \\
\hline & & 0.61 & 1.22 & -1.56 & $\mathrm{~N}-\mathrm{S}$ & $w$ & 73 & moderate fracture \\
\hline & LBT 2 & 0.61 & 1.22 & -1.67 & SE-NW & NE & 76 & major open fracture \\
\hline & LBT 2 & 0.61 & 1.22 & -1.82 & SE-NW & $\mathrm{NE}$ & 76 & major open fracture \\
\hline & & 0.61 & 1.22 & -3.82 & & & & horizontal fracture \\
\hline & & 0.61 & 1.22 & -4.26 & & & & horizontal fracture \\
\hline & & 0.61 & 1.22 & -5.02 & & & & top subhorizontal \\
\hline & & 0.61 & 1.22 & -5.20 & & & & base subhorizontal \\
\hline \multicolumn{9}{|l|}{ E10 } \\
\hline & & 0.61 & 1.83 & 0.15 & & & & horizontal fracture \\
\hline & LBTI & 0.61 & 1.83 & -0.36 & & & 34 & top large subhorizontal \\
\hline & LBT1 & 0.61 & 1.83 & -0.38 & & & 34 & base large subhorizontal \\
\hline & & 0.61 & 1.83 & 0.15 & $\mathrm{~N}-\mathrm{S}$ & $W$ & 81 & major open fracture \\
\hline & & 0.61 & 1.83 & -0.10 & $\mathrm{~N}-\mathrm{S}$ & $W$ & 81 & major open fracture \\
\hline & & 0.61 & 1.83 & -1.25 & $\mathrm{~N}-\mathrm{S}$ & $W$ & 63 & major open fracture \\
\hline & & 0.61 & 1.83 & -1.32 & $\mathrm{~N}-\mathrm{S}$ & $W$ & 63 & major open fracture \\
\hline & LBT16 & 0.61 & 1.83 & -1.27 & $\mathrm{~N}-\mathrm{S}$ & $W$ & 81 & major open fracture \\
\hline & LBT16 & 0.61 & 1.83 & -1.50 & $\mathrm{~N}-\mathrm{S}$ & $W$ & 81 & major open fracture \\
\hline & LBT 12 & 0.61 & 1.83 & -2.77 & $E-W$ & $S$ & 78 & major open fracture \\
\hline & LBT 12 & 0.61 & 1.83 & -2.95 & $E-W$ & $\mathrm{~s}$ & 78 & major open fracture \\
\hline & LBT 2 & 0.61 & 1.83 & -3.07 & SE-NW & $\mathrm{NE}$ & 79 & major open fracture \\
\hline & LBT 2 & 0.61 & 1.83 & -3.28 & SE-NW & $\mathrm{NE}$ & 79 & major open fracture \\
\hline & LBT14 & 0.61 & 1.83 & -3.23 & & & & open horizontal fracture \\
\hline & & 0.61 & 1.83 & -3.71 & & & & horizontal fracture \\
\hline & & 0.61 & 1.83 & -3.84 & & & & horizontal fracture \\
\hline & & 0.61 & 1.83 & -5.11 & SW-NE & $\mathrm{SE}$ & & minor fracture \\
\hline \multicolumn{9}{|l|}{$\mathrm{EHI}$} \\
\hline & LBT21 & 2.84 & 0.30 & -2.74 & & & 90 & $\begin{array}{l}\text { moderate vertical open } \\
\text { fracture }\end{array}$ \\
\hline & & 2.64 & 0.30 & -2.74 & & & 90 & minor vertical closed fracture \\
\hline & & 2.51 & 0.30 & -2.74 & N6OE & SE & 80 & angled partly open fracture \\
\hline & & 2.44 & 0.30 & -2.74 & NGOE & SE & 80 & angled partly open fracture \\
\hline
\end{tabular}




\begin{tabular}{|c|c|c|c|c|c|c|c|c|}
\hline Hole & $\begin{array}{l}\text { Surface } \\
\text { feature }\end{array}$ & $\begin{array}{l}\text { Model } \\
\text { east }\end{array}$ & $\begin{array}{l}\text { Model } \\
\text { north }\end{array}$ & $\begin{array}{l}\text { Model } \\
\text { depth }\end{array}$ & $\begin{array}{l}\text { Strike } \\
\text { direct }\end{array}$ & $\begin{array}{l}\text { Dip } \\
\text { direct }\end{array}$ & $\begin{array}{c}\text { TRUE } \\
\text { dip }\end{array}$ & Comments \\
\hline & & 2.54 & 0.30 & -2.74 & & & & $\begin{array}{l}\text { closed, healed subhorizontal } \\
\text { fracture }\end{array}$ \\
\hline & & 2.11 & 0.30 & -2.74 & & & & $\begin{array}{l}\text { closed, healed subhorizontal } \\
\text { fracture }\end{array}$ \\
\hline & & 1.98 & 0.30 & -2.74 & & & 90 & minor vertical fracture \\
\hline & & 1.60 & 0.30 & -2.74 & & & 90 & $\begin{array}{l}\text { moderate vertical open } \\
\text { fracture }\end{array}$ \\
\hline & LBT2 & 1.57 & 0.30 & -2.74 & N50W & NE & 80 & $\begin{array}{l}\text { major open angled fracture, } \\
\text { subvertical }\end{array}$ \\
\hline & LBT2 & 1.52 & 0.30 & -2.74 & N50W & $\mathrm{NE}$ & 80 & $\begin{array}{l}\text { major open angled fracture, } \\
\text { subvertical }\end{array}$ \\
\hline & & 1.50 & 0.30 & -2.74 & & & 90 & minor vertical fracture \\
\hline & & 1.45 & 0.30 & -2.74 & N3OE & & 90 & major open fracture, vertical \\
\hline & & 1.42 & 0.30 & -2.74 & $\mathrm{~N} 30 \mathrm{E}$ & & 90 & major open fracture, vertical \\
\hline & & 0.74 & 0.30 & -2.74 & NE-SW & & & $\begin{array}{l}\text { minor subvertical closed } \\
\text { fracture }\end{array}$ \\
\hline \multicolumn{9}{|l|}{$\mathrm{EH} 2$} \\
\hline & LBT21 & 2.79 & 0.91 & -2.74 & & & 90 & significant vertical fracture \\
\hline & & 2.74 & 0.91 & -2.74 & & & 90 & $\begin{array}{l}\text { significant vertical fracture. } \\
\text { open }\end{array}$ \\
\hline & & 1.89 & 0.91 & -2.74 & & & & $\begin{array}{l}\text { minor subvertical closed } \\
\text { fracture }\end{array}$ \\
\hline & & 1.57 & 0.91 & -2.74 & NE-SW & & & subvertical open fracture \\
\hline & & 1.57 & 0.91 & -2.74 & $\mathrm{~N} 30 \mathrm{~W}$ & SW & 70 & moderate open fracture \\
\hline & & 1.55 & 0.91 & -2.74 & N3OW & SW & 70 & moderate open fracture \\
\hline & & 1.14 & 0.91 & -2.74 & NW-SE & SW & & $\begin{array}{l}\text { moderate partly open } \\
\text { subvertical fracture }\end{array}$ \\
\hline & & 0.74 & 0.91 & -2.74 & & & & $\begin{array}{l}\text { minor random irregular } \\
\text { closed fractures }\end{array}$ \\
\hline & & 0.30 & 0.91 & -2.74 & & & & $\begin{array}{l}\text { minor random irregular } \\
\text { closed fractures }\end{array}$ \\
\hline \multicolumn{9}{|l|}{$\mathrm{EH} 3$} \\
\hline & & 2.79 & 1.52 & -2.74 & & & 90 & minor vertical closed fracture \\
\hline & & 2.67 & 1.52 & -2.74 & & & 90 & $\begin{array}{l}\text { minor vertical closed } \\
\text { fracture, partly open }\end{array}$ \\
\hline & & 2.44 & 1.52 & -2.74 & & & 90 & minor vertical closed fracture \\
\hline & & 1.85 & 1.52 & -2.74 & & & 90 & minor vertical closed fracture \\
\hline & & 1.40 & 1.52 & -2.74 & & & 90 & minor vertical closed fracture \\
\hline & & 1.37 & 1.52 & -2.74 & NIOE & & 90 & major open vertical fracture \\
\hline & & 1.19 & 1.52 & -2.74 & & & 90 & $\begin{array}{l}\text { minor partly open vertical } \\
\text { fracture }\end{array}$ \\
\hline & & 0.97 & 1.52 & -2.74 & & & 90 & $\begin{array}{l}\text { minor partly open vertical } \\
\text { fracture }\end{array}$ \\
\hline & & 0.66 & 1.52 & -2.74 & N40W & $\mathrm{NE}$ & 70 & angled vertical tight fracture \\
\hline & & 0.61 & 1.52 & -2.74 & N4OW & NE & 70 & angled vertical tight fracture \\
\hline \multicolumn{9}{|l|}{$\mathrm{EH} 4$} \\
\hline & & 2.79 & 2.13 & -2.74 & & & 90 & $\begin{array}{l}\text { minor vertical partly open } \\
\text { fracture }\end{array}$ \\
\hline
\end{tabular}




\begin{tabular}{|c|c|c|c|c|c|c|c|c|}
\hline Hole & $\begin{array}{l}\text { Surface } \\
\text { feature }\end{array}$ & $\begin{array}{l}\text { Model } \\
\text { east }\end{array}$ & $\begin{array}{l}\text { Model } \\
\text { north }\end{array}$ & $\begin{array}{l}\text { Model } \\
\text { depth }\end{array}$ & $\begin{array}{l}\text { Strike } \\
\text { direct }\end{array}$ & $\begin{array}{l}\text { Dip } \\
\text { direct }\end{array}$ & $\begin{array}{l}\text { TRUE } \\
\text { dip }\end{array}$ & Comments \\
\hline & & 2.77 & 2.13 & -2.74 & & & 90 & $\begin{array}{l}\text { minor vertical partly open } \\
\text { fracture }\end{array}$ \\
\hline & LBT6 & 2.64 & 2.13 & -2.74 & N1OW & & 90 & major open vertical fracture \\
\hline & & 2.57 & 2.13 & -2.74 & NW-SE & & 90 & $\begin{array}{l}\text { minor-moderate angled } \\
\text { partly open vertical fracture }\end{array}$ \\
\hline & & 2.54 & 2.13 & -2.74 & $N W-S E$ & & 90 & $\begin{array}{l}\text { minor-moderate angled } \\
\text { partly open vertical fracture }\end{array}$ \\
\hline & & 2.57 & 2.13 & -2.74 & NE-SW & & & minor subvertical fracture \\
\hline & & 2.51 & 2.13 & -2.74 & NE-SW & & & minor subvertical fracture \\
\hline & & 2.06 & 2.13 & -2.74 & & & 90 & major open vertical fracture \\
\hline & & 2.03 & 2.13 & -2.74 & & & 90 & $\begin{array}{l}\text { major open vertical fracture, } \\
\text { less significant then above }\end{array}$ \\
\hline & & 1.78 & 2.13 & -2.74 & N2OE & NW & 90 & major vertical open fracture \\
\hline & & 1.65 & 2.13 & -2.74 & & & 90 & minor vertical fracture \\
\hline & & 1.50 & 2.13 & -2.74 & & & 90 & $\begin{array}{l}\text { minor fine fracture, vertical } \\
\text { barely open }\end{array}$ \\
\hline & & 1.45 & 2.13 & -2.74 & & & 90 & $\begin{array}{l}\text { minor fine fracture, vertical } \\
\text { barely open }\end{array}$ \\
\hline & & 1.40 & 2.13 & -2.74 & & & 90 & $\begin{array}{l}\text { minor fine fracture, vertical } \\
\text { barely open }\end{array}$ \\
\hline & & 1.32 & 2.13 & -2.74 & & & 90 & $\begin{array}{l}\text { open vertical fracture, more } \\
\text { significant then above }\end{array}$ \\
\hline & & 1.09 & 2.13 & -2.74 & N60E & SE & 50 & major angled open fracture \\
\hline & & 1.04 & 2.13 & -2.74 & N6OE & SE & 50 & major angled open fracture \\
\hline & & 0.99 & 2.13 & -2.74 & NGOE & SE & 45 & major angled open fracture \\
\hline & & 0.91 & 2.13 & -2.74 & N60E & SE & 45 & major angled open fracture \\
\hline & LBT23 & 0.81 & 2.13 & -2.74 & $\mathrm{~N} 20 \mathrm{E}$ & NW & 45 & major angled open fracture \\
\hline & LBT23 & 0.76 & 2.13 & -2.74 & N2OE & NW & 45 & major angled open fracture \\
\hline \multicolumn{9}{|l|}{ EH5 } \\
\hline & & 2.84 & 2.74 & -2.74 & & & 90 & major open vertical fracture \\
\hline & & 2.82 & 2.74 & -2.74 & $\mathrm{~N}-\mathrm{S}$ & $E$ & 20 & $\begin{array}{l}\text { minor tight closed } \\
\text { subhorizontal healed fracture }\end{array}$ \\
\hline & & 2.69 & 2.74 & -2.74 & $N-S$ & $\bar{E}$ & 20 & $\begin{array}{l}\text { minor tight closed } \\
\text { subhorizontal healed fracture }\end{array}$ \\
\hline & & 2.69 & 2.74 & -2.74 & & & 90 & $\begin{array}{l}\text { minor vertical partly open } \\
\text { fracture }\end{array}$ \\
\hline & & 2.64 & 2.74 & -2.74 & N3OE & SE & 80 & $\begin{array}{l}\text { moderate angled open } \\
\text { fracture }\end{array}$ \\
\hline & & 2.62 & 2.74 & -2.74 & N3OE & SE & 80 & $\begin{array}{l}\text { moderate angled open } \\
\text { fracture }\end{array}$ \\
\hline & & 2.29 & 2.74 & -2.74 & $\mathrm{~N}-\mathrm{S}$ & $w$ & & $\begin{array}{l}\text { minor tight subvertical } \\
\text { fracture }\end{array}$ \\
\hline & & 2.26 & 2.74 & -2.74 & $\mathrm{~N}-\mathrm{S}$ & $W$ & & $\begin{array}{l}\text { minor tight subvertical } \\
\text { fracture }\end{array}$ \\
\hline & LBT6 & 2.13 & 2.74 & -2.74 & N2OW & & 90 & $\begin{array}{l}\text { major open vertical fracture } \\
\text { zone }\end{array}$ \\
\hline & & 1.88 & 2.74 & -2.74 & $\mathrm{~N}-\mathrm{S}$ & $E$ & 20 & $\begin{array}{l}\text { moderate subhorizontal } \\
\text { fracture, with assoc. other }\end{array}$ \\
\hline
\end{tabular}




\begin{tabular}{|c|c|c|c|c|c|c|c|c|}
\hline Hole & $\begin{array}{l}\text { Surface } \\
\text { feature }\end{array}$ & $\begin{array}{l}\text { Model } \\
\text { east }\end{array}$ & $\begin{array}{l}\text { Model } \\
\text { north }\end{array}$ & $\begin{array}{l}\text { Model } \\
\text { depth }\end{array}$ & $\begin{array}{l}\text { Strike } \\
\text { direct }\end{array}$ & $\begin{array}{l}\text { Dip } \\
\text { direct }\end{array}$ & $\begin{array}{l}\text { TRUE } \\
\text { dip }\end{array}$ & Comments \\
\hline & & & & & & & & minors \\
\hline & & 1.73 & 2.74 & -2.74 & $N-S$ & $E$ & 20 & $\begin{array}{l}\text { moderate subhorizontal } \\
\text { fracture, with assoc. other } \\
\text { minors }\end{array}$ \\
\hline & & 1.60 & 2.74 & -2.74 & & & 90 & minor vertical fracture \\
\hline & LBT23 & 1.49 & 2.74 & -2.74 & N3OE & & 80 & $\begin{array}{l}\text { moderate open vertical } \\
\text { fracture }\end{array}$ \\
\hline & & 1.22 & 2.74 & -2.74 & & & 90 & $\begin{array}{l}\text { major open vertical sharp } \\
\text { fracture }\end{array}$ \\
\hline & & 0.99 & 2.74 & -2.74 & $\mathrm{~N}-\mathrm{S}$ & $E$ & 20 & $\begin{array}{l}\text { moderate subhorizontal } \\
\text { closed fracture }\end{array}$ \\
\hline & & 0.89 & 2.74 & -2.74 & $N-S$ & $E$ & 20 & $\begin{array}{l}\text { moderate subhorizontal } \\
\text { closed fracture }\end{array}$ \\
\hline & & 0.91 & 2.74 & -2.74 & N8OW & & 80 & $\begin{array}{l}\text { angled moderate partly } \\
\text { open fractures }\end{array}$ \\
\hline & & 0.71 & 2.74 & -2.74 & N8OW & & 80 & $\begin{array}{l}\text { angled moderate partly } \\
\text { open fractures }\end{array}$ \\
\hline & & 0.61 & 2.74 & -2.74 & & & & $\begin{array}{l}\text { minor subvertical closed } \\
\text { fracture, healed }\end{array}$ \\
\hline & & 0.53 & 2.74 & -2.74 & & & & $\begin{array}{l}\text { minor subvertical closed } \\
\text { fracture }\end{array}$ \\
\hline \multicolumn{9}{|l|}{ E03 } \\
\hline & LBT7 & 2.93 & 2.74 & -3.96 & & & 90 & vertical open fracture \\
\hline & & 2.54 & 2.74 & -3.96 & $\mathrm{~N}-\mathrm{S}$ & $E$ & 30 & moderate closed fracture \\
\hline & & 2.49 & 2.74 & -3.96 & $\mathrm{~N}-\mathrm{S}$ & $\bar{E}$ & 30 & moderate closed fracture \\
\hline & & 2.46 & 2.74 & -3.96 & N6OW & NW & & $\begin{array}{l}\text { angled moderate fracture, } \\
\text { open, subvertical }\end{array}$ \\
\hline & & 2.44 & 2.74 & -3.96 & N6OW & NW & & $\begin{array}{l}\text { angled moderate fracture, } \\
\text { open, subvertical }\end{array}$ \\
\hline & & 2.36 & 2.74 & -3.96 & $E-W$ & & 90 & $\begin{array}{l}\text { moderate partly open } \\
\text { fracture }\end{array}$ \\
\hline & & 2.31 & 2.74 & -3.96 & $E-W$ & & 90 & $\begin{array}{l}\text { moderate partly open } \\
\text { fracture }\end{array}$ \\
\hline & & 2.24 & 2.74 & -3.96 & & & 90 & minor vertical fracture \\
\hline & & 2.04 & 2.74 & -3.96 & N45E & & 90 & angled minor closed fracture \\
\hline & & 1.98 & 2.74 & -3.96 & N45E & & 90 & angled minor closed fracture \\
\hline & LBT6 & 1.78 & 2.74 & -3.96 & N70W & $\mathrm{NE}$ & 80 & major angled fracture \\
\hline & LBT6 & 1.65 & 2.74 & -3.96 & N70W & $\mathrm{NE}$ & 80 & major angled fracture \\
\hline & & 1.47 & 2.74 & -3.96 & N3OE & & 90 & $\begin{array}{l}\text { major angled locally open } \\
\text { fracture }\end{array}$ \\
\hline & & 1.42 & 2.74 & -3.96 & N3OE & & 90 & $\begin{array}{l}\text { major angled locally open } \\
\text { fracture }\end{array}$ \\
\hline & & 1.42 & 2.74 & -3.96 & & & & $\begin{array}{l}2 \text { open moderate subvertical } \\
\text { fractures }\end{array}$ \\
\hline & & 1.40 & 2.74 & -3.96 & & & & $\begin{array}{l}2 \text { open moderate subvertical } \\
\text { fractures }\end{array}$ \\
\hline & & 1.30 & 2.74 & -3.96 & & & 90 & $\begin{array}{l}\text { major large open vertical } \\
\text { fractures }\end{array}$ \\
\hline & & 0.99 & 2.74 & -3.96 & & & & minor subvertical tight \\
\hline
\end{tabular}




\begin{tabular}{|c|c|c|c|c|c|c|c|c|}
\hline Hole & $\begin{array}{l}\text { Surface } \\
\text { feature }\end{array}$ & $\begin{array}{l}\text { Model } \\
\text { east }\end{array}$ & $\begin{array}{l}\text { Model } \\
\text { north }\end{array}$ & $\begin{array}{l}\text { Model } \\
\text { depth }\end{array}$ & $\begin{array}{l}\text { Strike } \\
\text { direct }\end{array}$ & $\begin{array}{l}\text { Dip } \\
\text { direct }\end{array}$ & $\begin{array}{c}\text { TRUE } \\
\text { dip }\end{array}$ & Comments \\
\hline & & & & & & & & fractures \\
\hline & & 0.97 & 2.74 & -3.96 & & & & $\begin{array}{l}\text { minor subvertical tight } \\
\text { fractures }\end{array}$ \\
\hline & & 0.66 & 2.74 & -3.96 & $\sim \mathrm{N}-\mathrm{S}$ & $E$ & 10 & $\begin{array}{l}\text { major open subhorizontal } \\
\text { fracture, complex system }\end{array}$ \\
\hline & & 0.38 & 2.74 & -3.96 & $\sim \mathrm{N}-\mathrm{S}$ & $E$ & 10 & $\begin{array}{l}\text { major open subhorizontal } \\
\text { fracture, complex system }\end{array}$ \\
\hline \multicolumn{9}{|r|}{ 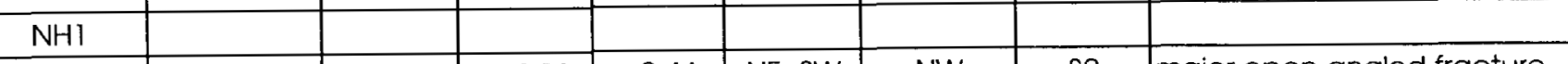 } \\
\hline & LBT22 & 2.13 & 2.72 & -2.44 & NE-SW & NW & 80 & major open angled fracture \\
\hline & LBT22 & 2.13 & 2.06 & -2.44 & NE-SW & NW & 80 & major open angled fracture \\
\hline & & 2.13 & 1.80 & -2.44 & $E-W$ & & 90 & minor tight vertical fracture \\
\hline & & 2.13 & 1.09 & -2.44 & $E-W$ & & 90 & $\begin{array}{l}\text { moderate-major vertical } \\
\text { partly open fracture }\end{array}$ \\
\hline & & 2.13 & 0.66 & -2.44 & N3OW & SW & 60 & $\begin{array}{l}\text { minor-moderate angled tight } \\
\text { fracture }\end{array}$ \\
\hline & & 2.13 & 0.28 & -2.44 & N3OW & SW & 60 & $\begin{array}{l}\text { minor-moderate angled tight } \\
\text { fracture }\end{array}$ \\
\hline \multicolumn{9}{|r|}{ 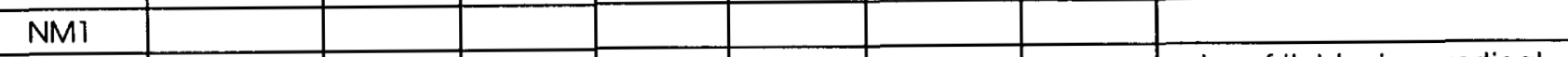 } \\
\hline & & 2.74 & 2.64 & -3.81 & & & & $\begin{array}{l}\text { series of tight minor vertical } \\
\text { to subvertical fractures }\end{array}$ \\
\hline & & 2.74 & 2.49 & -3.81 & & & & $\begin{array}{l}\text { series of tight minor vertical } \\
\text { to subvertical fractures }\end{array}$ \\
\hline & LBT8 & 2.74 & 1.80 & -3.81 & N80E & $S$ & 70 & major subvertical fracture \\
\hline & LBT8 & 2.74 & 1.73 & -3.81 & N8OE & $S$ & 70 & major subvertical fracture \\
\hline & & 2.74 & 1.73 & -3.81 & & & & $\begin{array}{l}\text { series of minor subvertical } \\
\text { fractures }\end{array}$ \\
\hline & & 2.74 & 1.03 & -3.81 & & & & $\begin{array}{l}\text { series of minor subvertical } \\
\text { fractures }\end{array}$ \\
\hline & LBT3 & 2.74 & 1.50 & -3.81 & & & 90 & $\begin{array}{l}\text { minor-moderate partly open } \\
\text { vertical fracture }\end{array}$ \\
\hline & & 2.74 & 1.09 & -3.81 & N45W & SW & 80 & $\begin{array}{l}\text { angled minor subvertical } \\
\text { fracture }\end{array}$ \\
\hline & & 2.74 & 0.99 & -3.81 & $\mathrm{~N} 45 \mathrm{~W}$ & SW & 80 & $\begin{array}{l}\text { angled minor subvertical } \\
\text { fracture }\end{array}$ \\
\hline \multicolumn{9}{|l|}{ NM2 } \\
\hline & & 0.91 & 3.02 & -2.44 & $E-W$ & & & $\begin{array}{l}\text { minor-moderate tight } \\
\text { fracture }\end{array}$ \\
\hline & & 0.91 & 2.72 & -2.44 & $E-W$ & & & $\begin{array}{l}\text { minor-moderate tight } \\
\text { fracture }\end{array}$ \\
\hline & & 0.91 & 2.29 & -2.44 & $E-W$ & & 90 & major open vertical fracture \\
\hline & & 0.91 & 1.93 & -2.44 & $E-W$ & & & $\begin{array}{l}\text { tough to see, but looks like a } \\
\text { minor fracture }\end{array}$ \\
\hline & & 0.91 & 1.83 & -2.44 & & & & $\begin{array}{l}\text { minor tight subvertical } \\
\text { fracture }\end{array}$ \\
\hline & & 0.91 & 1.73 & -2.44 & & & & $\begin{array}{l}\text { moderate open subvertical } \\
\text { fracture }\end{array}$ \\
\hline & LBT2 & 0.91 & 1.12 & -2.44 & N45W & SW & 70 & major angled open fracture \\
\hline & LBT2 & 0.91 & 0.99 & -2.44 & N45W & SW & 70 & major angled open fracture \\
\hline
\end{tabular}




\begin{tabular}{|c|c|c|c|c|c|c|c|c|}
\hline Hole & $\begin{array}{l}\text { Surface } \\
\text { feature }\end{array}$ & $\begin{array}{c}\text { Model } \\
\text { east }\end{array}$ & $\begin{array}{l}\text { Model } \\
\text { north }\end{array}$ & $\begin{array}{l}\text { Model } \\
\text { depth }\end{array}$ & $\begin{array}{l}\text { Strike } \\
\text { direct }\end{array}$ & $\begin{array}{l}\text { Dip } \\
\text { direct }\end{array}$ & $\begin{array}{c}\text { TRUE } \\
\text { dip }\end{array}$ & Comments \\
\hline & LBT3 & 0.91 & 0.97 & -2.44 & $E-W$ & & 90 & $\begin{array}{l}\text { moderate-major vertical } \\
\text { open fracture }\end{array}$ \\
\hline & & 0.91 & 0.61 & -2.44 & $E-W$ & & & minor tight fracture \\
\hline & & 0.91 & 0.28 & -2.44 & $E-W$ & & & minor tight fracture \\
\hline \multicolumn{9}{|l|}{$\mathrm{NM} 3$} \\
\hline & LBT24 & 0.30 & 2.39 & -0.91 & NE-SW & NW & & $\begin{array}{l}\text { moderate angled tight } \\
\text { fracture }\end{array}$ \\
\hline & LBT24 & 0.30 & 2.21 & -0.91 & NE-SW & NW & & $\begin{array}{l}\text { moderate angled tight } \\
\text { fracture }\end{array}$ \\
\hline & LBT2 & 0.30 & 1.24 & -0.91 & NW-SE & $\mathrm{NE}$ & & major open angled fracture \\
\hline & & 0.30 & 1.12 & -0.91 & NW-SE & $\mathrm{NE}$ & & major open angled fracture \\
\hline & LBT3 & 0.30 & 1.07 & -0.91 & & & 90 & $\begin{array}{l}\text { moderate open vertical } \\
\text { fracture }\end{array}$ \\
\hline & & 0.30 & 0.48 & -0.91 & $E-W$ & & 90 & $\begin{array}{l}\text { moderate-major vertical } \\
\text { open wet fracture }\end{array}$ \\
\hline \multicolumn{9}{|r|}{ ti } \\
\hline & & 2.13 & 2.87 & -0.91 & NW-SE & NE & & $\begin{array}{l}\text { moderate-major angled } \\
\text { partly open fracture }\end{array}$ \\
\hline & & 2.13 & 2.79 & -0.91 & NW-SE & NE & & $\begin{array}{l}\text { moderate-major angled } \\
\text { partly open fracture }\end{array}$ \\
\hline & LBT8 & 2.13 & 2.24 & -0.91 & NW-SE & & 90 & $\begin{array}{l}\text { minor tight nearly vertical } \\
\text { fracture }\end{array}$ \\
\hline & & 2.13 & 2.18 & -0.91 & & & 90 & $\begin{array}{l}\text { moderate vertical open wet } \\
\text { fracture }\end{array}$ \\
\hline & & 2.13 & 2.11 & -0.91 & & & 90 & $\begin{array}{l}\text { minor vertical open wet } \\
\text { fracture }\end{array}$ \\
\hline & & 2.13 & 1.83 & -0.91 & & & 90 & $\begin{array}{l}\text { minor nearly vertical closed } \\
\text { fracture }\end{array}$ \\
\hline & & 2.13 & 1.30 & -0.91 & NW-SE & SW & & $\begin{array}{l}\text { moderate-major angled } \\
\text { fracture partly open }\end{array}$ \\
\hline & & 2.13 & 1.17 & -0.91 & NW-SE & SW & & $\begin{array}{l}\text { moderate-major angled } \\
\text { fracture partly open }\end{array}$ \\
\hline & & 2.13 & 1.02 & -0.91 & & & 90 & $\begin{array}{l}\text { moderate open wet vertical } \\
\text { fracture }\end{array}$ \\
\hline & & 2.13 & 0.56 & -0.91 & & & 90 & vertical partly open fracture \\
\hline & & 2.13 & 0.33 & -0.91 & & & 90 & $\begin{array}{l}\text { vertical open moderate- } \\
\text { major fracture }\end{array}$ \\
\hline \multicolumn{9}{|l|}{ NN2 } \\
\hline & LBT6 & 2.13 & 2.91 & -1.98 & & & & $\begin{array}{l}\text { major, healed vertical } \\
\text { fracture }\end{array}$ \\
\hline & & 2.13 & 2.72 & -1.98 & & & & minor tight vertical fracture \\
\hline & & 2.13 & 2.64 & -1.98 & N45E & & & $\begin{array}{l}\text { angled minor tight } \\
\text { subvertical fracture }\end{array}$ \\
\hline & & 2.13 & 2.62 & -1.98 & N45E & & & $\begin{array}{l}\text { angled minor tight } \\
\text { subvertical fracture }\end{array}$ \\
\hline & & 2.13 & 2.24 & -1.98 & N45E & & & $\begin{array}{l}\text { moderate tight angled } \\
\text { subvertical fracture }\end{array}$ \\
\hline & & 2.13 & 2.18 & -1.98 & N45E & & & $\begin{array}{l}\text { moderate tight angled } \\
\text { subvertical fracture }\end{array}$ \\
\hline
\end{tabular}




\begin{tabular}{|c|c|c|c|c|c|c|c|c|}
\hline Hole & $\begin{array}{l}\text { Surface } \\
\text { feature }\end{array}$ & $\begin{array}{c}\text { Model } \\
\text { east }\end{array}$ & $\begin{array}{l}\text { Model } \\
\text { north }\end{array}$ & $\begin{array}{l}\text { Model } \\
\text { depth }\end{array}$ & $\begin{array}{l}\text { Strike } \\
\text { direct }\end{array}$ & $\begin{array}{l}\text { Dip } \\
\text { direct }\end{array}$ & $\begin{array}{c}\text { TRUE } \\
\text { dip }\end{array}$ & Comments \\
\hline & & 2.13 & 1.93 & -1.98 & $E-W$ & & 90 & $\begin{array}{l}\text { minor/moderate tight } \\
\text { fracture }\end{array}$ \\
\hline & & 2.13 & 1.35 & -1.98 & $E-W$ & & 90 & $\begin{array}{l}\text { minor/moderate tight } \\
\text { fracture }\end{array}$ \\
\hline & & 2.13 & 1.23 & -1.98 & N3OW & SW & 70 & $\begin{array}{l}\text { minor-moderate partly open } \\
\text { angled fracture }\end{array}$ \\
\hline & & 2.13 & 1.04 & -1.98 & N3OW & SW & 70 & $\begin{array}{l}\text { minor-moderate partly open } \\
\text { angled fracture }\end{array}$ \\
\hline & & 2.13 & 1.17 & -1.98 & & W & & $\begin{array}{l}\text { major-moderate fracture } \\
\text { (leaves same side of hole) }\end{array}$ \\
\hline & & 2.13 & 1.07 & -1.98 & & $w$ & & $\begin{array}{l}\text { major-moderate fracture } \\
\text { (leaves same side of hole) }\end{array}$ \\
\hline & LBT3 & 2.13 & 1.09 & -1.98 & & & 90 & major vertical open fracture \\
\hline & & 2.13 & 0.71 & -1.98 & N45E & SE & 70 & minor closed angled fracture \\
\hline & & 2.13 & 0.67 & -1.98 & N45E & SE & 70 & minor closed angled fracture \\
\hline \multicolumn{9}{|l|}{ NN3 } \\
\hline & & 2.13 & 2.91 & -3.81 & N45E & & 90 & major open vertical fracture \\
\hline & & 2.13 & 2.39 & -3.81 & NE-SW & & 90 & $\begin{array}{l}\text { major angled vertical } \\
\text { fracture }\end{array}$ \\
\hline & & 2.13 & 2.60 & -3.81 & NE-SW & & 90 & $\begin{array}{l}\text { major angled vertical } \\
\text { fracture }\end{array}$ \\
\hline & & $?$ & $?$ & $?$ & & & 90 & major open vertical fracture \\
\hline & & 2.13 & 1.75 & -3.81 & NW-SE & & & highly fractured zone \\
\hline & & 2.13 & 1.60 & -3.81 & NW-SE & & & highly fractured zone \\
\hline & & 2.13 & 1.60 & -3.81 & $E-W$ & & & $\begin{array}{l}\text { major partly open } \\
\text { subhorizontal fracture }\end{array}$ \\
\hline & & 2.13 & 1.30 & -3.81 & $E-W$ & & & $\begin{array}{l}\text { Major partly open } \\
\text { subhorizontal fracture }\end{array}$ \\
\hline & LBT3 & 2.13 & 1.04 & -3.81 & $E-W$ & & & $\begin{array}{l}\text { major subvertical open } \\
\text { fracture }\end{array}$ \\
\hline & & 2.13 & 1.04 & -3.81 & $E-W$ & $S$ & 15 & $\begin{array}{l}\text { major continuous horizontal } \\
\text { fracture }\end{array}$ \\
\hline & & 2.13 & 0.74 & -3.81 & $E-W$ & $S$ & 15 & $\begin{array}{l}\text { major continuous horizontal } \\
\text { fracture }\end{array}$ \\
\hline & & 2.13 & 0.84 & -3.81 & $E-W$ & $N$ & 15 & $\begin{array}{l}\text { major open subhorizontal } \\
\text { fracture }\end{array}$ \\
\hline & & 2.13 & 0.51 & -3.81 & $E-W$ & $N$ & 15 & $\begin{array}{l}\text { major open subhorizontal } \\
\text { fracture }\end{array}$ \\
\hline & & 2.13 & 0.51 & -3.81 & & & & $\begin{array}{l}\text { wandering minor } \\
\text { subhorizontal fracture }\end{array}$ \\
\hline & & 2.13 & 0.28 & -3.81 & & & & $\begin{array}{l}\text { wandering minor } \\
\text { subhorizontal fracture }\end{array}$ \\
\hline \multicolumn{9}{|l|}{ NN4 } \\
\hline & & 0.91 & 2.92 & -0.91 & NW-SE & $\mathrm{NE}$ & & $\begin{array}{l}\text { moderate-major angled } \\
\text { fracture, open }\end{array}$ \\
\hline & & 0.91 & 2.84 & -0.91 & NE-SW & NW & & $\begin{array}{l}\text { moderate-major open } \\
\text { angled fracture }\end{array}$ \\
\hline & & 0.91 & 2.77 & -0.91 & NE-SW & NW & & $\begin{array}{l}\text { moderate-major open } \\
\text { angled fracture }\end{array}$ \\
\hline
\end{tabular}




\begin{tabular}{|c|c|c|c|c|c|c|c|c|}
\hline Hole & $\begin{array}{l}\text { Surface } \\
\text { feature }\end{array}$ & $\begin{array}{l}\text { Model } \\
\text { east }\end{array}$ & $\begin{array}{l}\text { Model } \\
\text { north }\end{array}$ & $\begin{array}{l}\text { Model } \\
\text { depth }\end{array}$ & $\begin{array}{l}\text { Strike } \\
\text { direct }\end{array}$ & $\begin{array}{l}\text { Dip } \\
\text { direct }\end{array}$ & $\begin{array}{l}\text { TRUE } \\
\text { dip }\end{array}$ & Comments \\
\hline & & 0.91 & 2.77 & -0.91 & $E-W$ & & 90 & $\begin{array}{l}\text { moderate open wet vertical } \\
\text { fracture }\end{array}$ \\
\hline & & 0.91 & 2.31 & -0.91 & NW-SE & SW & & minor angled closed fracture \\
\hline & & 0.91 & 1.57 & -0.91 & NE-SW & SE & & $\begin{array}{l}\text { minor tight subvertical } \\
\text { fracture }\end{array}$ \\
\hline & & 0.91 & 1.50 & -0.91 & $\sim N-S$ & W & & $\begin{array}{l}\text { major open angled fracture, } \\
\text { trending along side of hole } \\
\end{array}$ \\
\hline & & 0.91 & 1.09 & -0.91 & $\sim N-S$ & W & & $\begin{array}{l}\text { major open angled fracture, } \\
\text { trending along side of hole }\end{array}$ \\
\hline & LBT3 & 0.91 & 1.07 & -0.91 & & & 90 & $\begin{array}{l}\text { major open vertical wet } \\
\text { fracture }\end{array}$ \\
\hline & & 0.91 & 0.89 & -0.91 & $\sim \mathrm{N}-\mathrm{S}$ & $W$ & & $\begin{array}{l}\text { moderate-major fracture } \\
\text { barely grazing side of hole }\end{array}$ \\
\hline & & 0.91 & 0.79 & -0.91 & $\sim \mathrm{N}-\mathrm{S}$ & W & & $\begin{array}{l}\text { moderate-major fracture } \\
\text { barely grazing side of hole }\end{array}$ \\
\hline & & 0.91 & 0.71 & -0.91 & NW-SE & SSW & & major angled open fracture \\
\hline & & 0.91 & 0.53 & -0.91 & NW-SE & SSW & & major angled open fracture \\
\hline & LBT2 & 0.91 & 0.41 & -0.91 & NW-SE & $\mathrm{NE}$ & & $\begin{array}{l}\text { moderate-major open } \\
\text { angled fracture }\end{array}$ \\
\hline \multicolumn{9}{|l|}{ NN5 } \\
\hline & & 0.91 & 2.64 & -1.98 & N45E & SE & 80 & major open fracture, angled \\
\hline & & 0.91 & 2.54 & -1.98 & N45E & SE & 80 & major open fracture, angled \\
\hline & & 0.91 & 2.54 & -1.98 & & & 90 & $\begin{array}{l}\text { major vertical fracture } \\
\text { trending along strike of hole... }\end{array}$ \\
\hline & & 0.91 & 2.26 & -1.98 & & & 90 & $\begin{array}{l}\text { major vertical fracture } \\
\text { trending along strike of hole... }\end{array}$ \\
\hline & & 0.91 & 2.24 & -1.98 & NW-SE & $\mathrm{NE}$ & & $\begin{array}{l}\text { moderate tight subvertical } \\
\text { partly open fracture }\end{array}$ \\
\hline & & 0.91 & 2.03 & -1.98 & NW-SE & NE & & $\begin{array}{l}\text { moderate tight subvertical } \\
\text { partly open fracture }\end{array}$ \\
\hline & & 0.91 & 2.03 & -1.98 & $E-W$ & & 90 & $\begin{array}{l}\text { moderate vertical open } \\
\text { fracture }\end{array}$ \\
\hline & & 0.91 & 1.68 & -1.98 & $E-W$ & & & $\begin{array}{l}\text { minor tight subvertical } \\
\text { fracture }\end{array}$ \\
\hline & & 0.91 & 1.04 & -1.98 & $E-W$ & & 90 & $\begin{array}{l}\text { moderate-major open } \\
\text { vertical fracture }\end{array}$ \\
\hline & LBT2 & 0.91 & 0.94 & -1.98 & $\mathrm{~N} 45 \mathrm{~W}$ & NE & 90 & major open vertical fracture \\
\hline & LBT2 & 0.91 & 0.94 & -1.98 & N45W & NE & 90 & major open vertical fracture \\
\hline \multicolumn{9}{|l|}{ NN6 } \\
\hline & & 0.91 & 2.79 & -3.81 & N2OE & & 90 & $\begin{array}{l}\text { angled tight fracture along } \\
\text { the wall surface }\end{array}$ \\
\hline & & 0.91 & 2.67 & -3.81 & & & 90 & minor vertical closed fracture \\
\hline & & 0.91 & 2.54 & -3.81 & & & 90 & minor vertical closed fracture \\
\hline & & 0.91 & 2.26 & -3.81 & & & 90 & major open vertical fracture \\
\hline & & 0.91 & 2.07 & -3.81 & $N-S$ & $E$ & 45 & $\begin{array}{l}\text { open fracture parallels the } \\
\text { hole }\end{array}$ \\
\hline & & 0.91 & 1.96 & -3.81 & $\mathrm{~N}-\mathrm{S}$ & $E$ & 45 & $\begin{array}{l}\text { open fracture parallels the } \\
\text { hole }\end{array}$ \\
\hline
\end{tabular}




\begin{tabular}{|c|c|c|c|c|c|c|c|c|}
\hline Hole & $\begin{array}{l}\text { Surface } \\
\text { feature }\end{array}$ & $\begin{array}{l}\text { Model } \\
\text { east }\end{array}$ & $\begin{array}{l}\text { Model } \\
\text { north }\end{array}$ & $\begin{array}{l}\text { Model } \\
\text { depth }\end{array}$ & $\begin{array}{l}\text { Strike } \\
\text { direct }\end{array}$ & $\begin{array}{l}\text { Dip } \\
\text { direct }\end{array}$ & $\begin{array}{l}\text { TRUE } \\
\text { dip }\end{array}$ & Comments \\
\hline & LBT2 & 0.91 & 1.63 & -3.81 & & & 90 & $\begin{array}{l}\text { major vertical open fracture } \\
\text { (is grout exiting fracture) }\end{array}$ \\
\hline & & 0.91 & 1.57 & -3.81 & N45W & & & $\begin{array}{l}\text { major subvertical angled } \\
\text { open fracture }\end{array}$ \\
\hline & & 0.91 & 1.24 & -3.81 & $\mathrm{~N} 30 \mathrm{~W}$ & NW & 90 & angled major fracture \\
\hline & & 0.91 & 1.04 & -3.81 & $\mathrm{~N} 30 \mathrm{~W}$ & NW & 90 & angled major fracture \\
\hline & & 0.91 & 1.14 & -3.81 & $E-W$ & $S$ & 10 & $\begin{array}{l}\text { major subhorizontal open } \\
\text { fracture }\end{array}$ \\
\hline & & 0.91 & 0.81 & -3.81 & $E-W$ & $S$ & 10 & $\begin{array}{l}\text { major subhorizontal open } \\
\text { fracture }\end{array}$ \\
\hline & & 0.91 & 0.81 & -3.81 & $E-W$ & $S$ & 20 & $\begin{array}{l}\text { minor wandering } \\
\text { subhorizontal fracture }\end{array}$ \\
\hline & & 0.91 & 0.51 & -3.81 & $E-W$ & $S$ & 20 & $\begin{array}{l}\text { minor wandering } \\
\text { subhorizontal fracture }\end{array}$ \\
\hline \multicolumn{9}{|l|}{$\mathrm{NOI}$} \\
\hline & & 2.13 & 2.64 & -4.11 & $E-W$ & $S$ & 90 & $\begin{array}{l}\text { moderate-major open to } \\
\text { partly open vertical fracture }\end{array}$ \\
\hline & & 2.13 & 2.59 & -4.11 & $E-W$ & & 90 & $\begin{array}{l}\text { moderate-major open to } \\
\text { partly open vertical fracture }\end{array}$ \\
\hline & & 2.13 & 2.08 & -4.11 & N70E & & 90 & angled major open fracture \\
\hline & & 2.13 & 2.04 & -4.11 & N7OE & & 90 & angled major open fracture \\
\hline & & 2.13 & 1.74 & -4.11 & $E-W$ & & 90 & $\begin{array}{l}\text { series of significant open } \\
\text { fractures }\end{array}$ \\
\hline & & 2.13 & 1.60 & -4.11 & $E-W$ & & 90 & $\begin{array}{l}\text { series of significant open } \\
\text { fractures }\end{array}$ \\
\hline & & 2.13 & 1.45 & -4.11 & N2OW & & 90 & angled large fracture \\
\hline & & 2.13 & 1.22 & -4.11 & $\mathrm{~N} 2 \mathrm{OW}$ & & 90 & angled large fracture \\
\hline & LBT3 & 2.13 & 1.07 & -4.11 & & & 90 & $\begin{array}{l}\text { minor vertical fracture, looks } \\
\text { closed }\end{array}$ \\
\hline & & 2.13 & 0.89 & -4.11 & N2OW & & 90 & $\begin{array}{l}\text { angled moderate-minor tight } \\
\text { closed fracture }\end{array}$ \\
\hline & & 2.13 & 0.79 & -4.11 & N2OW & & 90 & $\begin{array}{l}\text { angled moderate-minor tight } \\
\text { closed fracture }\end{array}$ \\
\hline & & 2.13 & 0.61 & -4.11 & N3OE & NW & & $\begin{array}{l}\begin{array}{l}\text { angled minor tight closed } \\
\text { fracture }\end{array} \\
\end{array}$ \\
\hline & & 2.13 & 0.56 & -4.11 & $\mathrm{~N} 30 \mathrm{E}$ & NW & & $\begin{array}{l}\text { angled minor tight closed } \\
\text { fracture }\end{array}$ \\
\hline & & 2.13 & 0.53 & -4.11 & & & 90 & $\begin{array}{l}\text { minor vertical partly open } \\
\text { fracture }\end{array}$ \\
\hline & & 2.13 & 0.41 & -4.11 & $E-W$ & $s$ & & closed subvertical fracture \\
\hline & & 2.13 & 0.36 & -4.11 & $E-W$ & $s$ & & closed subvertical fracture \\
\hline \multicolumn{9}{|l|}{$\mathrm{NO} 2$} \\
\hline & & 0.91 & 2.84 & -4.11 & N3OE & S-SE & 30 & $\begin{array}{l}\text { complex series of } \\
\text { subhorizontal open fractures }\end{array}$ \\
\hline & & 0.91 & 2.50 & -4.11 & $\mathrm{~N} 30 \mathrm{E}$ & S-SE & 30 & $\begin{array}{l}\text { Complex series of } \\
\text { subhorizontal open fractures }\end{array}$ \\
\hline & & 0.91 & 2.24 & -4.11 & N7OE & & 90 & $\begin{array}{l}\text { significant open subvertical } \\
\text { fracture }\end{array}$ \\
\hline & & 0.91 & 1.73 & -4.11 & NoOW & SW & 70 & minor angled closed fracture \\
\hline
\end{tabular}




\begin{tabular}{|c|c|c|c|c|c|c|c|c|}
\hline Hole & $\begin{array}{l}\text { Surface } \\
\text { feature }\end{array}$ & $\begin{array}{l}\text { Model } \\
\text { east }\end{array}$ & $\begin{array}{l}\text { Model } \\
\text { north }\end{array}$ & $\begin{array}{l}\text { Model } \\
\text { depth }\end{array}$ & $\begin{array}{l}\text { Strike } \\
\text { direct }\end{array}$ & $\begin{array}{l}\text { Dip } \\
\text { direct }\end{array}$ & $\begin{array}{c}\text { TRUE } \\
\text { dip }\end{array}$ & Comments \\
\hline & & 0.91 & 1.68 & -4.11 & N6OW & SW & 70 & minor angled closed fracture \\
\hline & & 0.91 & 1.55 & -4.11 & & & 90 & major open vertical fracture \\
\hline & & 0.91 & 1.40 & -4.11 & N3OW & SW & 70 & minor angled closed fracture \\
\hline & & 0.91 & 1.30 & -4.11 & N3OW & SW & 70 & minor angled closed fracture \\
\hline & & 0.91 & 1.30 & -4.11 & & & 90 & minor vertical fracture \\
\hline & & 0.91 & 1.30 & -4.11 & N3OW & SW & 80 & $\begin{array}{l}\text { angled moderate-minor } \\
\text { partly open fracture }\end{array}$ \\
\hline & & 0.91 & 1.17 & -4.11 & N3OW & SW & 80 & $\begin{array}{l}\text { angled moderate-minor } \\
\text { partly open fracture }\end{array}$ \\
\hline & & 0.91 & 1.14 & -4.11 & N3OE & NW & & $\begin{array}{l}\text { angled minor-moderate } \\
\text { vertical fracture }\end{array}$ \\
\hline & & 0.91 & 0.97 & -4.11 & N3OE & NW & & $\begin{array}{l}\text { angled minor-moderate } \\
\text { vertical fracture }\end{array}$ \\
\hline & & 0.91 & 0.97 & -4.11 & & & 90 & $\begin{array}{l}\text { moderate vertical partly } \\
\text { open fracture }\end{array}$ \\
\hline & & 0.91 & 0.64 & -4.11 & NE-SW & & 90 & $\begin{array}{l}\text { moderate vertical partly } \\
\text { open fracture }\end{array}$ \\
\hline & & 0.91 & 0.66 & -4.11 & & & 90 & $\begin{array}{l}\text { major open angled vertical } \\
\text { fracture-wandering }\end{array}$ \\
\hline & & 0.91 & 0.43 & -4.11 & & & 90 & $\begin{array}{l}\text { major open angled vertical } \\
\text { fracture-wandering }\end{array}$ \\
\hline & & 0.91 & 0.43 & -4.11 & N3OE & SE & 80 & angled fracture \\
\hline & & 0.91 & 0.30 & -4.11 & $\mathrm{~N} 30 \mathrm{E}$ & SE & 80 & angled fracture \\
\hline \multicolumn{9}{|l|}{ NTI } \\
\hline & LBT22 & 2.74 & 2.97 & -2.44 & N3OE & NW & 70 & angled fracture \\
\hline & LBT22 & 2.74 & 2.84 & -2.44 & N3OE & NW & 70 & angled fracture \\
\hline & & 2.74 & 2.74 & -2.44 & & & 90 & $\begin{array}{l}\text { moderate/major vertical } \\
\text { open fracture }\end{array}$ \\
\hline & & 2.74 & 2.26 & -2.44 & N70E & NW & 80 & angled minor fracture \\
\hline & & 2.74 & 2.24 & -2.44 & N70E & NW & 80 & angled minor fracture \\
\hline & & 2.74 & 2.13 & -2.44 & N70E & NW & 80 & angled minor fracture \\
\hline & & 2.74 & 2.11 & -2.44 & $\mathrm{~N} 70 \mathrm{E}$ & NW & 80 & angled minor fracture \\
\hline & & 2.74 & 2.13 & -2.44 & N6OE & NW & 80 & angled minor fracture \\
\hline & & 2.74 & 2.06 & -2.44 & N6OE & NW & 80 & angled minor fracture \\
\hline & & 2.74 & 2.11 & -2.44 & N3OW & NW & 80 & $\begin{array}{l}\text { moderate-major open } \\
\text { fracture }\end{array}$ \\
\hline & & 2.74 & 2.01 & -2.44 & N3OW & NW & 80 & $\begin{array}{l}\text { moderate-major open } \\
\text { fracture }\end{array}$ \\
\hline & & 2.74 & 1.91 & -2.44 & NE-SW & NW & & minor tight angled fracture \\
\hline & & 2.74 & 1.88 & -2.44 & NE-SW & NW & & minor tight angled fracture \\
\hline & & 2.74 & 1.80 & -2.44 & & & 90 & minor vertical tight fracture \\
\hline & & 2.74 & 1.50 & -2.44 & $\mathrm{~N} 15 \mathrm{~W}$ & SW & 60 & major angled open fracture \\
\hline & & 2.74 & 1.24 & -2.44 & $\mathrm{~N} 15 \mathrm{~W}$ & SW & 60 & major angled open fracture \\
\hline & & 2.74 & 1.24 & -2.44 & & & 90 & $\begin{array}{l}\text { minor vertical partly open } \\
\text { fracture }\end{array}$ \\
\hline & & 2.74 & 1.09 & -2.44 & N45E & SE & 70 & $\begin{array}{l}\text { moderate partly open } \\
\text { angled fracture }\end{array}$ \\
\hline
\end{tabular}




\begin{tabular}{|c|c|c|c|c|c|c|c|c|}
\hline Hole & $\begin{array}{l}\text { Surface } \\
\text { feature }\end{array}$ & $\begin{array}{l}\text { Model } \\
\text { east }\end{array}$ & $\begin{array}{l}\text { Model } \\
\text { north }\end{array}$ & $\begin{array}{l}\text { Model } \\
\text { depth }\end{array}$ & $\begin{array}{l}\text { Strike } \\
\text { direct }\end{array}$ & $\begin{array}{l}\text { Dip } \\
\text { direct }\end{array}$ & $\begin{array}{l}\text { TRUE } \\
\text { dip }\end{array}$ & Comments \\
\hline & & 2.74 & 1.03 & -2.44 & N45E & SE & 70 & $\begin{array}{l}\text { moderate partly open } \\
\text { angled fracture }\end{array}$ \\
\hline & LBT3 & 2.74 & 0.94 & -2.44 & & & 90 & $\begin{array}{l}\text { moderate open vertical wet } \\
\text { fracture }\end{array}$ \\
\hline \multicolumn{9}{|l|}{ NT2 } \\
\hline & & 0.91 & 2.41 & -0.61 & NW-SE & & & $\begin{array}{l}\text { minor tight subvertical } \\
\text { fracture }\end{array}$ \\
\hline & & 0.91 & 2.31 & -0.61 & & & 90 & minor tight vertical fracture \\
\hline & & 0.91 & 1.55 & -0.61 & NW-SW & SSW & & moderate open fracture \\
\hline & & 0.91 & 1.32 & -0.61 & NW-SW & SSW & & moderate open fracture \\
\hline & & 0.91 & 1.09 & -0.61 & $E-W$ & & 90 & $\begin{array}{l}\text { minor vertical generally } \\
\text { closed fracture }\end{array}$ \\
\hline & & 0.91 & 1.07 & -0.61 & $E-W$ & & 90 & $\begin{array}{l}\text { minor vertical generally } \\
\text { closed fracture }\end{array}$ \\
\hline & & 0.91 & 0.69 & -0.61 & & & 90 & minor vertical closed fracture \\
\hline \multicolumn{9}{|l|}{ NT3 } \\
\hline & & 0.91 & 2.72 & -3.20 & & & 90 & tight, minor vertical fracture \\
\hline & & 0.91 & 2.79 & -3.20 & N3OW & NE & 80 & $\begin{array}{l}\text { minor, subvertical fracture, } \\
\text { angled }\end{array}$ \\
\hline & & 0.91 & 2.72 & -3.20 & $E-W$ & W & & $\begin{array}{l}\text { minor, subvertical fracture, } \\
\text { angled }\end{array}$ \\
\hline & & 0.91 & 2.41 & -3.20 & $E-W$ & & 90 & major, open vertical fracture \\
\hline & & 0.91 & 1.85 & -3.20 & N45E & NW & & $\begin{array}{l}\text { minor-moderate subvertical } \\
\text { fracture, partly open }\end{array}$ \\
\hline & & 0.91 & 1.73 & -3.20 & N45E & NW & & $\begin{array}{l}\text { minor-moderate subvertical } \\
\text { fracture, partly open }\end{array}$ \\
\hline & & 0.91 & 1.73 & -3.20 & $E-W$ & & 90 & major open vertical fracture \\
\hline & & 0.91 & 1.52 & -3.20 & $E-W$ & $\mathrm{SE} / \mathrm{S}$ & & $\begin{array}{l}\text { moderate-major open } \\
\text { subvertical fracture }\end{array}$ \\
\hline & & 0.91 & 1.35 & -3.20 & NW-SE & SW & 90 & $\begin{array}{l}\text { moderate-major open } \\
\text { vertical fracture }\end{array}$ \\
\hline & & 0.91 & 1.17 & -3.20 & & & & $\begin{array}{l}\text { minor subvertical closed } \\
\text { fracture }\end{array}$ \\
\hline & & 0.91 & 0.97 & -3.20 & $E-W$ & & 90 & $\begin{array}{l}\text { moderate vertical fracture, } \\
\text { partly open }\end{array}$ \\
\hline \multicolumn{9}{|l|}{ NT4 } \\
\hline & LBT24 & 0.30 & 2.46 & -2.44 & NE-SW & NW & 70 & $\begin{array}{l}\text { major-moderate angled } \\
\text { sharp open fracture }\end{array}$ \\
\hline & LBT24 & 0.30 & 2.41 & -2.44 & NE-SW & NW & 70 & $\begin{array}{l}\text { major-moderate angled } \\
\text { sharp open fracture }\end{array}$ \\
\hline & & 0.30 & 2.44 & -2.44 & NE-SW & NW & 70 & $\begin{array}{l}\text { major-moderate angled } \\
\text { sharp open fracture }\end{array}$ \\
\hline & & 0.30 & 2.39 & -2.44 & NE-SW & NW & 70 & $\begin{array}{l}\text { major-moderate angled } \\
\text { sharp open fracture }\end{array}$ \\
\hline & & 0.30 & 2.39 & -2.44 & NE-SW & NW & 70 & $\begin{array}{l}\text { major-moderate angled } \\
\text { sharp open fracture }\end{array}$ \\
\hline & & 0.30 & 2.34 & -2.44 & NE-SW & NW & 70 & $\begin{array}{l}\text { major-moderate angled } \\
\text { sharp open fracture }\end{array}$ \\
\hline & & 0.30 & 2.03 & -2.44 & NE-SW & NW & 80 & moderate angled partly \\
\hline
\end{tabular}




\begin{tabular}{|c|c|c|c|c|c|c|c|c|}
\hline Hole & $\begin{array}{l}\text { Surface } \\
\text { feature }\end{array}$ & $\begin{array}{c}\text { Model } \\
\text { east }\end{array}$ & $\begin{array}{l}\text { Model } \\
\text { north }\end{array}$ & $\begin{array}{l}\text { Model } \\
\text { depth }\end{array}$ & $\begin{array}{l}\text { Strike } \\
\text { direct }\end{array}$ & $\begin{array}{l}\text { Dip } \\
\text { direct }\end{array}$ & $\begin{array}{c}\text { TRUE } \\
\text { dip }\end{array}$ & Comments \\
\hline & & & & & & & & open fracture \\
\hline & & 0.30 & 2.01 & -2.44 & NE-SW & NW & 80 & $\begin{array}{l}\text { moderate angled partly } \\
\text { open fracture }\end{array}$ \\
\hline & & 0.30 & 1.98 & -2.44 & N8OE & & & $\begin{array}{l}\text { major open vertical- } \\
\text { subvertical fracture }\end{array}$ \\
\hline & & 0.30 & 1.85 & -2.44 & N2OE & NW & 45 & $\begin{array}{l}\text { moderate-major discrete } \\
\text { sharp partly open/closed }\end{array}$ \\
\hline & & 0.30 & 1.68 & -2.44 & N2OE & NW & 45 & $\begin{array}{l}\text { moderate-major discrete } \\
\text { sharp partly open/closed }\end{array}$ \\
\hline & LBT23 & 0.30 & 1.35 & -2.44 & NE-SW & NW & 45 & major partly open fracture \\
\hline & LBT23 & 0.30 & 0.89 & -2.44 & NE-SW & NW & 45 & major partly open fracture \\
\hline & LBT3 & 0.30 & 1.02 & -2.44 & & & 90 & $\begin{array}{l}\text { moderate open vertical } \\
\text { fracture }\end{array}$ \\
\hline & & 0.30 & 0.86 & -2.44 & NW-SE & SS & 55 & $\begin{array}{l}\text { minor tight/healed angled } \\
\text { fracture }\end{array}$ \\
\hline & & 0.30 & 0.71 & -2.44 & NW-SE & $S$ & 55 & $\begin{array}{l}\text { minor tight/healed angled } \\
\text { fracture }\end{array}$ \\
\hline \multicolumn{9}{|r|}{ t } \\
\hline & & 0.66 & 1.68 & -1.22 & & & 90 & major open vertical fracture \\
\hline & & 1.09 & 1.68 & -1.22 & SW-NE & & & $\begin{array}{l}\text { angled minor/moderate } \\
\text { subhorizontal party open } \\
\text { fracture }\end{array}$ \\
\hline & & 1.78 & 1.68 & -1.22 & & & 90 & $\begin{array}{l}\text { minor vertical partly open } \\
\text { fracture }\end{array}$ \\
\hline & & 2.08 & 1.68 & -1.22 & $\sim \mathrm{SW}-\mathrm{NE}$ & $\bar{E}$ & & $\begin{array}{l}\text { moderate subhorizontal } \\
\text { closed fracture }\end{array}$ \\
\hline & & 2.18 & 1.68 & -1.22 & $\sim \mathrm{SW}-\mathrm{NE}$ & $E$ & & $\begin{array}{l}\text { moderate subhorizontal } \\
\text { closed fracture }\end{array}$ \\
\hline \multicolumn{9}{|l|}{ WH2 } \\
\hline & & 0.48 & 1.68 & -2.29 & & & 90 & minor tight vertical fracture \\
\hline & & 0.69 & 1.68 & -2.29 & NE-SW & & 90 & minor tight vertical fracture \\
\hline & & 1.27 & 1.68 & -2.29 & & & 90 & minor tight vertical fracture \\
\hline & & 1.47 & 1.68 & -2.29 & $\mathrm{~N}-\mathrm{S}$ & & 90 & $\begin{array}{l}\text { major open wet vertical } \\
\text { fracture }\end{array}$ \\
\hline & & 1.83 & 1.68 & -2.29 & & & 90 & minor tight vertical fracture \\
\hline & & 1.98 & 1.68 & -2.29 & & & 90 & minor tight vertical fracture \\
\hline & & 2.41 & 1.68 & -2.29 & NW-SE & SW & & moderate tight fracture \\
\hline & & 2.77 & 1.68 & -2.29 & NW-SE & SW & & moderate tight fracture \\
\hline & & 2.49 & 1.68 & -2.29 & & & 90 & minor tight vertical fracture \\
\hline \multicolumn{9}{|l|}{ WMI } \\
\hline & & 1.78 & 2.13 & -3.96 & & $E-N E$ & & $\begin{array}{l}\text { major subhorizontal fracture, } \\
\text { partly open }\end{array}$ \\
\hline & & 2.13 & 2.13 & -3.96 & & E-NE & & $\begin{array}{l}\text { major subhorizontal fracture, } \\
\text { partly open }\end{array}$ \\
\hline & & 2.67 & 2.13 & -3.96 & & & 90 & $\begin{array}{l}\text { moderate-major vertical } \\
\text { open fracture }\end{array}$ \\
\hline & & 2.44 & 2.13 & -3.96 & & & 90 & minor vertical closed fracture \\
\hline & & 2.34 & 2.13 & -3.96 & & & 90 & major vertical open fracture \\
\hline
\end{tabular}




\begin{tabular}{|c|c|c|c|c|c|c|c|c|}
\hline Hole & $\begin{array}{l}\text { Surface } \\
\text { feature }\end{array}$ & $\begin{array}{l}\text { Model } \\
\text { east }\end{array}$ & $\begin{array}{l}\text { Model } \\
\text { north }\end{array}$ & $\begin{array}{l}\text { Model } \\
\text { depth }\end{array}$ & $\begin{array}{l}\text { Strike } \\
\text { direct }\end{array}$ & $\begin{array}{l}\text { Dip } \\
\text { direct }\end{array}$ & $\begin{array}{c}\text { TRUE } \\
\text { dip }\end{array}$ & Comments \\
\hline & & 1.55 & 2.13 & -3.96 & & & 90 & major open vertical fracture \\
\hline & & 1.50 & 2.13 & -3.96 & & & 90 & major open vertical fracture \\
\hline & & 1.55 & 2.13 & -3.96 & & & & $\begin{array}{l}\text { series of generally minor } \\
\text { vertical to subvertical tight } \\
\text { fractures }\end{array}$ \\
\hline & & 1.09 & 2.13 & -3.96 & & & & $\begin{array}{l}\text { series of generally minor } \\
\text { vertical to subvertical tight } \\
\text { fractures }\end{array}$ \\
\hline & & 0.81 & 2.13 & -3.96 & NE-SW & SW & & $\begin{array}{l}\text { moderate partly open } \\
\text { subvertical fracture }\end{array}$ \\
\hline & LBT2 & 0.53 & 2.13 & -3.96 & $\mathrm{~N}-\mathrm{S}$ & & 90 & minor vertical fracture \\
\hline & & 0.15 & 2.13 & -3.96 & & & 90 & $\begin{array}{l}\text { moderate-major vertical } \\
\text { open fracture }\end{array}$ \\
\hline \multicolumn{9}{|l|}{ WM1? } \\
\hline & & 0.43 & 2.13 & -3.96 & & & & $\begin{array}{l}\text { minor tight } \\
\text { subvertical/vertical fracture }\end{array}$ \\
\hline & & 0.66 & 2.13 & -3.96 & $\mathrm{~N}-\mathrm{S}$ & & 90 & minor tight vertical fracture \\
\hline & & 1.09 & 2.13 & -3.96 & & & 90 & minor vertical tight fracture \\
\hline & & 1.35 & 2.13 & -3.96 & & & 90 & minor vertical tight fracture \\
\hline & & 1.77 & 2.13 & -3.96 & & & 90 & minor vertical tight fracture \\
\hline & & 2.06 & 2.13 & -3.96 & & & 90 & minor vertical tight fracture \\
\hline & & 2.34 & 2.13 & -3.96 & $\mathrm{~N} 45 \mathrm{~W}$ & SW & 70 & angled major open fracture \\
\hline & & 2.41 & 2.13 & -3.96 & N45W & SW & 70 & angled major open fracture \\
\hline & & 2.46 & 2.13 & -3.96 & & & & $\begin{array}{l}\text { major open } \\
\text { vertical/subvertical } \\
\text { fracture-rubbly }\end{array}$ \\
\hline & & 2.46 & 2.13 & -3.96 & & & & $\begin{array}{l}\text { major series of horizontal to } \\
\text { subhorizontal open fractures }\end{array}$ \\
\hline & & 2.77 & 2.13 & -3.96 & & & & $\begin{array}{l}\text { major series of horizontal to } \\
\text { subhorizontal open fractures }\end{array}$ \\
\hline \multicolumn{9}{|l|}{ WM2 } \\
\hline & LBT2 & 0.66 & 0.91 & -1.22 & & & & $\begin{array}{l}\text { moderate subhorizontal } \\
\text { fracture-hard to see }\end{array}$ \\
\hline & & 0.97 & 0.91 & -1.22 & N2OW & NE & 20 & $\begin{array}{l}\text { major subhorizontal closed to } \\
\text { open fracture }\end{array}$ \\
\hline & & 1.52 & 0.91 & -1.22 & N2OW & NE & 20 & $\begin{array}{l}\text { major subhorizontal closed to } \\
\text { open fracture }\end{array}$ \\
\hline & & 1.80 & 0.91 & -1.22 & & & & $\begin{array}{l}\text { minor mostly closed vertical } \\
\text { fracture }\end{array}$ \\
\hline & & 2.08 & 0.91 & -1.22 & & & 90 & minor vertical closed fracture \\
\hline & & 2.41 & 0.91 & -1.22 & & & 90 & minor vertical closed fracture \\
\hline WM3 & \multicolumn{3}{|c|}{$\begin{array}{l}\text { Cannot see the depths on } \\
\text { tape }\end{array}$} & & & & & \\
\hline \multicolumn{9}{|l|}{ WN2 } \\
\hline & & 0.10 & 2.13 & -1.68 & N3OE & SE & 70 & $\begin{array}{l}\text { angled minor closed/healed } \\
\text { subvertical fracture }\end{array}$ \\
\hline & & 0.13 & 2.13 & -1.68 & N3OE & SE & 70 & $\begin{array}{l}\text { angled minor closed/healed } \\
\text { subvertical fracture }\end{array}$ \\
\hline
\end{tabular}




\begin{tabular}{|c|c|c|c|c|c|c|c|c|}
\hline Hole & $\begin{array}{l}\text { Surface } \\
\text { feature }\end{array}$ & $\begin{array}{c}\text { Model } \\
\text { east }\end{array}$ & $\begin{array}{l}\text { Model } \\
\text { north }\end{array}$ & $\begin{array}{l}\text { Model } \\
\text { depth }\end{array}$ & $\begin{array}{l}\text { Strike } \\
\text { direct }\end{array}$ & $\begin{array}{l}\text { Dip } \\
\text { direct }\end{array}$ & $\begin{array}{c}\text { TRUE } \\
\text { dip }\end{array}$ & Comments \\
\hline & & 0.20 & 2.13 & -1.68 & & & 90 & minor vertical fracture \\
\hline & & 0.51 & 2.13 & -1.68 & $\sim \mathrm{N}-\mathrm{S}$ & $W$ & 80 & $\begin{array}{l}\text { major open fracture, with } \\
\text { some associated fractures }\end{array}$ \\
\hline & & 0.53 & 2.13 & -1.68 & $\sim N-S$ & $W$ & 80 & $\begin{array}{l}\text { major open fracture, with } \\
\text { some associated fractures }\end{array}$ \\
\hline & & 0.84 & 2.13 & -1.68 & N45W & SW & 40 & $\begin{array}{l}\text { major angled open to partly } \\
\text { open fracture }\end{array}$ \\
\hline & & 0.66 & 2.13 & -1.68 & $N 45 W$ & SW & 40 & $\begin{array}{l}\text { major angled open to partly } \\
\text { open fracture }\end{array}$ \\
\hline & & 1.04 & 2.13 & -1.68 & & & 90 & minor vertical closed fracture \\
\hline & & 1.24 & 2.13 & -1.68 & & & 90 & minor vertical closed fracture \\
\hline & & 1.27 & 2.13 & -1.68 & $N-S$ & $W$ & 20 & $\begin{array}{l}\text { major/moderate angled long } \\
\text { fracture, healed }\end{array}$ \\
\hline & & 1.55 & 2.13 & -1.68 & $\mathrm{~N}-\mathrm{S}$ & $w$ & 20 & $\begin{array}{l}\text { major/moderate angled long } \\
\text { fracture, healed }\end{array}$ \\
\hline & & 1.47 & 2.13 & -1.68 & & & 90 & major open vertical fracture \\
\hline & & 1.65 & 2.13 & -1.68 & & & 90 & $\begin{array}{l}\text { moderate vertical partly } \\
\text { open fracture (displaced by } \\
\text { a fault) }\end{array}$ \\
\hline & & 1.80 & 2.13 & -1.68 & N8OW & SW & 70 & $\begin{array}{l}\text { major long angled } \\
\text { fracture/fault }\end{array}$ \\
\hline & & 1.55 & 2.13 & -1.68 & N8OW & SW & 70 & $\begin{array}{l}\begin{array}{l}\text { major long angled } \\
\text { fracture/fault }\end{array} \\
\end{array}$ \\
\hline & & 1.75 & 2.13 & -1.68 & & & 90 & $\begin{array}{l}\text { minor vertical fracture } \\
\text { (displaced by fault) }\end{array}$ \\
\hline & & 2.03 & 2.13 & -1.68 & & & 90 & minor vertical closed fracture \\
\hline & & 2.29 & 2.13 & -1.68 & & & 90 & $\begin{array}{l}\text { moderate vertical sharp } \\
\text { open fracture }\end{array}$ \\
\hline & & 2.62 & 2.13 & -1.68 & & & & $\begin{array}{l}\text { minor subvertical closed } \\
\text { fracture }\end{array}$ \\
\hline \multicolumn{9}{|l|}{ WN3 } \\
\hline & LBT2 & 0.88 & 1.68 & -3.96 & & & 90 & $\begin{array}{l}\text { major vertical open wet } \\
\text { fracture }\end{array}$ \\
\hline & & 1.00 & 1.68 & -3.96 & & & 90 & $\begin{array}{l}\text { Sharp open wet vertical } \\
\text { moderate fracture }\end{array}$ \\
\hline & & 1.21 & 1.68 & -3.96 & & & 90 & $\begin{array}{l}\text { major open wet vertical } \\
\text { fracture }\end{array}$ \\
\hline & & 1.35 & 1.68 & -3.96 & & & 90 & $\begin{array}{l}\text { major open wet vertical } \\
\text { fracture }\end{array}$ \\
\hline & & 2.77 & 1.68 & -3.96 & & & 90 & $\begin{array}{l}\text { vertical fracture-gaping } \\
\text { hole }\end{array}$ \\
\hline & & 1.30 & 1.68 & -3.96 & N7OE & SE & 80 & top sharp open fracture \\
\hline & & 1.50 & 1.68 & -3.96 & N7OE & SE & 80 & base sharp open fracture \\
\hline & & 1.50 & 1.68 & -3.96 & N6OW & $W$ & 45 & $\begin{array}{l}\text { top moderate partially open } \\
\text { fracture (shallow) }\end{array}$ \\
\hline & & 1.63 & 1.68 & -3.96 & N6OW & $w$ & 45 & $\begin{array}{l}\text { base moderate partially } \\
\text { open fracture (shallow) }\end{array}$ \\
\hline & & 1.55 & 1.68 & -3.96 & & $w$ & & $\begin{array}{l}\text { subvertical partially open } \\
\text { fracture }\end{array}$ \\
\hline
\end{tabular}




\begin{tabular}{|c|c|c|c|c|c|c|c|c|}
\hline Hole & $\begin{array}{l}\text { Surface } \\
\text { feature }\end{array}$ & $\begin{array}{c}\text { Model } \\
\text { east }\end{array}$ & $\begin{array}{l}\text { Model } \\
\text { north }\end{array}$ & $\begin{array}{l}\text { Model } \\
\text { depth }\end{array}$ & $\begin{array}{l}\text { Strike } \\
\text { direct }\end{array}$ & $\begin{array}{l}\text { Dip } \\
\text { direct }\end{array}$ & $\begin{array}{c}\text { TRUE } \\
\text { dip }\end{array}$ & Comments \\
\hline & & 1.88 & 1.68 & -3.96 & & & & $\begin{array}{l}\text { subvertical moderate wet } \\
\text { open fracture }\end{array}$ \\
\hline & & 1.96 & 1.68 & -3.96 & N7OW & SW & 80 & $\begin{array}{l}\text { top major partly open } \\
\text { angled fracture }\end{array}$ \\
\hline & & 2.34 & 1.68 & -3.96 & N7OW & SW & 80 & $\begin{array}{l}\text { base major partly open } \\
\text { angled fracture }\end{array}$ \\
\hline & & 2.11 & 1.68 & -3.96 & & & & $\begin{array}{l}\text { major subvertical open wet } \\
\text { fracture }\end{array}$ \\
\hline & & 2.34 & 1.68 & -3.96 & & & & $\begin{array}{l}\text { major subvertical open wet } \\
\text { fracture }\end{array}$ \\
\hline & & 2.55 & 1.68 & -3.96 & $\mathrm{~N} 45 \mathrm{~W}$ & SW & 70 & top tight closed fracture \\
\hline & & 2.59 & 1.68 & -3.96 & N45W & SW & 70 & base tight closed fracture \\
\hline & & 2.67 & 1.68 & -3.96 & & & & $\begin{array}{l}\text { top subvertical wet open } \\
\text { fracture }\end{array}$ \\
\hline & & 2.69 & 1.68 & -3.96 & & & & $\begin{array}{l}\text { base subvertical wet open } \\
\text { fracture }\end{array}$ \\
\hline \multicolumn{9}{|r|}{$\begin{array}{lll} & \\
\end{array}$} \\
\hline & & 0.56 & 0.91 & -1.68 & & & 90 & $\begin{array}{l}\text { minor sharp vertical fracture, } \\
\text { thin maybe partly open }\end{array}$ \\
\hline & & 0.76 & 0.91 & -1.68 & NW-SE & NE & & major partly open fracture \\
\hline & LBT2 & 0.80 & 0.91 & -1.68 & NW-SE & $\mathrm{NE}$ & & major partly open fracture \\
\hline & & 1.50 & 0.91 & -1.68 & $\mathrm{~N}-\mathrm{S}$ & $\mathrm{S}$ & 45 & $\begin{array}{l}\text { major partly open angled } \\
\text { fracture }\end{array}$ \\
\hline & & 1.57 & 0.91 & -1.68 & $\mathrm{~N}-\mathrm{S}$ & $\mathrm{s}$ & 45 & $\begin{array}{l}\text { major partly open angled } \\
\text { fracture }\end{array}$ \\
\hline & & 1.70 & 0.91 & -1.68 & & & 90 & $\begin{array}{l}\text { moderate vertical open } \\
\text { fracture }\end{array}$ \\
\hline & & 1.75 & 0.91 & -1.68 & $\mathrm{~N}-\mathrm{S}$ & & 90 & minor vertical fracture \\
\hline & & 1.78 & 0.91 & -1.68 & $\mathrm{~N}-\mathrm{S}$ & & 90 & minor vertical fracture \\
\hline & & 2.01 & 0.91 & -1.68 & & & 90 & $\begin{array}{l}\text { vertical mostly closed } \\
\text { fracture }\end{array}$ \\
\hline & & 2.03 & 0.91 & -1.68 & & & 90 & minor tight vertical fracture \\
\hline & & 2.31 & 0.91 & -1.68 & & & 90 & $\begin{array}{l}\text { moderate sharp, vertical } \\
\text { open fracture, wet }\end{array}$ \\
\hline \multicolumn{9}{|l|}{ W05 } \\
\hline & & 0.09 & 0.91 & -3.96 & & & 90 & $\begin{array}{l}\text { vertical moderate wet open } \\
\text { fracture }\end{array}$ \\
\hline & & 0.10 & 0.91 & -3.96 & $\mathrm{~N}-\mathrm{S}$ & $W$ & & top shallow tight fracture \\
\hline & & 0.14 & 0.91 & -3.96 & N05E & NW & 20 & $\begin{array}{l}\text { base major open angled wet } \\
\text { fracture }\end{array}$ \\
\hline & & 0.25 & 0.91 & -3.96 & NO5E & NW & 20 & $\begin{array}{l}\text { top major open angled wet } \\
\text { fracture }\end{array}$ \\
\hline & & 0.42 & 0.91 & -3.96 & NW-SE & & & $\begin{array}{l}\text { generally minor subvertical } \\
\text { tight fracture }\end{array}$ \\
\hline & & 0.55 & 0.91 & -3.96 & NE-SW & NW & & $\begin{array}{l}\text { base angled tight fracture } \\
\text { (top not seen) }\end{array}$ \\
\hline & & 0.58 & 0.91 & -3.96 & N3OW & $\mathrm{NE}$ & 80 & moderate wet open fracture \\
\hline & & 0.61 & 0.91 & -3.96 & N3OW & $\mathrm{NE}$ & 80 & moderate wet open fracture \\
\hline
\end{tabular}




\begin{tabular}{|c|c|c|c|c|c|c|c|c|}
\hline Hole & $\begin{array}{l}\text { Surface } \\
\text { feature }\end{array}$ & $\begin{array}{l}\text { Model } \\
\text { east }\end{array}$ & $\begin{array}{l}\text { Model } \\
\text { north }\end{array}$ & $\begin{array}{l}\text { Model } \\
\text { depth }\end{array}$ & $\begin{array}{l}\text { Strike } \\
\text { direct }\end{array}$ & $\begin{array}{l}\text { Dip } \\
\text { direct }\end{array}$ & $\begin{array}{c}\text { TRUE } \\
\text { dip }\end{array}$ & Comments \\
\hline & & 0.61 & 0.91 & -3.96 & $\mathrm{~N}-\mathrm{S}$ & & & $\begin{array}{l}\text { subvertical tight minor } \\
\text { fracture }\end{array}$ \\
\hline & & 0.64 & 0.91 & -3.96 & $\sim E-W$ & & 90 & $\begin{array}{l}\text { base major open angled } \\
\text { fracture wet alteration halo }\end{array}$ \\
\hline & & 1.17 & 0.91 & -3.96 & $\sim E-W$ & & & $\begin{array}{l}\text { top major wandering } \\
\text { subvertical fracture }\end{array}$ \\
\hline & & 0.72 & 0.91 & -3.96 & & & & $\begin{array}{l}\text { partly open wet minor } \\
\text { subvertical fracture on north } \\
\text { side of hole }\end{array}$ \\
\hline & & 0.79 & 0.91 & -3.96 & & & & $\begin{array}{l}\text { major open wet subvertical } \\
\text { fracture }\end{array}$ \\
\hline & & 0.93 & 0.91 & -3.96 & $N-S$ & & & $\begin{array}{l}\text { major open wet subvertical } \\
\text { fracture }\end{array}$ \\
\hline & & 0.99 & 0.91 & -3.96 & & & & $\begin{array}{l}\text { minor wet partly open } \\
\text { fracture subvertical on north } \\
\text { side of hole }\end{array}$ \\
\hline & & 1.08 & 0.91 & -3.96 & $\mathrm{~N}-\mathrm{S}$ & & & $\begin{array}{l}\text { moderate partly open wet } \\
\text { subvertical fracture }\end{array}$ \\
\hline & & 1.46 & 0.91 & -3.96 & & & 90 & $\begin{array}{l}\text { fracture zone wet open } \\
\text { some vertical and angled }\end{array}$ \\
\hline & & 1.32 & 0.91 & -3.96 & & & 90 & $\begin{array}{l}\text { fracture zone wet open } \\
\text { some vertical and angled }\end{array}$ \\
\hline & & 1.60 & 0.91 & -3.96 & & & & $\begin{array}{l}\text { subvertical major open } \\
\text { fracture along the north wall }\end{array}$ \\
\hline & & 1.52 & 0.91 & -3.96 & & & & $\begin{array}{l}\text { subvertical major open } \\
\text { fracture along the north wall }\end{array}$ \\
\hline & & 1.63 & 0.91 & -3.96 & & & & $\begin{array}{l}\text { small patch of same } \\
\text { subvertical major open } \\
\text { fracture along the north wall }\end{array}$ \\
\hline & & 1.73 & 0.91 & -3.96 & $\mathrm{~N}-\mathrm{S}$ & & & $\begin{array}{l}\text { tight subvertical fracture } \\
\text { mainly on the north side of } \\
\text { hole }\end{array}$ \\
\hline & & 1.84 & 0.91 & -3.96 & N45W & SW & 45 & tight minor angled fracture \\
\hline & & 1.91 & 0.91 & -3.96 & $\mathrm{~N} 45 \mathrm{~W}$ & SW & 45 & tight minor angled fracture \\
\hline & & 1.88 & 0.91 & -3.96 & $\mathrm{~N}-\mathrm{S}$ & & & $\begin{array}{l}\text { subvertical partly open wet } \\
\text { fracture }\end{array}$ \\
\hline & & 2.25 & 0.91 & -3.96 & $\mathrm{~N}-\mathrm{S}$ & & & $\begin{array}{l}\text { major open wet subvertical } \\
\text { fracture }\end{array}$ \\
\hline & & 2.32 & 0.91 & -3.96 & $\mathrm{~N}-\mathrm{S}$ & & & $\begin{array}{l}\text { major open wet subvertical } \\
\text { fracture }\end{array}$ \\
\hline & & 2.53 & 0.91 & -3.96 & $\mathrm{~N}-\mathrm{S}$ & & & subvertical tight fracture \\
\hline \multicolumn{9}{|l|}{ WTI } \\
\hline & & 0.79 & 1.68 & -0.76 & N2OE & SE & 80 & $\begin{array}{l}\text { major open subvertical } \\
\text { fracture }\end{array}$ \\
\hline & & 1.14 & 1.68 & -0.76 & & & 90 & minor tight vertical fracture \\
\hline & & 1.85 & 1.68 & -0.76 & & & 90 & $\begin{array}{l}\text { moderate open wet vertical } \\
\text { fracture }\end{array}$ \\
\hline & & 1.88 & 1.68 & -0.76 & & & & $\begin{array}{l}\text { minor tight subvertical } \\
\text { fracture }\end{array}$ \\
\hline & & 2.08 & 1.68 & -0.76 & & & & moderate sharp subvertical \\
\hline
\end{tabular}




\begin{tabular}{|c|c|c|c|c|c|c|c|c|}
\hline Hole & $\begin{array}{l}\text { Surface } \\
\text { feature }\end{array}$ & $\begin{array}{c}\text { Model } \\
\text { east }\end{array}$ & $\begin{array}{l}\text { Model } \\
\text { north }\end{array}$ & $\begin{array}{l}\text { Model } \\
\text { depth }\end{array}$ & $\begin{array}{l}\text { Strike } \\
\text { direct }\end{array}$ & $\begin{array}{l}\text { Dip } \\
\text { direct }\end{array}$ & $\begin{array}{c}\text { TRUE } \\
\text { dip }\end{array}$ & Comments \\
\hline & & & & & & & & open wet fracture \\
\hline \multicolumn{9}{|l|}{ WT2 } \\
\hline & LBT2 & 0.23 & 1.68 & -1.68 & $\sim N-S$ & $E$ & 80 & $\begin{array}{l}\text { subvertical angled } \\
\text { moderate, partly open } \\
\text { fracture }\end{array}$ \\
\hline & LBT2 & 0.25 & 1.68 & -1.68 & $\sim N-S$ & $\bar{E}$ & 80 & $\begin{array}{l}\text { lubvertical angled } \\
\text { moderate, partly open } \\
\text { fracture }\end{array}$ \\
\hline & & 0.51 & 1.68 & -1.68 & & & 90 & $\begin{array}{l}\text { vertical, moderate-major } \\
\text { fracture }\end{array}$ \\
\hline & & 1.04 & 1.68 & -1.68 & N6OE & SE & 80 & $\begin{array}{l}\text { sharp angled minor- } \\
\text { moderate, open fracture }\end{array}$ \\
\hline & & 1.09 & 1.68 & -1.68 & N60E & SE & 80 & $\begin{array}{l}\text { sharp angled minor- } \\
\text { moderate, open fracture }\end{array}$ \\
\hline & & 1.57 & 1.68 & -1.68 & $\mathrm{~N}-\mathrm{S}$ & $W$ & & $\begin{array}{l}\text { moderate subvertical open } \\
\text { fracture }\end{array}$ \\
\hline & & 1.60 & 1.68 & -1.68 & $\mathrm{~N}-\mathrm{S}$ & $w$ & 90 & $\begin{array}{l}\text { moderate vertical open } \\
\text { fracture, but less open }\end{array}$ \\
\hline & & 1.70 & 1.68 & -1.68 & N2OW & $E$ & 80 & minor subvertical fracture \\
\hline & & 1.85 & 1.68 & -1.68 & & & 90 & minor tight vertical fracture \\
\hline \multicolumn{9}{|l|}{ WT3 } \\
\hline & & 0.11 & 1.68 & -3.05 & & & 90 & top zone vertical fractures \\
\hline & & 0.20 & 1.68 & -3.05 & & & 90 & base zone vertical fractures \\
\hline & LBT 2 & 0.66 & 1.68 & -3.05 & N2OW & & 90 & open, wet vertical fracture \\
\hline & & 0.81 & 1.68 & -3.05 & N2OW & $E$ & & top tight subhorizontal \\
\hline & & 0.85 & 1.68 & -3.05 & $\mathrm{~N} 20 \mathrm{~W}$ & $E$ & & base tight subhorizontal \\
\hline & & 0.94 & 1.68 & -3.05 & $\mathrm{~N} 20 \mathrm{~W}$ & $W$ & 90 & $\begin{array}{l}\text { top partly open, vertical } \\
\text { fracture }\end{array}$ \\
\hline & & 0.99 & 1.68 & -3.05 & N2OW & $W$ & 90 & $\begin{array}{l}\text { base partly open, vertical } \\
\text { fracture }\end{array}$ \\
\hline & & 1.12 & 1.68 & -3.05 & N50W & $\mathrm{s}$ & 45 & moderate \\
\hline & & 1.27 & 1.68 & -3.05 & N5OW & $\mathrm{s}$ & 45 & moderate \\
\hline & & 1.30 & 1.68 & -3.05 & N70E & $\mathrm{N}$ & 80 & $\begin{array}{l}\text { top fracture (cannot see } \\
\text { base) }\end{array}$ \\
\hline & & 1.33 & 1.68 & -3.05 & $\mathrm{~N}-\mathrm{S}$ & & 90 & vertical, wet, open fracture \\
\hline & LBT13 & 1.65 & 1.68 & -3.05 & N3OW & $W$ & & top subvertical fracture \\
\hline & \begin{tabular}{|l|} 
LBT13 \\
\end{tabular} & 1.68 & 1.68 & -3.05 & N3OW & $W$ & & base subvertical fracture \\
\hline & & 1.65 & 1.68 & -3.05 & $\mathrm{~N}-\mathrm{S}$ & $E$ & & top open, wet, subhorizontal \\
\hline & . & 1.79 & 1.68 & -3.05 & $\mathrm{~N}-\mathrm{S}$ & $E$ & & \begin{tabular}{|l|}
$\begin{array}{l}\text { base open, wet, } \\
\text { subhorizontal }\end{array}$ \\
\end{tabular} \\
\hline & & 2.25 & 1.68 & -3.05 & N1OW & & 90 & fracture \\
\hline & & 2.25 & 1.68 & -3.05 & N6OW & $S$ & 70 & top open, wet fracture \\
\hline & & 2.36 & 1.68 & -3.05 & N60W & $\mathrm{S}$ & 70 & base open, wet fracture \\
\hline & & 2.46 & 1.68 & -3.05 & $\mathrm{~N}-\mathrm{S}$ & & 90 & vertical fracture \\
\hline & & 2.51 & 1.68 & -3.05 & $\mathrm{~N}-\mathrm{S}$ & & 90 & tight, vertical fracture \\
\hline & & 2.59 & 1.68 & -3.05 & NIOW & $\mathrm{S}$ & 80 & top tight fracture \\
\hline & & 2.51 & 1.68 & -3.05 & N10W & $\mathrm{S}$ & 80 & base tight fracture \\
\hline
\end{tabular}




\begin{tabular}{|c|c|c|c|c|c|c|c|c|}
\hline Hole & $\begin{array}{l}\text { Surface } \\
\text { feature }\end{array}$ & $\begin{array}{l}\text { Model } \\
\text { east }\end{array}$ & $\begin{array}{l}\text { Model } \\
\text { north }\end{array}$ & $\begin{array}{l}\text { Model } \\
\text { depth }\end{array}$ & $\begin{array}{l}\text { Strike } \\
\text { direct }\end{array}$ & $\begin{array}{l}\text { Dip } \\
\text { direct }\end{array}$ & $\begin{array}{c}\text { TRUE } \\
\text { dip }\end{array}$ & Comments \\
\hline & & 2.74 & 1.68 & -3.05 & & & 90 & open vertical, wet fracture \\
\hline \multicolumn{9}{|c|}{ UE25FRPTC\# 1} \\
\hline & LBT8 & 1.22 & 2.73 & -2.29 & & & subvert & $\begin{array}{l}\text { top major open/closed } \\
\text { fracture }\end{array}$ \\
\hline & LBT8 & 1.22 & 2.60 & -2.29 & & & subvert & $\begin{array}{l}\text { base major open/closed } \\
\text { fracture }\end{array}$ \\
\hline & & 1.22 & 2.57 & -2.29 & & & 90 & minor closed fracture \\
\hline & & 1.22 & 1.85 & -2.29 & & & & $\begin{array}{l}\text { major open void/clean } \\
\text { fracture }\end{array}$ \\
\hline & & 1.22 & 1.72 & -2.29 & & & & hole intersection fracture \\
\hline & & 1.22 & 1.26 & -2.29 & & & subhor & $\begin{array}{l}\text { top moderate-major closed } \\
\text { fracture }\end{array}$ \\
\hline & & 1.22 & 1.06 & -2.29 & & & subhor & $\begin{array}{l}\text { base moderate-major closed } \\
\text { fracture }\end{array}$ \\
\hline & LBT3 & 1.22 & 1.06 & -2.29 & & & 90 & $\begin{array}{l}\text { moderate-major } \\
\text { open/closed fracture }\end{array}$ \\
\hline & LBT33 & 1.22 & 0.93 & -2.29 & & & subhor & $\begin{array}{l}\text { top minor-moderate closed } \\
\text { fracture }\end{array}$ \\
\hline & LBT33 & 1.22 & 0.46 & -2.29 & & & subhor & $\begin{array}{l}\text { base minor-moderate closed } \\
\text { fracture }\end{array}$ \\
\hline & LBT2 & 1.22 & 0.67 & -2.29 & & & 90 & $\begin{array}{l}\text { top moderate closed } \\
\text { fracture }\end{array}$ \\
\hline & LBT2 & 1.22 & 0.54 & -2.29 & & & 90 & $\begin{array}{l}\text { base moderate closed } \\
\text { fracture }\end{array}$ \\
\hline & & 1.22 & 0.21 & -2.29 & & & 90 & $\begin{array}{l}\text { top minor-moderate closed } \\
\text { fracture }\end{array}$ \\
\hline & & 1.22 & 0.08 & -2.29 & & & 90 & $\begin{array}{l}\text { base minor-moderate closed } \\
\text { fracture }\end{array}$ \\
\hline \multicolumn{9}{|c|}{ UE25FRPTC\#2 } \\
\hline & & 1.22 & 3.03 & -2.01 & & & & top major open fracture \\
\hline & & 1.22 & 2.91 & -1.99 & & & & base major open fracture \\
\hline & & 1.22 & 2.78 & -1.97 & & & subhor & top minor closed fracture \\
\hline & & 1.22 & 2.60 & -1.94 & & & subhor & base minor closed fracture \\
\hline & & 1.22 & 2.48 & -1.92 & & & & pre-test borehole \\
\hline & & 1.22 & 1.95 & -1.82 & & & 90 & $\begin{array}{l}\text { minor-moderate closed } \\
\text { fracture }\end{array}$ \\
\hline & & 1.22 & 1.79 & -1.80 & & & & pre-test borehole \\
\hline & & 1.22 & 1.24 & -1.70 & & & & pre-test borehole \\
\hline & & 1.22 & 1.04 & -1.66 & & & 90 & moderate open fracture \\
\hline & & 1.22 & 0.86 & -1.63 & & & subhor & top major open fracture \\
\hline & & 1.22 & 0.56 & -1.58 & & & subhor & base major open fracture \\
\hline & & 1.22 & 0.71 & -1.60 & & & $\sim 45$ & $\begin{array}{l}\text { top moderate closed } \\
\text { fracture }\end{array}$ \\
\hline & & 1.22 & 0.45 & -1.56 & & & -45 & $\begin{array}{l}\text { base moderate closed } \\
\text { fracture }\end{array}$ \\
\hline & & 1.22 & 0.58 & -1.58 & & & & pre-test borehole \\
\hline & LBT2 & 1.22 & 0.43 & -1.56 & & & $\sim 45$ & $\begin{array}{l}\text { top moderate-major } \\
\text { closed/open fracture }\end{array}$ \\
\hline
\end{tabular}




\begin{tabular}{|c|c|c|c|c|c|c|c|c|}
\hline Hole & $\begin{array}{l}\text { Surface } \\
\text { feature }\end{array}$ & $\begin{array}{c}\text { Model } \\
\text { east }\end{array}$ & $\begin{array}{l}\text { Model } \\
\text { north }\end{array}$ & $\begin{array}{l}\text { Model } \\
\text { depth }\end{array}$ & $\begin{array}{l}\text { Strike } \\
\text { direct }\end{array}$ & $\begin{array}{c}\text { Dip } \\
\text { direct }\end{array}$ & $\begin{array}{l}\text { TRUE } \\
\text { dip }\end{array}$ & Comments \\
\hline & LBT2 & 1.22 & 0.23 & -1.52 & & & -45 & $\begin{array}{l}\text { base moderate-major } \\
\text { closed/open fracture }\end{array}$ \\
\hline \multicolumn{9}{|c|}{ UE25FRPTC\#3 } \\
\hline & & 1.22 & 2.99 & -2.56 & & & & $\begin{array}{l}\text { top moderate-major } \\
\text { open/closed fracture }\end{array}$ \\
\hline & & 1.22 & 2.64 & -2.63 & & & & $\begin{array}{l}\text { base moderate-major } \\
\text { open/closed fracture }\end{array}$ \\
\hline & & 1.22 & 2.49 & -2.65 & & & & $\begin{array}{l}\text { minor-moderate closed } \\
\text { fracture }\end{array}$ \\
\hline & & 1.22 & 2.47 & -2.66 & & & subvert & $\begin{array}{l}\text { top moderate open/closed } \\
\text { fracture }\end{array}$ \\
\hline & & 1.22 & 2.37 & -2.67 & & & subvert & $\begin{array}{l}\text { base moderate open/closed } \\
\text { fracture }\end{array}$ \\
\hline & & 1.22 & 2.29 & -2.69 & & & & post-test borehole \\
\hline & & 1.22 & 2.04 & -2.73 & & & & post-test borehole \\
\hline & & 1.22 & 1.92 & -2.75 & & & & post-test borehole \\
\hline & & 1.22 & 1.85 & -2.77 & & & & post-test borehole \\
\hline & & 1.22 & 2.02 & -2.74 & & & subvert & top minor closed fracture \\
\hline & & 1.22 & 1.87 & -2.76 & & & subvert & base minor closed fracture \\
\hline & & 1.22 & 1.97 & -2.74 & & & subhor & top major open fracture \\
\hline & & 1.22 & 1.82 & -2.77 & & & subhor & base major open fracture \\
\hline & & 1.22 & 1.85 & -2.77 & & & & $\begin{array}{l}\text { possible major open } \\
\text { subvertical fracture }\end{array}$ \\
\hline & & 1.22 & 1.75 & -2.78 & & & 90 & minor closed fracture \\
\hline & LBT23 & 1.22 & 1.75 & -2.78 & & & & $\begin{array}{l}\text { top minor open/closed } \\
\text { fracture }\end{array}$ \\
\hline & LBT23 & 1.22 & 1.55 & -2.82 & & & & $\begin{array}{l}\text { base minor open/closed } \\
\text { fracture }\end{array}$ \\
\hline & & 1.22 & 1.57 & -2.82 & & & & $\begin{array}{l}\text { horizontal borehole } \\
\text { intersection }\end{array}$ \\
\hline & & 1.22 & 1.25 & -2.87 & & & & vertical borehole intersection \\
\hline & & 1.22 & 1.50 & -2.83 & & & subvert & top minor closed fracture \\
\hline & & 1.22 & 1.20 & -2.88 & & & subvert & base minor closed fracture \\
\hline & & 1.22 & 1.27 & -2.87 & & & subvert & top minor closed fracture \\
\hline & & 1.22 & 1.12 & -2.89 & & & subvert & base minor closed fracture \\
\hline & LBT3 & 1.22 & 1.07 & -2.90 & & & 90 & $\begin{array}{l}\text { moderate open/closed } \\
\text { fracture }\end{array}$ \\
\hline & & 1.22 & 1.00 & -2.92 & & & subvert & top minor fracture \\
\hline & LBT2 & 1.22 & 0.92 & -2.93 & & & subvert & top moderate open fracture \\
\hline & LBT2 & 1.22 & 0.80 & -2.95 & & & subvert & $\begin{array}{l}\text { base moderate open } \\
\text { fracture }\end{array}$ \\
\hline & & 1.22 & 0.62 & -2.98 & & & & vertical borehole intersection \\
\hline & & 1.22 & 0.10 & -3.07 & & & subvert & $\begin{array}{l}\text { top major open/closed } \\
\text { fracture }\end{array}$ \\
\hline & & 1.22 & 0.00 & -3.09 & & & subvert & $\begin{array}{l}\text { base major open/closed } \\
\text { fracture }\end{array}$ \\
\hline
\end{tabular}




\begin{tabular}{|c|c|c|c|c|c|c|c|c|}
\hline Hole & $\begin{array}{l}\text { Surface } \\
\text { feature }\end{array}$ & $\begin{array}{l}\text { Model } \\
\text { east }\end{array}$ & $\begin{array}{l}\text { Model } \\
\text { north }\end{array}$ & $\begin{array}{l}\text { Model } \\
\text { depth }\end{array}$ & $\begin{array}{l}\text { Strike } \\
\text { direct }\end{array}$ & $\begin{array}{l}\text { Dip } \\
\text { direct }\end{array}$ & $\begin{array}{c}\text { TRUE } \\
\text { dip }\end{array}$ & Comments \\
\hline \multicolumn{9}{|c|}{ UE25FRPTC\#4 } \\
\hline & & 1.22 & 2.44 & -1.51 & & & & pre-test borehole \\
\hline & & 1.22 & 2.04 & -1.37 & & & -90 & $\begin{array}{l}\text { moderate open/closed } \\
\text { fracture }\end{array}$ \\
\hline & & 1.22 & 1.74 & -1.25 & & & & post-test borehole \\
\hline & & 1.22 & 1.64 & -1.22 & & & & post-test borehole \\
\hline & & 1.22 & 1.24 & -1.08 & & & & minor-moderate fracture \\
\hline & LBT3 & 1.22 & 0.94 & -0.96 & & & subhor & top major open fracture \\
\hline & LBT3 & 1.22 & 1.13 & -1.03 & & & subhor & base major open fracture \\
\hline & & 1.22 & 0.59 & -0.84 & & & & pre-test borehole \\
\hline & & 1.22 & 0.47 & -0.79 & & & Angled & top major open fracture \\
\hline & & 1.22 & 0.26 & -0.72 & & & Angled & base major open fracture \\
\hline \multicolumn{9}{|c|}{ UE25FRPTC\#5 } \\
\hline & & 1.22 & 2.96 & -2.87 & & & -90 & major open fracture \\
\hline & & 1.22 & 2.60 & -3.00 & & & $\sim 90$ & major open fracture \\
\hline & LBT8 & 1.22 & 2.60 & -3.00 & & & 90 & $\begin{array}{l}\text { moderate open/closed } \\
\text { fracture }\end{array}$ \\
\hline & & 1.22 & 2.55 & -3.02 & & & & pre-test borehole \\
\hline & & 1.22 & 2.55 & -3.02 & & & & top moderate open fracture \\
\hline & & 1.22 & 2.34 & -3.10 & & & & $\begin{array}{l}\text { base moderate open } \\
\text { fracture }\end{array}$ \\
\hline & LBT8 & 1.22 & 2.58 & -3.01 & & & & $\begin{array}{l}\text { top moderate open/closed } \\
\text { fracture }\end{array}$ \\
\hline & LBT8 & 1.22 & 2.24 & -3.13 & & & & $\begin{array}{l}\text { base moderate open/closed } \\
\text { fracture }\end{array}$ \\
\hline & LBT12 & 1.22 & 2.10 & -3.19 & & & & $\begin{array}{l}\text { top minor-moderate closed } \\
\text { fracture }\end{array}$ \\
\hline & LBT12 & 1.22 & 2.02 & -3.21 & & & & $\begin{array}{l}\text { base minor-moderate closed } \\
\text { fracture }\end{array}$ \\
\hline & & 1.22 & 1.97 & -3.23 & & & & post-test borehole \\
\hline & & 1.22 & 1.90 & -3.26 & & & & post-test borehole \\
\hline & & 1.22 & 1.83 & -3.28 & & & $\sim 90$ & $\begin{array}{l}\text { top minor-moderate } \\
\text { open/closed fracture }\end{array}$ \\
\hline & & 1.22 & 1.78 & -3.30 & & & -90 & $\begin{array}{l}\text { base minor-moderate } \\
\text { open/closed fracture }\end{array}$ \\
\hline & & 1.22 & 1.73 & -3.32 & & & -90 & $\begin{array}{l}\text { top minor-moderate } \\
\text { open/closed fracture }\end{array}$ \\
\hline & & 1.22 & 1.71 & -3.33 & & & -90 & $\begin{array}{l}\text { base minor-moderate } \\
\text { open/closed fracture }\end{array}$ \\
\hline & & 1.22 & 1.69 & -3.34 & & & & top moderate open fracture \\
\hline & & 1.22 & 1.47 & -3.42 & & & & $\begin{array}{l}\text { base moderate open } \\
\text { fracture }\end{array}$ \\
\hline & & 1.22 & 1.61 & -3.36 & & & -80 & top major open fracture \\
\hline & & 1.22 & 1.18 & -3.52 & & & -80 & base major open fracture \\
\hline & & 1.22 & 1.30 & -3.48 & & & & pre-test borehole \\
\hline & LBT2 & 1.22 & 1.18 & -3.52 & & & & $\begin{array}{l}\text { top moderate-major open } \\
\text { fracture }\end{array}$ \\
\hline
\end{tabular}




\begin{tabular}{|c|c|c|c|c|c|c|c|c|}
\hline Hole & $\begin{array}{l}\text { Surface } \\
\text { feature }\end{array}$ & $\begin{array}{l}\text { Model } \\
\text { east }\end{array}$ & $\begin{array}{l}\text { Model } \\
\text { north }\end{array}$ & $\begin{array}{l}\text { Model } \\
\text { depth }\end{array}$ & $\begin{array}{l}\text { Strike } \\
\text { direct }\end{array}$ & $\begin{array}{l}\text { Dip } \\
\text { direct }\end{array}$ & $\begin{array}{l}\text { TRUE } \\
\text { dip }\end{array}$ & Comments \\
\hline & LBT2 & 1.22 & 1.11 & -3.55 & & & & $\begin{array}{l}\text { base moderate-major open } \\
\text { fracture }\end{array}$ \\
\hline & & 1.22 & 1.04 & -3.57 & & & $\sim 90$ & $\begin{array}{l}\text { moderate open/closed } \\
\text { fracture } \\
\end{array}$ \\
\hline & & 1.22 & 0.65 & -3.71 & & & & pre-test borehole \\
\hline & & 1.22 & 0.63 & -3.72 & & & subhor & $\begin{array}{l}\text { top major open/closed } \\
\text { fracture }\end{array}$ \\
\hline & & 1.22 & 0.29 & -3.84 & & & subhor & $\begin{array}{l}\text { base major open/closed } \\
\text { fracture }\end{array}$ \\
\hline & LBT15 & 1.22 & 0.48 & -3.77 & & & & top minor closed fracture \\
\hline & LBT15 & 1.22 & 0.36 & -3.82 & & & & base minor closed fracture \\
\hline & & 1.22 & 0.05 & -3.93 & & & & possible breakout or fracture \\
\hline \multicolumn{9}{|c|}{ UE25FRPTC\#6 } \\
\hline & & 1.22 & 3.04 & -1.40 & & & subvert & $\begin{array}{l}\text { top minor/tight closed } \\
\text { fracture }\end{array}$ \\
\hline & & 1.22 & 2.67 & -1.19 & & & subvert & $\begin{array}{l}\text { base minor/tight closed } \\
\text { fracture }\end{array}$ \\
\hline & & 1.22 & 2.44 & -1.05 & & & & pre-test borehole \\
\hline & & 1.22 & 1.88 & -0.73 & & & & pre-test borehole \\
\hline & & 1.22 & 1.64 & -0.59 & & & & top major open fracture \\
\hline & & 1.22 & 1.30 & -0.40 & & & & base major open fracture \\
\hline & & 1.22 & 1.25 & -0.37 & & & & pre-test borehole \\
\hline & & 1.22 & 1.21 & -0.35 & & & subvert & $\begin{array}{l}\text { top minor-moderate } \\
\text { open/closed fracture }\end{array}$ \\
\hline & & 1.22 & 1.17 & -0.32 & & & subvert & $\begin{array}{l}\text { base minor-moderate } \\
\text { open/closed fracture }\end{array}$ \\
\hline & LBT3 & 1.22 & 1.15 & -0.31 & & & subvert & top minor-moderate fracture \\
\hline & & 1.22 & 1.10 & -0.29 & & & subvert & $\begin{array}{l}\text { base minor-moderate } \\
\text { fracture }\end{array}$ \\
\hline & & 1.22 & 1.08 & -0.27 & & & -90 & minor closed fracture \\
\hline & & 1.22 & 0.87 & -0.15 & & & -90 & minor closed fracture \\
\hline \multicolumn{2}{|c|}{ UE25FRPTC\#7 } & \multicolumn{7}{|c|}{ very dirty hole with mudcake on the walls } \\
\hline & & \begin{tabular}{|l|}
1.22 \\
\end{tabular} & 2.75 & -4.05 & & & & pre-test borehole \\
\hline & & 1.22 & 2.55 & -4.24 & & & & pre-test borehole \\
\hline & LBT8 & 1.22 & 2.18 & -4.59 & & & & top minor closed fracture \\
\hline & LBT8 & 1.22 & 2.09 & -4.68 & & & & base minor closed fracture \\
\hline & & 1.22 & 1.91 & -4.86 & & & & pre-test borehole \\
\hline & & 1.22 & 1.89 & -4.87 & & & & top minor closed fracture \\
\hline & & 1.22 & 1.82 & -4.94 & & & & base minor closed fracture \\
\hline & & 1.22 & 1.78 & -4.98 & & & & top minor closed fracture \\
\hline & & 1.22 & 1.71 & -5.05 & & & & base minor closed fracture \\
\hline & & 1.22 & 1.44 & -5.31 & & & subhor & $\begin{array}{l}\text { top moderate closed } \\
\text { fracture }\end{array}$ \\
\hline & & 1.22 & 1.31 & -5.44 & & & subhor & $\begin{array}{l}\text { base moderate closed } \\
\text { fracture }\end{array}$ \\
\hline & & 1.22 & 1.00 & -5.74 & & & & top minor closed fracture \\
\hline & & 1.22 & 0.96 & -5.77 & & & & base minor closed fracture \\
\hline
\end{tabular}




\begin{tabular}{|c|c|c|c|c|c|c|c|c|}
\hline Hole & $\begin{array}{l}\text { Surface } \\
\text { feature }\end{array}$ & $\begin{array}{c}\text { Model } \\
\text { east }\end{array}$ & $\begin{array}{l}\text { Model } \\
\text { north }\end{array}$ & $\begin{array}{l}\text { Model } \\
\text { depth }\end{array}$ & $\begin{array}{l}\text { Strike } \\
\text { direct }\end{array}$ & $\begin{array}{l}\text { Dip } \\
\text { direct }\end{array}$ & $\begin{array}{c}\text { TRUE } \\
\text { dip }\end{array}$ & Comments \\
\hline & & 1.22 & 0.91 & -5.82 & & & subvert & top minor closed fracture \\
\hline & & 1.22 & 0.69 & -6.03 & & & subvert & base minor closed fracture \\
\hline & & 1.22 & 0.71 & -6.02 & & & subvert & major open fracture \\
\hline & & 1.22 & 0.44 & -6.28 & & & subvert & major open fracture \\
\hline \multicolumn{9}{|c|}{ UE25FRPTC\#8 } \\
\hline & & 1.22 & 2.57 & -0.61 & & & $\sim 45$ & top major closed fracture \\
\hline & & 1.22 & 2.41 & -0.48 & & & -45 & base major closed fracture \\
\hline & LBTI & 1.22 & 2.45 & -0.51 & & & -45 & $\begin{array}{l}\text { top major open/closed } \\
\text { fracture }\end{array}$ \\
\hline & LBTI & 1.22 & 2.35 & -0.43 & & & -45 & $\begin{array}{l}\text { base major open/closed } \\
\text { fracture }\end{array}$ \\
\hline & & 1.22 & 2.37 & -0.44 & & & & pre-test borehole \\
\hline & & 1.22 & 2.37 & -0.44 & & & 90 & minor-moderate fracture \\
\hline & & 1.22 & 2.22 & -0.31 & & & & top minor closed fracture \\
\hline & & 1.22 & 2.08 & -0.20 & & & & base minor closed fracture \\
\hline & & 1.22 & 2.24 & -0.33 & & & $\sim 80$ & $\begin{array}{l}\text { top minor-moderate closed } \\
\text { fracture }\end{array}$ \\
\hline & & 1.22 & 1.98 & -0.11 & & & $\sim 80$ & $\begin{array}{l}\text { base minor-moderate closed } \\
\text { fracture }\end{array}$ \\
\hline \multicolumn{2}{|c|}{ UE25FRPTC\#9 } & \multicolumn{7}{|c|}{ locally muddy hole(covering tape) } \\
\hline & & 1.75 & 2.70 & -2.29 & & & 90 & $\begin{array}{l}\text { moderate open/closed } \\
\text { Discrete fracture }\end{array}$ \\
\hline & & 1.82 & 2.48 & -2.29 & & & & post-test borehole \\
\hline & LBT8 & 1.85 & 2.36 & -2.29 & & & -45 & top minor closed fracture \\
\hline & LBT8 & 1.87 & 2.29 & -2.29 & & & $\sim 45$ & base minor closed fracture \\
\hline & & 1.87 & 2.29 & -2.29 & & & & top minor closed fracture \\
\hline & & 1.91 & 2.16 & -2.29 & & & & base minor closed fracture \\
\hline & & 1.99 & 1.89 & -2.29 & & & -90 & minor closed fracture \\
\hline & & 2.02 & 1.77 & -2.29 & & & & post-test borehole \\
\hline & & 2.07 & 1.60 & -2.29 & & & & pre-test borehole \\
\hline & & 2.53 & 0.00 & -2.29 & & & -80 & top minor closed fracture \\
\hline & & 2.16 & 1.28 & -2.29 & & & -80 & base minor closed fracture \\
\hline & & 2.21 & 1.13 & -2.29 & & & $>85$ & major open fracture \\
\hline & & 2.33 & 0.69 & -2.29 & & & -80 & top minor closed fracture \\
\hline & & 2.36 & 0.59 & -2.29 & & & $\sim 80$ & base minor closed fracture \\
\hline \multicolumn{2}{|c|}{ UE25FRPTC\#10 } & \multicolumn{7}{|c|}{ difficult hole to log } \\
\hline & & 0.88 & 1.83 & -0.41 & & & -90 & top major open fracture \\
\hline & & 0.98 & 1.83 & -0.33 & & & $\sim 90$ & base major open fracture \\
\hline & & 1.15 & 1.83 & -0.20 & & & & pre-test borehole \\
\hline \multicolumn{2}{|c|}{ UE25FRPTC\#11 } & & & & & & & pre-test borehole \\
\hline & & 0.24 & 1.83 & -1.14 & & & -45 & $\begin{array}{l}\text { top major open/closed } \\
\text { fracture }\end{array}$ \\
\hline & & 0.33 & 1.83 & -1.08 & & & $\sim 45$ & $\begin{array}{l}\text { base major open/closed } \\
\text { fracture }\end{array}$ \\
\hline & & 1.05 & 1.83 & -0.62 & & & -45 & $\begin{array}{l}\text { top minor-moderate closed } \\
\text { fracture }\end{array}$ \\
\hline
\end{tabular}




\begin{tabular}{|c|c|c|c|c|c|c|c|c|}
\hline Hole & $\begin{array}{l}\text { Surface } \\
\text { feature }\end{array}$ & $\begin{array}{l}\text { Model } \\
\text { east }\end{array}$ & $\begin{array}{l}\text { Model } \\
\text { north }\end{array}$ & $\begin{array}{l}\text { Model } \\
\text { depth }\end{array}$ & $\begin{array}{l}\text { Strike } \\
\text { direct }\end{array}$ & $\begin{array}{l}\text { Dip } \\
\text { direct }\end{array}$ & $\begin{array}{c}\text { TRUE } \\
\text { dip }\end{array}$ & Comments \\
\hline & & 1.14 & 1.83 & -0.55 & & & $\sim 45$ & $\begin{array}{l}\text { base minor-moderate closed } \\
\text { fracture }\end{array}$ \\
\hline & & 1.55 & 1.83 & -0.29 & & & & pre-test borehole \\
\hline \multicolumn{9}{|c|}{ UE25FRPTC\#12 } \\
\hline & & 0.10 & 1.83 & -1.46 & & & subvert & top major closed fracture \\
\hline & & 0.14 & 1.83 & -1.44 & & & subvert & base major closed fracture \\
\hline & & 0.24 & 1.83 & -1.39 & & & 290 & minor closed fracture \\
\hline & & 0.38 & 1.83 & -1.32 & & & & pre-test borehole \\
\hline & & 0.43 & 1.83 & -1.29 & & & & $\begin{array}{l}\text { top moderate-major open } \\
\text { fracture }\end{array}$ \\
\hline & & 0.59 & 1.83 & -1.21 & & & & $\begin{array}{l}\text { base moderate-major open } \\
\text { fracture }\end{array}$ \\
\hline & & 1.06 & 1.83 & -0.97 & & & & pre-test borehole \\
\hline & & 0.87 & 1.83 & -1.07 & & & & top minor closed fracture \\
\hline & & 1.25 & 1.83 & -0.87 & & & & base minor closed fracture \\
\hline & & 1.32 & 1.83 & -0.84 & & & & post-test borehole \\
\hline & & 1.41 & 1.83 & -0.79 & & & & post-test borehole \\
\hline & LBT1 & 1.77 & 1.83 & -0.61 & & & subhor & top major open! fracture \\
\hline & LBTI & 1.91 & 1.83 & -0.54 & & & subhor & base major open! fracture \\
\hline & & 1.98 & 1.83 & -0.50 & & & & pre-test borehole \\
\hline & & 2.28 & 1.83 & -0.35 & & & subhor & $\begin{array}{l}\text { top moderate closed } \\
\text { fracture }\end{array}$ \\
\hline & & 2.40 & 1.83 & -0.29 & & & subhor & $\begin{array}{l}\text { base moderate closed } \\
\text { fracture }\end{array}$ \\
\hline & & 2.45 & 1.83 & -0.26 & & & & $\begin{array}{l}\text { top moderate closed } \\
\text { fracture }\end{array}$ \\
\hline & & 2.61 & 1.83 & -0.18 & & & & $\begin{array}{l}\text { base moderate closed } \\
\text { fracture }\end{array}$ \\
\hline & & 2.59 & 1.83 & -0.19 & & & & pre-test borehole \\
\hline \multicolumn{9}{|c|}{ UE25FRPTC\#13 } \\
\hline & & -0.05 & 1.83 & -1.75 & & & subvert & $\begin{array}{l}\text { top minor-moderate } \\
\text { open/closed fracture }\end{array}$ \\
\hline & & 0.03 & 1.83 & -1.72 & & & subvert & $\begin{array}{l}\text { base minor-moderate } \\
\text { open/closed fracture }\end{array}$ \\
\hline & & 0.45 & 1.83 & -1.57 & & & & minor-moderate fracture \\
\hline & & 1.03 & 1.83 & -1.35 & & & & post-test borehole \\
\hline & & 1.08 & 1.83 & -1.34 & & & & pre-test borehole \\
\hline & & 1.72 & 1.83 & -1.10 & & & & pre-test borehole \\
\hline & & 2.36 & 1.83 & -0.87 & & & & pre-test borehole \\
\hline & & 2.53 & 1.83 & -0.81 & & & subhor & $\begin{array}{l}\text { top major open/closed } \\
\text { fracture }\end{array}$ \\
\hline & & 2.39 & 1.83 & -0.86 & & & subhor & $\begin{array}{l}\text { base major open/closed } \\
\text { fracture }\end{array}$ \\
\hline \multicolumn{9}{|c|}{ UE25FRPTC\#14 } \\
\hline & LBT2 & 0.14 & 1.83 & -1.87 & & & -90 & top major open fracture \\
\hline & LBT2 & 0.21 & 1.83 & -1.85 & & & -90 & base major open fracture \\
\hline & & 1.22 & 1.83 & -1.60 & & & & pre-test borehole \\
\hline
\end{tabular}




\begin{tabular}{|c|c|c|c|c|c|c|c|c|}
\hline Hole & $\begin{array}{l}\text { Surface } \\
\text { feature }\end{array}$ & $\begin{array}{l}\text { Model } \\
\text { east }\end{array}$ & $\begin{array}{l}\text { Model } \\
\text { north }\end{array}$ & $\begin{array}{l}\text { Model } \\
\text { depth }\end{array}$ & $\begin{array}{l}\text { Strike } \\
\text { direct }\end{array}$ & $\begin{array}{l}\text { Dip } \\
\text { direct }\end{array}$ & $\begin{array}{c}\text { TRUE } \\
\text { dip }\end{array}$ & Comments \\
\hline & LBT12 & 1.57 & 1.83 & -1.51 & & & -45 & top major open fracture \\
\hline & LBT12 & 1.69 & 1.83 & -1.48 & & & -45 & base major open fracture \\
\hline & & 1.77 & 1.83 & -1.47 & & & & pre-test borehole \\
\hline & LBT13 & 1.84 & 1.83 & -1.45 & & & -45 & top minor closed fracture \\
\hline & LBTI3 & 1.99 & 1.83 & -1.41 & & & -45 & base minor closed fracture \\
\hline & & 2.43 & 1.83 & -1.30 & & & & pre-test borehole \\
\hline & & 2.51 & 1.83 & -1.28 & & & $\sim 45$ & $\begin{array}{l}\text { top minor-moderate } \\
\text { open/closed fracture }\end{array}$ \\
\hline & & 2.63 & 1.83 & -1.25 & & & $\sim 45$ & $\begin{array}{l}\text { base minor-moderate } \\
\text { open/closed fracture }\end{array}$ \\
\hline & & 2.60 & 1.83 & -1.26 & & & -45 & $\begin{array}{l}\text { top minor-moderate } \\
\text { open/closed fracture }\end{array}$ \\
\hline & & 2.73 & 1.83 & -1.23 & & & -45 & $\begin{array}{l}\text { base minor-moderate } \\
\text { open/closed fracture }\end{array}$ \\
\hline & & 2.73 & 1.83 & -1.23 & & & -60 & $\begin{array}{l}\text { top minor-moderate } \\
\text { open/closed fracture }\end{array}$ \\
\hline & & 2.80 & 1.83 & -1.21 & & & $\sim 60$ & $\begin{array}{l}\text { base minor-moderate } \\
\text { open/closed fracture }\end{array}$ \\
\hline \multicolumn{9}{|c|}{ UE25FRPTC\#15 } \\
\hline & LBT2O & 0.16 & 1.83 & -2.26 & & & & top major open fracture \\
\hline & LBT20 & 0.51 & 1.83 & -2.25 & & & & base major open fracture \\
\hline & & 0.35 & 1.83 & -2.25 & & & 90 & $\begin{array}{l}\text { top moderate closed } \\
\text { fracture }\end{array}$ \\
\hline & & 0.93 & 1.83 & -2.24 & & & $\sim 80$ & $\begin{array}{l}\text { base moderate closed } \\
\text { fracture }\end{array}$ \\
\hline & & 1.12 & 1.83 & -2.24 & & & & \\
\hline & & 1.06 & 1.83 & -2.24 & & & subvert & $\begin{array}{l}\text { top moderate-major closed } \\
\text { fracture }\end{array}$ \\
\hline & & 1.57 & 1.83 & -2.23 & & & subvert & $\begin{array}{l}\text { base moderate-major closed } \\
\text { fracture }\end{array}$ \\
\hline & & 1.78 & 1.83 & -2.23 & & & & post-test borehole \\
\hline & & 1.91 & 1.83 & -2.23 & & & & post-test borehole \\
\hline & & 1.96 & 1.83 & -2.23 & & & subvert & $\begin{array}{l}\text { top moderate closed } \\
\text { fracture }\end{array}$ \\
\hline & & 2.41 & 1.83 & -2.22 & & & subvert & $\begin{array}{l}\text { base moderate closed } \\
\text { fracture }\end{array}$ \\
\hline & & 2.41 & 1.83 & -2.22 & & & & pre-test borehole \\
\hline \multicolumn{9}{|c|}{ UE25FRPTC\#16 } \\
\hline & & 0.24 & 1.83 & -2.60 & & & & minor closed fracture \\
\hline & LBT2 & 0.37 & 1.83 & -2.62 & & & subvert & $\begin{array}{l}\text { top moderate open/closed } \\
\text { fracture }\end{array}$ \\
\hline & LBT2 & 0.45 & 1.83 & -2.63 & & & subvert & $\begin{array}{l}\text { base moderate open/closed } \\
\text { fracture }\end{array}$ \\
\hline & & 0.52 & 1.83 & -2.65 & & & & pre-test borehole \\
\hline & & 0.57 & 1.83 & -2.66 & & & subvert & $\begin{array}{l}\text { top moderate closed } \\
\text { fracture }\end{array}$ \\
\hline & & 0.93 & 1.83 & -2.72 & & & subvert & $\begin{array}{l}\text { base moderate closed } \\
\text { fracture }\end{array}$ \\
\hline
\end{tabular}




\begin{tabular}{|c|c|c|c|c|c|c|c|c|}
\hline Hole & $\begin{array}{l}\text { Surface } \\
\text { feature }\end{array}$ & $\begin{array}{l}\text { Model } \\
\text { east }\end{array}$ & $\begin{array}{l}\text { Model } \\
\text { north }\end{array}$ & $\begin{array}{l}\text { Model } \\
\text { depth }\end{array}$ & $\begin{array}{l}\text { Strike } \\
\text { direct }\end{array}$ & $\begin{array}{l}\text { Dip } \\
\text { direct }\end{array}$ & $\begin{array}{c}\text { TRUE } \\
\text { dip }\end{array}$ & Comments \\
\hline & & 1.15 & 1.83 & -2.76 & & & & post-test borehole \\
\hline & & 1.28 & 1.83 & -2.78 & & & -90 & $\begin{array}{l}\text { minor-moderate closed } \\
\text { fracture }\end{array}$ \\
\hline & & 1.58 & 1.83 & -2.83 & & & subvert & $\begin{array}{l}\text { top moderate open/closed } \\
\text { fracture }\end{array}$ \\
\hline & & 1.63 & 1.83 & -2.84 & & & subvert & $\begin{array}{l}\text { base moderate open/closed } \\
\text { fracture }\end{array}$ \\
\hline & & 1.73 & 1.83 & -2.86 & & & & post-test borehole \\
\hline & & 2.01 & 1.83 & -2.91 & & & -90 & major open/closed fracture \\
\hline & & 2.39 & 1.83 & -2.98 & & & & pre-test borehole \\
\hline & & 2.19 & 1.83 & -2.94 & & & 90 & minor closed fracture \\
\hline & LBT8 & 2.74 & 1.83 & -3.04 & & & 90 & $\begin{array}{l}\text { minor-moderate } \\
\text { open/closed fracture }\end{array}$ \\
\hline \multicolumn{9}{|c|}{ UE25FRPTC\#17 } \\
\hline & & 0.06 & 1.83 & -2.86 & & & & $\begin{array}{l}\text { moderate-major open } \\
\text { fracture }\end{array}$ \\
\hline & & 0.39 & 1.83 & -2.98 & & & subvert & major open fracture \\
\hline & & 0.18 & 1.83 & -2.90 & & & -90 & top major open! fracture \\
\hline & & 0.63 & 1.83 & -3.07 & & & -90 & base major open! fracture \\
\hline & & 0.39 & 1.83 & -2.98 & & & subvert & $\begin{array}{l}\text { top moderate-major open } \\
\text { fracture }\end{array}$ \\
\hline & & 0.46 & 1.83 & -3.01 & & & subvert & $\begin{array}{l}\text { base moderate-major open } \\
\text { fracture }\end{array}$ \\
\hline & & 0.80 & 1.83 & -3.13 & & & subvert & $\begin{array}{l}\text { top moderate-major open } \\
\text { fracture }\end{array}$ \\
\hline & & 0.94 & 1.83 & -3.18 & & & subvert & $\begin{array}{l}\text { base moderate-major open } \\
\text { fracture }\end{array}$ \\
\hline & & 1.13 & 1.83 & -3.25 & & & & post-test borehole \\
\hline & & 1.28 & 1.83 & -3.31 & & & subvert & moderate-major fracture \\
\hline & LBT13 & 1.47 & 1.83 & -3.38 & & & subvert & major! open/closed fracture \\
\hline & LBT13 & 1.56 & 1.83 & -3.41 & & & subvert & major! open/closed fracture \\
\hline & & 1.68 & 1.83 & -3.45 & & & & post-test borehole \\
\hline & & 1.68 & 1.83 & -3.45 & & & subvert & $\begin{array}{l}\text { minor-moderate closed } \\
\text { fracture }\end{array}$ \\
\hline & & 2.02 & 1.83 & -3.58 & & & subvert & $\begin{array}{l}\text { minor-moderate closed } \\
\text { fracture }\end{array}$ \\
\hline & & 1.85 & 1.83 & -3.51 & & & subvert & minor closed fracture \\
\hline & & 1.90 & 1.83 & -3.53 & & & subvert & minor closed fracture \\
\hline & & 2.09 & 1.83 & -3.60 & & & subvert & moderate closed fracture \\
\hline & & 2.83 & 1.83 & -3.87 & & & subvert & moderate closed fracture \\
\hline & & 2.02 & 1.83 & -3.58 & & & & pre-test borehole \\
\hline & & 2.43 & 1.83 & -3.72 & & & subvert & moderate closed fracture \\
\hline & & 2.93 & 1.83 & -3.91 & & & subvert & moderate closed fracture \\
\hline & & 2.71 & 1.83 & -3.83 & & & & pre-test borehole \\
\hline \multicolumn{9}{|c|}{ UE25FRPTC\#18 } \\
\hline & & 0.45 & 1.83 & -3.67 & & & -subver & minor fracture \\
\hline & & 0.75 & 1.83 & -3.87 & & & subhor & top moderate open/closed \\
\hline
\end{tabular}




\begin{tabular}{|c|c|c|c|c|c|c|c|c|}
\hline Hole & $\begin{array}{l}\text { Surface } \\
\text { feature }\end{array}$ & $\begin{array}{l}\text { Model } \\
\text { east }\end{array}$ & $\begin{array}{l}\text { Model } \\
\text { north }\end{array}$ & $\begin{array}{l}\text { Model } \\
\text { depth }\end{array}$ & $\begin{array}{l}\text { Strike } \\
\text { direct }\end{array}$ & $\begin{array}{l}\text { Dip } \\
\text { direct }\end{array}$ & $\begin{array}{c}\text { TRUE } \\
\text { dip }\end{array}$ & Comments \\
\hline & & & & & & & & fracture \\
\hline & & 0.93 & 1.83 & -4.01 & & & subhor & $\begin{array}{l}\text { base moderate open/closed } \\
\text { fracture }\end{array}$ \\
\hline & & 0.85 & 1.83 & -3.95 & & & & top minor closed fracture \\
\hline & & 0.98 & 1.83 & -4.04 & & & & base minor closed fracture \\
\hline & & 0.89 & 1.83 & -3.98 & & & subhor & top minor closed fracture \\
\hline & & 1.00 & 1.83 & -4.05 & & & subhor & base minor closed fracture \\
\hline & & 1.37 & 1.83 & -4.31 & & & subvert & $\begin{array}{l}\text { top moderate closed } \\
\text { fracture }\end{array}$ \\
\hline & & 1.48 & 1.83 & -4.39 & & & subvert & $\begin{array}{l}\text { base moderate closed } \\
\text { fracture }\end{array}$ \\
\hline & & 1.52 & 1.83 & -4.42 & & & subvert & top moderate open fracture \\
\hline & & 1.56 & 1.83 & -4.45 & & & subvert & $\begin{array}{l}\text { base moderate open } \\
\text { fracture }\end{array}$ \\
\hline & & 1.62 & 1.83 & -4.49 & & & subvert & $\begin{array}{l}\text { top minor open/closed } \\
\text { fracture }\end{array}$ \\
\hline & & 1.65 & 1.83 & -4.51 & & & subvert & $\begin{array}{l}\text { base minor open/closed } \\
\text { fracture }\end{array}$ \\
\hline & & 1.73 & 1.83 & -4.56 & & & subvert & $\begin{array}{l}\text { top minor open/closed } \\
\text { fracture }\end{array}$ \\
\hline & & 1.81 & 1.83 & -4.62 & & & subvert & $\begin{array}{l}\text { base minor open/closed } \\
\text { fracture }\end{array}$ \\
\hline & & 2.00 & 1.83 & -4.75 & & & & post-test borehole \\
\hline & & 2.59 & 1.83 & -5.16 & & & & pre-test borehole \\
\hline \multicolumn{9}{|c|}{ UE25FRPTC\#19 } \\
\hline & & 0.27 & 1.83 & -2.16 & & & subvert & top moderate-major fracture \\
\hline & & 0.38 & 1.83 & -2.15 & & & subvert & $\begin{array}{l}\text { base moderate-major } \\
\text { fracture }\end{array}$ \\
\hline & & 0.45 & 1.83 & -2.15 & & & subvert & top minor fracture \\
\hline & & 0.50 & 1.83 & -2.14 & & & subvert & base minor fracture \\
\hline & & 1.06 & 1.83 & -2.11 & & & subvert & top minor fracture \\
\hline & & 1.16 & 1.83 & -2.10 & & & subvert & base minor fracture \\
\hline & & 1.46 & 1.83 & -2.08 & & & 90 & major fracture \\
\hline & & 1.74 & 1.83 & -2.06 & & & & pre-test borehole \\
\hline & & 2.12 & 1.83 & -2.03 & & & & pre-test borehole \\
\hline & & 2.69 & 1.83 & -1.99 & & & -90 & top major fracture \\
\hline & & 2.80 & 1.83 & -1.98 & & & -90 & base major fracture \\
\hline \multicolumn{9}{|c|}{ UE25FRPTC\#19a } \\
\hline & & 0.46 & 1.26 & -2.15 & & & & pre-test borehole \\
\hline & & 0.54 & 1.24 & -2.14 & & & -90 & top major open fracture \\
\hline & & 0.64 & 1.21 & -2.13 & & & $\sim 90$ & base major open fracture \\
\hline & & 0.85 & 1.15 & -2.12 & & & & pre-test borehole \\
\hline & LBT3 & 1.00 & 1.10 & -2.11 & & & -90 & top major open fracture \\
\hline & LBT3 & 1.23 & 1.04 & -2.09 & & & -90 & base major open fracture \\
\hline & & 1.62 & 0.93 & -2.07 & & & -90 & $\begin{array}{l}\text { moderate-major } \\
\text { open/closed fracture }\end{array}$ \\
\hline & & 2.01 & 0.82 & -2.04 & & & subhor & top moderate fracture \\
\hline
\end{tabular}




\begin{tabular}{|c|c|c|c|c|c|c|c|c|}
\hline Hole & $\begin{array}{c}\text { Surface } \\
\text { feature }\end{array}$ & $\begin{array}{c}\text { Model } \\
\text { east }\end{array}$ & $\begin{array}{c}\text { Model } \\
\text { north }\end{array}$ & $\begin{array}{c}\text { Model } \\
\text { depth }\end{array}$ & $\begin{array}{c}\text { Strike } \\
\text { direct }\end{array}$ & $\begin{array}{c}\text { Dip } \\
\text { direct }\end{array}$ & $\begin{array}{c}\text { TRUE } \\
\text { dip }\end{array}$ & Comments \\
\hline & & 2.17 & 0.77 & -2.03 & & & subhor & base moderate fracture \\
\hline & & 2.12 & 0.79 & -2.03 & & & & pre-test borehole \\
\hline
\end{tabular}

